Stirring and Mixing

1999 Summer Study Program in Geophysical Fluid Dynamics

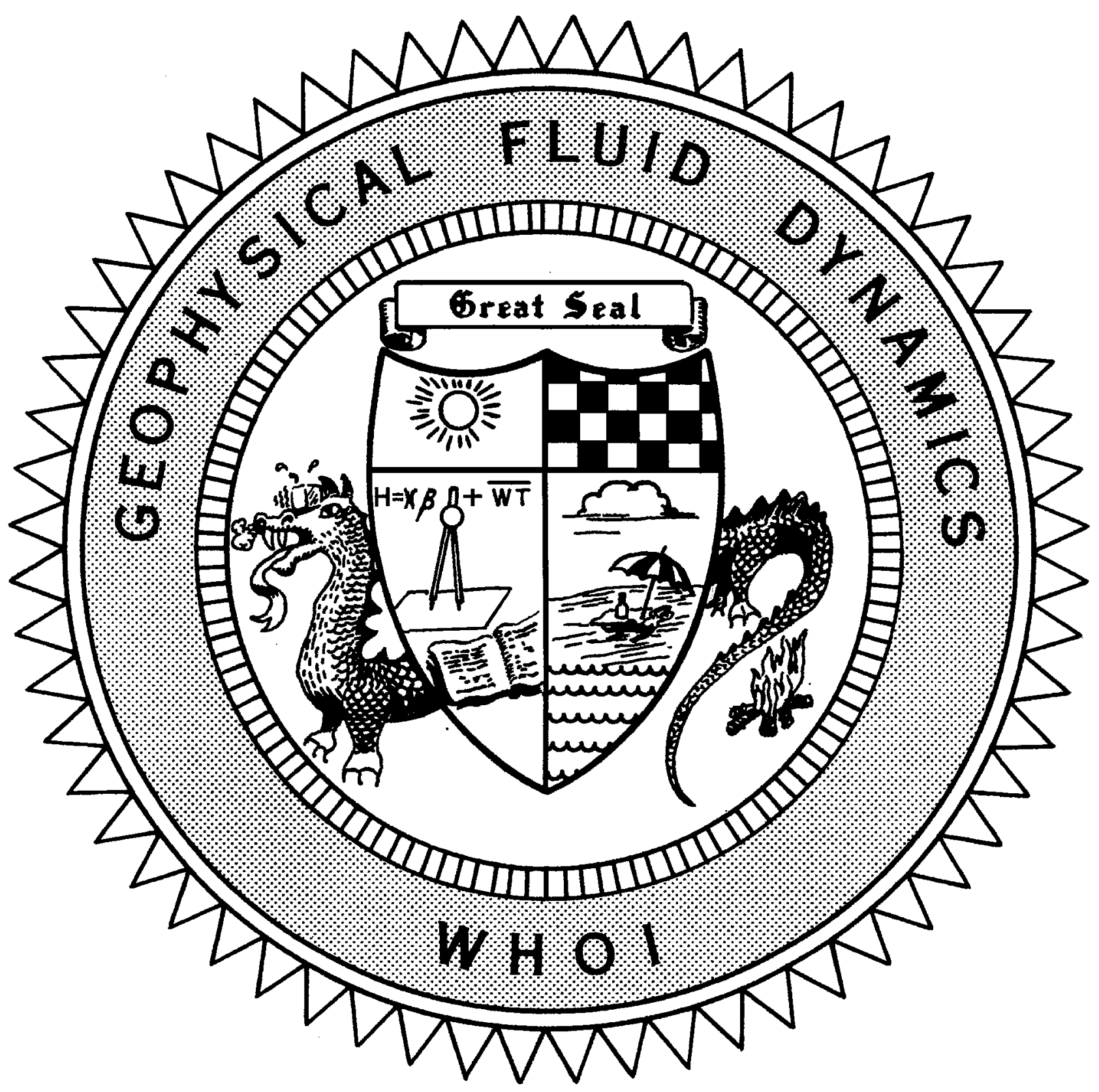

Course Lectures

Fellows Project Reports 


\title{
Stirring and Mixing: 1999 Program of Summer Study in Geophysical Fluid Dynamics
}

\author{
by \\ Neil J. Balmforth, Director \\ William R. Young, Principal Lecturer \\ Janet Fields, Administrator \\ Jean-Luc Thiffeault and Claudia Pasquero, Editors
}

July 2000

\section{Technical Report}

Funding was provided by the National Science Foundation under Grant No. OCE-9810647 and the Office of Naval Research under Grant No. N00014-97-1-0934.

\section{Reproduction in whole or in part is permitted for any purpose of the \\ United States Government. This report should be cited as \\ Woods Hole Oceanog. Inst. Tech. Rept., WHOI-2000-07.}

\begin{abstract}
Approved for public release; distribution unlimited.
\end{abstract}
Approved for Distribution:

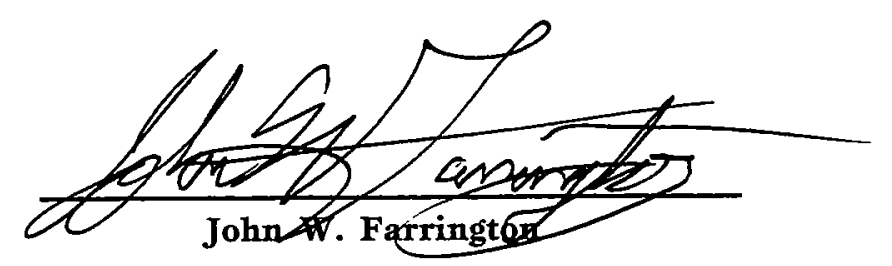

Associate Director for Education

Dean of Graduate Studies 



\section{PREFACE}

Mixing and Stirring took place at the 1999 Geophysical Fluid Dynamics Summer Study Program. William R. Young (Scripps Institution of Oceanography) gave the principal lectures, with Ray Pierre-Humbert (the University of Chicago) adding two special lectures (only one was scheduled; the second was given at the request of the audience). These lectures served us an introduction to the general physical principles of the subject and its many applications in a large number of fields, and provided us with essential mathematical technology.

As usual, we had a variety of seminars throughout the weeks following the lectures, covering the many subjects in which mixing and stirring plays a role. Week 4, organized by Jim Ledwell (Woods Hole Oceanographic Institution) with great success, focussed the attention of the participants on the oceanic problem. Several visitors came to participate solely in this week, and we saw many familiar faces from past summers.

This year was also the \$40th birthday of the Program. In celebration, the Oceanographic Institution graciously held a picnic for all the participants, for the people at Woods Hole connected to the Program, and for any fellows from previous summers that were able to visit for an afternoon. George Veronis gave a memorable speech with a historical perspective. The picnic ended with a customary sight - a GFD softball game - and the afternoon, thanks to W.H.O.I., was a very pleasant experience.

This year's fellows proved to be a group that meshed together especially well (including group triathlons each morning for the hardier fellows). Their academic accomplishments can be viewed elsewhere in this volume. One notable feature of their efforts is that they are all, pretty uniformly, commendable projects; sometimes, it has to be admitted that the program doesn't work for everyone, but this year, I think it worked as well as it could. Consequently, I think this summer was notably successful.

Special thanks go to Bill Young for his tireless efforts in preparing and giving the principal lectures, and for advising so many of the fellows. This summer could not have been so successful without Bill. Also, Eric Chassignet and Glenn Flierl spent many selfless hours with the computers, and Jack Whitehead dealt magnificently with many administrative matters throughout the whole year preceding the Program. Jean-Luc Thiffeault and Claudia Pasquero must be thanked for their important contributions to creating this volume.

And last, but by no means least, I thank W.H.O.I. Education, who continue to provide a perfect atmosphere in which to run the program. I specially thank Marcey Simon for all her efforts to organize the program, and our two staff assistants, Veta Green and Janet Fields, who stepped in to replace the veteran, Lee Campbell, when she moved off to other enterprises.

N. J. Balmforth

Director 


\section{TABLE OF CONTENTS}

Page

PREFACE

CONTENTS

.ii

II

PARTICIPANTS iv

III

LECTURE SCHEDULE ix

IV

\section{PRINCIPAL LECTURES - Stirring and Mixing}

Lectures One Through Four Presented by William R. Young, Scripps Institution of Oceanography, University of California, San Diego Lecture Six Presented by Raymond T. Pierrehumbert, University of Chicago

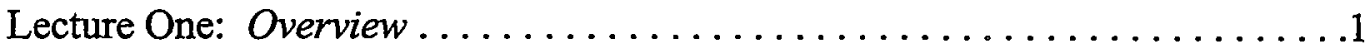

Lecture Two: Duffusion ............................. 20

Lecture Three: Stretching .......................... 42

Lecture Four: Anomalous Diffusion .......................... 63

Lecture Six: The Batchelor Spectrum and Tracer Cascade............90

\section{FELLOW'S LECTURES}

Fellow's Report One:

On Stratified Kolmogorov Flow

Yuan-Nan Young, University of Chicago. . . . . . . . . . . . . . . . 97

Fellow's Report Two:

What Goes Around Comes Around: A Bug's Life

Jennifer Curtis, University of Chicago.

Fellow's Report Three:

Coupled Nonlinear Oscillators

Roberto Sassi, Politecnico di Milano, ITALY.

Fellow's Report Four:

Effect of a Simple Storm on a Simple Ocean

Jeffrey Moehlis, University of California at Berkeley 
Fellow's Report Five:

Stirring \& Mixing by Vortical Mode

Jennifer MacKinnon, University of Washington. . . . . . . . . . . . . . . 191

Fellow's Report Six:

Stationary Vortices in a Keplerian Shear Flow

Pascale Garaud, University of Cambridge, UNITED KINGDOM. . . . . . . . .215

Fellow's Report Seven:

Some Analysis of a Two-Dimensional Double Diffusion Experiment

David Osmond, The Australian National University, AUSTRALIA . . . . . . . 240

Fellow's Report Eight:

Scalar Dispersion in a Two-Dimensional Random Flow Field

Meredith Metzger, University of Utah. . . . . . . . . . . . . . . . 257

Fellow's Report Nine:

The Temperature - Salinity Relation in the Mixed Layer

Raffaele Ferrari, Scripps Inst. of Oceanography,

University of California, San Diego. . . . . . . . . . . . . . . . . . . . 279 


\section{GFD FELLOWS, STAFF AND VISITORS}

\section{Fellows}

Jennifer E. Curtis

Raffaele Ferrari

Pascale Garaud

Jennifer A. MacKinnon

Meredith M. Metzger

Jeffrey M. Moehlis

David I. Osmond

Roberto Sassi

Yuan-nan Young
University of Chicago

Scripps Inst. of Oceanography, Univ. of Calif., San Diego

University of Cambridge, UK

University of Washington

University of Utah

University of California at Berkeley

The Australian National University, AUSTRALIA

Politecnico di Milano, ITALY

University of Chicago

\section{Staff and Visitors}

\author{
Alistair J. Adcroft \\ James L. Anderson \\ Hassan Aref \\ Neil J. Balmforth \\ Andrew J. Bernoff \\ Emmanuel Boss \\ John Bush \\ Paola Cessi \\ Claudia Cenedese \\ Eric P. Chassignet \\ Misha Chertkov \\ Predrag Cvitanovic \\ Sarah L. Dance \\ Charles Doering \\ Glenn Flierl \\ Rupert Ford \\ Christopher Garrett \\ Sarah Gille \\ Anand Gnanadesikan \\ Jost-Diedrich Graf von Hardenberg \\ Steve Griffies \\ Alonso Hernandez-Guerra \\ Louis N. Howard \\ Yongyun $\mathrm{Hu}$ \\ Rui Xin Huang \\ Eric Itsweire \\ Joseph B. Keller \\ Eric Kunze \\ Joseph LaCasce \\ Norman R. Lebovitz \\ James Ledwell \\ Sonya Legg
}

Massachusetts Institute of Technology

Stevens Institute of Technology

University of Illinois, Urbana-Champaign

Scripps Inst. of Oceanography, Univ. of Calif., San Diego

Harvey Mudd College

Oregon State University

Massachusetts Institute of Technology

Scripps Inst. of Oceanography, Univ. of Calif., San Diego

Woods Hole Oceanographic Institution

University of Miami

Los Alamos National Laboratory, DENMARK

Northwestern University

Brown University

University of Michigan

Massachusetts Institute of Technology

Imperial College, UK

University of Victoria, CANADA

University of California, Irvine

Princeton University

Istituto di Cosmogeofisica, ITALY

Princeton University

Universidad de Las Palmas, de Gran Canaria, SPAIN

Florida State University

University of Chicago

Woods Hole Oceanographic Institution

National Science Foundation

Stanford University

University of Washington

Woods Hole Oceanographic Institution

University of Chicago

Woods Hole Oceanographic Institution

Woods Hole Oceanographic Institution 
Stefan G. Llewellyn Smith

Manuel Lopez

Willem V.R. Malkus

Trevor McDougall

Richard L. Mc Laughlin

Philip J. Morrison

Bruce R. Morton

Walter Munk

Joseph Pedlosky

Francesco Paparella

Jeffrey D. Parsons

Claudia Pasquero

Raymond T. Pierrehumbert

Kurt Polzin

Antonello Provenzale

Anthony J. Roberts

Claes G. Rooth

Richard L. Salmon

Roger Samelson

Raymond W. Schmitt

Edwin Schneider

Vitalli Sheremet

Edward A. Spiegel

Melvin Stern

Esteban Tabak

Amit Tandon

Louis Tao

Jean-Luc Thiffeault

John Toole

J. Stewart Turner

Eli Tziperman

Geoffrey K. Vallis

George Veronis

Martin Visbeck

Judith Wells

John Wettlaufer

Jack Whitehead

Jonathan Wylie

Huijun Yang

William R. Young
University of Cambridge, UK

CICESE, MEXICO

Massachusetts Institute of Technology

CSIRO Div. of Marine Research, AUSTRALIA

University of North Carolina, Chapel Hill

University of Texas at Austin

Fitzroy North, AUSTRALIA

Scripps Inst. of Oceanography, Univ. of Calif., San Diego

Woods Hole Oceanographic Institution

Woods Hole Oceanographic Institution

Massachusetts Institute of Technology

Istituto di Cosmogeofisica/CNR, ITALY

University of Chicago

Woods Hole Oceanographic Institution

Istituto di Cosmogeofisica/CNR, ITALY

University of Southern Queensland, AUSTRALIA

University of Miami

Scripps Inst. of Oceanography, Univ. of Calif., San Diego

Oregon State University

Woods Hole Oceanographic Institution

Center for Ocean-Land Atmospheric Studies

Woods Hole Oceanographic Institution

Columbia University

Florida State University

New York University

University of California, Santa Cruz

Columbia University

Columbia University

Woods Hole Oceanographic Institution

Australian National University, AUSTRALIA

Weizmann Institute of Science, ISRAEL

Princeton University,

Yale University

Lamont-Doherty Earth Observatory, Columbia University

Woods Hole Oceanographic Institution

University of Washington

Woods Hole Oceanographic Institution

Woods Hole Oceanographic Institution

University of South Florida

Scripps Inst. of Oceanography, Univ. of Calif., San Diego 

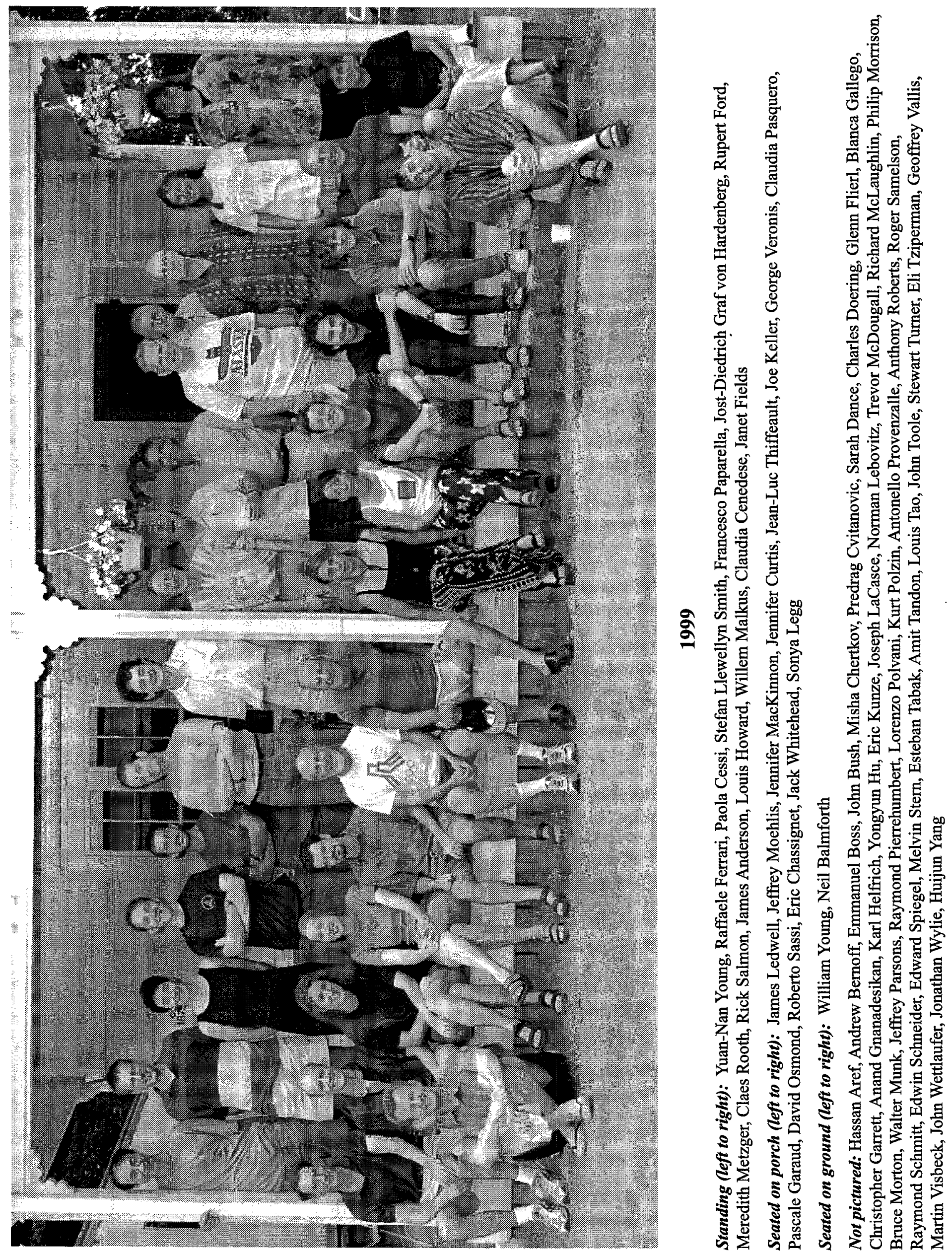


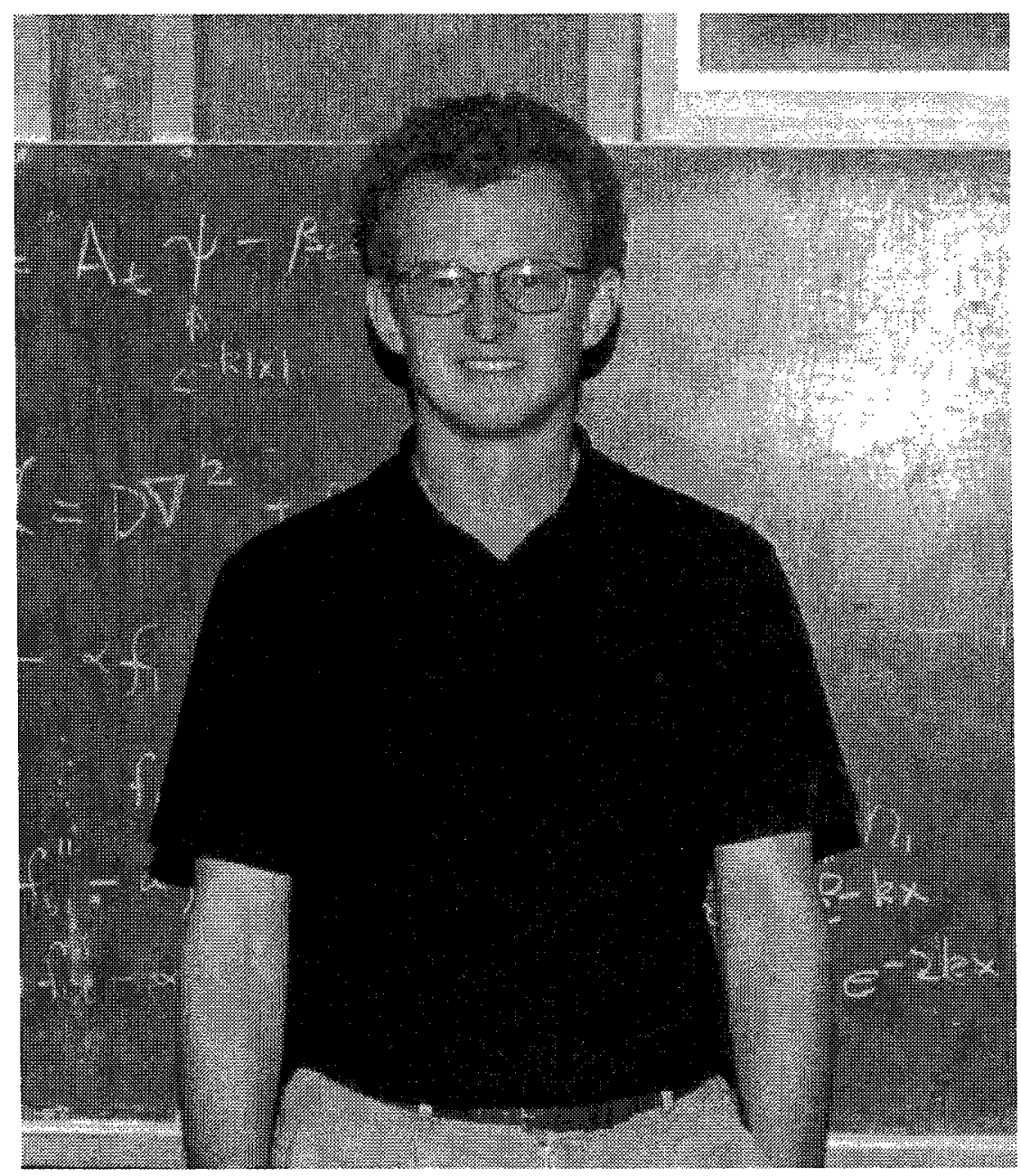

1999 GFD Principal Lecturer

William Young 


\section{GFD 1999 Lecture Schedule}

\section{Week of June $21-25,1999$}

Monday, June 21 10:00 am

Tuesday, June 22 10:00 am

Wednesday, June 23 10:00 am

Thursday, June 24 10:00 am

Friday, June 25 10:30 am 2:30 pm
William R Young, Scripps Institution of Oceanography, University of California, San Diego Eddy Diffusivity - Single Particle Statistics

William R Young

Lyapunov Exponents - Two-Particle Statistics

William R Young

Shear Dispersion and Density Driven Flows

William R Young

Correlation Functions and Type I Anomalous Diffusion

Bruce Morton, Fitzroy North, AUSTRALIA

Jets Deflected in Cross-Flow as Event Mixing

Project Discussions

Week of June 28 - July 2, 1999

Monday, June 28

10:00 am

2:30 pm

Tuesday, June 29

10:00 am

Wednesday, June 30

10:00 am
Ray Pierre-Humbert, University of Chicago

The Batchelor Spectrum and Tracer Cascades

Project Discussions

William R Young

Gradient Expulsion and Type II Anomalous Diffusion

William R Young

The Multi-Scale Method I: Passive Scalars 
Thursday, July 1

10:00 am William $R$ Young

The Multi-Scale Method II: Vorticity

Friday, July 2

10:00 am William R Young

Two-Dimensional Turbulence and Vortices

Week of July $6-J u l y, 9,1999$

Tuesday, July 6

10:30 am

Anand Gnanadesikan, Princeton University

Mixing and Stirring and the Overturning Circulation

$1: 30 \mathrm{pm}$

Yongyun $\mathrm{Hu}$, University of Chicago

Looking for the Universal Statistics of a Passive Tracer in the

Lower Stratosphere: Probability Distribution Functions of Tracer

Concentration, Gradients, and Increments

Wednesday, July 7

10:30 am

Hassan Aref, University of Illinois, Urbana-Champaign

Three-Vortex Motion and Two-Dimensional Turbulence

Thursday, July 8

10:30 am

Glenn Flierl, Massachusetts Institute of Technology

Mesoscale Turbulence and Ocean Biology

2:00 pm

Edwin Schneider, Center for Ocean-Land Atmospheric Studies A Dissipation Integral with Application to Ocean Diffusivities and Structure

Friday, July 9

10:30 am

Amit Tandon, University of California, Santa Cruz

The Role of Mixed Layer Entrainment in Water Mass Formation

Week of July 12 -July 16,1999 - Mini Symposium on Ocean Mixing

( All talks held in Redfeld Anditortum during the Mini Symposium)

Monday, July 12

10:00 am

John Toole, Woods Hole Oceanographic Institution

Mixing in the Ocean Interior

2:00 pm

James Ledwell, Woods Hole Oceanographic Institution

Tracer Studies of Ocean Mixing

Tuesday, July 13

9:30 am

Walter Munk, Scripps Institution of Oceanography,

University of California at San Diego

The Moon and Mixing 
3:00 pm

Wednesday, July 14

10:00 am

2:00 pm

Thursday, July 15

10:30 am

2:00 pm

Friday, July 16

$10: 30 \mathrm{am}$

2:00 pm
Trevor McDougall, CISRO, AUSTRALIA

Physical Oceanography Department Seminar,

Parameterizing the Effects of Eddies and of limited Horizontal

Resolution in Ocean GCMs: the

Horizontal and Temporal-Residual-Mean Circulation

Chris Garrett, University of Victoria, CANADA

Marginal Mixing Ideas

Kurt Polzin, Woods Hole Oceanographic Institution

A Rough Recipe for Mixing

Eric Kunze, University of Washington

Evidence for Potential Vorticity Finestructure and its Role in Horizontal Mixing

Ray Schmitt, Woods Hole Oceanographic Institution Secrets of Double Diffusion

J Stewart Turner, Australian National University, AUSTRALIA

The Neglect of Two Dimensional Double-Diffusive Processes in Studies of Ocean Circulation

Trevor McDougall, CSIRO Marine Research, AUSTRALIA

Consequences of the Nonlinear Nature of the Equation of State

\section{Week of July 19 - July 23,1999}

Monday, July 19

10:30 am

Tuesday, July 20

$10: 30$ am

Wednesday, July 21

10:30 am

Thursday, July 22

10:30 am
Joseph B Keller, Stanford University

Flow in Random Porous Media

Eric Chassignet, University of Miami

Can we learn from OGCMs?

Andrew J. Bernoff, Harvey Mudd College

The Interactions of Shear and Diffusion in the Mixing of Passive Scalars and Vorticity

Sonya Legg, Woods Hole Oceanographic Institution Temperature, Salinity and Spice in Convection 


\section{2:00 pm}

Friday, July 23

10:30 am

$2: 30$ pm
Jost-Diedrich Graf von Hardenberg, Istituto di Cosmogeofisica, ITALY Vortex Merging in QG Flows

A Fedorov Princeton University Nonlinear Gravity-Capillary Waves with Surface Forcing and Viscous Dissipation: A Theoretical Model Based on a Viscous Boundary Layer Approximation

Willem Malkus, Massachusetts Institute of Technology Boundary Reynolds Number for Turbulent Plane Couette Flow

Week of July 26 - July 30,1999

Monday, July 26

10:30 am

Tuesday, July 27

10:30 am

Wednesday, July 28

2:00 pm

Thursday, July 29

10:30 am

2:00 pm

Friday, July 30

10:30 am
John Wettlaufer, University of Washington When Oceans Freeze

Emmanuel Boss, Oregon State University

Lagrangian Dynamics and Tracer Evolution Near an Unstable Jet

Rupert Ford, Imperial College, UNITED KINGDOM

Influence of Topography on a Baroclinic Western Boundary Current

John Bush, Massachusetts Institute of Technology

Vortex Generation by Line Plumes in a Rotating Stratified Flow

Jeffrey D. Parsons, Massachusetts Institute of Technology

Mixing Mechanisms at Gravity Current Fronts

Antonello Provenzale, Istituto di Cosmogeofisica/CNR, ITALY

Impure Mixing

Week of August 2 - August 6, 1999

Monday, August 2

10:30am

Tuesday, August 3

10:30 am
Misha Chertkov, Los Alamos National Laboratory, DENMARK

Lagrangian View of Turbulence

Huijun Yang, University of South Florida

Transport, Mixing and chaos in Large-Scale Geophysical Flows 
Wednesday, August 4

10:30 am

2:30 pm

Thursday, August 5

10:30 am
Charles Doering, University of Michigan

Energy Dissipation in a Shear Layer with Suction: Boundary

Conditions That Suck

Richard McLaughlin, University of North Carolina, Chapel Hill

Scalar Intermittency in Shear Models and the Rigorous

Calculation of a PDF Tail

Joseph Pedlosky, Woods Hole Oceanographic Institution

The Propagation of Rossby Waves through Barriers: The Kelvin

Tunnel

Friday, August 6

10:30 am
Jack Whitehead, Woods Hole Oceanographic Institution

Multiple Equilibria Flows

\section{Week of August 9-August 13,1999}

Monday, August 9

10:30 am

Tuesday, August 10

10:30 am

$1: 30 \mathrm{pm}$

Wednesday, August 11

10:30 am

$4: 30 \mathrm{pm}$

Thursday, August 12

10:30 am

Friday, August 13

10:30 am
Esteban Tabak, New York University

Form Drag Balance Through Shocks and the Antarctic

Circumpolar Current

Predrag Cvitanovic, Northwestern University

Hopf's Last Hope

Claudia Pasquero, Istituto di Cosmogeofísica/CNR, ITALY

Statistical Properties of Velocity Field in Barotropic Turbulence

Norman Lebovitz, University of Chicago

Instabilities of Exact Time Periodic Solutions of the

Incompressible Euler Equation

Edward Spiegel, Columbia University

The Bifurcation of Species

Jean-Luc Thiffeault, Columbia University

Differential Geometry and Chaotic Advection

Louis Howard, Florida State University

Continued Fractions and Hydrodynamic Stability 
Francesco Paparella, Woods Hole Oceanographic Institution Multiple Equilibrium in Fingering Convection?

Week of August 23 - August 26, 1999- Fellows Lectures

Monday, August 23

No Lecture

Tuesday, August 24

11:00 am

Yuan-Nan Young, University of Chicago Stratified Shear Flow

$2: 30 \mathrm{pm}$

Jennifer Curtis, University of Chicago

What Goes Around Comes Around

3:30 pm

Roberto Sassi, Politecnico di Milano, ITALY

Coupled Oscillators

Wednesday, August 25

11:00 am

Jeffrey Moehlis, University of California at Berkeley Effect of a Simple Storm on a Simple Ocean

2:30 pm

Jennifer MacKinnon, University of Washington Vortical Mode Stirring \& Mixing

$3: 30 \mathrm{pm}$

Pascale Garaud, University of Cambridge, UNITED KNNGDOM Stationary Vortices in Accretion Disks

Thursday, August 26

11:00 am

David Osmond, The Australian National University, AUSTRALIA

Some Analysis of a $2 D$ Double Diffusion Experiment

$2: 30 \mathrm{pm}$

Meredith Metzger, University of Utah

A Stirring Story of Waves

$3: 30$ pm

Raffaele Ferrari, Scripps Inst. of Oceanography, University of California, San Diego

The Temperature - Salinity Relation in the Mixed Layer 


\section{Lecture 1: Overview}

\section{Diffusion by discontinous movements}

In 1827 Robert Brown, observed that suspended pollen grains are in an uninterrupted and irregular "swarming" motion. Brown was a botanist and at first he believed that only organic materials exhibited this agitation. But very soon he extended his observations to particles of inorganic material, such as a ground-up fragment of the Sphinx. Through the nineteenth century there was a intermittent discussion concerning the cause of this Brownian motion, and in 1877 Delsaux suggested that the impact of molecules on a macroscopic particle produces observable displacements. In 1905, after nearly a century of debate, Einstein definitively explained this phenomenon $[6,7]$.

\subsection{Einstein's derivation of the diffusion equation}

Our interest here is in Einstein's derivation of the diffusion equation, which is very different from that of Fourier. We consider one-dimensional Brownian motion by projecting the location of the particle onto a straight line which we call the $x$-axis.

Einstein's assumptions are the following: (i) the particles move independently of one another; (ii) we observe particle positions at time intervals $\tau$ which are much greater than the time intervals between molecular collisions. As a result, the motion in one interval is independent of what happened in the previous interval.

In the interval $\tau$ each particle has a random displacement $\Delta$ along the $x$-axis. The probability density function (PDF) of $\Delta$ is $\phi(\Delta)$. This means that if we observe $N \gg 1$ particles for a time $\tau$ then the number of particles which are displaced through a distance which lies between $\Delta$ and $\Delta+\mathrm{d} \Delta$ is

$$
\mathrm{d} N=N \phi(\Delta) \mathrm{d} \Delta .
$$

The PDF $\phi(\Delta)$ does not change from interval to interval, and $\phi$ is symmetric and normalised:

$$
\phi(\Delta)=\phi(-\Delta), \quad \int_{-\infty}^{\infty} \phi(\Delta) \mathrm{d} \Delta=1
$$

The symmetry of $\phi$ implies that the displacements are unbiased. The average of any function of $\Delta$, $f(\Delta)$, is

$$
\bar{f} \equiv \int_{-\infty}^{\infty} f(\Delta) \phi(\Delta) \mathrm{d} \Delta
$$

In particular, $\overline{\Delta^{2}}$ is the mean square displacement in a single step.

If the concentration of particles at time $t$ is denoted by $c(x, t)$, then the evolution of $c$ is determined from the master equation:

$$
c(x, t+\tau)=\int_{-\infty}^{\infty} c(x-\Delta, t) \phi(\Delta) \mathrm{d} \Delta .
$$




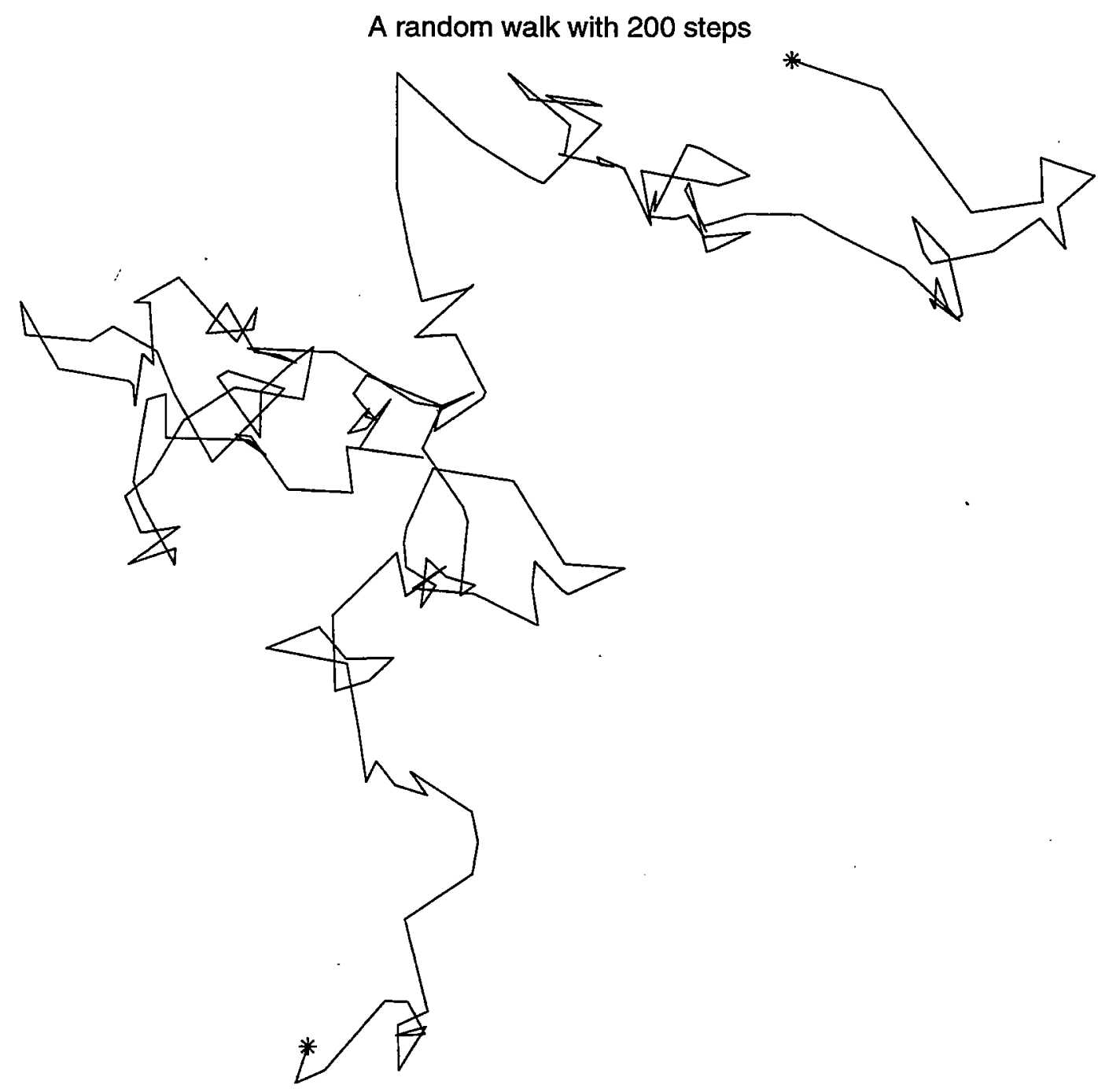

Figure 1: Simulated Brownian motion using MATLAB; the routine rand is used to generate a sequence of 200 random displacements.

The integral over $\Delta$ is a sum over the prior locations at time $t$ of the particles that are at $x$ at time $t+\tau$. Thus, the number of particles in the interval $(x-\Delta, x-\Delta+\mathrm{d} \Delta)$ is $c(x-\Delta, t) \mathrm{d} \Delta$ and $\phi(\Delta)$ is the fraction of these particles which jump from $x-\Delta$ onto $x$.

If the concentration $c(x, t)$ changes on a length scale which is much greater than the root mean square displacement, then we can approximate the integral equation (4) by the diffusion equation. This assumption that $c$ is slowly varying means that it it is sensible to use a Taylor series expansion

$$
c(x, t)+\tau c_{t}(x, t) \approx \int_{-\infty}^{\infty} \phi(\Delta)\left[c(x, t)-\Delta c_{x}(x, t)+\frac{\Delta^{2}}{2} c_{x x}(x, t)\right] \mathrm{d} \Delta .
$$

Next, using (2), we reduce (5) to

$$
c_{t}(x, t) \approx D c_{x x}(x, t), \quad D \equiv \frac{\overline{\Delta^{2}}}{2 \tau}
$$


This is the diffusion equation, and $D$ is the diffusivity.

The greatness of Einstein's contribution to this subject is not the derivation above but rather his formula for the diffusivity of a macroscopic particle

$$
D=\frac{R T}{6 \pi N_{\mathrm{a}} \nu a}
$$

where $R$ is the gas constant, $T$ the absolute temperature, $N_{\mathrm{a}}$ the Avogadro number, $\nu$ the coefficient of viscosity and $a$ the radius of the particle. Coincidentally, (7) was also discovered in 1905 by William Sutherland in Australia. This relation enabled Perrin to determine Avogadro's number by observing Brownian displacements [7].

The diffusion equation is an approximation of the more exact master equation. As we try to design parameterizations of nonlocal mixing processes, in which scale separation assumptions are shaky, we should pay more attention to this history and consider the possibility of using integral equations such as (4). Notice also that if the Taylor expansion in (5) is continued to higher order then one will usually (i.e. for most kernels $\phi$ ) obtain a hyperdiffusive term such as $c_{x x x x}$.

\subsection{The method of moments}

As a check on the derivation of (6), we take a different approach using the method of moments. A moment of the concentration is an integral of the form

$$
\int_{-\infty}^{\infty} x^{n} c(x, t) \mathrm{d} x
$$

The zeroth moment, $n=0$ in (8), is the total number of particles:

$$
N=\int_{-\infty}^{\infty} c(x, t) \mathrm{d} x
$$

The first and second moments can be interpreted as the center of mass and moment of inertia of the concentration profile.

We expect that $N$ is constant, and it is educational to verify this conservation law for both the master equation and the diffusion equation by "taking the zeroth moment". Integrating (4) from $x=-\infty$ to $x=+\infty$, and changing the order of the integrals on the right-hand side gives

$$
N(t+\tau)=\int_{-\infty}^{\infty} \mathrm{d} \Delta \phi(\Delta) \int_{-\infty}^{\infty} \mathrm{d} x c(x-\Delta, t)
$$

Changing variables to $x^{\prime}=x-\Delta$ in the inner integral, and using (2), gives the particle conservation law $N(t+\tau)=N(t)$. The diffusive analog of particle conservation is easily obtained by integrating the diffusion equation (6) from $x=-\infty$ to $x=+\infty$. Provided that $D c_{x}$ vanishes at $x= \pm \infty$ (physically, there is no flux of particles from infinity), one immediately finds that $N_{t}=0$.

Extending the procedure above to higher moments, we can make a comparison between the exact results for $\int x^{n} c \mathrm{~d} x$ and the diffusive approximation of these same integrals. To take the first moment of the diffusion equation, multiply (6) by $x$ and integrate from $x=-\infty$ to $x=+\infty$. Once again, we use integration by parts and assume that terms such as $x c_{x}$ and $c$ vanish as $x \rightarrow \pm \infty$. Thus we find that the center of mass is stationary

$$
\frac{\mathrm{d}}{\mathrm{d} t} \int_{-\infty}^{\infty} x c(x, t) \mathrm{d} x=0 .
$$

The same result can be obtained by taking the first moment of the master equation. The center of mass is stationary because in (2) we assume that the PDF of displacements is symmetric. 
Continuing, we come to the second moment. For the diffusion equation we obtain

$$
\frac{\mathrm{d}}{\mathrm{d} t} \int_{-\infty}^{\infty} x^{2} c \mathrm{~d} x=2 D \int_{-\infty}^{\infty} c \mathrm{~d} x=2 D N
$$

where, as before, the terms which fall outside the integration by parts are zero because of the rapid decay of $c$ as $x \rightarrow \pm \infty$. The student should show that from the master equation

$$
\int_{-\infty}^{\infty} x^{2} c(x, t+\tau) \mathrm{d} x-\int_{-\infty}^{\infty} x^{2} c(x, t) \mathrm{d} x=\int_{-\infty}^{\infty} \Delta^{2} \phi(\Delta) \mathrm{d} \Delta
$$

Recalling the definition of the diffusivity in (6), we see that in the limit $\tau \rightarrow 0$ the difference equation in (13) can be approximated by the differential equation in (12).

The law in (12), that the mean square displacement of a cloud of particles grows linearly with time, is often taken to be the defining characteristic of diffusion. As we will see later, there are dispersive processes which have other power-laws, such as $\int x^{2} c \mathrm{~d} x \propto t^{1 / 2}$. These processes are referred to as "anomalous diffusion".

\section{Diffusion by continuous movements}

\subsection{Lagrangian time series}

In 1922 Taylor [11] analyzed the diffusing power of a velocity field. The basic concept here is that of a Lagrangian time series, such as the $x$-velocity of a tagged fluid particle, $u(t)$, as a function of time. This data is Lagrangian (i.e., following a "float"), not Eulerian (i.e, obtained from a "current meter" fixed in space). The velocity time series might look like figure 2. Clearly there is some regularity: evenly spaced maxima and minima are obvious, and we might guess that there is a wave which is producing oscillatory displacements. At the same time, the velocity is not completely predictable, and there is no obvious law by which we can anticipate all details of the future using observations of the past.

The simplest assumption we can make to analyze the process in figure 2 is that the velocity is statistically stationary. This means that average properties of the velocity, such as the mean square velocity, are not changing with time. In operational terms, the assumption of stationarity means that if we take nonoverlapping and well-separated subsamples of the time series in figure 2 then the statistical properties of the subsamples are identical.

If the time series is long enough we can chop it into $N$ chunks, each of length $T$. We define an ensemble average by considering each of the $N$ chunks as a single realization of a random process. This procedure introduces the additional assumptions that there is a decorrelation time $\tau \ll T$, and that time averages are equivalent to ensemble averages. Thinking of dispersion, Taylor imagined that each chunk was an independent particle, labeled $n=1,2, \ldots, N$, executing continuous movements. "Continuous" in this context means that the velocity of particle $n, u_{n}(t)$, is a relatively smooth function of time, at least in comparison with the jittery motion in figure 1 .

We denote the position of particle $n$ by $x_{n}(t)$, so that if all the particles begin at $x=0$ then

$$
\frac{\mathrm{d} x_{n}}{\mathrm{~d} t}=u_{n}(t), \quad \Longrightarrow \quad x_{n}(t)=\int_{0}^{t} u_{n}\left(t^{\prime}\right) \mathrm{d} t^{\prime}
$$

We use angular brackets $\langle$ to denote the ensemble average. As an example of this notation, the average velocity of the $N$ particles is

$$
\langle u\rangle \equiv \frac{1}{N} \sum_{n=1}^{N} u_{n}(t) .
$$




\section{A time series of Lagrangian velocity}

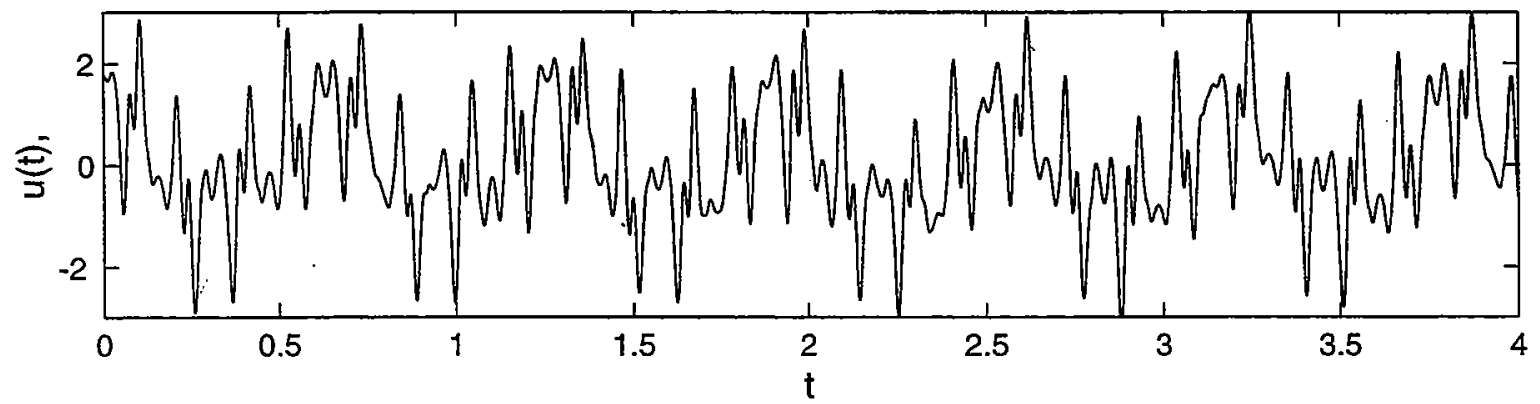

Figure 2: A time series with a spectral peak.

Because of the stationarity assumption, $\langle u\rangle$ is independent of time, and we can refer all displacements relative to the position of the center of mass by writing $x^{\prime}=x-\langle u\rangle t$ and $u_{n}^{\prime}=u_{n}-\langle u\rangle$. To save decorating all our subsequent $x$ 's and $u$ 's with primes we now assume that $\langle u\rangle=0$.

\subsection{Taylor's formula}

The simplest measure of dispersion about the center of mass is the mean square displacement, $\left\langle x^{2}\right\rangle$. We can calculate the rate of change of this quantity by first noting that:

$$
\frac{\mathrm{d} x_{n}^{2}}{\mathrm{~d} t}=2 x_{n} u_{n}, \quad \text { and }(14) \Longrightarrow \quad \frac{\mathrm{d} x_{n}^{2}}{\mathrm{~d} t}=2 \int_{0}^{t} u_{n}(t) u_{n}\left(t^{\prime}\right) \mathrm{d} t^{\prime} .
$$

We now ensemble average (16). Because of stationarity, $\left\langle u(t) u\left(t^{\prime}\right)\right\rangle$ depends only on the time difference $t-t^{\prime}$. Thus, we introduce the correlation function

$$
\mathcal{C}\left(t-t^{\prime}\right) \equiv\left\langle u(t) u\left(t^{\prime}\right)\right\rangle
$$

and, after a change of variables, write the ensemble average of (16) as

$$
\frac{\mathrm{d}\left\langle x^{2}\right\rangle}{\mathrm{d} t}=2 \int_{0}^{t} \mathcal{C}\left(t^{\prime}\right) \mathrm{d} t^{\prime}
$$

Equation (18) is Taylor's formula, which relates the variance in particle displacement $\left\langle x^{2}\right\rangle$ to an integral of the Lagrangian velocity autocorrelation function $\mathcal{C}(t)$.

In the simplest situations the correlation function $\mathcal{C}(t)$ decreases rapidly to zero as $t \rightarrow \infty$ so that the integral in (18) converges. In this case, the dispersion of the ensemble at large times is characterized by a diffusivity $\left\langle x^{2}\right\rangle \sim 2 D t$, where the diffusivity $D$ is related to the correlation function by:

$$
D=\int_{0}^{\infty} \mathcal{C}(t) \mathrm{d} t
$$

In statistical physics, (19) is known as the Green-Kubo formula.

Taylor did not claim that turbulent dispersion was governed by the diffusion equation, (6). We will return to this point later. For the moment notice that (6) is an approximation valid only for sufficently long times that the integral in (18) has converged to the constant $D$. This restriction is related to Einstein's assumption that particle positions are observed at time intervals $\tau$ which are much greater than the decorrelation time. 


\section{Diffusion and anomalous diffusion}

In the previous sections we emphasized that the diffusion equation (6) is only valid on times long compared to the decorrelation time $\tau$, and only if the concentration $c(x, t)$ varies on length scales greater than the width of the density $\phi(\Delta)$. These assumptions of scale separation in both time and space are often not satisfied in real flows. Thus, dispersion experiments over the last ten years have revealed behaviours which are much richer than those suggested by the arguments of Einstein and Taylor. Experiments often show that the growth of variance is described by a power law

$$
\left\langle x^{2}\right\rangle \propto t^{\xi}
$$

In some cases $\xi=1$ (diffusion), but sometimes $\xi \neq 1$, in which case the process is referred to as anomalous diffusion.

\subsection{Rayleigh-Bénard convection}

As an example of hydrodynamic diffusion $(\xi=1)$ and transient subdiffusion $(\xi=2 / 3)$ we mention the experiments of Solomon and Gollub $[9,8]$ on the dispersion of passive scalar (either methylene blue or uranine dye, or small latex spheres) along a chain of Rayleigh-Bénard convection cells (see figure 3). We refer to the passive scalar generically as "tracer".

Following the experimental procedure in figure 3, suppose that all of the tracer is initially released in a single cell. The main question is: how many cells, $N(t)$, have been invaded by tracer at time $t$ ? If this dispersive process is described by diffusion then we expect that $N(t) \propto t^{1 / 2}$. With certain interesting restrictions, this $t^{1 / 2}$-law is the experimental result.

The Rayleigh-Bénard flow can be approximately described using a two-dimensional and incompressible velocity field, $(u, v)$, obtained from the streamfunction

$$
\psi=k^{-1} A \sin [k(x+B \sin \omega t)] W(z), \quad(u, v)=\left(-\psi_{y}, \psi_{x}\right) .
$$

The parameter $A$ controls the amplitude of the flow, $k=2 \pi / \lambda$ is the wavenumber, and $W(z)$ is a function which satisfies the no-slip boundary conditions at $z=0$ and $z=H$. The term $B \sin \omega t$ is a simple model of the lateral oscillation of the roll pattern which results from an instability which occurs when the convection is driven sufficiently strongly. Because the flow in (21) is simple, highly structured and deterministic, this is not an example of turbulent dispersion. Nonetheless, the experimental results can be summarized using the notion of an effective diffusivity.

The Péclet number is

$$
P \equiv \frac{A}{k \kappa},
$$

where $\kappa$ is the molecular diffusivity of the tracer, is a nondimensional parameter which measures the importance of molecular diffusivity to advection. The Péclet number can be considered as the ratio of the time it takes a molecule to orbit around a convection cell to the diffusion time across a cell. In the experiments described here, $P$ is large and molecules make many circuits around a convection cell before Brownian motion jostles them through a distance as large as $k^{-1}$.

There are two cases which must be carefully distinguished:

Steady rolls The rolls are steady if either $\omega=0$ or $B=0$ in (21). In either case, tracer can pass from one roll to a neighbour only via molecular diffusion. But, because molecules are advected through a distance $k^{-1}$, the dye is transported along the array of cells with an effective diffusivity $D_{\text {eff }} \propto \sqrt{A \kappa / k} \gg \kappa$. Because $D_{\text {eff }} \rightarrow 0$ if $\kappa \rightarrow 0$, the transport is limited by molecular diffusion.

Unsteady rolls If $B$ and $\omega$ are both nonzero then advection (rather than molecular diffusion) can take particles through the time-averaged position of the cell boundaries. In this case, there is the possibility of transport unlimited by weak molecular diffusion. 

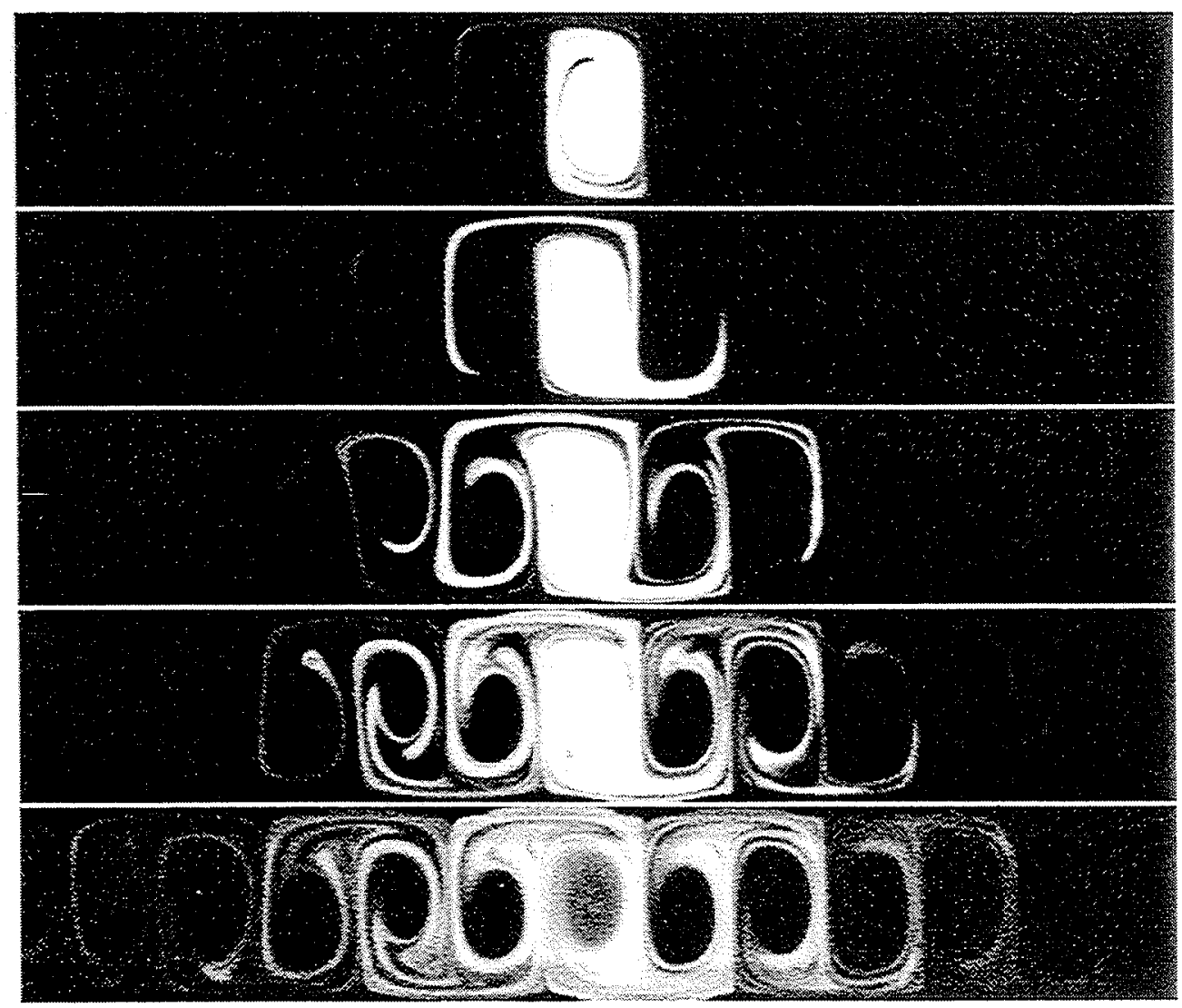

Figure 3: Transport of uranine dye along an array of convection cells with $k B=0.12$; time (from the top): 1, 2, 4 and 10 periods of oscillation. (Figure courtesy of Tom Solomon [10].)

In the unsteady case, Solomon and Gollub show that trajectories of particles computed with the model streamfunction (21) are similar to the patterns observed experimentally. In both the numerics and the experiments, provided that $\omega B \neq 0$, the transport of particles along the array of cells (in the $x$-direction) is due to chaotic advection in the neighbourhood of the roll boundaries. This process is strikingly shown in figure 3 .

A rough summary of the results is that in both the steady and the unsteady cases the dye spreads via a one-dimensional diffusive process, $\xi=1$ in (20), with a local effective diffusivity $D_{\text {eff }}$. The number of invaded cells is $N(t) \propto \sqrt{D_{\text {eff }} t}$. In the unsteady case $D_{\text {eff }}$ is independent of the molecular diffusivity $\kappa$, while in the steady case $D_{\text {eff }} \propto \sqrt{\kappa}$. The effective diffusivity in the unsteady case is enhanced by 1 to 3 orders of magnitude over the effective diffusivity of the steady case (which in turn is much greater than the molecular diffusivity, $\kappa$ ).

The summary in the previous paragraph omits many interesting details. One of the more important caveats is that the effective diffusivity in the steady case only describes the dispersion process at very long times:

$$
N(t) \propto t^{1 / 2} \text { when } t \gg \frac{1}{k^{2} \kappa} .
$$

The time $1 / k^{2} \kappa$ is an estimate of the time taken for molecular diffusion to transport tracer through a distance of order $k^{-1}$, from the edge of a cell to the center ${ }^{1}$. In this long time limit, the evolution

\footnotetext{
${ }^{1}$ We assume that the aspect ratio of the cells is of order unity, $k H=O(1)$.
} 
of the tracer is slower than the intracellular diffusion time $1 / k^{2} \kappa$ and consequently the concentration is uniform within each roll. The concentration changes rapidly at diffusive boundary layers (with thickness proportional to $\kappa^{1 / 2}$ ) which are located at the roll boundaries. The intercellular flux across these boundary layers is responsible for the spread of the tracer from one roll to the next.

The scenario described above does not have time to become established until $t \gg 1 / k^{2} \kappa$. When $t \ll 1 / k^{2} \kappa$ there is still a significant dispersion of tracer through many cells which is described by the anomalous diffusion law

$$
N(t) \propto t^{1 / 3} \quad \text { when } \quad t \ll \frac{1}{k^{2} \kappa} .
$$

The anomalous process above relies on molecular diffusion passing tracer quickly across the cell boundaries before there has been time to reach the center of newly invaded cells $[3,4,13]$. Thus there is a transient regime of subdiffusion which preceeds the final asymptotic diffusive law in (23).

\subsection{Anomalous diffusion in two-dimensional turbulence}

Cardoso et al. [2] conducted an experimental study of dispersion in a quasi-two-dimensional turbulent flow. The experimental apparatus is a shallow pan of fluid, $30 \mathrm{~cm}$ by $30 \mathrm{~cm}$, and $3 \mathrm{~mm}$ deep. The pan is filled with salty water and flow is driven electromagnetically ( $\boldsymbol{E} \times \boldsymbol{B}$ forcing). The forcing is arranged so that the basic flow is a square lattice of $30 \times 30$ counter-rotating vortices. This flow is almost two-dimensional because of the large disparity between the horizontal dimensions $(30 \mathrm{~cm})$ and the vertical dimension $(3 \mathrm{~mm})$.

Although the forcing produces a regular array of vortices, this simple pattern is unstable and a two-dimensional turbulent flow emerges. Visualization of the turbulence, using tracer particles, shows that in the statistically equilibrated state there is a population of vortices whose size is two or three times the injection scale of the forcing. Each vortex emerges, moves, merges with other vortices, and eventually disappears.

Cardoso et al. [2] injected dye into this vortex mess and observed the two-dimensional dispersion of the dye in the horizontal plane. To measure the growth of the dye blob, they defined

$$
R_{m} \equiv \int \sqrt{x^{2}+y^{2}} c(x, y, t) \mathrm{d} x \mathrm{~d} y / \int c(x, y, t) \mathrm{d} x \mathrm{~d} y
$$

and

$$
R_{g} \equiv \sqrt{\int\left(x^{2}+y^{2}\right) c(x, y, t) \mathrm{d} x \mathrm{~d} y / \int c(x, y, t) \mathrm{d} x \mathrm{~d} y} .
$$

The experimental scaling law is

$$
\left(R_{g}, R_{m}\right) \sim t^{0.32 \pm 0.04} .
$$

The exponent $0.32 \neq 1 / 2$ indicates anomalous diffusion - specifically subdiffusion, because the dispersion is slower than diffusion.

By examining typical particle trajectories, such as the one in figure 4, Cardoso et al.explained the subdiffusive growth in terms of an interrupted random walk. Consider a random walker who pauses between steps. The length of the pause, $\tau$, is a random variable; in the experiment of Cardoso et al.the pause is a trapping event in which a molecule is sequestered in the core of a stationary vortex. If the average duration of a pause is well defined then one can simply use Einstein's formula (6) with $\tau$ replaced by the average time between steps. However, if the pausing times are very broadly distributed then the average duration of a pause may be infinite and consequently the dispersion is subdiffusive. We explore this in more details in the next section. 


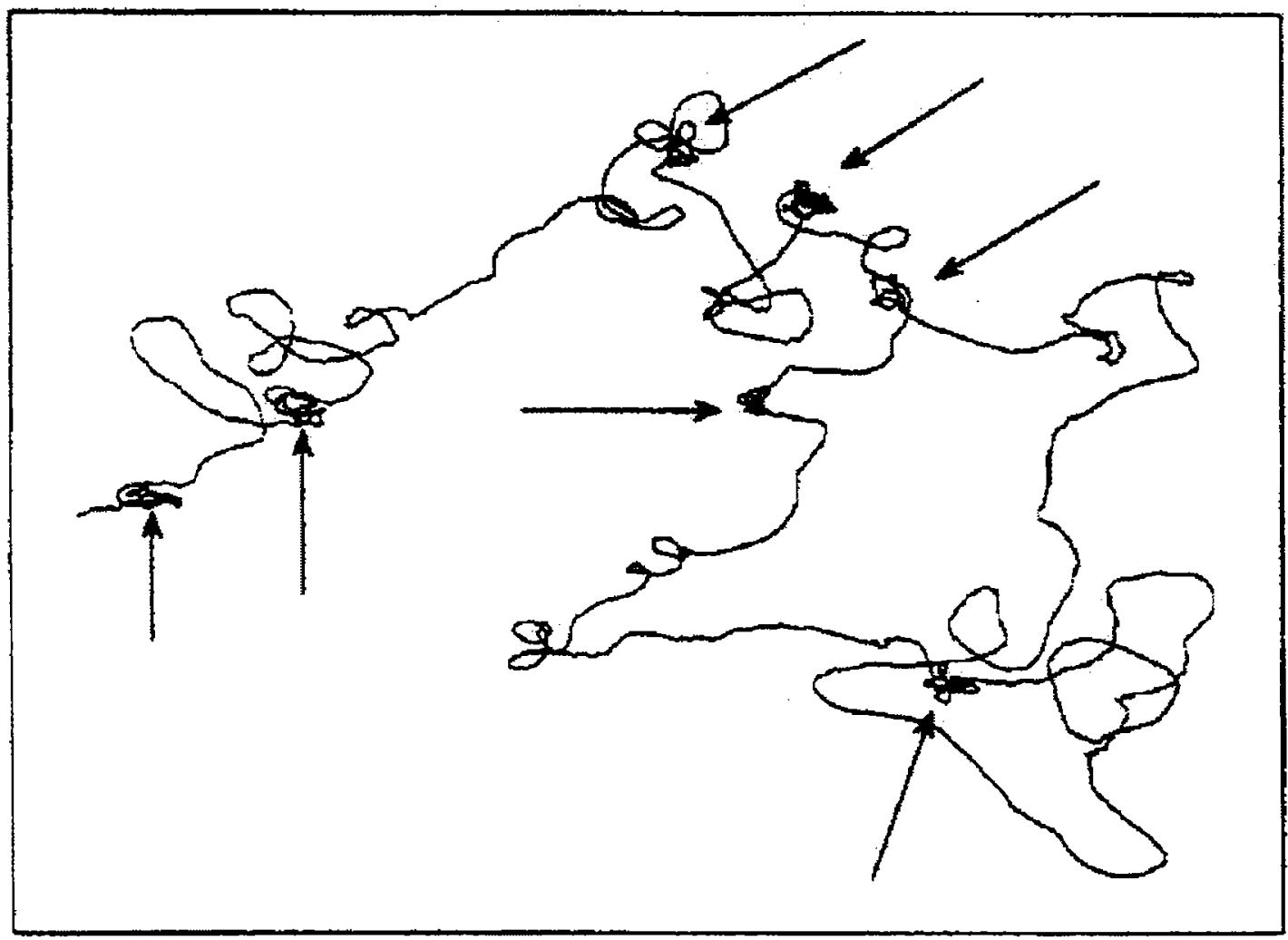

Figure 4: The trajectory of a single particle shows a sequence of long flights interrupted by trapping events in which the particle circles around a vortex. The vortex trapping events are indicated by the arrows. (From Cardoso et al. [2])

\subsection{Random walk with pauses}

Consider a random walk in which the walker pauses for a random time $\tau$ between steps. The various $\tau$ 's have a probability density function $W(\tau)$ (the waiting time PDF). This PDF is normalised,

$$
\int_{0}^{\infty} W(\tau) \mathrm{d} \tau=1,
$$

and the average waiting time spent between steps is

$$
\bar{\tau}=\int_{0}^{\infty} \tau W(\tau) \mathrm{d} \tau
$$

Motivated by the experiments of Cardoso et al., we entertain the notion that $\bar{\tau}$ is infinite because the integral in (29) diverges. For example, suppose that for large $\tau, W(\tau) \sim \tau^{-\mu}$. Then $\bar{\tau}=\infty$ if $\mu \leq 2$.

However, if we only observe a finite number of steps, then we do not sample the entire density $W(\tau)$. Specifically, suppose that after $N$ steps, we have experienced pauses of duration $\tau_{1}, \tau_{2}, \ldots, \tau_{N}$. We want to estimate the likely value of $\tau_{\max }(N) \equiv \max \left\{\tau_{1}, \tau_{2}, \ldots, \tau_{N}\right\}$. The quantity $\tau_{\max }(N)$ is useful because we can argue that the structure of $W(\tau)$ for $\tau>\tau_{\max }(N)$ cannot be significant for the displacement after $N$ steps. 
To determine $\tau_{\max }(N)$, we turn to probability theory. Consider a random variable $\theta$ uniformly distributed in the interval $[0,1]$. That is, the PDF of $\theta$ is $P(\theta)=1$ if $0<\theta<1$ and $P(\theta)=0$ otherwise. Suppose we take $N$ samples, $\theta_{1}, \cdots, \theta_{N}$ and define $\theta_{\min }(N) \equiv \min \left\{\theta_{1}, \cdots, \theta_{N}\right\}$. In this simple case it is plausible that $\theta_{\min } \sim N^{-1}$ as $N \rightarrow \infty$.

Now the trick is to use $\theta$ to represent $\tau$ : we write $\theta=\tau^{p}$, and adjust $p$ so that the power-law tail of $W(\tau) \sim \tau^{-\mu}$ corresponds to the simple structure of $P(\theta)=1$. In fact,

$$
P(\theta)=W(\tau)\left|\frac{\mathrm{d} \tau}{\mathrm{d} \theta}\right|, \quad \Longrightarrow \quad 1 \sim \tau^{1-\mu-p}
$$

or $p=1-\mu$. Because the minimum value of $\theta$ maps to the maximum value of $\tau$, it follows that

$$
\tau_{\max }(N) \sim N^{1 /(\mu-1)} .
$$

Now we return to (29) to estimate the effective average pause time after $N$ pauses:

$$
\bar{\tau}_{\text {eff }}=\int_{0}^{\tau_{\max }} \tau W(\tau) \mathrm{d} \tau \sim \tau_{\max }^{2-\mu} .
$$

It is also plausible that the total time $t$ spent on this random walk is given by

$$
t \sim N \bar{\tau}_{\text {eff }}
$$

Combining (31), (32) and (33) yields the following scaling relationships:

$$
N \sim t^{\mu-1}, \quad \bar{\tau}_{\text {eff }} \sim t^{2-\mu}, \quad \tau_{\max } \sim t .
$$

The final relation is worthy of comment: it implies a form of self-similarity of the random walk.

To conclude, the total displacement of our random walk is proportional to $\sqrt{N}$. But, with the random pauses, the scaling against time has been altered to

$$
\text { RMS displacement } \propto \sqrt{N} \sim t^{(\mu-1) / 2} .
$$

This theory can be used to interpret the experiment of Cardoso et al:: because the RMS displacement grows as $t^{1 / 3}$ it follows that $\mu \approx 5 / 3$. Cardoso et al.successfully tested this prediction by measuring the PDF of trapping times inside vortices.

\section{Stirring and mixing}

\subsection{Coffee and cream}

Appealing to the everyday experience of mixing cream into coffee, Eckart [5] argued that the homogenization of two fluids occurs in three stages. The distinction between the stages is the value of the concentration gradient averaged over the domain.

Initial: there are distinct interfaces separating globules of cream and coffee. Within each globule, the concentration of cream is nearly constant and the concentration gradient is close to zero. There is a very large concentration gradient between regions of coffee and cream. But the interfaces between coffee and cream are small in number and not of great area, so the average gradient in the coffee mug is small.

Stirring: the cream is mechanically swirled and folded, and molecular diffusion is unimportant. During this second stage the concentration gradients increase. 

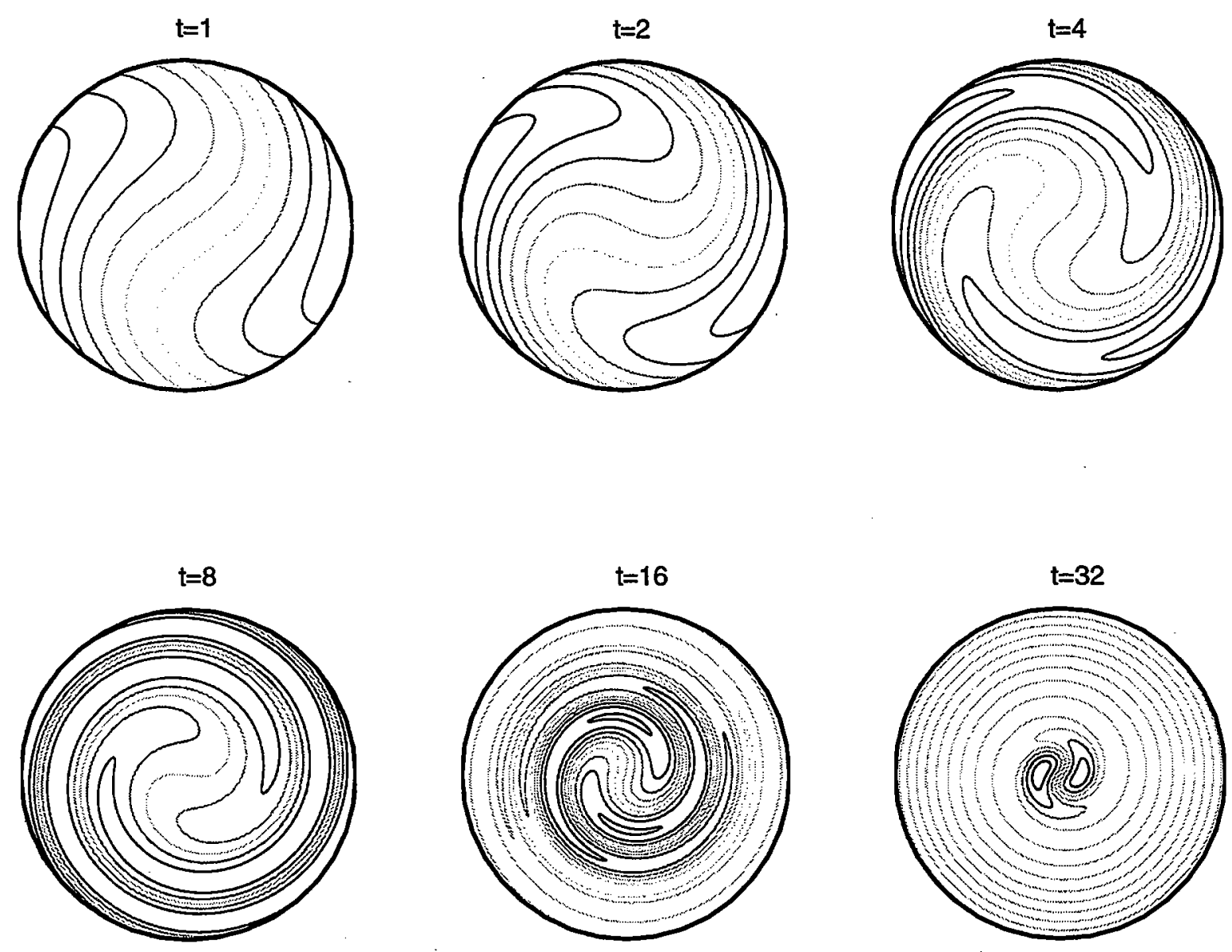

Figure 5: Solution of $c_{t}+\left(1-r^{2}\right) c_{\theta}=\left(8 \times 10^{-4}\right) \nabla^{2} c$. The initial condition is $c(x, y, 0)=x$.

Mixing: the gradients suddenly disappear and the fluid becomes homogeneous; molecular diffusion is responsible for the sudden mixing.

In a chemical reaction, molecules of different species must come into contact for the reaction to occur. Thus, when the species are initially separated, the reaction will not begin until the final mixing stage is reached. In this sense there is an important distinction between coarse-grained homogenization, occuring solely as a result of stirring, and mixing at the molecular scale.

To illustrate these concepts figure 5 shows a solution of the advection diffusion equation

$$
c_{t}+\left(1-r^{2}\right) c_{\theta}=\kappa \nabla^{2} c, \quad c(r, \theta, 0)=r \sin \theta
$$

where $\kappa=8 \times 10^{-4}$. A particle at a distance $r$ from the origin completes a rotation in a time $2 \pi /\left(1-r^{2}\right)$. Thus particles at smaller values of $r$ will overtake particles at larger values of $r$ and so the concentration is twisted into spirals by differential advection (stirring).

The increase in gradient during the stirring phase is evident in the figure. But at approximately $t=16$, mixing starts to dominate, and diffusion rapidly reduces the average gradient. From the initial condition, an estimate of the time it would take unassisted diffusion to homogenize the fluid is $T_{D} \sim 1 / \kappa=1250$. It is only through the initial process of stirring that the concentration gradient is amplified or, alternatively, that the spirals are stretched out so that small diffusion homogenizes the tracer at $t=32 \ll T_{D}$. 


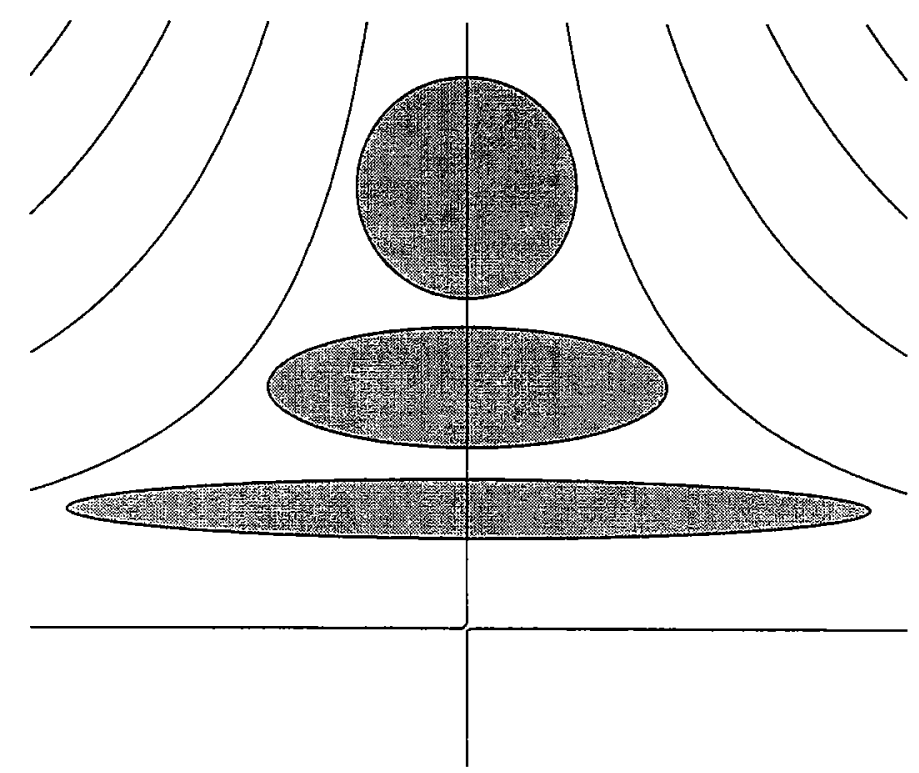

Figure 6: The straining flow described by the streamfunction $\psi=-\alpha x y$. The figure shows how a circular patch of tracer is stretched out along the $x$-axis by the hyperbolic strain. If $\kappa=0$ the major axis of the ellipse grows as $\exp (\alpha t)$ and the minor axis reduces as $\exp (-\alpha t)$ so that the area remains constant.

\subsection{A straining flow}

A simple example of a two-dimensional flow which amplifies concentration gradients is the hyperbolic strain shown in figure 6 . The streamfunction is $\psi=-\alpha x y$ and so the advection diffusion equation is

$$
c_{t}+\alpha x c_{x}-\alpha y c_{y}=\kappa \nabla^{2} c .
$$

Notice the dimensions here: $\alpha^{-1}$ has dimensions "time" and $\kappa$ has dimensions (length) ${ }^{2} /($ time). From these two quantities we can build a combination with the dimensions of (length):

$$
\ell \equiv \sqrt{\frac{\kappa}{\alpha}}
$$

The length $\ell$ will appear prominently in the sequel.

We begin our discussion of hyperbolic strain by obtaining a solution in which $c$ is independent of both $x$ and $t$. In this special case the solution of (37) is

$$
c_{y}=A \exp \left[-\frac{y^{2}}{2 \ell^{2}}\right], \quad c(x, \pm \infty, t)= \pm \sqrt{2 \pi} A \ell .
$$

The concentration profile is the error function shown in figure 7 . The solution shows the steady state balance between advection and diffusion: with $\sqrt{2 \pi} A \ell=1$, the concentration $c$ changes smoothly between $c=+1$ as $y \rightarrow+\infty$ to $c=-1$ as $y \rightarrow-\infty$. The transition occurs in a front of width $\ell$.

We can give an intuitive discussion of how the steady state profile in figure 7 is established as the solution of an initial value problem. Suppose we had started with the initial condition such as $c(x, y, 0)=\operatorname{sgn}(y)$ in which the transition between $c=-1$ and $c=+1$ occurs in a distance much less than $\ell$. Then the discontinuity in $c$ initially diffuses freely, growing like $\sqrt{\kappa t}$. Once the width of the front becomes comparable to $\ell$, that is when

$$
\sqrt{\kappa t} \sim \ell, \quad \Longrightarrow \quad t \sim \alpha^{-1}
$$




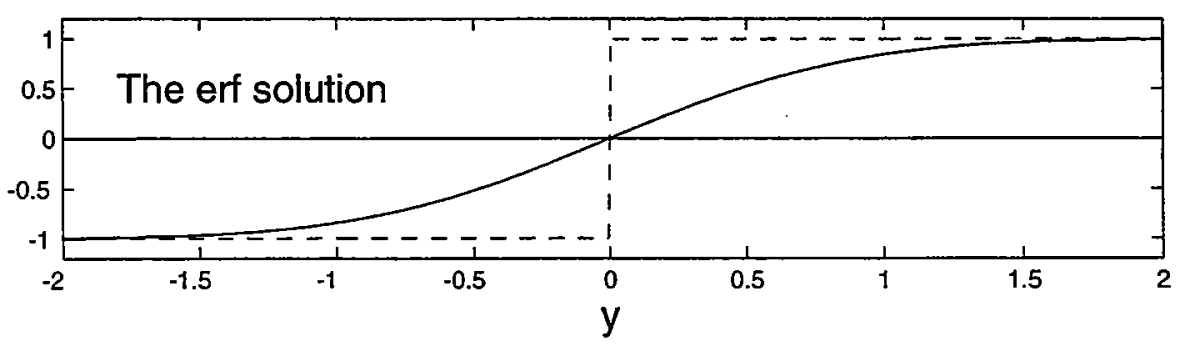

Figure 7: The time independent error function solution to equation (37).

the spread is arrested and the steady state in figure 7 is established.

On the other hand, we can also consider an initial condition in which the transition between $c=1$ and $c=-1$ occurs on a scale $L_{0} \gg \ell$. In this case the front is initially compressed by the hyperbolic strain so that the width is reduced exponentially, $L=L_{0} \exp (-\alpha t)$. Because $L_{0} \gg \ell$ the diffusion is unimportant until the exponential reduction in scale reaches $\ell$. That is,

$$
L_{0} e^{-\alpha t} \sim \ell, \quad \Longrightarrow \quad t \sim \alpha^{-1} \ln \left(L_{0} / \ell\right) .
$$

These considerations illustrate the fundamental importance of $\ell$ as the scale on which advection and diffusion come into balance.

\subsection{Lagrangian coordinates: a simple example}

The hyperbolic strain also provides a painless illustration of some mathematical techniques which can be used in more complicated problems. We begin by considering the solution of (37) with $\kappa=0$. With no diffusion $c$ is tied to fluid particles. The position of a fluid particle is related to its initial position $(a, b)$, by solving the differential equations

$$
(\dot{x}, \dot{y})=\alpha(x,-y), \quad \Longrightarrow \quad(x, y)=\left(e^{\alpha t} a, e^{-\alpha t} b\right)
$$

The solution of (37) can now be obtained by arguing that the particle which is at the point $(x, y)$ at time $t$ began at $(a, b)=(\exp (-\alpha t) x, \exp (\alpha t) y)$ at $t=0$. Because the a particle carries the concentration it follows that the solution of (37) as an initial value problem is

$$
c(x, y, t)=c_{0}[\exp (-\alpha t) x, \exp (\alpha t) y],
$$

where $c_{0}(x, y)$ is the initial condition. The philosophy of this method is that we care where fluid particles come from, but not where they are going to.

The solution above seems to rely crucially on the restriction that $\kappa=0$. But now look what happens if we use the Lagrangian coordinates $(a, b)$ in (42) as new independent variables in (37). As an accounting device, it is comforting to define $\tau=t$ and consider that $\partial_{\tau}$ as the time derivative with $(a, b)$ fixed. Thus the transformation rules are

$$
\left(\partial_{x}, \partial_{y}\right)=\left(\frac{\partial a}{\partial x}, \frac{\partial a}{\partial y}\right) \partial_{a}+\left(\frac{\partial b}{\partial x}, \frac{\partial b}{\partial y}\right) \partial_{b}=\left(e^{-\alpha \tau} \partial_{a}, e^{\alpha \tau} \partial_{b}\right)
$$

and

$$
\partial_{t}=\frac{\partial \tau}{\partial t} \partial_{\tau}+\frac{\partial a}{\partial t} \partial_{a}+\frac{\partial b}{\partial t} \partial_{b}=\partial_{\tau}-\alpha a \partial_{a}+\alpha b \partial_{b}
$$

The punchline is that

$$
\partial_{t}+\alpha x \partial_{x}-\alpha y \partial_{y}=\partial_{\tau}
$$


which shows that the change to a Lagrangian description makes the convective derivative trivial.

Substituting the transformations above into (37) gives:

$$
c_{t}=\kappa e^{-2 \alpha t} c_{a a}+\kappa e^{2 \alpha t} c_{b b} .
$$

Naturally, if $\kappa=0$, we recover our earlier solution in (43). But even if $\kappa \neq 0$ it is often easier to solve (47) than the Eulerian form in (37). For example, Fourier transforming (47), with $\left(\partial_{a}, \partial_{b}\right) \rightarrow \mathrm{i}(p, q)$, gives a simple ordinary differential equation in time.

It is instructive to use the method above to solve (37) with the initial condition

$$
c(x, y, 0)=\delta(x) \delta(y) .
$$

Physically, this is a spot of dye released in a straining flow. When $\alpha t \ll 1$ the spot spreads diffusively, with a diameter which grows as $\sqrt{\kappa t}$. However when $\alpha t \sim 1$ the diameter of the spot becomes comparable to $\ell \equiv \sqrt{\kappa / \alpha}$, and then the spot stops expanding against the compressive direction of the strain. However the spot continues to stretch along the extensive direction. Thus, when $\alpha t>1$, the spot becomes a filament with an equilibrium width of order $\ell$ and an exponentially growing length. These intuitive arguments are supported by the exact solution:

$$
c(x, y, t)=\frac{1}{4 \pi f g} \exp \left[-\frac{x^{2}}{4 f^{2}}-\frac{y^{2}}{4 g^{2}}\right],
$$

where $f(t)$ and $g(t)$ are

$$
f^{2} \equiv \frac{\kappa}{2 \alpha}\left(e^{2 \alpha t}-1\right), \quad g^{2} \equiv \frac{\kappa}{2 \alpha}\left(1-e^{-2 \alpha t}\right)
$$

Notice that the peak concentration ultimately decreases like $e^{-2 \alpha t}$.

\subsection{An example of sudden mixing}

As a final look at the hyperbolic straining How, we note that a solution of (37) is

$$
c(x, y, t)=A(t) \cos \left(k e^{-\alpha t} x\right) \cos \left(k e^{\alpha t} y\right)
$$

where

$$
A(t)=\exp \left[-\ell^{2} k^{2} \sinh 2 \alpha t\right] .
$$

One route to this exact solution is to look for separable solutions of (47), and then transform back to the Eulerian coordinates (e.g., Young, Rhines \& Garrett,1982).

The mean value of the square of the concentration gradient varies with time as:

$$
\{\nabla c \cdot \nabla c\}=\frac{k^{2}}{2} \cosh (2 \alpha t) \exp \left[-2 \ell^{2} k^{2} \sinh (2 \alpha t)\right],
$$

where \{\} denotes an average over a large area. $\{\nabla c \cdot \nabla c\}$ is plotted in figure 8 for various values of $k \ell$. Recalling Eckart's description of stirring as increasing the concentration gradient, and mixing as decreasing the concentration gradient, we can see the transition between the two phases occurs at the peaks of the various curves. If $k \ell \ll 1$, then the time it takes to reach this peak is given by $t_{*}$, where

$$
\alpha t_{*} \sim-\ln (k \ell) .
$$

Once again, this is the time taken for the exponential factor $e^{-\alpha t}$ to reduce initial length of the tracer field, $k^{-1}$, down to the length $\ell$ on which strain and diffusion balance. 


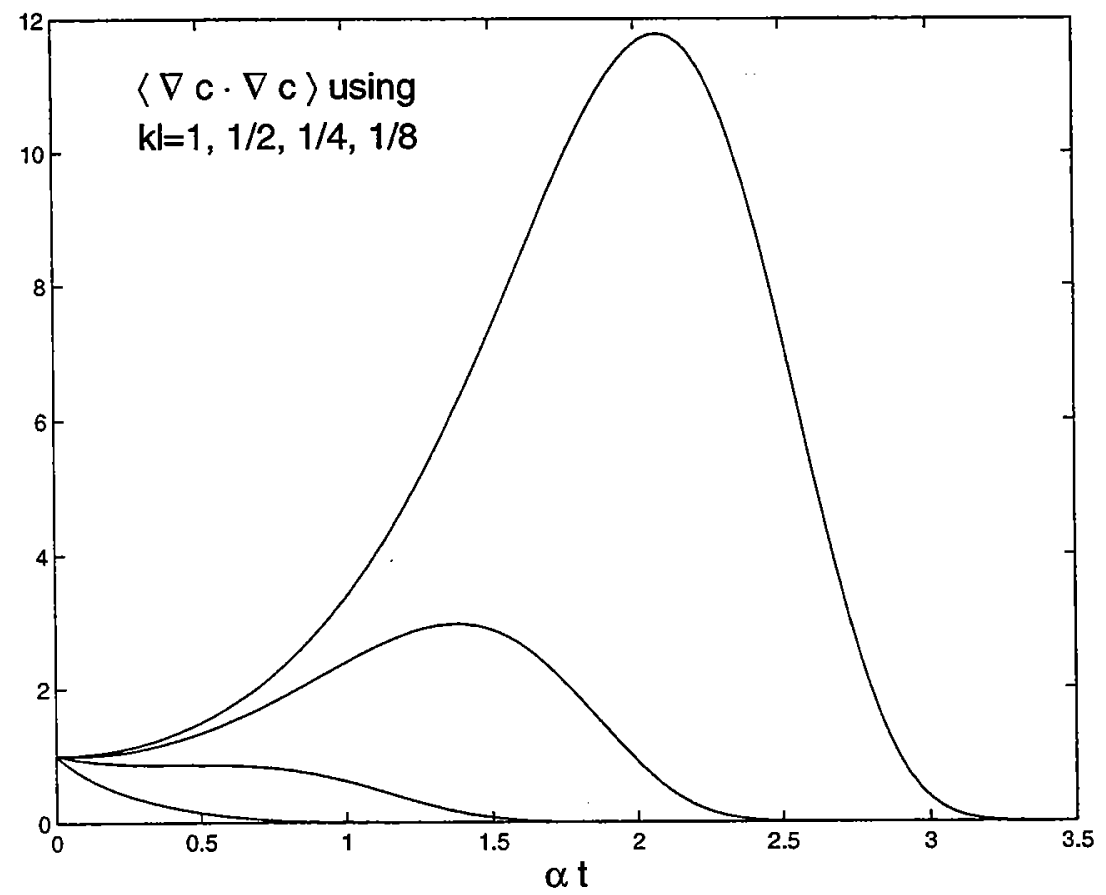

Figure 8: The mean square of the concentration gradient. If $k \ell \ll 1$ then the concentration gradient grows until $t=t_{*}$ in (54) and then decreases precipitiously. If $k \ell \geq 1 / 2$ then diffusion always overpowers strain and the mean square gradient decreases monotonically to zero.

\subsection{A Welander scrapbook}

Stirring was beautifully illustrated in a 1955 paper of Welander's [12]. This paper is notable also because of its discussion of the importance of coarse-grained averages. Figures 9,10 and 11 reproduced from Welander (1955) show that simple velocity fields produce spectacular distortion of passive scalars.

In figures 9,10 and 11 , some dimensions of the scalar blob are stretched out while other dimensions are contracted. Batchelor (1952) [1] argued that in turbulent flows random stretching results in an exponential growth of the separation between two initially adjacent fluid elements. That is, if we consider two material elements separated by a distance $s_{0}$ which is much less than the scale of the velocity field, then Batchelor argues that the separation grows as

$$
s \sim s_{0} e^{\gamma t} .
$$

The time-scale $\gamma^{-1}$ is analogous to $\alpha^{-1}$ in (37), though in figures 10 and 11 the exponential straining is driven by a random and unsteady velocity, rather than the simple hyperbolic field in figure 6 . Note particularly that the exponential law in (55) is valid until the separation $s(t)$ becomes comparable to the length scale over which the velocity varies. 

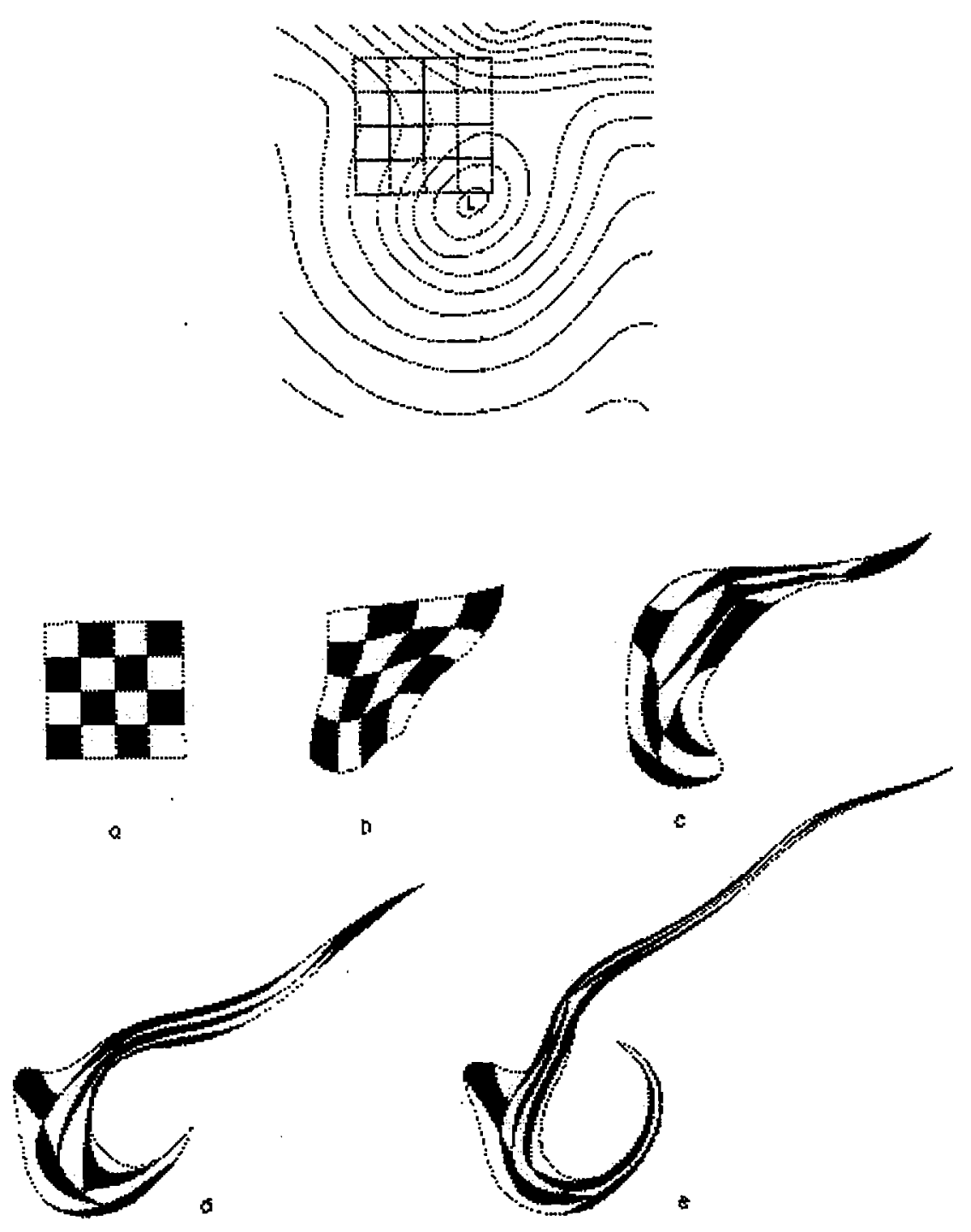

Figure 9: Welander's numerical solution illustrating differential advection by a simple velocity field. A checkerboard pattern is deformed by a quasigeostrophic barotropic solution which models atmospheric flow at the $500 \mathrm{mb}$ level. The initial streamline pattern is shown at the top and the subsequent figures are at 6 hours, 12 hours, 24 hours and 36 hours, respectively. Notice that each square of the checkerboard maintains constant area as it deforms.

\section{References}

[1] G.K. Batchelor. The effect of turbulence on material lines and surfaces. Proc. Roy. Soc. London $A, 213: 349-366,1952$.

[2] O. Cardoso, B. Gluckmann, O. Parcollet, and P. Tabeling. Dispersion in a quasi-two-dimensional turbulent flow: an experimental study. Phys. Fluids, 8(1):209-214, January 1996. 


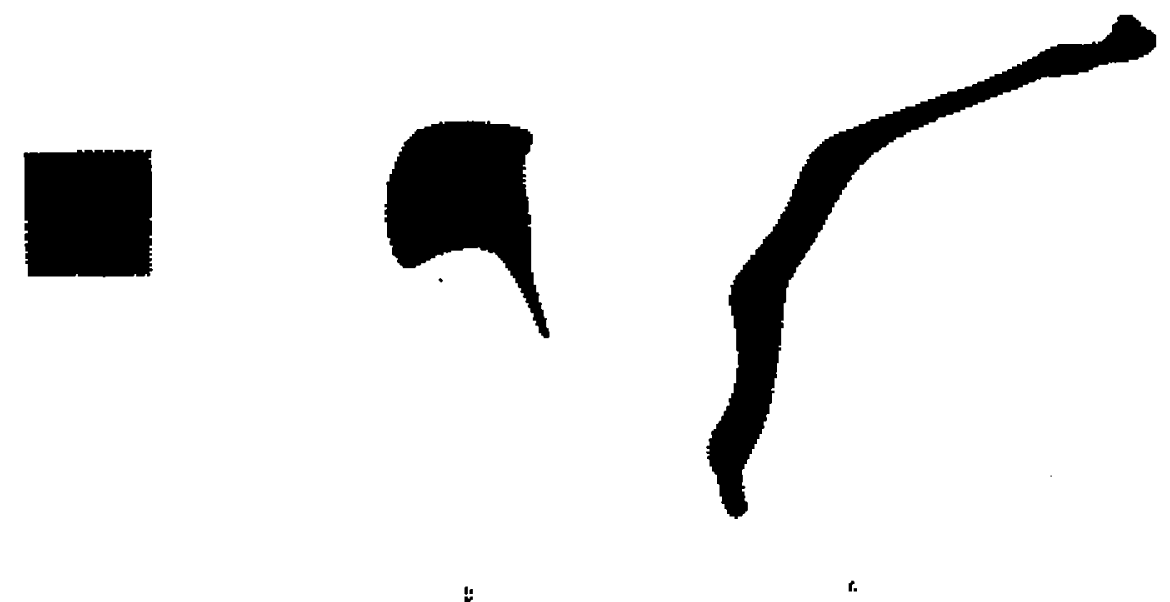

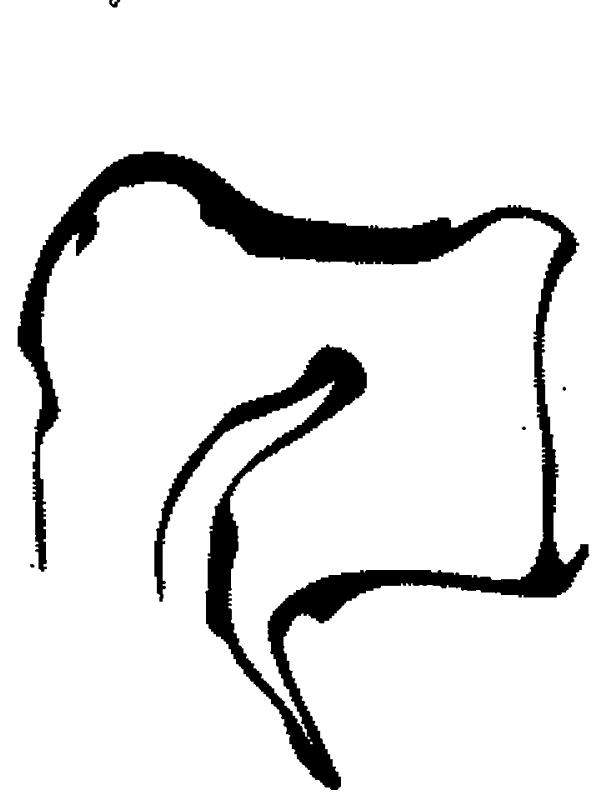

$s$

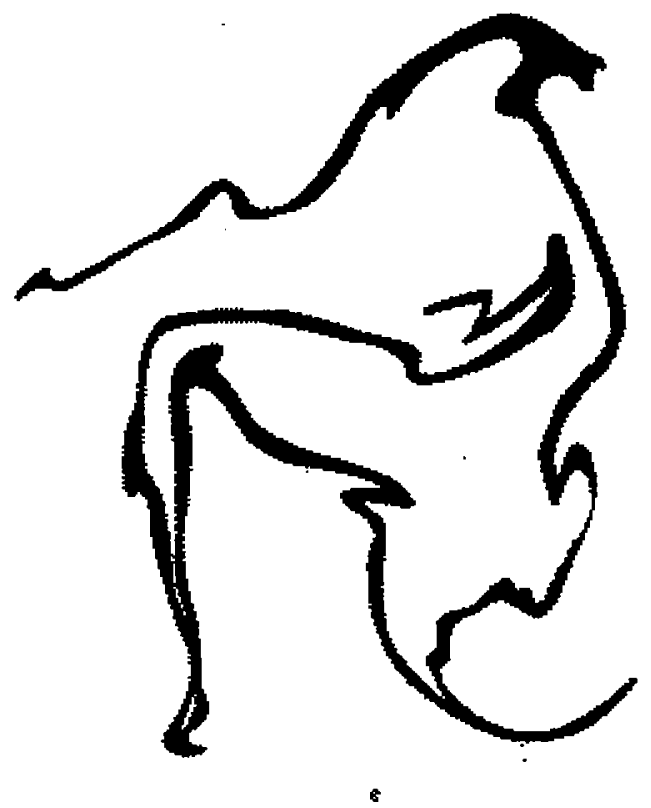

Figure 10: Welander's experimental illustration of the deformation of a small coloured square element of a fluid surface. To suppress three dimensional turbulence, a vessel of water is brought into solid body rotation. A floating film of butanol is divided into square elements by means of a metal grid and one of these square elements is coloured with methyl-red. The solid body rotation is disturbed by stirring the water and the grid is removed.

[3] O. Cardoso and P. Tabeling. Anomalous diffusion in a linear array of vortices. Europhys. Lett., $7(3): 225-230,1988$.

[4] O. Cardoso and P. Tabeling. Anomalous diffusion in a linear system of vortices. Euro. J. Mech. B/Fluids, 8(6):459-470, 1989.

[5] C. Eckart. An analysis of stirring and mixing processes in incompressible fluids. J. Mar. Res., 7:265-275, January 1948. 


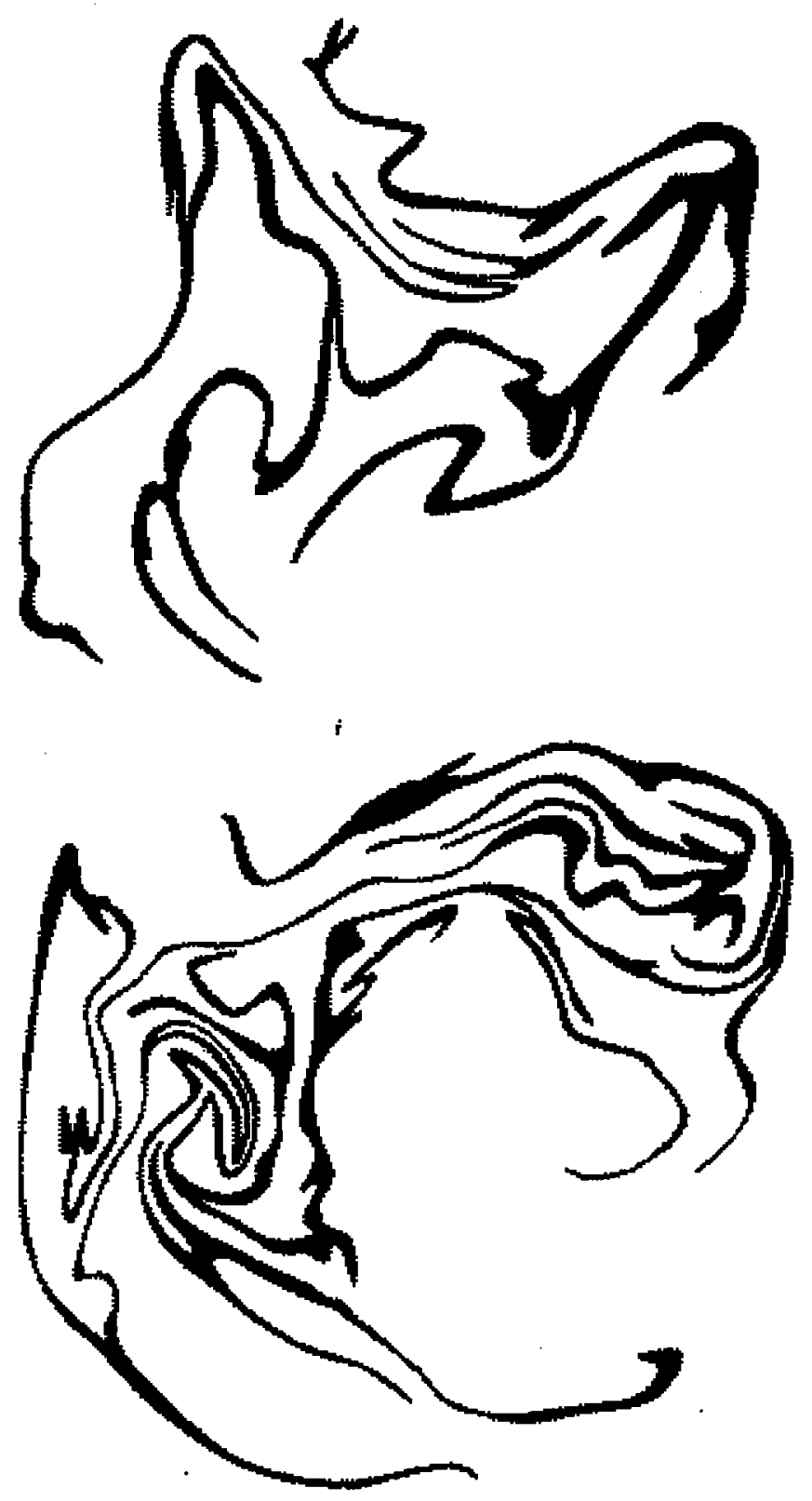

Figure 11: Further deformation of the butanol square in figure 10. According to Batchelor [1], the length of the filaments increases exponentially with $t$.

[6] A. Einstein. Investigations on the theory of the Brownian Movement. Dover, New York, 1956.

[7] A. Pais. 'Subtle is the Lord...'. Oxford University Press, Oxford and New York, 1982.

[8] T.H. Solomon and J.P. Gollub. Chaotic particle transport in time dependent Rayleigh-Bénard convection. Phys. Rev. A, 38:6280, 1988.

[9] T.H. Solomon and J.P. Gollub. Passive transport in steady Rayleigh-Bénard convection. Phys. Fluids A, 31:1372-???, 1988. 
[10] T.H. Solomon, S. Tomas, and J.L. Warner. Chaotic mixing of immiscible impurities in a twodimensional flow. Phys. Fluids A, 10:342-350, 1998.

[11] G.I. Taylor. Diffusion by continuous movements. Proc. London Math. Soc., 20:196-212, 1921.

[12] P. Welander. Studies on the general development of motion in a two-dimensional, ideal fluid. Tellus, 7:141-156, 1955.

[13] W.R. Young, A. Pumir, and Y. Pomeau. Anomalous diffusion of tracer in convection rolls. Phys. Fluids A, 1:462-469, 1989.

[14] W.R. Young, P.B. Rhines, and C. Garrett. Shear-flow dispersion, internal waves and horizontal mixing in the ocean. J. Phys. Ocean., 12:515-527, 1982. 


\section{Lecture 2: Diffusion}

\section{Introduction}

Perhaps you have heard that turbulence is the most difficult problem in fluid mechanics and, according to some, the greatest unsolved problem in physics. One indication of the difficulty is that it is impossible to give a satisfactory definition of a "turbulent flow". But everyone agrees that one property of turbulence is greatly enhanced transport of passive contaminants. For example, relying only on molecular agitation, a dissolved sugar molecule takes years to diffuse across a coffee cup, and on that time-scale the coffee will surely evaporate. With a spoon the coffee drinker can create eddies that transport dissolved sugar throughout the cup in less than one second. This is an example of eddy diffusivity.

Fluid mechanics textbooks often often justify eddy diffusivity by appealing to an analogy between turbulent eddies and molecular diffusion - perhaps this notion originates with G.I. Taylor's 1905 paper entitled "Eddy motion in the atmosphere" [4]. In any event, the molecular analogy, supplemented with some hand-waving, leads to the notion of an eddy diffusivity and for many scientists this is the end of the turbulence problem.

Our goal in this lecture is to explain very explicitly the assumptions behind Taylor's "proof by analogy" and to illustrate the interesting points at which the analogy fails. We will pursue this program by working with some very simple model flows for which analytic results, such as expressions for the eddy diffusivity, are available. As you will soon see, these model flows do not greatly resemble turbulence, but then neither does molecular motion! Our excuse is that soluble examples are always diverting and educational.

\section{The renovating wave model}

\subsection{A recipe for constructing soluble models}

The main problem in analyzing transport is solving the differential equations which describe the motion of particles in even very simple flows. However there is a class of flows for which this task is trivial. These are steady and unidirectional flows, such as $u=\sin y$. A particle which starts at $(a, b)$ at $t=0$ finds itself at $(a+\tau \sin b, b)$ at $t=\tau$. This is dull, but it becomes more interesting if at intervals of $\tau$ we "renovate" the flow by randomly picking a new direction along which the velocity acts. In this way we can construct a sequence of iterated random maps and calculate diffusivities, and other statistical properties, by averaging the exact solution. I learned of this trick from the literature on dynamo theory. The book Stretch, Twist, Fold: the Fast Dynamo is highly recommended for students interested in all aspects of stirring and mixing [1].

\subsection{The renovating wave (RW) model}

As a particular example we now formulate the renovating wave (RW) model. We divide the time axis into intervals

$$
I_{n} \equiv\{t:(n-1) \tau<t<n \tau\}
$$


and in each interval we apply a velocity, $\mathbf{u}=\left(-\psi_{y}, \psi_{x}\right)$, derived from the streamfunction

$$
\psi_{n}(x, y, t)=k^{-1} U \cos \left[k \cos \theta_{n} x+k \sin \theta_{n} y+\varphi_{n}\right],
$$

where $\theta_{n}$ and $\varphi_{n}$ are independent random variables uniformly distributed in the interval $[-\pi, \pi]$. Thus in each $I_{n}$ there is a steady, unidirectional velocity with sinusoidal profile (a single wave). There is sudden and complete loss of all information about the past velocity at $t=n \tau$ because at these instants we "renovate" the velocity by picking new random angles $\theta$ and $\varphi$. (This means that the velocity correlation function, $\mathcal{C}(t)$, is zero if $t>\tau$.)

The renovating wave model can be nondimensionalized by using $k^{-1}$ as a unit of length and and $1 /(U k)$ as a unit of time. With this choice, the model contains a single dimensionless parameter, $\tau_{*} \equiv \tau k U$, which is a measure of the persistence of the motion. Much of the literature on random advection-diffusion uses model velocity fields which are $\delta$-correlated in time. We can recover this limit as a special case by taking $\tau_{*} \rightarrow 0$.

Using dimensionless variables, a particle which is at $\mathbf{x}_{n}=\left(x_{n}, y_{n}\right)$ at $t_{n}=n \tau_{*}$ moves to $\mathbf{x}_{n+1}$ at $t=(n+1) \tau_{*}$, where

$$
\left(x_{n+1}, y_{n+1}\right)=\left(x_{n}, y_{n}\right)+\tau_{*} \sin \left(c_{n} x+s_{n} y+\varphi_{n}\right)\left(s_{n},-c_{n}\right) .
$$

with $s_{n} \equiv \sin \theta_{n}$ and $c_{n} \equiv \cos \theta_{n}$. Thus motion in the renovating wave problem is equivalent to an iterated sequence of random maps.

\subsection{The single-particle diffusivity}

It is very easy to calculate the diffusivity in the RW model (and much more difficult to interpret the answer). The average of a function of the two random angles $\theta$ and $\varphi$ (suppress the subscript $n$ ) is defined by

$$
\langle f\rangle=\oint \frac{\mathrm{d} \varphi}{2 \pi} \oint \frac{\mathrm{d} \theta}{2 \pi} f(\theta, \varphi)
$$

Therefore, using (3),

$$
\left\langle\left(x_{n+1}-x_{n}\right)^{2}\right\rangle=\frac{\tau_{*}^{2}}{4} .
$$

The computation is trivial if the integral over $\varphi$ is evaluated first.

In (5), following our previous discussion based on Einstein's derivation of the diffusion equation, we are computing the statistics of dispersion along the $x$-axis. Because the renovating wave model is isotropic, dispersion in the $y$-direction is identical to that in the $x$-direction.

Because all of the waves are independent and identically distributed it follows that after $n$ renovation cycles

$$
\left\langle\left(x_{n}-x_{0}\right)^{2}\right\rangle=n \frac{\tau_{*}^{2}}{4}
$$

But $t=n \tau_{*}$, and $\left\langle\left(x_{n}-x_{0}\right)^{2}\right\rangle=2 D t$, so that using dimensionless variables the diffusivity is

$$
D=\frac{\tau_{*}}{8} \text {. }
$$

Sometimes $D$ is referred to as the single-particle diffusivity. "Single-particle" emphasizes that $D$ strictly applies only to the RMS displacement of a particle from its initial position; $D$ contains no information concerning the deformation of a patch of tracer, nor of any other quantity involving correlated motion. Thus, using dimensional variables, the diffusivity in (7) is $D=U^{2} \tau / 8$, which is independent of $k$. Because $D$ is independent of the scale of the wave, even a spatially uniform, but random-in-time velocity (the case $k=0$ ), has a single-particle diffusivity. 

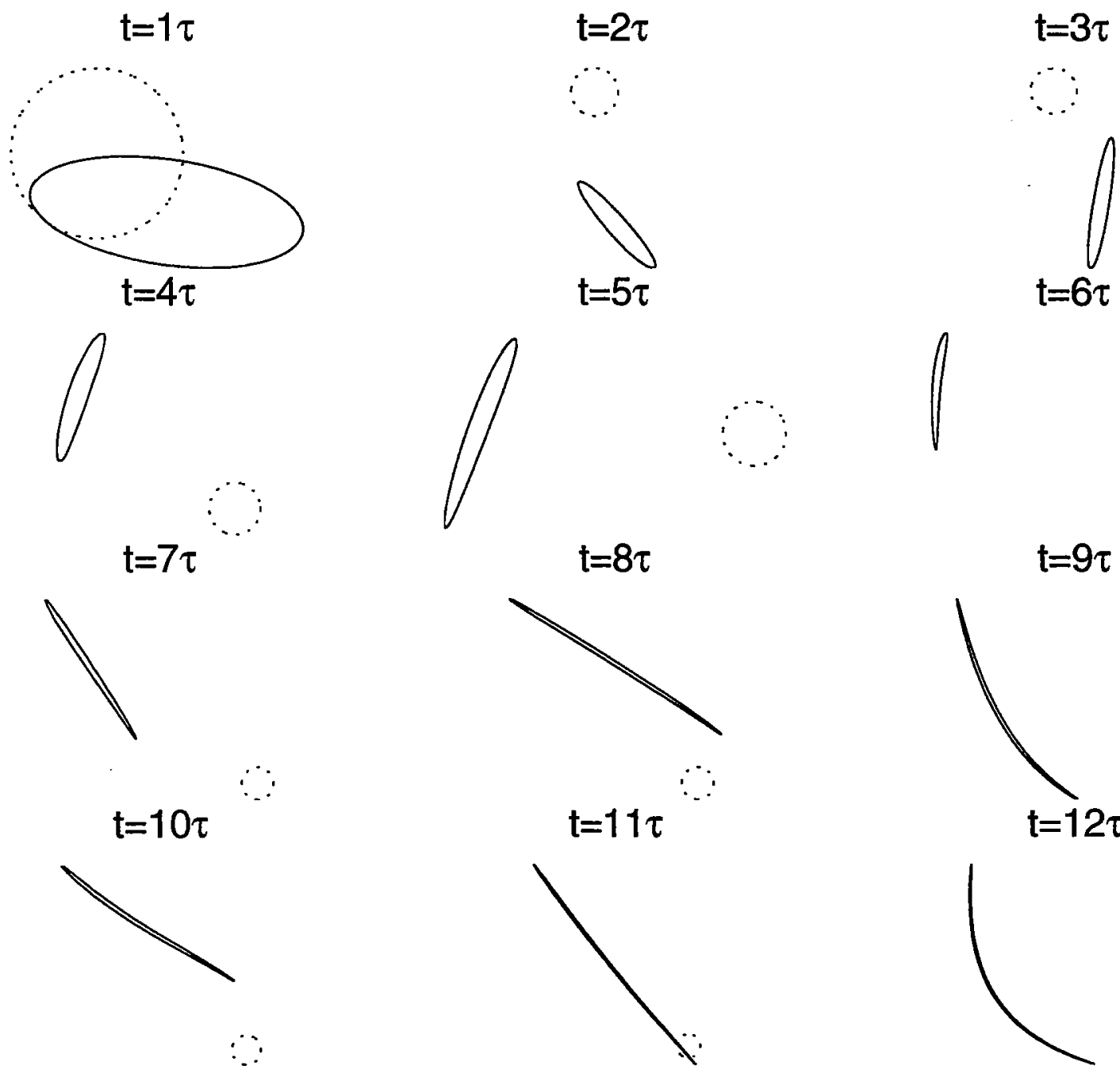

Figure 1: Stretching of a small spot, $r \ll 1$ where $r$ is the initial radius of the spot, by a succession of random sinusoidal flows. The dotted circle is the initial spot.
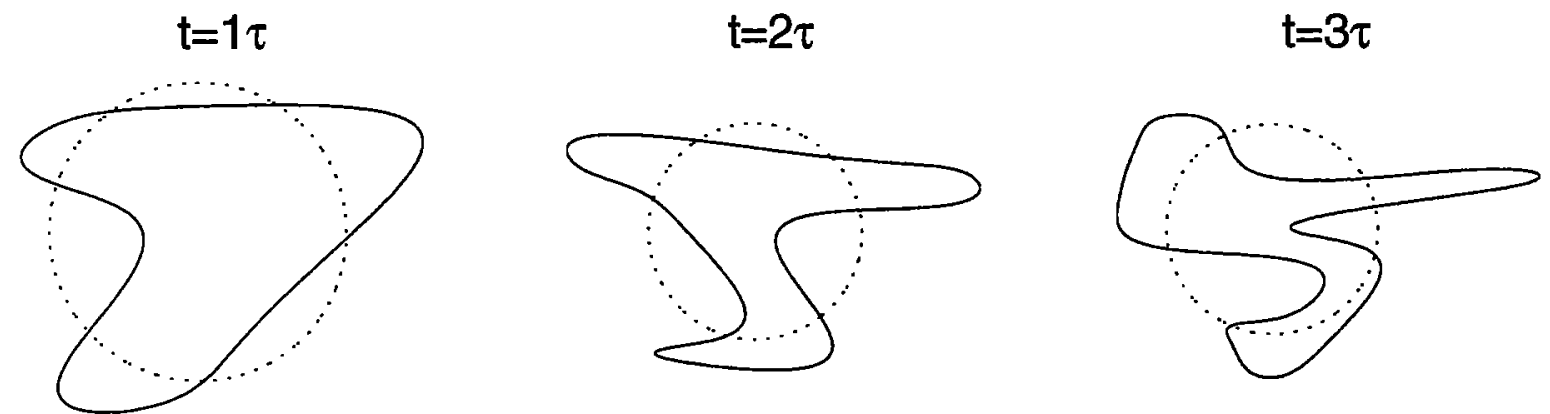

Figure 2: Stretching of a blob with $r=1$, where $r$ is the initial radius. The dotted circle is the initial patch. 

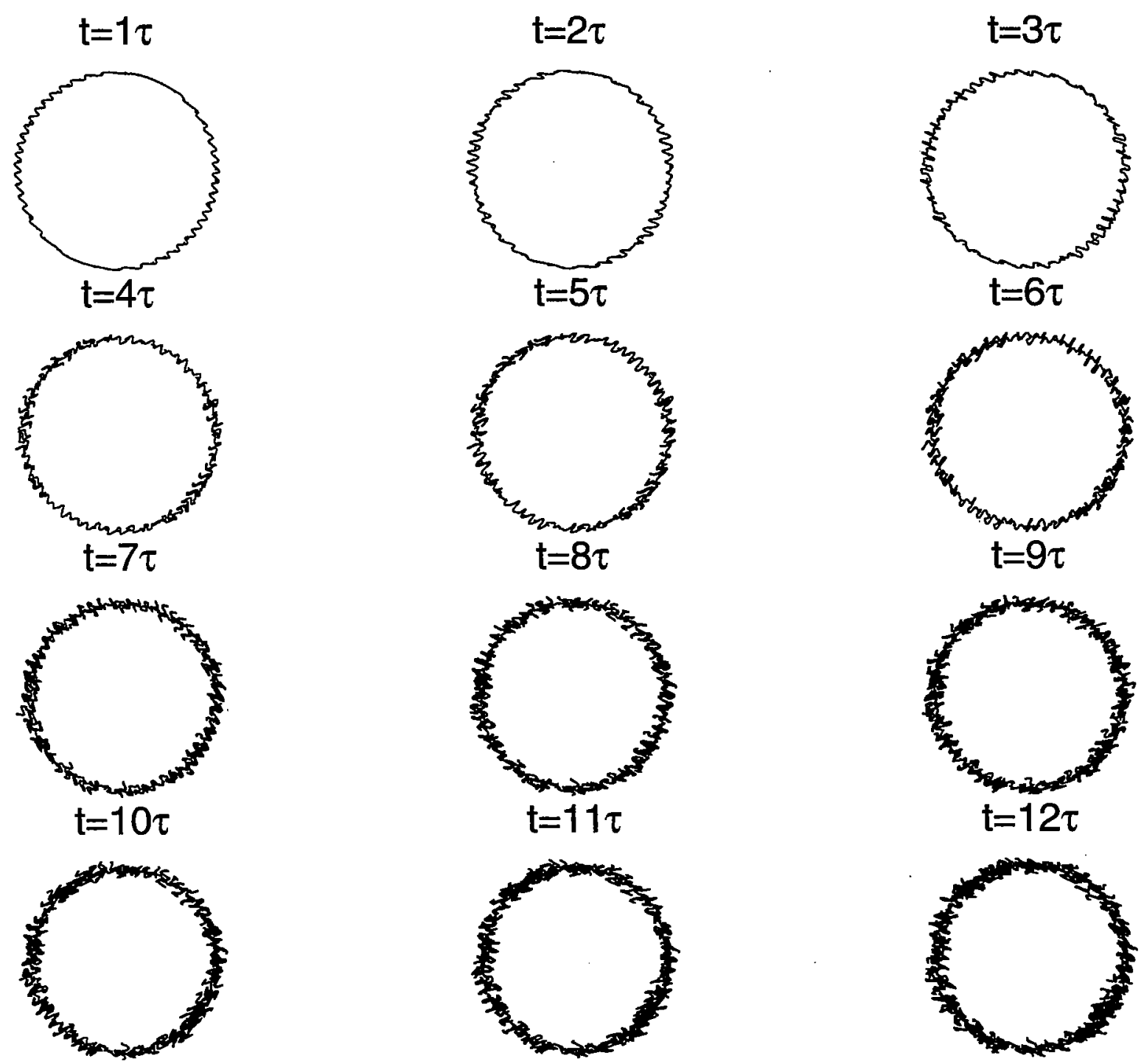

Figure 3: Stretching of a big blob $r \gg 1$, where $r$ is the initial radius of the blob. The dotted circle representing the initial patch may not be visible beneath the wiggly boundary of the blob.

\subsection{Deformation of variously sized blobs}

To emphasize the importance of understanding more than single-particle diffusivities we take a digression and illustrate how the deformation of an initially circular blob of fluid depends on the blob radius $r$. (Recall that we have used $k^{-1}$ as unit of length; in terms of dimensional variables the relevant nondimensional parameter is $k r$.)

If the initial blob is much smaller than the wavelength of the velocity then on the scale of the blob the velocity profile is a linear function of the coordinates. Because of this simplicity, the first few iterations deform the circular blob into an ellipse which must have the same area as the initial circle. We will see in the next lecture that the major axis of the ellipse grows exponentially while the minor axis shrinks so that the area is fixed. Once the dimensions of the ellipse are comparable to the wavenumber of the flow, more complicated deformations occur. Ultimately the blob will be stretched 
into a folded filament as in figure 1.

The blob has the same scale as the velocity field if $r \sim 1$. Because there is no scale separation there is no easy description of the action of the flow on the blob, see figure 2.

If $r \gg 1$ then we are in the "eddy diffusivity" limit in which the scale of the velocity field is much smaller than the scale of the tracer. This case is shown in figure 3 . The action of the waves perturbs the edge of the blob, making it look "fuzzy". In fact, the area is preserved, but the circumference of the blob grows exponentially. We will be discussing this type of problem for the remainder of the lecture.

\subsection{The Lagrangian correlation function}

In (7) we gave the diffusivity of particles moving in an ensemble of renovating waves. How do we. obtain the Lagrangian velocity autocorrelation function and verify Taylor's formula that

$$
D=\int_{0}^{\infty} \mathcal{C}(t) \mathrm{d} t ?
$$

Considering this question, we encounter an annoying technical difficulty: our derivation of (8) assumes that the velocity statistics are stationary. But the renovating wave ensemble, as we defined it back in (1) and (2), is not a stationary stochastic process. This is because with our original definition all members of the ensemble renovate at the same instants $t=\tau, t=2 \tau$ etcetera. In order to obtain a stationary process we should initiate different realizations at uniformly distributed points during the renovation cycle. Thus, for realization number $j$, we pick a random time $\tau^{(j)}$ which is uniformly distributed in the interval $[0, \tau]$ and renovate first at $t=\tau^{(j)}$ and then subsequently at $t=\tau^{(j)}+\tau$, $t=\tau^{(j)}+2 \tau$ etcetera. With this new and improved formulation of the RW model the Lagrangian correlation function of $u(t)$ is a "triangular" function:

$$
\mathcal{C}(t)=\frac{U^{2}}{4}\left(1-\frac{t}{\tau}\right) H(\tau-t)
$$

where $H$ is the step function and $U$ is the velocity in (2). The area under this correlation function is $D=U^{2} \tau / 8$.

\section{The eddy diffusion equation}

\subsection{The ensemble averaged Green's function}

Now that we have obtained the RW diffusivity in (7) we turn to the derivation of the eddy diffusion equation. For each realization we introduce the Green's function which is

$$
G_{t}+\mathbf{u} \cdot \nabla G=0, \quad \text { with } \quad G\left(\mathbf{x}, \mathbf{x}_{0}, 0\right)=\delta\left(\mathbf{x}-\mathbf{x}_{0}\right) .
$$

The solution of the problem above is

$$
G\left(\mathbf{x}, \mathbf{x}_{0}, t\right)=\delta\left(\mathbf{x}_{t}-\mathbf{x}_{0}\right),
$$

where $\mathbf{x}_{t}$ is the position at time $t$ (in a particular realization of $\mathbf{u}$ ) of the particle which started at $\mathbf{x}_{0}$.

The ensemble averaged Green's function is

$$
g(r, t)=\left\langle G\left(\mathbf{x}, \mathbf{x}_{0}, t\right)\right\rangle, \quad r \equiv\left|\mathbf{x}-\mathbf{x}_{0}\right|,
$$

where we have assumed that the random velocity is isotropic, homogeneous and stationary so that $g$ can depend only on the distance $r$ and the elapsed time $t$. 
Possessing $g(r, t)$, we can then represent the ensemble-averaged solution of the initial value problem

$$
c_{t}+\mathbf{u} \cdot \nabla c=0, \quad c(\mathbf{x}, 0)=c_{0}(\mathbf{x})
$$

as the convolution

$$
\langle c\rangle(\mathbf{x}, t)=\int c_{0}\left(\mathbf{x}-\mathbf{x}^{\prime}\right) g\left(\left|\mathbf{x}^{\prime}\right|, t\right) \mathrm{d} \mathbf{x}^{\prime} .
$$

(We are assuming that the initial condition $c_{0}$ is the same for all realizations.)

At this point, the analogy between (14) and the master equation of lecture 1 is obvious. With the master equation in mind, we can anticipate that a variant of Einstein's derivation of the diffusion equation can be applied to (14). Rather than develop a general derivation we prefer to use the renovating wave model as a concrete illustration of how one can obtain $g$, and then pass from the integral equation in (14) to an approximate diffusion equation.

\subsection{The averaged Green's function of the RW model}

There are at least two ways of obtaining $g(r)$ in (12) for the RW model: the hard, straightforward way (see the appendix) and the easy, devious way. Let us be devious.

We begin by calculating the probability density function (PDF) of displacements in a single pulse of the RW model. Because the ensemble of velocities is isotropic and homogeneous there is no harm in supposing that the particle is at the origin and the $x$-axis is aligned with the direction of the velocity. That is, put $\left(x_{n}, y_{n}\right)=(0,0)$ and $\theta_{n}=\pi / 2$ in (3). Thus, the displacement $r$ produced by a single pulse is

$$
x_{n+1}-x_{n}=\tau_{*} \sin \varphi_{n}, \quad \text { and } \quad r=\left|x_{n+1}-x_{n}\right| \text {. }
$$

The PDF of the random variable $r$ can be obtained from the PDF of $\varphi$, that is $P(\varphi)=1 / 2 \pi$, using the rule for transforming probabilities:

$$
P(r)=\sum P(\varphi)\left|\frac{\mathrm{d} \varphi}{\mathrm{d} r}\right|, \quad \Longrightarrow \quad P(r)=\frac{2}{\pi} \frac{H\left(\tau_{*}-r\right)}{\sqrt{\tau_{*}^{2}-r^{2}}}
$$

In (16) $H\left(\tau_{*}-r\right)$ is a Heaviside step function which ensures that there are no displacements greater than $\tau_{*}$. (The sum in (16) is because there are four values of $\varphi$ corresponding to a single value of $r$.)

The averaged Green's function is now given by

$$
g(r)=\frac{P(r)}{2 \pi r}, \quad \Longrightarrow \quad g(r)=\frac{1}{\pi^{2}} \frac{H\left(\tau_{*}-r\right)}{r \sqrt{\tau_{*}^{2}-r^{2}}}
$$

The geometric factor $2 \pi r$ is included because $g(r)$ is a concentration. That is, $P(r) \mathrm{d} r$ the expected number of particles which fall into the differential annulus between $r$ and $r+\mathrm{d} r$ and $g(r)$ is the expected number of particles per area in this same annulus; see figure 4.

Now that we have the averaged Green's function of a single pulse we can obtain the evolution the ensemble averaged concentration, $\langle c\rangle$, over many pulses. Because each pulse is independent of the preceeding pulses we have

$$
\langle c\rangle\left(\mathbf{x},(n+1) \tau_{*}\right)=\int\langle c\rangle\left(\mathbf{x}-\mathbf{x}^{\prime}, n \tau_{*}\right) g\left(\left|\mathbf{x}^{\prime}\right|\right) \mathrm{d} \mathbf{x}^{\prime} .
$$

The master equation above, with $g(r)$ in (17), is an exact description of the evolution of $\langle c\rangle$ under advection by the RW model. 

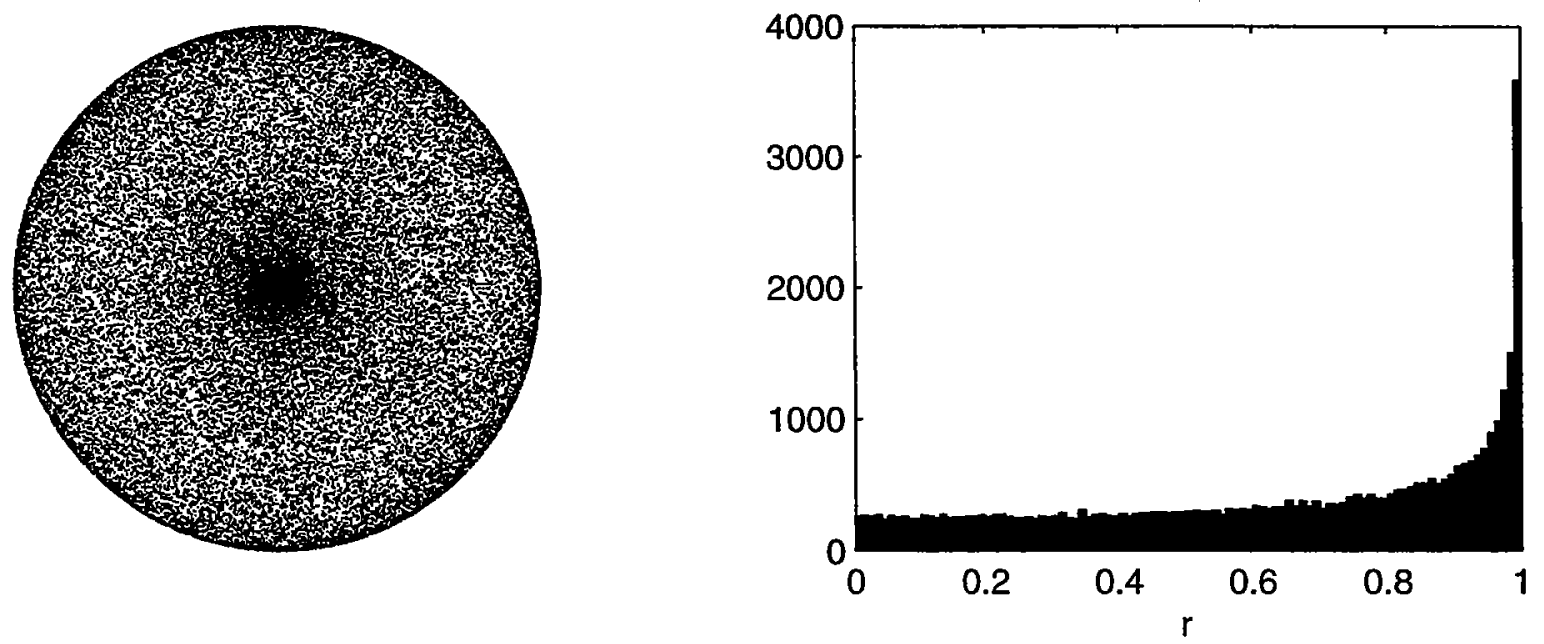

Figure 4: Displacements of 40,000 particles in independent realizations of the the RW model. The left panel shows the final position of particles which all start at the center of the the circle after one pulse of the wave. The density of points corresponds to $g(r)$ in (17). The histogram on the right shows the number of particles at a distance $r$ from the center; this is the function $P(r)$ in (16).

\subsection{The diffusion equation}

With the master equation (18) in hand, we can use Einstein's approximations to obtain the diffusion equation. Using the dimensionless variables of the renovating wave model, we have

$$
\langle c\rangle_{t} \approx \frac{\tau_{*}}{8} \nabla^{2}\langle c\rangle \text {. }
$$

We leave this as a homework exercise and instead we take a different route to (19).

Because the Fourier transform of a convolution is the product of the Fourier transforms, we can simplify (18) by transforming. The Fourier transform of $f(\mathbf{x})$ is defined here ${ }^{1}$ as

$$
\tilde{f}(\mathbf{k})=\int e^{-\mathbf{i k} \cdot \mathbf{x}} f(\mathbf{x}) \mathrm{d} x, \quad f(\mathbf{x})=\frac{1}{2 \pi} \int e^{\mathbf{i k} \cdot \mathbf{x}} \tilde{f}(\mathbf{k}) \mathrm{d} k .
$$

Applying the transform to (18) we obtain

$$
\widetilde{\langle c\rangle}\left(\mathbf{k}, n \tau_{*}\right)=\tilde{g}(k)^{n} \tilde{c}_{0}(\mathbf{k}), \quad k \equiv|\mathbf{k}| .
$$

With a good table of integrals one can discover that the Fourier transform of the averaged Green's function, $g(r)$ in (17), is

$$
\tilde{g}(k)=J_{0}^{2}\left(k \tau_{*} / 2\right)
$$

where $J_{0}$ is the Bessel function.

The diffusion equation describes the evolution of large spatial scales, which is the same as small wavenumbers. This means that we simplify (21) by taking $k \tau_{*} / 2 \ll 1$ and using the approximation $J_{0}\left(k \tau_{*} / 2\right) \approx 1-\left(k^{2} \tau_{*}^{2} / 16\right)$ to write

$$
\widetilde{\langle c\rangle}\left(\mathbf{k}, n \tau_{*}\right) \approx \exp \left\{n \ln \left[1-\left(k^{2} \tau_{*}^{2} / 8\right)\right]\right\} \tilde{c}_{0}(\mathbf{k}) .
$$

\footnotetext{
${ }^{1}$ By denoting the wavenumber with $k$ we are recycing notation used in (2).
} 
But now, since $n=t / \tau_{*}$ and $\ln \left[1-\left(k^{2} \tau_{*}^{2} / 8\right)\right] \approx-k^{2} \tau_{*}^{2} / 8$, we have

$$
\widetilde{\langle c\rangle}(\mathbf{k}, t)=e^{-D k^{2} t} \tilde{c}_{0}(\mathbf{k})
$$

where, as in (19), $D=\tau_{*} / 8$. Equation (24) is the equivalent to the decay of Fourier components given by (19).

This derivation based on Fourier analysis explicitly recognizes that the diffusion approximation is valid only for wavenumbers which satisfy $k \tau_{*} / 2 \ll 1$. This is a precise statement of the scale separation assumption which underlies Einstein's approach.

\section{Ensemble averages and single realizations}

In hydrodynamic dispersion, particles which begin at neighbouring points have similar histories in any single realization. Marbled endpapers in old books were produced by floating coloured inks on water, stirring the surface, and then capturing the swirls by carefully lowering a sheet of paper onto the inky film [3]. This technique, probably originating in Persia in the 1400s, presses hydrodynamic correlations into the service of art. Fortunately for printers, and distressingly for statisticians, a single realization does not resemble the blurry diffusion equation.

\subsection{Eddy diffusion of a front}

Figure 5 shows a single realization of the evolution of a "front" under the RW advection process. The front is the sharp border which separates white from dark; initially this line coincides with the $y$-axis. We suppose that the concentration is $c=-1$ for $x<0$ and $c=+1$ for $x>0$. Successive pulses of the renovating wave produce an increasingly folded front and the $c=-1$ fluid invades the region $x>0$ in long thin tendrils. The central question is:

\section{How well is the process in figure 5 described by the diffusion equation?}

We know that given many realizations of this process, the long-time ensemble average of these realizations will follow the diffusion equation $\langle c\rangle_{t}=D\langle c\rangle_{x x}$, with the initial conditions $c(x, 0)= \pm 1$. The solution of this problem is

$$
\langle c\rangle=\operatorname{erf} \eta, \quad \text { where } \quad \eta=\frac{x}{2 \sqrt{D t}} .
$$

Figure 6 shows this smooth erf solution which, of course, looks nothing like figure 5 . If the dark fluid in figure 5 contained radioactive contaminant, and we wanted to estimate the maximum exposure of at some value of $x>0$, then the erf solution in (25) is not useful.

On the other hand, diffusivities are useful if we want to know how many particles are at such-andsuch a distance from their initial location. Thus, figure 7 shows a histogram of the positions of 10,000 particles which all start on the line $x=0$ (the initial front). The Gaussian curve in figure 7 is the corresponding prediction for the PDF of positions which is obtained by solving (19) with the initial condition $\langle c\rangle=\delta(x)$ :

$$
c(x, t)=\frac{1}{\sqrt{4 \pi D t}} \exp \left[-\frac{x^{2}}{4 D t}\right], \quad D=\frac{\tau_{*}}{8}
$$

The histograms converge slowly to this Gaussian prediction. This asymptotic success shows that the diffusion equations correctly predicts the dispersion of particles when $t \gg \tau_{*}$.

An amusing aspect of the simple problem in figure 5 is that we can easily calculate the RMS fluctuations of $c$ around the ensemble average concentration in (25). Because $c= \pm 1$ we have $\left\langle c^{2}\right\rangle=1$. Therefore, defining the fluctuation as

$$
c^{\prime}=c-\langle c\rangle
$$



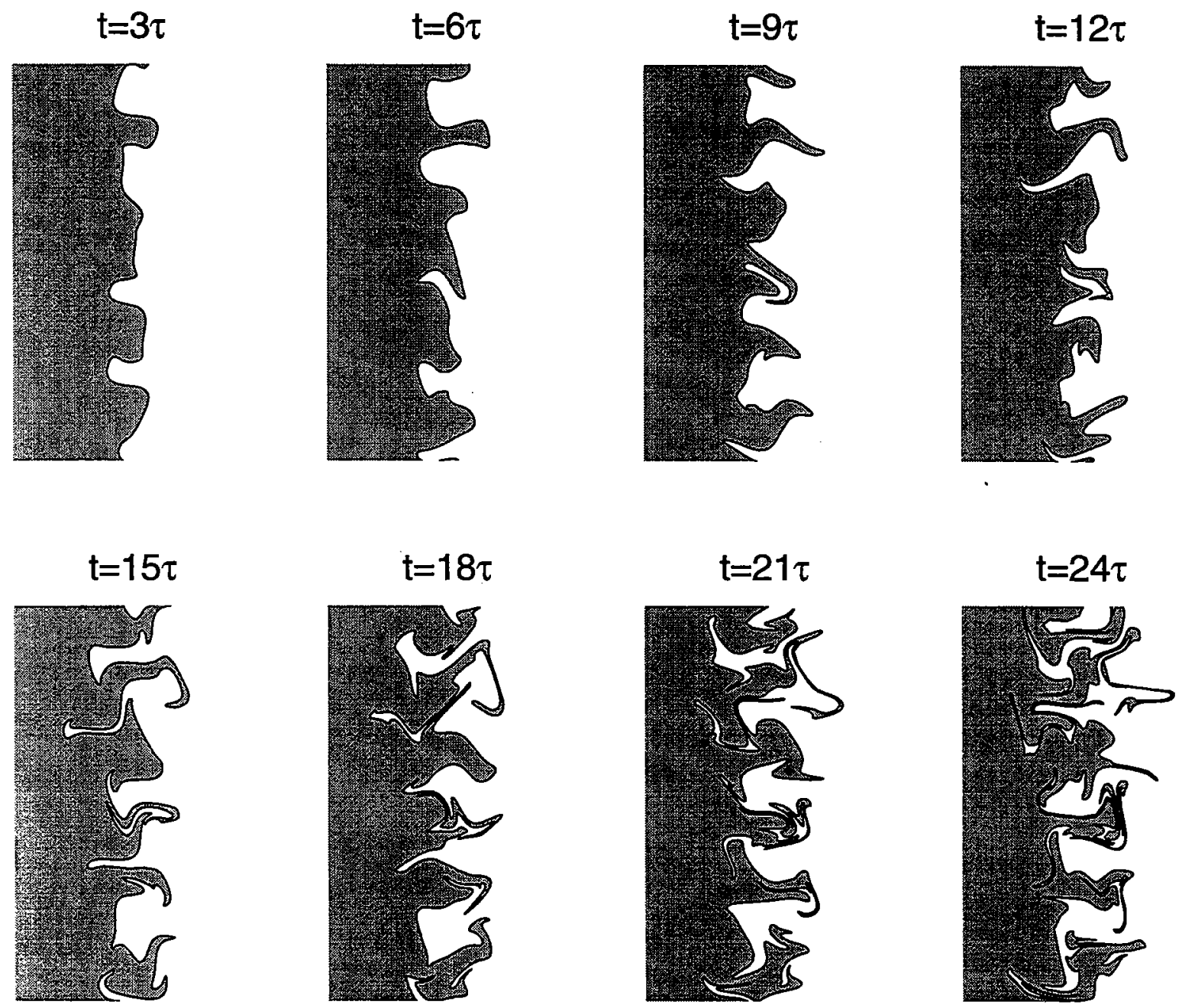

Figure 5: Evolution of a front under the advection by the RW model. The front initially coincides with the $y$-axis.

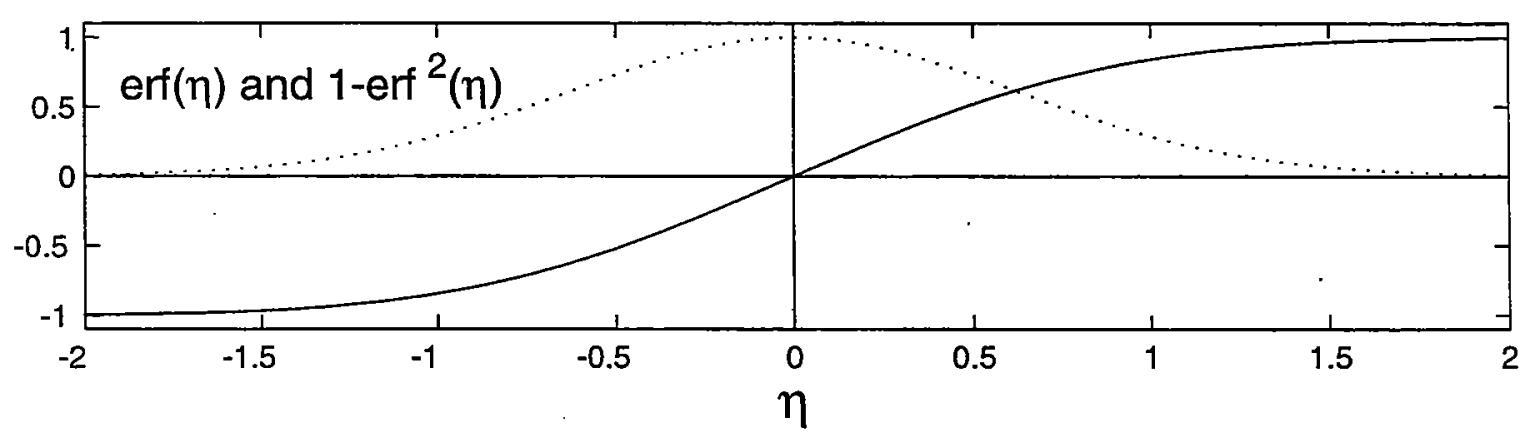

Figure 6: Evolution of the ensenble-averaged concentration $c$ and its variance during the evolution of the front underthe RRW model. Note how most of the variance is localised around $x=0$. 

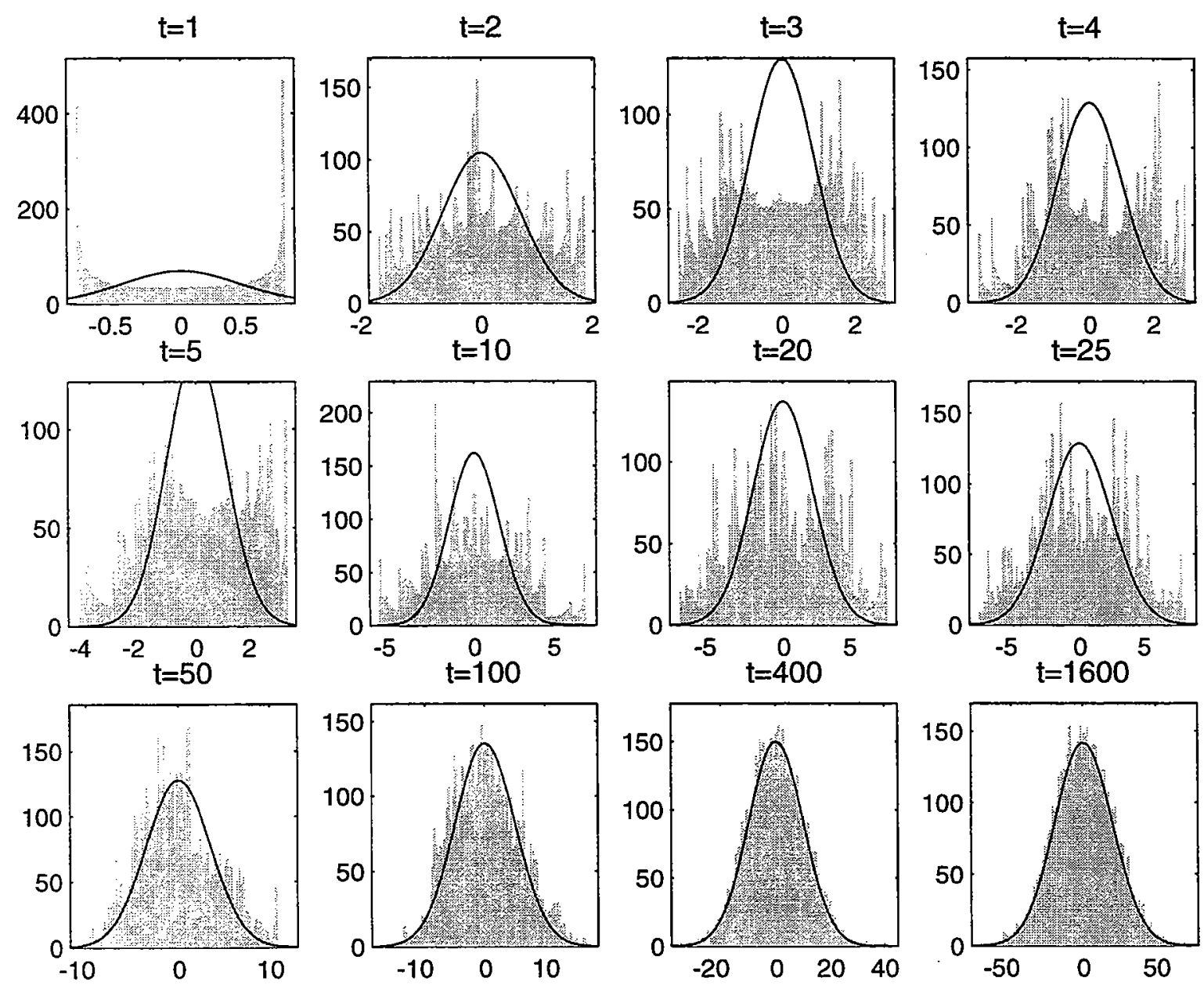

Figure 7: At $t=0$ the front in figure 5 is tagged by placing 10000 particles along the $y$-axis. The historgram above shows the subsequent $x$-locations of these marker particles as the front is distorted by the RW model with $\tau_{*}=1$. The Gaussian curve is given by (26).

we have

$$
\left\langle c^{\prime 2}\right\rangle=\left\langle c^{2}\right\rangle-\langle c\rangle^{2}=1-\operatorname{erf}^{2}(\eta)
$$

The variance $\left\langle c^{\prime 2}\right\rangle$ is also indicated in figure 6 .

\subsection{Coarse grained averages and spatial filters}

The process in figure 5 is translationally invariant in the $y$-direction and so using only a single realization we can calculate a spatially averaged concentration

$$
\bar{c}(x, t) \equiv \lim _{L \rightarrow \infty} \frac{1}{2 L} \int_{-L}^{L} c(x, y, t) \mathrm{d} y .
$$

The evolution of $\bar{c}$ will be asymptotically described by the diffusion equation. 
In a general case, in which there is no statistical symmetry along a particular direction, one can take a single realization and define a coarse-grained or low-pass filtered concentration by:

$$
\hat{c}(\mathbf{x}, t) \equiv \int K\left(\mathbf{x}-\mathbf{x}^{\prime}\right) c\left(\mathbf{x}^{\prime}, t\right) \mathrm{d}^{2} \mathbf{x}^{\prime} ; \quad K(|\mathbf{x}|) \text { is a filter. }
$$

The hope is that scale separation between the width of the erf and the swirls will ensure that $\hat{c} \approx\langle c\rangle$. Thus the kernel of the filter, $K$ in (30), might be a Gaussian with a width which is at once much smaller than the thickness of the erf transition zone and much greater than an individual swirl in figure 5 .

Scale separation is essential here because the filtering operation defined by the convolution in (30) is not strictly an "average". Some of the properties we take for granted when we use averages are

$$
\left\langle c^{\prime}\right\rangle=0, \quad\langle\langle c\rangle\rangle=\langle c\rangle, \quad\langle\langle a\rangle\langle b\rangle\rangle=\langle a\rangle\langle b\rangle .
$$

For the ensemble average, as indicated in (31), everything works.

For a filter, such as "in (30), we can define the fluctuation concentration $c^{\prime \prime}$ in analogy with (27):

$$
c^{\prime \prime} \equiv c-\hat{c} \text {. }
$$

But then $\widehat{c^{\prime \prime}} \neq 0$ and none of the other desiderata in (31) follow. In other words, spatial filtering instead of the ensemble averaging introduces a host of extra assumptions which should be carefully assessed (but almost never are).

\subsection{A digression: Brownian bugs}

I have hinted darkly at problems associated with spatial filters. These issues are largely ignored by optimistic scientists. The hope is that scale separation justifies the application of diffusive closures to the coarse-grained version of a single realization. Perhaps a justification of this optimistic approach is that the alternative seems so repellent. Nonetheless, it is important to realize that interpreting coarse-grained distributions as ensemble averages involves a nontrivial assumption. The best way of exposing this assumption is to exhibit a problem in which spatial filters and ensemble averages are very different. Accordingly, as a model of biological processes, we consider random walkers which both die and reproduce. We refer to these biological walkers as Brownian bugs.

The model is formulated by first placing $N \gg 1$ Brownian bugs randomly in the unit square; the boundary conditions are periodic in both directions. Each cycle of the simulation begins with a random walk step in which bug $k$, located at $\mathbf{x}_{k}=\left(x_{k}, y_{k}\right)$, is displaced to a new position

$$
\left(x_{k}^{\prime}, y_{k}^{\prime}\right)=\bmod \left[\left(x_{k}, y_{k}\right)+\left(\delta x_{k}, \delta y_{k}\right) ; 1\right] \text {. }
$$

In (33), $\delta x_{k}$ and $\delta y_{k}$ are Gaussian random variables and the "mod" is to enforce the periodic boundary conditions and keep each bug in the unit square. After this random walk step, the second part of the cycle is a "coin toss" which results in either death (heads) or division (tails). When a lucky bug divides, the offspring is placed at the same position as the parent. This cycle of random displacement and random birth/death is repeated many times in order to simulate many generations of reproduction, death and dispersion.

The simulation shown in figure 8 was implemented in MATLAB using these rules. The striking result is that the density of bugs spontaneously develops large-scale clumps and voids. Figure 8 seems to show an inverse cascade of patch sizes: patches emerge on small scales in panel (b) and then, after more cycles, panels (c) and (d) show that the patches have expanded in scale. To quantify this impression, we have computed one-dimensional concentration spectra which show that an increasingly red spectrum develops.

A seemingly innocuous ingredient of the brownian-bug model is that deaths can occur anywhere, but births are always adjacent to a living bug. This asymmetry between birth and death is crucial for 
(a) Initial condition, $\mathrm{N}=20,000$

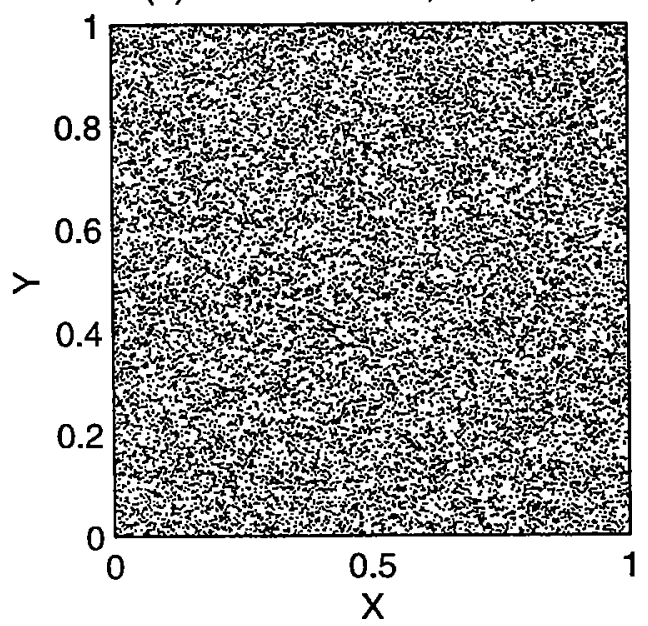

(c) 100 cycles, $\mathrm{N}=17,728$

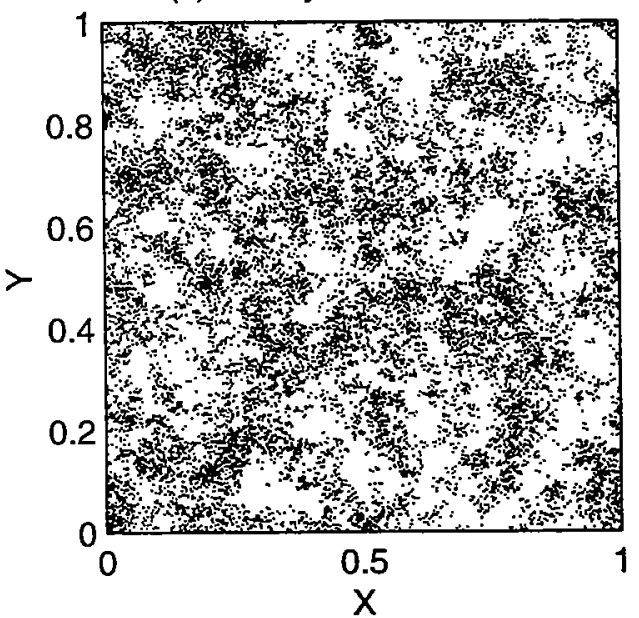

(b) 10 cycles, $\mathrm{N}=19,692$

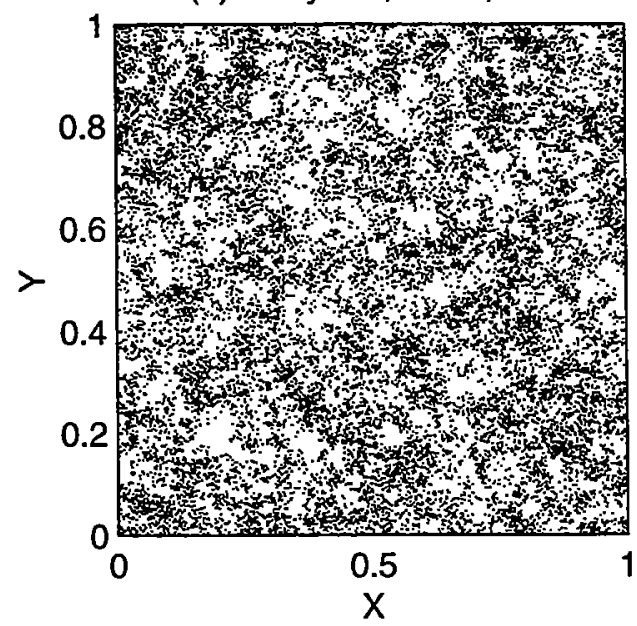

(d) 1000 cycles, $\mathrm{N}=21,814$

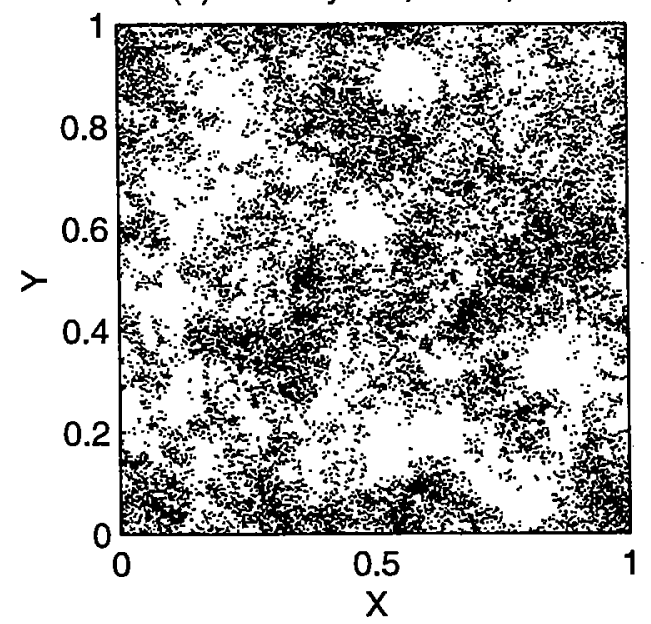

Figure 8: (a) The initial condition is $N=20,000$ randomly located bugs in the unit square. Panels (b), (c) and (d) then show the development of patches after 10,100 and 1000 cycles of random displacement followed by random birth/death. As the panel titles indicate, there are random fluctuations in the total size, $N$, of the population. The RMS step length of the underlying random walk is $\left\langle\delta x_{k}^{2}\right\rangle^{1 / 2}=\left\langle\delta y_{k}^{2}\right\rangle^{1 / 2}=0.005$.

the spontaneous development of the voids and patches evident in figure 8: if one simulates birth by randomly placing the new bugs in the unit square then no patches form. This subtle point shows that making the births coincide with living bugs - surely a realistic feature of the model - introduces pair correlations. From another perspective, one can view the voids in figure 8 as the result of random extinctions which create voids. The step length of the random walk in figure 8 is such that diffusion is not strong enough to fill in the voids created by extinction.

The ensemble average of the Brownian bug process is described by

$$
\langle c\rangle_{t}=D \nabla^{2}\langle c\rangle+(\lambda-\mu)\langle c\rangle .
$$

where $\lambda$ is the birthrate and $\mu$ the deathrate. However if the coin-toss is fair then births and deaths are equiprobable and consequently $\lambda=\mu$. In this case the solution of (34) which satisfies the initial 
condition is

$$
\lambda=\mu, \quad \Longrightarrow \quad\langle c\rangle=1 / N
$$

The uniform density above is the correct answer for the ensemble average concentration: the location of the voids and patches in figure 8 are accidently determined by the MATIAB random number generator. If we ensemble average many such patterns then the patches and voids must disappear because the process is spatially homogeneous.

On the other hand, the spatial average of a single realization, such as that in figure 8 , will still show concentration patches ${ }^{2}$. Thus, in this Brownian bug example, $\hat{c} \neq\langle c\rangle$. Indeed, the patches are surely an important feature of the "real" answer. The correct but useless result in (35) exposes a failure of ensemble averaging. What do we make of this example? Are biological problems, with reproduction and death, so fundamentally different from the advection-diffusion of chemical tracers? I am not prepared to answer that question in these lectures and I leave further development of this example to the students.

\section{Variance budgets}

In this section we return to basics and present an alternative view of eddy-diffusivity. The following arguements emphasize the importance of the concentration variance equation.

\subsection{The Reynolds' decomposition}

Our point of departure is the advection-diffusion equation

$$
c_{t}+\mathbf{u} \cdot \nabla c=\kappa \nabla^{2} c+s
$$

where $\kappa$ is the molecular diffusivity of $c$ and $\mathbf{u}$ is an incompressible $(\boldsymbol{\nabla} \cdot \mathbf{u}=0)$ velocity field. In (36) we have included a source term, $s(\mathbf{x}, t)$, which forces the system.

The velocity $\mathbf{u}$ in (36) is a single realization selected from an ensemble of velocity fields. Then we can introduce the "Reynolds' decomposition":

$$
c=\langle c\rangle+c^{\prime}
$$

where \langle\rangle is the ensemble average and $c^{\prime}$ is the fluctuation from $\langle c\rangle$ which arises in a single realization. Taking the ensemble average of $(36)$ gives

$$
\langle c\rangle_{t}+\langle\mathbf{u}\rangle \cdot \nabla\langle c\rangle+\nabla \cdot\left\langle\mathbf{u}^{\prime} c^{\prime}\right\rangle=\kappa \nabla^{2}\langle c\rangle+s .
$$

(The source $s$ is taken to be deterministic, $\langle s\rangle=s$. )

Subtracting the ensemble average in (38) from (36) gives the fluctuation equation

$$
c_{t}^{\prime}+\langle\mathbf{u}\rangle \cdot \nabla c^{\prime}+\nabla \cdot\left[\mathbf{u}^{\prime} c^{\prime}-\left\langle\mathbf{u}^{\prime} c^{\prime}\right\rangle\right]-\kappa \nabla^{2} c^{\prime}=-\mathbf{u}^{\prime} \cdot \nabla\langle c\rangle .
$$

Equation (39) has been organized by taking the source term to the right hand side. Thus we see that advective distortion of the mean gradient, $\nabla\langle c\rangle$, generates the fluctuation $c^{\prime}$.

\footnotetext{
${ }^{2}$ If the width of the kernel, $K$ in (30), is larger than the dimension of the patches then filtering will remove the patches. However, since the patches expand in scale, eventually they will become so large that they survive the blurring power of the filter.
} 


\subsection{Consequences of linearity}

If $c^{\prime}=0$ at $t=0$ then, because (39) is linear, $c^{\prime}$ and $\nabla\langle c\rangle$ will be linearly related. It follows that the eddy flux $\left\langle\mathbf{u}^{\prime} c^{\prime}\right\rangle$ will also be linearly related to the mean gradient $\nabla\langle c\rangle$. These simple observations, in alliance with the scale separation assumption, can be used to extract a surprising amount of information [2].

Because of the scale separation, it is plausible that this linear relation between eddy flux and mean gradient can be developed in a series of the form

$$
\left\langle u_{i}^{\prime} c^{\prime}\right\rangle=-\mathcal{D}_{i j}^{(1)} *\langle c\rangle_{, j}-\mathcal{D}_{i j k}^{(2)} *\langle c\rangle_{, j k}+\cdots
$$

The comma subscripts denote partial derivatives, $a_{, j} \equiv \partial a / \partial x_{j}$. We are also using the Einstein sum convention, where repeated indices are summed. The $*$ in $(40)$ indicates that the product also involves convolutions in time, such as

$$
\mathcal{D}_{i j}^{(1)} *\langle c\rangle_{, j}=\int_{0}^{t} \mathcal{D}_{i j}^{(1)}\left(t^{\prime}\right)\langle c\rangle_{, j}\left(t-t^{\prime}\right) \mathrm{d} t^{\prime} .
$$

If the mean field is varying slowly over an eddy decorrelation time then the convolution above approximates to

$$
\left\langle u_{i}^{\prime} c^{\prime}\right\rangle \approx-\mathcal{D}_{i j}^{(1)} *\langle c\rangle_{, j} \approx-\int_{0}^{\infty} \mathcal{D}_{i j}^{(1)}\left(t^{\prime}\right) \mathrm{d} t^{\prime}\langle c\rangle_{, j}(t)
$$

In the simplest cases $^{3}$

$$
\int_{0}^{\infty} \mathcal{D}_{i j}^{(1)}\left(t^{\prime}\right) \mathrm{d} t^{\prime}=D_{e} \delta_{i j}
$$

where $D_{e}$ is the eddy diffusivity. Using (43) the flux gradient relation is

$$
\left\langle\mathbf{u}^{\prime} c^{\prime}\right\rangle-\kappa \nabla\langle c\rangle=-D \nabla\langle c\rangle, \quad D \equiv D_{e}+\kappa,
$$

and the evolution of the average concentration is determined by

$$
\langle c\rangle_{t} \approx D \nabla^{2}\langle c\rangle+s .
$$

This is a general version of the specific diffusion equation derived in Section 3.3 for the renovating wave model.

\subsection{The $\mathrm{G} \cdot \mathrm{x}$-trick}

The tensors $\mathcal{D}^{(n)}(t)$ are determined by the linear operator on the left-hand side of (39). Thus, these tensors depend on (i) the statistical properties of $\mathbf{u}^{\prime}$; (ii) the mean advection $\langle\mathbf{u}\rangle$; (iii) the molecular diffusion $\kappa$. The essential point is that these tensors do not depend on $\langle c\rangle$. At least for the first term in the series, $\mathcal{D}_{i j}^{(1)}$, we can exemplify this by noting that there is a special solution of (36) in which $\langle\mathbf{u}\rangle=s=0$ and concentration has the form

$$
c=\mathbf{G} \cdot \mathbf{x}+c^{\prime} .
$$

\footnotetext{
3 "Simple" means that the velocity ensemble is isotropic, homogeneous and reflexionally invariant. The last requirement means that the mirror image of a particular realization of $\mathbf{u}^{\prime}$ is just as probable as $\mathbf{u}^{\prime}$. If the ensemble is reflexionally invariant then $\mathcal{D}_{i j}^{(1)}$ is a symmetric tensor. This subtle point will be illustrated later in this lecture series.
} 
In (46) the mean concentration is simply $\langle c\rangle=\mathbf{G} \cdot \mathbf{x}$ and the fluctuation $c^{\prime}$ is determined from a reduced version of $(39)$ :

$$
c_{t}^{\prime}+\mathbf{u}^{\prime} \cdot \nabla c^{\prime}-\kappa \nabla^{2} c^{\prime}=-\mathbf{G} \cdot \mathbf{u}^{\prime} .
$$

As emphasized above, the advection of the mean gradient appears as a source term for $c^{\prime}$ on the right hand side of (47). Because (47) is linear, and $\mathbf{G}$ is constant, the solution $c^{\prime}$ will be proportional to the large-scale gradient $\mathbf{G}$ and otherwise independent of $\mathbf{G}$.

This $\mathbf{G} \cdot \mathbf{x}$-trick enforces the platonic ideal of scale separation between the eddies and the mean field. If the concept of an eddy diffusivity is to have any validity, then it must work in the simplified context of (47). In fact, the $\mathbf{G} \cdot \mathbf{x}$-trick is used in doubly-periodic turbulence simulation to calculate eddy diffusivities. In that context, $\mathbf{u}^{\prime}=(u, v)$ and $c^{\prime}$ are efficiently represented by Fourier series. Then (47) is solved using a spectral code and the eddy flux is estimated by computing the integral

$$
\left\langle\mathbf{u}^{\prime} c^{\prime}\right\rangle=A^{-1} \iint \mathbf{u}^{\prime} c^{\prime} \mathrm{d} x \mathrm{~d} y,
$$

over the computational domain. (In (48) $A$ is the total area of the domain so $\langle 1\rangle=1$ ). Notice that in (48) the ensemble average is identified with an integral over the domain. Later in these lectures we will use this same procedure to analytically calculate the eddy diffusivities of some spatially periodic velocity fields.

\subsection{The concentration variance equation}

An equation for the concentration variance,

$$
\mathcal{Z} \equiv \frac{1}{2}\left\langle c^{\prime 2}\right\rangle
$$

is obtained by multiplying (39) by $c^{\prime}$ and ensemble averaging. The result is

$$
\mathcal{Z}_{t}+\langle\mathbf{u}\rangle \cdot \nabla \mathcal{Z}+\nabla \cdot\left\langle\frac{1}{2} \mathbf{u}^{\prime} c^{2}\right\rangle-\kappa \nabla^{2} \mathcal{Z}=-\kappa\left\langle\nabla c^{\prime} \cdot \nabla c^{\prime}\right\rangle-\left\langle\mathbf{u}^{\prime} c^{\prime}\right\rangle \cdot \nabla\langle c\rangle
$$

The terms on the left-hand side of $(50)$ can be interpreted as fluxes of $\mathcal{Z}$. The two terms on the right hand side of (50) are respectively dissipation of variance by molecular diffusion, $\kappa$, and a source of variance due to advective distortion of the mean gradient.

\subsection{Heuristic closure arguments}

In (50) there are three terms which we would like to relate to the mean quantities $\langle c\rangle$ and $\mathcal{Z}$. First, there is $-\left\langle\mathbf{u}^{\prime} c^{\prime}\right\rangle \cdot \nabla\langle c\rangle=D_{e} \nabla\langle c\rangle \cdot \nabla\langle c\rangle$. The remaining two terms are $\left\langle\mathbf{u}^{\prime} c^{\prime 2} / 2\right\rangle$ and $\kappa\left\langle\nabla c^{\prime} \cdot \nabla c^{\prime}\right\rangle$.

The correlation $\left\langle\mathbf{u}^{\prime} c^{\prime 2} / 2\right\rangle$ in (50) is an eddy-flux of ${c^{\prime}}^{2}$, just as $\left\langle\mathbf{u} c^{\prime}\right\rangle$ is an eddy flux of $c^{\prime}$. Thus, by analogy with (44), we can argue that

$$
\frac{1}{2}\left\langle\mathbf{u}^{\prime} c^{2}\right\rangle=-D_{e} \nabla \mathcal{Z}
$$

This heuristic argument is discussed further in appendix B.

The final term in (50) is the dissipation of variance by molecular diffusivity, $\kappa\left\langle\nabla c^{\prime} \cdot \nabla c^{\prime}\right\rangle$. The simplest closure assumption we can make about this term is that

$$
\kappa\left\langle\nabla c^{\prime} \cdot \nabla c^{\prime}\right\rangle \approx \beta \mathcal{Z},
$$


where $\beta$ has the dimensions of time. The closure above relies on dimensional analysis and the linearity of (36). However, in anticipation of a later discussion of the Batchelor spectrum, we now make some heuristic arguments in support of (52) which suggest that $\beta$ is independent of the molecular diffusivity.

Suppose that the mean field $\langle c\rangle$ has a length scale $L$ and that the velocity field $\mathbf{u}^{\prime}$ has a length scale $L_{\mathbf{u}}$ (in the RW example $L_{\mathbf{u}}=k^{-1}$ ). The scale separation assumption is that

$$
L \gg L_{\mathbf{u}} \text {. }
$$

The inequality in (53) is exemplified in idealized case of (46) in which $L$ is infinite. If follows that advective distortion of $\nabla\langle c\rangle$ generates $c^{\prime}$ first on the scale $L_{\mathbf{u}}$. Then, following our arguments in lecture 1 , the scale of $c^{\prime}$ will be exponentially reduced, like $\exp (-\gamma t)$, where $\gamma$ is roughly proportional to the RMS strain of $\mathbf{u}^{\prime}$. This exponential contraction continues until the cascade is halted by molecular diffusion at the scale

$$
\ell \equiv \sqrt{\frac{\kappa}{\gamma}}
$$

Using arguments from lecture 1 , we can estimate that the time taken for this arrest at $\ell$ is

$$
t_{\ell} \approx \gamma^{-1} \ln \left(L_{\mathbf{u}} / \ell\right)
$$

Then the smallest length scale in the $c^{\prime}$-field is $\ell$ and, plausibly, the gradient is $\nabla c^{\prime} \sim c_{R M S}^{\prime} / \ell$ where $c_{R M S}^{\prime} \equiv \sqrt{2 \mathcal{Z}}$. We now have a simple estimate $\kappa\left\langle\nabla c^{\prime} \cdot \nabla c^{\prime}\right\rangle \sim \gamma \mathcal{Z}$. This rough argument leads to the closure in (52), with $\beta \propto \gamma$, and the caveat that $t>t_{\ell}$.

We can summarize the arguments above by rewriting the variance equation (50) as

$$
\mathcal{Z}_{t}+\langle\mathbf{u}\rangle \cdot \nabla \mathcal{Z}-D \nabla^{2} \mathcal{Z}=D_{e} \nabla\langle c\rangle \cdot \nabla\langle c\rangle-\beta \mathcal{Z}, \quad\left(\text { if } t \geq t_{\ell}\right) .
$$

The most dubious approximation is probably (52). To conclude this discussion we will interpret the variance equation in two specific examples.

\subsection{Example 1: the dispersing front}

First consider the dispersing front in figure 5. In this example $s=\kappa=\langle\mathbf{u}\rangle=0$ and we have already know from (28) that

$$
\mathcal{Z}=\frac{1}{2}\left[1-\operatorname{erf}^{2}(\eta)\right], \quad \eta=\frac{x}{2 \sqrt{D t}} .
$$

On the other hand, since $\kappa=0$, it follows that $D=D_{e}$ and $\beta=0$. With these simplifications the variance equation $(50)$ reduces to

$$
\mathcal{Z}_{t}-D \mathcal{Z}_{x x}=D \nabla\langle c\rangle \cdot \nabla\langle c\rangle
$$

where $\langle c\rangle$ is the erf-solution in (25). As a consistency check, one can show that (57) is the solution of the variance equation in (58).

This example shows that the destruction of variance by molecular diffusivity is not required in order to prevent an accumulation of variance: the source on the right-hand side of (58) is balanced by eddy diffusion.

\subsection{Example 2: a large-scale source}

In this second example the tracer is injected by a source $s=\cos q x$ in (36). We also take $\langle\mathbf{u}\rangle=0$ so that the mean concentration field is obtained by solving

$$
\langle c\rangle_{t}-D \nabla^{2}\langle c\rangle=\cos q x, \quad \Longrightarrow \quad\langle c\rangle=\frac{1}{D q^{2}}\left[1-e^{-D q^{2} t}\right] \cos q x
$$



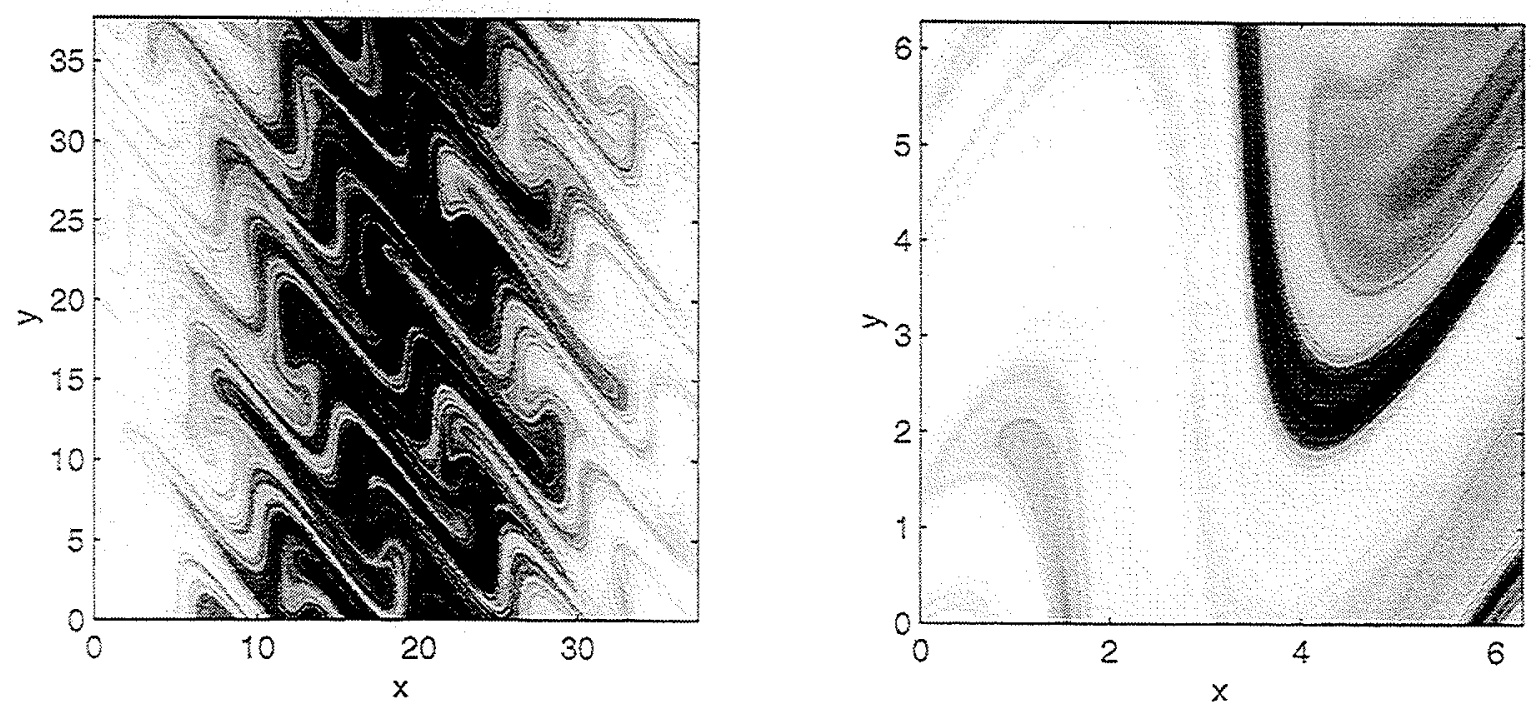

Figure 9: A simulation of the source problem, with $s=\cos (x / 6)$, using the RW model to generate $\mathbf{u}$ in (36). There is no molecular diffusivity $(\kappa=0)$. The left-hand panel shows the whole domain (the length of the side is $12 \pi$ ) while the right hand panel shows a smaller subdomain (the length of the side is $2 \pi$ ). The concentration fields were generated by 10 pulses of the renovating wave using $\tau_{*}=3$ (that is, $t=30$ ).

(To apply the diffusion equation the scale of the source, $q^{-1}$, must be much larger than the scale of the velocity field.) A steady mean concentration pattern is established when $D q^{2} t \gg 1$.

The concentration variance is determined by solving the variance equation (56)

$$
\mathcal{Z}_{t}-D \nabla^{2} \mathcal{Z}=\frac{1}{2} \frac{D_{e}}{D^{2} q^{2}}\left[1-e^{-D q^{2} t}\right]^{2}(1-\cos 2 q x)-\kappa\left\langle\nabla c^{\prime} \cdot \nabla c^{\prime}\right\rangle
$$

In (60), the solution in (59) has been used to evaluate the source term on the right hand side and we have left the diffusive sink in its exact form.

It is clear from (60) that the molecular diffusion, $\kappa$, plays an important role. If $\kappa=0$ then the long time solution of $(60)$ has a component which eventually grows linearly with time:

$$
\kappa=0, \quad \Longrightarrow \quad \mathcal{Z} \propto t / 2 D q^{2} .
$$

Thus, without molecular diffusion, there is "runaway variance". Ultimately, in a single realization, the mean field in (59) will be buried under enormous fluctuations.

To give an intuitive derivation of (61) we argue that with $\kappa=0$ the concentration on each fluid element is determined by solving the Lagrangian equation

$$
\frac{D c}{D t}=\cos q x(t)
$$

where $x(t)$ is the randomly changing $x$-position of the particle. Thus, the concentration on each particle is undergoing a random walk along the $c$-axis, which is induced by the random motion of the particle through the $\cos q x$ source function. The decorrelation time of this walk is the time it takes a particle to diffuse through a distance of order $q^{-1}$, which is $1 / D q^{2}$. Thus, in a time $t$, there are roughly $N(t) \sim D q^{2} t$ independent steps along the $c$-axis. But because the source acts coherently for a time $1 / D q^{2}$ with a strength of order unity, the step length of this random walk is roughly $\Delta c \sim 1 / D q^{2}$. Thus, the mean square displacement of $c$ is:

$$
\left\langle c^{2}\right\rangle \sim(\Delta c)^{2} N(t) \sim \frac{t}{D q^{2}},
$$


Concentration at $\mathrm{t}=200 \tau=400$
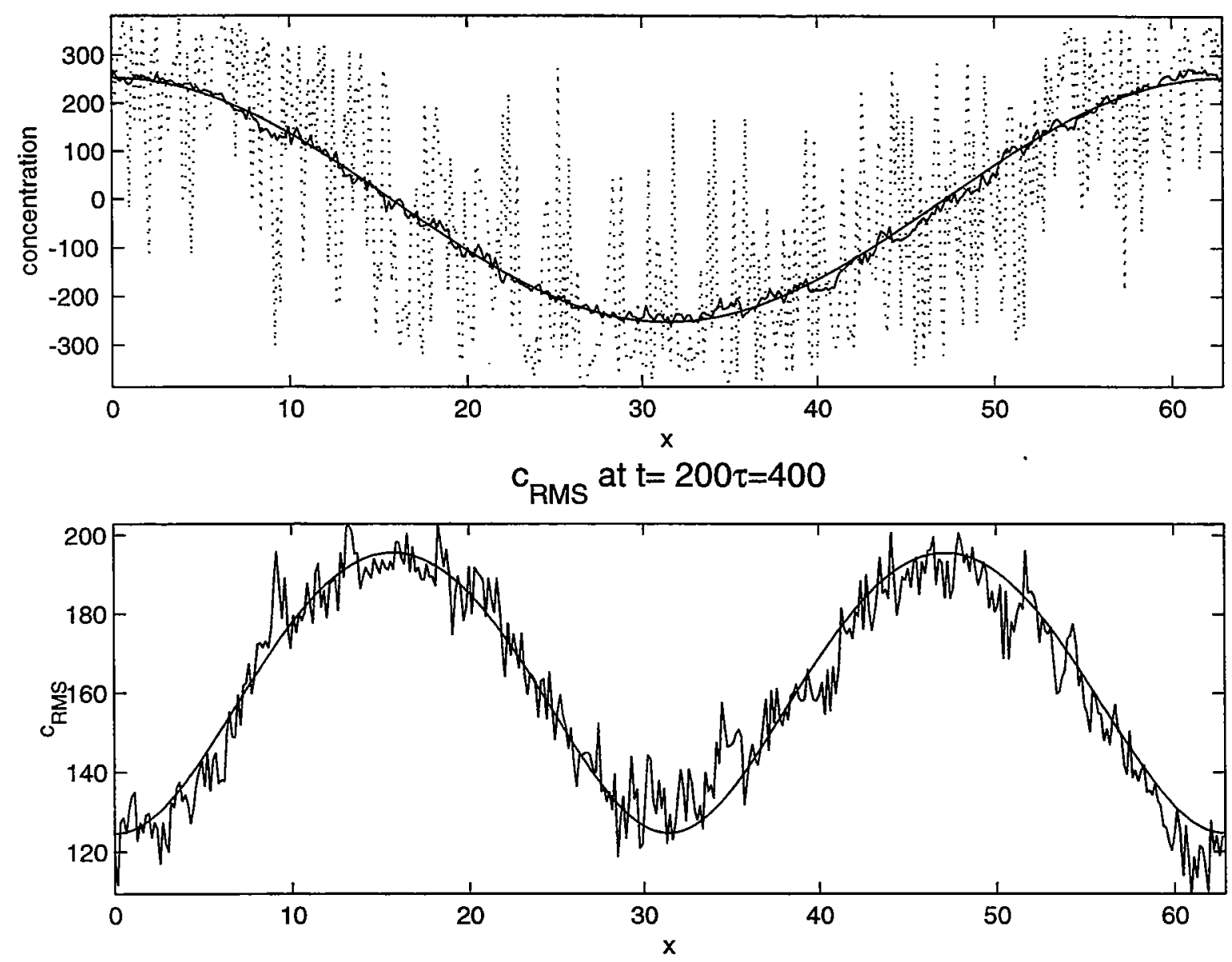

Figure 10: This figure compares analytic results with a numerical solution of (62), taking $q=1 / 10$, and using the RW model to generate $u$. The persistence parameter is $\tau_{*}=2$ and the results are at $t=400$ (that is, 200 renovation cycles). The concentration $c(x, 400)$ is calculated on a $400 \times 400$ grid using the method in appendix $B$. In the top panel there are three curves: the concentration as a function of $0<x<20 \pi$ along the line $y=0$ (the jagged dotted curve); the $y$-averaged concentration defined in (64); the analytic result in (59) (the smooth sinusoid). The bottom panel compares the $c_{R M S}=\sqrt{2 \mathcal{Z}}$ obtained by solving (60) analytically with $c_{R M S}$ estimated using (64).

which is the final result in (61).

It is interesting to compare the analytic results in (59) and (60) with a numerical solution of (62). Thus we must compute the spatial averages

$$
\bar{c}(x, t) \equiv \frac{1}{L} \int_{0}^{L} c(x, y, t) \mathrm{d} y, \quad c_{R M S}^{2}(x, t) \equiv \frac{1}{L} \int_{0}^{L}[c(x, y, t)-\bar{c}]^{2} \mathrm{~d} y
$$

using the numerical solution, and compare these with the analytic results for $\langle c\rangle$ and $\mathcal{Z}=c_{R M S}^{2} / 2$. The best way to make this comparison is to obtain $c(x, y, t)$ on a regular grid in the $(x, y)$-plane. As a bonus, one can then also use contouring routines to make pretty pictures of the concentration field (see figure 9).

The concentration field is calculated on a regular grid using the procedure described in Appendix $\mathrm{C}$ 
(essentially the method of characteristics). Figure 10 shows good agreement between this simulation and analytic results. Notice that in figure 10 the variance $\mathcal{Z}$ peaks where $\nabla(c)$ is greatest. This illustrates that concentration fluctuations are produced by advective distortion of the mean gradient: where the mean gradient is large there is lots of variance. But $\mathcal{Z} \neq 0$ even where $\nabla\langle c\rangle=0$ (for example, at $x=0$ and $x=10 \pi$ in figure 10). Thus, where the source term on the right hand side of (60) vanishes, the diffusive term $D \nabla^{2} \mathcal{Z}$ supplies variance.

\subsection{Cautionary remarks}

In the both examples above there is no molecular diffusion $(\kappa=0)$ and consequently there is no destruction of variance by the term $\kappa\left\langle\nabla c^{\prime} \cdot \nabla c^{\prime}\right\rangle$ in (50). As a project for a student, include molecular diffusion in the RW model (perhaps by pulsing diffusion in alternation with advection) and assess the efficacy of this process. In particular, can the closure in (52) be justified?

\section{References}

[1] S. Childress and A. D. Gilbert. Stretch, Twist, Fold: The Fast Dynamo. Springer, Berlin, 1995.

[2] H.K.Moffatt. Transport effects associated with turbulence with particular attention to the influence of helicity. Rep. Prog. Phys., 46:621-664, 1983.

[3] W.A. Medeiros. Marbling Techniques. Watson-Guptill, New York, 1994.

[4] G.I. Taylor. Eddy motion in the atmosphere. Phil. Trans. Roy. Soc. London, 215:1-16, 1915.

\section{A Calculation of the RW Green's function}

In this appendix we present an alternative calculation of the RW ensemble averaged Green's function, $g(r)$, in (17). The unaveraged Green's function, $G\left(\mathbf{x}, \mathbf{x}_{0}, t\right)$, is the solution of $(10)$. Because the process is spatially homogeneous it is harmless to take $\mathbf{x}_{0}=0$ so that

$$
G\left(\mathbf{x}, 0, \tau_{*}\right)=\delta\left[x-\tau_{*} s \sin \varphi\right] \delta\left[y+\tau_{*} c \sin \varphi\right]
$$

where $(s, c) \equiv(\sin \theta, \cos \theta)$. The ensemble average of $(65)$ is computed by integration over $\varphi$ and $\theta$, as in (4). It is very pleasant that there are two integrals and two $\delta$-functions. Thus, we first do the $\varphi$-integral by noting that $\delta\left[x-\tau_{*} s \sin \varphi\right]$ is nonzero at the two values of $\varphi$ where $\sin \varphi=x / \tau_{*} s$, and at those positions:

$$
\frac{\mathrm{d}}{\mathrm{d} \varphi}\left[x-\tau_{*} s \sin \varphi\right]= \pm \sqrt{\tau_{*}^{2} s^{2}-x^{2}} .
$$

Using the standard rule for changing variables in a $\delta$-function, we find that the average of (65) over $\varphi$ alone is

$$
\langle G\rangle_{\varphi}=\frac{1}{\pi} \frac{\delta(y+\cot \theta x)}{\sqrt{\tau_{*}^{2} \sin ^{2} \theta-x^{2}}} .
$$

The second integral over $\theta$ is performed by noting that $\delta(y+\cot \theta x)$ is nonzero at the two values of $\theta$ where $\cot \theta=-y / x$, and at those positions

$$
\sin ^{2} \theta=\frac{x^{2}}{x^{2}+y^{2}}, \quad \frac{\mathrm{d}}{\mathrm{d} \theta}[y+x \cot \theta]=-\frac{x^{2}+y^{2}}{x} .
$$

After changing variables in the $\delta$-function we recover $g(r)$ in (17). 


\section{B Eddy diffusion of variance}

Ignoring small molecular diffusion ( $\kappa=0$ ), if $c$ satisfies the advection equation then any function of $c$ satisfies the same equation. That is to say

$$
\frac{D c}{D t}=0, \quad \Longrightarrow \quad \frac{D f}{D t}=0,
$$

where $f(c)=c^{2}$, or $\exp (c)$, etcetera. Taking an ensemble average, and making the same arguments for $f(c)$ as for $c$, we have that

$$
\langle f\rangle_{t}=D \nabla^{2}\langle f\rangle \text {. }
$$

In the particular case $f=c^{2} / 2,\langle f\rangle=\langle c\rangle^{2} / 2+\mathcal{Z}$ and (70) reduces to

$$
\mathcal{Z}_{t}=D \nabla^{2} \mathcal{Z}+D \nabla\langle c\rangle \cdot \nabla\langle c\rangle
$$

Matching the terms in (71) with those in (50) we conclude that $\left\langle\mathbf{u}^{\prime} c^{\prime 2} / 2\right\rangle=-D \nabla \mathcal{Z}$.

\section{Numerical simulation of the RW process}

Drawing figures 9 and 10 requires that we obtain the solution of (62) on a regular grid in the $(x, y)$ plane. This is an opportunity to use the method of characteristics and learn some MATLAB programming techniques.

Equation (3) shows how the movement of a particle in the RW velocity field is equivalent to a random map which determines the position at $(n+1) \tau_{*}$ in terms of the previous position at $n \tau_{*}$. If this particle carries a concentration, $c(x, t)$, which changes because of the $\cos q x$ source in (62), then the concentration changes can also be calculated and expressed as a map in discrete time.

Thus, suppose that the concentration on a particle at time $t=n \tau_{*}$ is $c_{n}$. Then the change in concentration during $n \tau_{*}<t<(n+1) \tau_{*}$ is obtained by integrating

$$
\frac{D c}{D t}=\cos \left[q x_{n}+q u_{n}\left(t-n \tau_{*}\right)\right]
$$

where the constant $x$-velocity of the particle is $u_{n}=s_{n} \sin \left(c_{n} x_{n}+s_{n} y_{n}+\varphi_{n}\right)$, with $\left(s_{n}, c_{n}\right) \equiv$ $\left(\sin \theta_{n}, \cos \theta_{n}\right)$. The integral of (72) can be written as

$$
c_{n+1}=c_{n}+\frac{\sin \left(q x_{n+1}\right)-\sin \left(q x_{n}\right)}{q u_{n}} .
$$

With equations (73) and (3) we can advance forward in time and so determine the concentration on a particle at $t=n \tau_{*}$.

However we need to determine the concentration at $t=n \tau_{*}$ at a specified grid point $\mathbf{x}$. The trick is illustrated in the Matlab program below.

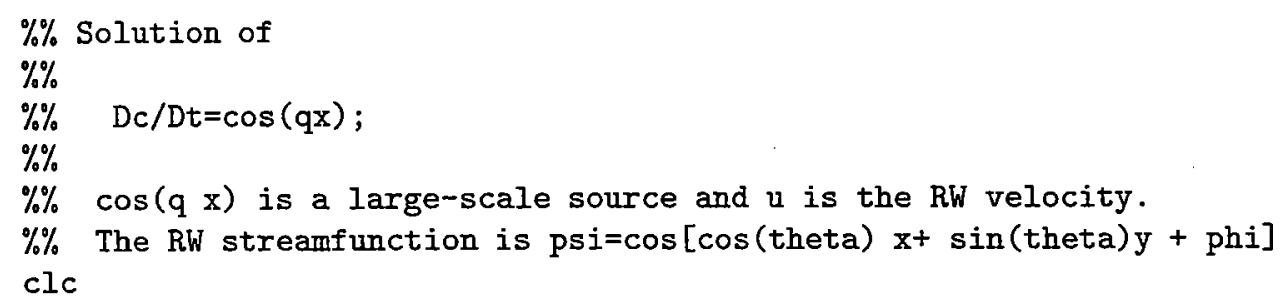




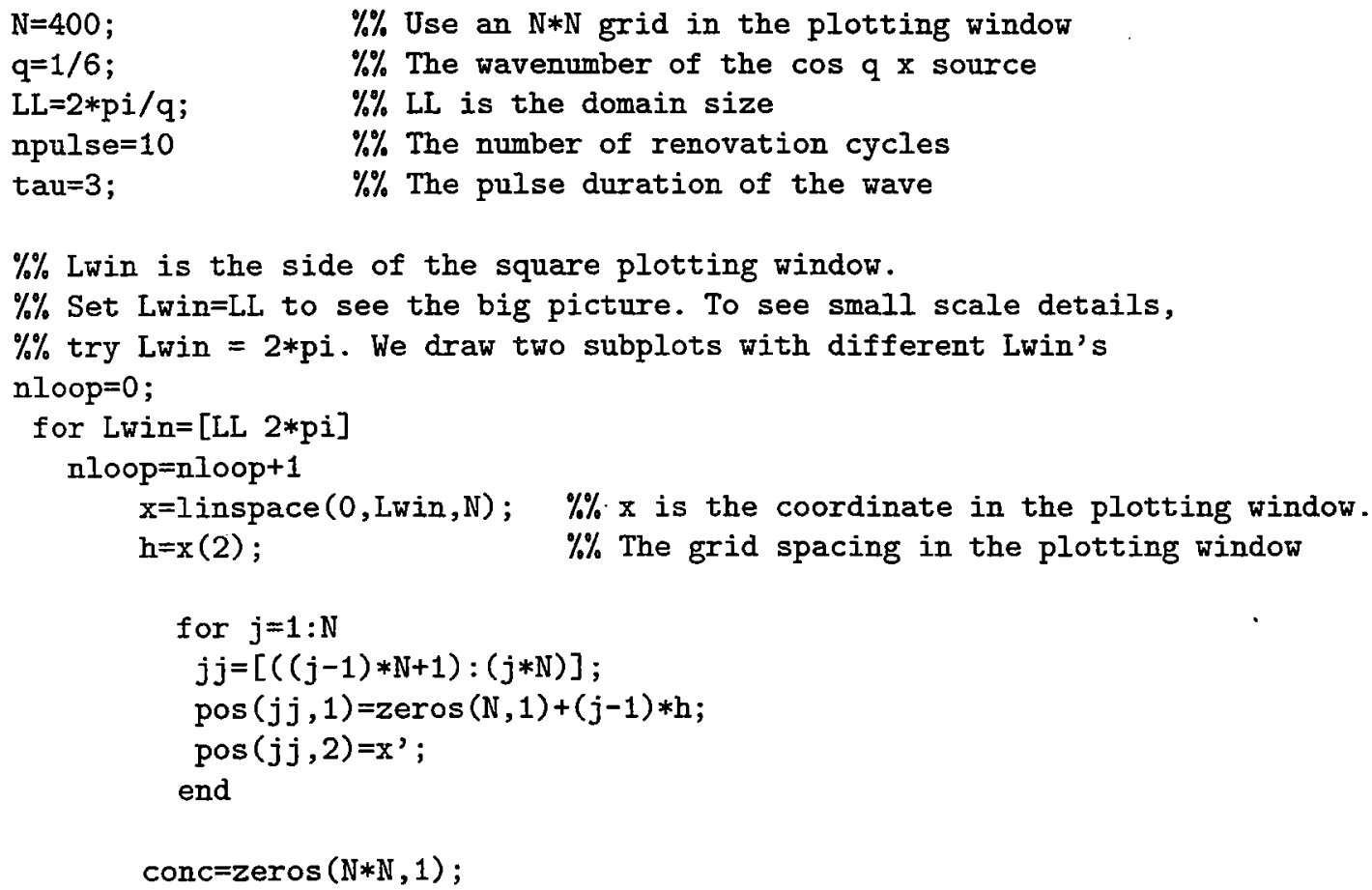

$\% \%$ Lwin is the side of the square plotting window.

$\%$ Set Lwin=LI to see the big picture. To see small scale details,

$\%$ try Lwin $=2 * p i$. We draw two subplots with different Iwin's

nloop=0;

for Lwin=[LL $2 *$ pi]

$\mathrm{nloop}=\mathrm{nloop}+1$

$\mathrm{x}=$ linspace $(0, \mathrm{Lwin}, \mathrm{N}) ; \% \mathrm{x}$ is the coordinate in the plotting window.

$\mathrm{h}=\mathrm{x}(2)$;

$\% \%$ The grid spacing in the plotting window

$\% \%$ The position of the $\mathrm{N}^{\wedge} 2$ particles are stored in pos with

$\% \% \mathrm{~N}^{\wedge} 2$ rows and 2 columns. Each vertical segment of

$\% \%$ length $\mathrm{N}$ in pos contains particles with the same initial $\mathrm{x}$-position.

$\% \%$ the column vector conc contains the concentration on the

$\% \% \mathrm{~N} * \mathrm{~N}$ particles in pos. Initially, conc $=0$ at the $\mathrm{N} * \mathrm{~N}$

$\% \%$ grid points. Then we integrate

$\% \%$ backwards in time to find the concentration change.

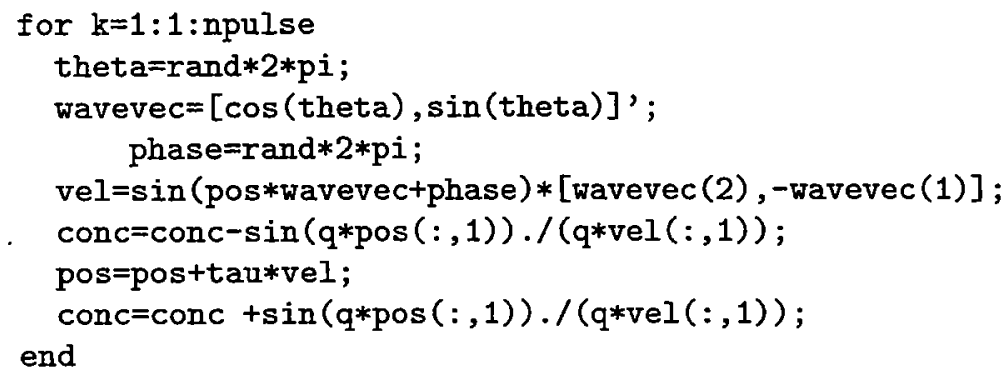

$\% \%$ Emerging from this loop, we have the the new positions

$\% \%$ and the new concentration

conc $=$ reshape $($ conc $, N, N) ; \%$ conc is reshaped into an $\mathrm{N} * \mathrm{~N}$ matrix

$\mathrm{hh}=\operatorname{subplot}(1,2, \mathrm{nloop})$

colormap ('gray')

imagesc $(x, x$, conc $)$

axis equal

$x$ label (' $x$ ')

ylabel ('y') 
$\operatorname{axis}$ ([0 Lwin 0 Lwin])

set (hh, 'ydir', 'norm')

end 


\section{Lecture 3: Stretching}

\section{Line stretching}

In the previous lecture we emphasized that the destruction of tracer variance by molecular diffusivity relies on the increase of $\nabla c$ by stirring. Thus the term $\kappa\left\langle\nabla c^{\prime} \cdot \nabla c^{\prime}\right\rangle$ in the variance budget eventually becomes important, even though the molecular diffusivity $\kappa$ is very small. One goal of this lecture is to understand in more detail how tracer gradients in a moving fluid are amplified by simple velocity fields. We will assume that $\kappa=0$ so that there is stirring without mixing. This is a good approximation provided that the smallest scale in the tracer field is much greater than the length $\ell=\sqrt{\kappa / \alpha}$ that we identified in lecture 1.

Gradient amplification is closely related to the stretching of material lines, a subject that was opened by Batchelor in 1952. A material line is a curve that consists always of the same fluid particles. Batchelor's main conclusion is that there is a timescale governing the ultimate growth of an infinitesimal line element, but no length scale other than that of the element itself. These dimensional considerations force the conclusion that the element grows exponentially,

$$
\ell=\ell_{0} \mathrm{e}^{\gamma t},
$$

where $\gamma$ is a constant with dimensions of inverse time, related to the timescale that Batchelor had in mind.

Just as some close particle pairs separate exponentially, other pairs starting at distant points are brought close together. This might seem paradoxical until one recalls the folded tracer patterns evident in Welander's 1955 experiments (see the final figures in lecture 1). If two closely approaching particles are carrying different values of $c$ then the gradient $\nabla c$ will be amplified. Thus, as a corollary of (1) we expect that $|\nabla c| \sim\left|\nabla c_{0}\right| \exp (\gamma t)$. It is through this exponential amplification of the concentration gradients that the small molecular diffusivity $\kappa$ is able eventually to destroy tracer variance.

\subsection{Material line elements and tracer gradients}

Using a geometric argument (see figure 1) we can give a proof-by-intimidation that a material line element, $\boldsymbol{\xi}(\boldsymbol{x}, t)$, attached to a fluid element evolves according to

$$
\frac{D \boldsymbol{\xi}}{D t}=(\boldsymbol{\xi} \cdot \nabla) \boldsymbol{u} .
$$

Here the "convective derivative" is $D / D t=\partial / \partial t+u \cdot \nabla$. The field of line elements can be visualized a collection of tiny straight arrows attached to each moving particle of fluid. Then (2) describes the evolution of this collection of arrows. Notice that (2) refers to an infinitesimal line element $\xi$. If the length of a material line is comparable to the scale of $u$ there is no longer a simple relation between the stretching of the material line and local properties of $u$, such as $\nabla \boldsymbol{u}$.

Taking the gradient of the tracer equation

$$
\frac{D c}{D t}=0
$$




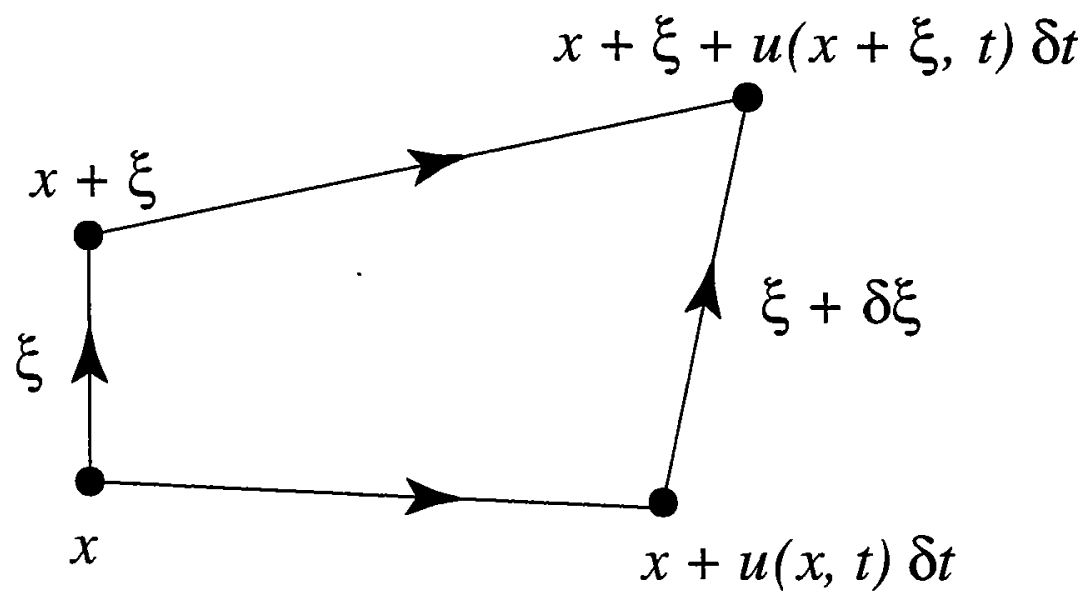

Figure 1: The line element $\boldsymbol{\xi}$ is short enough to remain straight and to experience a strain that is uniform over its length during the time $\delta t$. Proof by intimidation of $(2): \delta \xi=[\boldsymbol{u}(\boldsymbol{x}+\boldsymbol{\xi}, t)-\boldsymbol{u}(\boldsymbol{x}, t)] \delta t$, and take $(\delta t, \boldsymbol{\xi}, \delta \boldsymbol{\xi}) \rightarrow 0$.

gives

$$
\frac{D \nabla c}{D t}=-(\nabla c \cdot \nabla) u
$$

Despite the difference in the sign of the right hand sides of (2) and (4) there is a close connection between the solutions of the two equations.

To emphasize the connection between $\nabla c$ and $\xi$, we mention the conservation law

$$
\frac{D}{D t}(\nabla c \cdot \xi)=0 \text {. }
$$

(Meteorologists and oceanographers might recognize (5) as a relative of potential vorticity conservation.) Later in this lecture (5) is used to deduce $\nabla c$ from $\xi$.

The easy way to prove (5) is to consider a pair of particles separated by a small displacement $\xi$. If the concentration carried by the first particle is $c_{1}$, and that of the second particle is $c_{2}=c_{1}+\mathrm{d} c_{\text {, }}$ then $\mathrm{d} c=\xi \cdot \nabla c$. Thus (5) is equivalent to the "obvious" fact that $\mathrm{d} c$ is conserved as the two particles move.

The difficult way to prove (5) is to take the dot product of $\nabla c$ with (2) and add this to the dot product of $\boldsymbol{\xi}$ with (4). Performing some nonobvious algebra, perhaps with Mathematica or Maple, one can eventually simplify the mess to (5). Suffering through this tedious exercise will convince the student that the earlier, easy proof is worthy of serious attention.

\subsection{Eulerian versus Lagrangian: the golden rule}

Particle trajectories, $\boldsymbol{x}=\boldsymbol{x}\left(\boldsymbol{x}_{0}, t\right)$, are determined by solving the differential equations

$$
\frac{D x}{D t}=u(x, t), \quad x(0)=\dot{x}_{0} .
$$

The solution of the differential equation above defines the particle position, $x$, as a function of the two independent variables, $x_{0}$ and $t$. Using this time-dependent mapping between $x$ and $x_{0}$, we can take a problem posed in terms of $x$ and $t$ (the Eulerian formulation) and change variables to obtain an equivalent formulation in terms of $x_{0}$ and $t$ (the Lagrangian formulation). 
In the Eulerian view, the independent variables are $x=(x, y, z)$ and $t$. The convective derivative,

$$
\frac{D}{D t}=\frac{\partial}{\partial t}+u \frac{\partial}{\partial x}+v \frac{\partial}{\partial y}+w \frac{\partial}{\partial z}
$$

is a differential operator involving all of the independent variables.

In the Lagrangian view, the independent variables are $x_{0}$ and $t^{\prime}$ and $\boldsymbol{x}\left(\boldsymbol{x}_{0}, t^{\prime}\right)$ is a dependent variable. As an accounting device, the time variable is decorated with a prime to emphasize that a $t^{\prime}$-derivative means that the independent variables are $x_{0}$. To move between the Eulerian and Lagrangian representations notice that

$$
\frac{\partial t}{\partial t^{\prime}}=1, \quad \text { and } \quad \frac{\partial}{\partial t^{\prime}}(x, y, z)=(u, v, w) .
$$

The second equation above is the definition of velocity, $\boldsymbol{u}=(u, v, w)$.

Using (8), the rule for converting partial derivatives is

$$
\frac{\partial}{\partial t^{\prime}}=\frac{\partial}{\partial t}+\frac{\partial x}{\partial t^{\prime}} \frac{\partial}{\partial x}+\frac{\partial y}{\partial t^{\prime}} \frac{\partial}{\partial y}+\frac{\partial z}{\partial t^{\prime}} \frac{\partial}{\partial z}=\frac{D}{D t} .
$$

Equation (9) is the golden rule that enables us to interpret expressions such as

$$
\frac{D}{D t} \text { (unknown) }=\text { RHS }
$$

in either Eulerian or Lagrangian terms. Using the golden rule we can dispense with the prime that decorates the Lagrangian time variable.

In the Eulerian interpretation we must express the RHS in (10) as a function of $x, y, z$ and $t$ and use the Eulerian definition of the convective derivative in (7). Then (10) is a partial differential equation for the unknown.

In the Lagrangian interpretation $D / D t$ is the same as a simple time derivative and we must express the RHS of (10) as a function of $x_{0}, y_{0}, z_{0}$ and $t$. Then (10) is a ordinary differential equation for the unknown.

As an illustration of the transformation between Eulerian and Lagrangian variables, consider the steady, unidirectional velocity field $u=[u(y), 0]$. The solution of $(6)$ is

$$
x=x_{0}+u(y) t, \quad y=y_{0} .
$$

In this example it is a simple matter to express $(x, y)$ in terms of $\left(x_{0}, y_{0}\right)$ and vice versa.

The line-stretching equation, (2), has the same form as (10). For the same unidirectional velocity field, using components, $\boldsymbol{\xi}=(\xi, \eta)$, we have in Lagrangian variables

$$
\frac{D \xi}{D t}=\eta u^{\prime}\left(y_{0}\right), \quad \frac{D \eta}{D t}=0 \text {. }
$$

(We have used the golden rule (9) above.) Equation (12) is an ordinary differential equation and the solution is

$$
\xi=\xi_{0}\left(x_{0}, y_{0}\right)+t \eta_{0}\left(x_{0}, y_{0}\right) u^{i}\left(y_{0}\right), \quad \eta=\eta\left(x_{0}, y_{0}\right) .
$$

Using (11), we can write (13) in terms of Eulerian variables as

$$
\xi=\xi_{0}[x-u(y) t, y]+t \eta_{0}[x-u(y) t, y] u^{\prime}(y), \quad \eta=\eta_{0}[x-u(y) t, y] .
$$

We can alternatively view (12) in terms of Eulerian variables and in this case we are confronted with the partial differential equations

$$
\frac{\partial \xi}{\partial t}+u(y) \frac{\partial \xi}{\partial x}=\eta u^{\prime}(y), \quad \frac{\partial \eta}{\partial t}+u(y) \frac{\partial \eta}{\partial x}=0
$$

It is easy to check by substitution that (14) is the solution of (15). 


\subsection{Motion is equivalent to mapping}

We obtained (2) using the geometric argument in figure 1 . Now we admire some different scenery by taking an algebraic path to (2). Our itinerary emphasizes that the solutions of (6) define a mapping of the space $x_{0}$ of initial coordinates onto the space $x$, and hence the title of this section.

Using indicial notation (summation implied over repeated indices), it follows from the chain rule that

$$
\mathrm{d} \boldsymbol{x}_{i}=\frac{\partial \boldsymbol{x}_{i}}{\partial \boldsymbol{x}_{0 j}} \mathrm{~d} \boldsymbol{x}_{0 j}
$$

Taking the time derivative of (16), and keeping in mind that $x_{0 j}$ is independent of $t$, gives

$$
\frac{D}{D t}\left(\mathrm{~d} \boldsymbol{x}_{i}\right)=\frac{\partial u_{i}}{\partial x_{0 j}} \mathrm{~d} x_{0 j}=\frac{\partial u_{i}}{\partial x_{0 j}} \frac{\partial x_{0 j}}{\partial x_{k}} \mathrm{~d} x_{k}=\frac{\partial u_{i}}{\partial x_{j}} \mathrm{~d} x_{j} .
$$

(We have used the golden rule.) Making the identification $\mathrm{d} x \rightarrow \boldsymbol{\xi}$ we obtain (2).

The motion of a fluid defines a family of mappings from the space of initial coordinates, $x_{0}$, onto the space of coordinates $\boldsymbol{x}$. At $t=0$ this is just the identity map but as $t$ increases the map from $x_{0}$ to $x$ can become very complicated. Equation (16) defines the Jacobian matrix,

$$
\mathcal{J}_{i j} \equiv \frac{\partial \boldsymbol{x}_{i}}{\partial \boldsymbol{x}_{0 j}}
$$

of the map.

With these algebraic formalities we have given an alternative derivation of (2) and, as a bonus, we have also found a representation of the solution:

$$
\xi=\mathcal{J} \xi_{0} .
$$

The solution above is known as Cauchy's solution.

In (19) there is no assumption that the flow is incompressible. If the flow is incompressible (i.e., if $\boldsymbol{\nabla} \cdot \boldsymbol{u}=0$ ) then mapping from $\boldsymbol{x}_{0}$ to $\boldsymbol{x}$ conserves volume. In this case, $\operatorname{det} \mathcal{J}=1$.

\section{Two-dimensional incompressible flow}

In the case of a two-dimensional incompressible flow there is a streamfunction $\psi=\psi(\boldsymbol{x}, t)$ such that $\boldsymbol{u}=(u, v)=\left(-\psi_{y}, \psi_{x}\right)$. In terms of $\psi,(2)$ can be written as:

$$
\frac{D \boldsymbol{\xi}}{D t}=\boldsymbol{W} \boldsymbol{\xi}, \quad \text { where } \quad \boldsymbol{W} \equiv\left(\begin{array}{cc}
-\psi_{x y} & -\psi_{y y} \\
\psi_{x x} & \psi_{x y}
\end{array}\right) .
$$

The trace of $\boldsymbol{W}$ is zero and the determinant is $\operatorname{det}(\boldsymbol{W})=\psi_{x x} \psi_{y y}-\psi_{x y}^{2}$. The solution of (20) can be written as

$$
\xi=\exp \left(\int_{0}^{t} W\left(t^{\prime}\right) \mathrm{d} t^{\prime}\right) \xi_{0}
$$

Thus, using (19), we obtain a fundamental connection between $\mathcal{J}(t)$ and $\boldsymbol{W}(t)$ :

$$
\mathcal{J}(t)=\exp \left(\int_{0}^{t} W\left(t^{\prime}\right) \mathrm{d} t^{\prime}\right)
$$

Because $\operatorname{tr} W=0$ it follows ${ }^{1}$ that $\operatorname{det} \mathcal{J}=1$. This is, of course, just another way of saying that if the flow is incompressible then the map from $x_{0}$ to $x$ is area preserving.

\footnotetext{
${ }^{1}$ For a square matrix $M$
}

$$
\operatorname{det} \mathrm{e}^{M}=\mathrm{e}^{\operatorname{tr} M} .
$$


$\operatorname{det} W>0$

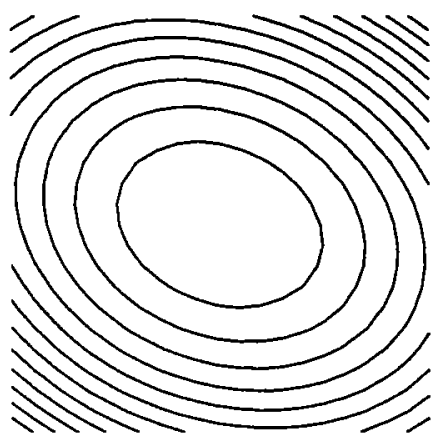

$\operatorname{det} W<0$

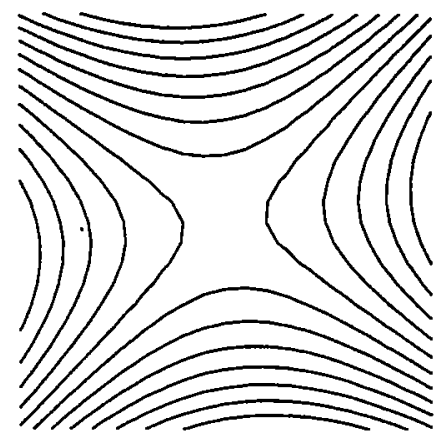

$\operatorname{det} W=0$

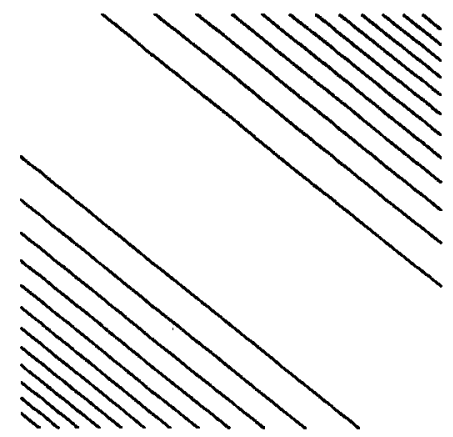

Figure 2: The sign of $\operatorname{det}(\boldsymbol{W})=\psi_{x x} \psi_{y y}-\psi_{x y}^{2}$ determines the streamline pattern.

\subsection{The steady case}

Because (20) is linear the solution is straightforward if the velocity field in the Lagrangian frame is steady. Thus

$$
\xi(t)=\mathrm{e}^{\gamma t} \hat{\xi}, \quad \Rightarrow \quad \gamma= \pm \sqrt{-\operatorname{det} \boldsymbol{W}}
$$

where

$$
\operatorname{det} \boldsymbol{W}=\psi_{x x} \psi_{y y}-\psi_{x y}^{2} .
$$

There are three cases, which correspond to the three panels in figure 2:

Elliptic: If $\operatorname{det} \boldsymbol{W}>0$, then $\gamma$ is imaginary and the local streamfunction has elliptic streamlines; $\boldsymbol{\xi}$ changes periodically in time and there is no exponential stretching.

Hyperbolic: If $\operatorname{det} W<0$ then $\gamma$ is real and the streamfunction is locally hyperbolic. Then, as in lecture 1, material line elements will be stretched exponentially in one direction and compressed in the other.

Transitional: If $\operatorname{det} \boldsymbol{W}=0$ then $|\boldsymbol{\xi}|$ grows linearly with time.

Following Okubo (1970) and Weiss (1991), the sign of det $W$ has been used to diagnose twodimensional turbulence simulations (e.g., McWilliams 1984). Assuming that $\operatorname{det} W$ is changing slowly in the Lagrangian frame, one argues that the result in (23) applies "quasistatically". For instance, using simulations of two-dimensional turbulence, McWilliams shows that in the core of a strong vortex $\psi_{x x} \psi_{y y}-\psi_{x y}^{2}>0$. The interpretation is that there is no exponential stretching of line elements in vortex cores, which indicates that these regions are isolated patches of laminar flow. This so-called Okubo-Weiss criterion is only a rough guide to the stretching properties of complicated flows; for a critique and more refined results see Hua and Klein (1999).

One pleasant aspect of the steady two-dimensional case is that it is possible to explicitly calculate the matrix exponential $\mathcal{J}(t)=\exp (t \boldsymbol{W})$. (This is not the case in three dimensions.) Begin by noting that

$$
W^{2}+(\operatorname{det} W) \mathcal{I}=0,
$$

where $\mathcal{I}$ is the $2 \times 2$ identity matrix. The result above is easily checked by direct evaluation, but (25) is also a consequence of $\operatorname{tr} W=0$ and the Cayley-Hamilton theorem. When (25) is substituted into 
the definition of the matrix exponential:

$$
\mathcal{J}=\exp (t W)=\mathcal{I}+t W+\frac{t^{2}}{2} W^{2}+\frac{t^{3}}{6} W^{3}+\cdots
$$

the sum collapses to

$$
\mathcal{J}=\cos (\sqrt{\operatorname{det} W} t) \mathcal{I}+\frac{\sin (\sqrt{\operatorname{det} W} t)}{\sqrt{\operatorname{det} W}} \boldsymbol{W} .
$$

We now use the result above to formulate a renovation model.

\subsection{The $\sigma-\zeta$ model}

We construct the " $\sigma-\zeta$ " model using the matrix equation in (20). The idea is to define an ensemble of stretching flows in which the $2 \times 2$ matrix $W$ is piecewise constant in the intervals $\mathcal{I}_{n}=\{t:(n-1) \tau<$ $t<n \tau\} ; \tau$ is the "decorrelation time". We use the following representation of $W$ in the interval $\mathcal{I}_{n}$ :

$$
\boldsymbol{W}_{n}=\frac{\zeta_{n}}{2}\left(\begin{array}{cc}
0 & -1 \\
1 & 0
\end{array}\right)+\frac{\sigma_{n}}{2}\left(\begin{array}{cc}
-\cos 2 \theta_{n} & \sin 2 \theta_{n} \\
\sin 2 \theta_{n} & \cos 2 \theta_{n}
\end{array}\right) .
$$

$\zeta_{n}$ is the vorticity and $\sigma_{n}$ the strain. Isotropy is ensured by picking the random angle $0<\theta_{n}<2 \pi$ from a uniform density. (We use $2 \theta_{n}$ because the principal strain axes are at angle $\theta_{n}$ to the coordinate axes, and tbey specify a orientation but not an direction. That is, the strain axes are like vectors without an arrow.)

Because $W_{n}$ is constant in $\mathcal{I}_{n}$ the calculation of stretching rates can be reduced to a product of random matrices. The terms in the product are $\exp \left(\tau \boldsymbol{W}_{n}\right)$ and, using (27), one can obtain this matrix exponential analytically. There is an extensive and difficult literature devoted to calculating the statistical properties of products of random matrices (e.g., Crisanti, Paladin \& Vulpiani, 1993). It is fortunate that we can avoid these complications by using the isotropy of the $\sigma-\zeta$ model to reduce averages of matrix products to averages of scalar products.

Two important properties of $\boldsymbol{W}_{n}$ are easily related to the vorticity and the strain:

$$
\operatorname{det} \boldsymbol{W}_{n}=\frac{1}{4}\left(\zeta_{n}^{2}-\sigma_{n}^{2}\right), \quad \operatorname{tr}\left(\boldsymbol{W}_{n}^{\mathrm{T}} \boldsymbol{W}_{n}\right)=\frac{1}{2}\left(\zeta_{n}^{2}+\sigma_{n}^{2}\right)
$$

In the examples that follow we will use $\sigma-\zeta$ ensembles which model spatially homogeneous flows, for which $\left\langle\sigma^{2}\right\rangle=\left\langle\zeta^{2}\right\rangle$ (by the way: this is not obvious). In this case $\left\langle\operatorname{det} W_{n}\right\rangle=0$ and "on average" the Okubo-Weiss criterion is zero.

We employ (27) to obtain an explicit expression for the matrix $\mathcal{J}_{n}=\exp \left(\tau \boldsymbol{W}_{n}\right)$. It turns out that we do not need the full details: all that is required is

$$
\frac{1}{2} \operatorname{tr}\left(\mathcal{J}_{n}^{\mathrm{T}} \mathcal{J}_{n}\right)=1+\Xi\left(\sigma_{n}, \tau_{n}, \tau\right)
$$

where

$$
\Xi(\sigma, \zeta, \tau) \equiv \frac{\sigma^{2}}{\zeta^{2}-\sigma^{2}}\left[1-\cos \left(\sqrt{\zeta^{2}-\sigma^{2}} \tau\right)\right] .
$$

The "trace formula" above should be known to experts on two-dimensional stretching problems, but I have not found (30) in the literature. 


\subsubsection{Stretching of squared length}

Consider the first interval $\mathcal{I}_{1}$, and suppose that at $t=0, \xi=\ell_{0}(\cos \chi, \sin \chi)$. At $t=\tau$ we have

$$
\ell_{1}^{2}=\xi_{0}^{\mathrm{T}} \mathcal{J}_{1}^{\mathrm{T}} \mathcal{J}_{1} \xi_{0}
$$

Now we use isotropy to average (32) over the random direction $\chi$ of the element $\xi_{0}$. A trivial calculation gives

$$
\left\langle\left(\ell_{1} / \ell_{0}\right)^{2}\right\rangle_{\chi}=\frac{1}{2} \operatorname{tr}\left(\mathcal{J}_{1}^{\mathrm{T}} \mathcal{J}_{1}\right)
$$

The RHS of (33) is given explicitly in (30). We must still average over the random variables $\sigma$ and $\zeta$ : This gives

$$
\left\langle\left(\ell_{1} / \ell_{0}\right)^{2}\right\rangle=1+\iint \mathcal{P}(\sigma, \zeta) \Xi(\sigma, \zeta, \tau) \mathrm{d} \sigma \mathrm{d} \zeta
$$

where $\mathcal{P}(\sigma, \zeta)$ is the joint PDF of $\sigma$ and $\zeta^{2}$.

We are now well on our way to computing the rate at which $\ell^{2}$ grows with the number of renovation cycles, $n$. The average stretching of $\ell^{2}$ in each $\mathcal{I}_{n}$ is independent of the previous $\mathcal{I}^{\prime}$ s. Thus, to compute the growth of $\ell^{2}$ over $n$ renovation cycles, we can simply raise the average $\ell^{2}$-stretching factor in a single $\mathcal{I}$ to the $n^{\prime}$ th power:

$$
\left\langle\left(\ell_{n} / \ell_{0}\right)^{2}\right\rangle=\left\{1+\iint \mathcal{P}(\sigma, \zeta) \Xi(\sigma, \zeta, \tau) \mathrm{d} \sigma \mathrm{d} \zeta\right\}^{n}
$$

The stretching rate $\gamma_{2}$ is defined by

$$
\gamma_{2} \equiv \lim _{t \rightarrow \infty} \frac{1}{t} \ln \left[\left\langle\left(\ell_{n} / \ell_{0}\right)^{2}\right\rangle^{1 / 2}\right] .
$$

The notation $\gamma_{2}$ anticipates section 4 in which we will define a stretching rate $\gamma_{p}$ which measures the growth of $\left\langle\left(\ell_{n} / \ell_{0}\right)^{p}\right\rangle$.

Using $n=t / \tau$, we have from (35)

$$
\gamma_{2}=\frac{1}{2 \tau} \ln \left\{1+\iint \mathcal{P}(\sigma, \zeta) \Xi(\sigma, \zeta, \tau) \mathrm{d} \sigma \mathrm{d} \zeta\right\}
$$

To further simplify the integral above we must specify the probability density function $\mathcal{P}(\sigma, \zeta)$ (examples follow).

\subsubsection{Randomly oriented Couette flows}

As a first example, suppose that in each $\mathcal{I}_{n}$ the random variables $\zeta_{n}$ and $\sigma_{n}$ are independently and identically distributed, each equal to $\pm \beta$ with equal probability. In this case

$$
\mathcal{P}(\sigma, \zeta)=\frac{1}{4}[\delta(\sigma+\beta)+\delta(\sigma-\beta)][\delta(\zeta+\beta)+\delta(\zeta-\beta)] .
$$

This ensemble is a set of randomly oriented Couette flow, such as the third case in figure 2. According to the Okubo-Weiss criterion there should be no stretching because $\operatorname{det} \boldsymbol{W}$ is identically zero. However, this is wrong.

\footnotetext{
2If $\sigma$ and $\zeta$ are independent and identically distributed random varaibles then $\mathcal{P}(\sigma, \zeta)=\hat{\mathcal{P}}(\sigma) \hat{\mathcal{P}}(\zeta)$.
} 


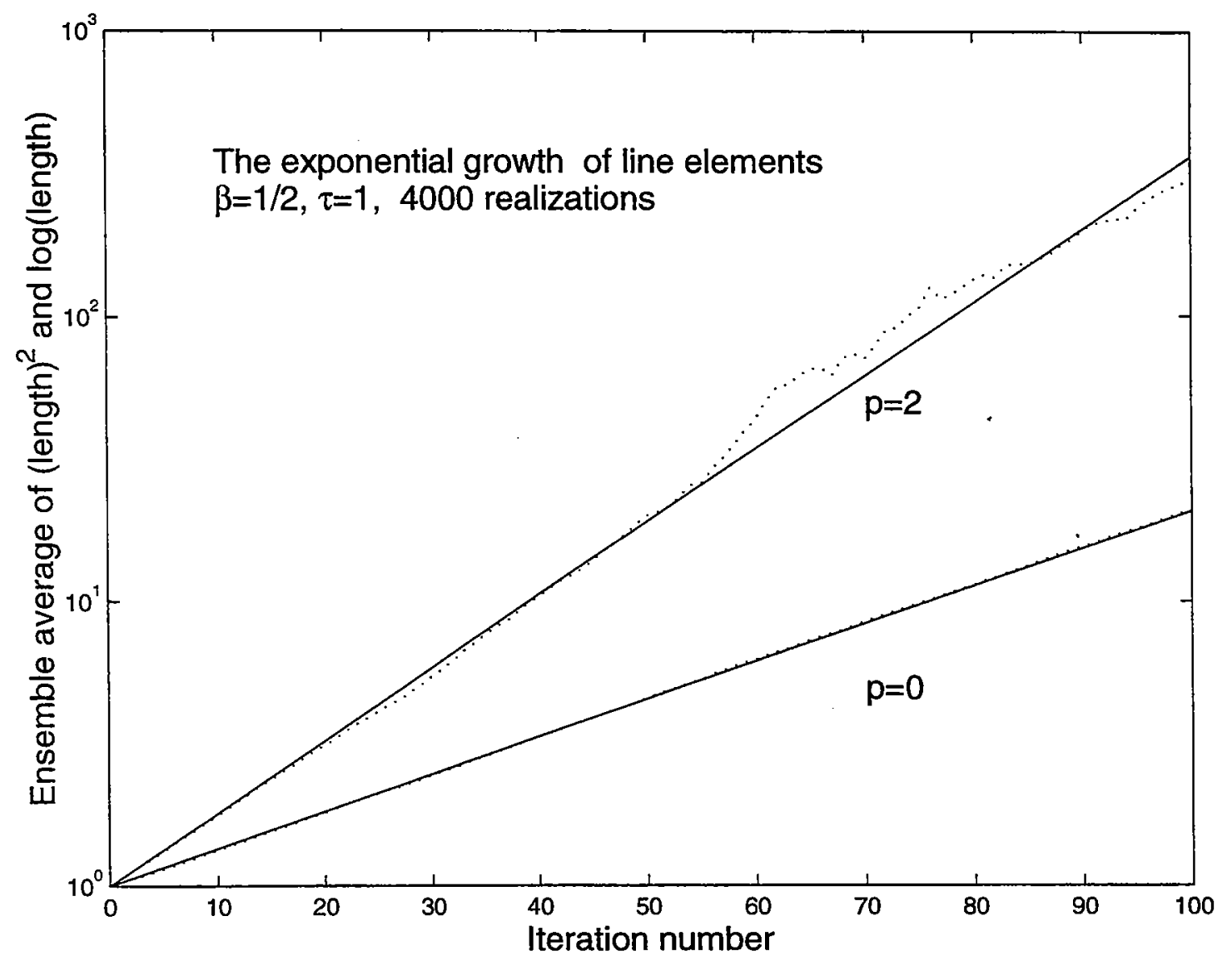

Figure 3: A comparison of the exponent $\gamma_{2}$ in (40) with a simulation (the dotted curves) of the random Couette flow. The simulation is conducted by creating random matrices according to the recipe in (28) and (38), and then computing the matrix product. The iteration number is the number of matrices in the product. To get reasonable agreement between the simulation and the analytic result in (40) one must ensemble average over a large number of realizations ( 4000 in the figure above). The discrepancies evident at large iteration number can be reduced by using more realizations. The figure also shows a comparison of the exponent $\gamma_{0}$ in (73) with simulation.

The recipe in (38) leads to trivial calculations because $\zeta_{n}^{2}=\sigma_{n}^{2}$ and $\Xi=\beta^{2} \tau^{2} / 2$. Thus, even without averaging over $\sigma$ and $\zeta$,

$$
\frac{1}{2} \operatorname{tr}\left(\mathcal{J}_{n}^{\mathrm{T}} \mathcal{J}_{n}\right)=1+\frac{1}{2} \beta^{2} \tau^{2}
$$

and it follows that

$$
\gamma_{2}=\frac{1}{2 \tau} \ln \left(1+\frac{\beta^{2} \tau^{2}}{2}\right)
$$

(See Figure 3.) The nonzero exponential stretching, which occurs even though $\operatorname{det} \boldsymbol{W}=0$, is due to the realignment of a material element with respect to the direction of extension of the velocity field which occurs at $t=n \tau$. In the limit of a very slowly changing velocity field, $\tau \rightarrow \infty$, the stretching rate vanishes because there are fewer realignment events. This is the revenge of the Okubo-Weiss criterion. 


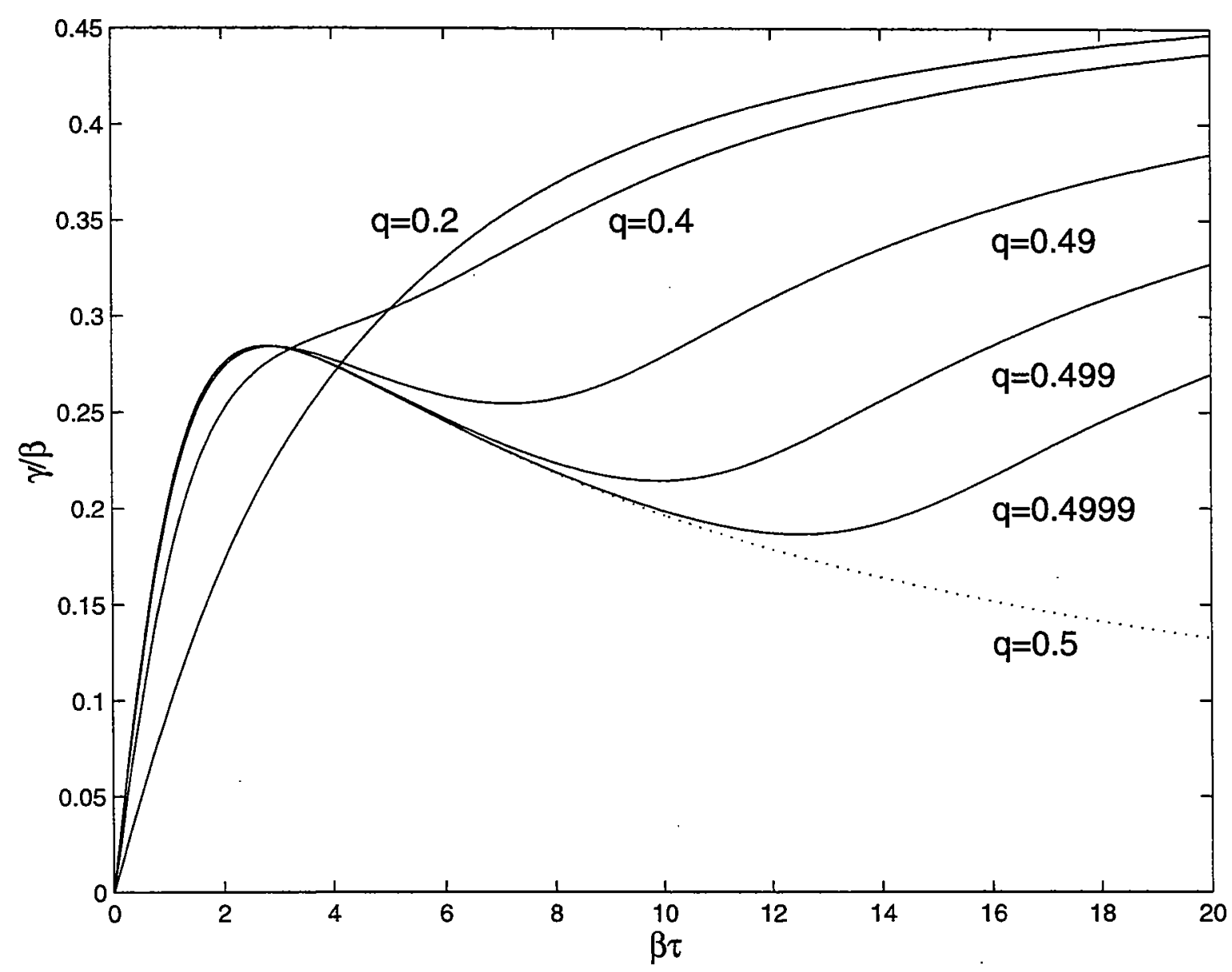

Figure 4: The nondimensional stretching exponents $\gamma_{2} / \beta$ in (41) as a function of $\beta \tau$ for various values of $q$. If $q=1 / 2$, then $\operatorname{det} W$ is zero identically and $\gamma \rightarrow 0$ as $\tau \rightarrow \infty$. When slightly less than $1 / 2$, and $\tau$ is sufficiently large, the occasional hyperbolic points can make a large contribution to the stretching exponent $\gamma_{2}$.

\subsubsection{An example with $\operatorname{det} W \neq 0$}

A more interesting stretching ensemble is defined by taking $\sigma_{n}$ and $\zeta_{n}$ to be identical and independently distributed random variables equal to $\beta$ with probability $q,-\beta$ with probability $q$, or zero with probability $1-2 q$. With this prescription there is a hyperbolic point in $\mathcal{I}_{n}$, as in the middle panel of figure 2, with probability $2 q(1-2 q)$.

One can calculate $\gamma_{2}$ in (37) by enumeration and averaging over the nine possible pairs $\left(\sigma_{n}, \zeta_{n}\right)$. Calculation gives

$$
\gamma_{2}=\frac{1}{2 \tau} \ln \left\{1+2 q^{2} \beta^{2} \tau^{2}+2 q(1-2 q)(\cosh \beta \tau-1)\right\} .
$$

Figure 4 shows the nondimensional $\gamma_{2} / \beta$ as a function of $\beta \tau$ for various values of $q$. From figure 4 we conclude that while instantaneous hyperbolic points are not essential for exponential stretching, they do help, especially if the correlation time $\tau$ is long. 


\subsubsection{The Batchelor and Kraichnan limits}

The calculation of stretching exponents in this section does not follow the historical path. The pioneering papers by Batchelor (1959) and Kraichnan (1974) considered limiting cases - slowly decorrelating in the case of Batchelor and rapidly decorrelating in the case of Kraichnan - in which stretching rates can be calculated approximately. A major advantage of these approximations is that they work equally well in two and three dimensional space. On the other hand, by considering exactly soluble two-dimensional models we can extract the Batchelor and Kraichnan limits as special cases.

Batchelor (1959) considered stretching by slowly decorrelating velocity fields. This is the limit in which $\zeta \tau$ and $\sigma \tau$ are large. Batchelor's main conclusion is that in this quasisteady limit the net stretching is dominated by hyperbolic straining events. Indeed, this conclusion is illustrated by the exact result for $\gamma_{2}$ which is plotted in figure 4 .

Kraichnan (1974) considered the opposite limit in which $\zeta \tau$ and $\sigma \tau$ are small. In this rapidly decorrelating limit we can simplify the exact expression in (37) by noting that $\Xi \approx(\sigma \tau)^{2} / 2 \ll 1$. Thus, simplifying (37), we find that the stretching rate is

$$
\gamma_{2} \approx \frac{1}{4}\left\langle\sigma^{2}\right\rangle \tau
$$

independent of the vorticity.

\subsection{The renovating wave model}

In this section we calculate the average growth of $\ell^{2}$ using the renovating wave (RW) model. It is interesting to see how this calculation can be done without using matrix identities such as (27).

Begin by recalling the definition of the RW model. The RW streamfunction is

$$
\mathcal{I}_{n}=(n-1) \tau_{*}<t<n \tau_{*}: \quad \psi_{n} \equiv \cos \left[\cos \theta_{n} x+\sin \theta_{n} y+\varphi_{n}\right] .
$$

In (43), $\theta_{n}$ and $\varphi_{n}$ are random phases and $\tau_{*}$ is the decorrelation time. The random phases are reinitialized at $t=n \tau_{*}$ so there is the complete and sudden loss of memory at these instants. (In this section we use the dimensionless version of the RW model; the parameter $\tau_{*} \equiv \tau k U$ is the ratio of the correlation time $\tau$ to the maximum shear of the sinusoidal wave $k U$.)

The renovating wave model is equivalent to the random map

$$
\left(x_{n+1}, y_{n+1}\right)=\left(x_{n}, y_{n}\right)+\left(s_{n},-c_{n}\right) \sin \left[c_{n} x_{n}+s_{n} y_{n}+\varphi_{n}\right] \tau_{*},
$$

where $\left(c_{n}, s_{n}\right) \equiv\left(\cos \theta_{n}, \sin \theta_{n}\right)$. The Jacobian matrix can easily be obtained by differentiation of (44):

$$
\mathcal{J}^{(n)}=\exp \left(\tau_{*} W^{(n)}\right)=\left[\begin{array}{cc}
1+c_{n} s_{n} \tau_{*} \psi_{n} & s_{n}^{2} \tau_{*} \psi_{n} \\
-c_{n}^{2} \tau_{*} \psi_{n} & 1-c_{n} s_{n} \tau_{*} \psi_{n}
\end{array}\right]
$$

Notice that $\operatorname{det} \mathcal{J}^{(n)}=1$ : the map is area preserving.

Using $\mathcal{J}^{(n)}$ we can track the stretching of an infinitesimal material element as

$$
\xi_{n+1}=\mathcal{J}^{(n)} \xi_{n}, \quad \Rightarrow \quad \ell_{n+1}^{2}=\xi_{n+1}^{\mathrm{T}} \xi_{n+1}=\xi_{n}^{\mathrm{T}} \mathcal{K}^{(n)} \xi_{n}
$$

where $\mathcal{K}^{(n)}=\mathcal{J}^{(n) \mathrm{T}} \mathcal{J}^{(n)}$. Explicitly:

$$
\mathcal{K}^{(n)}=\left[\begin{array}{cc}
\left(1+c_{n} s_{n} \psi_{n} \tau_{*}\right)^{2}+c_{n}^{4} \psi_{n}^{2} \tau_{*}^{2} & \left(s_{n}^{2}-c_{n}^{2}\right) \psi_{n} \tau_{*}+c_{n} s_{n} \psi_{n}^{2} \tau_{*}^{2} \\
\left(s_{n}^{2}-c_{n}^{2}\right) \psi_{n} \tau_{*}+c_{n} s_{n} \psi_{n}^{2} \tau_{*}^{2} & \left(1-c_{n} s_{n} \psi_{n} \tau_{*}\right)^{2}+s_{n}^{4} \psi_{n}^{2} \tau_{*}^{2}
\end{array}\right] .
$$


To compute the stretching rate we consider an element which has length $\ell_{0}$ at $t=0$. Because the problem is isotropic, it is harmless to choose the coordinate system so that this element lies along the $x$-axis: $\xi_{0}=\ell_{0}(1,0)$. After the first iteration of the map:

$$
\ell_{1}^{2}=\mathcal{K}_{11}^{(1)} \ell_{0}^{2}=\left[\left(1+c_{1} s_{1} \psi_{1} \tau_{*}\right)^{2}+c_{1}^{4} \psi_{1}^{2} \tau_{*}^{2}\right] \ell_{0}^{2} .
$$

Averaging (48) over the phases $\theta_{1}$ and $\varphi_{1}$ gives

$$
\left\langle\left(\ell_{1} / \ell_{0}\right)^{2}\right\rangle=\left(1+\frac{\tau_{*}^{2}}{4}\right)
$$

If you are suspicious of the argument above, then you might prefer to align the initial material element at an arbitrary angle, say $\xi_{0}=\ell_{0}(\cos \chi, \sin \chi)$. Repeating the calculation, we now find that

$$
\ell_{1}^{2}=\left(\mathcal{K}_{11}^{(1)} \cos ^{2} \chi+\mathcal{K}_{22}^{(1)} \sin ^{2} \chi\right) \ell_{0}^{2}
$$

Averaging (50) over $\theta_{1}$ and $\varphi_{1}$, we recover (49).

Because each $\mathcal{J}^{(n)}$ is independent of the earlier $\mathcal{J}$ 's the average growth of $\ell^{2}$ is

$$
\left\langle\left(\ell_{n} / \ell_{0}\right)^{2}\right\rangle=\left(1+\frac{\tau_{*}^{2}}{4}\right)^{n}
$$

Using $t=n \tau_{*},(51)$ can be written as

$$
\left\langle\left(\ell_{n} / \ell_{0}\right)^{2}\right\rangle^{1 / 2}=\mathrm{e}^{\gamma_{2} t}, \quad \gamma_{2} \equiv \frac{1}{2 \tau_{*}} \ln \left(1+\frac{\tau_{*}^{2}}{4}\right) .
$$

Aside from notational differences, the expression above for $\gamma_{2}$ is identical to (40).

\section{Amplification of concentration gradients}

In this section we discuss the amplification of $\nabla c$ which occurs when a passive scalar is advected by a random flow in two dimensions.

Back in (4) we noted that the quantity $\xi \cdot \nabla c$ satisfies the conservation equation

$$
\frac{D}{D t}(\xi \cdot \nabla c)=0 .
$$

Equation (53) enables us to use our earlier results concerning the stretching of material elements to analyze gradient amplification. In fact, using (53), we can obtain $\nabla c$ from $\xi$. The first step is to construct a basis by considering the following initial value problem:

$$
\frac{D \boldsymbol{\xi}_{k}}{D t}=\left(\boldsymbol{\xi}_{k} \cdot \boldsymbol{\nabla}\right) \boldsymbol{u}, \quad \text { with initial conditions } \quad \boldsymbol{\xi}_{1}(\boldsymbol{x}, 0)=\hat{\boldsymbol{x}}, \quad \boldsymbol{\xi}_{2}(\boldsymbol{x}, 0)=\hat{\boldsymbol{y}}
$$

where the unit vectors of the coordinate system are $\hat{\boldsymbol{x}}, \hat{\boldsymbol{y}}, \hat{\boldsymbol{z}}$. As the fluid moves, the parallelogram spanned by $\xi_{1}$ and $\xi_{2}$ will deform. But because $u$ is incompressible, the area of the parallelogram is constant and so

$$
\xi_{1} \times \xi_{2}=\hat{z}, \quad(\text { for all } t) .
$$

If we can solve (54) for $\xi_{1}$, then we can use (53) and (55) to calculate $\xi_{2}$ and $\nabla c$. 

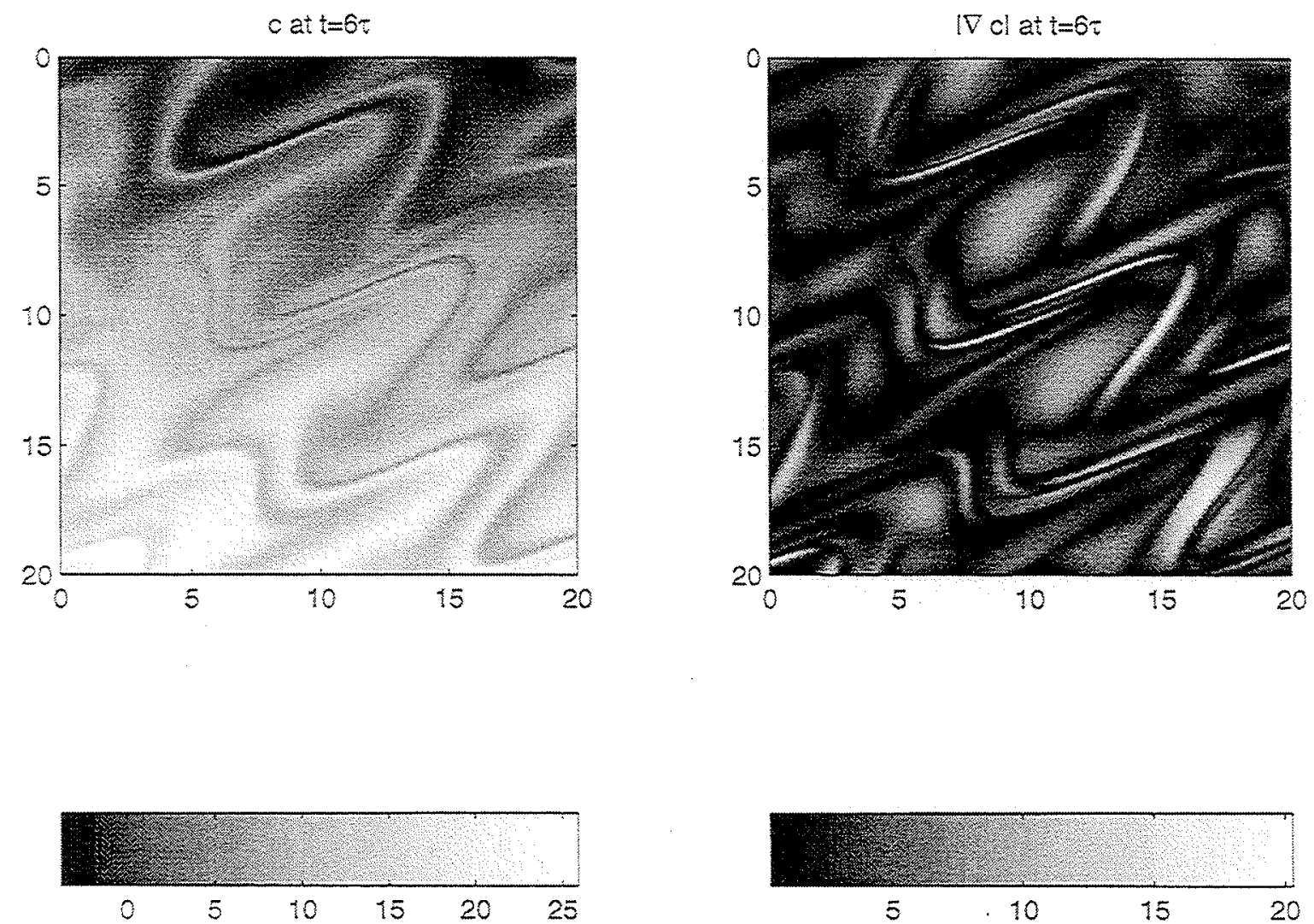

Figure 5: Numerical solution of the renovating wave model with $\tau=2$. The initial condition is $c(x, y, 0)=y$. Already, at $t=6 \tau,|\nabla c|$ is greatly amplified in some regions. 
c
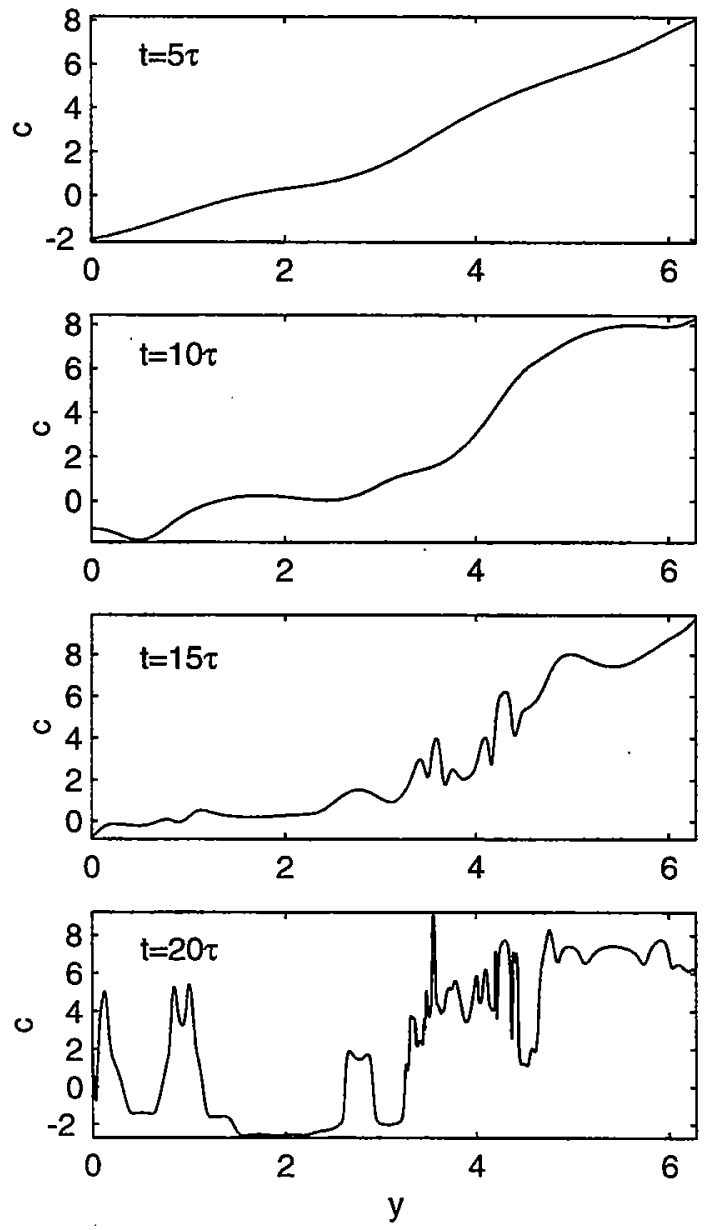

$\left|\nabla c l, l_{x}\right|$ and $l c_{y} \mid$
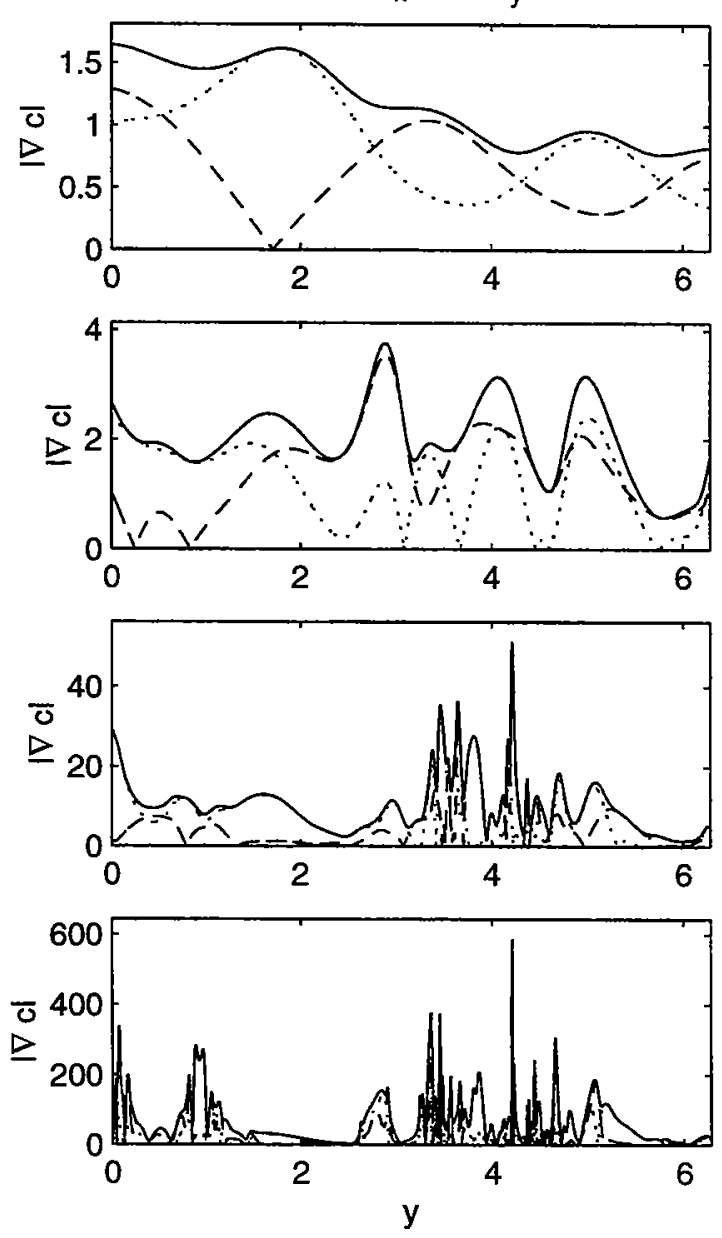

Figure 6: A numerical solution of the renovating wave model with $\tau=1$. The initial condition is $c(x, y, 0)=$ $y$. The plots show the values of $c$ and $|\nabla c|$ along the slice $x=0$. After 20 iterations, $|\nabla c|$ has developed strong spatial intermittency. 
As an example of this procedure, suppose that the initial condition is $c(x, 0)=y$. Then it follows from (53) that:

$$
\xi_{1} \cdot \nabla c=0 \quad \text { and } \quad \xi_{2} \cdot \nabla c=1 \quad(\text { for all } t) .
$$

Using (55) and (56) we see that

$$
\nabla c=\hat{z} \times \xi_{1}
$$

Thus, in this example, once we calculate $\xi_{1}$ we obtain $\nabla c$ as a bonus.

Figure 5 displays the numerical solution for $c$ and $|\nabla c|$ after 6 iterations of the renovating wave model. The initial condition is $c(\boldsymbol{x}, 0)=y$, so that $\boldsymbol{\nabla c}(\boldsymbol{x}, 0)=\hat{\boldsymbol{y}}$; the decorrelation time is $\tau=2$. The field in figure 5 is obtained using a $256 \times 256$ grid. To find $c$ at the grid point $x$ at time $t=n \tau$, one iterates the renovating wave model backwards in time till the initial location $(a, b)$ is determined, and then $c(\boldsymbol{x}, t)=b$. In parallel with this backwards iteration, $\boldsymbol{\xi}(\boldsymbol{x}, n \tau)$ is computed by matrix multiplication of the $\mathcal{J}^{(n)}$ defined in (45), and then $\nabla c$ is given by (57).

An important feature of stirring is the development of intermittency in the concentration gradient, $|\nabla c|$. In figure 6 the development of intermittency is illustrated, again using the renovating wave model. After 20 iterations there are "hotspots" in which large values of $|\nabla c|$ are concentrated. Without diffusion, the gradient of $c$ condenses onto a fractal set as the number of iterations increases (Városi, Antonsen \& Ott 1991).

\section{Multiplicative random variables}

In our solution of the $\sigma-\zeta$ model in section 2 we used isotropy to reduce a product of random matrices to a product of random scalars e.g., see equations (33), (34) and the following discussion. The main point of this section is that the statistical properties of isotropic line-element stretching are bedevilled by the large fluctuations which are characteristic of products of random variables. Indeed, figure 6 shows that there are large fluctuations in $\ell^{2}=|\xi|^{2}=|\nabla c|^{2}$. If one is attempting to measure the variance dissipation, $\kappa\langle\nabla c \cdot \nabla c\rangle$, then the intermittent structure of $\nabla c$ in figure 5 might pose a sampling problem. Imagine steering a ship through the field in figure 5 and making occasional point measurements of $\nabla c$. If the density of measurements is too low then one might easily miss the gradient hot-spots and so grossly underestimate $\kappa\langle\nabla c \cdot \nabla c\rangle$.

\subsection{Most probable values versus mean values}

We begin by stepping back from the stirring problem, and making some general remarks about multiplicative random processes. Suppose that a random quantity, $X$, is formed by taking the product of $N$ independent and identically distributed random variables

$$
X=x_{1} x_{2} \cdots x_{N}
$$

What can we say about the statistical properties of $X$ ?

The most nonintuitive aspect of $X$ in (58) is the crucial distinction which must be made between the mean value of $X$ and the most probable value of $X$. As an illustration, it is useful to consider an extreme case in which each $x_{k}$ in (58) is either $x_{k}=0$ or $x_{k}=2$ with equal probability. Then the sample space consists of $2^{N}$ sequences of zeros and two's. For all but one those sequences, $X=0$; in the remaining single case $X=2^{N}$. Thus, the most probable (that is, most frequently occuring) value of $X$ is

$$
X_{\mathrm{mp}}=0
$$


On the other hand, the mean of $X$ is

$$
\langle X\rangle \equiv \frac{\text { sum all the } X \text { 's from different realizations }}{\text { number of realizations }}=1
$$

Notice that one can also calculate $\langle X\rangle$ by arguing that $\left\langle x_{k}\right\rangle=1$ and, since the $x_{k}$ 's are independent, $\langle X\rangle=\left\langle x_{k}\right\rangle^{N}=1$

The example above is representative of multiplicative processes in that extreme events, although exponentially rare if $N \gg 1$, are exponentially different from typical or most probable events. Thus, for the product of $N$ random variables the ratio $\langle X\rangle / X_{\mathrm{mp}}$ diverges exponentially as $N \rightarrow \infty$. On the other hand, for the sum of $N$ random variables the most probable outcome is a good approximation of the mean outcome. Perhaps this is why people have an intuitive appreciation of sums, but find products confusing.

Now let us consider a more realistic example in which each $x_{k}$ is either $\alpha$ or $1 / \alpha$ with probability $1 / 2$. In this case the $p^{\prime}$ th moment of $X$ is

$$
\left\langle x_{k}^{p}\right\rangle=\frac{1}{2}\left(\alpha^{p}+\alpha^{-p}\right), \quad \Rightarrow \quad\left\langle X^{p}\right\rangle=\left(\frac{\alpha^{p}+\alpha^{-p}}{2}\right)^{N} .
$$

Before continuing, the student will profit from showing that the most probable value of $X$ is $X_{\mathrm{mp}}=1$ (for $N$ even). For example, if $\alpha=2$ then $\langle X\rangle=(5 / 4)^{N}$, while $X_{\mathrm{mp}}=1$. Again, the most probable value differs exponentially from the mean value as $N \rightarrow \infty$.

\subsection{The log-normal distribution}

Because $X_{\mathrm{mp}}$ is so different from the $\langle X\rangle$ the problem of determining $\langle X\rangle$ via Monte Carlo simulation is difficult: one may have to exhaust nearly all of the $2^{N}$ cases in order to obtain a reliable estimate of $\langle X\rangle$. This exhaustion is necessary for the first example, in which $x_{k}=0$ or 2 . In the example of equation (61), provided that $\alpha \approx 1$, we can get a pretty good estimate of $\langle X\rangle$ with less than exhaustive enumeration of all sequences of the $x_{n}$ 's.

Begin by noting that

$$
\ln X=\ln x_{1}+\ln x_{2}+\cdots+\ln x_{N}
$$

and so if $\ln x_{k}$ has finite variance then it follows from the Central Limit Theorem (CLT) that $\Lambda \equiv \ln X$ becomes normally distributed as $N \rightarrow \infty$.

The pitfall is in concluding that all the important statistical properties of $\Lambda$, and therefore of $X=\exp (\Lambda)$, can be calculated using the asymptotic log-normal distribution. of $X$. This not the case because the PDF of $\Lambda, \mathcal{P}(\Lambda)$, is approximated by a Gaussian only in a central scaling region in which $|\Lambda|<c N^{1 / 2}$, where $c$ is some constant which depends on the PDF of $x_{k}$. On the other hand, a reliable calculation of $\left\langle X^{p}\right\rangle=\langle\exp (p \Lambda)\rangle$ may require knowledge of the tail-structure of $\mathcal{P}(\Lambda)$.

To illustrate these difficulties, we use the example in which $\ln x_{k}= \pm \ln \alpha$ and $\left\langle\ln ^{2} x_{k}\right\rangle=\ln ^{2} \alpha$. Invoking the Central Limit Theorem, the asymptotic PDF of $\Lambda$ is therefore

$$
\mathcal{P}_{\mathrm{CLT}}(\Lambda)=\frac{1}{\sqrt{2 \pi N \ln ^{2} \alpha}} \exp \left(-\Lambda^{2} / 2 N \ln ^{2} \alpha\right) .
$$

In the central scaling region, $\mathcal{P}(\Lambda) \approx \mathcal{P}_{\mathrm{CLT}}(\Lambda)$.

To determine $X_{\mathrm{mp}}$ we can consider $\Lambda=\ln X$, which is an additive process for which the mean and most probable coincide, so that

$$
\langle\ln X\rangle=\ln X_{\mathrm{mp}}, \quad \Rightarrow \quad X_{\mathrm{mp}}=\mathrm{e}^{(\ln X\rangle} .
$$




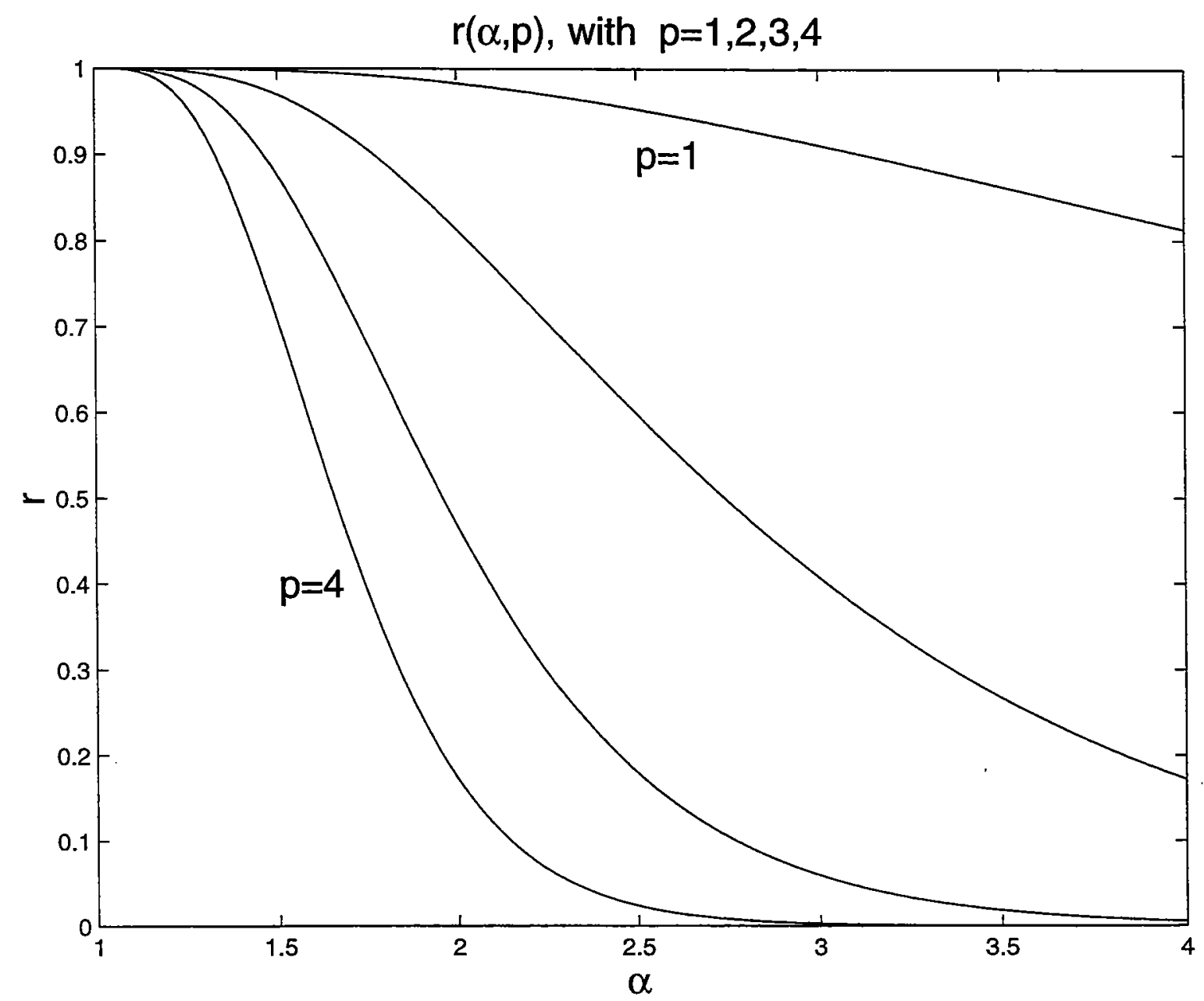

Figure 7: The function $r(\alpha, p)$ defined in (67). In order to accurately estimate $\left\langle X^{p}\right\rangle$ using the CLT one must have $r \approx 1$.

In our example with $\ln x_{k}= \pm \ln \alpha,\langle\ln X\rangle=0$ and therefore $X_{\mathrm{mp}}=1$. (This is one way of solving the problem posed in the previous section; another is to obtain the exact $\mathcal{P}(\Lambda)$ using the binomial density.)

With hope in our hearts, we now attempt to recover the exact result in (61) by substituting (63) into

$$
\left\langle X^{p}\right\rangle \equiv \int_{-\infty}^{\infty} \mathrm{e}^{p \Lambda} \mathcal{P}(\Lambda) \mathrm{d} \Lambda
$$

After the integration, one finds that

$$
\left\langle X^{p}\right\rangle_{\mathrm{CLT}}=\exp \left(N p^{2} \ln ^{2} \alpha / 2\right) .
$$

To assess the error we form the ratio of the exact result to the approximation:

$$
\left\langle X^{p}\right\rangle /\left\langle X^{p}\right\rangle_{\mathrm{CLT}}=r^{N}, \text { where } r \equiv \frac{1}{2} \exp \left(-p^{2} \ln ^{2} \alpha / 2\right)\left(\alpha^{p}+\alpha^{-p}\right) .
$$


When $r(\alpha, p)$ is close to 1 , the error is tolerable in the sense that $\ln \left\langle X^{p}\right\rangle_{\text {CLT }}$ is close to $\ln \left\langle X^{p}\right\rangle$.

For example, with $\alpha=2$, the exact result is $\langle X\rangle=(5 / 4)^{N}$ while $\langle X\rangle_{\mathrm{CLT}}=(1.27)^{N}$. However the second moment $p=2$, is seriously in error. As a general rule, $\left\langle X^{p}\right\rangle_{\text {CLT }}$ is a reliable estimate of $\left\langle X^{p}\right\rangle$ provided that $p^{2}\left\langle\ln ^{2} x_{k}\right\rangle<c$, where $c$ is the constant which determines the width of central scaling region, $|\Lambda|<c N^{1 / 2}$, in which $\mathcal{P}(\Lambda) \approx \mathcal{P}_{\mathrm{CLT}}(\Lambda)$. We conclude that the complete analysis of a random multiplicative quantity cannot be reduced to the Central Limit Theorem merely by taking a logarithm.

\subsection{Stretching exponents}

Equation (64) is a very important result for multiplicative random variables: to obtain the most probable value of $X$, one can exponentiate $\langle\ln X\rangle$. This explains why there is so much attention paid to $\left\langle\ln \left[\ell(t) / \ell_{0}\right]\right\rangle$ in the literature on random line element stretching: knowing the average of the logarithm enables one to estimate the stretching of a typical line element. Of course, the typical line element may not make a large contribution to the dissipation $\kappa\left\langle\nabla c^{\prime} \cdot \nabla c^{\prime}\right\rangle$. Thus our earlier focus on $\ell^{2}$ was not wasted, but it was not complete either.

A good characterization of random stretching is provided by the complete set of stretching exponents. Following Drummond \& Münch, we define the stretching exponents, $\gamma_{p}$, as

$$
\gamma_{p} \equiv \lim _{t \rightarrow \infty} \frac{1}{p\left\langle\ell^{p}\right\rangle} \frac{\mathrm{d}\left\langle\ell^{p}\right\rangle}{\mathrm{d} t}, \quad p>0
$$

and

$$
\gamma_{0}=\lim _{p \rightarrow 0} \gamma_{p}=\lim _{t \rightarrow \infty} \frac{\mathrm{d}}{\mathrm{d} t}\langle\ln \ell\rangle
$$

Knowing all these $\gamma$ 's, the asymptotic growth of line elements is characterized by

$$
\left\langle\ell^{p}\right\rangle^{1 / p} \sim \ell_{0} \mathrm{e}^{\gamma_{p} t} .
$$

Back in section 2 we calculated only $\gamma_{2}$ (e.g., see (33) and the subsequent discussion). To conclude this section I will discuss the calculation of the other stretching exponents, particularly $\gamma_{0}$.

\subsection{The stretching exponent $\gamma_{0}$ of the $\sigma-\zeta$ model}

As an example of the difference between $\gamma_{0}$ and $\gamma_{2}$ we return to the $\sigma-\zeta$ model. In section 2 we obtained a general expression for $\gamma_{2}$ in (37). Now consider the problem of determining $\gamma_{0}$. Taking the $\log$ of (32), writing $\xi_{0}=\ell_{0}(\cos \chi, \sin \chi)$, and then integrating ${ }^{3}$ over $\chi$, we have after some travail,

$$
\left\langle\ln \left(\ell_{1} / \ell_{0}\right)\right\rangle_{\chi}=\frac{1}{2} \ln \left(1+\frac{\Xi}{2}\right)
$$

where $\Xi(\sigma, \zeta, \tau)$ is given in (31). Averaging over $\sigma$ and $\zeta$, and using $\gamma_{0}=\tau^{-1}\left\langle\ln \left(\ell_{1} / \ell_{0}\right)\right\rangle$, gives

$$
\gamma_{0}=\frac{1}{2 \tau} \iint \mathcal{P}(\sigma, \zeta) \ln \left[1+\frac{1}{2} \Xi(\sigma, \zeta, \tau)\right] \mathrm{d} \sigma \mathrm{d} \zeta
$$

The expression above should be compared with that for $\gamma_{2}$ in (37).

\footnotetext{
${ }^{3}$ The integral

$$
\int_{0}^{\pi} \ln (a \pm b \cos x) \mathrm{d} x=\pi \ln \left[\left(a+\sqrt{a^{2}-b^{2}}\right) / 2\right]
$$
}

is useful. 
With the ensemble of random Couette flows in section 2.2.2 we can evaluate the integrals in (37) and (72). Thus, we find that

$$
\gamma_{0}=\frac{1}{2 \tau} \ln \left(1+\frac{\beta^{2} \tau^{2}}{4}\right), \quad \gamma_{2}=\frac{1}{2 \tau} \ln \left(1+\frac{\beta^{2} \tau^{2}}{2}\right) .
$$

Notice that $\gamma_{2}>\gamma_{0}$. This is a illustration of the general result that $\gamma_{p}$ is an increasing function of $p$ (Childress \& Gilbert 1995). Figure 3 compares the expressions for $\gamma_{0}$ and $\gamma_{2}$ in (73) with simulation.

\subsection{Stretching in one-dimension}

One-dimensional compressible velocity fields provide striking examples of the nontrivial dependence of $\gamma_{p}$ on $p$. We conclude this lecture with a model of random one-dimensional stretching for which the $\gamma_{p}$ 's can be obtained analytically.

\subsubsection{A sinusoidal velocity}

With the one-dimensional velocity $u=\sin x$, the equation governing line element stretching, (2), is

$$
\xi_{t}+\sin x \xi_{x}=\xi \cos x, \quad \xi(x, 0)=1 .
$$

The initial condition above is that the line elements attached to different fluid particles all have the same initial length. Because the fluid is compressible, the fluid density $\rho(x, t)$ satisfies

$$
\rho_{t}+(\sin x \rho)_{x}=0 \quad \rho(x, 0)=1 .
$$

It is easy to show by substitution that the solutions of (74) and (75) are related $\rho(x, t)=1 / \xi(x, t)$. The physical interpretation of this result should be obvious...

To solve (74), we follow the route outlined in section 1.3 by determining the mapping from the initial space, $x_{0}$, to the space $x\left(x_{0}, t\right)$. This means we solve

$$
\frac{D x}{D t}=\sin x, \quad x\left(0, x_{0}\right)=x_{0} .
$$

Using separation of variables we find that

$$
\tan (x / 2)=\mathrm{e}^{t} \tan \left(x_{0} / 2\right)
$$

which enables us to determine $x$ given $x_{0}$, or vice versa. Figure 8 shows how the mapping from $x_{0}$ to $x$ evolves as $t$ increases.

In this one-dimensional example, the Jacobian of the mapping is simply

$$
\frac{\mathrm{d} x}{\mathrm{~d} x_{0}}=\frac{1}{\cosh t-\cos x_{0} \sinh t}=\cosh t+\cos x \sinh t .
$$

It is easy to check by substitution that $\xi=\mathrm{d} x / \mathrm{d} x_{0}$ is the solution of (74).

\subsubsection{A one-dimensional renovating model}

Using the previous one-dimensional illustration of the Cauchy solution, we can formulate a renovating model that illustrates some of the subtleties involved in random stretching problems. Consider an ensemble of random renovating one-dimensional velocity fields in which

$$
u=\sin \left(x+\varphi_{n}\right) \quad \text { if } \quad(n-1) \tau<t<n \tau .
$$



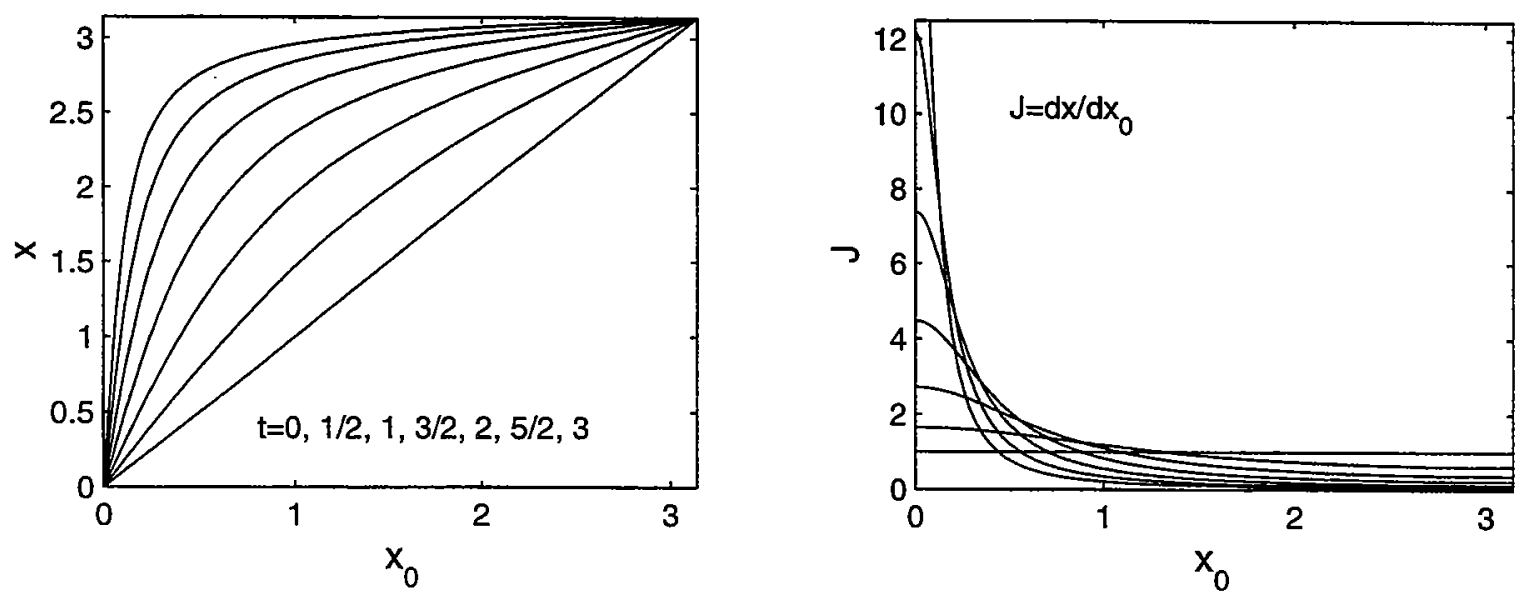

Figure 8: The left panel shows the mapping from $x_{0}$ to $x$ at the indicated times. The interval $0<x_{0}<\pi$ is compressed into the neighbourhood of $x=\pi$. The right panel shows $J\left(x_{0}, t\right)$ at the same times. Notice that an element that starts at say, $x_{0}=1 / 2$, is first stretched $(J>1)$ but then ultimately compressed $(J<1)$ as the particle approaches $x=\pi$.

The random phase, $0<\varphi_{n}<2 \pi$, is reset at $t=n \tau$. Notice that there is no preferred location on the $x$-axis; that is, the statistical properties of the process are spatially homogeneous.

Now, suppose we follow the stretching of a line element attached to a particle that moves in a particular realization of this velocity field. We denote location of this particle at $t=n \tau$ by $a_{n}$, and the length of the attached line element at this time by $\ell_{n}$. Then, using the solution from the previous section, the stretching of the line element is given by the random product

$$
\ell_{n}=J\left(a_{n-1}\right) J\left(a_{n-2}\right) \cdots J\left(a_{0}\right) \ell_{0},
$$

where the Jacobian is

$$
J(a) \equiv \frac{1}{\cosh \tau-\cos a \sinh \tau} .
$$

Because the phase is reset at $t=n \tau$, each $J\left(a_{n}\right)$ in (80) is independent of the others. Moreover, because of spatial homogeneity, each $a_{n}$ is uniformly distributed with $0<a_{n}<2 \pi$.

Equation (80) expresses the length of a material line element at $t=n \tau$ as a product of $n$ random numbers. Following our earlier discussion of multiplicative random variables, we first calculate $\gamma_{0}$ by taking the logarithm of $(80)$ :

$$
\ln \left(\ell_{n} / \ell_{0}\right)=\sum_{k=0}^{n-1} \ln J\left(a_{k}\right)
$$

Thus, the mean of $\ln \left(\ell_{n} / \ell_{0}\right)$ is

$$
\left\langle\ln \left(\ell_{n} / \ell_{0}\right)\right\rangle=n\langle\ln J\rangle
$$

where

$$
\langle\ln J\rangle=\oint \ln [J(a)] \frac{\mathrm{d} a}{2 \pi}=-\ln [\cosh (\tau / 2)] .
$$

Because $\left\langle(\ln J)^{2}\right\rangle$ is finite, the central limit theorem applies and we conclude that as $n \rightarrow \infty, \ln \left(\ell_{n} / \ell_{0}\right)$ is approximately normally distributed with the mean value $n\langle\ln J\rangle$. 


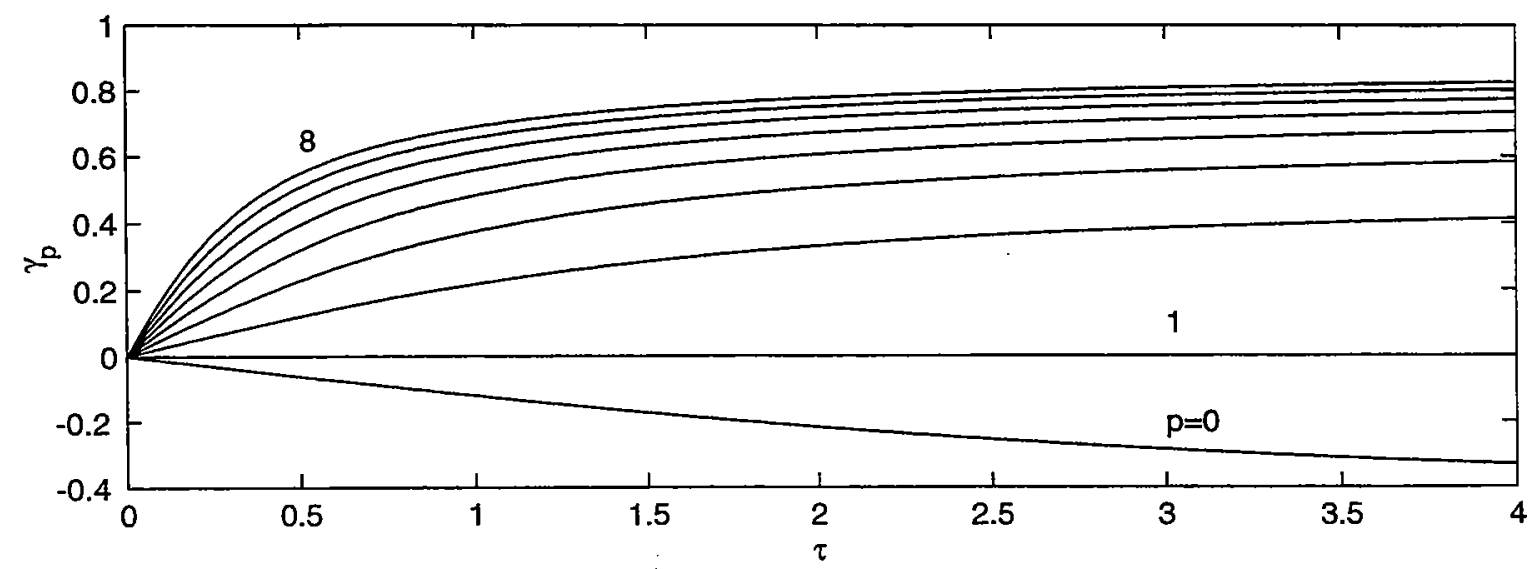

Figure 9: The stretching exponents $\gamma_{p}(\tau)$, with $p=0,1, \cdots, 8$ calculated using (89) .

Moreover, we can conclude from the central limit theorem that the most probable value of $\ell_{n} / \ell_{0}$ is

$$
\left(\ell_{n} / \ell_{0}\right)_{\mathrm{mp}} \approx \mathrm{e}^{\left\langle\ln \left(\ell_{n} / \ell_{0}\right)\right\rangle}=\mathrm{e}^{\gamma_{0} t},
$$

where, since $n=t / \tau$,

$$
\gamma_{0}=-\ln [\cosh (\tau / 2)] / \tau<0 .
$$

The result in (85) is remarkable because it implies that most of the line elements in this compressible flow exponentially contract (rather than stretch) as $t \rightarrow \infty$ !

Exponential contraction of most material lines is incomplete disagreement with the spirit of Batchelor's result in (1), where $\gamma>0$. The result above, that $\gamma_{0}<0$, is a special consequence of the compressible velocity field used in (79). (For a discussion of compressible velocities in a space of arbitrary dimension, see Chertkov et al. (1998).) In any event, this example shows that one cannot take exponential stretching for granted, no matter how intuitive it seems on the basis of experiments, such as those of Welander (1955).

How is contraction in the length of most material elements compatible with conservation of the total length of the $x$-axis? The answer is that even though most elements become exponentially small as $t \rightarrow \infty$, a few elements become exponentially large. Thus most of the length accumulates in exponentially rare, but exponentially long, line elements. This is an elementary example of an inverse cascade i.e., the spontaneous appearence of large-scale structures (big line elements).

To demonstrate length conservation, we can compute the mean (as opposed the most probable) length of an element. The mean length is

$$
\left\langle\ell_{n}\right\rangle=\langle J\rangle^{n} \ell_{0}
$$

where $J(a)$ is defined in $(81)$ and

$$
\langle J\rangle=\oint J(a) \frac{\mathrm{d} a}{2 \pi}=1
$$

Thus, the mean length of an element is constant, even though most elements exponentially contract.

As an exercise, I suggest showing that for integer values of $p$ the stretching exponents of this one-dimensional model are given by

$$
\gamma_{p}=\ln \left[P_{p-1}(\cosh \tau)\right] / p r,
$$

where $P_{m}$ is the $m$ 'th Legendre polynomial. Thus, in this particular example, there is a nice analytic characterization of the rate at which different moments stretch (see figure 9 ). 


\section{References}

[1] A.Okubo. Horizontal dispersion of floatable particles in the vicinity of velocity singularity such as convergences. Deep-Sea Res., 17:445-454, 1970.

[2] G. K. Batchelor. Small-scale variation of convected quantities like temperature in turbulent fluid. part 1. general discussion and the case of small conductivity. J. Fluid Mech., 5:113-133, 1959.

[3] G.K. Batchelor. The effect of turbulence on material lines and surfaces. Proc. Roy. Soc. London $A, 213: 349-366,1952$.

[4] B.L.Hua and P. Klein. An exact criterion for the stirring properties of nearly two-dimensional turbulence. Physica D, 113:98-110, 1999.

[5] M. Chertkov, I. Kolokolov, and M. Vergassola. Inverse versus direct cascades in turbulent advection. Phys. Rev. Lett., 80(3):512-515, 1998.

[6] S. Childress and A. D. Gilbert. Stretch, Twist, Fold: The Fast Dynamo. Springer, Berlin, 1995.

[7] W.J. Cocke. Turbulent hydrodynamic line stretching: consequences of isotropy. Phys. Fluids, 12:2488-2492, 1969.

[8] A. Crisanti, G. Paladin, and A. Vulpiani. Products of Random Matrices. Springer-Verlag, Berlin, 1993.

[9] I.T. Drummond and W. Münch. Turbulent stretching of line and surface elements. J. Fluid Mech., 215:45-59, January 1990.

[10] R.H. Kraichnan. Convection of a passive scalar by a quasi-uniform random stretching field. $J$. Fluid Mech., 64:737-762, 1974.

[11] J.C. McWilliams. The emergence of isolated coherent vortices in turbulent flows. J. Fluid Mech., 198:199-230, 1984.

[12] S.A. Orszag. Comments on 'turbulent hydrodynamic line stretching: consequences of isotropy. Phys. Fluids, 13:2203-2204, 1970.

[13] F. Varosi, T.M. Antonsen, and E. Ott. The spectrum of fractal dimensions of passively convected scalar gradients. Phys. Fluids A, 3:1017-1028, 1991.

[14] J. Weiss. The dynamics of enstrophy transfer in two-dimensional hydrodynamics. Physica D, 48:273-294, 1991.

[15] P. Welander. Studies on the general development of motion in a two-dimensional, ideal fluid. Tellus, 7:141-156, 1955. 


\section{Lecture 4: Anomalous diffusion}

In this lecture we discuss stochastic models of correlated random walks. By "correlated" we mean that that if a particle is headed in one direction then there is nonzero probability that it continues in that same direction for some time and this probability fades to zero as the time interval increases. This is, of course, the situation envisaged by Taylor (1921).

The distinction between normal and anomalous diffusion made in lecture 1 can be understood by examining the rate at which velocity correlation decrease to zero. Normal diffusion occurs if the velocity correlation decrease rapidly while anomalous diffusion results from processes in which particles move coherently for long times with infrequent changes of direction. Roughly speaking, this distinction is quantified by the tail behaviour of the velocity autocorrelation function. For example, if the correlation function decays exponentially then there is normal diffusion, whereas if the correlation function decays algebraically then there is the possibility of anomalous diffusion.

The definition of anomalous diffusion is based only on the behaviour of the second moment, $\left\langle x^{2}\right\rangle$. But we usually want to know more about the distribution of a tracer than simply the second moment. In the case of normal diffusion, detailed information concerning the tracer distribution is obtained by solving the diffusion equation

$$
c_{t}=D c_{x x} .
$$

Can we obtain continuum models, analogous to (1), which provide the same detailed information for anomalously diffusing tracer? The main goal of this lecture is to develop partial differential equation models which can be used for this purpose.

\section{Superdiffusion and subdiffusion}

\subsection{Taylor's formula and long tails}

Yet again we recall Taylor's formula which relates the growth of position variance to an integral of the Lagrangian velocity autocorrelation function, $\operatorname{corr}(t)$,

$$
\frac{\mathrm{d}\left\langle x^{2}\right\rangle}{\mathrm{d} t}=2 \int_{0}^{t} \operatorname{corr}\left(t^{\prime}\right) \mathrm{d} t^{\prime} .
$$


In order to obtain $\left\langle x^{2}\right\rangle$ we must integrate (2). Standard manipulations turn the resulting double integral of corr $(t)$ into a single integral

$$
\left\langle x^{2}\right\rangle=2 \int_{0}^{t}\left(t-t^{\prime}\right) \operatorname{corr}\left(t^{\prime}\right) \mathrm{d} t^{\prime} .
$$

The result (3), which is not in Taylor's original paper, will prove to be very useful.

We usually have in mind situations in which $\operatorname{corr}(t)$ decreases to zero as $t \rightarrow \infty$ so that the integrals in (2) and (3) converge to nonzero values. An example is the renovating wave model, with its "triangular" correlation function, from lecture 2. Later in this lecture I will introduce the telegraph model which has an exponentially decaying correlation function, $\operatorname{corr}(t)=U^{2} \exp (-2 \alpha t)$. These are both examples in which correlations decrease very rapidly so that normal diffusion occurs. But now consider the possibility that $\operatorname{corr}(t)$ decreases so slowly that the integrals in (3) diverge.

Suppose, for instance, that as $t \rightarrow \infty, \operatorname{corr}(t) \sim t^{-\eta}$ with $0<\eta<1$. Even though the diffusivity no longer exists, it still follows from (3) that

$$
\left\langle x^{2}\right\rangle \sim t^{2-\eta} .
$$

In this case there is superdiffusion: the variance of the particle displacement grows faster than linearly with time because $2-\eta>1$.

Taylor's formula also contains the possibility of subdiffusion. This case is subtle because, like the example of the sea-surface mentioned in lecture 1 , it requires that the integral defining $D$ is zero. But suppose additionally that the remaining integral in (3) diverges. This can happen if $\operatorname{corr}(t) \sim c t^{-\eta}$ with $1<\eta<2$. The condition that $1<\eta$ ensures that $\int_{0}^{\infty} \operatorname{corr}\left(t^{\prime}\right) \mathrm{d} t^{\prime}$ converges (to zero). The second inequality, $\eta<2$, ensures that $\int_{0}^{t} t^{\prime} \operatorname{corr}\left(t^{\prime}\right) \mathrm{d} t^{\prime}$ diverges. Using (3), we again find the scaling law in (4). However this time, because $2-\eta<1$, there is subdiffusion.

At first glance two possibilities above appear as unlikely exceptions to the more natural cases in which both integrals in (3) converge. However there are examples in fluid mechanics in which either subdiffusion or superdiffusion is observed experimentally or computationally. Thus (4) cannot be dismissed as an unlikely pathology.

\subsection{The Texas experiments}

An experiment illustrating anomalous diffusion has been conducted in Swinney's laboratory at University of Texas; see Solomon, Weeks \& Swinney (1994) and Weeks, Urbach \& Swinney (1996). These investigators study the dispersion of particles in an almost two-dimensional flow in annular tank (see figure 1). The tank is rotating at about 1 or $2 \mathrm{Hertz}$ and the bottom is sloped to simulate the $\beta$-effect. Because of the rapid rotation the flow is quasi two-dimensional.

The flow is forced by pumping fluid through the tank. If the pumping rate is sufficiently large then this azimuthal flow is unstable to a vortex-forming instability. 


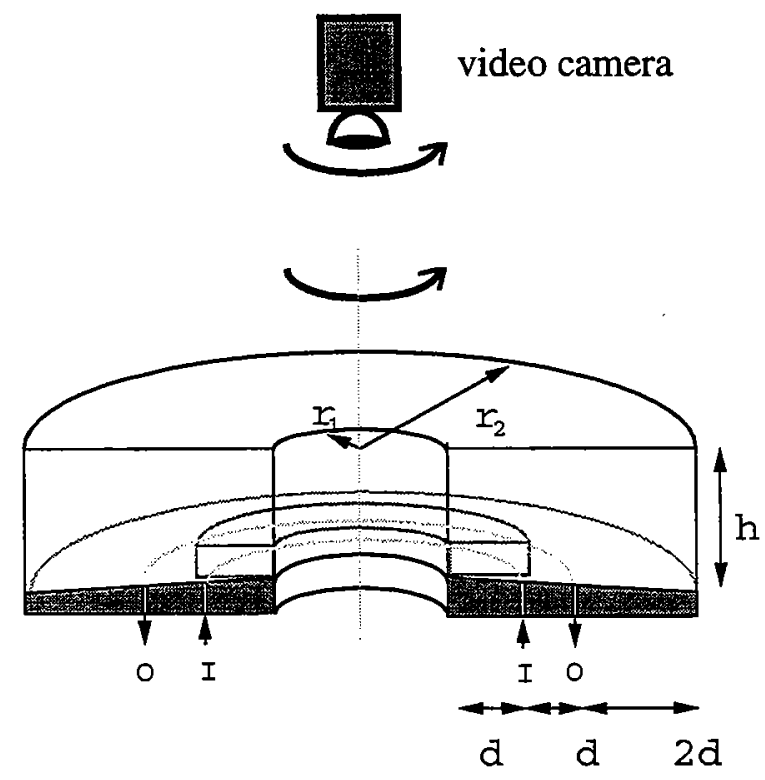

Figure 1: A sketch of the rotating annulus; the rotation rate is about $1 \mathrm{~Hz}$. Flow is forced by pumping water in through the ring of holes marked by $I$ and withdrawing the same volume through the other ring marked $\mathrm{O}$. As a consequence of the strong Coriolis forced acting on the radial flow between these concentric rings there is an azimuthal flow around the annulus. The experiment is viewed from above using a video camera. Figure courtesy of Eric Weeks.

A typical flow pattern in the rotating frame is shown in figure 2. Evident also in this figure is the azimuthal jet which runs all the way around the tank. The vortex pattern can be perturbed experimentally by making the strength of the pumping depend on azimuth. In this fashion, one can drive an unsteady flow and observe chaotic particle trajectories.

Automated image processing techniques are used to follow nearly neutrally buoyant tracer particles suspended in such flow. Typical particle trajectories are shown in figure 3. Particles within a vortex remain trapped for very long time (stick). Particles in the azimuthal jet experience prolonged fights around the circumference of the tank. Because the vortex pattern is not perfectly stationary particles alternate, apparently randomly, between flying in jets and sticking in vortices.

One can change the pattern of jets and vortices by altering the diameters of the circular barriers which confine the flow. Thus it is possible to create a flow with two oppositely directed jets separated by a vortex chain. In this case the dispersion process is more symmetric than in figure 4 because the flights go in both directions around the tank.

During a flight the angular displacement is proportional to the time elapsed since 


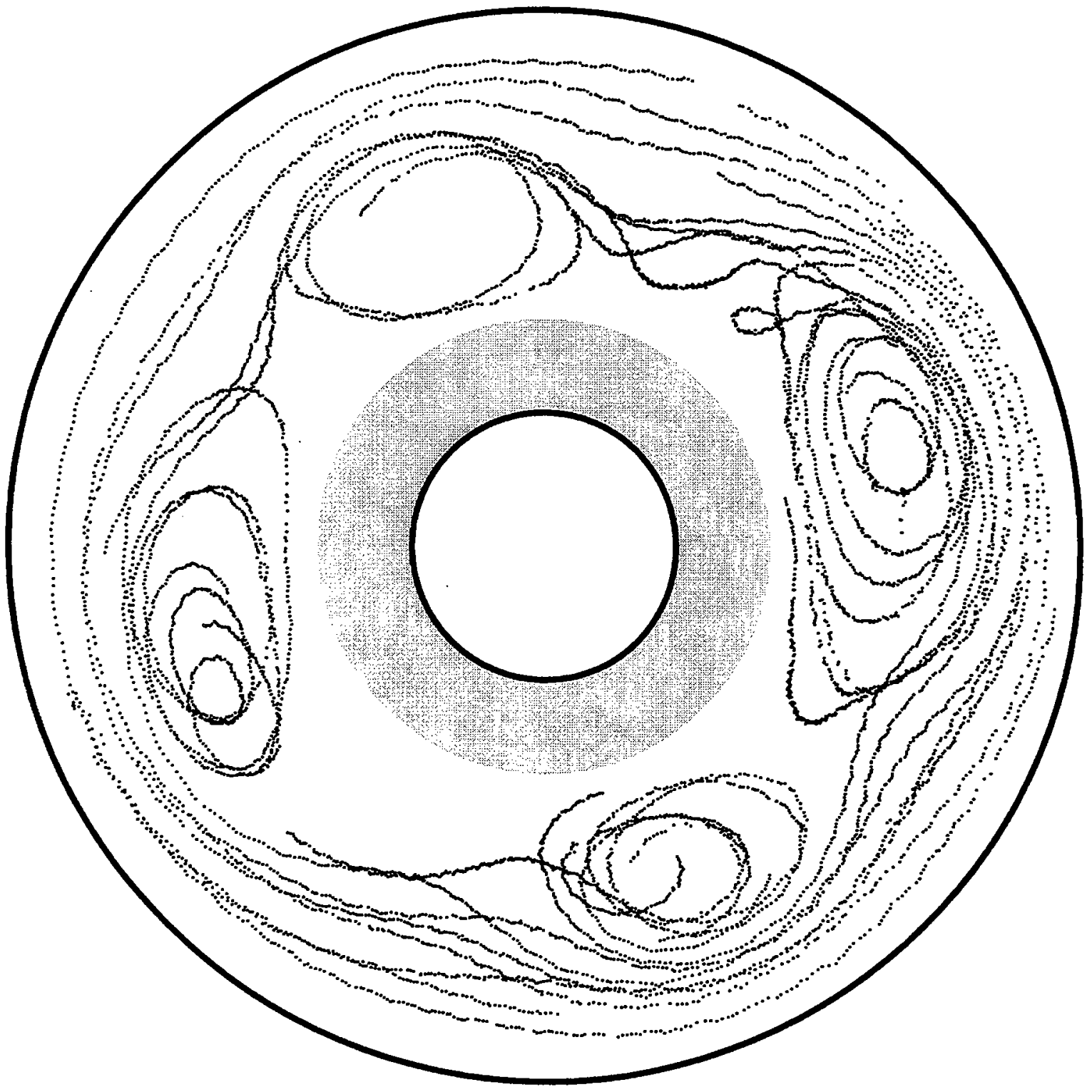

Figure 2: Streaks formed by 100s trajectories of 12 particles reveal four vortices. Weeks et al. show that the motion of these coherent vortices is chaotic. That is, a velocity spectrum, obtained by measuring velocity with a hot film probe, is broad band. Figure courtesy of Eric Weeks. 

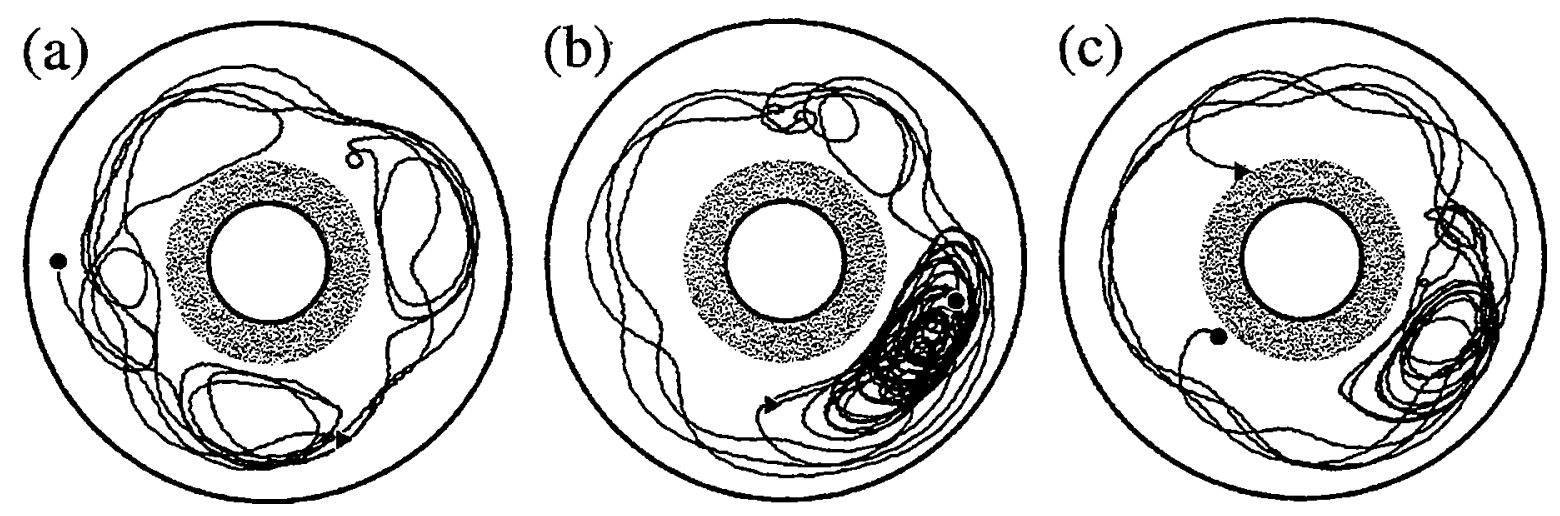

Figure 3: Trajectories of three tracer particles in the flow shown in figure 2. The beginning of each trajectory is indicated by a triangle and the end by with a circle. In (b) the particle spends most of its life trapped in a single vortex. However, this vortex wobbles erratically because the flow is chaotic. In parts (a) and (c) the particles experience several episodes of trapping within a vortex and flight around the tank in the jet. Figure courtesy of Eric Weeks.

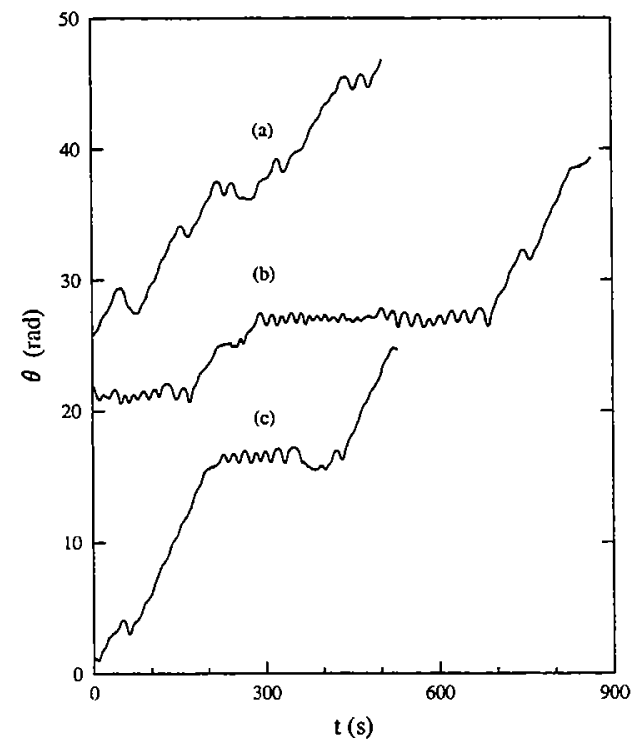

Figure 4: Angular displacement, $\theta(t)$ for the trajectories in figure 3 . There is an obvious distinction between the flights and the sticking events. The small oscillations during the sticking events correspond to particle motion within a vortex. Figure courtesy of Eric Weeks. 
the flight began:

$$
\Delta \theta \approx U t
$$

The displacement, $\Delta \theta$, is essentially zero during a sticking event (see figure 4).

The experiments show that the dispersion of an ensemble of particles is superdiffusive. That is

$$
\left\langle(\theta-\langle\theta\rangle)^{2}\right\rangle \sim t^{\gamma}
$$

where $\gamma>1$; typical values are $\gamma \approx 1.4$ to 1.7 depending on the experimental configuration. (It is also possible to observe normal diffusion, $\gamma=1$, by strongly forcing the flow and breaking the azimuthal symmetry of the forcing.)

To characterize the motion Solomon et al. used sticking and flying PDFs:

$$
\mathcal{P}_{F}(a) \mathrm{d} a=\text { Probability that a flight has a duration } \in(a, a+\mathrm{d} a) .
$$

Later in this lecture we will refer to $a$ as the "lifetime" of a particle in the flying or sticking state. We figuratively speak of a particle being born into the flying state and moving coherently for a lifetime $a$ so that the total angular displacement during the flight is $\Delta \theta=U a$.

The PDF $\mathcal{P}_{F}$ is normalized by $\int_{0}^{\infty} \mathcal{P}_{F}(a) \mathrm{d} a=1$ and

$$
\tau_{F}=\int_{0}^{\infty} a \mathcal{P}_{F}(a) \mathrm{d} a=\text { average duration of a flight } .
$$

The PDF of sticking times, $\mathcal{P}_{S}(a)$, and the average sticking time, $\tau_{S}$, are defined analogously.

Experiments show that as $a \rightarrow \infty, \mathcal{P}_{F}$ and $\mathcal{P}_{S}$ have algebraically decaying tails:

$$
\mathcal{P}_{F}(a) \sim a^{-\mu_{F}}, \quad \mathcal{P}_{S}(a) \sim a^{-\mu_{S}},
$$

with

$$
2<\left(\mu_{F}, \mu_{S}\right)<3 .
$$

Because of this slow algebraic decay the variance of the lifetimes, defined by

$$
\left\langle a^{2}\right\rangle_{F, S} \equiv \int_{0}^{\infty} a^{2} \mathcal{P}_{F, S}(a) \mathrm{d} a,
$$

diverges.

The divergence of $\left\langle a^{2}\right\rangle_{F}$ is significant because invoking Einstein's formula for the diffusivity

$$
D=\frac{\left\langle(\Delta \theta)^{2}\right\rangle}{\tau}
$$


and using $\Delta \theta=U a$, we conclude that $D \propto\left\langle(\Delta \theta)^{2}\right\rangle=U^{2}\left\langle a^{2}\right\rangle_{F}=\infty$. The divergence of $D$ is symptomatic of superdiffusion.

Notice that the denominator $\tau$ in (12) is related to the average flying and sticking times, $\tau_{F}$ and $\tau_{S}$, which are both finite. Thus, in the Texas experiments, we can say that anomalous diffusion occurs because the numerator of (12) is divergent. In other cases it is the denominator which causes trouble.

The Texas experiments show that anomalous diffusion occurs in realistic and geophysically relevant systems. Several theoretical questions suggest themselves. How do the algebraic tails of $\mathcal{P}_{S}$ and $\mathcal{P}_{F}$ arise, and can we make a microscopic models which exhibits this phenomenon? Can we relate the exponents $\gamma, \mu_{F}$ and $\mu_{S}$ ? (From section 4 , the answer to the last question is $\gamma=4-\mu_{F}$.)

\section{The telegraph model}

The key issue raised by anomalous diffusion is decay of velocity correlations. Thus our goal is to formulate models for which we can explicitly calculate velocity statistics and understand the decay of correlations. Our first attempt is not very ambitious: we begin with the telegraph model, which is the simplest example of a continuous-time correlated random walk.

\subsection{The Lagrangian formulation of the telegraph model}

In a telegraph process the velocity of particle $n$, denoted by $u_{n}(t)$, can have only one of two possible values, $+U$ and $-U$. The velocity of each particle, $u_{n}(t)$, flips randomly back and forth between $\pm U$ with a transition probability $\alpha$ per time. This means that in a time $\mathrm{d} t$ a fraction $\alpha \mathrm{d} t$ of the ensemble switches velocity. Because the transition rate, $\alpha$, is constant we can say that a particle has no "memory" of when it first arrived in its present state. Thus this telegraph model is Markovian.

We refer to the prescription for constructing a telegraph process as model $A$. There is a variant, model $B$, discussed below.

With the prescription above, the velocity of a particle is a discontinuous function of time as shown in figure 5 . The correlation function and the diffusivity are

$$
\operatorname{corr}(t)=U^{2} e^{-2 \alpha|t|}, \quad D=\int_{0}^{\infty} \operatorname{corr}(t) \mathrm{d} t=\frac{U^{2}}{2 \alpha}, \quad \text { (model A). }
$$

Notice that the corr $_{t t}$ is infinite at $t=0$; this is because the acceleration is infinite at the discontinuities in figure 5 .

To obtain (13), return to the definition of the correlation function

$$
\operatorname{corr}(t)=\frac{1}{N} \sum_{n=1}^{N} u_{n}(0) u_{n}(t),
$$



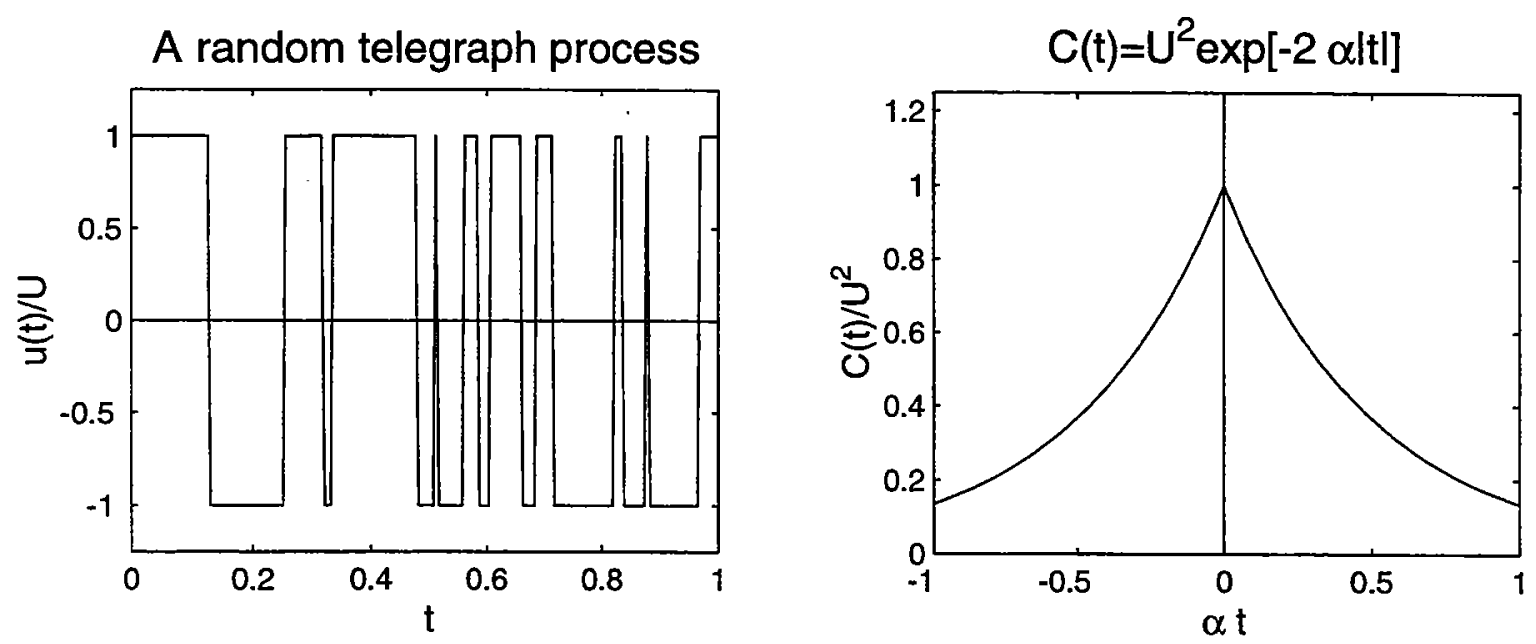

Figure 5: An example of a telegraph time series, and the telegraph correlation function.

where $N$ is the total number of particles in the ensemble. Suppose that at $t$ the sum on the right hand side has $P(t)$ positive terms, all equal to $U^{2}$, and $N-P(t)$ negative terms, all equal to $-U^{2}$. Thus

$$
\operatorname{corr}(t)=\frac{U^{2}}{N}[2 P(t)-N]
$$

In a time $\mathrm{d} t, P \alpha \mathrm{d} t$ of the positive terms become negative and $(N-P) \alpha \mathrm{d} t$ of the negative terms become positive. Thus, at $t+\mathrm{d} t$,

$$
P(t+\mathrm{d} t)=P(t)(1-2 \alpha \mathrm{d} t)+N \alpha \mathrm{d} t
$$

and the analog of (15) is:

$$
\operatorname{corr}(t+\mathrm{d} t)=\frac{U^{2}}{N}[2 P(t)(1-2 \alpha \mathrm{d} t)+2 N \alpha \mathrm{d} t-N] .
$$

Taking the limit $\mathrm{d} t \rightarrow 0$ in (17) gives corr $_{t}=-2 \alpha$ corr; the solution of this differential equation is (13).

An alternative telegraph process (model B) is constructed by imagining that at random instants each particle flips at coin. The flipping rate is $\alpha$ so that in a time $\mathrm{d} t$, there are $N \alpha \mathrm{d} t$ coin flips. After each flip, the velocity is $+U$ if there is a head and $-U$ if a tail. With this prescription, a particle will change direction on average once out of every two tosses. On the other tosses the particle continues in the same direction and the result is as if nothing happened. Thus with model B we simply replace $\alpha$ by $\alpha / 2$ in our earlier calculations and consequently the correlation function and diffusivity are

$$
\operatorname{corr}(t)=U^{2} e^{-\alpha|t|}, \quad D=\int_{0}^{\infty} \operatorname{corr}(t) \mathrm{d} t=\frac{U^{2}}{\alpha}, \quad \text { (model B). }
$$


The difference between model $\mathrm{A}$ and model $\mathrm{B}$ is trivial. However the distinction between the two cases will plague us later.

If we are searching for a model of anomalous diffusion then the telegraph model is a disappointment: the exponentially decaying correlation function ensures that $D$ is finite and that the displacement variance ultimately grows diffusively. We continue our investigation of the telegraph model in order to better understand "ultimately" and because in section 4 the telegraph model is used as the foundation of more elaborate models which do show anomalous diffusion.

\subsection{The Eulerian formulation of the telegraph model}

Now we ignore the Lagrangian information contained in the correlation function (14) and instead we give an Eulerian formulation of the telegraph process. Let $R(x, t)$ denote the density (particles/length) of particles moving to the right with velocity $+U$ and $L(x, t)$ denote the density of left-moving particles with velocity $-U$. The coupled conservations laws are

$$
R_{t}+U R_{x}=\alpha(L-R), \quad L_{t}-U L_{x}=\alpha(R-L) .
$$

These equations should be self-evident...

We can put (19) into a revealing alternative form by defining the total concentration, $C(x, t)$, and the flux, $F(x, t)$, as

$$
C \equiv R+L, \quad F \equiv U(R-L) .
$$

In terms of these new variables the model is

$$
C_{t}+F_{x}=0, \quad F_{t}+2 \alpha F=-U^{2} C_{x} .
$$

The first equation is conservation of particles and the second equation is the fluxgradient relation.

Notice that in (21) Fick's law does not apply - the flux $F$ is not instantaneously related to the gradient $C_{x}$. Equation (21b), which might be called Cattaneo's law (see the 1989 review by Joseph and Preziosi), can be solved as a first-order differential equation for $F(x, t)$. Thus, the flux at $x$ is expressed as weighted integral over the past history of the gradient at $x$ :

$$
F(x, t)=-U^{2} \int_{-\infty}^{t} e^{-2 \alpha\left(t-t^{\prime}\right)} C_{x}\left(x, t^{\prime}\right) \mathrm{d} t^{\prime} .
$$

The flux has a "fading memory" of the gradient and the exponential in (22) is the fading factor which strongly weights the most recent values of the gradient.

Next, if we eliminate $F$ from (21), we obtain

$$
C_{t t}+2 \alpha C_{t}-U^{2} C_{x x}=0 .
$$



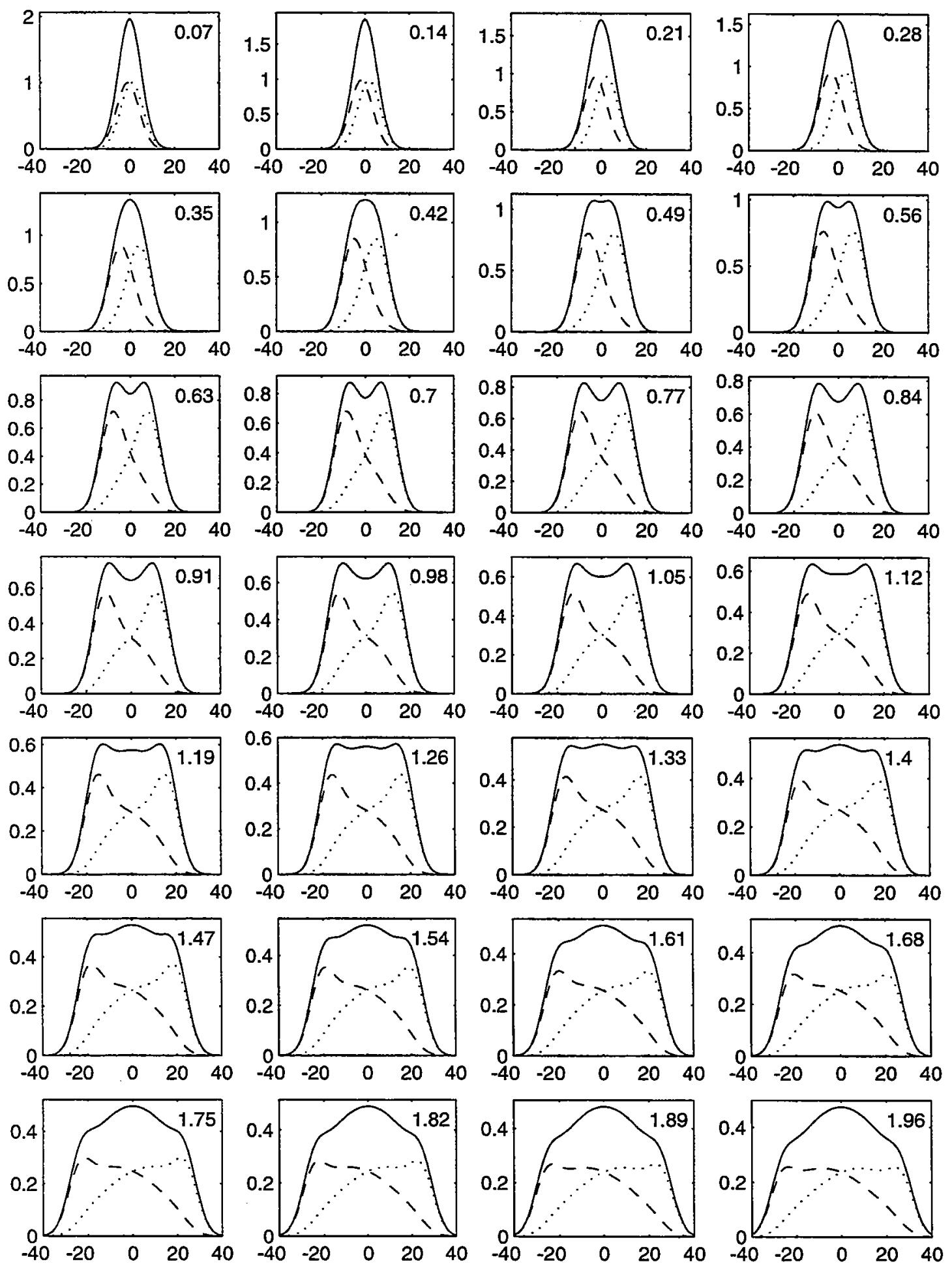

Figure 6: Solution of the telegraph equation. $\alpha t$ is in the top corner of the panel. At $t=0$, $R=L=\exp \left(-x^{2} / 50\right)$. The solid curve is $C=R+L$, and $R$ and $L$ are shown as dotted and dashed curves. 
This is the telegraph equation; the diffusion equation is obtained only as an approximation which applies to the low frequency and wavenumber components of $C(x, t)$. On these large and slowly evolving scales one can neglect the term $C_{t t}$ in (23) and so obtain the approximation

$$
C_{t} \approx D C_{x x}, \quad D=\frac{U^{2}}{2 \alpha} .
$$

The diffusivity $D$ in (24) was anticipated in (13).

Figure 6 shows a numerical solution of (23) starting with an initial condition of the form

$$
R(x, 0)=L(x, 0)=e^{-\mu^{2} x^{2}} .
$$

At small times the density $C$ develops a double peaked structure as the left and right going populations separate. This behaviour is transient, and at longer times the central part of the concentration relaxes to the well-known Gaussian solution of the diffusion equation.

According to (23) the disturbance travels at a finite speed: these are the "heat waves" discussed by Joseph and Preziosi (1989), and also evident in figure 6. The approximate diffusion equation (24) makes the unrealistic prediction that disturbances are propagated at infinite speed. This unphysical consequence of the diffusion equation motivated Cattaneo to propose (21b) as an alternative to Fick's law.

These considerations shows that one cannot blithely assert the validity of the diffusion equation (24) as an exact description of dispersion. The diffusion equation applies only as an approximate description of low frequencies and long wavelengths.

\subsection{Discretization of the telegraph model}

This section is a digression. Read on if you want to learn how to solve the telegraph equation using a simple numerical scheme. (This is how I drew figure 6.)

We reformulate the telegraph model in terms of discrete variables: divide the $x$-axis is divided into segments of length $\delta x$ separated by "scattering sites" at $x_{n}=n \delta x$. Time is also discretized in units of $\delta t$ so that $t=T \delta t$ where $T$ is an integer $T=0,1,2 \cdots$ The walkers move along the $x$-axis with a velocity that is either $+\delta x / \delta t$ or $-\delta x / \delta t$. In terms of the continuous model in (19)

$$
U=\frac{\delta x}{\delta t} .
$$

When a walker reaches the scattering site at $x_{n}=n \delta x$ he is "backwards scattered" or reflected with probability $b$ and "forward scattered" or transmitted with probability $1-b$. Because the probability of a change in direction, $b$, is the same for left as for right moving walkers there is no mean velocity along the line. 


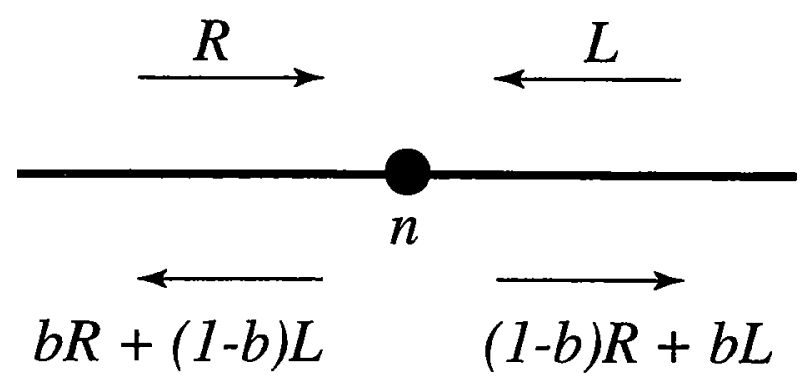

Figure 7: A steady state with constant flux, $f=U(1-b)(R-L)$, passing through site $n$.

Let $R_{n}(T) \delta x$ be the number of right walkers in the segment $n, n \delta x<x<(n+1) \delta x$. The number of left walkers, $L_{n}(T) \delta x$, is defined analogously. $R_{n}$ and $L_{n}$ are the discrete analogs of the continuous densities used in (19).

With these rules and definitions, the discrete evolution equations for the ensemble are

$$
\begin{gathered}
R_{n}(T)=(1-b) R_{n-1}(T-1)+b L_{n}(T-1) \\
L_{n}(T)=(1-b) L_{n+1}(T-1)+b R_{n}(T-1)
\end{gathered}
$$

For instance, in the first equation above, the number of right movers in segment $n$ is equal to the number in segment $n-1$ at the previous time that successfully passed through scattering site $n$, plus the number of left movers previously in segment $n$ that were reflected at this same site. Figure 6 shows the result of iterating the discrete system above.

One exact solution of the difference equations above is

$$
R_{n}=L_{n}=L_{n+1}=R_{n+1}=\cdots
$$

This solution is steady: $R_{n}(T)=R_{n}(T-1)$. In fact, (29) is the discrete analog of the equilibrium solution of the diffusion equation. The distribution of walkers is spatially uniform with equal numbers going left and right in each interval and there are no concentration gradients. An individual walker is moving to and fro, but the ensemble is in steady state.

Next, we consider the constant-flux solution. In figure $7, R$ right walkers impinge on site $n$ from the left and $L$ left walkers impinge on $n$ from the right. In steady state it must be that on the left of $n$ there are $b R+(1-b) L$ left walkers moving away, while to the right there are $b L+(1-b) R$ right walkers moving away. Thus the flux to the right of the site is

$$
f=U[(1-b) R+b L]-U L=\frac{\delta x}{\delta t}(1-b)(R-L)
$$


where we have used $U=\delta x / \delta t$. Because there is a steady state, calculating the flux to the left of the site gives exactly the same result and so there is a nondivergent flux of walkers along the line.

Next, we can calculate the concentration difference across the site in figure 7 . To the right of the site the total density of walkers is

$$
c^{+}=L+b L+(1-b) R,
$$

while on the left the density is

$$
c^{-}=R+b R+(1-b) L .
$$

Combining (31) and (32) we have for the concentration jump across the site

$$
\delta c=c^{+}-c^{-}=2 b(L-R)
$$

Thus, using (30), the flux-gradient relation in steady state is

$$
f=-D \frac{\delta c}{\delta x}, \quad D=\frac{(1-b)}{2 b} \frac{(\delta x)^{2}}{\delta t} .
$$

Does it seem obvious to you that the diffusivity should diverge as $b \rightarrow 0$ ? If you think of the diffusivity as the area under the correlation functions then this divergence should be intuitive. It is an instructive exercise to obtain $D$ in (34b) using Taylor's formula. (Hint: consider $N \gg 1$ right walkers which initially set out together. At $t=T \delta t$, after $T$ encounters with scattering sites, how many of these walkers have changed direction an even number of times, and how many odd?)

Comparing the equation above with our earlier expression for the diffusivity, $D$ in (13) and (24), we conclude that

$$
\alpha \delta t=\frac{b}{1-b} .
$$

Thus, with (26) and (35), we can express the parameters of the discrete model, $(\delta x, \delta t, b)$, in terms of the parameters characterizing the continuous model, $U$ and $\alpha$.

\section{Age-stratified populations}

The telegraph model from section 2 is Markovian. This means that each particle has a constant probability per unit time, $\alpha$, of switching direction. Thus, no matter how long a particle has been moving to the right (say), its probability of switching direction in the next $\mathrm{d} t$ is always $\alpha \mathrm{d} t$. Consequently an exponentially decreasing number of particles move coherently for long intervals and the telegraph model in (19) does not exhibit anomalous diffusion. 
A satisfactory description of anomalous diffusion demands a non-Markovian model in which particles have some memory of their past motion. To obtain superdiffusion it is necessary that a right-moving particle is less and less likely to change direction as it spends more and more time moving right ${ }^{1}$.

Such memory effects are implicit in the models discussed by Weeks et al (1996), and in several of the articles in the conference proceedings edited by Schlesinger, Zaslavsky \& Frisch (1994). The stochastic models discussed in Schlesinger et al. draw heavily on statistical physics. In this lecture we are going to develop the theory from scratch using a formalism which is accessible to people whose background is in fluid mechanics. The climb begins with an excursion into the theory of age-stratified populations.

Consider a population of items with a finite lifetimes and a death rate which depends on age, $a$. For example, light bulbs in a large building, or the population of the United States. At time $t$ the age structure of the population is characterized by a density function for which $f(a, t) \mathrm{d} a$ is the number of items whose age is between $a$ and $a+\mathrm{d} a$. In terms of $f$, the total number of items in the population, $N(t)$, and the average age, $\bar{a}(t)$, are given by

$$
N(t)=\int_{0}^{\infty} f(a, t) \mathrm{d} a, \quad \bar{a}(t) \equiv N^{-1} \int_{0}^{\infty} a f(a, t) \mathrm{d} a .
$$

The density function evolves according to

$$
f_{t}+f_{a}+\alpha f=0
$$

where $\alpha(a)$ is the death-rate. The term $f_{a}$ in (37) says that the population translates along the age-axis at a rate one year every year. To completely specify the problem we must supply an initial condition, and also a boundary condition at $a=0$. The boundary condition at $a=0$ has an obvious interpretation:

$$
f(0, t)=\text { the birth (or replacement) rate. }
$$

In the case of a population of people, the boundary condition above is a flux of babies into the system.

The Markovian limit is the special case in which $\alpha$ is independent of $a$. This model of $\alpha$ is unrealistic for both light-bulbs and people, though it might apply to a population of radioactive molecules. The Markovian case is very simple because one can integrate (37) over $a$ and obtain a closed equation for $N(t)$ :

$$
N_{t}+\alpha N=f(0, t) \text {. }
$$

Thus if $\alpha$ is constant and we need only the total number of functional items at $t$ then we do not need to solve partial differential equations and deal with the age structure of the population.

\footnotetext{
${ }^{1} \mathrm{~A}$ popular metaphor for the Markovian case is radioactive decay: a molecule has a constant probability per unit time of decaying. As a metaphor for the non-Markovian case, imagine entering an enormous maze and then trying to find your way back to the entrance. The longer one has wandered, the less the chance of stumbling on the exit in the next $\mathrm{d} t$.
} 


\subsection{The steady-state solution}

As a first illustrative example, suppose that the replacement rate is adjusted so that $N$ is constant. (Janitors replace light bulbs as soon as they burn-out.) In this case the equilibrium solution of (37) is

$$
f(a)=N \tau^{-1} \Psi(a)
$$

where

$$
\Psi(a) \equiv \exp \left(-\int_{0}^{a} \alpha\left(a^{\prime}\right) \mathrm{d} a^{\prime}\right), \quad \tau \equiv \int_{0}^{\infty} \Psi(a) \mathrm{d} a .
$$

The function $\Psi$, and its integral $\tau$, will occur frequently in the sequel. Notice that the replacement rate is $f(0)=N / \tau$ and this suggests that $\tau$ should be the average lifetime of an item. On the other hand, $\tau$ will not usually be equal to $\bar{a}$ in (36). I suggest brooding on this "paradox" and, as an exercise, see if you can resolve this confusion to your satisfaction by the end of this section.

In (41) we assume that the death rate $\alpha(a)$ is such that as $a \rightarrow \infty, \Psi(a) \rightarrow 0$ fast enough to ensure that $\tau$ is finite. For instance, if $\alpha$ is constant (this is the Markovian case) then $\Psi(a)=\exp (-\alpha a)$ and $\tau=\bar{a}=1 / \alpha$.

If the death rate $\alpha$ decreases with age then the average liftetime $\tau$ might not be finite. For example, consider the specific model

$$
\alpha=\frac{\nu}{\theta+a}, \quad \Rightarrow \quad \Psi(a)=\left(\frac{\theta}{\theta+a}\right)^{\nu} .
$$

Provided that $\nu>1$ then the integral of $\Psi(a)$ converges and $\tau=\theta /(\nu-1)$.

If $\nu<1$ then $\tau=\infty$ and there is no steady solution. To understand this curious result we must solve an initial value problem (see appendix A). Here we just remark that if $\nu<1$ then the average lifetime of a bulb is infinite. Detailed solution of the initial value problem in appendix $\mathrm{A}$ shows that in this case the replacement rate is $f(0, t) \propto t^{\nu-1}$. That is, the total number of new bulbs which have been installed at time $t$ grows like $t^{\nu} \ll t$. The hypothetical manufacturer of lightbulbs with $\nu<$ 1 is threatened with bankruptcy: sales decrease with time, even though every bulb eventually fails.

\subsection{A cohort of babies}

Imagine a cohort of babies leaving the maternity ward together, or a box of new lightbulbs shipped fresh from the factory. These items will function for varying amounts of time, and so we can speak of the PDF of lifetimes. We denote this PDF by $\mathcal{P}(a)$, and our goal is to relate $\mathcal{P}(a)$ to the death rate $\alpha(a)$. 
Consider a group of $N$ items which all start with $a=0$ at $t=0$. What fraction of this cohort survives at $t>0$ ? The surviving fraction is also the fraction of lifetimes longer than $t$ and so

$$
\text { surviving fraction at } t \equiv \Psi(t)=\int_{t}^{\infty} \mathcal{P}(a) \mathrm{d} a .
$$

To calculate the surviving fraction; we solve (37) with the initial and boundary conditions

$$
f(a, 0)=N \delta(a), \quad f(0, t)=0 .
$$

The solution of (37) and (44) is

$$
f(a, t)=N \Psi(t) \delta(a-t),
$$

where $\Psi$ is defined in (41). Thus $\Psi(t)$ is the fraction of the cohort which is still alive at time $t$; we refer to $\Psi$ as the survival function.

It now follows from (43) that the PDF of lifetimes of new items is

$$
\mathcal{P}(a)=-\Psi_{a}=\alpha \Psi .
$$

The average lifetime, $\tau$, is given by the equivalent expressions:

$$
\tau=\int_{0}^{\infty} a \mathcal{P}(a) \mathrm{d} a=-\int_{0}^{\infty} a \Psi_{a} \mathrm{~d} a=\int_{0}^{\infty} \Psi(a) \mathrm{d} a .
$$

Thus, as was suggested in the discussion following (41), to keep a population in equilibrium the replacement rate is equal to the size of the population, $N$, divided by the average lifetime of new items, $\tau$.

\subsection{Extinction of a population}

As a final example, suppose that at $t=0$ we have the steady-state lightbulb population in (40). If the janitors then go on strike, so that bulbs burn out without replacement, then how many bulbs are still operating at $t>0$ ? In this example we must solve (37) with the initial and boundary conditions that

$$
f(a, 0)=N \tau^{-1} \Psi(a), \quad f(0, t)=0 .
$$

The solution is

$$
f(a, t)=H(a-t) N \tau^{-1} \Psi(a),
$$


where $H(a-t)$ is the step function. Thus the fraction of surviving bulbs at $t$ is

$$
\Theta(t)=\tau^{-1} \int_{t}^{\infty} \Psi(a) \mathrm{d} a=\tau^{-1} \int_{t}^{\infty}(a-t) \mathcal{P}(a) \mathrm{d} a .
$$

Using the specific model of $\alpha$ in (42), the surviving fraction is

$$
\Theta(t)=\left(1+\theta^{-1} t\right)^{1-\nu} .
$$

$\Theta(t)$ is the most slowly decaying function we have seen so far: as $t \rightarrow \infty, \Theta(t) \gg$ $\Psi(t) \gg \mathcal{P}(t)$. This model may be relevant to the very slow extinction of professors once the supply of graduate students is cut-off.

Comparing the results in sections 3.1 and 3.2 , we see that the steady state population in section 3.1 contains more long-lived items than are in a cohort of new items section 3.2. This means that the average lifetime of the light bulbs currently operating in the Empire State building is longer than the average lifetime of bulbs shipped from the factory. The reason is obvious: items with brief lifetimes fail quickly, and will likely be replaced with items whose lifetime is closer to the mean. Thus, fragile individuals are underrepresented in an operational population.

\section{The generalized telegraph model}

\subsection{Formulation}

Using the machinery from the previous section we now construct a generalization of the telegraph model which exhibits anomalous diffusion. In this generalization particles switch randomly between moving with $u(t)=+U, u(t)=0$ and $u(t)=-U$. The transition probabilities between these states are functions of the time since the last transition. In other words, each particle carries an "age", $a$, which is the time elapsed since the particle transitioned into its present state. We denote the density of right moving particles at $(x, t)$, with age $a$, by $\mathcal{R}(a, x, t)$. For left-moving particles the density is $\mathcal{L}(a, t, x)$, and for the stationary particles the density is $\mathcal{S}(a, x, t)$. We refer to the left and right-movers collectively as "flying particles" while the stationary particles are "stickers".

The flying particles satisfy the conservational laws

$$
\mathcal{R}_{t}+\mathcal{R}_{a}+U \mathcal{R}_{x}+\alpha_{F} \mathcal{R}=0, \quad \mathcal{L}_{t}+\mathcal{L}_{a}-U \mathcal{L}_{x}+\alpha_{F} \mathcal{L}=0
$$

while the sticking particles have

$$
\mathcal{S}_{t}+\mathcal{S}_{a}+\alpha_{S} \mathcal{S}=0
$$

The death rates of flying and sticking particles, $\alpha_{F}$ and $\alpha_{S}$ respectively, are functions of age $a$; it is through this device that particles have a memory of their previous 
history. The price we pay for this nonMarkovian memory is that there are now three independent variables, $(a, t, x)$.

Stationary particles are born when left and right-moving particles die. And, conversely, when a stationary particle dies it is reborn as either a left moving particle or a right moving particle with equal probability. Notice that in order for a right-moving particle to become a left-moving particle it must pass through the intermediate state with $u=0$. These karmic rules are enforced by boundary conditions at $a=0$ :

$$
\mathcal{L}(0, t, x)=\mathcal{R}(0, t, x)=\frac{1}{2} \int_{0}^{\infty} \alpha_{S}(a) \mathcal{S}(a, t, x) \mathrm{d} a,
$$

and

$$
\mathcal{S}(0, t, x)=\int_{0}^{\infty} \alpha_{F}(a)[\mathcal{L}(a, t, x)+\mathcal{R}(a, t, x)] \mathrm{d} a .
$$

Trajectories of particles moving with this generalized telegraph process are shown in figure 8.

The model we have formulated here is a generalization of the telegraph model in two ways. First, there are three states: left, right and stationary. This minor embellishment is motivated by the Texas experiments in which trapping in a vortex corresponds to the stationary particles. The nontrivial generalization is the introduction of the age variable used to capture memory effects. As an exercise, the student can show that if $\alpha_{F}$ and $\alpha_{S}$ are independent of $a$ then one can easily integrate over $a$ and reduce (52) through (55) to a three-state telegraph model. (This exercise shows how the boundary condition at $a=0$ works.) As a sequel to this exercise, discuss $\alpha_{S} \rightarrow \infty$ and show that in this limit one obtains effectively a two-state telegraph model. Are you surprised that the diffusivity is given by (18)? That is, why do we recover model $\mathrm{B}$, rather than model $\mathrm{A}$, when the sojourns in the intermediate state $u=0$ are very brief?

In order to model slowly fading velocity correlations and anomalous diffusion we use

$$
\alpha_{F}(a)=\frac{\nu_{F}}{\theta_{F}+a}, \quad \alpha_{S}(a)=\frac{\nu_{S}}{\theta_{S}+a} .
$$

With the form above, the transition rates decrease as particles age. Numerical simulations of the three-state model using the transition rates in (56) show that many particles move in the same direction for a long time (see figure 9).

The main point of (56) is that if $a \gg 1$ then the transition rates $\alpha_{F}$ and $\alpha_{S}$ are proportional to $a^{-1}$. This inverse dependence on age ensures that the flying and sticking PDFs, $\mathcal{P}_{F}$ and $\mathcal{P}_{S}$ in (7), decay algebraically. Thus (56) incorporates important experimental information into the model ${ }^{2}$. One can make a dimensional argument in

${ }^{2}$ As far as scaling exponents are concerned, only the $a \gg 1$ structure of $\alpha_{F}$ and $\alpha_{S}$ matter. We use the specific functional form in (56) for simplicity. 


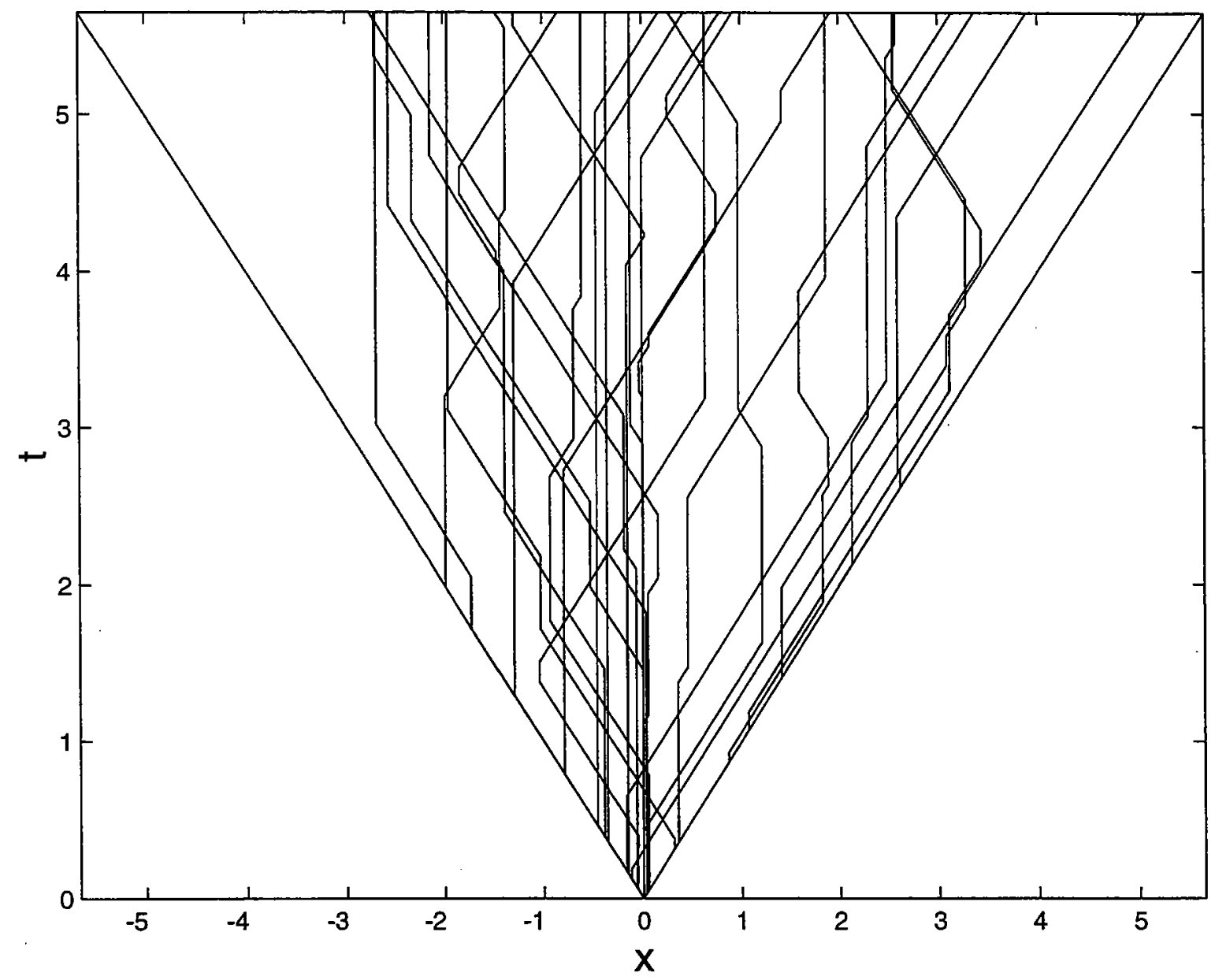

Figure 8: Trajectories of particles in the generalized telegraph random process. All particles are released from $x=0$.

support of (56): $\alpha_{F}$ and $\alpha_{S}$ have the dimensions of inverse time. If the only time-scale relevant for long-lived particles is the particle age, $a$, then it follows that $\alpha_{F}$ and $\alpha_{S}$ are inversely proportional to $a$. We now show that the parameters $\nu_{F}$ and $\nu_{S}$ are easily related to the experimentally measured exponents $\mu_{F}$ and $\mu_{S}$ in (9).

\subsection{The equilibrium solution}

The system (52) through (55) has a solution which is homogeneous $\left(\partial_{x}=0\right)$ and steady $\left(\partial_{t}=0\right)$. This equilibrium solution is

$$
\mathcal{R}(a, x, t)=\mathcal{L}(a, t, x)=r \Psi_{F}(a), \quad \mathcal{S}(a, t, x)=2 r \Psi_{S}(a)
$$


(a) $t=10^{3}$

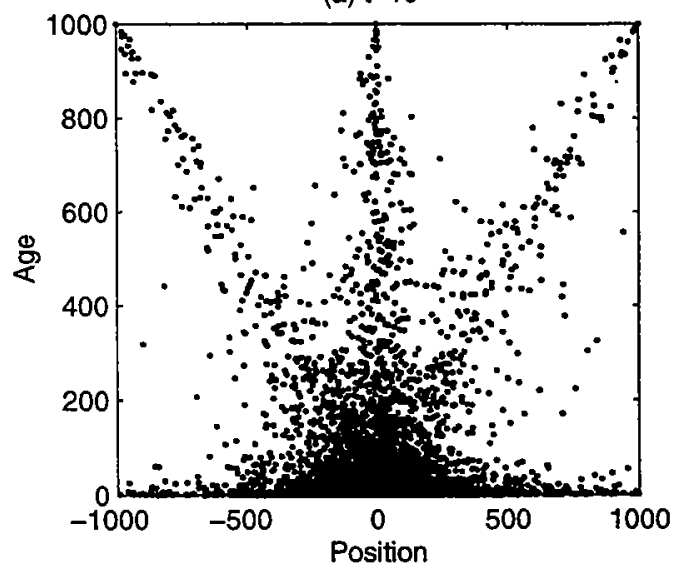

(c) $t=10^{3}$

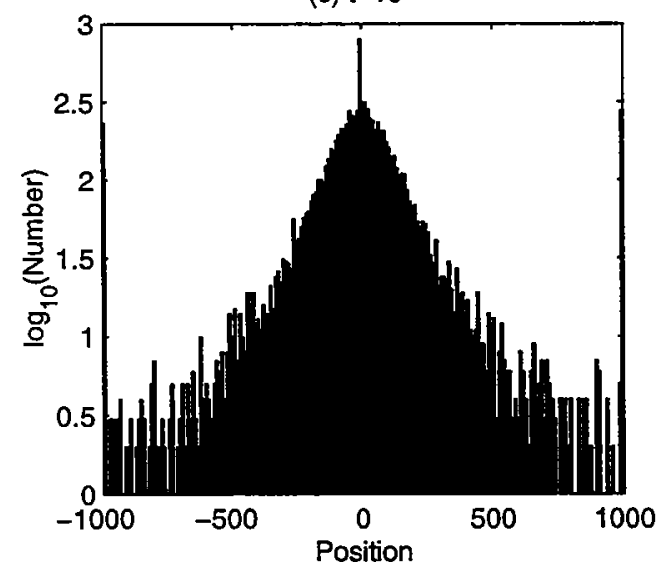

(e) $t=10^{3}$

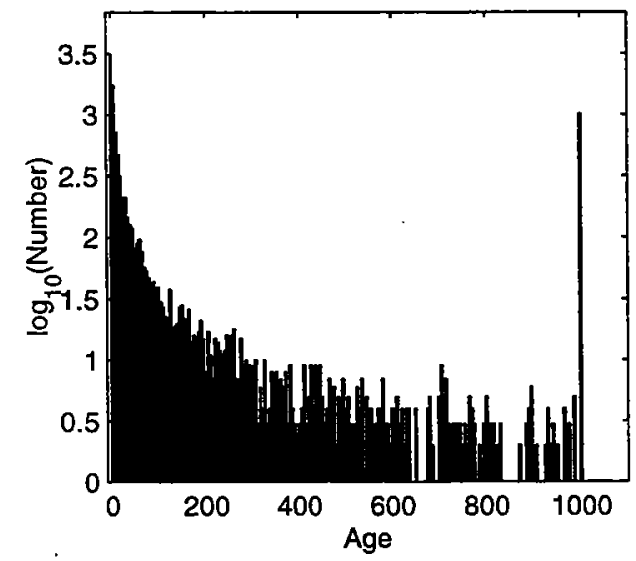

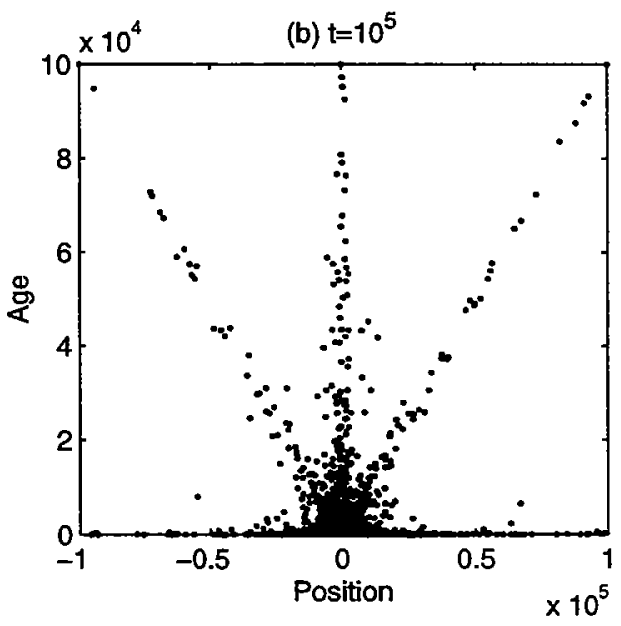

(d) $t=10^{5}$

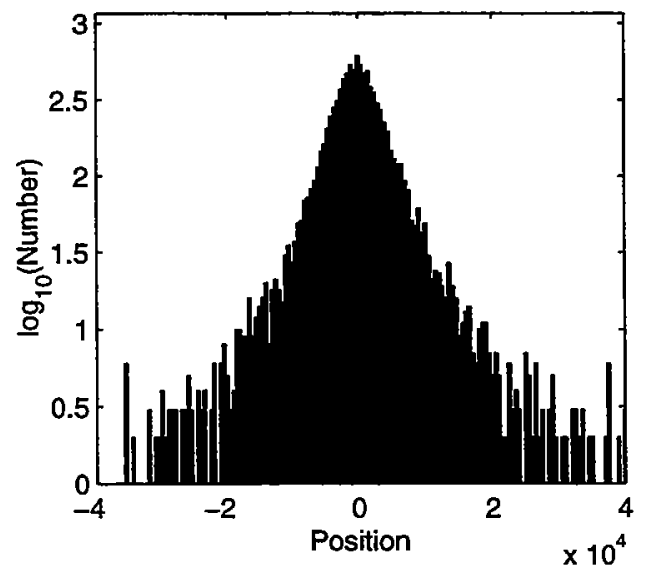

(f) $t=10^{5}$

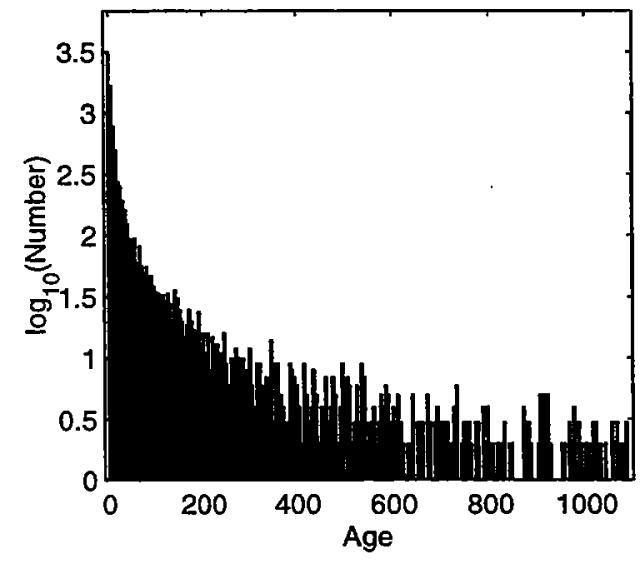

Figure 9: A simulation with $N=10^{4}$ particles; $\alpha_{F}(a)=\alpha_{S}(a) \sim 1.35 / a$. Upper panels: PDFs as a function of age and position show that there are many particles that either stick or move at a constant velocity for nearly the whole simualtion. Center panels: PDFs of the position of particles develop tails larger than Gaussians as time goes on. Lower panels: PDFs of the age of particles have a spike at large times, because there is a fraction of particles that never die. 
where $\Psi_{F, S}(a)$ is

$$
\Psi_{F, S}(a) \equiv \exp \left(-\int_{0}^{a} \alpha_{F, S}\left(a^{\prime}\right) \mathrm{d} a^{\prime}\right) .
$$

The constant $r$ in (57) is the transition rate between the different states; $r$ is determined by the normalization condition:

$$
N=2 r\left(\tau_{S}+\tau_{F}\right), \quad \tau_{F, S} \equiv \int_{0}^{\infty} \Psi_{F, S}(a) \mathrm{d} a .
$$

We can use the results from section 3 to interpret $\tau_{F}$ and $\tau_{S}$ as the average lifetimes in the flying and sticking states respectively. Using (46), the PDF of lifetimes in those states is given by

$$
\mathcal{P}_{F, S}=\alpha_{F, S} \Psi_{F, S}
$$

Using the expression in (56) for $\alpha_{F, S}$, we see that as $a \rightarrow \infty$, the survival functions decay algebraically with $\Psi_{F, S} \sim a^{-\nu_{F, S}}$, and so $\mathcal{P}_{F, S} \sim a^{-\nu_{F, S}-1}$. It follows that the exponents $\mu_{F}$ and $\mu_{S}$ defined in (9) are related to $\nu_{F}$ and $\nu_{S}$ by

$$
\mu_{F, S}=\nu_{F, S}+1 \text {. }
$$

We can summarize our arguments to this point by observing that the experiments provide the flying velocity, $U$; the average lifetime in the flying and sticking states, $\tau_{F, S}$, and the exponents $\mu_{F, S}$. These five experimental data determine the five parameters in the generalized telegraph model, namely $\left(U, \nu_{F, S}, \theta_{F, S}\right)$.

\subsection{Formulation of the initial value problem}

Now that we have determined the model parameters using experimental constraints it is time to do some mathematics and use the model to predict the exponent $\gamma$ in (6). The simplest intial value problem we can consider is (52) through (55) with

$$
[\mathcal{R}(a, 0, x), \mathcal{S}(a, 0, x), \mathcal{L}(a, 0, x)]=r\left[\Psi_{F}(a), 2 \Psi_{S}(a), \Psi_{F}(a)\right] \delta(x) .
$$

The constant $r$ is given in (57). Thus, the initial population has an equilibrium distribution of ages and is released at $x=0$. Because of the symmetry between left and right moving particles

$$
\mathcal{R}(a, t, x)=\mathcal{L}(a, t,-x), \quad \mathcal{S}(a, t, x)=\mathcal{S}(a, t,-x) .
$$

Equation (63) greatly simplifies subsequent algebra.

One technical point (which I confess confuses me) is using the equilibrium age distribution as the initial condition in (62). This choice leads to simple calculations 
below. And perhaps the gross details of the dispersion process, such the exponent $\gamma$, are independent of the initial distribution of ages? As an excercise I suggest solving the initial value problem using other initial conditions e.g., $\mathcal{R}(a, 0, x)=\delta(a) \delta(t)$ etcetera. Are there any significant differences in the $t \rightarrow \infty$ structure of the solution?

Our strategy will be to obtain a closed hierarchy of spatial moments by multiplying the conservation laws (52) and (53) by $x^{n}$ and integrating over $x$. It is possible to solve the first few members of the hierarchy and show that if $\alpha_{F, S}$ and has the form in (56) with $1<\nu<2$ then as $t \rightarrow \infty$

$$
\left\langle x^{2}\right\rangle=\int_{0}^{\infty} \int_{-\infty}^{\infty} x^{2}[\mathcal{R}(a, t, x)+\mathcal{S}(a, t, x)+\mathcal{L}(a, t, x)] \mathrm{d} x \mathrm{~d} a \propto t^{3-\nu_{F}} .
$$

Before entering this calculation, we give a simple argument which suggests how the anomalous exponent $3-\nu_{F}>1$ arises in (64).

The variance $\left\langle x^{2}\right\rangle$ in (64) can alternatively be written as

$$
\left\langle x^{2}\right\rangle=\frac{1}{N} \sum_{n=1}^{N} x_{n}^{2}
$$

At time $t>0$ some of the $N$ particles will have moved coherently with unchanging velocity (either $+U$ or $-U$ ) ever since $t=0$; half of these particles will be at $x=U t$ and the other half at $x=-U t$. These "coherent particles" each contribute a term $U^{2} t^{2}$ to the sum on the right hand side of (65). The number of coherent flying particles is just $\Theta(t) N$ where $\Theta(t)$ is given by (51) with $\nu$ replaced by $\nu_{F}$. Thus, because every term in the sum in (65) is positive, one has

$$
\left\langle x^{2}\right\rangle>\Theta(t) U^{2} t^{2} \sim U^{2} \theta_{F}^{\nu-1} t^{3-\nu_{F}}
$$

The inequality (66) has teeth only if $3-\nu_{F}>1$ : then we learn that the coherent particles alone produce a superdiffusive contribution to the variance.

The argument above may suggest to you that superdiffusion is due solely to the few extreme particles which move without changes in direction. This is an overstatement: the lower bound in (66) is generously less than the exact result for $\left\langle x^{2}\right\rangle$ which we obtain in the next section. Thus "nearly-coherent" particles, meaning particles which change direction only once or twice, also make a large contribution to the sum in (65). This is an essential point, because in their analysis of the experiments Solomon et al. discarded all coherent particles from the data set. ${ }^{3}$. Thus the exponent measured by Solomon et al reflects only the contribution of nearly coherent particles.

\footnotetext{
${ }^{3}$ This drastic procedure is necessary because some fraction of the experime ntal particles are in integrable regions and will fly forever. Retaining all these particles will ultimately lead to the trival ballistic exponent $\gamma=2$.
} 


\subsection{Solution of the initial value problem}

This is a dry section which contains the details of the analytic calculation of $\left\langle x^{2}\right\rangle$. The main point of interest here is that a lot of the algebra can be avoided by proving (75) below. (I suggest this as an exercise.)

The spatial moments are defined by

$$
\left[\mathcal{R}_{n}(a, t), S_{n}(a, t), \mathcal{L}_{n}(a, t)\right] \equiv \int_{-\infty}^{\infty} x^{n}[\mathcal{R}(a, t, x), \mathcal{S}(a, t, x) \mathcal{L}(a, t, x)] \mathrm{d} x,
$$

Because of the symmetry in (63)

$$
\mathcal{R}_{n}(a, t)=(-1)^{n} \mathcal{L}_{n}(a, t), \quad \mathcal{S}_{n}(a, t)=0 \text { if } n \text { is odd. }
$$

The result above allows us to work exclusively with $\mathcal{R}_{n}$ and $\mathcal{S}_{n}$ while retaining full information about the distribution. Using the symmetry, the variance can be written as

$$
\left\langle x^{2}\right\rangle=\int_{0}^{\infty} 2 R_{2}+S_{2} \mathrm{~d} a .
$$

The zeroth moment of (52) through (55), with the initial condition in (62) is

$$
\left[\mathcal{R}_{0}(a, t), \mathcal{S}_{0}(a, t), \mathcal{L}_{0}(a, t)\right]=r\left[\Psi_{F}(a), 2 \Psi_{S}(a), \Psi_{F}(a)\right] .
$$

That is, the zeroth moment is just the equilibrium solution. (This is why using the equilibrium age distribution as the initial condition is so convenient.)

Using (68), the first spatial moment is $\mathcal{S}_{1}=0, \mathcal{L}_{1}(a, t)=-\mathcal{R}_{1}(a, t)$ and

$$
\mathcal{R}_{1 t}+\mathcal{R}_{1 a}+\alpha_{F} \mathcal{R}_{1}=U r \Psi_{F}, \quad \mathcal{R}_{1}(0, t)=0, \quad \mathcal{R}_{1}(a, 0)=0 .
$$

The solution of the initial value problem (71) is

$$
\mathcal{R}_{1}(a, t)=U r \Psi_{F}(a) \min (a, t) .
$$

The second moment equations are $\mathcal{L}_{2}=\mathcal{R}_{2}$ and

$$
\mathcal{R}_{2 t}+\mathcal{R}_{2 a}+\alpha_{F} \mathcal{R}_{2}=2 U \mathcal{R}_{1}, \quad \mathcal{S}_{2 t}+\mathcal{S}_{2 a}+\alpha_{S} \mathcal{S}_{2}=0,
$$

with the $a=0$ boundary condition that

$$
2 \mathcal{R}_{2}(0, t)=\int_{0}^{\infty} \alpha_{S}(a) \mathcal{S}_{2}(a, t) \mathrm{d} a, \quad \mathcal{S}_{2}(0, t)=2 \int_{0}^{\infty} \alpha_{F}(a) \mathcal{R}_{2}(a, t) \mathrm{d} a .
$$

To obtain the variance in (69) we do not need the complete solution of (73) and (74). Instead, after some judicious integration over $a$, one finds that

$$
\frac{\mathrm{d}}{\mathrm{d} t}\left\langle x^{2}\right\rangle=4 U \int_{0}^{\infty} \mathcal{R}_{1}(a, t) \mathrm{d} a .
$$


Substituting (72) into the result above we obtain

$$
\frac{\mathrm{d}}{\mathrm{d} t}\left\langle x^{2}\right\rangle=4 U^{2} r\left[\int_{0}^{t} a \Psi_{F}(a) \mathrm{d} a+t \int_{t}^{\infty} \Psi_{F}(a) \mathrm{d} a\right] .
$$

If the right hand side of (76) approaches a constant as $t \rightarrow \infty$ then the variance grows diffusively. Otherwise there is anomalous diffusion.

With (76) in hand, one can easily determine if particular models of $\alpha_{F}$ and $\Psi_{F}$ lead to anomalous diffusion. For example, with the model in (56), evaluating the integrals in (42) gives a pleasant exact solution

$$
\frac{\mathrm{d}}{\mathrm{d} t}\left\langle x^{2}\right\rangle=4 U^{2} r \theta_{F}^{2}\left[\frac{(1+\tilde{t})^{2-\nu_{F}}}{\left(2-\nu_{F}\right)\left(\nu_{F}-1\right)}+\frac{1}{\left(\nu_{F}-1\right)\left(\nu_{F}-2\right)}\right]
$$

where $\tilde{t} \equiv t / \theta$.

The asymptotic behaviour at large time depends crucially on $\nu_{F}$. If $\nu_{F}>2$ then there is normal diffusion:

$$
\frac{\mathrm{d}}{\mathrm{d} t}\left\langle x^{2}\right\rangle \approx \frac{4 U^{2} r \theta_{F}^{2}}{\left(\nu_{F}-1\right)\left(\nu_{F}-2\right)}+O\left(t^{2-\nu_{F}}\right)
$$

If $1<\nu_{F}<2$, there is superdiffusion

$$
\frac{\mathrm{d}}{\mathrm{d} t}\left\langle x^{2}\right\rangle \approx \frac{4 U^{2} r \theta_{F}^{2} \tilde{t}^{2-\nu_{F}}}{\left(2-\nu_{F}\right)\left(\nu_{F}-1\right)}+O(1) .
$$

(At $\nu_{F}=2$ there is a logarithmic term.)

Notice the minor role of $\alpha_{S}(a)$ in the solution above: if $\nu_{S}>1$, so that the mean sticking time is finite, then the parameters $\nu_{S}$ and $\theta_{S}$ occur only in $r$.

\subsection{An exercise for the diligent student}

Consider an asymmetric two-state model

$$
\mathcal{L}_{t}+\mathcal{L}_{a}+U_{L} \mathcal{L}_{x}+\alpha_{L}(a) \mathcal{L}=0, \quad \mathcal{R}_{t}+\mathcal{R}_{x}+U_{R} \mathcal{R}_{x}+\alpha_{R}(a) \mathcal{R}=0,
$$

with the boundary conditions

$$
\mathcal{L}(0, t, x)=\int_{0}^{\infty} \alpha_{R}(a) \mathcal{R}(a, t, x) \mathrm{d} a, \quad \mathcal{R}(0, t, x)=\int_{0}^{\infty} \alpha_{L}(a) \mathcal{L}(a, t, x) \mathrm{d} a .
$$

Show that the average velocity is

$$
\bar{U}=\frac{\tau_{L} U_{L}+\tau_{R} U_{R}}{\tau_{L}+\tau_{R}}, \quad \tau_{L, R} \equiv \int_{0}^{\infty} \Psi_{L, R}(a) \mathrm{d} a,
$$


where $\Psi_{L}$ and $\Psi_{R}$ are defined by analogy with (41). Show that the Laplace transform of the velocity autocorrelation function is given by

$$
\operatorname{côrr}(s)=U_{R M S}^{2}\left[\frac{1}{s}-\frac{\tau_{L}+\tau_{R}}{\tau_{L} \tau_{R}} \frac{\left(1-\hat{\psi}_{L}\right)\left(1-\hat{\psi}_{R}\right)}{s^{2}\left(1-\hat{\psi}_{L} \hat{\psi}_{R}\right)}\right],
$$

where

$$
U_{R M S}^{2} \equiv \frac{\tau_{L}+\tau_{R}}{\sqrt{\tau_{L} \tau_{R}}} U_{R} U_{L} .
$$

(If you use the moment method, you will need Laplace transforms to solve the integral equation which arises at $n=1$.) Using the model

$$
\alpha_{R, L}(a)=\frac{\nu_{R, L}}{\theta_{R, L}+a},
$$

perform an asymptotic analysis of (83) to identify the anomalous diffusion exponents which occur if either or both of $\nu_{L}$ and $\nu_{R}$ are less than 2.

\section{References}

[1] D.D. Joseph and L. Preziosi. Heat waves. Rev. Mod. Phys., 61:41-73, 1989.

[2] M. Schlesinger, G.M. Zaslavsky, and U. Frisch. Lévy Flights and Related Topics in Physics. Springer, Berlin, 1994.

[3] T.H. Solomon, E. Weeks, and H.L. Swinney. Chaotic advection in a two-dimensional flow: Lévy flights and anomlaous diffusion. Physica D, 76:70-84, 1994.

[4] G.I. Taylor. Diffusion by continuous movements. Proc. London Math. Soc., 20:196$212,1921$.

[5] E.R. Weeks, J.S. Urbach, and H.L. Swinney. Anomalous diffusion in asymmetric random walks with a quasi-geostrophic example. Physica D, 97:291-310, 1996.

\section{A Solution of an initial value problem}

In this appendix we discuss the issue raised at the end of section 3.1 and analyze a problem in which the death rate of old items is so small that the average lifetime $\tau$ is infinite. For example, this is the case $\nu<1$ in (42). Specifically, consider the initial value problem posed by (37) with the initial and boundary conditions

$$
f(a, 0)=N \delta(a), \quad f(0, t)=r(t) .
$$


In (86) the replacement rate $r(t)$ is determined by requiring that

$$
N=\int_{0}^{\infty} f(a, t) \mathrm{d} a .
$$

The solution of (37) and (86) is

$$
f=N \Psi(t) \delta(a-t)+\Psi(a) r(t-a) .
$$

The first term on the right hand side of the equation above is the cohort of initial items aging and dying. The second term is influx of new items. Imposing (87) on (88), we obtain an integral equation for $r$ :

$$
N=N \Psi(t)+\int_{0}^{t} \Psi(a) r(t-a) \mathrm{d} a .
$$

The integral relation above is known as the renewal equation

Because of the convolution in (89), the Laplace transform

$$
[\hat{\Psi}(s), \hat{r}(s)] \equiv \int_{0}^{\infty} e^{-s a}[\Psi(a), r(a)] \mathrm{d} a,
$$

is gratifying. In this way we find from (89) that

$$
\hat{r}=N \frac{1-s \hat{\Psi}}{s \hat{\Psi}} .
$$

The large-time behaviour of $r(t)$ can be obtained from (91) using standard asymptotic methods.

If $\alpha(a) \propto 1 / a$ as $a \rightarrow \infty$, then the rightmost singularity of $\hat{\Psi}(s)$ in the complex $s$-plane is the branch-point at $s=0$. We show below in (94) through (97) that the structure of $\hat{\Psi}$ at this branch-point is

$$
\Psi(s)=\varpi s^{\nu-1}+\tau+\cdots
$$

If $\nu<1$ then the singular term involving $s^{\nu-1}$ dominates the constant $\tau$ as $s \rightarrow 0$. In this case, from(91),

$$
\hat{r}(s) \sim \frac{N}{\varpi s^{\nu}}, \quad \Rightarrow \quad r(t) \sim \frac{N t^{\nu-1}}{\varpi \Gamma(\nu)}, \quad \text { as } \quad t \rightarrow \infty
$$

Because $\nu<1$ the replacement rate vanishes as $t \rightarrow \infty$.

To explain the small-s expansion in (92), we use the model death-rate in (42), which produces the survival function

$$
\Psi(a)=\left(\frac{\theta}{\theta+a}\right)^{\nu} .
$$


The Laplace transform in (90) is then

$$
\hat{\Psi}(s)=\theta^{\nu} s^{\nu-1} e^{\theta s} \Gamma(1-\nu, \theta s),
$$

where $\Gamma(a, x)$ is the incomplete $\Gamma$-function defined by Abramowitz \& Stegun in their article 6.5.3. This Laplace transform can be rewritten as

$$
\hat{\Psi}(s)=\theta^{\nu} s^{\nu-1} e^{\theta s} \Gamma(1-\nu)-\theta \Gamma(1-\nu) \sum_{n=0}^{\infty} \frac{(\theta s)^{n}}{\Gamma(2-\nu+n)} .
$$

The form above is convenient because the singular terms containing $s^{\nu-1}$ are localized in the first function on the right hand side. When $s \ll 1$ the expansion of (96) is

$$
\hat{\Psi}(s)=\theta^{\nu} \Gamma(1-\nu) s^{\nu-1}+\frac{\theta}{\nu-1}+O\left(s, s^{\nu}\right),
$$

which is the form assumed in (92). 


\title{
Lecture 6: \\ The Batchelor spectrum and tracer cascade
}

\author{
R. T. Pierrehumbert
}

\section{The advection-diffusion equation}

In this lecture we consider the problem of determining the spectrum of a passive tracer advected by a velocity field. The evolution of the tracer is described by the equation

$$
\partial_{t} \Theta+\mathbf{u} \cdot \nabla \Theta=\kappa \nabla^{2} \Theta
$$

where $\kappa$ is the molecular diffusivity, $\mathbf{u}$ the advection velocity field and $\partial_{t}$ denotes a partial derivative with respect to time. Depending on the particular experimental situation, the tracer $\Theta$ could be temperature or density of a passive contaminant such as ink. By $\Theta$ being passive, we mean that the dynamics of the velocity does not couple with $\Theta$. Thus equation (1) is truly linear in $\Theta$. We confine our attention to the case where $\mathbf{u}$ is non-divergent. Most of the mathematical details will be carried out in the two dimensional case, though many of the techniques and arguments admit ready generalizations to any number of dimensions. Throughout, we employ cartesian coordinates $(x, y)$.

Batchelor [1] realized that the general increase of gradients of $\Theta$ accompanying the stirring action of the velocity field, which is a consequence of the quadratic term of (1), can also be thought of as a transfer between different Fourier components of the spectrum of $\Theta$. If both $\mathbf{u}$ and $\Theta$ are written in the form of Fourier integrals, the term $\mathbf{u} \cdot \nabla \Theta$ leads to the generation of new harmonics of $\Theta$ and the growth of ever-increasing wavenumbers. The transfer of tracer variance from low wavenumbers to high wavenumbers is mathematically similar to that hypothetised by Kolomogorov for the velocity variance in a turbulent field.

The Fourier components of the tracer $\Theta$, if the flow is spatially unbounded, are defined by

$$
\Theta(x, y)=\frac{1}{(2 \pi)^{2}} \int_{-\infty}^{+\infty} \int_{-\infty}^{+\infty} d k d l \hat{\Theta}(k, l) e^{i k x+i l y} .
$$

In the present context, it is convenient to define a spectrum function for $\Theta$ as

$$
\mathcal{C}(K)=\frac{1}{V} \frac{1}{(2 \pi)^{2}} \int_{0}^{2 \pi} d \phi K|\hat{\Theta}(K, \phi)|^{2},
$$

where $\hat{\Theta}(K, \phi)$ are the Fourier components of $\Theta$ in polar coordinates $K=\sqrt{k^{2}+l^{2}}$ and $\phi=$ $\arctan (l / k)$. If the tracer field is isotropic the integral is trivial and $\mathcal{C}(K)=K \mid \hat{\Theta}(K))\left.\right|^{2} / 2 \pi V$. The normalization factor $V=\iint_{V} d x d y$ ensures that the spectrum has units of variance of $\Theta$ per unit wavenumber. In fact it follows from Parseval's equality that the variance of $\Theta$ is the integral of $\mathcal{C}(K)$ in wavenumber space

$$
\left\langle\Theta^{2}\right\rangle=\lim _{V \rightarrow \infty} \frac{1}{V} \iint_{V} d x d y \Theta^{2}(x, y)=\int_{0}^{+\infty} d K \mathcal{C}(K) .
$$


Following the line of argument of Kolmogorov and Batchelor, we suppose that the diffusivity $\kappa$ is so small as to make the effect of diffusion appreciable only at the large wavenumber end of the spectrum. The part of the equilibrium range of wavenumbers for which the Fourier components of $\mathbf{u}$ are independent of viscosity is usually termed the "inertial subrange" and an appropriate term for the part of the equilibrium range for which the Fourier components of $\Theta$ are independent of molecular diffusion is the "advection subrange". No actual destruction of tracer variance takes place at wavenumbers in, or smaller than those in the advection subrange. All the destruction takes place at high wavenumbers as a result of the action of molecular diffusion. The total rate of destruction of variance per unit volume is calculated by integrating the advection-diffusion equation over the whole domain

$$
\partial_{t}\left\langle\Theta^{2}\right\rangle=-2 \kappa\left\langle|\nabla \Theta|^{2}\right\rangle=-\chi .
$$

This relation shows that the quadratic terms in (1) makes no contribution to $\partial_{t}\left\langle\Theta^{2}\right\rangle$. Thus when one Fourier component of the spectrum of $\Theta$ is changed by the interaction between $\Theta$ and $\mathbf{u}$, other Fourier components are changed simultaneously in such a way that the sum of the contributions to $\left\langle\Theta^{2}\right\rangle$ from all Fourier components remains the same. This shows that $\Theta$ variance is simply transferred from small to large wavenumbers in the advection subrange and $\chi$ is a given constant quantity.

If the velocity field $\mathbf{u}$ is characterized by a single time scale $\tau$, it is possible to predict the spectrum of $\Theta$ on dimensional grounds. In fact the spectrum $\mathcal{C}(K)$ at a wavenumber $K$ is determined by $\chi$, the mean rate at which variance is cascaded, the time scale $\tau$, and the local wavenumber $K$. There is only one combination of these three parameters with dimensions of $\left\langle\Theta^{2}\right\rangle$ per unit wavenumer and this combination gives the functional form of the spectrum,

$$
\mathcal{C}(K) \sim \chi \tau K^{-1}
$$

Batchelor noticed that this result is of physical interest for tracers whose diffusivity is much smaller than the viscosity of the advecting fluid. In this case the velocity field at scales shorter than the viscous cutoff is extremely smooth and the primary effect of the advection on variations of $\Theta$ is a uniform straining rate of strength $\tau^{-1}$.

A characterization of the spectrum of $\Theta$ can be obtained also at scales larger than the viscous cutoff, if the velocity spectrum has a power law dependence on the wavenumber. The spectrum of velocity $\mathcal{E}(K)$ is the variance of velocity as a function of wavenumber and is defined in a way analogous to the spectrum of $\Theta$ such that

$$
\frac{1}{2}\langle\mathbf{u} \cdot \mathbf{u}\rangle=\int_{0}^{+\infty} d K \mathcal{E}(K)
$$

If $\mathcal{E}(K) \sim K^{-\alpha}$, a time-scale $\tau$ for the velocity field is given by the turnover time at scale $K$,

$$
\tau \sim \frac{K^{-1}}{\sqrt{K \mathcal{E}(K)}} \sim K^{-(3-\alpha) / 2} .
$$

The eddy turnover time decreases with scale for $\alpha<3$ and the picture of larger eddies feeding smaller eddies is appropriate. For $\alpha>3$ the cascade of energy through different scales cannot be considered local in wavenumber space and the estimate of $\tau$ must be corrected to include the presence of non local effects, but this is beyond the scope of this lecture. Given an estimate for $\tau$, the same dimensional argument used for the case of a velocity field characterized by only one time scale implies

$$
\mathcal{C}(K) \sim \chi K^{-(5-\alpha) / 2} .
$$

According to Kolmogorov's arguments on isotropic and homogeneous turbulence, the spectrum of velocity in the inertial subrange scales as $\mathcal{E}(K) \sim K^{-5 / 3}$. In this case the tracer spectrum scales as $\mathcal{C}(K) \sim K^{-5 / 3}$ as well and is known as the Obukhov-Corrsin spectrum $[2,3]$. 


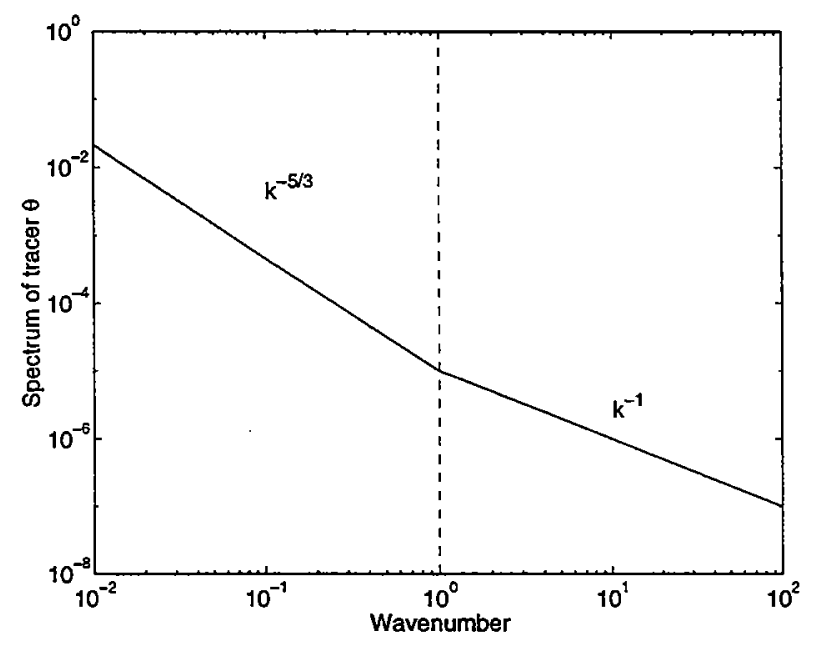

Figure 1: Spectrum of a passive tracer advected by a 3D turbulent field. Wavenumbers are normalized by the cutoff viscous scale (the scale at which momentum is dissipated).

For tracers advected by a turbulent, three-dimensional velocity field, with diffusivity $\kappa$ much smaller than the viscosity of the fluid $\nu$, we expect to see a spectrum $\mathcal{C}(K) \sim K^{-5 / 3}$ at scales larger than the viscous cutoff, and a spectrum $\mathcal{C}(K) \sim K^{-1}$ at smaller scales, as shown in Figure 1 . The scaling breaks off at large wavenumbers at the dissipation cutoff (the scale at which the tracer is dissipated, here assumed smaller than the scale at which momentum is dissipated) and at small wavenumbers at the scale of the domain, if the domain is finite, or at the scale of forcing if there is some forcing feeding variance in $\Theta$.

\section{The 2-point correlation function: what can it tell us about the properties of the flow.}

\subsubsection{Definition and uses of the 2-point correlation function}

The aim of the two point correlation function $Z_{2}(r)$ of a concentration field is to yield information about the typical variation of the concentration over a distance $r$. Let's therefore define it as

$$
Z_{2}(r ; \ldots)=\left\langle|\Theta(\mathbf{x}+r \hat{\mathbf{n}})-\Theta(\mathbf{x})|^{2}\right\rangle
$$

where $\hat{\mathbf{n}}$ is a unit vector in the chosen direction, and the average can be a time, spatial, or ensemble average. Depending on the average chosen, $Z_{2}(r ; \ldots)$ may also depend on the time $t$ (for spatial or ensemble average), or the starting position $\mathbf{x}$ (for the time average if the fluid is not homogeneous). If the flow is anisotropic, the correlation function depends on $\hat{\mathbf{n}}$ as well.

The 2-point correlation function can also be rewritten as

$$
\begin{aligned}
Z_{2}(r, \hat{\mathbf{n}}) & =\left\langle\Theta(\mathbf{x})^{2}\right\rangle+\left\langle\Theta(\mathbf{x}+r \hat{\mathbf{n}})^{2}\right\rangle-2\langle\Theta(\mathbf{x}) \Theta(\mathbf{x}+r \hat{\mathbf{n}})\rangle \\
& =2\left\langle\Theta^{2}\right\rangle-2\langle\Theta(\mathbf{x}) \Theta(\mathbf{x}+r \hat{\mathbf{n}})\rangle
\end{aligned}
$$

if the flow is spatially homogeneous. More generally, one could define correlation functions of any order:

$$
Z_{q}(r)=\left\langle|\Theta(\mathbf{x}+r \hat{\mathbf{n}})-\Theta(\mathbf{x})|^{q}\right\rangle
$$


As $q$ becomes larger, $Z_{q}$ is increasingly dominated by the most extreme fluctuations (i.e. $\Theta(\mathbf{x}+r \hat{\mathbf{n}})-$ $\Theta(\mathbf{x})$ locally very large).

The 2-point correlation function yields precious information about the global spatial structure of the flow. For instance,

- if the flow is smooth at all points, then the concentration gradient exists everywhere and $|\nabla \Theta|<+\infty$. As a result, for small enough $r$, we get $Z_{2}(r) \approx\left\langle|\nabla \Theta|^{2}\right\rangle r^{2}$;

- for a flow containing well separated jumps/steps in the concentration field, then $Z_{2}(r) \propto r$;

- for a non-singular fractal flow, with a possible local accumulation of steps, the correlation function becomes $Z_{2}(r) \propto r^{\nu}$; where $1<\nu<2$. Smaller values of $\nu$ arise form singularities in the tracer field-places where the tracer value becomes infinite;

- if the flow contains integrable singularities, then $Z_{2}(r) \propto r^{\nu}$ with $0<\nu<1$, whereas nonintegrable singularities have $\nu<0$;

- for a flow with a white noise spectrum, there is no correlation between any two points, so that the correlation function is constant $Z_{2}(r) \propto r^{0}$.

To summarize, non-singular flows have 2-point correlation functions given by $Z_{2}(r) \propto r^{\nu}$ where $0<\nu<2$. There exists no interesting flows with $\nu>2$ since this would require the gradient of the velocity to be 0 everywhere.

\subsection{Relation between the 2-point correlation function and the power spec- trum}

We saw that the flow variance can be decomposed onto the spectral modes as

$$
\left\langle\Theta^{2}\right\rangle=\int_{0}^{\infty} \mathcal{C}(K) \mathrm{d} K
$$

where, say, $\mathcal{C}(K) \propto K^{-\alpha}$. In the specific case of $1-\mathrm{D}$, one can show that the correlation function can also be rewritten as $[4$, p. 95]

$$
Z_{2}(r)=2 \int_{0}^{\infty} \mathcal{C}(K)(1-\cos (K r)) \mathrm{d} K
$$

so that

$$
\begin{aligned}
Z_{2}(r) & =2 \int_{0}^{1 / r} \mathcal{C}(K) K^{2} r^{2} \mathrm{~d} K+2 \int_{1 / r}^{\infty} \mathcal{C}(K)(1-\cos (K R)) \mathrm{d} K \\
& \approx 2 \int_{0}^{1 / r} \mathcal{C}(K) K^{2} r^{2} \mathrm{~d} K+2 \int_{1 / r}^{\infty} \mathcal{C}(K) \mathrm{d} K \\
& \approx 2 r^{2}\left[\frac{K^{3-\alpha}}{3-\alpha}\right]_{0}^{1 / r}+2\left[\frac{K^{1-\alpha}}{1-\alpha}\right]_{1 / r}^{\infty}
\end{aligned}
$$

Note that only the tail of $\mathcal{C}(K)$ varies like $K^{-\alpha}$, so that the 0 bound of the integral does not actually pose any problems, and is a given constant, say $\zeta_{0}$. Various cases can occur.

- in the case where $\alpha>3$ then the integral is dominated by the first term, and so $Z_{2}(r) \approx$ $\frac{4 \quad \cdot}{(3-\alpha)(\alpha-1)} r^{\alpha-1}+2 r^{2} \zeta_{0}$. For small $r$ the dominant term is therefore $Z_{2}(r) \propto r^{2}$. 
- in the case $1<\alpha<3$ then the integral is

$$
Z_{2}(r) \approx 2 r^{2} \frac{r^{\alpha-3}}{3-\alpha}+2 r^{2} \zeta_{0}+2 \frac{r^{\alpha-1}}{\alpha-1}=r^{\alpha-1} \frac{4}{(3-\alpha)(\alpha-1)}+2 r^{2} \zeta_{0}
$$

For very small $r$, the dominant term is $Z_{2}(r) \propto r^{\alpha-1}$.

- in the case where $\alpha<1$ the second integral is not convergent; $Z_{2}(r)$ is not defined in that case. However, in the limit $\alpha \rightarrow 1$ and $\alpha>1$, the dominant terms are

$$
Z_{2}(r) \approx \frac{4}{(3-\alpha)(\alpha-1)}+\frac{4}{(3-\alpha)} \ln r \propto \ln \left(\frac{r}{r_{\star}}\right)
$$

so that the length-scale $r_{\star}$ appears as a cutoff below which the flow is smooth on all scales. The limit $\alpha \rightarrow 1$ corresponds to the Batchelor spectrum, and $r_{\star}$ is the dissipation scale.

Note that in the case where $\alpha$ is greater than 3, the correlation function increases faster than $r^{2}$, which cannot correspond to any physical flow. We also see that provided $1<\alpha<3$ the following simple scaling argument applies: $Z_{2}(r)$ is related to the variance on a scale of $r$, so

$$
\begin{aligned}
Z_{2}(r) & \approx \mathcal{C}(K) \delta K \approx K^{-\alpha} K \text { where } K=1 / r \\
& \propto r^{\alpha-1} .
\end{aligned}
$$

\section{Determination of $Z_{2}(r)$ from the mixing properties of the flow}

\subsection{The non-diffusive case}

In the case where the equation governing the spread of concentration contains no source terms, or diffusive terms, we have

$$
\frac{\mathrm{D} \Theta}{\mathrm{D} t}=0
$$

so in order to know $\Theta(x, t)$ we only need to track the trajectories back in time to the initial conditions. The correlation between the concentrations of 2 points $\mathbf{x}$ and $\mathbf{x}^{\prime}$ in the fluid is equal to the correlation of the concentration of $\mathbf{x}_{0}$ and $\mathbf{x}_{0}^{\prime}$ in the initial conditions, where $\mathbf{x}_{0}$ and $\mathbf{x}_{0}^{\prime}$ are the initial positions of $x$ and $x^{\prime}$. However, we also know from the stretching properties of the fluid that any 2 points grow exponentially further apart as time evolves (either forwards or backwards). If $\mathbf{x}$ and $\mathbf{x}^{\prime}$ are separated by $r$ at a time $t, \mathbf{x}_{0}$ and $\mathbf{x}_{0}^{\prime}$ were separated, on average, by $r_{0}=r \exp (\Lambda t)$ at $t_{0}=0$, where $\Lambda$ is the finite time Lyapunov exponent of the flow. If the initial conditions of the flow have a typical correlation length-scale $L$ (for instance, the length-scale of the initial forcing in a decaying turbulence experiment), then there will be 2 regimes:

- either $r \exp (\Lambda t)<L$, then the particles have remained in the same "eddy", within the same correlation length-scale, so that we simply have

$$
\Delta_{r} \Theta \approx|\nabla \Theta|_{0} r \exp (\Lambda t)
$$

where $|\nabla \Theta|_{0}$ is the typical gradient of the eddy at $t=0$.

- on the other hand, if $r \exp (\Lambda t)>L$ the points are uncorrelated and so $\left\langle\left|\Delta_{\tau} \Theta\right|^{2}\right\rangle=\left.2\left\langle\Theta^{2}\right\rangle\right|_{0}$, where $\left.\left\langle\Theta^{2}\right\rangle\right|_{0}$ is the variance of the initial flow.

The resulting profile is shown in in Fig. 2; as one can see, there seems to be no logarithmic profile appearing, which would have been the sign of the Batchelor regime. This is typical of the initial value problem, where the flow keeps a memory of the initial condition for some period of time before reaching a random flow. 


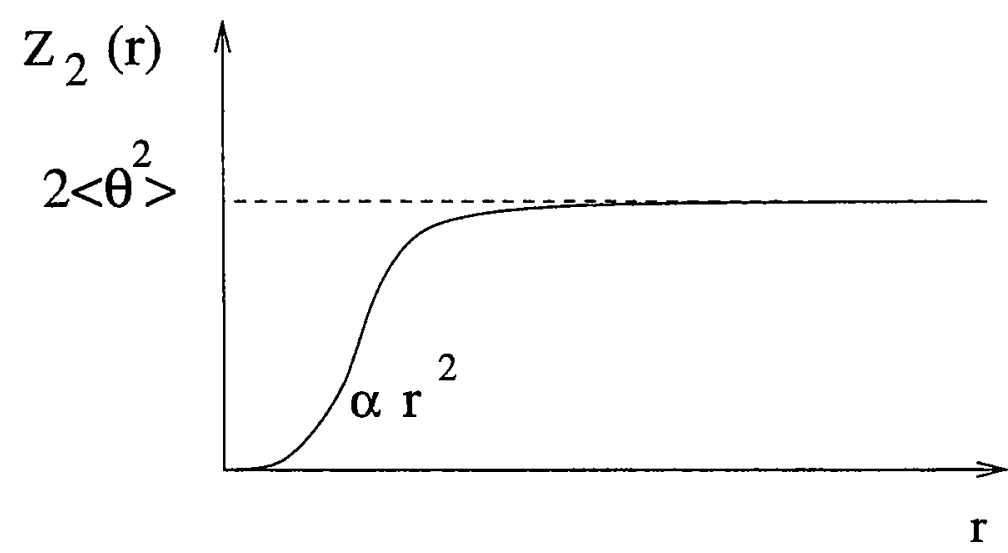

Figure 2: two-point correlation function in the unforced, non-diffusive case.

\subsection{The forced case}

This time we have

$$
\frac{\mathrm{D} \Theta}{\mathrm{D} t}=\kappa \nabla^{2} \Theta+S(\mathbf{x}, t)
$$

where $S(\mathbf{x}, t)$ is a source term, with a finite correlation length-scale, but no time correlation, i.e.

$$
\left\langle S(\mathbf{x}, t) S\left(\mathbf{x}^{\prime}, t^{\prime}\right)\right\rangle=S_{0}^{2}\left(\left|\mathbf{x}-\mathbf{x}^{\prime}\right|\right) \delta\left(t-t^{\prime}\right)
$$

where $S_{0}\left(\left|\mathbf{x}-\mathbf{x}^{\prime}\right|\right)$ decays on a lengthscale $L$. One can distinguish 2 regimes. In the initial phase the flow is mixed without dissipation; since the forcing is uncorrelated in time, the system behaves like a random walk process, so that

$$
\left\langle\Theta^{2}\right\rangle \propto S_{0}^{2} t
$$

Later on, the flow reaches a steady state with a balance between the forcing and the mixing/dissipation terms. The dissipation scale $l_{\star}$ for which the strain balances the dissipation is $l_{\star}=\sqrt{\kappa / \Lambda}$ (cf. Bill's lecture). The time $t_{\star}$ needed for the flow to create structures on the dissipation scale is $t_{\star}=\frac{1}{\Lambda} \ln \left(L / l_{\star}\right)$. The time-scale $t_{\star}$ can be seen as the memory of the system. Trajectories with small Lyapunov exponent have a large memory, the flow remains correlated for longer times. The 2-point correlation function will mostly depend on scales of order of $L$. Indeed, if 2 points are separated by $r<L$, they must come from regions which have the same source term, so that the quantity $Z_{2}(r)$ is likely to be small. On the other hand, if $r>L$ then the correlation function of the concentration field will be similar to that of the source terms. As before, let's consider 2 points at $\mathbf{x}$ and $\mathbf{x}^{\prime}$ at a time $t$ and trace their trajectories backward in time. Assuming that the statistics of the forced flow are stationary, the flow builds up correlation when the separation of the 2 points is larger than $L$, but loses correlation for times larger than the dissipation time-scale. The 2-point correlation function will therefore depend on the difference $t_{L}-t_{\star}$, where $t_{L}$ is the amount of time necessary to reach scales of order $L$ starting from and initial separation $r: t_{L}=\frac{1}{\Lambda} \ln L / r$. This is illustrated in Fig. 2.

Hence

$$
Z_{2}(r) \propto t_{L}-t_{\star} \propto \frac{1}{\Lambda} \ln \left(r / l_{\star}\right)
$$

This logarithmic dependence in $r$ of the correlation function shows that the forced case is consistent with a Batchelor regime. For more details on this subject, see Refs. [5] and [6]. 


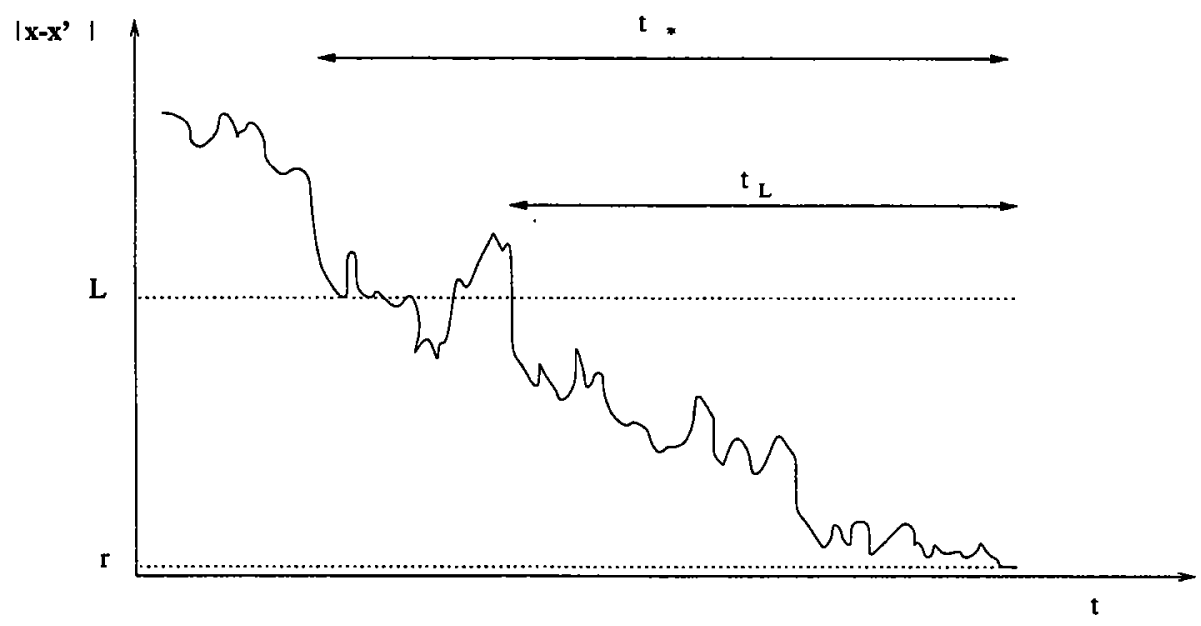

Figure 3: Evolution of the separation of 2 points, and relevant time-scales.

\section{References}

[1] G. K. Batchelor, "Small-scale variation of convected quantities like temperature in turbulent fluid. part 1. general discussion and the case of small conductivity," J. Fluid Mech. 5, 113 (1959).

[2] A. M. Obukhov, "Structure of the temperature field in turbulent flow," Izv. Akad. Nauk. Geogr. Geofiz. 13, 58 (1949).

[3] S. Corrsin, "On the spectrum of isotropic temperature fluctuations in an isotropic turbulence," J. Appl. Phys 22, 469 (1951).

[4] A. S. Monin and A. M. Yaglom, Statistical Fluid Mechanics (MIT Press, Cambridge, MA, 1981).

[5] R. T. Pierrehumbert, "Lattice models of advection-diffusion," Chaos 10, 61 (2000).

[6] I. K. M. Chertkov, G. Falkovich and I.Lebed, "Statistics of a passive scalar advected by a largescale two-dimensional velocity field: and analytic solution.," Phys. Rev. E 51, 5609 (1995). 


\title{
On Stratified Kolmogorov Flow
}

\author{
Yuan-nan Young
}

\begin{abstract}
In this study we investigate the stability of the weakly stratified Kolmogorov shear flow. We derive the amplitude equations for this system and solve them numerically to explore the effect of weak stabilizing stratification. We then explore the non-diffusive limit of this system and derive amplitude equations in this limit.
\end{abstract}

\section{Introduction}

The Kolmogorov flow - a two-dimensional viscous sinusoidal flow induced by a unidirectional external force field - has been studied in the context of generation of large scale turbulence in two-dimension. Various aspects of the Kolmogorov flow, such as the generation of $2 \mathrm{D}$ turbulence [1], vortex merging [2], and the negative viscosity in the role of large scale formation in $2 \mathrm{D}$ turbulence [3], have been widely applied to geophysical [4] and laboratory systems [5, 6].

In this study we impose a weak, stabilizing temperature gradient and investigate the temperature evolution associated with the flow instability. We first adopt Sivashinsky's approach and derive the finite-amplitude equation for the case of infinite domain (periodic boundary conditions) and finite Peclet numbers. We then solve the amplitude equations (both 1D and 2D) numerically and investigate the buoyancy effect on the structure formation of the flow. We also investigate large Peclet number cases, where the critical layers in the scalar field plays a key role for the flow instability and dynamics. We also make comparison between fully numerical simulations and results from the weakly nonlinear analyses.

\section{Formulation and linear analysis}

\subsection{Formulation}

The Kolmogorov shear flow is more generally defined as a sinusoidal shear flow, whether the fluid is viscous or inviscid. In our 2-D formulation of the problem, where the incompressible flow can be written as a stream function, we couple this background shear flow to a stabilizing temperature. Without loss of generality, we write the total background state as a sinusoidal stream function and a linear temperature profile:

$$
\Psi_{0}=U_{0} l \cos (z / l), \quad T_{0}=\frac{\triangle T}{l} z,
$$

where $U_{0}$ is the amplitude of the background shear flow, $l$ is the periodicity of the shear flow, and $\triangle T$ is the temperature difference across distance $l$. Denoting $\psi$ as the stream function 
disturbance and $\theta$ as the temperature disturbance, we first write the momentum equation and the advection diffusion equation as follows:

$$
\begin{gathered}
\partial_{t} \nabla^{2} \psi+\partial_{x}\left(\partial_{z} \Psi_{0} \nabla^{2} \psi-\partial_{z}^{3} \Psi_{0} \psi\right)-J\left(\psi, \nabla^{2} \psi\right)=\nu \nabla^{4} \psi-g \alpha \theta_{x} \\
\partial_{t} \theta+\partial_{z} \Psi_{0} \partial_{x} \theta-\partial_{x} \psi \partial_{z} T_{0}-J(\psi, \theta)=\kappa \nabla^{2} \theta
\end{gathered}
$$

We nondimensionalize the above equations such that the background shear flow $u_{0}=-\sin z$ and the background stabilizing thermal gradient is equal to 1 (Re $\equiv U_{0} l / \nu$ is the Reynolds number, $\mathrm{Pe} \equiv U_{0} l / \kappa$ is the Peclet number, and $\mathrm{Ri} \equiv g \alpha \delta T l^{2} / U_{0}^{2}$ is the Richardson number), and equations 2 and 3 thus read:

$$
\begin{aligned}
\partial_{t} \nabla^{2} \psi-\sin z\left(\nabla^{2} \psi+\psi\right)_{x}-J\left(\psi, \nabla^{2} \psi\right) & =\frac{1}{\operatorname{Re}} \nabla^{4} \psi-\operatorname{Ri} \theta_{x} \\
\partial_{t} \theta-(\sin z \theta+\psi)_{x}-J(\psi, \theta) & =\frac{1}{\mathrm{Pe}} \nabla^{2} \theta
\end{aligned}
$$

In the following subsections we first present results from the usual linear analysis on cases where the periodicity of the shear flow is the same as the domain considered (integer periodicity). We then consider cases where the perturbations are products of periodic and exponential functions (Floquet system) and may exhibit parametric resonance.

\subsection{Linear analysis on the stratified Kolmogorov flow}

In this section we present results of linear analysis on equations 4 and 5 for shear flow of periodicity the same as the domain size. The linearized version of equations 4 and 5 read

$$
\begin{aligned}
\partial_{t} \nabla^{2} \psi-\sin z \partial_{x}\left(\nabla^{2} \psi+\psi\right) & =\frac{1}{\operatorname{Re}} \nabla^{4} \psi-\operatorname{Ri} \theta_{x} \\
\partial_{t} \theta-(\sin z \theta+\psi)_{x} & =\frac{1}{\mathrm{Pe}} \nabla^{2} \theta
\end{aligned}
$$

Without the stabilizing temperature, the non-stratified Kolmogorov shear flow is known to be unstable to long wave length perturbation for Reynolds numbers $\operatorname{Re}>\sqrt{2}$ : the critical wave number $k_{c}=0$ and the critical Reynolds number $\operatorname{Re}_{c}=\sqrt{2}$. Also for small horizontal wave numbers $(k \ll 1)$ the growth rate $\lambda$ can be obtained via the following dispersion relation:

$$
\lambda=\left(1-\frac{\operatorname{Re}^{2}}{2}\right) k^{2}+\operatorname{Re}^{2}\left(1+\frac{\operatorname{Re}^{2}}{4}\right) k^{4}+\mathcal{O}\left(k^{6}\right) .
$$

We numerically solve the above linearized equations with periodic boundary conditions (in both the horizontal and the vertical directions). Figure 1 shows marginal curves for weak stratification (see caption for the corresponding stratification strength for each curve) and figure 2 shows the critical Reynolds numbers and wave numbers as functions of Richardson numbers for Prandtl number $\operatorname{Pr} \equiv \nu / \kappa=10$. As shown in the figures, the critical wave number $k_{c}$ increases rapidly as we increase the Richardson number above. As the Richardson 


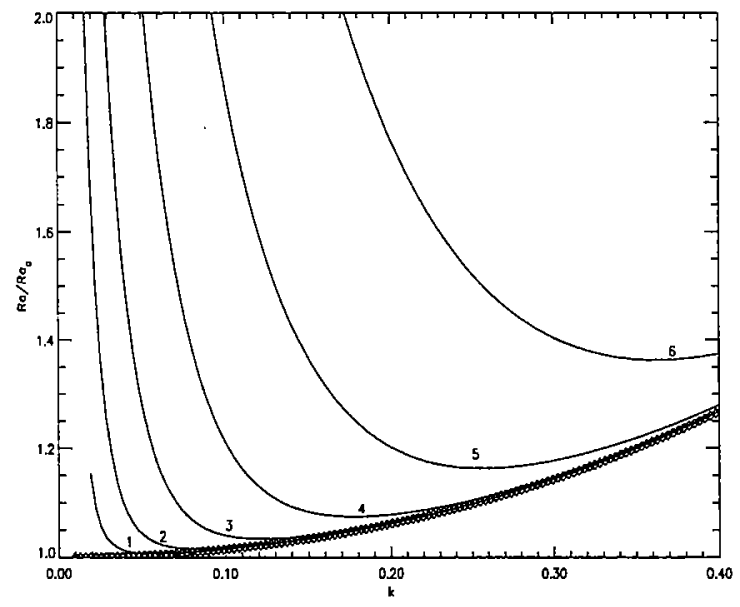

(a)

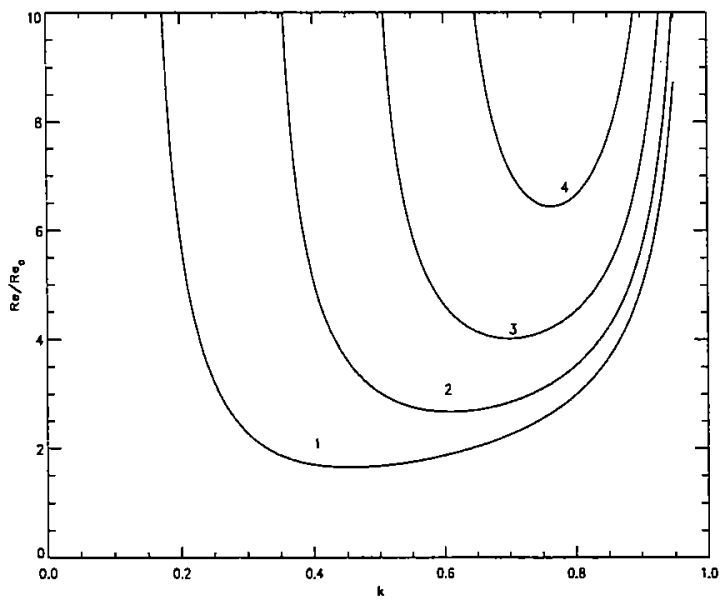

(b)

Figure 1: Marginal curves for the unbounded stratified Kolmogorov flow for weak stratification and Prandtl number $\sigma=1$. Curves are labeled by their Richardson numbers. In Panel (a) from curve 1 to curve 6 , the Richardson number is, respectively, $10^{-7}, 10^{-6}, 10^{-5}, 10^{-4}$, $10^{-3}$, and $10^{-2}$. In Panel (b), the Richardson number is $0.01,0.05,0.1$ and 0.15 for curve 1 to curve 4 , respectively. 


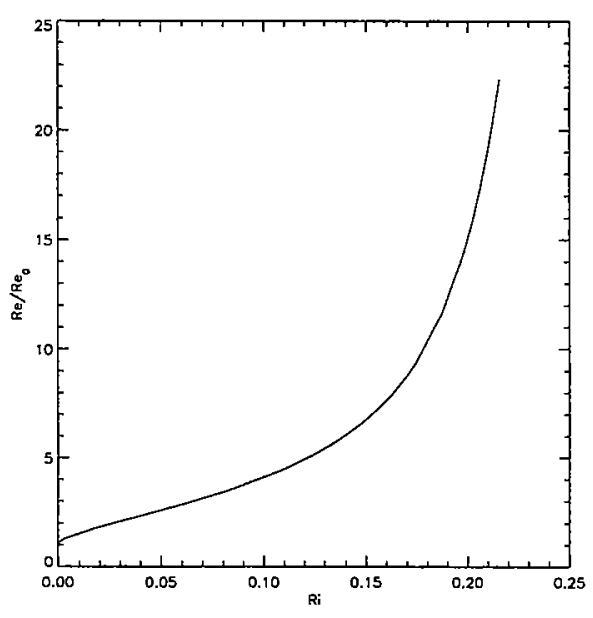

(a)

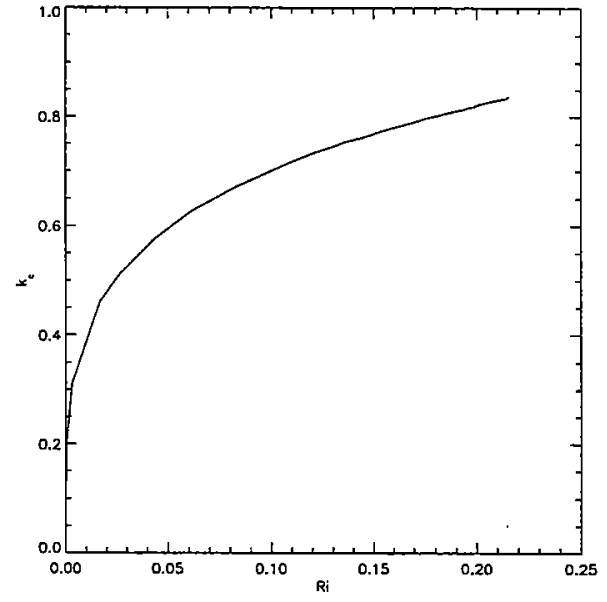

(b)

Figure 2: Critical Reynolds number (a) and critical wave number (b) as functions of Richardson number for $\operatorname{Pr}=10$ for the periodic case.

number increases above $10^{-5}$, the critical wave number $k_{c}$ increases significantly from 0 towards some finite value $(\sim 0.1)$. This also implies that the inverse cascade observed in the nonstratified Kolmogorov shear flow is at risk, namely, the large scale perturbation now has been stabilized by the existence of the stably stratified temperature. As will be shown in the numerical results, the inverse cascade is indeed prevented by the stabilizing temperature and we will discuss this in detail via the tool of Lyapunov functional.

\subsection{Linear analysis on the stratified Kolmogorov flow: Floquet calculation}

We now show results from the Floquet calculation for the stratified Kolmogorov shear flow. We perturb the system with perturbation of the form: $e^{i q z+i k x} \psi(z, t)$, where $0 \leq q \leq 0.5$ is the Floquet multiplier (Bloch number) and $k$ is the horizontal wave number. With the definition of $\nabla^{\prime 2}$ in equation 9 ,

$$
\nabla^{\prime 2} \psi \equiv-\left(k^{2}+q^{2}\right) \psi+2 i q \partial_{z} \psi+\partial_{z}^{2} \psi
$$

equations 6 and 7 then take the following form:

$$
\begin{aligned}
\partial_{t} \nabla^{\prime 2} \psi-\sin z i k\left(\nabla^{\prime 2} \psi+\psi\right) & =\frac{1}{\operatorname{Re}} \nabla^{\prime 4} \psi-i k \operatorname{Ri} \theta \\
\partial_{t} \theta-i k(\sin z \theta+\psi) & =\frac{1}{\mathrm{Pe}} \nabla^{\prime 2} \theta
\end{aligned}
$$

Solving equations 10 and 11 numerically with periodic boundary conditions, we obtain the parametric marginal curves for various values of $q$. Figure 3 shows the critical Reynolds number as a function of $q$ for the non-stratified $(\mathrm{Ri}=0)$ case. We first note that $\operatorname{Re}_{c}(q) \geq$ 
$\operatorname{Re}_{c}(q=0)$, and at around $q=0.35$ the minimum moves from one branch to the other, thus a cuspy transition at $q=0.35$. From figure 3 we also note that the most unstable mode has the same periodicity as the background shear flow. Therefore, we do not need to perform the same Floquet calculation for the stratified case. Having shown that perturbations of

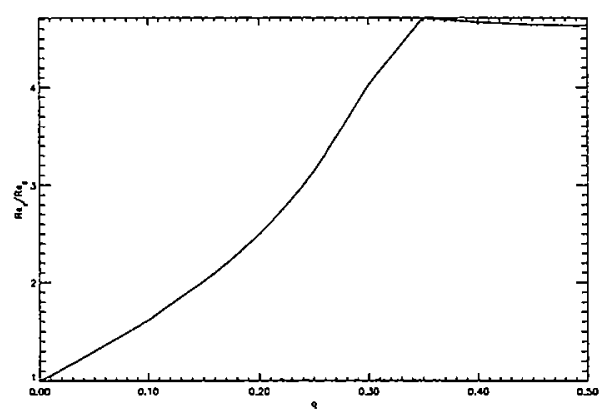

(a)

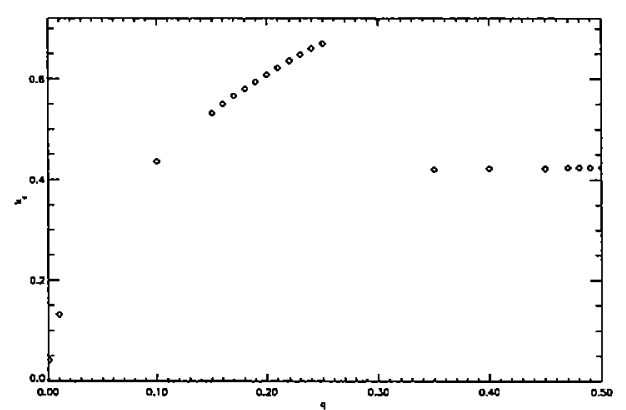

(b)

Figure 3: Critical Reynolds number (a) and critical wave number (b) as functions of the Floquet multiplier $q$ for the non-stratified Kolmogorov shear flow.

the same periodicity are the most unstable, we then proceed to uncover the effect of weak stratification on the nonlinear behavior of the flow. By this we in particular mean that we are going to perform amplitude expansion around the $k=0$ mode for small $\mathrm{Ri}$. From the linear analysis, we have observed that the critical wave number $k_{c}$ increase from zero (for no stratification, $\mathrm{Ri}=0$ ) to finite value (for strong stratification). As $k_{c}$ transitions from 0 to finite values, the amplitude equation changes from a Cahn-Hilliard like equation [4] for long wavelength instability to a Ginzburg-Landau equation for finite wavelength instability. In our weakly nonlinear analysis, we focus on the weak stratification limit where the system still inherits the instability to long wavelength perturbation. To have buoyancy $(\theta)$ appear at the desired order in the amplitude equation, we rescale $\theta$ and put $\mathrm{Ri}$ to small numbers such that $\mathrm{Ri} \equiv \epsilon^{6} F_{6}$ and $b \equiv \operatorname{Ri} \theta / \epsilon^{5}=\epsilon F_{6} \theta$. Equations 4 and 5 , in the new scaling, take the following form:

$$
\begin{gathered}
\epsilon^{4} \partial_{\tau} \nabla^{2} \psi-\epsilon J_{\xi}\left(\psi, \nabla^{2} \psi\right)-\epsilon \sin z\left(\nabla^{2} \psi+\psi\right)_{\xi}=\frac{1}{\operatorname{Re}}\left(1-\epsilon^{2}\right) \nabla^{4} \psi-\epsilon^{6} b_{\xi}, \\
\epsilon^{4} \partial_{\tau} b-\epsilon J_{\xi}(\psi, b)-\epsilon^{2} F_{6} \psi_{\xi}-\epsilon \sin z b_{\xi}=\frac{1}{\mathrm{Pe}} \nabla^{2} b
\end{gathered}
$$

where $J_{\xi}$ is the usual Jacobian with respect to $\xi$ and $z$.

\section{Weakly nonlinear analysis: $\mathrm{Pe} \sim \mathcal{O}(1)$}

In this section we first construct the amplitude equations for the stratified shear flow with $\mathrm{Pe} \sim \mathcal{O}(1)$. We remark here that we are mostly interested in two ranges of Pe: $\mathrm{Pe} \sim \mathcal{O}(1)$ and $\mathrm{Pe} \gg 1$. The range $\mathrm{Pe} \ll 1$ is where molecular diffusivity dominates the dynamics, and 
to first few orders in the expansion, there appears to be no coupling between the temperature and the flow, and thus is of no interests in our analysis. In real physical systems (salty water, for example), the Pelect number for a small Reynold number of $\sqrt{2}$ is already on the order of a thousand, and thus cases where $P e \gg 1$ are of more physical relevance. In subsection 3.3 we present numerical solutions to the amplitude equations for the $\mathrm{Pe} \sim \mathcal{O}(1)$ case. In the following section, we derive the amplitude equation for the nondiffusive case $(\mathrm{Pe} \gg 1)$.

\subsection{Construction of the amplitude equations:}

$\mathrm{Pe} \sim \mathcal{O}(1)$

Adopting the scaling discussed in section 2.2, and expanding $\psi$ and $\theta$ as follows

$$
\begin{aligned}
\psi & =\psi_{0}+\epsilon \psi_{1}+\epsilon^{2} \psi_{2}+\epsilon^{3} \psi_{3}+\cdots, \\
\theta & =\theta_{0}+\epsilon \theta_{1}+\epsilon^{2} \theta_{2}+\epsilon^{3} \theta_{3}+\cdots,
\end{aligned}
$$

we substitute the above expansions into equations 12 and 13. Collecting terms order by order, $\left(\psi_{i}, \theta_{i}\right)$ that satisfy the periodic boundary conditions (for the "fast variable" $z$ ) are obtained, and the solvability condition at each order gives rise to relationships between $\psi_{i}$ and $\theta_{i}$. At the zeroth order $\mathcal{O}\left(\epsilon^{0}\right)$, the equations are:

$$
\psi_{0 z z z z}=0, \quad \frac{1}{\mathrm{Pe}} b_{0 z z}=0,
$$

and the periodic solutions are

$$
\psi_{0}=A(\xi, \tau), \quad b_{0}=B(\xi, \tau) .
$$

At the first order $\mathcal{O}\left(\epsilon^{1}\right)$, we obtain the following equations

$$
\begin{aligned}
\frac{1}{\operatorname{Re}} \psi_{1 z z z z} & =-A_{\xi} \sin z \\
\psi_{0 z} B_{\xi}-\psi_{0 \xi} B_{z} & =\frac{1}{\mathrm{Pe}} b_{1 z z}
\end{aligned}
$$

and the periodic solutions

$$
\psi_{1}=-\operatorname{Re} A_{\xi} \sin z+A_{1}(\xi, \tau), \quad b_{1}=\mathrm{Pe} B_{\xi} \sin z+B_{1}(\xi, \tau) .
$$

At the second order $\mathcal{O}\left(\epsilon^{2}\right)$, the equations are as follows:

$$
\begin{gathered}
\frac{1}{\mathrm{Re}} \psi_{2 z z z z}=-A_{1 \xi} \sin z-\operatorname{Re} A_{\xi}^{2} \cos z \\
\frac{1}{\mathrm{Pe}} b_{2 z z}=-B_{1 \xi} \sin z-(\mathrm{Re}+\mathrm{Pe}) A_{\xi} B_{\xi} \cos z-\left(\frac{\mathrm{Pe}}{2}+\frac{1}{\mathrm{Pe}}\right) B_{\xi \xi}-F_{6} A_{\xi}+\frac{\mathrm{Pe}}{2} B_{\xi \xi} \cos 2 z .
\end{gathered}
$$

At this order $\mathcal{O}\left(\epsilon^{2}\right)$, the solvability condition for $\psi_{2}$ gives rise to the critical Reynolds number $\mathrm{Re}=\mathrm{R}_{0} \equiv \sqrt{2}$. The solvability condition for $\theta_{2}$ gives us the following relationship between $B\left(\equiv \theta_{0}\right)$ and $A\left(\equiv \psi_{0}\right)$ :

$$
\left(\frac{\mathrm{Pe}}{2}+\frac{1}{\mathrm{Pe}}\right) B_{\xi \xi}+F_{6} A_{\xi}=0 .
$$


The solutions at this order are:

$$
\begin{aligned}
& \psi_{2}=-\mathrm{R}_{0} A_{1 \xi} \sin z-\mathrm{R}_{0}^{2} A_{\xi}^{2} \cos z+A_{2}(\xi, \tau) \\
& b_{2}=\operatorname{Pe} B_{1 \xi} \sin z+\mathrm{Pe}\left(\mathrm{R}_{0}+\mathrm{Pe}\right) A_{\xi} B_{\xi} \cos z-\frac{\mathrm{Pe}^{2}}{8} \cos 2 z B_{\xi \xi} .
\end{aligned}
$$

Going on to the 3rd order in $\epsilon$, we have the following equations for $\psi_{3}$ :

$$
\frac{1}{\mathrm{R}_{0}} \psi_{3 z z z z}=\left[\mathrm{R}_{0}^{2} A_{\xi}^{3}-3 A_{\xi \xi \xi}-A_{\xi}-A_{\xi}^{2}\right] \sin z-2 \mathrm{R}_{0}^{2} A_{1 \xi} A_{\xi} \cos z
$$

and the solution $\psi_{3}$ is easily obtained as follows:

$$
\psi_{3}=\mathrm{R}_{0}\left[\mathrm{R}_{0}{ }^{2} A_{\xi}^{3}-3 A_{\xi \xi \xi}-A_{\xi}-A_{2 \xi}\right] \sin z-2 \mathrm{R}_{0}{ }^{3} A_{1 \xi} A_{\xi} \cos z+A_{3}(\xi, \tau) .
$$

The solvability condition at this order gives us the amplitude equation:

$$
\left(A_{\xi \xi}\right)_{\tau}+\frac{3 \mathrm{R}_{0}}{2} A_{\xi \xi \xi \xi \xi \xi}+\left\{\left[\mathrm{R}_{0}-\frac{\mathrm{R}_{0}^{3}}{3} A_{\xi}^{2}\right] A_{\xi}\right\}_{\xi \xi \xi}-\frac{F_{6}}{\mathrm{Pe} / 2+1 / \mathrm{Pe}} A=0 .
$$

Following Sivashinsky, if we write $\partial_{z}=\partial_{z}+\epsilon^{3} \partial_{\eta}$, we obtain identical solutions till the second order and obtain the following amplitude equation at third order:

$$
\begin{aligned}
\left(A_{\xi \xi}\right)_{\tau}= & -\frac{3 \mathrm{R}_{0}}{2} A_{\xi \xi \xi \xi \xi \xi}-\left\{\left[\mathrm{R}_{0}-\frac{\mathrm{R}_{0}{ }^{3}}{3} A_{\xi}^{2}\right] A_{\xi}\right\}_{\xi \xi \xi} \\
& -A_{\eta} A_{\xi \xi \xi}+A_{\xi} A_{\xi \xi \eta}+\frac{\mathrm{R}_{0}^{2}}{2}\left(A_{\xi}\right)_{\xi \eta}^{2}+\frac{F_{6}}{\mathrm{Pe} / 2+1 / \mathrm{Pe}} A .
\end{aligned}
$$

We note that the buoyancy amplitude $B$ is completely slaved to the stream function amplitude $A$ as the effect of the stabilizing temperature gradient is put to higher order $\left(\epsilon^{6}\right)$. Writing $\rho=\xi+c \eta$, we can turn equation 29 into a uni-directional amplitude equation [1] in terms of $\rho$ and $\tau$ as follows:

$$
\left(A_{\rho \rho}\right)_{\tau}=-\frac{3 \mathrm{R}_{0}}{2} A_{\rho \rho \rho \rho \rho \rho}-\left\{\left[\mathrm{R}_{0}-\frac{\mathrm{R}_{0}^{3}}{3} A_{\rho}^{2}\right] A_{\rho}\right\}_{\rho \rho \rho}+\frac{c \mathrm{R}_{0}^{2}}{2}\left(A_{\rho}\right)_{\rho \rho}^{2}+\frac{F_{6}}{\mathrm{Pe} / 2+1 / \mathrm{Pe}} A,
$$

where $c$ is the aspect ratio of the characteristics of the uni-directional flow.

\subsection{Lyapunov functional}

In this subsection we derive a Lyapunov functional for the $1 \mathrm{D}$ amplitude equation (equation 28). Following [7], we try to find an energetic functional of amplitude $A$ such that the evolution of the amplitude can be described by the functional. To be more specific, we seek a Lyapunov functional $V[A]$ such that

$$
\partial_{\tau} A=-\frac{\delta V[A]}{\delta A}, \quad \partial_{\tau} V=-\int\left(A(\xi, \tau)_{\tau}\right)^{2} d \xi
$$


which then implies that the system cannot sustain oscillatory motion and has to settle down to a stationary equilibrium. For the 1D amplitude equation for the weakly stratified case, it is straightforward to find a functional for the amplitude equation 28 if we rewrite it as follows:

$$
\left(A_{\xi \xi}\right)_{\tau}=-\partial_{\xi}^{2} \frac{\delta F}{\delta A}+\frac{F_{6}}{\mathrm{Pe} / 2+1 / \mathrm{Pe}} A
$$

where

$$
F[A] \equiv-\frac{1}{4} \int 4 A_{\xi}^{2}-A_{\xi}^{4}-2 A_{\xi \xi}^{2} d \xi .
$$

Putting $C_{\xi \xi} \equiv A$, we write down the evolution equation for $F$ as:

$$
\frac{d F}{d \tau}=-\int A_{\tau}^{2} d \xi-\frac{F_{6}}{\mathrm{Pe} / 2+1 / \mathrm{Pe}} \partial_{\tau} \int \frac{C_{\xi}^{2}}{2} d \xi
$$

This indicates that the new functional $G \equiv F+\frac{F_{6} \mathrm{Pe} C_{\xi}^{2}}{\mathrm{Pe}^{2}+2}$ is decaying in time and there is a stationary solution for arbitrary initial conditions. In the absence of the stabilizing stratification, random perturbation of small scales will reach a stationary solution with minimum number of nodes within the domain, i.e., the scale of the stationary solution is the size of the domain. This is the essence of inverse cascade: the evolution of the amplitude is such that the spatial scale increases until it reaches the scale comparable to the size of the computation domain. In the nonstratified case, since the functional is expressed in the gradient of $A$, an the fact that $A$ is periodic in $\xi$, we conclude that the stationary solution $A(\xi)$ should have only one bump inside the domain. However, this inverse cascade is arrested by the presence of stabilizing stratification, as the additional term $C_{\xi}$ included in the functional prevents the inverse cascade process. This will be demonstrated in the following subsection.

\subsection{Numerical solution}

In this section we present numerical results from solving the amplitude equations using a pseudo-spectral code. First we show results for the 1D version of the amplitude equation (equation 28). Figure 4 demonstrates the stabilizing effect of the temperature: the amplitude decreases and the structure tends to be of smaller scale as we increase the strength of stratification. As shown in the previous subsection, we can find a Lyapunov functional for this equation in terms of the gradient of the amplitude, therefore, we display the temporal evolution of the amplitude gradient (figure 5). Figures 5 show the time-space plots for the gradients of the amplitudes without any stratification in (a) and with an $F_{6}=0.1$ in (b). We note that the inverse cascade manifested in panel (a) is arrested by the presence of stabilizing stratification in panel (b), in agreement with the conclusion we draw from the Lyapunov functional. The numerical solutions to the uni-directional amplitude equation (equation 30) are displayed in figures 6 , where the time evolution is for the amplitudes, not the amplitude gradients. In panel (a) of figures 6 , where there is no stratification, we see the chaotic behavior of the flow due to the extra nonlinear term. Yet in panel (b) where $F_{6}=0.1$, we see the stratification diminishes the chaotic behavior and reduces the flow to spatially periodic. 


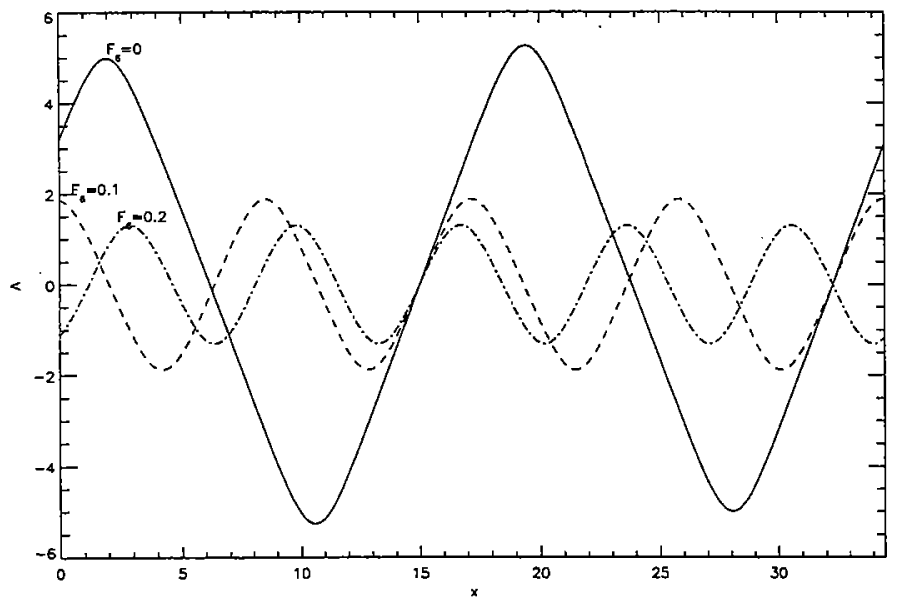

Figure 4: Numerical solutions at $t=300$ for various strengths of weak stratification.

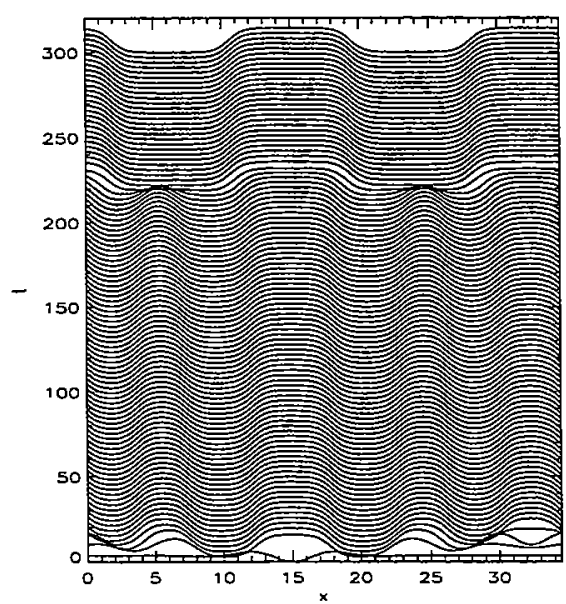

(a)

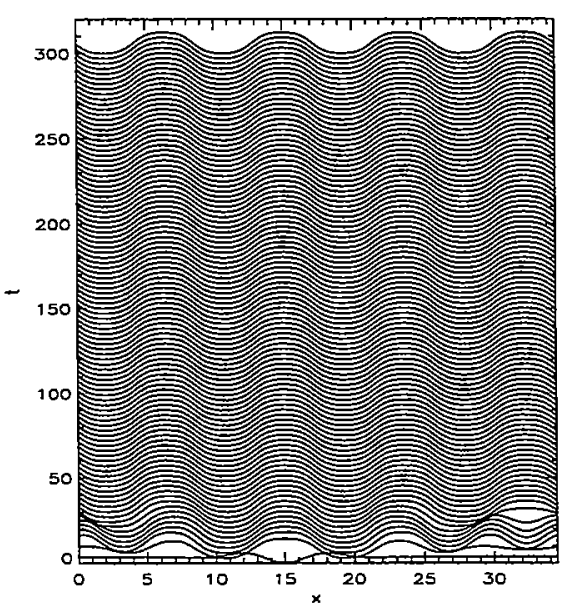

(b)

Figure 5: Time-space plots of the amplitude gradient with $F_{6}=0$ (a) and $F_{6}=0.1(\mathrm{~b})$. 


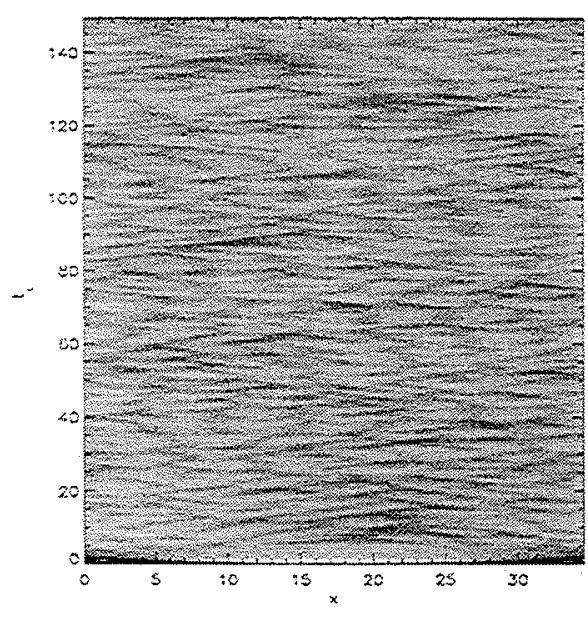

(a)

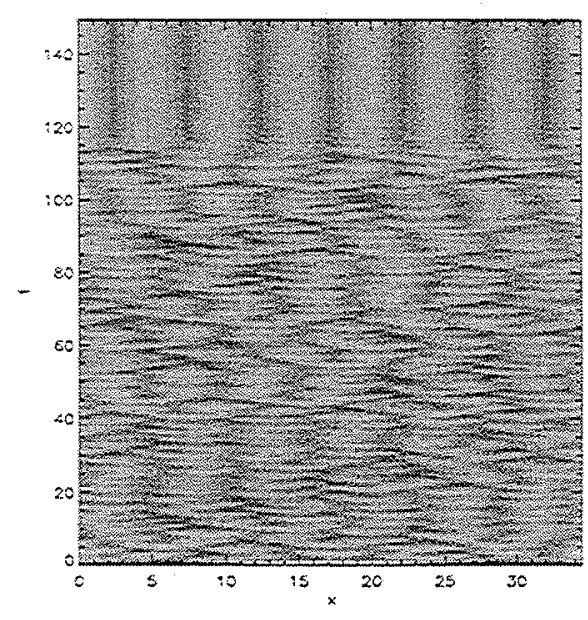

(b)

Figure 6: Time-space plots of the amplitude for the uni-directional flow with $F_{6}=0$ (a) and $F_{6}=0.1(\mathrm{~b})$.

In figures 7 we show the solutions $\left(\Psi_{0}+A(\xi, \eta, \tau)\right)$ to the 2-D amplitude equation, where the computation domain has been scaled by an aspect ratio $c=20$ as suggested in [3]. The only difference between these two snapshots of the stream functions is the stratification strength. We note that the effect of stratification is manifested not only by the change in the amplitude of the flow but also the flow patterns: the stronger the stratification, the smaller the scales are for the flow patterns.

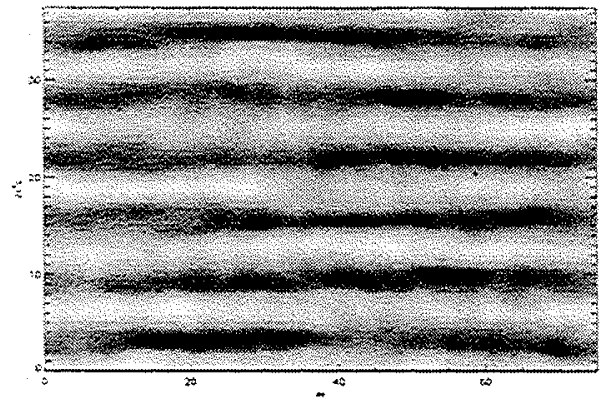

(a)

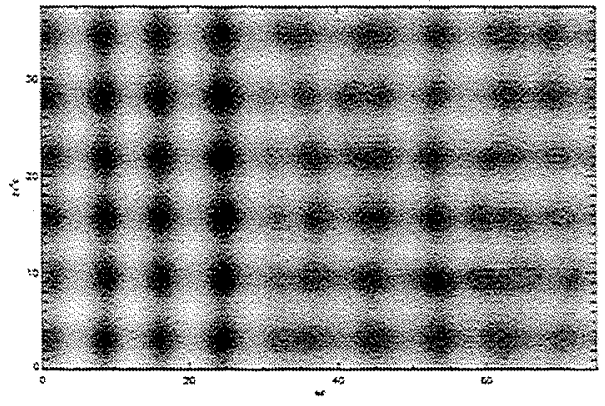

(b)

Figure 7: Stream function (zeroth order) from the 2D amplitude equation for (a) $F_{6}=0.01$ at $t=15$ and (b) $F_{6}=0.1$.

In figures 8 and 9 we show the horizontal average of the temperature to demonstrate the potential of layer formation in the temperature. Figure 8 is a snapshot of the horizontal average temperature: $\overline{T_{0}(z)+B(\xi, \eta, t)}$. We note that in Panel (a) of figure 9, layers disappear 


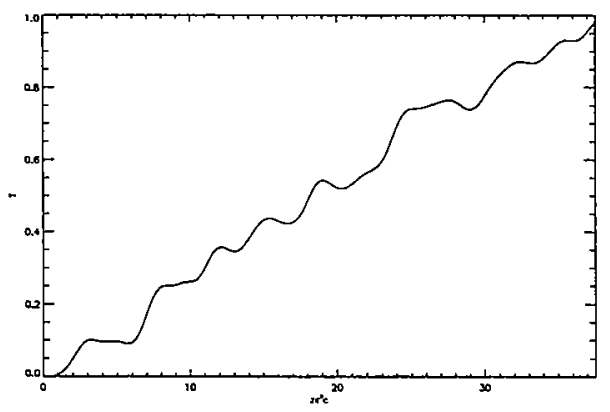

Figure 8: Snapshot of the horizontal average of the total temperature profile for $F_{6}=0.01$.

and re-appear randomly, while in Panel (b) layer structures eventually disappear due to the stabilizing stratification which diminishes the flow.

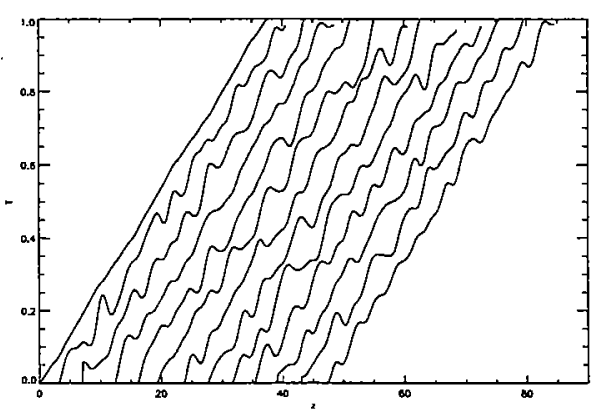

(a)

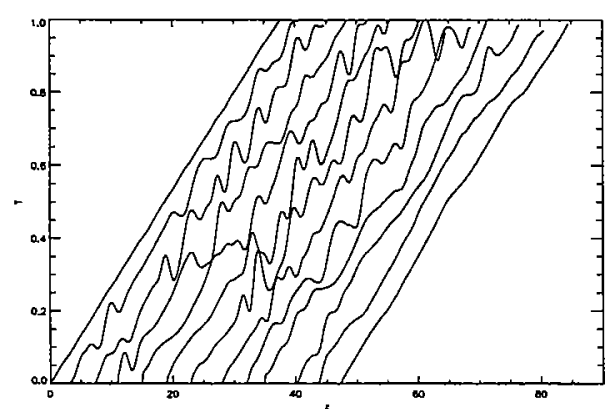

(b)

Figure 9: Horizontal average of the total temperature profile at various times for (a) $F_{6}=0.01$ and (b) $F_{6}=0.1$.

\section{Internal Boundary Layer for large Peclet numbers: $\mathrm{Pe} \rightarrow \infty$}

In this section we focus on the instability of the stratified shear flow in the large Peclet number limit. Figure 10 displays characteristics of the eigenfunctions of the stratified shear flow: the stream function disturbance reaches local minima while the temperature peaks at the inflection point of the background shear flow.

The above structure reminds one of the no-slip, no-flux boundary layer: velocities vanish at the walls and so does the density flux. This is similar to what we observe from the eigen function (for the unbounded case) except that there is a constant background vertical velocity if the horizontal wave number $k$ is not zero. Figures 11 show the internal boundary layer structure as we vary the Prandtl number. We observe the decrease of the internal boundary layer thickness as we decrease the molecular diffusivity. By balancing the advective term 


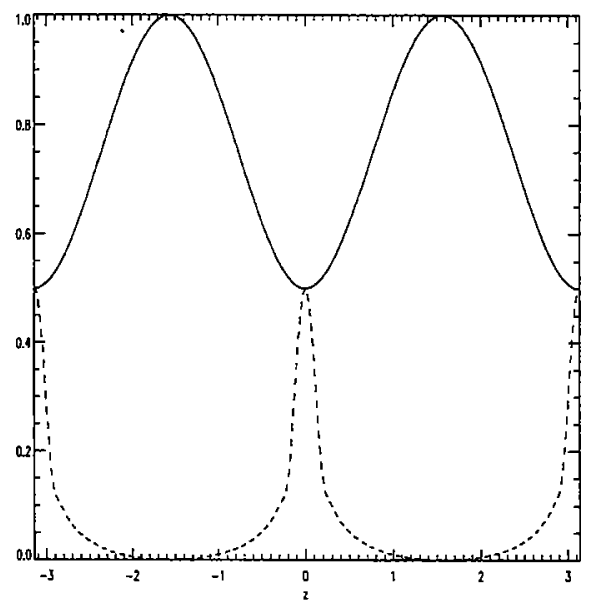

Figure 10: Eigenfunctions for stratified shear flow. The Prandtl number $\operatorname{Pr}=10^{4}$ and $\sin z$ is the background shear flow. The solid line is for the stream function disturbance $\psi$ and the dashed line is the temperature disturbance $\theta$.

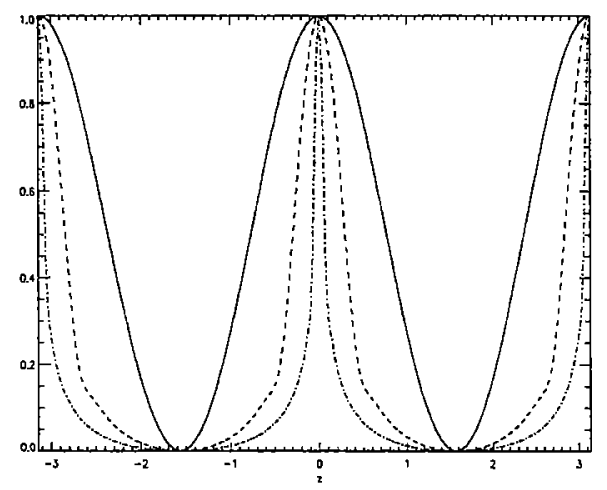

(a)

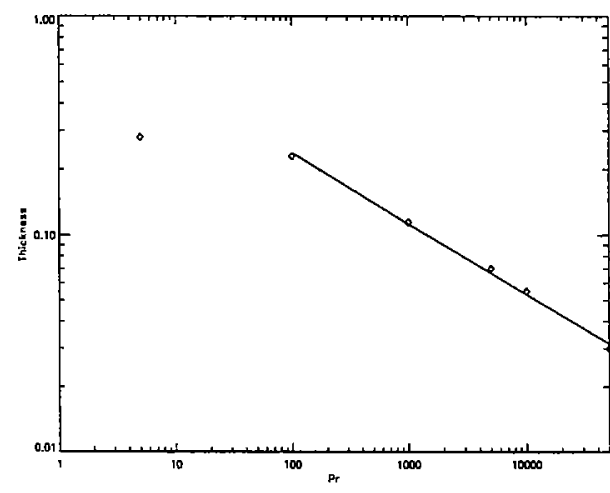

(b)

Figure 11: (a) Internal boundary layer structure as the Prandtl number Pr increases. The Prandtl numbers for the solid, dashed, and dash-dotted lines are, respectively, $10,10^{3}$, and $10^{5}$. (b) The boundary layer thickness as a function of Pr (note that since the Reynolds number is fixed, the Peclet number is proportional to the Prandtl number). The solid line is the best fit for the last five points, which indicate that the thickness scales to $\mathrm{Pe}^{-0.326}$. The Richardson number $\mathrm{Ri}$ is fixed at 0.01 and the Reynolds number is $\mathrm{Re}=1.92$. 
(associated with the background shear flow) and the diffusive term, we obtain a naive scaling of the boundary layer thickness $(l)$ with the Peclet number Pe:

$$
l \sim \mathrm{Pe}^{-1 / 3},
$$

which is in fair agreement with the empirical fit obtained from the numerical solutions to the linearized equations. Also in this limit of large (or infinite) Peclet number, the scaling and. expansion used in previous section to derive the amplitude equations no longer work inside the "internal boundary layer" as terms of different orders are mixed up. Thus we need to find a new scaling inside the internal boundary layer and perform asymptotic matching across the internal boundary layer. We first perform asymptotic matching for infinite Peclet number cases, and then relax the infinite Peclet number limit to large Peclet number limit $\left(\epsilon^{10}\right)$ and derive the dispersion relations and general linear solutions in subsection 4.2.

\subsection{Scaling and asymptotic matching for the internal boundary layer}

We first focus on the linearized version of equation 13 and put right hand side to zero:

$$
\epsilon^{2} \partial_{\tau} \theta+\sin z \theta_{\xi}-\psi_{\xi}=0
$$

The zeroth order solution is ( $A$ is the amplitude for the stream function disturbance as defined in 3.1)

$$
\theta_{0}=\frac{\psi_{0}}{\sin z}=\frac{A}{\sin z}
$$

The solutions for the first and the second order are:

$$
\theta_{1}=\frac{\psi_{1}}{\sin z}, \quad \theta_{2}=\frac{\psi_{2}}{\sin z} .
$$

The third order solution takes the following form:

$$
\theta_{3 \xi}=\frac{\psi_{3 \xi}}{\sin z}-\frac{\psi_{0 \tau}}{\sin ^{2} z}
$$

As the background shear flow goes to zero at $z=0$, the "outer" solutions shown above are no longer regular. We therefore need to find different scaling around $z=0$ to avoid this embarrassment by matching the above "outer" solution to the "inner" solution, to be derived in the following with the new scaling. The new scaling we adopt is as follows: around $z=0$ we scale $z=\epsilon^{3} Z$ and $\theta=\epsilon^{-3} \Theta$. The rescaled, linearized equation takes the following form:

$$
\partial_{\tau} \Theta+Z \Theta_{\xi}-\psi_{\xi}=0
$$

where we have replaced $\sin z$ with $\epsilon^{3} Z$. To perform the matching between inner and outer solutions, we first write the inner solution $\Theta$ as

$$
\Theta=\frac{A}{Z}+\frac{B}{Z^{2}}+\cdots
$$


where $A$ is the stream function amplitude and $B$ is to be determined by matching the inner solution to the outer solution. We then express the outer solution (full solution to the third order) in terms of the rescaled coordinate $Z$ inside the internal boundary layer:

$$
\begin{aligned}
\theta & =\frac{A}{\sin z}+\epsilon \frac{\mathrm{R}_{0} A_{\xi} \sin z}{\sin z}+\epsilon^{2} \frac{\mathrm{R}_{0} A_{1 \xi} \sin z+\mathrm{R}_{0}^{2} A_{\xi}^{2} \cos z+A_{2}}{\sin z}+\epsilon^{3}\left(\frac{\psi_{3}}{\sin z}-\frac{C}{\sin ^{2} z}\right) \\
& =\frac{A}{\epsilon^{3} Z}+\epsilon \mathrm{R}_{0} A_{\xi}+\epsilon^{2}\left[\mathrm{R}_{0} A_{1 \xi}+\frac{\mathrm{R}_{0}^{2} A_{\xi}^{2}\left(1-\epsilon^{6}\right)+A_{2}}{\epsilon^{3} Z}\right]+\epsilon^{3}\left(\frac{\psi_{3}}{\epsilon^{3} Z}-\frac{C}{\epsilon^{6} Z^{2}}\right)
\end{aligned}
$$

where $C_{\xi}=A_{\tau}$. The leading order term in equation 43 (order $\epsilon^{-3}$ )

$$
\theta \sim \frac{1}{\epsilon^{3}}\left(\frac{A}{Z}-\frac{C}{Z^{2}}\right)+\mathcal{O}\left(\epsilon^{-1}\right)
$$

gives us the undetermined $B$ as follows:

$$
B_{\xi}+A_{\tau}=0
$$

Having shown how the asymptotic matching works in the internal boundary layer, we press on to find the consistent scaling for the Peclet number. Adopting the same scaling for the inner solution above, we have to put $\mathrm{Pe}$ to order $\epsilon^{-10}$ to have the diffusive term appeared at the first order in the equation for $\Theta$ inside the internal boundary layer:

$$
\partial_{\tau} \Theta+(Z \Theta-\psi)_{\xi}=\frac{1}{P_{10}} \Theta_{Z Z}
$$

We first note that the boundary conditions for the above linear equation have to be found by matching the inner solution to the outer solution. Secondly, we note that the zeroth order term $\Theta_{0}$ has non trivial $Z$ and $\xi$ dependence, in contrary to what we have found for $\mathrm{Pe} \sim \mathcal{O}(1)$ cases.

\subsection{Amplitude equation and the dispersion relation}

The previous analysis shows that, in the limit of large Peclet number, $\Theta$ depends on $Z$ as well as $\xi$ and $\tau$. With this in mind, we proceed from equations 12 and 13 to write down the amplitude equation for the internal boundary layer. First we note that as the strength of stratification is put to $\epsilon^{6}$, the solutions for the stream function obtained in section 3.1 are still valid in the internal boundary layer. We then only need to concentrate on the heat equation for $\theta$ :

$$
\partial_{t} \theta-J(\psi, \theta)+\sin z \partial_{x} \theta-\partial_{x} \psi=\frac{1}{\mathrm{Pe}} \nabla^{2} \theta
$$

Adopting the scaling $\partial_{t}=\epsilon^{4} \partial_{\tau}, \partial_{x}=\epsilon \partial_{\xi}, \partial_{z}=\epsilon^{-3} \partial_{Z}$ and $\theta=\epsilon^{-3} \Theta$ the above equation takes the following form:

$$
\epsilon \partial_{\tau} \Theta-\epsilon^{-5} J(\psi, \Theta)+\epsilon\left(Z \Theta_{\xi}-\psi_{\xi}\right)=\frac{\epsilon}{P_{10}} \Theta_{Z Z}
$$


Rescaling $\Theta^{\prime}=\epsilon^{6} \Theta, \psi^{\prime}=\epsilon^{6} \psi$ and dropping the primes, we arrive at the following equation for the temperature disturbance inside the internal boundary layer:

$$
\partial_{\tau} \Theta-J(\psi, \Theta)+Z \Theta_{\xi}-\psi_{\xi}=\frac{1}{P_{10}} \Theta_{Z Z}
$$

The stream function amplitude $A$ satisfies the same equation 28 except that inside the internal boundary layer, the average of $\Theta_{x}$ over $Z$ is not simply $\Theta_{x}$ as $\Theta$ depends on $Z$ as well. Also, we have to rescale $A$ accordingly inside the internal boundary layer, so all the nonlinear terms in equation 28 drop out and we obtain the following equation:

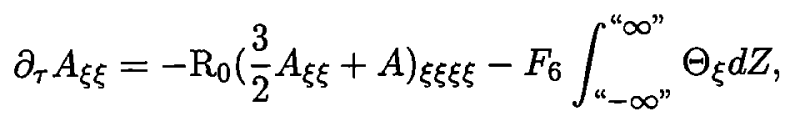

where the integral range (" $-\infty$ ", " $\infty$ ") is referred to the scaled internal boundary layer. To zeroth order in $\epsilon$, we obtain the following equation:

$$
\partial_{\tau} \Theta-A_{\xi} \Theta_{Z}+Z \Theta_{\xi}-A_{\xi}=\frac{1}{P_{10}} \Theta_{Z Z}
$$

where $A=A(\xi, \tau)$ is the amplitude for the stream function. Equations 50 and 51 are the amplitude equations for the internal boundary layer. The linear equations for the internal boundary layer are

$$
\begin{aligned}
\partial_{\tau} A_{\xi \xi} & =-\mathrm{R}_{0}\left(\frac{3}{2} A_{\xi \xi}+A\right)_{\xi \xi \xi \xi}-F_{6} \int_{-\infty}^{\infty} \Theta_{\xi} d Z \\
\partial_{\tau} \Theta & =-Z \Theta_{\xi}+A_{\xi}+\frac{1}{P_{10}} \Theta_{Z Z} .
\end{aligned}
$$

We first derive the dispersion relation for the infinite Peclet number case. Replacing $\partial_{\xi}$ with $i k$ and $\partial_{\tau}$ with $s$, we obtain the following equations:

$$
\begin{aligned}
-k^{2}\left[s+\frac{3 \mathrm{R}_{0}}{2} k^{4}-\mathrm{R}_{0} k^{2}\right] A & =-F_{6} \int_{-\infty}^{\infty} i k \Theta d Z \\
(s+i Z k) \Theta & =i k A .
\end{aligned}
$$

Substituting equation 55 into 54, we obtain the dispersion relation for the infinite Peclet number case:

$$
s=-\frac{F_{6}}{|k|} \operatorname{sgn}(s) \pi-\frac{3 \mathrm{R}_{0}}{2} k^{4}+\mathrm{R}_{0} k^{2}
$$

In the case of finite, large Peclet numbers, we expand $\Theta$ in both $e^{i k x}$ and $e^{i q z}$ and obtain the following equations

$$
-\left(s+k q^{2}\right) \widetilde{\Theta}+\widetilde{\Theta}_{q}=-2 \pi i \widetilde{A} \delta(q)
$$


where $\widetilde{\Theta}$ and $\widetilde{A}$ are Fourier components in the $k-q$ spectral space. The solution to equation 57 is

$$
\begin{aligned}
\widetilde{\Theta} & =e^{\left(s q+k q^{3} / 3\right) / k} \int_{q}^{\infty} 2 \pi i \widetilde{A} \delta\left(q^{\prime}\right) e^{-\left(s q^{\prime}+k q^{\prime 3} / 3\right) / k} d q^{\prime} \\
& =e^{\left(s q+k q^{3} / 3\right) / k} 2 \pi i \widetilde{A} H(-q)
\end{aligned}
$$

where $H$ is the Heaviside function. Substituting the above normal mode solution for $\widetilde{\Theta}$ into 52 , we get the dispersion relation for the large, finite Peclet number case:

$$
s=-\frac{F_{6}}{|k|} \pi-\frac{3 \mathrm{R}_{0}}{2} k^{4}+\mathrm{R}_{0} k^{2}
$$

We note the difference between equation 56 and 60 is the existence of $\operatorname{sgn}(s)$, and we display the two dispersion relations in figure 12.

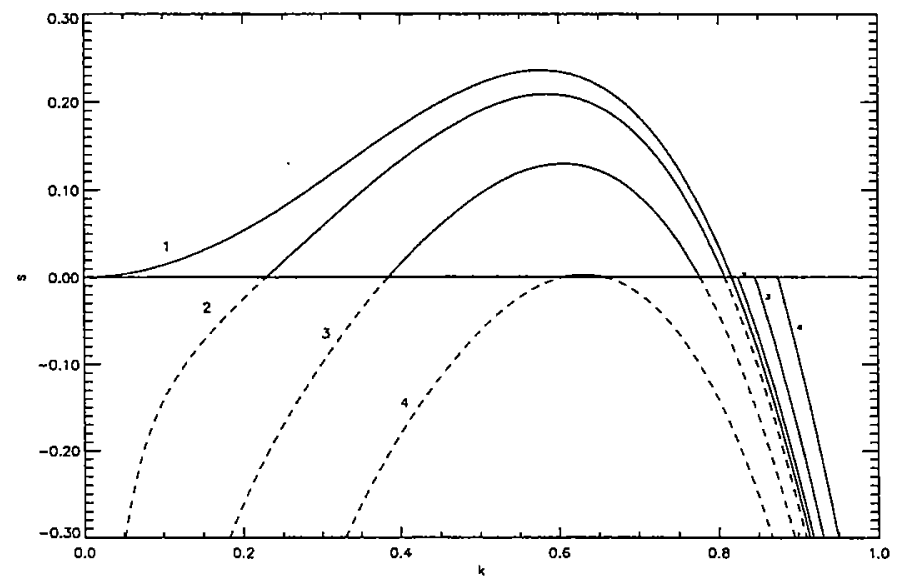

Figure 12: Dispersion relation for the internal boundary layer $\left(R_{0}=\sqrt{2}\right)$. Curves are labeled by the scaled Richardson number $F_{6}$ : From curve 1 to curve $4, F_{6}$ are, respectively, $0,0.005$, $0.02,0.045$. The solid lines are for the infinite Peclet number cases, and the dotted lines are for large Peclet number cases.

We also note that for any given Reynolds number, the value of $F_{6}$ such that the maximum growth rate $s$ is zero is proportional to the Reynolds number and the ratio is 0.0322 . We are now ready to find the general solution to $\Theta$ for large Peclet number cases. We rewrite equation 53 as follows:

$$
\kappa \Theta_{Z Z}-(s+i k Z) \Theta=i k A,
$$

where $\kappa=1 / P_{10}$. Dividing the above equation by $i k A$ and denote $f \equiv \Theta / i k A$, we obtain the following equation which allows us to find a closed-form solution:

$$
\kappa f_{Z Z}-i k(Z-i s / k) f=1 .
$$

The solution is the Yi function:

$$
\Theta=\frac{i \pi A}{k \kappa} \mathrm{Yi}[k(Z-i s / k)] .
$$




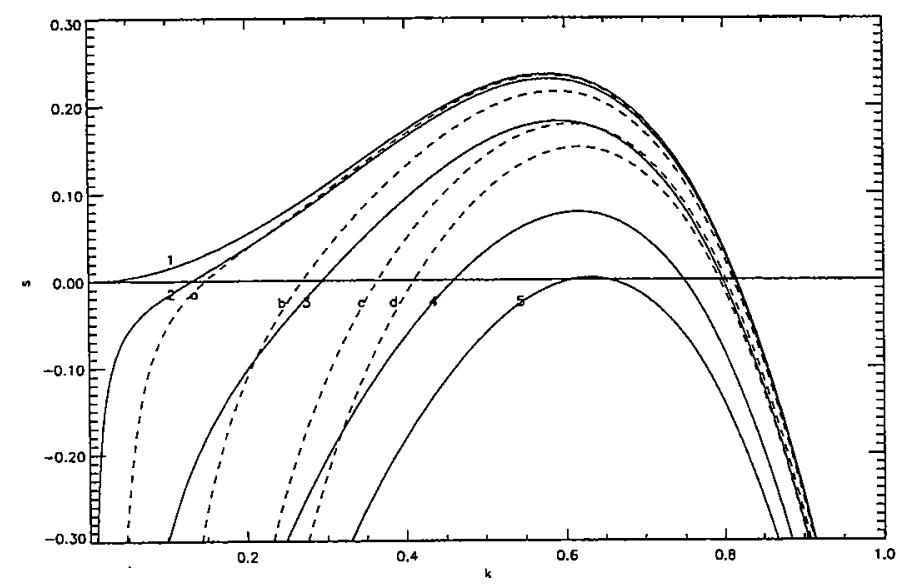

Figure 13: Dispersion relation for the internal boundary layer $\left(R_{0}=\sqrt{2}\right)$. Dashed lines are for order $\mathcal{O}(1)$ Peclet numbers and solid lines are for large Peclet numers. Curve ' 1 ' is for $F_{6}=0$, curves ' 2 ' and ' $a$ '. are for $F_{6}=0.001$, curves ' 3 ' and 'b' are for $F_{6}=0.01$, curves ' 4 ' and ' $c$ ' are for $F_{6}=0.03$, and curves ' 5 ' and 'd' are for $F_{6}=0.045$.

\section{Inviscid limit of the stratified, Kolmogorov shear flow}

In this section we conclude the report by presenting a brief study on the inviscid limit of the stratified Kolmogorov shear flow. A general review on linear analysis of the inviscid shear flow can be found in [8]. Here we provide a way to find neutral states for the unbounded, stratified Kolmogorov shear flow. We have numerically verified the marginal boundary presented in the following analyses, and it would be an interesting direction to provide analytic proofs that this is indeed the case. We should also point out that there may be hope to couple the critical layers (CL) associated with each inflection point in the background shear flow, and hence the interaction between CLs can be investigated.

\subsection{Linear analysis: analytical and numerical}

The inviscid, nondiffusive system $(1 / \mathrm{Pe}=0)$ is described as follows (where the shear flow is a sinusoidal $\sin z$ in a periodic domain):

$$
\begin{gathered}
\partial_{t} \nabla^{2} \psi-J\left(\psi, \nabla^{2} \psi\right)+\sin z \partial_{x}\left(\nabla^{2} \psi+\psi\right)=-F \partial_{x} \theta \\
\partial_{t} \theta-J(\psi, \theta)+\sin z \partial_{x} \theta-\partial_{x} \psi=0 .
\end{gathered}
$$

The linearized equations can be put into the following equation with the diffusivity being zero:

$$
(\sin z-c)\left(D^{2}-k^{2}\right) \psi+\sin z \psi=-\frac{F \psi}{\sin z-c},
$$

where $D \equiv \partial_{z}$ and $c$ is growth rate divided by the wave number. In this notation, the imaginary part of $c$ indicates instability: positive imaginary part means growing mode and 
negative imaginary part means decaying mode. We reorganize the above equation into a more familiar form usually found in the past literature:

$$
\psi^{\prime \prime}-k^{2} \psi+\frac{\sin z \psi}{\sin z-c}=-\frac{F \psi}{(\sin z-c)^{2}} .
$$

First we put $c=0$, though in general only the imaginary part is required to be zero on the neutral curve. Equation 67 now takes the following form:

$$
D^{2} \psi+\left(1-k^{2}+\frac{F}{\sin ^{2} z}\right) \psi=0
$$

The above equation can be solved as follows: first we put the left hand side of equation 68 as the product of two differential operators as defined as follows:

$$
\left(D^{2}+1-k^{2}+\frac{F}{\sin ^{2} z}\right) \psi=\mathcal{L} \mathcal{L}^{\dagger} \psi=0
$$

where $\mathcal{L}$ and $\mathcal{L}^{\dagger}$ are defined as

$$
\mathcal{L} \equiv D+\frac{a}{\cot z}, \quad \mathcal{L}^{\dagger} \equiv D-\frac{a}{\cot z}
$$

and $a$ is to be determined (in terms of $k$ and $F$ ). We note that $\mathcal{L} \mathcal{L}^{\dagger}=D^{2}-\left(f^{\prime}+f\right.$ ) where $f=\cot z$, and relationships between $a, k$, and $F$ are obtained as follows:

$$
a=1-k^{2}, \quad F=a-a^{2}=\sqrt{1-k^{2}}-\left(1-k^{2}\right) .
$$

The first solution $\psi_{1}$ is obtained by demanding $\mathcal{L} \psi_{1}=0$ and takes the following form:

$$
\psi_{1}=(\sin z)^{\sqrt{1-k^{2}}}, \quad z \geq 0 .
$$

The second solution $\psi_{2}$ satisfies the following equation

$$
\mathcal{L}^{\dagger} \psi_{2}=(\sin z)^{-\sqrt{1-k^{2}}}
$$

and is obtained as follows:

$$
\psi_{2}=(\sin z)^{\sqrt{1-k^{2}}} \int^{z}\left(\sin z^{\prime}\right)^{-2 \sqrt{1-k^{2}}} d z^{\prime} .
$$

For some value of $k$, the second solution is not periodic in $z$ and therefore is not of particular interest. $F$, as a function of $k$, is shown in figure 14. First we note that the maximum value of $F$ is $1 / 4$ when $k=\sqrt{3 / 4}$. We also note that $k$ goes from 0 to 1 as we are only interested in positive $F$. 


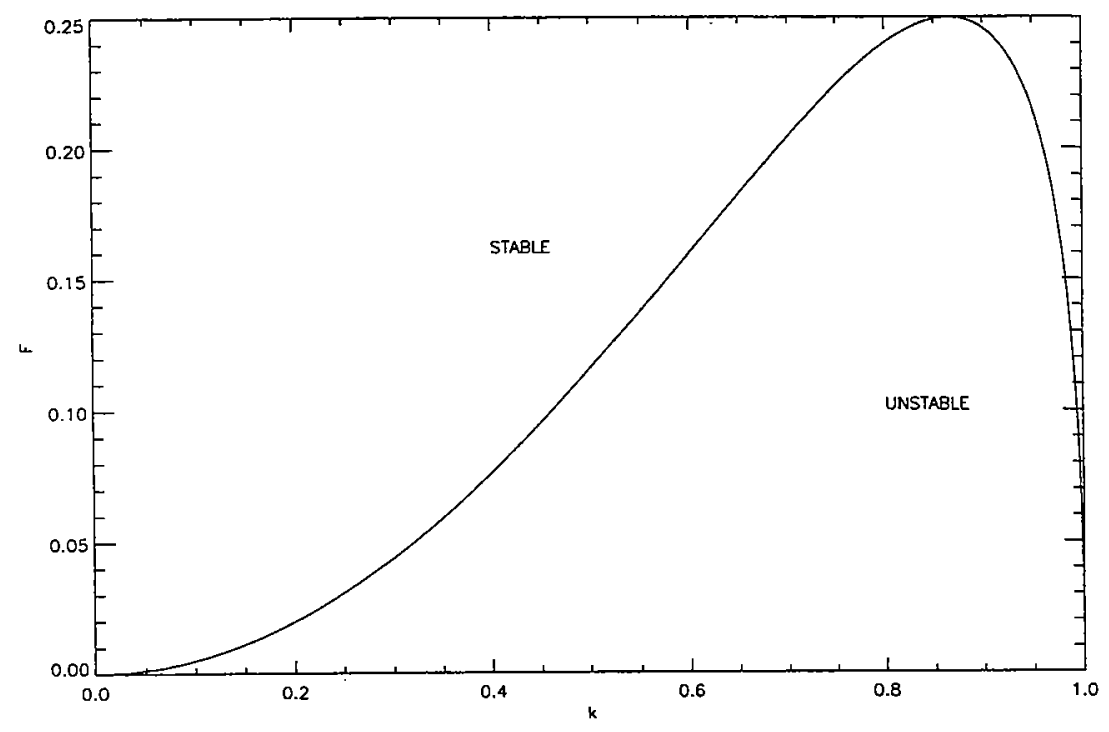

Figure 14: Stability boundary for the inviscid, non-diffusive limit.

\section{Conclusion}

We have investigated the effect of stabilizing stratification on the Kolmogorov shear flow in the weak limit where the long wavelength instability inherits from the non-stratified shear flow. Concentrating on cases where $\mathrm{Pe} \sim \mathcal{O}(1)$, we first derived amplitude equations for the weakly stratified Kolmogorov shear flow and demonstrated the stabilizing effects by numerically solving the amplitude equations. For the 1-D amplitude equation, the stabilizing gradient arrests the inverse cascade and weaken the flow. For the uni-directional amplitude equation, the gradient not only lessens the flow, but also diminishes the chaotic behavior of the unidirectional solution. The same phenomena have been observed for the large aspect ratio 2-d solutions to the full amplitude equation. For the nondiffusive limit $\left(\mathrm{Pe} \sim \epsilon^{-10}\right)$, the dynamics are dominated by the internal boundary layer. From the linear eigenfunctions, we are able to estimate an empirical scaling of boundary layer thickness with the Peclet number. We choose boundary layer scaling accordingly and derive amplitude equations for the internal boundary layer. Dispersion relations are derived and utilized for some preliminary analysis. The linear stability of the stratified, inviscid Kolmogorov shear flow has been investigated as a preliminary step to the weakly nonlinear analysis which is now under investigation.

\section{Acknowledgments}

The project literally started to take form in the summer of 1998 , during my first visit to GFD. Serious development of this project started only at the beginning of this summer under guidance from two key collaborators, Bill Young and Neil Balmforth. I would like to acknowledge, with full sincerity and gratitude, the principal lecturer Bill Young for his infinite patience during this collaboration. I would also like to thank the director Neil Balmforth for 
his insightful guidance all the way through the collaboration, and great advice he so sincerely provided for the whole summer. Thanks also go to Louis Howard and Willem Malkus for inspiring and useful conversations. Finally, I would like to thank all the staff (Claudia, especially) and my fellow fellows for making this summer quite an unforgettable experience.

\section{References}

[1] G. Sivashinsky, "Weak turbulence in periodic flows," Physica D 17, 243 (1985).

[2] Z. S. She, "Metastability and vortex pairing in the kolmogorov flow," Physics Letter A 124, 161 (1987).

[3] G. Sivashinsky and V. Yakhot, "Negative viscosity effect in large-scale flows," Phys. Fluids 28, 1040 (1985).

[4] A.J. Manfroi and W.R. Young, "Slow evolution of zonal jets on the beta plane," J. Atmos. Sci. 56, 784 (1999).

[5] Y.-G. Park, J.A. Whitehead and A. Gnanadeskian, "Turbulent mixing in stratified fluids: layer formation and energetics," J. Fluid Mech. 279, 279 (1994).

[6] N.J. Balmforth, S.G.L. Smith and W.R. Young, "Turbulent stratified fluids," J. Fluid Mech. 400, 500 (1995).

[7] C.J. Chapman and M.R.E. Proctor, "Nonlinear rayleigh-benard convection with poorly conducting boundaries," J. Fluid Mech. 101, 759 (1980).

[8] P. Drazin and L. Howard, "Hydrodynamic stability of parallel flow of inviscid fluid," Advances in Applied Mechanics 9, 1 (1996). 


\title{
What Comes Around Goes Around: A Bug's Life
}

\author{
Jennifer Curtis
}

\section{Introduction}

Many biological phenomena such as the spread of a favored gene, the population growth of species, ecological competition, and others are described by equations that contain the dominant physical processes of diffusion, convection, and a background reaction [6]. The model studied in this paper describes the limited universe of these forces and its influence on the lives and deaths one colony of photosynthetic bacteria living on an inhomogenous substrate.

Interestingly, the model reaches beyond the scope of biology to touch upon unexpected research areas in condensed matter physics, including vortices in superconductors [7] and semiconductor physics [5]. The bacteria and vortex systems are mathematically analogous, non-Hermitian models which have drawn a great deal of interest $[1],[3]$ due to their ability to undergo a delocalization transition in their eigenfunctions. Previously such transitions were believed to be impossible in one or two dimensional systems [5].

The goal of this paper is to continue the analysis of Dahmen, Nelson, and Shnerb (DNS) [4],[2], by studying the delocalization transition in the presence of a weakly non-linear saturation term. This term represents the crowding of the bacteria due to competition or deadly concentrations of toxins from their waste.

The model is new territory for the mathematical analysis of pattern formation and population dynamics in biology. With the exception of DNS, very little work has been done on this type of inhomogenous system. Our analysis leads to the very interesting result that the dynamics of the model is governed by a differential-delay equation. This delay equation is explored with the hopes that oscillations, quasi-periodicity or chaos might arise within the physical regime of the model. A little familiarity with delay-equations and a comparison with another familiar delay-equation of mathematical biology, the Glass-Mackey delay-equation [6] , suggests a structural method to quickly predict the dynamic behavior of a subset of such equations.

Finally, several efforts are made to reasonably modify the physical system to achieve interesting dynamics. These attempts include changing the form of the non-linear saturation and the increasing the spatial complexity of the system. The former is proved to be stable, while the latter remains an open-ended question. 


\section{The Modified Fisher Equation}

Imagine one species of bacteria living in a periodic, one-dimensional ring with coordinates in $x, 0<x<L$. Located at at the origin is an "oasis" where plentiful supplies of food and light support life. The rest of the ring is deadly to the bacteria. DNS call this region a "desert". The bacteria experience diffusion, as well as a convective drift due to a background current. They also compete with their fellow neighbors as mentioned above. This is the source of the non-linear term neglected by DNS. The equation governing this model is the Fisher equation with an extra term included to account for convection. Originally, the Fisher equation was proposed as a model for the spread of a favored gene. The modified Fisher equation is then

$$
c_{t}(x, t)+u c_{x}(x, t)=D c_{x x}(x, t)+[\beta \delta(x)-\alpha] c(x, t)-b c^{2}(x, t),
$$

where $\mathrm{c}(\mathrm{x}, \mathrm{t})$ is the concentration of the bacteria. A delta function of strength $\beta$ represents the oasis and the $-\alpha$ term represents the death rate of the desert. The combination of $\beta \delta(x)-\alpha$ is the spatial inhomogeniety of this particular system.

DS's biological motivation for suggesting this model was to study the effect of spatial inhomogenieties in the underlying medium. Disorder in the medium may be due to many things, including random diffusion constants, stochastic growth and death rates, or a random concentration of environimental factors such as food, toxins or illumination. Here we use the simplest choice, a random concentration of food and/or illumination.

A possible experiment suggested by DNS is to place the bacteria in a thin annular ring covered by a dark mask with a small slot cut to let light pass through. Turning the mask at a slow speed while the ring remains fixed would simulate the convection current. Currently DNS are talking with experimental biologists to do this experiment. Another practical system where this model may be applied is the circumpolar current around Anartica. It has been shown to carry photosynthetic plankton completely around the continent, with various patches of nutrient-rich upswellings supporting the plankton.

\section{Linear Stability Analysis}

The following section reviews the analysis of the linearized modified-Fisher equation. Here we become familiar with the delocalization transition that occurs in this biological model and with the associated behavior of the eigenspectrum. Linearizing about the fixed point $c=0$ leads to

$$
c_{t}(x, t)+u c_{x}(x, t)=D c_{x x}(x, t)+[\beta \delta(x)-\alpha] c(x, t)
$$

\subsection{Without the oasis: An example of delocalized modes}

Delocalized eigenfunctions are those solutions which have a form like $e^{i k x}$, where $k$ is complex and the real part is non-zero. The simplest example of a delocalization occurs if $\beta=0$. Solutions are of the form $c(x, t)=e^{s t} e^{i k x}$. Periodicity requires that $\mathrm{k}$ be quantized as $k=$ $2 \pi m / L$, where $m$ is an integer. 


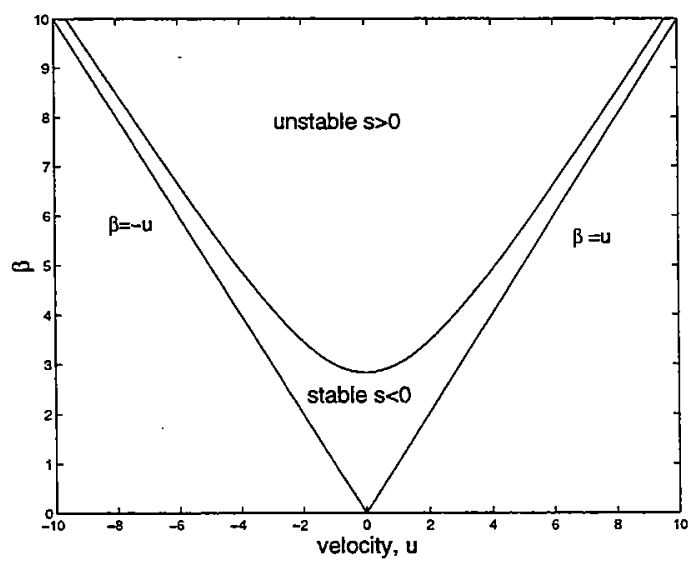

Figure 1: Regime diagram for an infinite ring

The associated dispersion relation is

$$
s=-D k^{2}+\alpha-i u k
$$

The growth rate, $s$, is a discrete set of complex numbers. The plot of $\operatorname{Re}(\mathrm{s})$ versus $\operatorname{Im}(\mathrm{s})$ is a parabola symmetric to the real axis. Increasing the velocity $u$ broadens the parabola. When $u=0$, the growth rate is real. The value of $\alpha$ determines the stability of the eigenfunctions. The growth rate of the $m^{\text {th }}$ eigenfunction will be positive if

$$
\alpha>D\left(\frac{2 \pi m}{L}\right)^{2}
$$

The eigenfunction with the largest positive growth rate $(k=0)$ will dominate the system at large times.

\subsection{An infinite ring}

We begin the linear analysis of our system for the case of an infinite ring because it has simple analytical results which clearly demonstrate the signature of delocalization. Assume $c(x, t)=e^{s t} c(x)$ to eliminate the time dependence in (1),

$$
s c(x)+u c_{x}(x)=D c_{x x}(x)+[\beta \delta(x)-\alpha] c(x) .
$$

It may also be written as

$$
c_{t}=\mathcal{L} c
$$

where the linear operator,

$$
\mathcal{L} c=D c_{x x}-u c_{x}+[\beta \delta(x)-\alpha] c,
$$


generates the time-evolution of the system. When $u=0$, the operator is Hermitian with real eigenvalues, and for strong enough disorder, all of its eigenfunctions are real and localized $[2]$. $k$,

Using $c(x)=e^{-k x}$, a dispersion relation is found to have two roots for the wavenumber

$$
\begin{array}{r}
D k^{2}+u k-s-\alpha=0 \\
k_{ \pm}=\frac{-u \pm \sqrt{u^{2}+4 D(s+\alpha)}}{2 D} .
\end{array}
$$

Periodicity is satisfied only if the eigenfunction $c(x)=e^{-k x}$ decays as $x \rightarrow \pm \infty$. Thus we use $k_{+}$when $x>0$ and $k_{-}$when $x<0$. This restriction is the equivalent of solving for only the localized eigenfunctions of this physical system, i.e. we are working in the regime where $k_{ \pm}$is real.

Using the appropriate eigenfunction to the left and right of the origin, we integrate (patch) across the delta function to acquire a value for the growth rate s:

$$
\begin{array}{r}
D\left[\left[c_{x}\right]+\beta=0,\right. \\
D\left[-k_{+}-\left(-k_{-}\right)\right]=-\beta, \\
\Rightarrow \quad s=\frac{\beta^{2}-u^{2}}{4 D}-\alpha .
\end{array}
$$

The expression for $k_{ \pm}(8)$ can now be simplified using the growth rate (9),

$$
\begin{array}{r}
k_{ \pm}=\frac{-u \pm \beta}{2 D}, \\
\Rightarrow \quad \beta>u \text { when } u>0, \\
\beta>-u \text { when } u<0 .
\end{array}
$$

The requirements on $k_{ \pm}$restrict the range of $u$, according to the given strength of the oasis $\beta$. We have one localized solution, although if the oasis were wider than a delta function, say a box, there would be many localized solutions.

A regime diagram (figure 1 ) maps the properties of this system as $\beta$ and $u$ vary. The two straight lines, $\beta=u$ and $\beta=-u$, are the boundaries which restrict $k_{ \pm}$. If crossed, the eigenfunctions will be in the delocalized regime.

The marginal stability curve is the hyperbola labeled $s=0$. As the parameters $u$ and $\beta$ tend to infinity, the marginal stability curve coincides with the delocalization transition. Inside of the hyperbola, $s$ is positive, and thus the eigenfunctions are unstable and grow in time. Outside of the hyperbola, $s$ is negative, and the eigenfunctions are stable and decay with time.

Larger values of $\alpha$ and $|u|$ shift the marginal stability curve upwards, increasing the regime of stability. This is because $\alpha$ is the size of the death rate; while in an infinite ring, larger velocities carry more bacteria and being carried away from the oasis is a sure death sentence in an infinite ring. 


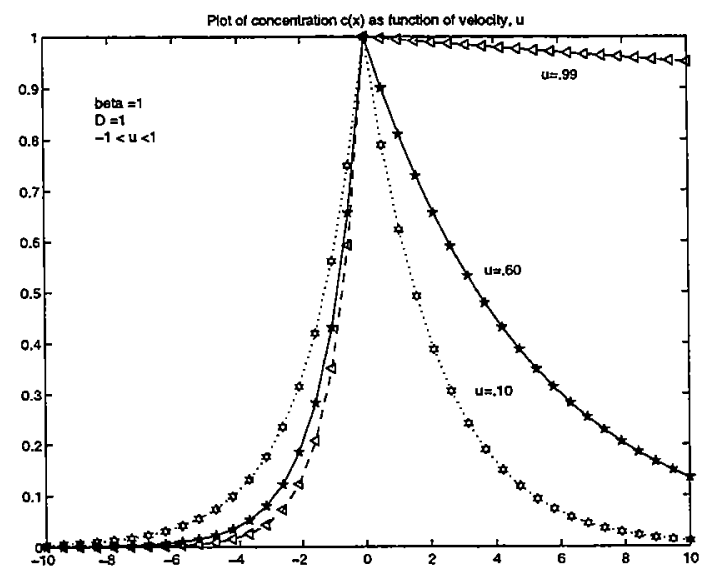

Figure 2: $c(x)$ for positive velocities

The behavior of the concentation $c(x)$ when there is positive background velocity is examined in figure (2). The plots are asymmetric due to the eigenfunction dependence on the sign of u:

$$
c\left(x_{ \pm}\right)=e^{\left(\frac{-u \pm \beta}{2 D}\right) x} .
$$

The velocity blows bacteria away from the orgin to the right, increasing their concentration in this region. To the left of the origin, the competing mechanisms of diffusion away from the oasis, and advection back into the oasis result in a thin boundary layer. If no wind or current were present, the distribution would be symmetric, decaying exponentially away from the orgin.

As the value of the velocity increases, the concentration becomes nearly constant across $x$ because more bacteria is being blown out of the origin. For this particular example, choices for the velocity $u$ are limited by the choice of $\beta=1$. At $u=.99$ for instance, the eigenfunction is nearly delocalized. If we surpassed $u=1$ we would be examing the delocalized spectrum.

The one localized mode, is unstable if

$$
\beta^{2}>4 D \alpha+u^{2}
$$

This section has been included to introduce the problem and gain some intuition for the delocalization transition, and its dependence on the physics $(D, \beta, u)$ of the system. Note that for this infinite ring case, the velocity carries the bacteria away from their haven, never returning them in time before they die. The velocity has a purely deadly effect. Thus, the inequality above, a requirement for instability, makes sense. Only if the life production in the oasis, $\beta$, is large enough to overcome the deadly effects of diffusion and convection, will the system grow in time.

\subsection{A finite ring with no convective drift}

We now study the linearized problem in a finite ring with no wind. When $u=0,(5)$ becomes

$$
s c=D c_{x x}+[\beta \delta(x)-\alpha] c .
$$



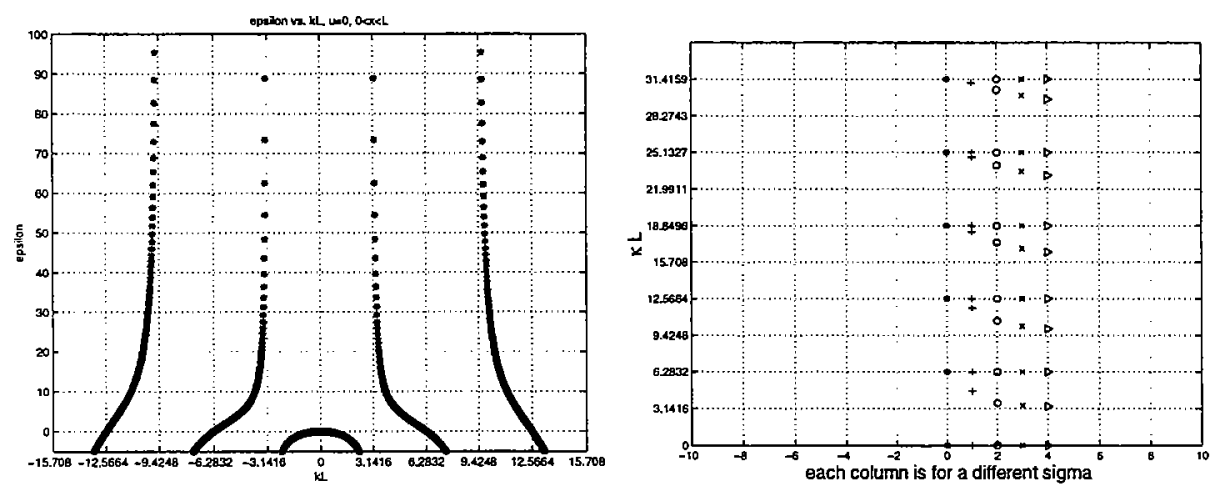

Figure 3: (a) $\sigma=\frac{\beta L}{2 D}$ vs $\kappa L$ when $u=0$ in finite ring (b) roots of $\sigma \sin (x)=0 . *=\sigma=0 ;+=\sigma=5 ; o=\sigma=15 ; x=\sigma=25 ;<=\sigma=35$

$x(1-\cos (x))+$

We arrange that the unknown concentration $c(x)$ will be equal to 1 at $x=0$. The eigenfunctions will then be of the form,

$$
c(x)=(1-A) e^{-k x}+A e^{k x} .
$$

Using periodicity, $c(0)=c(L)$, we find an expression for $\mathrm{A}$

$$
A=\frac{1-e^{-k L}}{2 \sinh k L} .
$$

Combining this result with (14), gives the expression for $c(x)$ :

$$
c(x)=e^{-k x}+\frac{\left(1-e^{-k L}\right)}{\sinh k L} \sinh k x .
$$

Patching across the delta function gives a transcendental relation for the wavenumber $k$ :

$$
2 k(1-\cosh k L)=-\frac{\beta}{D} \sinh k L .
$$

This is the same expression found for $\mathrm{k}$ by DNS [4], if one sets their velocity, $\mathrm{v}$, equal to zero. Their result was found by solving the problem for a periodic domain with an oasis that is a finite square well, and then taking the area of the well to zero.

To study the delocalized spectrum, this expression can be neatly rewritten by letting $k=i \kappa$ and $\sigma=\beta L /(2 D)$ :

$$
\kappa L(1-\cos (\kappa L))+\sigma \sin (\kappa L)=0 .
$$

In this form, we assume that the wavenumber $k$ is purely imaginary. The spectrum may be studied graphically by plotting $\sigma$ as a function of $k L$ (see figure 3(a)). The wavenumbers $\mathrm{k}$ for fixed $\sigma$ are found by drawing a line at one value of $\sigma$ and intersecting the curves. The numerical results are plotted in 3(b) for various values of $\sigma$. 
A double degeneracy exists in the solutions of the finite ring without a delta function $(\sigma=0)$. This degeneracy is not reflected in 3(a) because the dispersion relation was obtained after dividing out one extra factor of $\cosh (k L)$. The degeneracy of the eigenvalues is broken by turning on the delta function strength so that $\sigma \neq 0$. As $\sigma$ is increased, one of the two sets of eigenvalues moves away from the general oscillatory solutions $k=2 \pi m$ of section (3.1). As the strength of the delta function increases, the moving eigenvalues asympotote to $(2 m+1) \pi$. The other eigenvalue remains fixed at these values.

None of these delocalized modes are unstable in time, although we will see in the next section, that they can be unstable when there is a strong enough wind to help blow the bacteria around the ring before they die.

One localized mode exists. The solution can be found analytically if one assumes that $\sigma$ is very large so that $\cosh \sigma \approx \sinh \sigma$. In this limit, we find that $k L \rightarrow \sigma$. The localized mode can be unstable depending on the values of the parameters $k$ and $\alpha$. The dispersion relation for this system is

$$
s=D k^{2}-\alpha
$$

\subsection{Finite length and a constant wind: Traveling around the ring}

We now explore the full linearized problem in a finite ring. The relevant partial differential equation is

$$
s c(x)+u c_{x}(x)=D c_{x x}(x)+[\beta \delta(x)-\alpha] c(x) .
$$

We begin with the assumption of the form of the concentration $c(x)$, so that $c=1$ at the origin,

$$
c(x)=\left((1-A) e^{-k x}+A e^{k x}\right) e^{-\frac{u x}{2 D}} .
$$

Applying periodicity gives an expression for the constant $A$

$$
A=\frac{e^{\frac{u L}{2 D}}-e^{-k L}}{2 \sinh k L} .
$$

Now, to obtain an expression for $k$ similar to (17), we repeat patching. The $u c_{x}(x)$ term adds nothing new since it is zero when integrating over $x$; however the derivative of $c(x)$ is now more complicated due to the addition of $u / 2 D$ in the exponential. Using the definition, $\eta=u L / 2 D$ where $\eta$ is the Peclet number of fluid mechanics, we find

$$
2 k(\cosh \eta-\cosh k L)=-\frac{\beta}{D} \sinh k L .
$$

Note that if $u=0$, it is identical to (17) as it should be. Also, if $\operatorname{Re}(k)>u$ and $L \rightarrow \infty$ the dispersion relation reduces to (9).

Given the concentration in (21), the dispersion relation is

$$
s=D k^{2}-\frac{u^{2}}{4 D}-\alpha .
$$



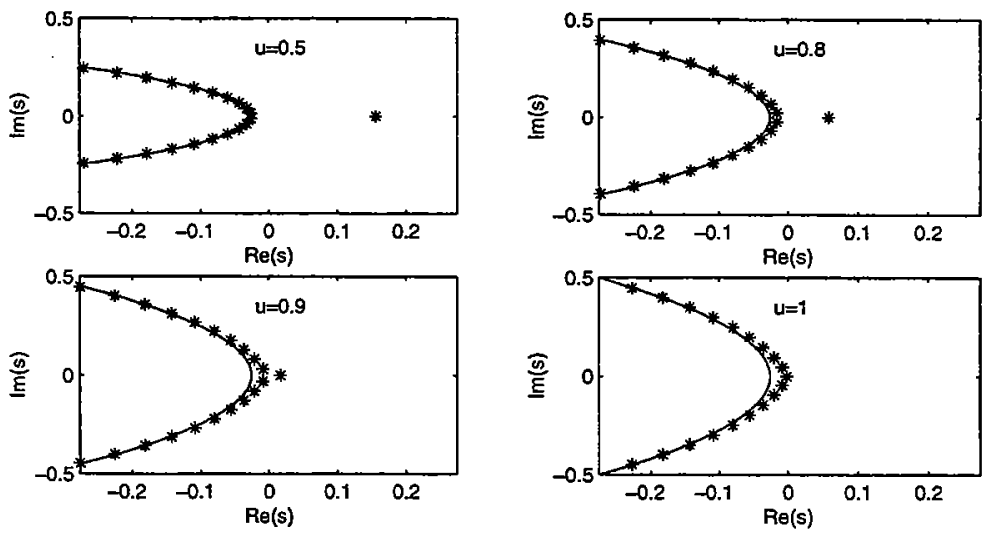

Figure 4: The movement of the localized mode into the delocalized mode with increasing velocity. Complex $\mathrm{s}$ is a signature of delocalization

To learn more about this new dispersion relation for $k$, let $k=i \kappa$ and use the definition for $\sigma:$

$$
\kappa L(\cosh \eta-\cos (\kappa L))+\sigma \sin \kappa L=0 .
$$

When $k$ is complex, the system is best studied using a numerical algorithim to solve for the growth rate s. The results are very interesting. We find that the eigenspectrum is a parabola on the complex plane as it was in section (3.1), but now we also have one real eigenvalue which varies in its distance to the parabola depending on the velocity $u$. This is the one localized mode that accompanies the delta function. As the velocity is increased, the eigenvalue moves towards and finally onto the apex of the parabola which remains fixed. This represents the same delocalization transition we experienced in section (3.2) when we crossed the boundaries $\beta= \pm u$. Figure 4(b) shows a series of spectra with increasing velocity. Here the critical delocalization velocity, the velocity at which the real eigenvalue moves onto the parabola, is determined by a more complicated relationship between $\beta$ and $u$ which we examine in the next section.

\subsection{An important limit for the finite ring with wind}

Here we present a nice way to represent the delocalization transiton for a large but finite ring. This is a new addition to the analysis done by DNS.

In the limit that $\mathrm{L}$ is large, $(23)$ reduces to the dispersion relation

$$
e^{-L\left(k-\frac{u}{2 D}\right)}=1-\frac{\beta}{2 k D} .
$$

We define a parameter $P$, which in the limit that $L \rightarrow \infty$, is a measure of our closeness to the delocalization threshold

$$
P=\frac{\beta}{u}-1 .
$$


In order to examine the complex eigenvalue spectrum, we rewrite $\mathrm{k}$ in terms of a complex parameter $\zeta$,

$$
k \equiv \eta[1+P \zeta]
$$

where for eventual simplicity, $\zeta$ is

$$
\zeta=1+\frac{x+i y}{P \eta}
$$

Then $k$ becomes

$$
k=\eta(1+P)+x+i y .
$$

Putting $k$ (28) into the dispersion relation (26) and simplifying gives the expression,

$$
e^{-P \eta}=1-\frac{P+1}{1+P \zeta}
$$

Making the assumption that $P \zeta<<1$, we have a final expression for $\zeta$

$$
\zeta=1+\frac{e^{-P \eta \zeta}}{P}
$$

Using the definitions for $\zeta(29,32)$ and plugging it into $k(28)$, gives a nice expression for the wavenumber

$$
k=\eta\left[1+P+e^{-\eta P} e^{-x-i y}\right] .
$$

Another useful relation is obtained by combining $(30,33)$,

$$
x+i y=\rho e^{-x-i y}
$$

where we have used the definition of $\rho$

$$
\rho \equiv \eta e^{-P \eta}
$$

Setting real and imaginary parts equal, we have two expressions for $x$ and $y$,

$$
\begin{aligned}
& x=\rho e^{-x} \cos y \\
& y=-\rho e^{-x} \sin y .
\end{aligned}
$$

A little maninpulation of these expressions gives the final form that we use to explore the delocalization transition,

$$
\begin{aligned}
x^{2}+y^{2} & =\rho^{2} e^{-2 x}, \\
\frac{y}{x} & =-\tan y .
\end{aligned}
$$



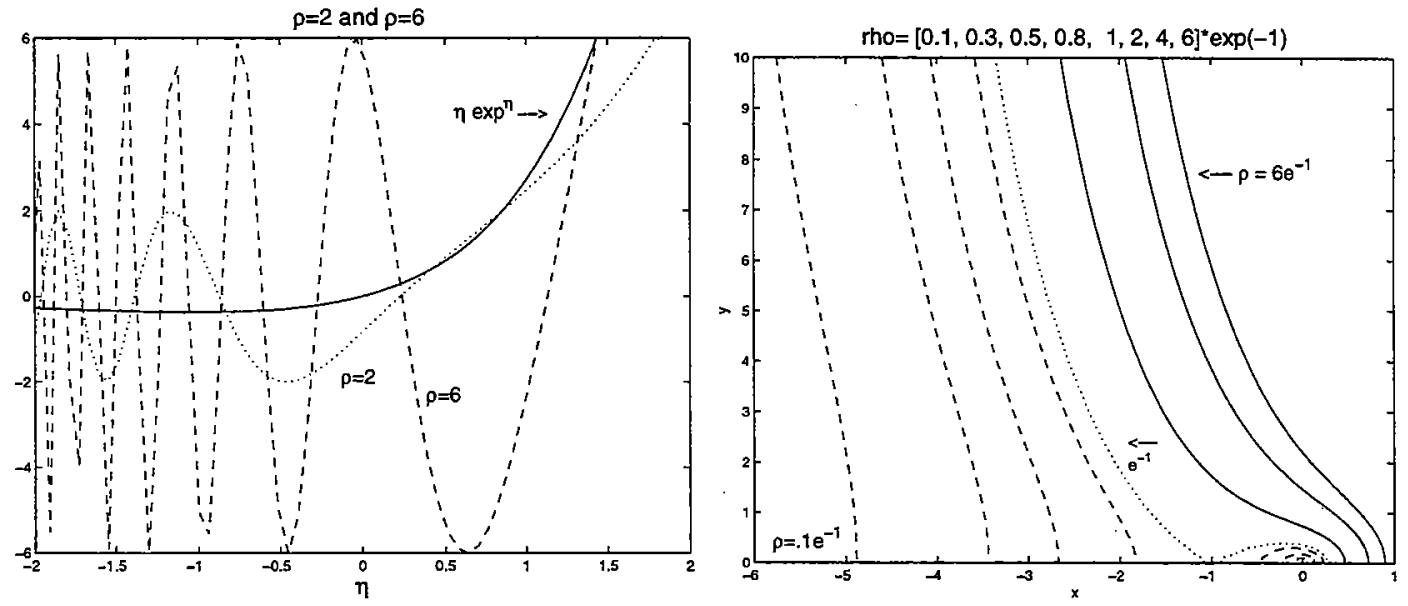

Figure 5: (a) A graphical solution of (37). The solid curve is $\eta e^{\eta}$ and the dashed curves show $\rho \cos \left[\sqrt{\rho^{2} e^{-2 \eta}-\eta^{2}}\right]$ for two values of $\rho=[2,6] * \exp (-1)$. (b) Eigenvalue loci obtained from (39) for various values of $\rho$. The dotted curve is $\rho=1 / e$ and the multi-branched dashed curves are loci with $\rho<1 / e$. The solutions obtained from the intersections of figure 5(a) can be placed on the appropriate curves above.

Figure (5)(b) plots contours of $\rho$ in the $x-y$ plane. The pair of values $(x, y)$ for each wavenumber $k$ is found by solving

$$
x=\rho e^{-x} \cos \sqrt{\rho^{2} e^{-2 x}-x^{2}},
$$

for a given $\rho$. The most unstable mode (the largest solution) is $x_{0}$, which is determined by $x_{0} e^{x_{0}}=\rho$. The cooresponding $y_{0}$ is always zero, so that the most unstable model has a real eignevalue. The higher modes occur in complex conjugate pairs.

For a given value of $\rho$, one can draw the eigenvalue locii in the $\mathrm{x}+\mathrm{iy}$ plane. It is found that when $\rho<\exp (-1)$, two contour curves exist in the $\mathrm{x}-\mathrm{y}$ plane, while if $\rho>\exp (-1)$, only one curve exists. The associated wavenumbers $k$ are represented as points $(x, y)$ on the curves, corresponding to the intersections in figure 5(a). If we are in the region where $\rho>\exp (-1)$, the contour of $\rho$ will be to the right of the set of half circles near the origin. An infinite set of discrete pairs of $(\mathrm{x}, \mathrm{y})$ (and thus wavenumbers $k$ ) are found along each contour in that set. If $\rho<\exp (-1)$, two curves exist, a half circle and a line somewhere to the left of the half circle. Only one pair $\left(x_{o}, y_{o}\right)$ exists on the associated half circle, representing the one localized mode. The second curve will have an infinite but discrete set of $(\mathrm{x}, \mathrm{y})$ pairs along it, representing the the delocalized spectrum. When $\rho=\exp (-1)$, we are right at the delocalization transition. This corresponds to the eigenvalue $s$ moving onto the apex of the parabola in figure 4(b). Thus, the value of the non-dimensional parameter $\rho$ determines at what velocity the delocalization transiton occurs, for a given $\beta, D, L$.

A similar condition restraint of the velocity like $|u|<\beta$ of the infinite ring, comes in the 
form of the transcendental equation

$$
\rho \equiv \frac{u L}{2 D} e^{(\beta-u)\left(\frac{L}{2 D}\right)}<\exp (-1)
$$

\section{Weakly non-linear anaylsis near the delocalization transition}

The goal of this work is to study the effect of the non-linear saturation term on the delocalization transition that we observed in the linear analysis. In the following sections we explore this effect in infinite and finite diameter rings.

The previous section reviewed the results of DNS and carefully studied the existance of the delocalization transition in our one-dimensional model. While DNS quickly discuss the effects of the non-linear term which represents the competition between the bacteria, they do not study it in depth. They suggest that the non-linear term is irrevelant, especially in the limit that the ring has an infinite diameter.

Here we consider the effect of the non-linear saturation term, $-b c^{2}$, on the delocalization transition and discuss its effects on the dynamics of our bacteria colony. The modified-Fisher equation is now examined in its entirity:

$$
c_{t}+u c_{x}=D c_{x x}+[\beta \delta(x)-\alpha] c-b c^{2}
$$

\subsection{Infinite ring on a windy day}

We start by studying the infinite ring. We remain near to the delocalization transition represented by the lines in figure (1), by keeping $\beta_{c}$ nearly equal to the critical beta, $\beta=u$,

$$
\beta=\beta_{c}(1+\epsilon)
$$

where $\epsilon$ is a small parameter. We also define a slow time $T$ and express the concentration as:

$$
T=\epsilon t, \quad c=\epsilon f .
$$

Rewritten with these scalings, the adjusted equation is

$$
\epsilon f_{T}+u f_{x}=D f_{x x}+\beta_{c}(1+\epsilon) \delta(x) f-\alpha f-b \epsilon f^{2} .
$$

Expanding the eigenfunction $c=\epsilon f$ :

$$
f=f_{0}+\epsilon f_{1}+\epsilon^{2} f_{2}+\ldots
$$

Substituting in the expansion of $f$ gives:

$$
\begin{gathered}
\epsilon\left(f_{0 T}+\epsilon f_{1 T}\right)+u\left(f_{0 x}+\epsilon f_{1 x}\right)=D\left(f_{0 x x}+\epsilon f_{1 x x}\right)+ \\
\beta_{c}(1+\epsilon) \delta(x)\left(f_{0}+\epsilon f_{1}\right)-\alpha\left(f+\epsilon f_{1}\right)-b \epsilon\left(f_{0}+\epsilon f_{1}\right)^{2} .
\end{gathered}
$$

The zeroth order equation in $\epsilon$ is:

$$
D f_{0 x x}-u f_{0 x}+\beta_{c} \delta(x) f_{0}-\alpha f_{0}=0 .
$$


It is useful to write $(47)$ in terms of the linear operator $\mathcal{L}(7)$ defined previously in section 3.2 , so that

$$
\mathcal{L} f_{0}=0
$$

The solution $f_{0}$ for this zeroth order homogeneous equation is

$$
f_{0}=A(T) e^{\left(\frac{u-\beta_{c}}{2 D} x\right)}
$$

where $A(T)$ is the time-dependent constant of the solution. This is the result we expect, since the zeroth order solution represents the eigenfunction at the delocalization transition. The constant concentration along $x$ is what would see when $\beta_{c}=u$ in figure (1). For now, we imagine that $\beta$ is not quite equal to $u$. The first-order equation in $\epsilon$ is

$$
f_{0 T}+u f_{1 x}=D f_{1 x x}+\beta_{c} \delta(x) f_{1}+\beta_{c} \delta(x) f_{0}-\alpha f_{1}-b f_{0}^{2} .
$$

Rewriting (50) in terms of the linear operator, $\mathcal{L}$,

$$
\mathcal{L} f_{1}=f_{0 T}-\beta_{c} \delta(x) f_{0}+b f_{0}^{2}
$$

The first order eigenfunctions may be solved for from (51), using the definition of $f_{0}$ found in (49).

To find the time-dependence of $f_{0}=A(T)$, we derive an amplitude equation. Using the adjoint of $f_{0}, f_{0}^{\dagger}$, and integration by parts, it is easy to show that

$$
\left\langle f_{0}^{\dagger} \mathcal{L} f_{1}\right\rangle=\left\langle f_{1} \mathcal{L}^{\dagger} f_{0}^{\dagger}\right\rangle=0
$$

We may take advantage of this fact if we multiply (51) by $f_{0}^{\dagger}$ and integrate over $x$. The left hand side dissapears and the right hand side reduces to the amplitude equation

$$
\left\langle f_{0}^{\dagger} \mathcal{L} f_{1}\right\rangle=\left\langle f_{1} \mathcal{L}^{\dagger} f_{0}^{\dagger}\right\rangle \equiv 0=\left\langle f_{0}^{\dagger} f_{0 T}\right\rangle+b\left\langle f_{0}^{\dagger} f_{0}^{2}\right\rangle-\left\langle\beta_{c} f_{0}^{\dagger} f_{0} \delta(x)\right\rangle
$$

Using our solution for the zeroth-order amplitude $f_{0}$ and performing the integrals results in the amplitude equation:

$$
A_{T}=\left(\frac{\beta_{c}^{2}}{2 D}\right) A+\frac{6 b \beta_{c}^{2} A^{2}}{u^{2}-9 \beta_{c}^{2}}
$$

This result is not unusual for a perturbative analyis of a non-linear problem, and is not of much interest except for comparison with the unique results of the next section. It is reassuring to note that if $\beta_{c}=u$, this amplitude equation blows up, as it should since none of the integrals we performed would have converged. The solution to (54) is found easily using the Bernoulli trick which transforms non-linear equations to solvable linear equations. As a check on this result, one may show that the analytical and perturbative energies agree at the zeroth and first orders. 


\subsection{Finite ring on a windy day: Part I The solution}

In this section we work with the entire physical model. At the origin there is an oasis where the bacteria grows; away from the origin the bacteria struggles for life, dying at a rate $\alpha$. The bacteria diffuses along concentration gradients and is advected by a constant background flow at speed $u$. Throughout the ring, the bacteria competes, dying if conditions become too crowded, thus adding to the the resultant death rate of the desert. Finally, the bacteria live in a finite domain with periodic boundary conditions, e.g. a ring.

The strategy here, is to expand near the delocalization threshold, as we have done for the infinite domain. It is also assumed that the ring is large, but finite. Obviously, this is not the most general consideration of the problem that can be made, but it allows one to proceed analytically. The meaning of a large domain will be discussed below.

We begin by non-dimensionalizing the modified-Fisher equation with the intention of having a firm grasp of the size of each term. Non-dimensionalizing may obscure the physics, but it clarifies the relative magnitudes of terms. It is natural to scale distance with the length of the ring and to scale time with the transit time:

$$
\hat{x}=\frac{x}{L}, \quad \hat{t}=t \frac{u}{L}, \quad \delta(x)=\delta(L \hat{x})=\frac{1}{L} \delta(\hat{x}) .
$$

The resulting equation is:

$$
c_{\hat{t}}+c_{\hat{x}}=\frac{L}{u}\left(\frac{1}{L} \beta \delta(\hat{x})-\alpha\right) c+\frac{D}{L u} c_{\hat{x} \hat{x}}-\frac{L b}{u} c^{2} .
$$

Several more useful non-dimensionalizations are:

$$
\hat{\alpha}=\frac{L}{u} \alpha, \quad \hat{\beta}=\frac{1}{u} \beta, \quad \hat{D}=\frac{D}{L u}, \quad \tilde{c}=\sqrt{\frac{b}{\hat{\alpha}}} \hat{c} .
$$

These non-dimensionalized constants contain the physical meaning of competing effects. $\hat{\alpha}$ is the ratio of the decay rate $\alpha$ to the advective transit time $L / u$. $\hat{D}$ is the inverse Peclet number, or a measure of the strength of diffusion versus advection. Finally, $\hat{\beta}$ is a measure to the nearness of the delocalization transition. If $|\hat{\beta}|=1$, we are at the transition.

The fully non-dimensionalized equation is

$$
\tilde{c}_{t}+\tilde{c}_{\hat{x}}=\hat{D} \tilde{c}_{\hat{x} \hat{x}}+[\hat{\beta} \delta(\hat{x})-\hat{\alpha}] \tilde{c}-\hat{\alpha} \tilde{c}^{2} .
$$

We are interested in the non-linearity near the delocalization transition, and so we expand $\hat{\beta}$ around 1. Using the non-dimensionalized diffusion coefficient $\hat{D}$ as the small parameter with which we expand, we have

$$
\hat{\beta}=1+\hat{D} \hat{\beta}_{1} .
$$

When $\beta<1$ there is a localized mode and this mode becomes delocalized if $\beta>1$. Thus, condition (59), in which $\beta_{1}$ is held fixed as $\hat{D} \rightarrow 0$, ensures that the system is operating close to this delocalization threshold. $\beta_{1}$ may be positive or negative, putting us in either the localized or delocalized regime, as long as $\hat{D}$ is small. Small $\hat{D}$ is the equivalent to large $L$ or large $u$ since $\hat{D}=D / L u(57)$. 


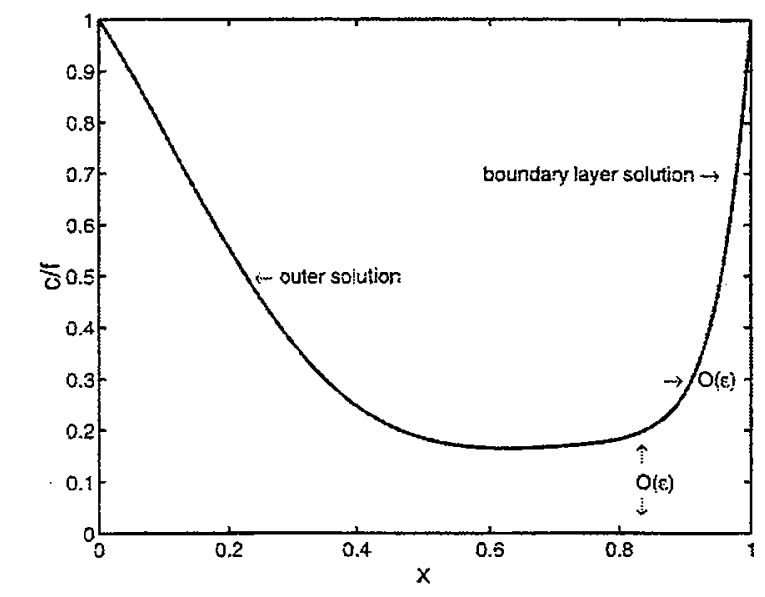

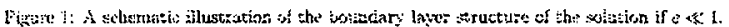

Figure 6: The outer and inner eigenfunctions. $\epsilon \equiv \hat{D}$

\subsubsection{The outer solution}

Dropping the tildes and hats for notational simplicity, and making an expansion in the small parameter $D, c \approx c_{0}+D c_{1},(58)$ becomes:

$$
\begin{aligned}
\left(c_{0 t}+\epsilon c_{1 t}\right)+\left(c_{0 x}+\epsilon c_{1 x}\right) & =(\beta \delta(x)-\alpha)\left(c_{0}+\epsilon c_{1}\right) \\
& +D\left(c_{0 x x}+\epsilon c_{1 x x}\right)-\alpha\left(c_{0}^{2}+2 \epsilon c_{0} c_{1}\right) .
\end{aligned}
$$

The zeroth order equation is then:

$$
c_{0 t}+c_{0 x}=-\alpha c_{0}-\alpha c_{0}^{2} .
$$

A series of tricks and substitutions are used to solve for the zeroth order concentration $c_{0}(x, t)$. The result is

$$
c_{0}(x, t)=\frac{f(t-x)}{\left(e^{\alpha x}-1\right) f(t-x)+e^{\alpha x}} .
$$

Checking this expression at $x=0$, reveals $c(0, t)=f(t)$. This implies that all higher-order terms must be zero at the origin. Meanwhile attempting to demonstrate the periodicity of the system at $x=1$,

$$
f(t)=\frac{f(t-1)}{\left(e^{\alpha}-1\right) f(t-1)+e^{\alpha}},
$$

requires a stringent restriction of $f(t-x)$, suggesting that there is a problem at $x=1$.

In fact, this solution is an "outer approximation", which is valid provided that $0 \leq x \leq$ $1-\mathcal{O}(\mathcal{D})$. The failure of the outer solution at the boundary becomes apparent, if we define 
the restricted parameter $\lambda$,

$$
\lambda \equiv \frac{e^{-\alpha}}{D}, \quad \text { or }, \quad \alpha=\log \left(\frac{1}{\hat{D} \lambda}\right)
$$

where $\lambda$ is fixed as $\hat{D} \rightarrow 0$. This means that $\alpha$ is large, but not very large. The parameter $\lambda$ is necessary for a satisfactory asymptotic development.

In the sequel we will treat $\alpha$ as $\mathcal{O}\left(D^{0}\right)$, except when it appears in exponentials, where it is $\mathcal{O}\left(D^{1}\right)$. This condition means that the population which is swept away from the oasis will decay to $\mathcal{O}(D)$ on its passage through the desert before revisiting the oasis.

In the limit $D \rightarrow 0$, with $\beta_{1}$ and $\lambda$ fixed, all details of the solution can be expressed in terms of $f(t-x)$ and simple functions of $x$. The form of the solution is indicated in figure (): there is a boundary layer of thickness $D$ immediately to the left of $x=1$.

Rewriting the zeroth order concentration $c_{0}$ in terms of $\lambda$ and expanding in terms of the small parameter $D$, we see why the outer approximation does not satisfy the periodicity requirement. At $x=1, c_{0}(x=1, t)$ is:

$$
c_{0}(x=1, t)=\frac{D \lambda f(t-1)}{1+f(t-1)-D \lambda f(t-1)} .
$$

Expanding gives,

$$
c_{0}(x=1, t) \approx \frac{D \lambda f(t-1)}{1+f(t-1)}-\frac{D^{2} \lambda^{2} f(t-1)^{2}}{1+f(t-1)}+\ldots \neq f(t) .
$$

Near $x=1$, this outer solution of the concentration of bacteria, $c_{0}$ has decayed to $O(D)$ and thus is inappropriate to describe this region of the ring. The role of the boundary layer at $x=1$ is to repair this failure, and so to determine the evolution of $f(t)$. This insight is the most difficult part of this asympototic expansion.

\subsubsection{The boundary layer}

We now turn to the "inner region", and introduce the stretched coordinate, $\xi=x / D$. In terms of $\xi$, equation (58) becomes

$$
D c_{t}+c_{\xi}=c_{\xi \xi}+D[\beta \delta(D \xi)-\alpha] c-D \alpha c^{2} .
$$

Making an expansion in $D$ of the concentration: $c(\xi, t) \approx c_{0}+D c_{1}$, we arrive at an equation to solve for the zeroth order, boundary layer concentration, $c_{0}(\xi, t)$ :

$$
c_{0 \xi \xi}-c_{0 \xi}=0
$$

The solution is

$$
c_{0}=f e^{\xi} .
$$

This solution satisfies the requirement that at $\xi=0$ we have $c(0, t)=f(t)$, implying that all higher order terms are zero at the origin. 
The first order boundary equation is:

$$
c_{1 \xi}-c_{1 \xi \xi}=-\alpha c_{0}-\alpha^{2} c_{0}^{2}-c_{0 t}
$$

Solving this inhomogeneous, partial differential equation with the help of the solution $c_{0}(\xi, t)$ in (70), gives the expression for the first order boundary layer $c_{1}(\xi, t)$ :

$$
c_{1}(\xi, t)=\left(f_{t}+\alpha f\right) \xi e^{\xi}+r(t)\left(1-e^{\xi}\right)+\frac{1}{2}\left(e^{2 \xi}-e^{\xi}\right) \alpha f^{2}
$$

where $r(t)$ is the constant of integration.

The constant $r(t)$ is found by matching the outer concentration with the boundary layer in the limit that $\xi \rightarrow-\infty$, which is equivalent to taking the limit where the boundary layer dissapears, $D \rightarrow 0$. The zeroth order boundary solution $c_{0}$ is zero, and all the terms but $r(t)$ are zero in the first order boundary layer in this limit. For the matching, the outer solution is evaluated at $x=1$, which is appropriate in this limit, since there is no boundary current for an infinite domain. This is an example of a "switchback" - the $\mathcal{O}\left(D^{1}\right)$ inner solution matches the leading order outer solution.

The resultant expression for $r(t)$ is:

$$
\begin{aligned}
c_{0}(\xi & =-\infty, t)+D c_{1}(\xi=-\infty, t)=c_{0}(x=1, t) \\
& \Rightarrow \\
r(t) & =\frac{\lambda f(t-1)}{1+f(t-1)}
\end{aligned}
$$

The first order expression for the boundary layer concentration is then:

$$
c_{1}(\xi, t)=\left(f_{t}+\alpha f\right) \xi e^{\xi}+\frac{\lambda f(t-1)}{1+f(t-1)}\left(1-e^{\xi}\right)+\frac{1}{2} \alpha f^{2}\left(e^{2 \xi}-e^{\xi}\right) .
$$

Thus $r(t)$ represents a time-delay of $t-1$. The origin of this term is interesting to note, as it will be the origin of the rest of our discussion.

As usual, an element of information has been neglected by excluding evaluation of the modified-Fisher equation at the origin. The patching condition contains this information, and can now be evaluated since we have expressions for the outer and inner solutions. This condition, obtained by integrating (68) about the orgin, is

$$
c_{\xi}(-)-c_{\xi}(+)=(1+D \beta) c(0),
$$

where the pluses and minuses indicate evaluation to the left and right of the orgin, and thus imply whether to use the outer or inner solution. For instance, $c_{\xi}(+)$ is actually the outer solution to the right of the origin, $D c(x=0, t)$. Expanding in $D$ gives

$$
c_{0 \xi}+D c_{1 \xi}-D c_{0 x}-D^{2} c_{1 x}=\left(1+D \beta_{1}\right)\left[c_{0}(x=0)+D c_{1}(x=0)\right]
$$

It is interesting to note that the zeroth order outer solution is related non-trivially to the first order boundary solution because of the role of the diffusion coeffcient $D$ as a small parameter. 
The zeroth order equation is trivially satisfied due to our choice of the constant in the solution (70). The non-trivial first order expression is:

$$
c_{x}(x=0, t)-c_{\xi}(\xi=0, t)+\left(1+\beta_{1}\right) c(x=0, t)=0 .
$$

Differentiating the appropriate expressions of the concentration and evaluating them at $x=0$ $(\xi=0)$ in (77) leads to a very interesting amplitude equation for $f(t)$,

$$
f_{t}=\left(\frac{\beta_{1}}{2}-\alpha\right) f-\frac{3}{4} \alpha f^{2}+\frac{\frac{1}{2} \lambda f(t-1)}{1+f(t-1)} .
$$

This amplitude equation is a differential-delay equation. The rate of change of $f$ at any time depends not only on the value of $f$ at that particular moment, but also on the particular value of $f$ at a specific earlier time, $t-1$. Not suprisingly, the time-delay is 1 time-unit, or the time required for transit around the ring. Comparison to the amplitude equation for the infinite ring reveals that we have the same Ginzburg-Landau type terms, while the timedelay piece is a result of the finite-size of the ring. We note that $\lambda$ is the only parameter that depends on the length of the ring $L$. As the ring becomes infinite in size, $\lambda \rightarrow 0$, so that the differential-delay equation reduces to the Ginzburg-Landau equation of the previous section with redimensionalization.

A good check is to verify that the first order energy obtained from (78) agrees with the analytic expression for the energy of the linear solution. The linearized version of (78) is:

$$
f_{t}=\left(\frac{\beta_{1}}{2}-\alpha\right) f+\left(\frac{e^{-\alpha}}{2 \sigma}\right) f(t-1)
$$

Consistent with linearization, we assume this is an eigenvalue problem and let $f=e^{s t}$. The expression for the first order energy is then:

$$
s=\frac{\beta_{1}}{2}-\alpha+\frac{e^{-\alpha-s}}{2 D} .
$$

Remembering that we have non-dimensionalized our results, we work the analytic result, (23, 24 ), into the same form. Non-dimensionalization leads to,

$$
\begin{array}{r}
2 D k\left(\cosh k-\cosh \frac{1}{2 D}\right)=(1+D \beta) \sinh k, \\
k \equiv \frac{1}{2 D} \sqrt{1+4 D(\alpha+s)} .
\end{array}
$$

To show the equivalence, we expand $k$ in $D$ and drop any terms which have $e^{-(1 /(2 \hat{D}))}$ since they are very small.

$$
k \approx \frac{1}{2 \hat{D}}+\alpha+s
$$

Using this in the transcendental relation for $\mathrm{k}(81)$, leaves us with the expression:

$$
-\frac{1}{2}+(\alpha D+s D)\left(e^{\alpha+s}-1\right)=D \frac{\beta_{1}}{2} e^{\alpha+s}
$$



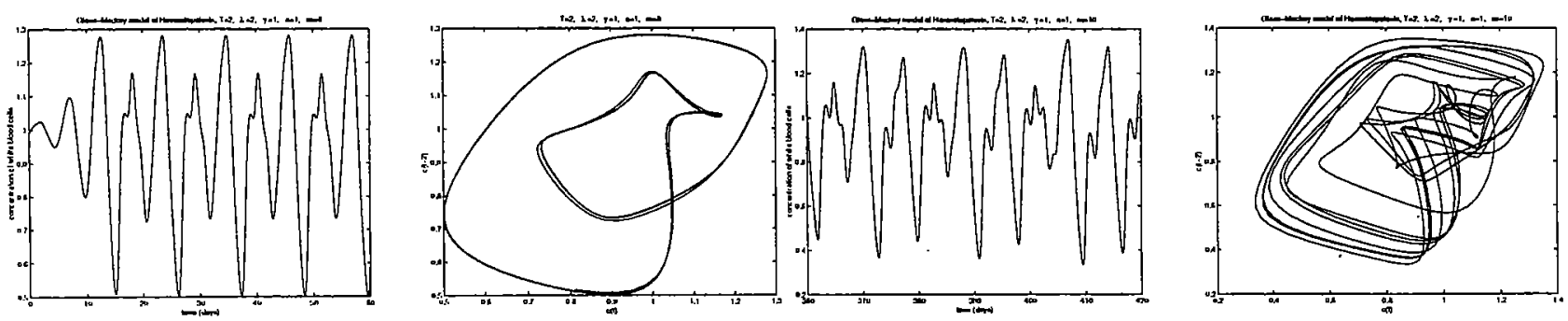

Figure 7: Periodic and chaotic behavior of the Glass-Mackey equation in various regimes (a) quasi-periodic state when $m=8$ (b) phase diagram, $m=8$ (c) chaotic state when $m=10$ (d) phase diagram, $m=10$

Again, we consider which terms are very small. Much smaller than any of the exponential terms, $s D$ is dropped, as is $\alpha D$, since $\alpha \sim \mathcal{O}(-\ln (D))$. Thus, we are left with an expression identical to the first order energy, if we rearrange the following

$$
-\frac{1}{2}+(\alpha+s) D e^{\alpha+s}=D \frac{\beta_{1}}{2} e^{\alpha+s}
$$

\subsection{Finite Ring on a Windy Day: Part II The Dynamics}

The next step of this analysis is obvious: we should study the stability of the steady state solutions of our amplitude equation. Before beginning this analysis, we set the tone of the rest of this project by suggesting the results that were expected.

\subsubsection{Interesting Zoology of Differential-Delay Equations}

Differential-delay equations are well know for their periodic, quasi-periodic, or chaotic behaviour, with examples often arising in biology. One such system is the model suggested by Glass and Mackey [6] to describe the regulation of white blood cells. The structure of the Glass-Mackey equation is similar to our differential delay equation,

$$
c_{t}=\frac{\lambda c(t-T)}{1+c^{m}(t-T)}-\gamma c
$$

and is nearly identical to ours, if $m=1$.

This differential-delay equation describes the change in time of the concentration of the white blood cells $c_{t}$. The rate at which cells die is proportional to $c$, e.g., $-\gamma c$. Meanwhile, the flux, $\lambda$ of new cells produced by bone marrow, is dependent on the concentration of the blood cells at some previous set time, $t-T$ due to a delay time $T$ in the production of white blood cells. This time delay exists because of the time costs of communciation and production. All the parameters, $\lambda, g, m, T$ are greater than zero. $m$ is a parameter determined experimentally, and if large enough, gives rise to limit cycles or chaos. See figure (7). 
In our system, we deal with our first exposure to a delay-equation and thus, a potentially chaotic system. The possiblility of chaotic dynamics of the concentration of bacteria seems like a fascinating result. We examine this possibility below.

\subsubsection{What about us? A stability analysis}

Thus, one might begin the stability analysis of the bacteria-ring system. Perhaps the three free parameters of our system, $\lambda, \beta$, and $\alpha$, can have both reasonable physical values and interesting dynamics. Simplifying the differential-delay equation (78) with the definitions

$$
\bar{\beta}=\frac{\beta_{1}}{2}-\alpha, \quad \bar{\alpha}=\frac{3}{4} \alpha, \quad \bar{\lambda}=\frac{1}{2} \lambda,
$$

the equation becomes

$$
f_{t}=\bar{\beta} f-\bar{\alpha} f^{2}+\frac{\bar{\lambda} f(t-1)}{1+f(t-1)} .
$$

The steady-states of (88) are found by letting $f_{t}=0$ where $f(t-1) \equiv f(t)$. This leads to a cubic equation for the roots, one of which is zero, and the other two roots are obtained from

$$
\bar{\alpha} f^{2}+(\bar{\alpha}-\bar{\beta}) f-(\bar{\lambda}+\bar{\beta})=0
$$

The roots are

$$
f_{ \pm}=\frac{(\bar{\beta}-\bar{\alpha}) \pm \sqrt{(\bar{\alpha}+\bar{\beta})^{2}+4 \bar{\alpha} \bar{\lambda}}}{2 \bar{\alpha}}
$$

Discarding the non-physical negative amplitude, we are left with two steady-states which the system may tend towards, $f=0$ and $f_{0}$. A study of the stability is necessary to understand the dynamics. Expanding around the steady-state solutions, we use $f=f_{0}+\epsilon f_{1}$. The delay term in (88) must also be expanded in terms of $\epsilon$. We find that the zeroth order equation for $f_{0}$ is just the cubic equation obtained earlier. The first order differential delay-equation defining $f_{1}$ is:

$$
f_{1 t}=\bar{\beta} f_{1}-2 \bar{\alpha} f_{0} f_{1}+\frac{\bar{\lambda} f_{1}(t-1)}{1+f_{0}}-\frac{f_{0} f_{1}(t-1)}{\left(1+f_{0}\right)^{2}} .
$$

It is useful to define a function $N$,

$$
\begin{gathered}
N=\frac{1}{1+f_{0}}, \\
N^{\prime}=-\frac{1}{\left(1+f_{0}\right)^{2}},
\end{gathered}
$$

to rewrite (91), where $N^{\prime}=d N / d f_{0}$. Since this is a linear stability analysis, $f=e^{s} t$, which gives

$$
s=\bar{\beta}-2 \bar{\alpha} f_{0}+\bar{\lambda} e^{-s}\left(N+f_{0} N^{\prime}\right)
$$



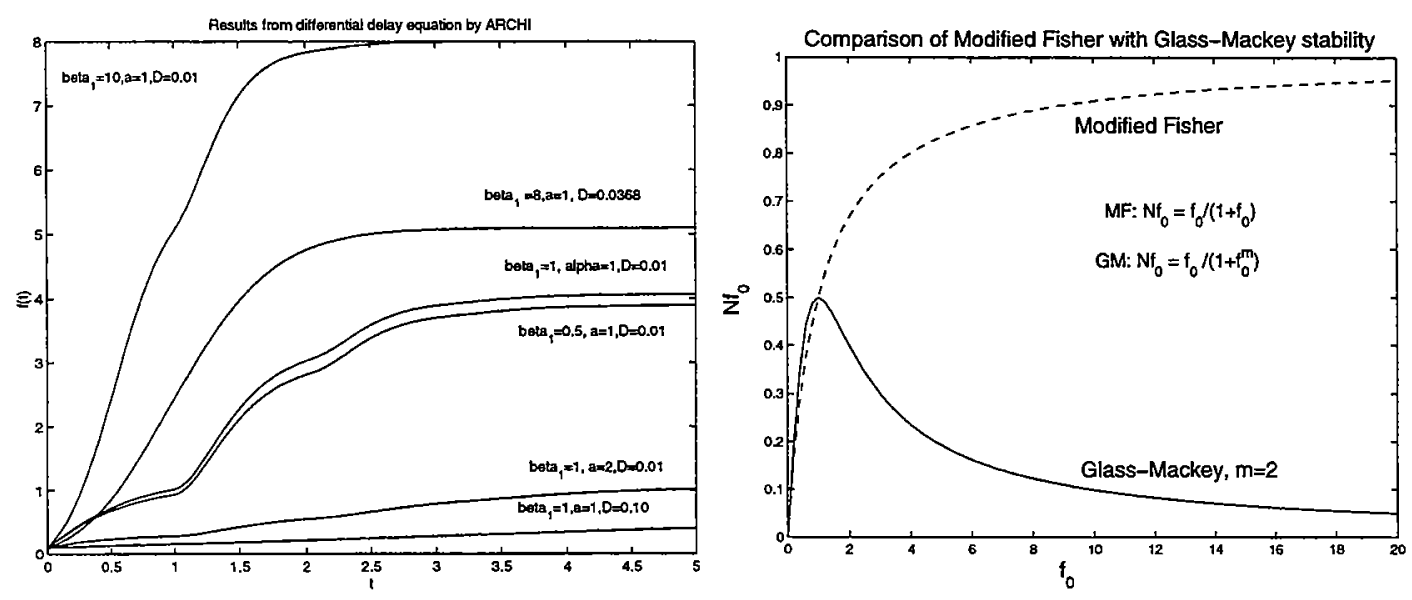

Figure 8: (a) Various steady-state solutions found numerically using ARCHI. Parameters are given with each curve. (b) Plot of $N f_{0}$ vs $f_{0}$ of the modified-Fisher equation and the GlassMackey equation. The leveling off of the modified-Fisher equation prevents any instability. The negative slope in the Glass-Mackey equation is what gives it the ability to be unstable. The higher the value of the Hill coeffient, $m$, the steeper the slope.

To study the growth rate $s$, let $s=\mu+i \omega$. (94) becomes,

$$
\mu+i \omega-\bar{\beta}+2 \bar{\alpha} f_{0}=\bar{\lambda} e^{-\mu-i \omega}\left(N+f_{0} N^{\prime}\right)
$$

Direct instabilities occur when $\mu$, the real part of $\mathbf{s}$, is greater than zero. For simplicity, let $\omega=0$ and examine if it is possible for $\mu>0$. We can show that $\bar{\beta}=\bar{\alpha} f_{0}-\bar{\lambda} N$, using the zeroth order equation, (89), so that

$$
\bar{\beta}-2 \bar{\alpha} f_{0}=-\bar{\alpha} f_{0}-\bar{\lambda} N
$$

Using (96) we arrive at:

$$
\mu-\bar{\alpha} f_{0}+\bar{\lambda} N\left(1-e^{-\mu}\right)+\bar{\lambda} e^{-\mu} N^{\prime}
$$

Assuming $u>0$, we find the expression on the right hand side to be negative for all parameters. This disproves our assumption. There can be no direct instability.

Hopf instabilities are the second possible type of linear instability. Breaking (95) up into its real and imaginary parts, squaring and combining them gives

$$
\left(\mu-\bar{\beta}+2 \bar{\alpha} f_{0}\right)^{2}+\omega^{2}=\bar{\lambda}^{2} e^{-2 \mu}\left(N+f_{0} N^{\prime}\right)^{2}
$$

For simplicity, assume $\mu=0$, and subtract $\omega^{2}$ from each side. Using (96), we have the inequality

$$
\left(\frac{\bar{\alpha} f_{0}}{\bar{\lambda}}+N\right)^{2}<\left(N+f_{0} N^{\prime}\right)^{2}
$$


If (99) is true, an instability exists. Further simplification leads to

$$
\begin{aligned}
\left|f_{0} N^{\prime}\right| & >2 N+\frac{\bar{\alpha} f_{0}}{\bar{\lambda}} \\
& \Rightarrow \bar{\alpha} f_{0}\left(1+f_{0}\right)^{2}>0 .
\end{aligned}
$$

This condition is obviously impossible since $f_{0}, \bar{\alpha}, \bar{\lambda}$ are all positive. Thus, there are no Hopf instabilities. This system is stable - an acceptable but dissapointing result for situation represented by an equation filled with such apparent possiblity. We check these results with a numerical results, exploring some of the 3-dimensional parameter space. The two agree, as shown in figure (8(a)), which plots several numerical results. One may note that the bumpiness in the amplitude corresponds with the time delay, so that at each time unit, the slope increases until the steady-state value is reached.

\subsubsection{Key to Instability: A comparison}

In hindsight, it is easy to predict that our system will be stable for all parameter space, despite the freedom of three independent parameters, $\lambda, \beta$, and $\alpha$. The hint is contained in the Glass-Mackey equation (86). Rewriting it in terms of a similar $N$, the $N_{G M}$ of the Glass-Mackey equation is,

$$
\begin{gathered}
N_{G M}\left(f_{0}\right)=\frac{1}{1+f_{0}^{m}}, \\
N_{G M}^{\prime}\left(f_{0}\right)=\frac{-m f_{0}^{m-1}}{\left(1+f_{0}^{m}\right)^{2}} .
\end{gathered}
$$

A similar stability analysis shows that direct instabilities are always impossible, while Hopf instabilities may exist if

$$
\left|f_{0} N^{\prime}\right|>2 N \text {. }
$$

This inequality is very similar to our the bug-ring system's inequality for Hopf bifurcations. The difference is due to the non-linear term in our amplitude equation (78). One might think that the non-linear term is what is preventing instability - that it is damping out oscillations - but comparison of these two inequalities reveal that the non-linear term only makes what is already impossible, more impossible. The stability is a result of the structure of $N$ and $N_{G M}$. Plot (8)(b) shows $N f_{0}$ versus $f_{0}$, where the slope is $X_{i}=N+f_{0} N^{\prime}$. For our system, the slope is always positive so that

$$
X_{1}=N+f_{0} N^{\prime}>0 .
$$

For Glass-Mackey,

$$
X_{2}=N_{G M}+f_{0} N_{G M}^{\prime}<0 .
$$

We see from (101) that for a Hopf instability to exist,

$$
0>X_{i}+N+\frac{\bar{\alpha} f_{0}}{\bar{\lambda}} .
$$

This is impossible unless the slope, $X_{i}<0$, since the other two terms are positive. And so we see that the structure of $N$ is crucial to the stability of these types of systems. 


\subsection{Other possiblilities for instability}

With the initial goals of this work accomplished, it is fun to continue along further lines of investigation. For instance, is it possible to modify the non-linear saturation term so that we may find a non-steady-state solution? Or what about increasing the spatial complexity? Or perhaps adding another delta function to the ring or many more, will lead to chaotic dynamics. What if the strength of the delta function(s) varies with time? The first two ideas are explored in the rest of this paper.

\subsubsection{Adjusting the non-linearity}

We begin with the simplest adjustment. What happens if we increase the non-linearity in the modified-Fisher equation from $-b c^{2}$ to $-b c^{n}$ ? Perhaps this will do something. However, it was shown that if we change the power of $c$ to any value $n$, that the structure of $\mathrm{N}$ will be

$$
N=\frac{1}{\left(1+f_{0}^{n}\right)^{\frac{1}{n}}} .
$$

Direct instabilities remain impossible, while this $\mathrm{N}$ still does not yield the negative slope $N^{\prime}$ necessary for a Hopf instability.

In fact, it appears that we must tailor a function which has a similiar behavior to the Glass-Mackey type N, so that it levels off at some lower value than it's maximum. For our system, this seems physically unreasonable.

\subsection{Two delta functions and more... Does spatial complexity breed instability?}

The second physically motivated suggestion, is to increase the complexity of the oasis and desert zones to acheive interesting dynamics. If one additional delta function is added to the ring, located at position $a^{\prime}$, possesing a strength $\beta_{1}^{\prime}$, while the first delta function is located at $a$ with strength $\beta_{1}$, a similar analysis to section (4) leads to two coupled differential-delay equations:

$$
\begin{aligned}
& f_{t}=\left(\frac{\beta_{1}}{2}-\alpha\right) f-\frac{3}{4} \alpha f^{2}+\frac{\frac{1}{2} \lambda g(t-a)}{1+g(t-a)} \\
& g_{t}=\left(\frac{\beta_{1}^{\prime}}{2}-\alpha\right) g-\frac{3}{4} \alpha g^{2}+\frac{\frac{1}{2} \lambda^{\prime} f\left(t-a^{\prime}\right)}{1+f\left(t-a^{\prime}\right)}
\end{aligned}
$$

where $\lambda=e^{-\alpha a} / 2 D$ and $\lambda^{\prime}=e^{-\alpha a^{\prime}} / 2 D$ In general, for independent parameters, these equations yield 8 steady-state solutions $\left(f_{0}, g_{0}\right)$, if we count $f_{0}, g_{0}=0$. A study of the stability is quite complex. A few things, however can be said:

1. No direct instabilities exist, for any set parameters.

2. Equal parameters, $\beta_{1}=\beta_{1}^{\prime}, a=a^{\prime}$, results in the amplitudes $f_{0}$ and $g_{0}$ always being equal. $\left(f_{0}=g_{0}\right)$. This case reduces to the 1 -delta function case, so there is no interesting zoology here. 
3. When the parameters are not equal, $f_{0}$ never equals $g_{0}$. Stability has not been proven for this case, although numerical tests suggest that the system is stable.

The first two results are also true a system composed of $n$ delta functions. The coupled delay-equations are straightforward to derive:

$$
\begin{gathered}
f_{1 t}=\bar{\beta}_{1} f-\bar{\alpha} f^{2}{ }_{1}+\frac{\bar{\lambda}_{n} f_{n}}{1+f_{n}}, \\
f_{2 t}=\bar{\beta}_{2} f-\bar{\alpha} f^{2}{ }_{2}+\frac{\bar{\lambda}_{1} f_{1}}{1+f_{1}}, \\
\cdots \\
f_{n t}=\bar{\beta}_{n} f-\bar{\alpha} f^{2}{ }_{n}+\frac{\bar{\lambda}_{n-1} f_{n-1}}{1+f_{n-1}} .
\end{gathered}
$$

It would be nice to develop a technique to study the stability of all the steady-state solutions for 2-delta functions (and then n-delta's) which is more straightforward than the usual algebraically complex method. Perhaps this will be accomplished as our familiarity with delay-equations grow, just as the simple discovery of $N$ made the analysis of the simplest case swifter and less convoluted.

\section{Conclusion}

In conclusion, the modified-Fisher equation and the delocalization transition has been studied in detail for a large ring and in the distinguished limit that $\lambda$ is $\mathcal{O}(1)$. While the differentialdelay equation was an unexpected result, it is an interesting property of the system which deserves more study in complex inhomogenous backgrounds. It is also suggested that a timedependent delta function could model oscillations of illumination due to cloud cover, or the day/night cycle, as well as lead to an interesting problem with a new delay-equation. Other work may also be done in different parameter regimes, especially in the small diameter limit.

\section{Acknowledgements}

None of the work in this paper could have been done without the guidance of Bill Young. He is a great inspiration and a pleasure to work with. Also many thanks go out to Stefan G.L. Smith and Neil J. Balmforth for their contributions and entertaining discussions regarding this work. Thanks to George Veronis for his coaching and friendship throughout the summer. Finally thanks to Claudia, Francesco, Jean Luc and this year's fellows. Your friendship is treasured, as is the memory of our 'filth and squalor' at Maury Lane!

\section{References}

[1] N.M. Shnerb D. R. Nelson. Non-hermitian delocalization and eigenfunctions. Phys. Rev. $B, 58(13): 8384-8390$, October 1998. 
[2] N.M. Shnerb D.R. Nelson. Non-hermitian localization and population biology. Phys. Rev. $E, 58(2): 1383-1403$, August 1998.

[3] K.B. Efetov. Phys. Rev. Lett., 79:491, 1997.

[4] N.M. Shnerb K.A. Dahmen, D.R. Nelson. Life and death near a windy oasis. unplublished, 1998.

[5] P.A. Lee and T.V. Ramakrishnan. Rev. Mod. Phys., 57:287, 1985.

[6] J.D. Murray. Mathematical Biology. Springer-Verlag, New York, 1993.

[7] D.R. Nelson N. Hatano. Vortex pinning and non-hermitian quantum mechanics. Phys. Rev. $B, 56(14): 8651-8673$, October 1997. 


\title{
Coupled Nonlinear Oscillators
}

\author{
Roberto Sassi
}

\section{Introduction}

Mutual synchronization is a common phenomenon in biology. It occurs at different levels, ranging from the small scale of the cardiac pace-maker cells of the SA (Sino-Atrial) and AV (Atrium-Ventricular) nodes in the human hearth that synchronously fire and give the pace to the whole muscle, to the coordinated behaviours of crickets that chirp in unison and of freflies that flash together in some parts of southeast Asia.

The dynamics of coupled oscillators is a very broad field of research; the approach we have chosen is only one of the many that are possible. The question we would like to answer is something like: "What special phenomena can we expect to arise from the rhythmical interaction of whole populations of periodic processes?" [1].

Winfree [1] was the first to underline the generality of the problem, fixing the first assumptions for a mathematical model. In his work each oscillating species (cell, or cricket, or firefly) is modeled as a nonlinear oscillator with a globally attracting limit cycle; The oscillators were assumed to be weakly coupled and their natural frequencies to be randomly distributed across the population.

Kuramoto [2] proposed the first model (called for this reason the Kuramoto model). His assumptions were that each oscillator is equal to the others, upto the frequency and phase, that the system has a mean field coupling and that the amplitudes of the oscillations are all the same (phase-only model). The equation of the model for the $n$ oscillator is:

$$
\frac{d \theta_{n}}{d t}=\omega_{n}+\frac{K}{N} \sum_{j=1}^{N} \sin \left(\theta_{j}-\theta_{n}\right)+\xi_{n},
$$

where $K$ is the coupling strength, $\omega_{n}$ is a random variable with probability density function $g(\omega)$ and $\xi_{n}$ is white noise.

Defining as order parameter the complex number,

$$
r e^{i \psi}=\frac{\sum_{j=1}^{N} e^{i \theta_{j}}}{N}
$$

it's possible to measure the synchronization among the oscillators phases: $r=0$ corresponds to the completely incoherent state, finite $r$ to synchronization.

Kuramoto determined that $r=0$ is always a steady solution; but there exists, in the case of no added random noise, a critical value of the coupling parameter $K_{c}=\frac{2}{\pi g(0)}$ below which only incoherent populations exist $(r=0)$. For $K>K_{c}$ a population of synchronized oscillators can exist $(r>0)$. 

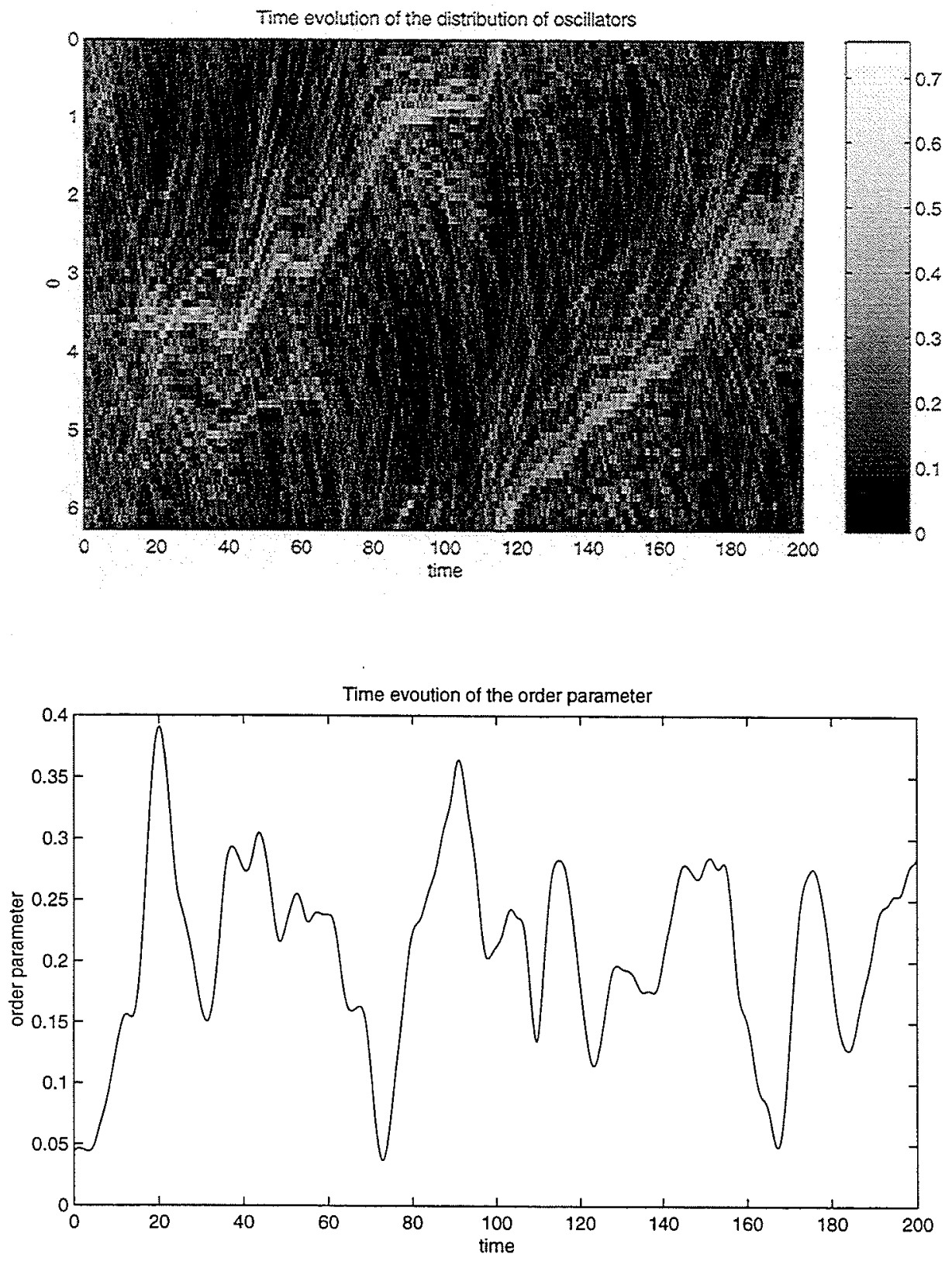

Figure 1: Numerical simulations on the discrete Kuramoto model with $N=256, D=0.01, K=0.65$; (upper) time evolution of the probability density function computed on the trajectories of the system splitting up the $\theta$ axe in sub-intervals; (lower) time evolution of the absolute value of the order parameter 

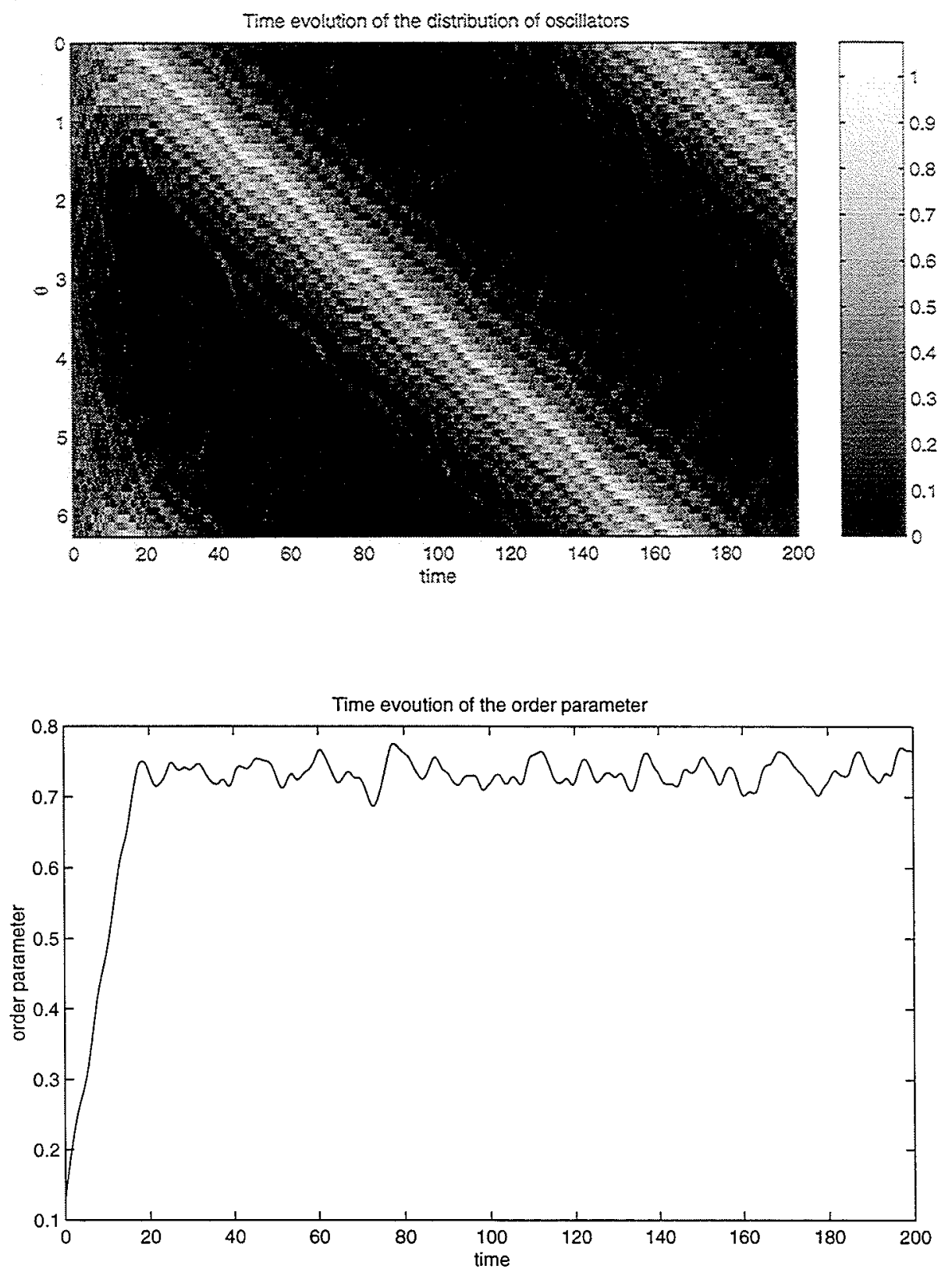

Figure 2: Numerical simulations on the discrete Kuramoto model with $N=256, D=0.01, K=0.8$; (upper) time evolution of the probability density function computed on the trajectories of the system splitting up the $\theta$ axe in sub-intervals; (lower) time evolution of the absolute value of the order parameter 
The results of the numerical simulations, performed solving equation (1) with $N=256$ and two different values of the coupling parameter $K$, are shown in figures (1) and (2); in the upper panels the time evolution of the discrete probability density function ${ }^{1}$ is plotted; in the lower panels, the time evolution of the order parameter is displayed. The initial condition is, in both cases, a population of oscillators with phases uniformly distributed in $[0,2 \pi]$.

When $K=0.8>K_{c}$, in a very short time, the phases of the oscillators gather together in a small range of angles and then begin drifting coherently. The order parameter grows quickly and exhibits small oscillations due to the random noise added to the system (figure (2)).

A different situation arises with $K=0.65<K_{c}$; a coherent behaviour never starts, even if small structures can be noticed: small population of oscillators synchronize and drift for short periods of time. This is reflected in the order parameter that oscillates between 0 and 0.3 and decreases only slowly (figure (1)).

\subsection{A continuous model}

Using the approach sketched in the previous paragraph, it's difficult to go much farther; it's not easy, for example, to answer questions such as "Is the coherent state ( $K>K_{c}$ ) stable?"

Strogatz \& Mirollo [3] introduced a partial differential equation that describes the behaviour of the Kuramoto model in the limit $N \rightarrow \infty$.

The idea is that, in the continuous limit, the state is described by a probability density function: $\rho(\theta, \omega, t)$. The Kuramoto equation (1) becomes:

$$
v=\omega+K \int_{-\infty}^{\infty} \int_{0}^{2 \pi} \sin (\phi-\theta) \rho(\phi, \omega, t) g(\omega) d \phi d \omega
$$

where $v$ is the velocity at the point $(\theta, \omega, t)$. Moreover, the density function $\rho$ has to satisfy, for each given $\omega$, a normalization law

$$
\int_{0}^{2 \pi} \rho d \theta=1
$$

and a Fokker-Plank-type conservation law ${ }^{2}$

$$
\frac{\partial}{\partial t} \rho(\theta, \omega, t)+\frac{\partial}{\partial \theta}(\rho(\theta, \omega, t) v(\theta, \omega t))=D \frac{\partial^{2}}{\partial \theta^{2}} \rho(\theta, \omega, t)
$$

\footnotetext{
${ }^{1}$ The $\theta$ axis is divided into 64 intervals and, at each instant of time, the normalized histogram of the phases of the oscillators is computed.

${ }^{2}$ The derivation of the two equations has the flavor of the BBGKY hierarchy in plasma physics and can be found in [4]. Some rationalization of equation (5) can be given on recollecting that because the probability is conserved,

$$
\begin{aligned}
\frac{\partial}{\partial t} \int_{\theta_{1}}^{\theta_{2}} \rho(\theta) d \theta & =\rho\left(\theta_{1}\right) v\left(\theta_{1}\right)-\rho\left(\theta_{2}\right) v\left(\theta_{2}\right) \\
& =-\int_{\theta_{1}}^{\theta_{2}} \frac{\partial}{\partial \theta}(\rho v) d \theta \\
\rho_{t} & =-\frac{\partial}{\partial \theta}(\rho v),
\end{aligned}
$$

and on remembering Einstein's derivation of the diffusion equation in his work on the explanation of the
} 
where $\theta \in[0 ; 2 \pi]$ and $\omega \in[-\infty ; \infty]$.

The order parameter (2), in the continuous limit, becomes:

$$
r e^{i \psi}=\int_{-\infty}^{\infty} \int_{0}^{2 \pi} e^{i \theta} \rho(\theta, \omega, t) g(\omega) d \theta d \omega
$$

\section{Linear Stability Theory}

Strogatz \& Mirollo [3] worked on the linear stability of the continuous Kuramoto equation. We will try to sketch the main results, useful for the discussion that follows.

With direct substitution into the equations (3) and (5), it can be seen that $\rho_{0}=\frac{1}{2 \pi}$ is a steady state solution for the system; it corresponds to the incoherent state with $r=0$.

By linearizing $\rho$ around the steady state solution, that is

$$
\rho=\rho_{0}+\epsilon\left(c(\omega, t) e^{i \theta}+c^{*}(\omega, t) e^{-i \theta}\right)+h . h .
$$

where $\epsilon$ is a small parameter and $c^{*}$ is the complex conjugate of $c$, then substituting into (3) and introducing the notation

$$
G(\omega, t)=\int_{-\infty}^{\infty} \int_{0}^{2 \pi} \sin (\phi-\theta) \rho(\phi, \omega, t) g(\omega) d \phi d \omega,
$$

it can be seen that $G$ is different from zero only for functions that have a component on the bases $e^{i \theta}$ and $e^{-i \theta}$. That is, the higher harmonics do not give any contribution and the linearized equation (5) becomes

$$
c_{t}=-(D+i \omega) c+\frac{K}{2} \int_{-\infty}^{\infty} c(\nu, t) g(\nu) d \nu
$$

The discrete spectrum can be computed by seeking solutions of the form $c(\omega, t)=b(\omega) e^{\lambda t}$. By substituting into equation (7), multiplying by $g(\omega)$ and integrating over $\omega$, one finds the dispersion relation,

$$
1=\frac{K}{2} \int_{-\infty}^{\infty} \frac{g(\nu)}{\lambda+D+i \nu} d \nu
$$

When $\lambda$ is negative, the order parameter decays and the system reverts to the incoherent state; vice versa for lambda positive, the order parameter exponentially grows and this is, in the coupled oscillators system, the onset of synchronization.

The system has, also, a continuous spectrum at $\omega=-i D$ (see [3]). As the dissipation is always positive, the modes in the continuous spectrum are either all decaying or, at most, neutrally stable when $D=0$.

Brownian motion [5], which indicates that

$$
\begin{aligned}
\rho_{t} & =2\left\langle\xi^{2}\right\rangle \rho_{\theta \theta} \\
& =D \rho_{\theta \theta}
\end{aligned}
$$




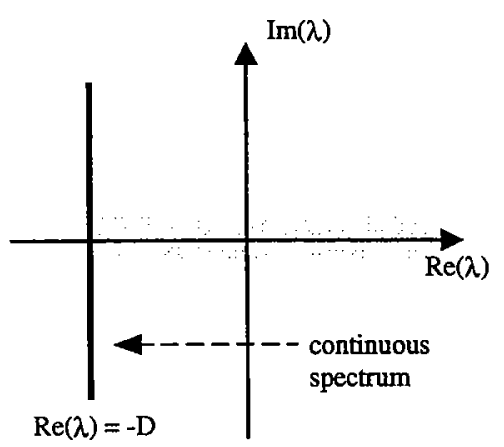

$(\mathrm{D} \neq 0)$

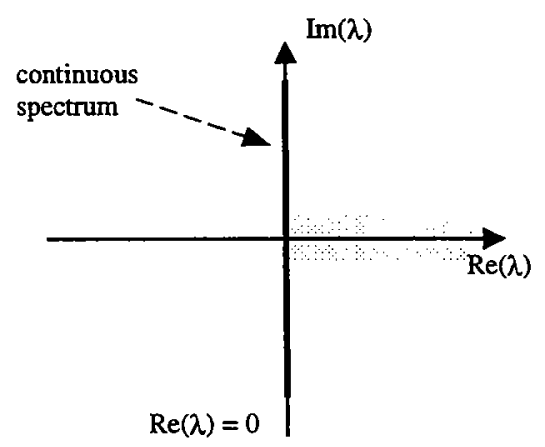

$(\mathrm{D}=0)$

Figure 3: Continuous spectrum for the dispersion relation (8) either for the case $D \neq 0$ (left) and for the noise-free case with $D=0$ (right). The discrete spectrum is composed of only one mode that exists for $K>K_{c}$ and lies to the right of the continuous spectrum (inside the grey region in the picture); in the noise-free case the discrete mode either is unstable or doesn't exist.

\subsection{An example}

Choosing as probability density function a lorentzian, that is

$$
g(\omega)=\frac{1}{\pi} \frac{1}{\omega^{2}+1}
$$

the dispersion relation can be solved analytically; the computed growing rate $\lambda$ is

$$
\operatorname{sgn}(\lambda+D) \lambda=\frac{K}{2}-1+D
$$

and the critical coupling (that is the value of $K$ at which the system is neutrally stable) is

$$
K_{c}=2(1+D) \text {. }
$$

If $D=0$, then $K_{c}=\frac{2}{\pi g(0)}$; this is the same result that Kuramoto found working on the discrete system, as described in the introduction.

Summarizing, for $K \leq 2$, the system does not have any discrete mode; for $2<K<K_{c}$ the system is stable and exponentially decaying; if $K=K_{c}$ it has a neutral mode and with $K>K_{c}$ an unstable growing mode (figure (3(left))).

It's interesting to notice that, if the dissipation is zero, the system can only be either unstable, with a growing mode $\left(K>K_{c}\right)$, or neutrally stable, with no mode $\left(K \leq K_{c}\right)$ (figure $(3($ right $)))$. But, looking at figure $(4($ right $))$, it can be seen that the order parameter is, however, decaying exponentially.

How can we explained this apparent contradiction? Let's look at the solution of the initial value problem with $K<K_{c}$ and the initial condition

$$
c(\omega, 0)=\frac{2}{\pi} \frac{1}{\omega^{2}+4}
$$



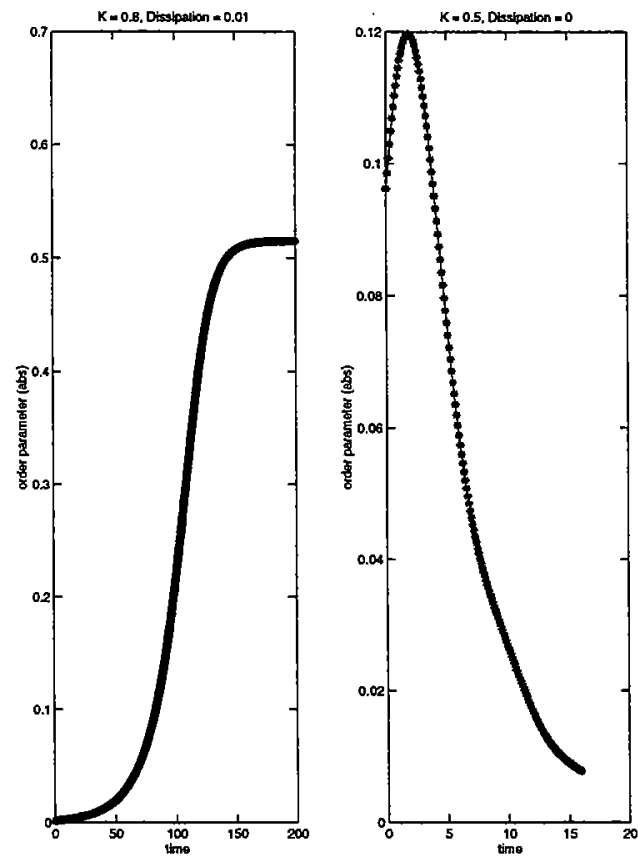

Figure 4: Time evolution of the order parameter for the Kuramoto continuous model; compact support within $[-1 ; 1]$ for $g(\omega)=\frac{1}{\pi-2} \frac{1-\omega^{2}}{1+\omega^{2}} ;$ initial condition $\tilde{\rho}_{0}=\frac{1}{2 \pi}$ and $\widetilde{\rho}_{1}=\xi \frac{2}{\pi} \frac{1}{4+\omega^{2}}$; (left) $D=0.01, K_{c}=0.739, \xi=0.001$ and $K=0.8$ : over-critical coupling, the order parameter grows linearly and then, when the nonlinearity becomes strong enough, saturates; (right) $D=0, K_{c}=2-\frac{4}{\pi}$ , $\xi=0.1$ and $K=0.5$ : under-critical coupling, the order parameter grows, initially, and then decays. 

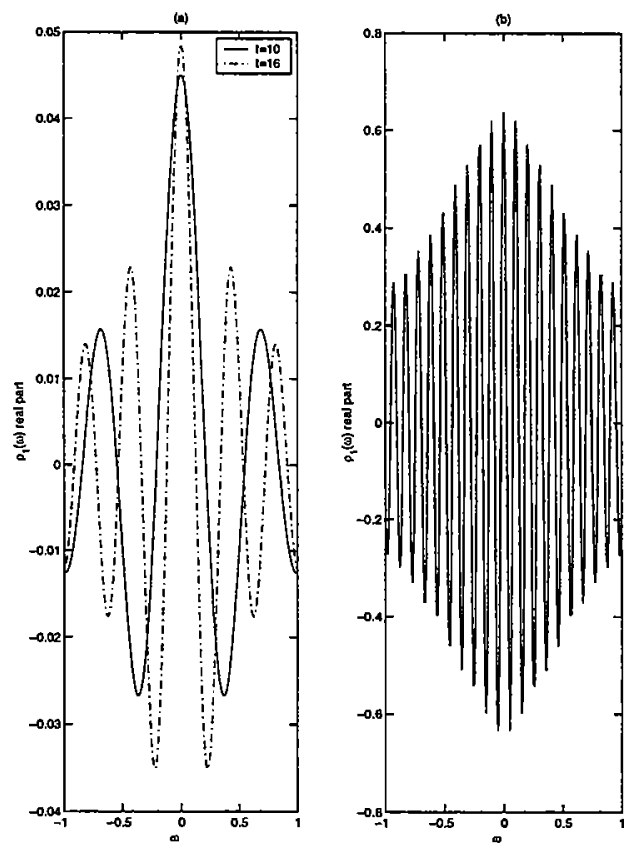

Figure 5: (a): real part of the first Fourier component of $\rho(\theta, \omega, t)$ at two different times, obtained with the numerical simulation described in figure (4(right)). (b): real part of the first Fourier component of $\rho(\theta, \omega, t)$ at time $t=60$ analytically computed. In both case is possible to observe the increasing number of oscillations.

Integrating equation (7) via Laplace's transform, we find that

$$
\begin{aligned}
c(\omega, t)= & \left\{\frac{2}{\pi} \frac{1}{\omega^{2}+4}-\frac{1}{3 \pi} \frac{5}{2 i \omega-1}-\frac{1}{3 \pi} \frac{1}{2-i \omega}\right\} e^{i \omega t} \\
& +\frac{5}{3 \pi} \frac{1}{2 i \omega-1} e^{-\left(\frac{K}{2}-1\right) t}+\frac{1}{3 \pi} \frac{1}{2-i \omega} e^{-2 t} .
\end{aligned}
$$

Evidently, the function $c(\omega, t)$ is proportional to the non-decaying and non-separable term $e^{i \omega t}$. As time goes on, this term becomes increasingly crenellated.

What we are seeing here is equivalent to the Landau damping in plasma physics; the order parameter is proportional to the integral of the function $c(\omega, t)$; even if the latter doesn't decay, as soon as it starts crenellating, the positive and negative part cancel and the integral decreases.

In fact, computing the absolute value of the order parameter, we have

$$
r=\frac{10}{3} e^{-\left(\frac{K}{2}-1\right) t}-\frac{4}{3} e^{-2 t}
$$

which decays exponentially as $t \rightarrow \infty$.

\section{Numerical Integration}

The integration of the discrete model (equation (1)) has been performed with a fixed step $(\Delta t=0.1)$, fully-implicit predictor-corrector scheme. The fixed time step is forced by the 
noise added to the derivatives that avoids the convergence of most adaptative methods.

In the continuous model (equations (5) and (3)) the density function $\rho$ is periodic with period $2 \pi$, so the latter can be expanded in the Fourier series:

$$
\rho(\theta, \omega, t)=\sum_{m=-\infty}^{\infty} \tilde{\rho}(\theta, t) e^{i m \theta}
$$

Substituting into the equations (5) and (3), we obtain the system of nonlinear ordinary differential equations for the Fourier coefficients $\widetilde{\rho}_{m}$ :

$$
\begin{aligned}
\tilde{\rho}_{0 t}= & 0, \\
\tilde{\rho}_{m t}= & -K \pi m\left\langle\widetilde{\rho}_{-1}\right) \widetilde{\rho}_{m+1}-\left(i m \omega+D m^{2}\right) \widetilde{\rho}_{m}+K \pi m\left\langle\widetilde{\rho}_{1}\right\rangle \widetilde{\rho}_{m-1} \\
& \text { for } m \neq 0
\end{aligned}
$$

where

$$
\langle f(\omega)\rangle=\int_{-\infty}^{\infty} f(\omega) g(\omega) d \omega
$$

For each value of $\omega$, truncating the Fourier series at $m=L$, the system can be efficiently integrated (a semi-implicit Adams-Bashfort-Moulton predictor-corrector scheme leads to the inversion of a tri-diagonal matrix). We used $L=16,32,64,128$ in the computations; the smaller the dissipation or the longer time windows considered, the bigger the number of Fourier components necessary to approximate properly the Kuramoto system. We found $L=32$ a good compromise in many situations.

A little more attention is needed for the evaluation of the integral (9). We found that the most efficient way of computing it is via Gauss-Legendre quadrature formulae, setting compact support for $g(\omega)$, and using the solution for $\rho$, produced at the previous available time step.

In figures (6) and (7) the solutions computed for $\rho(\theta, \omega, t)$ are shown; in the sub-critical case, when the coupling parameter $K$ is smaller than $K_{c}$, stripes of probability can be noticed, which increase in number and, slightly tilting, start shrinking. At fixed $\theta$, this is the same crenellation as described above and seen in figure (5).

When $K>K_{c}$, in the super-critical case, the probability gathers, initially, in a stripelike area, but immediately also starts to deplete from the central region. Unlike before, the number of stripes doesn't increase (in this case there's no Landau damping); two areas collect the whole probability. The process is reminiscent of the formation of a shock layer in the white regions in figure (7), but a truely weak solution does not form due to the dissipation introduced by the noise.

\section{A symmetry property}

Looking at the numerical results of the previous section, it can be observed that, starting from $\rho(\theta, \omega, 0)$ and $g(\omega)$ which are even functions in $\omega$, a symmetry is preserved during the evolution of the dynamics; that is $\rho(\theta, \omega, t)=\rho(-\theta,-\omega, t)$. This behaviour can be explained in a general way. 

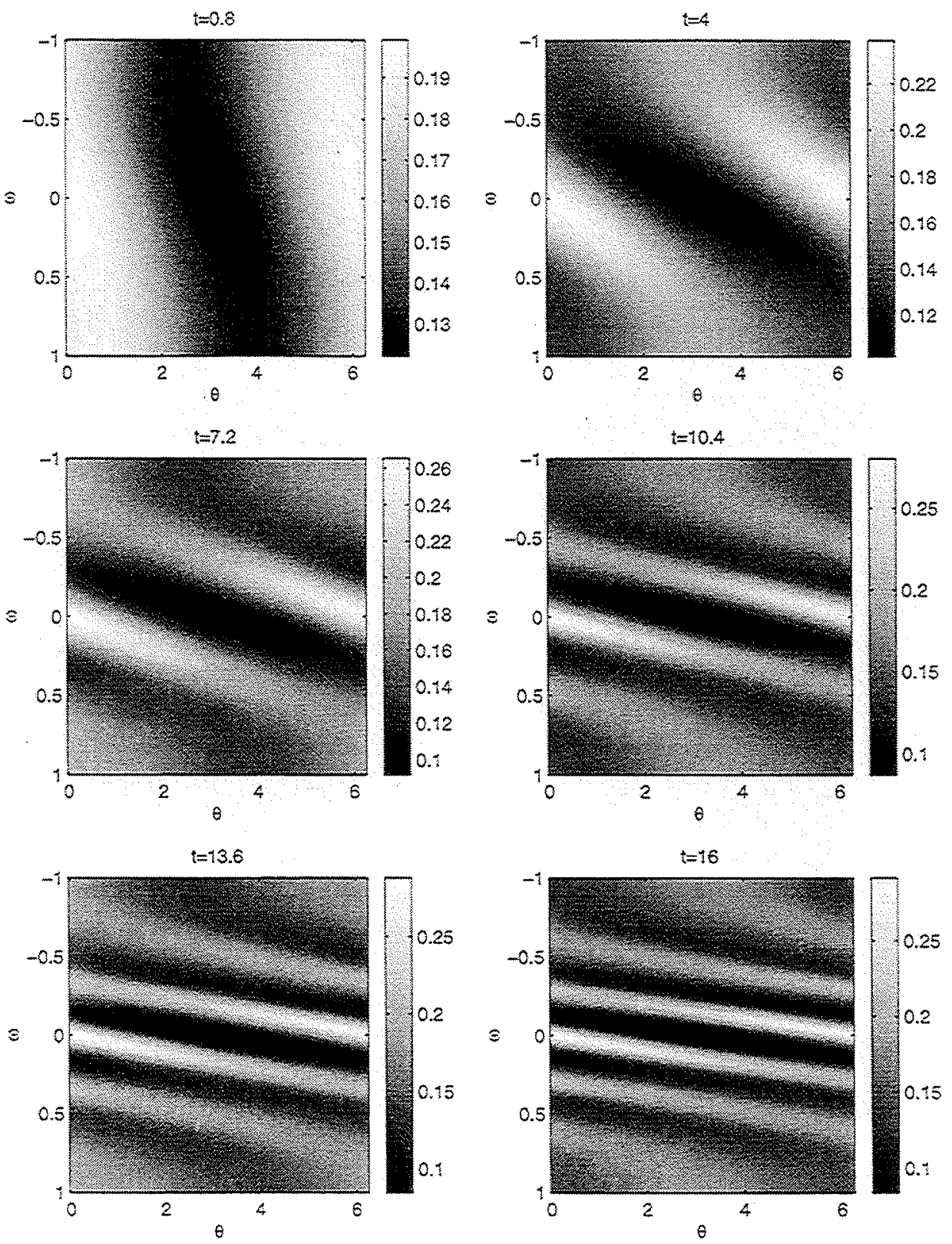

Figure 6: $\rho(\theta, \omega, t)$ at six successive instant of time obtained via numerical integration of the Kuramoto continuous model with $D=0$ and $K=0.5$ (see figure (4)(right) for further details). The coupling $K$ is sub-critical. 

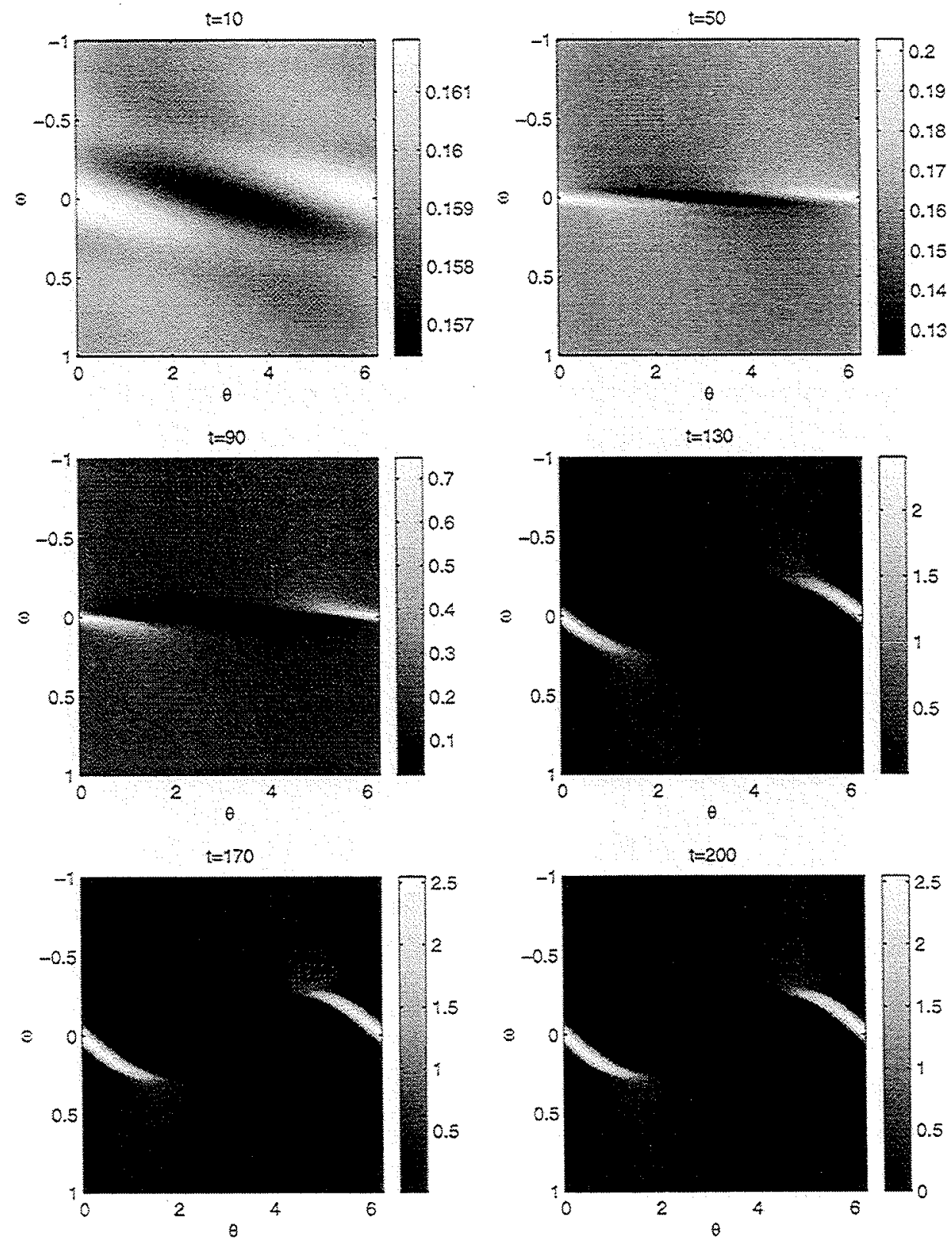

Figure 7: $\rho(\theta, \omega, t)$ at six successive instant of time obtained via numerical integration of the Kuramoto continuous model with $D=0.01$ and $K=0.8$ (see figure (4)(right) for further details). The coupling $K$ is super-critical. 
Let's start stating that the probability density function is so that, for each integer $n$,

$$
\rho(\theta, \omega, t)=\rho(\theta+2 \pi n, \omega, t)=\rho(2 \pi n-\theta,-\omega, t)=\rho(-\theta,-\omega, t) .
$$

The symmetry property will be proved by construction.

Let's expand $\sin (\phi-\theta)$ in equation (3); the first integral can be rewritten

$$
\begin{aligned}
\int_{-\infty}^{\infty} & \int_{-\pi}^{\pi} \sin (\phi) \rho(\phi, \omega, t) g(\omega) d \phi d \omega \\
= & \int_{-\infty}^{\infty} \int_{-\pi}^{0} \sin (\phi) \rho(\phi, \omega, t) g(\omega) d \phi d \omega+ \\
& \int_{-\infty}^{\infty} \int_{0}^{\pi} \sin (\phi) \rho(\phi, \omega, t) g(\omega) d \phi d \omega
\end{aligned}
$$

If we make the change of variables $\phi^{\prime}=-\phi$ and $\omega^{\prime}=-\omega$ and we use equation (10), expression (11) becomes

$$
\begin{aligned}
(11)= & -\int_{-\infty}^{\infty} \int_{0}^{\pi} \sin \left(\phi^{\prime}\right) \rho\left(\phi^{\prime}, \omega^{\prime}, t\right) g\left(\omega^{\prime}\right) d \phi^{\prime} d \omega^{\prime}+ \\
& \int_{-\infty}^{\infty} \int_{0}^{\pi} \sin (\phi) \rho(\phi, \omega, t) g(\omega) d \phi d \omega \\
= & 0 .
\end{aligned}
$$

At the end,

$$
\begin{aligned}
G(\theta, t) & =-\sin (\theta) \int_{-\infty}^{\infty} \int_{-\pi}^{\pi} \cos (\phi) \rho(\phi, \omega, t) g(\omega) d \phi d \omega \\
& =-\mathcal{G}(t) \sin (\theta)
\end{aligned}
$$

Substituting (12) into equation (5), it can be noticed that $\rho(-\theta,-\omega, t)$ is a solution of the Kuramoto model.

\section{$5 \quad$ Weakly Nonlinear Theory}

When $K=K_{c}$, the system is, by definition, linearly neutrally stable. In this situation and for $D \neq 0$, Bonilla \& al. [6] and Crawford [7] developed an asymptotic expansion around the equilibrium solution and derived a Landau-type ordinary differential equation for the amplitude of the perturbation.

Defining $\epsilon \ll 1$, they chose the scalings,

$$
\begin{aligned}
\epsilon^{2} t & =T \\
\partial_{t} & =\partial_{T} \epsilon^{2}
\end{aligned}
$$

where $T$ is the slow time (when $t$ is very big, $T$ is small),

$$
K=K_{c}+\epsilon^{2} K_{2}+\cdots
$$


and expanded the function $\rho$ such that

$$
\begin{aligned}
\rho(\theta, \omega, T) & =\frac{1}{2 \pi}+ \\
& =\epsilon\left(\rho_{1}(\omega) a_{1}(T) e^{i \theta}+\rho_{1}^{*}(\omega) a_{1}^{*}(T) e^{-i \theta}\right)+ \\
& =\epsilon^{2}\left(\rho_{2}(\omega) a_{2}(T) e^{i \theta}+\rho_{2}^{*}(\omega) a_{2}^{*}(T) e^{-i \theta}\right)+ \\
& =\epsilon^{3}\left(\rho_{3}(\omega) a_{3}(T) e^{i \theta}+\rho_{3}^{*}(\omega) a_{3}^{*}(T) e^{-i \theta}\right)+ \\
& =o\left(\epsilon^{4}\right) .
\end{aligned}
$$

From equations (5) and (3) it follows that

$$
\epsilon^{2} \frac{\partial \rho}{\partial T}=-\omega \frac{\partial \rho}{\partial \theta}-\frac{\partial}{\partial \theta} \rho K \int_{-\infty}^{\infty} \int_{0}^{2 \pi} \sin (\phi-\theta) \rho g(\omega) d \phi d \omega+D \frac{\partial^{2} \rho}{\partial \theta^{2}}
$$

and

$$
G=\epsilon G_{1}+\epsilon^{2} G_{2} \ldots
$$

At the first order $(O(\epsilon))$ :

$$
\rho_{1}=\frac{K \int_{-\infty}^{\infty} \rho_{1}(\nu) g(\nu) d \nu}{2(\omega-i D)}
$$

Continuing to $O\left(\epsilon^{3}\right)$, eventually, one derives the amplitude equation,

$$
a_{1 T}=\frac{K_{2}}{2} a_{1}+\left(K_{c} \pi\right)^{2} \mathcal{I}\left|a_{1}\right|^{2} a_{1}
$$

where

$$
\mathcal{I}=\int_{-\infty}^{\infty} \frac{\rho_{1} g(\omega)}{\omega-2 i D} d \omega=\text { constant }
$$

We can see from the expression for $\rho_{1}$ that this kind of approach is not satisfactory when $D=0 ; \rho$ diverges at $\omega=0$, but as long as it's a probability density, this can not have any physical meaning and has to be avoided.

\subsection{Weakly Nonlinear Theory: $D \neq 0$, but small}

In this section we try to sketch how it's possible to develop an asymptotic expansion for the case $1 \gg D \neq 0$. Let's choose the variables to scale as in the previous case (Hopf scaling) but let's say that $D=\epsilon^{2} D_{2}$. The motivation for this scaling can be found in the numerical experiments we performed and in the analysis by Daido and Crawford [4].

Substituting, as in the previous paragraph, into the equations (5) and (3) we have

$$
\epsilon^{2} \rho_{T}+\left(\frac{G_{\theta}}{2 \pi}+(\rho G)_{\theta}\right)\left(K_{c}+\epsilon^{2} K_{2}\right)=\epsilon^{2} D_{2} \rho_{\theta \theta}
$$


and at the first and second order we can derive the following expressions for $\rho_{1}$ and $\rho_{2}$ :

$$
\begin{aligned}
O(\epsilon): & \omega \rho_{1 \theta}+\frac{G_{1 \theta} K_{c}}{2 \pi}=0 \\
& \rho_{1}=-\frac{K_{c} G_{1}}{2 \pi \omega} \\
O\left(\epsilon^{2}\right): & \omega \rho_{2 \theta}+\frac{G_{2 \theta} K_{c}}{2 \pi}=-K_{c}\left(\rho_{1} G_{1}\right)_{\theta} \\
& \rho_{2}=\frac{K_{c}^{2} G_{1}^{2}}{2 \pi \omega^{2}}-\frac{K_{c} G_{2}}{2 \pi \omega} .
\end{aligned}
$$

Here is the problem we have to overcome; to treat properly the case $D \ll 1$ we need a critical layer around $\omega=0$; in fact, at this point, the asymptotic expansion breaks down and $\rho_{2}$ is bigger than $\rho_{1}$.

Inside the critical layer, we set the new independent and dependent variables

$$
\begin{aligned}
\omega & =\epsilon y \\
\rho & =Z(\theta, y, T)=Z_{0}+\epsilon Z_{1} .
\end{aligned}
$$

The equation in the inner region becomes

$$
\epsilon^{2} Z_{T}+\epsilon y Z_{\theta}+\left(\frac{G_{\theta}}{2 \pi}+(Z G)_{\theta}\right)\left(K_{c}+\epsilon^{2} K_{2}\right)=\epsilon^{2} D_{2} Z_{\theta \theta}
$$

and at the first two orders

$$
\begin{aligned}
O(\epsilon): & y Z_{0 \theta}+\frac{G_{1 \theta} K_{c}}{2 \pi}+K_{c}\left(Z_{0} G_{1}\right)_{\theta}=0 \\
& Z_{0}=-\frac{1}{2 \pi} \frac{K_{c} G_{1}}{y+K_{c} G_{1}} \\
O\left(\epsilon^{2}\right): \quad & y Z_{1 \theta}+\frac{G_{2 \theta} K_{c}}{2 \pi}+\left(Z_{1} G_{1}\right)_{\theta} K_{c}= \\
& -Z_{0 T}-\left(Z_{0} G_{2}\right)_{\theta} K_{c}+D_{2} Z_{0 \theta \theta}, \\
& \left(\left(y+K_{c} G_{1}\right) Z_{1}+\frac{K_{c} G_{2}}{2 \pi}\right)_{\theta}= \\
& \mathcal{F}\left(\frac{G_{1 T}}{\left(y+K_{c} G_{1}\right)^{2}}, \frac{G_{1 \theta}}{\left(y+K_{c} G_{1}\right)^{2}}, \frac{D_{2} G_{1 \theta}^{2}}{\left(y+K_{c} G_{1}\right)^{3}}\right)
\end{aligned}
$$

where $\mathcal{F}$ is a function, that for our purpose we not need derive.

Even in the inner region we still have trouble: when $y+K_{c} G_{1}=0, Z_{0}$ diverges and $Z_{1}$ diverges even more; the asymptotic expansion breaks down another time.

This is very peculiar; the first inner layer is not sufficient at all and it's necessary for another critical layer, a second, inside the first. Moreover the shape of this inner layer is peculiar: it develops around the curve $y+K_{c} G_{1}=0$. The whole situation is sketched in figure (8).

In this second inner layer we define the new independent variable

$$
y+K_{c} G_{1}=\epsilon^{\alpha} \xi
$$



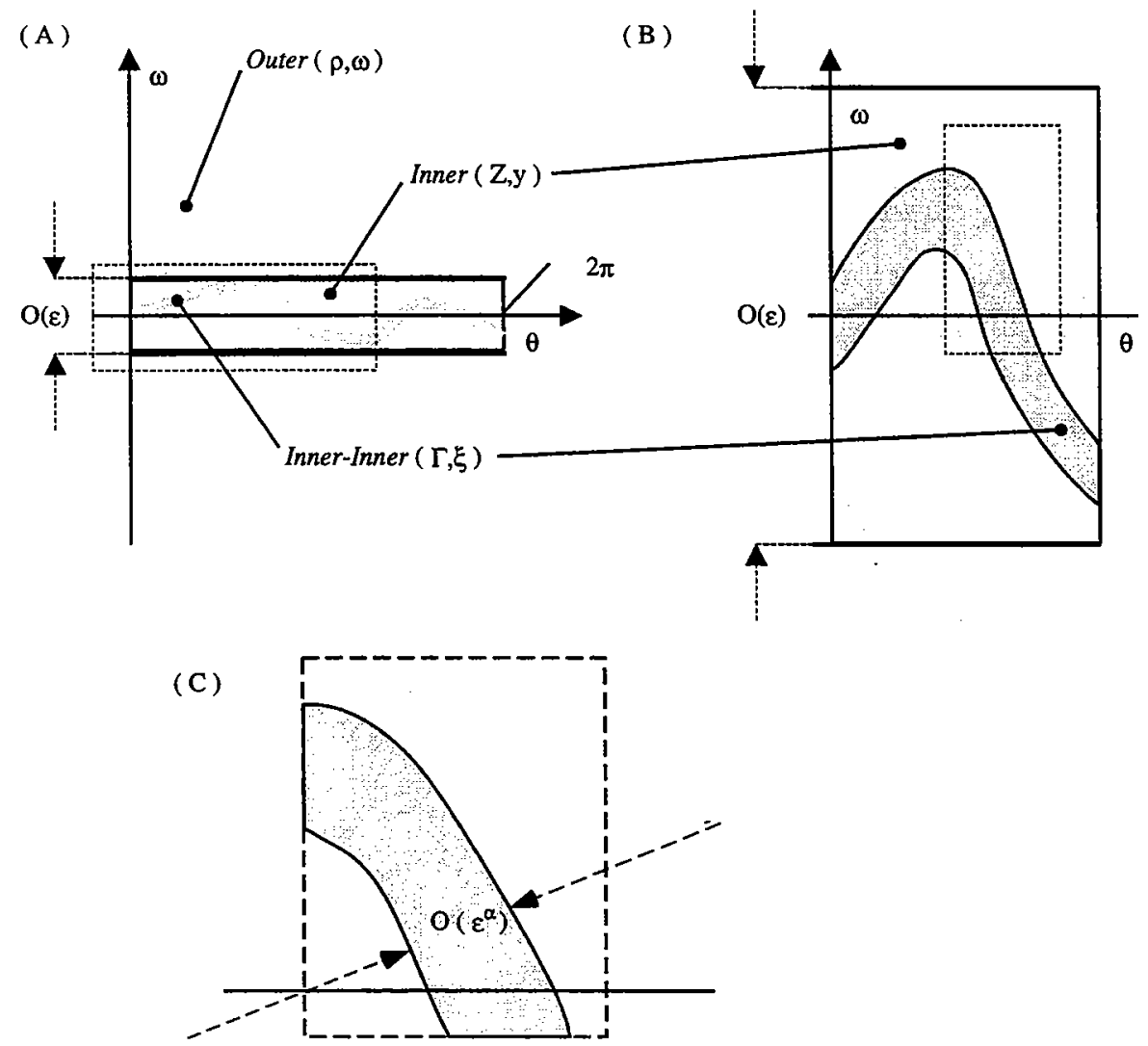

Figure 8: Weakly Nonlinear Theory. (A): When the dissipation is small $\left(O\left(\epsilon^{2}\right)\right)$ a critical layer becomes necessary around the frequency $\omega=0$ (light gray region); the width of this layer is of order $\epsilon$. (B): The sketched-box in (A) is enlarged: inside is possible to notice the second "snake-like" critical layer (inner-inner layer, dark gray region), that is necessary around the line $y+K_{c} G_{1}=0$. (C): The sketched-box in (B) is enlarged again: the width of the inner-inner layer is of order $\epsilon^{\alpha}(\alpha=1$ if $D_{2}=0 ; \alpha=1 / 2$ if $D_{2} \neq 0$ ). 
and the new dependent variable

$$
Z=\Gamma\left(\theta, \frac{y+K_{c} G_{1}}{\epsilon^{\alpha}}, T\right)
$$

Deriving a new equation, in this second inner layer, is very technical and we will not develop it. The reason lies in the strange relation between $T, \theta$ and $\xi$, imposed by the shape of the layer. On the change of variable to $\xi$, the $\theta$ and $T$ derivatives pick up additional $\xi$-derivatives,

$$
\begin{aligned}
& {\left[Z_{\theta}\right]_{y, T}=\left[\Gamma_{\theta}\right]_{\xi, T}+\frac{K_{c} G_{\theta}}{\epsilon^{\alpha}}\left[\Gamma_{\xi}\right]_{\theta, T}} \\
& {\left[Z_{T}\right]_{y, \theta}=\left[\Gamma_{T}\right]_{\xi, \theta}+\frac{K_{c} G_{\theta}}{\epsilon^{\alpha}}\left[\Gamma_{\xi}\right]_{T, \theta}}
\end{aligned}
$$

and this makes it much more complex to derive useful analytical expressions.

We conclude this section sketching an argument for determining the scaling parameter $\alpha$. We can deduce from the relations (13) the scaling for $Z_{0}$ and $Z_{1}$,

$$
\begin{aligned}
O(\epsilon) & : Z_{0} \sim O\left(\epsilon^{-\alpha}\right) \\
O\left(\epsilon^{2}\right) & : Z_{1} \sim O\left(\epsilon^{-2 \alpha} \text { OR } D_{2} \epsilon^{-3 \alpha}\right) .
\end{aligned}
$$

Hence $Z_{1}$ scales in different way depending on the value of $D_{2}$.

We now apply the condition that $Z_{0}$ and $\epsilon Z_{1}$ have to scale at the same order, which is where the expansion formally breaks down and we enter the innermost region. There are two different situations, depending upon the value of $D_{2}$ : if $D_{2}=0$, then $\alpha=1$, and when $D_{2} \neq 0$, then $\alpha=1 / 2$. The reason of the scaling we chose for $D$ becomes, now, more clear and we would have lost this double behaviour with a higher scaling of the noise term $\left(D \rightarrow \epsilon^{\beta} D\right.$ with $\beta>2$ ).

Summarizing the results in this section, when $D$ is finite but small, the derived asymptotic expansion reveals that we need, at least, two critical layers, one inside the other. The first, around $\omega=0$, is of order $\epsilon$; the second, snake-shaped, is inside the first and has a characteristic width that depends upon the value of $D_{2}$.

\section{$6 \quad$ Steadily propagating solutions}

In this section we look for steadily propagating solutions for $\rho$, that is

$$
\rho(\theta, \omega, t)=\rho(\theta-\Omega t, \omega)
$$

where $\Omega$ is the propagation velocity.

Substituting this expression into equation (3) and making the change of variable $\Phi=$ $\phi-\Omega t$, we find

$$
\begin{aligned}
G(\theta, t) & =\int_{-\infty}^{\infty} \int_{0}^{2 \pi} \sin (\phi-\theta) \rho(\phi-\Omega t, \omega) g(\omega) d \phi d \omega \\
& =\int_{-\infty}^{\infty} \int_{0}^{2 \pi} \sin (\Phi-(\theta-\Omega t)) \rho(\Phi, \omega) g(\omega) d \Phi d \omega \\
& =G(\theta-\Omega t, \omega)
\end{aligned}
$$


From this, we can observe that $G$ preserves the dependence on $(\theta-\Omega t)$ that $\rho$ is supposed to have.

Equation (5), in the situation $D=0$, becomes

$$
(\omega-\Omega) \rho_{\theta}+k(G \rho)_{\theta}=0
$$

integrating over $\theta$

$$
\rho=\frac{\mathcal{J}(\omega)}{\omega-\Omega+k G(\theta-\Omega t)}
$$

where $\mathcal{J}(\omega)$ is a generic function of $\omega$.

As N.J.B. says in these situations, we are in bad shape; as long as $\rho$ is a probability distribution function, it can not have singularity; as long as $\omega$ is a real variable that spans the entire real axis, the pole from the denominator of $\rho$ is hard to avoid.

This is an interesting point; if the dissipation is zero the system does not admit steadily propagating solutions. But what happens when the dissipation is small?

If $D \neq 0$, equation (13) has an additional term, and after the integration over $\theta$, we have

$$
(\omega-\Omega) \rho+k G \rho=\mathcal{J}(\omega)+D \rho_{\theta}
$$

For the sake of simplicity, let's say that we have fixed $\omega$ to a certain value; then there's a value of $\Theta=\theta-\Omega t$ at which the denominator of equation (14) vanishes; we call this value $\Delta$.

As $D \rightarrow 0, \rho$ scales as $1 /(\Theta-\Delta)$; hence we need an inner layer in the proximity of $\Theta-\Delta=0$ in which the dissipative term becomes important. For this reason we choose the following scaling and variables,

$$
\begin{aligned}
\Theta-\Delta & =\epsilon \delta \\
\rho & =\frac{1}{\epsilon} R \\
D & =\epsilon^{2}
\end{aligned}
$$

Substituting in equation (15) and noting that $\partial_{\theta}=\partial_{\Theta}=\epsilon^{-2} \partial_{\delta}$ we have

$$
k G_{\Theta}(\Delta) \delta R=\mathcal{J}(\omega)+R_{\delta}
$$

Integrating over $\delta$, we can find the expression of the probability distribution function in the inner layer and check the condition for the matching with the outer:

$$
\begin{aligned}
R_{\delta} & =k G_{\Theta}(\Delta) \delta R-\mathcal{J}(\omega) \\
\left(R e^{\frac{-k G_{\Theta}(\Delta) \delta^{2}}{2}}\right)_{\delta} & =-\mathcal{J}(\omega) e^{\frac{-k G_{\Theta}(\Delta) \delta^{2}}{2}} \\
R & =R_{0} e^{\frac{k G_{\Theta}(\Delta) \delta^{2}}{2}}-\mathcal{J}(\omega) e^{\frac{k G_{\Theta}(\Delta) \delta^{2}}{2}} \int_{-\infty}^{\delta} e^{\frac{-k G_{\Theta}(\Delta) \bar{\delta}^{2}}{2}} d \bar{\delta}
\end{aligned}
$$

The value of $R_{0}$ can be computed with the normalization condition (4). 


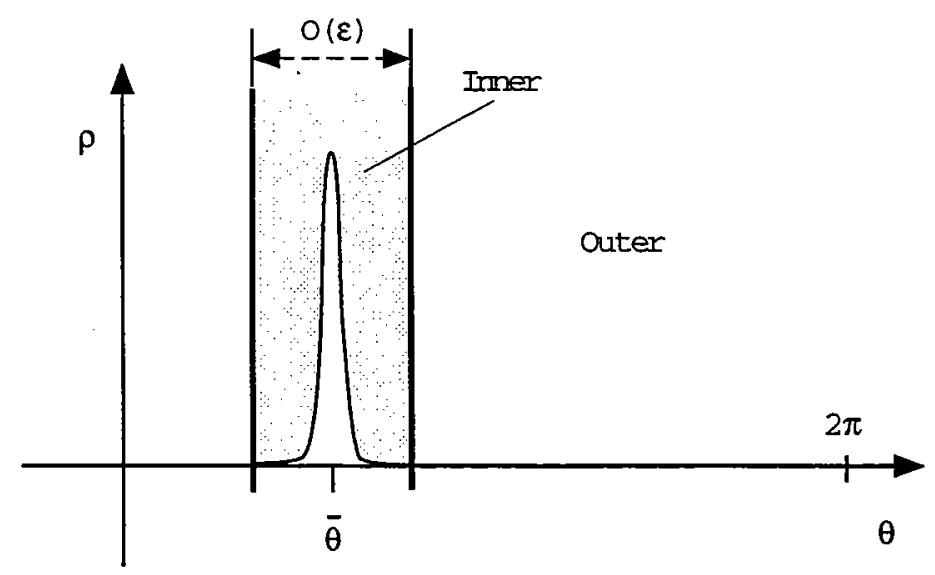

Figure 9: Steadily propagating solution with $\Omega=0$ : a sketch of $\rho(\theta, \omega)$. Near the phase $\bar{\theta}$ (where $\omega+K G(\bar{\theta})=0$ and $G^{\prime}(\bar{\theta})<0$ ) a critical layer (light gray region) is necessary. In the outer layer $\rho$ is zero everywhere.

This solution is potentially dangerous; it has to be bounded, otherwise it again diverges; moreover in the limit of $\delta$ going to infinity (that is, going out of the inner layer) $R$ has still to be limited, for a proper matching with $\rho$. Using these two conditions, we fix the value of $\mathcal{J}(\omega)$ and $R_{0}$

$$
\begin{array}{rlll}
\mathcal{J}(\omega)=0 & \text { for } & G_{\Theta}(\Delta)<0 \\
\mathcal{J}(\omega)=0, R_{0}=0 & \text { for } & G_{\Theta}(\Delta)>0
\end{array}
$$

The matching with the outer layer is straightforward; as $\delta$ goes to infinity, $R$ it's zero and $\rho$ is zero everywhere. In figure (9) is shown a sketch of the situation for $\Omega=0$.

Summarizing this result, steadily propagating solution can not develop for $D=0$; with $D$ finite, but small, they exist only in a small layer, that follows the line $(\omega-\Omega+k G(\theta-\Omega t))=0$ where $G_{\Theta}(\Delta)<0$.

The probability density $\rho$ is exponentially small everywhere in the outer layer; for this reason the integral for $G$ is limited to the inner, and

$$
\begin{aligned}
G(\Theta, t) & \left.=\int_{-\infty}^{\infty} \int_{0}^{2 \pi} \sin (\Phi-\Theta, t)\right) \rho(\Phi, \omega) g(\omega) d \Phi d \omega \\
& \left.=\int_{-\infty}^{\infty} \int_{-\infty}^{\infty} \sin (\Delta(\omega)-\Theta, t)\right) R_{0} e^{\frac{k G_{\Theta}(\Delta) \delta^{2}}{2}} g(\omega) d \delta d \omega
\end{aligned}
$$

\subsection{An example: $\Omega=0$}

Let's say that $\Omega=0$, so that we look for a steady solution of the kind $\rho(\theta, \omega)$. In this case, steadily propagating solutions can develop only along the line $\omega+K G(\theta)=0$.

Choosing a symmetric initial condition and applying relation (12), the curve becomes

$$
\omega=K \mathcal{G}(t) \sin (\theta) .
$$


Moreover, along the line, solutions can form only where $G_{\theta}<0$, that is

$$
\begin{aligned}
-\mathcal{G}(t) \frac{d \sin (\theta)}{d \theta} & <0 \\
\frac{d \sin (\theta)}{d \theta} & >0
\end{aligned}
$$

as $K$ and $\mathcal{G}$ are both positive. In figure (10) this prediction is compared to the actual solution, obtained with the numerical simulations. As it can be seen, the agreement is evident and this explains the depletion of probability noticed in the middle of the plane $\theta-\omega$, in figure (7).

\section{$7 \quad$ The case $g(\omega)=\delta(w)$}

In the previous two sections we appreciate that, if we set $D$ small, but different from zero, it's possible to perform an asymptotic expansion and steadily propagating solutions can develop. But what happens when $D=0$ ? The described approach only underlined that this is a critical situation. In this section, with $D=0$, we will show how is possible to solve analytically the Kuramoto equations in the case that $g(\omega)$ is a Dirac's delta function.

In this case all the oscillators share the same frequency $\omega=0$ and have different phases. Starting from a symmetric initial condition, that is $\rho(\theta, 0)=\rho(-\theta, 0)$, and using property (12), equations (5) and (3) become

$$
\begin{aligned}
\rho_{t}(\theta, 0, t)-k \partial_{\theta}\left(\rho(\theta, 0, t) \sin (\theta) \int_{0}^{2 \pi} \int_{-\infty}^{\infty} \cos (\phi) \rho(\phi, 0, t) \delta(\omega) d \omega d \dot{\phi}\right) & =0 \\
\rho_{t}(\theta, t)-k \partial_{\theta}\left(\rho(\theta, t) \sin (\theta) \int_{0}^{2 \pi} \cos (\phi) \rho(\phi, t) d \phi\right. & =0
\end{aligned}
$$

Setting

$$
\mathcal{F}(t)=\int_{0}^{2 \pi} \cos (\phi) \rho(\phi, t) d \phi
$$

the last relation becomes

$$
\rho_{t}-k \sin (\theta) \mathcal{F}(t) \rho_{\theta}=k \cos (\theta) \mathcal{F}(t) \rho .
$$

We solve this equation by the method of characteristics; for equation (17) the characteristic equation is

$$
\frac{d t}{1}=\frac{d \theta}{-k \sin (\theta) \mathcal{F}(t)}=\frac{d \rho}{k \cos (\theta) \mathcal{F}(t) \rho}
$$

Hence,

$$
\begin{aligned}
& \frac{d \theta}{d t}=-k \sin (\theta) \mathcal{F}(t) \\
& \frac{d \rho}{d t}=k \cos (\theta) \mathcal{F}(t) \rho
\end{aligned}
$$



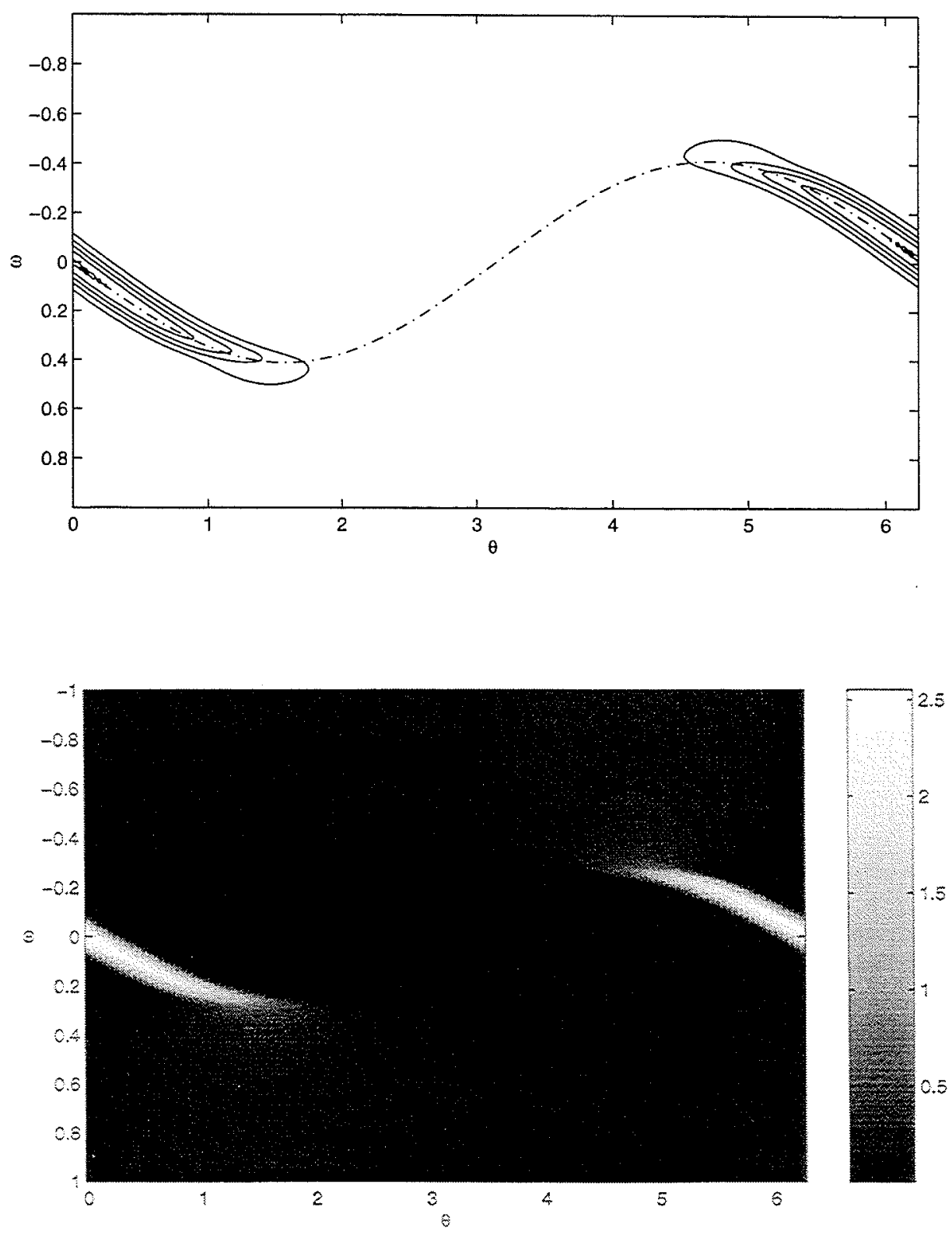

Figure 10: $\rho(\theta, \omega, t)$ at $t=180$ obtained via numerical integration of the Kuramoto continuous model with $D=0.01$ and $K=0.8$ (see figure (4)(left) for further details). UPPER: contour plot of $\rho$; the super-imposed sketched line is $\omega=K \mathcal{G}(t) \sin (\theta)$. The steadily propagating solution (with $\Omega=0$ ) can develop only where $G^{\prime}(\theta)<0$, as expected. LOWER: plot of $\rho$. Looking back to figure (7) is now possible to understand why the probability is zero in the middle of the pictures. 
Solving the first, we obtain

$$
\tan \left(\frac{\theta}{2}\right)=\tan \left(\frac{\theta_{0}}{2}\right) e^{-k \int_{0}^{t} \mathcal{F}\left(t^{\prime}\right) d t^{\prime}}
$$

dividing the first with the second

$$
\begin{aligned}
\frac{d \rho}{d \theta} & =-\rho \cot (\theta) \\
\rho \sin (\theta) & =\text { constant }=\rho_{0}\left(\theta_{0}(\theta, t)\right) \sin \left(\theta_{0}(\theta, t)\right) .
\end{aligned}
$$

The derivation here becomes a little technical:

$$
\begin{aligned}
\rho & =\rho_{0} \frac{\frac{2 \tan \left(\theta_{0} / 2\right)}{1+\tan ^{2}\left(\theta_{0} / 2\right)}}{2 \sin (\theta / 2) \cos (\theta / 2)}=\rho_{0} \frac{\frac{2 \tan (\theta / 2) \mathcal{E}(t)}{1+\tan ^{2}(\theta / 2) \mathcal{E}^{2}(t)}}{2 \sin (\theta / 2) \cos (\theta / 2)} \\
& =\rho_{0} \frac{e^{-k q}}{e^{-2 k q} \cos ^{2}(\theta / 2)+\sin ^{2}(\theta / 2)}
\end{aligned}
$$

where $q=\int_{0}^{t} \mathcal{F}\left(t^{\prime}\right) d t^{\prime}$ and $\mathcal{E}=e^{k q}$.

As time goes on, we expect the order parameter to saturate to a certain constant value; this is what we saw in the numerical simulations. The order parameter is, by definition, proportional to $G$ by a factor $2 \pi$. Calling $\overline{\mathcal{F}}$ the saturation value of $G$ then $q$, the integral of $\mathcal{F}$, becomes

$$
q \sim \overline{\mathcal{F}} t \quad \text { as } \quad t \rightarrow \infty
$$

Choosing, now, the initial condition $\rho_{0}=\cos \left(\theta_{0}\right)$, and recalling relation (18), we find

$$
\begin{aligned}
\rho_{0} & =\frac{1-\tan ^{2}\left(\theta_{0} / 2\right)}{1+\tan ^{2}\left(\theta_{0} / 2\right)}=\frac{1-\tan ^{2}(\theta / 2) \mathcal{E}^{2}}{1+\tan ^{2}(\theta / 2) \mathcal{E}^{2}} \\
& =\frac{e^{-2 k q} \cos ^{2}(\theta / 2)-\sin ^{2}(\theta / 2)}{e^{-2 k q} \cos ^{2}(\theta / 2)+\sin ^{2}(\theta / 2)}
\end{aligned}
$$

Finally, substituting $\rho_{0}$ into the expressions for $\rho$ and $q$ we have

$$
\rho=e^{-k q} \frac{e^{-2 k q} \cos ^{2}(\theta / 2)-\sin ^{2}(\theta / 2)}{\left(e^{-2 k q} \cos ^{2}(\theta / 2)+\sin ^{2}(\theta / 2)\right)^{2}}
$$

and

$$
\frac{d q}{d t}=e^{-k q} \int_{0}^{2 \pi} \cos (\theta) \frac{e^{-2 k q} \cos ^{2}(\theta / 2)-\sin ^{2}(\theta / 2)}{\left(e^{-2 k q} \cos ^{2}(\theta / 2)+\sin ^{2}(\theta / 2)\right)^{2}} d \theta
$$

The integral in (19) can be evaluated in the limit that $q$ goes to infinity. Making the change of variable $\theta=2 x e^{-k q}$ and expanding in Taylor's series the trigonometric functions, it becomes:

$$
\begin{aligned}
\frac{d q}{d t} & =\int_{-\infty}^{\infty} \frac{-2 x^{2}}{\left(1+x^{2}\right)^{2}} d x=-2 \pi \\
q & =-2 \pi t
\end{aligned}
$$


Summarizing, as the time goes on, $q$ grows linearly; $\rho$ becomes small everywhere except near $\theta=0$ where $\rho \sim e^{k q}$, so that it grows exponentially. The dynamics brings probability toward a singular phase (the point $\theta=0$ here); this is exactly what we saw in the numerical simulation, although with different $g(\omega)$, and along a curve on the $(\theta, \omega)$ plane.

Concluding, the solution is a spike-shaped shock-like object. This is also consistent with another fact, which is namely that, with $g(\omega)=\delta(\omega)$ and the alternative coupling, $\sin (\phi-$ $\theta) \rightarrow \delta(\phi-\theta)$, the Kuramoto model reduces to the Burger's equation,

$$
\rho_{t}(\theta, 0, t)+\left[K \rho^{2}(\theta, 0, t)\right]_{\theta}
$$

\section{Discrete vs. Continuous}

An important question is whether the continuous approximation, made in the limit $N \rightarrow \infty$, remains valid with a relatively small number of oscillators. That is, if the results, obtained in the previous sections, are applicable to equation (1).

We don't have a final answer to such a question, but, comparing figures (11) and (12), obtained from the discrete model with $N=256$, with (6) and (7), can be seen that the main behaviours are the same. In particular, in figure (12), we see the probability gathering in two symmetric areas of the plane, as in the continuous case of figure (7).

A peculiarity of the discrete case is that the concentration of probability drifts in the $\theta$ direction; we suppose this to be related to the fact that, although the probability density function, from which the initial condition is extracted, is symmetric, the actual initial condition is not.

\section{Conclusions and other remarks}

The Kuramoto model generates many interesting results; many more than what we were expecting. So, in this report, for brevity reasons, we have omitted several of the analyses we made. We mention two particular ones here:

First, we studied the issue of transient amplification: starting from a situation of equilibrium with a sub-critical coupling, and imposing perturbations of different intensities, one can find solutions to the linear initial-value problem that grow to arbitrarily large amplitude before decaying. One important question is whether this transient growth induces nonlinear behaviour in the full system before the disturbance can decay. But, from the numerical simulations, the system appears to be very robust, with a decay that is very much similar to what predicted by the linear theory no matter how big the transient growth. In other words, the nonlinearity of the system doesn't provide any new, unexpected behaviours.

Second, we also applied Nyquist methods to the linear stability problem: in the linear analysis section we computed analytically the value of the growth rate and the critical value of the coupling parameter for the Lorentzian. For general $g(\omega)$, the integral in (8) can be solved numerically to furnish similar results, but in many situations it is helpful to have a quick, general understanding. We used the Nyquist criteria to derive the following result 

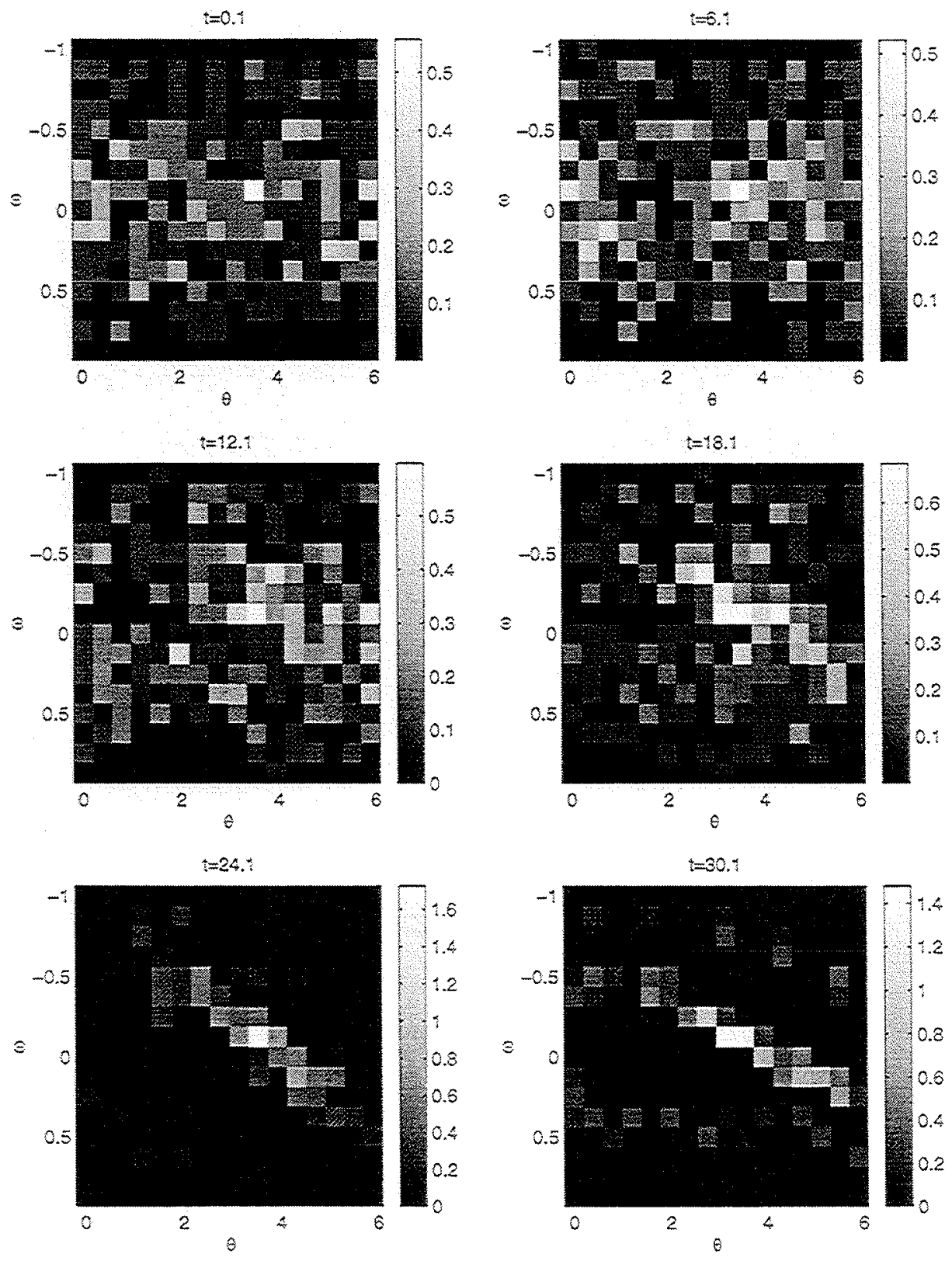

Figure 11: $\rho(\theta, \omega, t)$ at six successive instant of time obtained via numerical integration of the Kuramoto discrete model with $D=0.01$ and $K=0.65\left(K_{c}=0.739\right.$, sub-critical coupling). Both $\theta$ and $\omega$ axes are discretized in 16 intervals. Compact support within $[-1 ; 1]$ for $g(\omega)=\frac{1}{\pi-2} \frac{1-\omega^{2}}{1+\omega^{2}}$; initial condition $\widetilde{\rho}_{0}=\frac{1}{2 \pi}$ and $\widetilde{\rho}_{1}=\xi \frac{2}{\pi} \frac{1}{4+\omega^{2}}$ with $\xi=0.1$. 

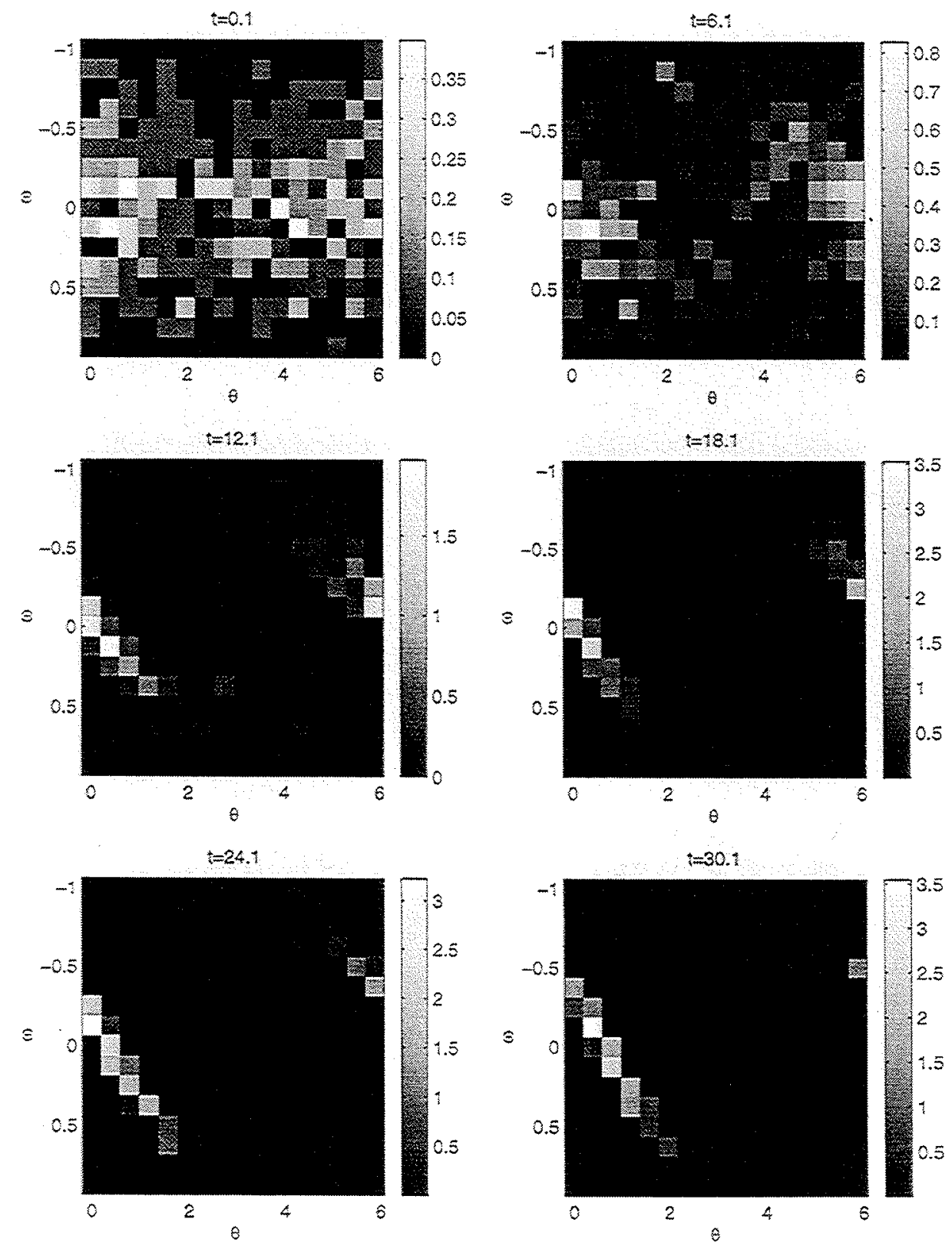

Figure 12: The same as in figure (11) except $K=0.8$ (super-critical coupling). 
that, here, we only state: the maximum number of unstable growing rates, for the linearized Kuramoto system, is the number of monotonic pieces of $g(\omega)$ divided by two.

On the other hand, there is further work to be done in at least two different directions:

- the comparison of the discrete and continuous model is only sketched and needs more numerical explorations. Moreover, do traveling solutions, as we saw in figure (12), exist in the continuous case? In figure (1) structures can be noticed, even if the coupling is sub-critical and we are expecting incoherence. What is the origin of these structures? Do they only depend on the initial condition?

- The change in the form of the coupling seems to be critical (see ad example [4] and [8]). What happen if the coupling is different than $\sin (\phi-\theta)$ ? Why?

\section{Acknowledgments}

The first person I like to thank is Neil J. Balmforth that suggested this work and followed me on each step, all the summer long. Then I'm grateful to Francesco Paparella that helped me with the numerical computations; to W. R. Young, Rick Salmon, Phil Morrison, Jean-Luc Thiffeault and Stefan G. Llewellyn Smith for aid and very useful conversations.

In particular thank to Ed Spiegel, that first suggested me to come to Woods Hole this summer and that helped me several times with his car.

The work I did this summer would have been impossible without the help of the other fellows. Apart from that, we lived almost together for ten weeks so there are thousands of reasons to say thank for. Among them I like to say thank to Claudia Pasquero that cooked for everyone so many times; to Yuan-Nan Young that shared with me a very small room in the Barn; to Pascale Garaud that, restless, organized a lot of nice after-dinner events; to David Osmond that introduced the fellows to the triathlon and showed me how to repair the bike; to Jennifer Jen MacKinnon for having explained me the difference between walnuts and pecans; to Raffaele Ferrari for listening me so many times about my non-working code; to Jennifer Niffer Curtis that made me DrPepper's freak; to Jeffrey Jeff Moehlis and his wife Allison for the GFD cooler; to Meredith Metzger for her internet radio; and again to Jean-Luc Thiffeault for being Canadian.

At the end, I like to thank George Veronis that taught how to play softball to the fellows and that was happy though we lost all the games we played; moreover he had his sailboat repaired so that I tried sailing, for the first time, on the pond in front of his cottage.

\section{References}

[1] A. T. Winfree, "Biological rhythms and the behavior of populations of coupled oscillators," J. Theoret. Biol. 16, 15 (1967). 
[2] Y. Kuramoto, in International symposium on mathematical problems in theoretical physics, No. 39 in Lecture Notes in Physics, edited by H. Araki (Springer, New York, 1975), pp. $420-422$.

[3] S. H. Strogatz and R. E. Mirollo, "Stability of incoherence of a population of coupled oscillators," J. Stat. Phys. 63, 613 (1991).

[4] J. D. Crawford and K. T. R. Davies, "Synchronization of globally coupled phase oscillators: singularities and scaling for general couplings," Phys. D 125, 1 (1999).

[5] A. Einstein, Investigations on the Theory of the Brownian Movement (Dover Publications, New York, 1985).

[6] L. L. Bonilla, J. C. Neu, and R. Spigler, "Nonlinear stability of incoherence and collective synchronization in a population of coupled oscillators," J. Stat. Phys. 67, 313 (1992).

[7] J. D. Crawford, "Amplitude expansions for instabilities in populations of globally-coupled oscillators," J. Stat. Phys. 74, 1047 (1994).

[8] L. L. Bonilla, C. J. P. Vicente, and R. Spigler, "Time-periodic phases in populations of nonlinearly coupled oscillators with bimodal frequency distributions," Phys. D 113, 79 (1998). 


\title{
Effect of a Simple Storm on a Simple Ocean
}

\author{
Jeff Moehlis
}

\section{Introduction: Near-Inertial Oscillations and Storms}

Horizontal motion of a free particle on the Earth's surface subject only to the Coriolis force is governed by the equations

$$
\frac{d u_{p}}{d t}-f v_{p}=0, \quad \frac{d v_{p}}{d t}+f u_{p}=0
$$

where $u_{p}$ and $v_{p}$ are respectively the eastward and northward components of the particle's velocity in the frame rotating with the Earth, $f \equiv 2 \Omega_{E} \sin \phi$ is the Coriolis parameter, $\Omega_{E}$ is the frequency of the Earth's rotation, and $\phi$ is the latitude (see, e.g., [4]). This has solution $u_{p}+i v_{p}=e^{-i f t}\left(u_{0}+i v_{0}\right)$, where $u_{0}$ and $v_{0}$ are the initial components of the velocity. This corresponds to the particle's velocity describing a circle of radius $\left(u_{0}^{2}+v_{0}^{2}\right)^{1 / 2} / f$ with frequency $f$. In the northern hemisphere, $f>0$ and the particle rotates in a clockwise direction when viewed from above. The inertial frequency $f$ is the low-frequency cutoff for internal waves in the ocean. An internal wave with frequency near $f$ is called a near-inertial oscillation (NIO). About half of the total kinetic energy associated with internal waves in the ocean is contained in NIOs [5].

There is much observational evidence, starting with [17, 14], that wind from storms can excite near-inertial currents in the mixed layer of the ocean; recent observations include $[8,13,15]$. Simple models which treat the mixed layer as a solid slab have been quite successful at explaining the process by which wind generates such currents (see, e.g., $[14,5]$ ). These currents decay away after the storm passes, with possible mechanisms for the decay including nonlinear interactions which transfer energy to other frequencies [12], turbulent dissipation [11], and the radiation of downward propagating NIOs excited by inertial pumping into the interior of the ocean [10]. The last mechanism will be the focus of this paper. Such downward propagation of NIOs is believed to be a significant mechanism for mixing in the upper ocean.

Observations give a time scale for the decay of the energy deposited by the passing storm on the order of ten to twenty days $[8,13,15]$. This time scale is in contrast with estimates such as that by [10] that near-inertial currents decaying through the downward propagation of NIOs and with a horizontal length scale typical of the atmospheric forcing mechanism can remain in the mixed layer for longer than a year. To account for this difference, several mechanisms for the enhancement of vertical propagation of NIOs have been suggested (these are nicely summarized in [16]), including smaller-scale fluctuations within the storms, the $\beta$ effect [6], and interaction with background geostrophic or quasigeostrophic flow (see, e.g., $[2,3,16])$. . 
This paper considers the vertical propagation of near-inertial energy and shear deposited into the mixed layer by a storm in the presence of the $\beta$ effect. The analysis uses the formalism of [18] which is briefly discussed in Section 2. In Section 3, a simplified model with three main assumptions is outlined. First, the background flow is assumed to be independent of longitude and the associated vorticity is assumed to be zero. Second, the buoyancy frequency is taken to be approximately zero in the mixed layer, and constant in the interior (i.e., beneath the mixed layer). Third, it is assumed that the storm has moved very rapidly across the ocean and has created a horizontally uniform near-inertial current to the east concentrated within the mixed layer. Section 4 uses the fact that the depth of the ocean is very much larger than the mixed layer depth to formulate and solve the model for an ocean which is (effectively) infinitely deep. Section 5 discusses the results and suggests directions for further investigation.

\section{Formalism}

Consider the ocean to be infinite in horizontal extent and of depth $D$, with the mixed layer being the portion of the ocean with $-H_{\text {mix }}<z<0$, and the interior the portion with $-D<z<-H_{\text {mix }}$. The $x$ and $y$ axes are taken to point to the east and north, respectively. The buoyancy frequency $N=N(z)$ is an arbitrary piecewise continuous function of depth $z$.

\subsection{Evolution Equation}

Young and Ben Jelloul [18] derive an evolution equation for a complex field $A(x, y, z, t)$ from which leading-order NIO motion in the presence of a steady barotropic background flow and the $\beta$ effect can be deduced:

$$
L A_{t}+\frac{\partial(\psi, L A)}{\partial(x, y)}+\frac{i}{2} f_{0} \nabla^{2} A+i\left(\beta y+\frac{1}{2} \zeta\right) L A=0
$$

where

$$
L A=\frac{\partial}{\partial z}\left(\frac{f_{0}^{2}}{N^{2}} \frac{\partial A}{\partial z}\right)
$$

$\psi$ is the streamfunction for the background flow, $\zeta \equiv \nabla^{2} \psi$ is the associated vorticity, and the Coriolis parameter $f=f_{0}+\beta y$. Here $\nabla$ is the horizontal gradient, and $\nabla^{2}=\partial_{x}^{2}+\partial_{y}^{2}$. Subscripts denote partial differentiation. The asymptotic expansion used in the derivation of equation (1) relies upon the frequency of near-inertial waves being close to the inertial frequency $f_{0}$. The NIO velocity field $(u, v, w)$, buoyancy $b$, and pressure $p$ are given by

$$
\begin{aligned}
u+i v & =e^{-i f_{0} t} L A \\
w & =-\frac{1}{2} f_{0}^{2} N^{-2}\left(A_{x z}-i A_{y z}\right) e^{-i f_{0} t}+c . c . \\
b & =\frac{i}{2} f_{0}\left(A_{x z}-i A_{y z}\right) e^{-i f_{0} t}+c . c . \\
p & =\frac{i}{2}\left(A_{x}-i A_{y}\right) e^{-i f_{0} t}+c . c .
\end{aligned}
$$


Here $b$ is related to the density $\rho$ by

$$
\rho=\rho_{0}\left[1-\frac{1}{g} \int_{0}^{z} N^{2}\left(z^{\prime}\right) d z^{\prime}-\frac{b}{g}\right]
$$

where $\rho_{0}$ is the reference density at the top of the ocean. Note that $p$ has been normalized by $\rho_{0}$.

The boundary conditions are that $w$ vanishes at the top and bottom of the ocean; this corresponds to the boundary condition $A_{z}=0$ at $z=0$ and $z=-D$. This boundary condition along with equation (3) implies that

$$
\int_{-D}^{0}(u+i v)=0
$$

Thus, the barotropic motion is not included in the analysis; note that [10] shows that the barotropic response to a storm is instantaneous and the associated currents are weak.

\subsection{Jump Conditions}

Suppose that the buoyancy frequency is discontinuous at $z=z_{d}$. Integrating equations (2) and (3) from $z=z_{d}-\delta$ to $z=z_{d}+\delta$, the following jump condition is obtained:

$$
\left[\frac{f_{0}^{2}}{N^{2}} \frac{\partial A}{\partial z}\right]_{z_{d}-\delta}^{z_{d}+\delta}=e^{i f_{0} t} \int_{z_{d}-\delta}^{z_{d}+\delta}(u+i v) d z .
$$

The left hand side tends to zero as $\delta \rightarrow 0$ provided $u$ and $v$ remain finite (which must be true on physical grounds). Thus, $\frac{1}{N^{2}} \frac{\partial A}{\partial z}$ is continuous, even when $N^{2}$ is discontinuous. Now assuming that $\psi$ and $\zeta$ have no $\delta$-function behavior in the $z$ direction, integrating equation (1) over the same interval in $z$ implies that

$$
\lim _{\delta \rightarrow 0} \int_{z_{d}-\delta}^{z_{d}+\delta} \nabla^{2} A d z=0
$$

Thus $\nabla^{2} A=A_{x x}+A_{y y}$ is continuous across $z=z_{d}$.

\subsection{Energy and Shear}

The quantities

$$
u^{2}+v^{2}=|L A|^{2}, \quad u_{z}^{2}+v_{z}^{2}=\left|\frac{\partial}{\partial z}(L A)\right|^{2}
$$

give local measures of the horizontal kinetic energy per unit mass (hereafter HKE) contained in near-inertial motion and the associated vertical shear, respectively. Using equation (1) 
and its complex conjugate,

$$
\begin{aligned}
\frac{\partial|L A|^{2}}{\partial t}= & L A_{t}^{*} L A+L A_{t} L A^{*} \\
= & \frac{i f_{0}}{2}\left[\nabla \cdot\left(L A \nabla A^{*}-L A^{*} \nabla A\right)\right]-\frac{\partial\left(\psi,|L A|^{2}\right)}{\partial(x, y)} \\
& +\frac{i f_{0}}{2}\left\{\frac{\partial}{\partial z}\left[\frac{f_{0}^{2}}{N^{2}}\left(\nabla A_{z}^{*} \cdot \nabla A-\nabla A_{z} \cdot \nabla A^{*}\right)\right]\right\},
\end{aligned}
$$

where the star denotes complex conjugation. In the following, it will be useful to integrate this over a volume in order to determine how the energy contained in horizontal near-inertial motion in the volume depends on the value of derivatives of $A$ evaluated on the surface of the volume. A general equation for the evolution of the shear is not given here, but will be for the simplified model considered next.

\section{A Simplified Model}

To simplify the analysis, it is assumed that $A$ and $\psi$ do not vary in the $x$ direction, and that $\zeta=0$. The analysis thus keeps the $\beta$ effect but neglects the effect of background barotropic vorticity. The buoyancy frequency profile is taken to be

$$
\begin{array}{ll}
N^{2}=\epsilon^{2} N_{0}^{2} & -H_{\operatorname{mix}}<z<0 \\
N^{2}=N_{0}^{2} & -D<z<-H_{\operatorname{mix}}
\end{array}
$$

where $\epsilon \ll 1$. Finally, the storm is assumed to produce the initial condition of a horizontally uniform near-inertial current to the east concentrated within the mixed layer.

Instead of approaching this problem by projecting onto normal modes (see, e.g., $[2,10]$ ), the problem will be formulated as an initial value problem on a semi-infinite domain corresponding to an effectively infinitely deep ocean. In order to formulate the problem properly for this limit, this section considers an ocean of finite depth. In Section 4 the solution in the limit that the depth of the interior is much greater than the mixed layer depth will be found.

\subsection{Nondimensionalization}

Quantities are nondimensionalized according to

$$
\hat{y}=\frac{y}{Y}, \quad \hat{z}=\frac{z}{H_{\text {mix }}}+1, \quad \hat{t}=\Omega t, \quad \hat{N}=\frac{N}{N_{0}}
$$

where

$$
Y \equiv\left(\frac{H_{\mathrm{mix}}^{2} N_{0}^{2}}{\beta f_{0}}\right)^{1 / 3}, \quad \Omega \equiv\left(\frac{\beta^{2} H_{\mathrm{mix}}^{2} N_{0}^{2}}{f_{0}}\right)^{1 / 3}
$$

Typical values $\beta \approx 10^{-11} \mathrm{~m}^{-1} \mathrm{~s}^{-1}, H_{\text {mix }} \approx 100 \mathrm{~m}, f_{0} \approx 10^{-4} \mathrm{~s}^{-1}, N_{0} \approx 10^{-2} \mathrm{~s}^{-1}$ give $Y \approx 10^{5} \mathrm{~m}$ and $\Omega \approx 10^{-6} \mathrm{~s}^{-1}$. The relevant time scale is thus $1 / \Omega \approx 11.5$ days. Also, with a view to 
specifying the initial velocity profile according to equation (3), the velocity and the field $A$ are nondimensionalized as

$$
(\hat{u}, \hat{v})=\frac{(u, v)}{U}, \quad \hat{A}=\frac{f_{0}^{2}}{U N_{0}^{2} H_{\mathrm{mix}}^{2}} A
$$

where $U$ is a characteristic value of the velocity. The hats will be dropped for ease of notation. With this nondimensionalization, the buoyancy frequency profile is

$$
\begin{array}{ll}
N^{2}=\epsilon^{2} & 0<z<1 \\
N^{2}=1 & -H \equiv-\frac{D}{H_{\operatorname{mix}}}+1<z<0
\end{array}
$$

and equation (1), the boundary conditions, and initial condition become

$$
\begin{aligned}
& A_{z z t}+\frac{i}{2} N^{2} A_{y y}+i y A_{z z}=0 \\
& A_{z}=0 \quad z=-H, \quad z=1 \\
& A_{z z}=N^{2} u \quad t=0 .
\end{aligned}
$$

The jump conditions in nondimensional form are

$$
\left.A_{z}\right|_{z=0^{+}}=\left.\epsilon^{2} A_{z}\right|_{z=0^{-}},\left.\quad A_{y y}\right|_{z=0^{+}}=\left.A_{y y}\right|_{z=0^{-}},
$$

where $z=0^{+}$and $z=0^{-}$are the limits as $z \rightarrow 0$ from positive and negative $z$ values, respectively.

This nondimensionalization allows some immediate conclusions to be drawn about the propagation of NIO energy and shear downwards. Most importantly, if $H_{\text {mix }}$ increases then the timescale $1 / \Omega$ decreases. Thus, assuming that the storm causes a uniform near-inertial current throughout the whole mixed layer, energy and shear transfer will be faster for a deeper mixed layer. This confirms the results of [10], which associated the more efficient transfer with a larger projection of the initial velocity profile on the first vertical mode.

\subsection{Integral Energy Relations and Energy Flux}

The nondimensional local kinetic energy per unit mass is $u^{2}+v^{2}=\left|A_{z z} / N^{2}\right|^{2}$. The nondimensional form of equation (6), with the assumptions of the simplified model, is

$$
\frac{\partial}{\partial t}\left|\frac{A_{z z}}{N^{2}}\right|^{2}=\frac{i}{2 N^{2}} \frac{\partial}{\partial y}\left(A_{z z} A_{y}^{*}-A_{z z}^{*} A_{y}\right)+\frac{i}{2 N^{2}} \frac{\partial}{\partial z}\left(A_{y z}^{*} A_{y}-A_{y z} A_{y}^{*}\right) \text {. }
$$

Let

$$
\int_{\mathrm{INT}} d V \equiv \int_{-H}^{0} d z \int_{-\infty}^{\infty} d x \int_{-\infty}^{\infty} d y, \quad \int_{\mathrm{ML}} d V \equiv \int_{0}^{1} d z \int_{-\infty}^{\infty} d x \int_{-\infty}^{\infty} d y
$$

be the integrals over the interior of the ocean and the mixed layer, respectively. Assuming $A_{z z} A_{y}^{*}-A_{z z}^{*} A_{y}$ vanishes for $|y| \rightarrow \infty$ and using equation (8) gives the following results:

$$
\frac{d}{d t} \int_{\mathrm{INT}}\left|A_{z z}\right|^{2} d V=\left.\frac{i}{2} \int_{-\infty}^{\infty} \int_{-\infty}^{\infty}\left(A_{y z}^{*} A_{y}-A_{y z} A_{y}^{*}\right)\right|_{z=0^{-}} d x d y
$$




$$
\frac{d}{d t} \int_{\mathrm{ML}}\left|\frac{A_{z z}}{\epsilon^{2}}\right|^{2} d V=-\left.\frac{i}{2 \epsilon^{2}} \int_{-\infty}^{\infty} \int_{-\infty}^{\infty}\left(A_{y z}^{*} A_{y}-A_{y z} A_{y}^{*}\right)\right|_{z=0^{+}} d x d y
$$

Equations (10),(12) and (13) may be combined to give

$$
\frac{d}{d t} \int_{\mathrm{INT}}\left|A_{z z}\right|^{2} d V+\frac{d}{d t} \int_{\mathrm{ML}}\left|\frac{A_{z z}}{\epsilon^{2}}\right|^{2} d V=0 .
$$

This is a statement of conservation of HKE in nondimensional form.

The quantity $\left.F_{E}(y, t) \equiv \frac{i}{2}\left(A_{y z}^{*} A_{y}-A_{y z} A_{y}^{*}\right)\right|_{z=0^{-}}$is the flux of HKE from the mixed layer to the interior of the ocean. Letting

$$
\int_{V_{d}} d V \equiv \int_{-H}^{-d} d z \int_{-\infty}^{\infty} d x \int_{-\infty}^{\infty} d y
$$

similar arguments show that

$$
\frac{d}{d t} \int_{V_{d}}\left|A_{z z}\right|^{2} d V=\int_{-\infty}^{\infty} \int_{-\infty}^{\infty} F_{E}(y, t ; d) d x d y
$$

where

$$
\left.F_{E}(y, t ; d) \equiv \frac{i}{2}\left(A_{y z}^{*} A_{y}-A_{y z} A_{y}^{*}\right)\right|_{z=-d}
$$

gives the flux of HKE from the region $z>-d$ to the region $z<-d$. Equation (12) is a special case of equation (15) with $F_{E}(y, t) \equiv F_{E}\left(y, t ; 0^{-}\right)$.

\subsection{Integral Shear Relations and Shear Flux}

In Section 3.4, it will shown that, to leading order in $\epsilon, A$ is independent of $z$ in the mixed layer, and thus there is no shear in the mixed layer. For $z<0$ the nondimensionalized buoyancy frequency is $N=1$, so the vertical shear from equation (5) may be written in nondimensional form as $u_{z}^{2}+v_{z}^{2}=\left|A_{z z z}\right|^{2}$. Similar arguments to those leading to equations (6) and (11) give the evolution equation

$$
\frac{\partial}{\partial t}\left|A_{z z z}\right|^{2}=\frac{i}{2} \frac{\partial}{\partial y}\left(A_{z z z} A_{y z}^{*}-A_{z z z}^{*} A_{y z}\right)+\frac{i}{2} \frac{\partial}{\partial z}\left(A_{y z z}^{*} A_{y z}-A_{y z z} A_{y z}^{*}\right)
$$

Assuming $A_{z z z} A_{y z}^{*}-A_{z z z}^{*} A_{y z}$ vanishes for $|y| \rightarrow \infty$,

$$
\frac{d}{d t} \int_{V_{d}}\left|A_{z z z}\right|^{2} d V=\int_{-\infty}^{\infty} \int_{-\infty}^{\infty} F_{S}(y, t ; d) d x d y
$$

where

$$
\left.F_{S}(y, t ; d) \equiv \frac{i}{2}\left(A_{y z z}^{*} A_{y z}-A_{y z z} A_{y z}^{*}\right)\right|_{z=-d}
$$

is the flux of vertical shear from the region $z>-d$ to the region $z<-d$. 


\subsection{Boundary Condition at Base of Mixed Layer}

For $0<z<1$, equation (7) becomes

$$
A_{z z t}+\frac{i}{2} \epsilon^{2} A_{y y}+i y A_{z z}=0 .
$$

Expanding $A(y, z, t)=A_{0}(y, z, t)+\epsilon^{2} A_{2}(y, z, t)+\mathcal{O}\left(\epsilon^{4}\right)$,

$$
A_{0 z z t}+i y A_{0 z z}=0 \text {. }
$$

Integrating this subject to the boundary condition that $A_{z}$ and thus $A_{0 z}$ vanishes at $z=1$ gives

$$
A_{0}=e^{-i y t} \int_{0}^{t} g\left(y, t^{\prime}\right) e^{i y t^{\prime}} d t^{\prime}
$$

for some function $g$. In particular, $A_{0}$ is independent of $z$. At $\mathcal{O}\left(\epsilon^{2}\right)$

$$
A_{2 z z t}+i y A_{2 z z}+\frac{i}{2} A_{0 y y}=0
$$

which may be integrated subject to the boundary condition that $A_{2 z}$ vanishes at $z=1$ to give

$$
A_{2 z t}+i y A_{2 z}+\frac{i}{2} A_{0 y y}(z-1)=0
$$

Evaluating at $z=0^{+}$and using

$$
A_{y y}=A_{0 y y}+\mathcal{O}\left(\epsilon^{2}\right), \quad A_{z}=\epsilon^{2} A_{2 z}+\mathcal{O}\left(\epsilon^{4}\right), \quad A_{z t}=\epsilon^{2} A_{2 z t}+\mathcal{O}\left(\epsilon^{4}\right)
$$

implies that

$$
A_{z t}+i y A_{z}-\frac{i \epsilon^{2}}{2} A_{y y}=\mathcal{O}\left(\epsilon^{4}\right) \quad z=0^{+}
$$

Finally, applying (10) gives the boundary condition

$$
A_{z t}+i y A_{z}-\frac{i}{2} A_{y y}=0 \quad z=0^{-}
$$

to leading order in $\epsilon$.

\subsection{Initial Condition}

Suppose that in a short time compared with the NIO wave propagation time the passing storm causes near-inertial currents in the mixed layer with no horizontal structure. For simplicity, the initial velocity is assumed to be piecewise constant with depth. Thus the initial velocity profile (consistent with equation (4)) is taken to be

$$
\begin{array}{ccc}
u=1 & 0<z<1, \\
u=-\frac{1}{H} & -H<z<0, \\
v=0 & -H<z<1 .
\end{array}
$$


Integrating equation (9) with respect to $z$ and using the boundary conditions (8) then gives at $t=0$

$$
\begin{array}{ll}
A_{z}=\epsilon^{2}(z-1) & 0<z<1 \\
A_{z}=-\frac{z+H}{H} & -H<z<0 .
\end{array}
$$

\section{Solution for an Infinitely Deep Ocean}

The total depth of the ocean is typically on the order of a hundred times the depth of the mixed layer; thus, the limit of infinite depth is considered. The initial condition is taken to be equation (21) with $H \rightarrow \infty$. The boundary condition for $z \rightarrow-\infty$ is taken to be $A_{z z} \rightarrow 0$, corresponding to the near-inertial velocities vanishing at infinite depth (see equation (3)). This limit does not invalidate the use of equation (1) which assumed hydrostatic balance and thus holds for the ocean having depth much smaller than the horizontal scales. The ocean still in reality has finite depth, but for depths just below the mixed layer it is effectively infinitely deep. Of course, this limit excludes the possibility of reflections off the bottom of the ocean which may be important (see, e.g., [9]); thus, the results should be viewed as what would happen in the absence of such reflections. Finally, the boundary condition for $z=0^{-}$ given by equation (19) is used. For convenience, the problem to be solved for the semi-infinite domain $z<0$ is summarized:

$$
\begin{array}{rl}
A_{z z t}+\frac{i}{2} A_{y y}+i y A_{z z}=0 & z<0 \\
A_{z t}+i y A_{z}-\frac{i}{2} A_{y y}=0 & z=0^{-} \\
A_{z z} \rightarrow 0 & z \rightarrow-\infty \\
A_{z}=-1 & t=0 .
\end{array}
$$

\subsection{Solution by Laplace Transforms}

Making the ansatz and definitions

$$
A(y, z, t)=e^{-i y t} B(z, t), \quad T \equiv \frac{t^{3}}{3}, \quad \tilde{B}(z, T) \equiv B(z, t)
$$

implies that

$$
\begin{array}{rl}
\tilde{B}_{z z T}-\frac{i}{2} \tilde{B}=0 & z<0 \\
\tilde{B}_{z T}+\frac{i}{2} \tilde{B}=0 & z=0^{-} \\
\tilde{B}_{z z} \rightarrow 0 & z \rightarrow-\infty \\
\tilde{B}_{z}=-1 & T=0 .
\end{array}
$$


Laplace transforming equations (23)-(25) in time gives

$$
\begin{array}{rlrl}
b(z, p) \equiv \mathcal{L}[B(z, t)]=\int_{0}^{\infty} & \tilde{B}(z, T) e^{-p T} d T \\
p b_{z z}-\tilde{B}_{z z}(z, 0)-\frac{i}{2} b & =0 & & z<0 \\
b_{z z} & \rightarrow 0 & & z \rightarrow-\infty \\
p b_{z}-\tilde{B}_{z}(z, 0)+\frac{i}{2} b & =0 & & z=0^{-}
\end{array}
$$

$\tilde{B}_{z z}(z, 0)=0$ from equation (26); thus the solution to equation (28) satisfying the boundary condition (29) is

$$
\begin{aligned}
b(z, p) & =f(p) \exp \left(\frac{\alpha z}{\sqrt{p}}\right) \\
\alpha & \equiv \frac{1}{\sqrt{2}} e^{i \pi / 4}=\frac{1}{2}(1+i) .
\end{aligned}
$$

Using boundary condition (30) with $\tilde{B}_{z}(z, 0)=-1$ from (26) determines $f(p)$, giving

$$
b(z, p)=-\frac{1}{\alpha} \frac{1}{\sqrt{p}+\alpha} \exp \left(\frac{\alpha z}{\sqrt{p}}\right) .
$$

In principle, the problem is solved at this stage; inverting this Laplace transform gives $\tilde{B}(z, T)$, then $A(y, z, t)$ is obtained from equation (22). This can then be differentiated in order to determine various quantities of interest. In practice, it is more convenient to first differentiate with respect to $z$ as appropriate, and then to invert the transform. This inversion may be done in three different ways: first, for $z=0^{-}$analytical expressions can be found, and these can be used to find analytical expressions for $0<z<1$; second, for other $z$ values the inversion may be done numerically; and third, the asymptotic behavior can be determined by the method of steepest descents. But first, expressions for the flux of energy and shear in terms of the field $B(z, t)$ are given.

\subsection{Flux of Energy and Shear}

The energy and shear fluxes given in equations (16) and (17) may be related to the field $B$ using equation (22), giving

$$
\begin{aligned}
F_{E}(t ; d) & =\left.\frac{i}{2} t^{2}\left(B_{z}^{*} B-B_{z} B^{*}\right)\right|_{z=-d} \\
& =\left.t^{2}\left[\operatorname{Im}\left(B_{z}\right) \operatorname{Re}(B)-\operatorname{Re}\left(B_{z}\right) \operatorname{Im}(B)\right]\right|_{z=-d} \\
F_{S}(t ; d) & =\left.\frac{i}{2} t^{2}\left(B_{z z}^{*} B_{z}-B_{z z} B_{z}^{*}\right)\right|_{z=-d} \\
& =\left.t^{2}\left[\operatorname{Im}\left(B_{z z}\right) \operatorname{Re}\left(B_{z}\right)-\operatorname{Re}\left(B_{z z}\right) \operatorname{Im}\left(B_{z}\right)\right]\right|_{z=-d}
\end{aligned}
$$


These fluxes are independent of $y$ so the dependence on this variable is suppressed. Also, initially $A_{z z}=0$ for $z<0$. Integrating equation (15) with respect to time then gives

$$
\int_{V_{d}}\left|A_{z z}\right|^{2} d V=\int_{-\infty}^{\infty} d x \int_{-\infty}^{\infty} d y \int_{0}^{t} F_{E}(t ; d) d t
$$

Thus

$$
E(t ; d) \equiv \int_{0}^{t} F_{E}(t ; d) d t
$$

is the total amount of $\mathrm{HKE}$ which has penetrated into the region $z<-d$. Calculating the initial amount of HKE in the mixed layer for the initial velocity profile shows that $E(t ; d) \rightarrow 1$ corresponds to all energy originally in the mixed layer having reached depths below $z=-d$. The quantity

$$
S(t ; d) \equiv \int_{0}^{t} F_{S}(t ; d) d t
$$

is the integrated shear flux which has penetrated into the region $z<-d$. Note that the initial value of the shear for $z<0$ and $z>0$ is zero, but the total initial shear is infinite because of the discontinuity in the initial velocity profile at $z=0$.

\subsection{Analytical Solution for $z=0^{-}$}

For $z=0^{-}$, inverse Laplace transforms are found in or deduced from a table in [1]. From equation (31),

$$
b\left(0^{-}, p\right)=-\frac{1}{\alpha} \frac{1}{\sqrt{p}+\alpha}
$$

and one obtains

$$
\tilde{B}\left(0^{-}, T\right)=\mathcal{L}^{-1}\left[b\left(0^{-}, p\right)\right]=-\frac{1}{\alpha \sqrt{\pi T}}+e^{\alpha^{2} T} \operatorname{erfc}(\alpha \sqrt{T})
$$

This is converted to the original time $t$ using equation (22):

$$
B\left(0^{-}, t\right)=-\frac{1}{\alpha}\left(\frac{3}{\pi t^{3}}\right)^{1 / 2}+e^{\alpha^{2} t^{3} / 3} \operatorname{erfc}\left(\frac{\alpha}{\sqrt{3}} t^{3 / 2}\right)
$$

Differentiating equation (31) with respect to $z$ and evaluating at $z=0^{-}$gives

$$
\begin{aligned}
& b_{z}\left(0^{-}, p\right)=-\frac{1}{p+\alpha \sqrt{p}} \Rightarrow B_{z}\left(0^{-}, t\right)=-e^{\alpha^{2} t^{3} / 3} \operatorname{erfc}\left(\frac{\alpha}{\sqrt{3}} t^{3 / 2}\right) \\
& b_{z z}\left(0^{-}, t\right)=-\frac{\alpha}{p(\sqrt{p}+\alpha)} \Rightarrow B_{z z}\left(0^{-}, t\right)=e^{\alpha^{2} t^{3} / 3} \operatorname{erfc}\left(\frac{\alpha}{\sqrt{3}} t^{3 / 2}\right)-1 \\
& b_{z z z}\left(0^{-}, p\right)=-\frac{\alpha^{2}}{p(p+\alpha \sqrt{p})} \\
& \Rightarrow \quad B_{z z z}\left(0^{-}, t\right)=1-e^{\alpha^{2} t^{3} / 3} \operatorname{erfc}\left(\frac{\alpha}{\sqrt{3}} t^{3 / 2}\right)-2 \alpha\left(\frac{t^{3}}{3 \pi}\right)^{1 / 2}
\end{aligned}
$$


The solid lines (labelled d $=0$ ) in Figure 1 show the quantities $F_{E}, F_{S}, E$, and $S$ calculated using the results of this section. $F_{E}$ peaks at the nondimensionalized time $t \approx 0.62$; for the typical values quoted in Section 3.1, this corresponds to about a week after the storm. From Figure 1(b) and using the fact that whatever energy flows through $z=0^{-}$must have initally been in the mixed layer, we see that by $t=1$ (approximately 11.5 days after the storm) nearly half of the energy associated with horizontal NIO currents caused by the storm has left the mixed layer. By $t=2$ (approximately 23 days after the storm) $82 \%$ has left, while by $t=3$ (just over a month after the storm) $93 \%$ has left. Although this simplified model cannot be expected to capture the full complexity of a real storm over the ocean, it does give reasonable estimates for the time scale for which the decay of NIO energy occurs: [8] found that the mixed layer inertial energy was reduced to background levels by 21 days after the storm. Both the shear flux $F_{S}$ and the integrated shear $S$ increase monotonically with time, an artifact of the initial velocity profile being discontinuous at $z=0$.
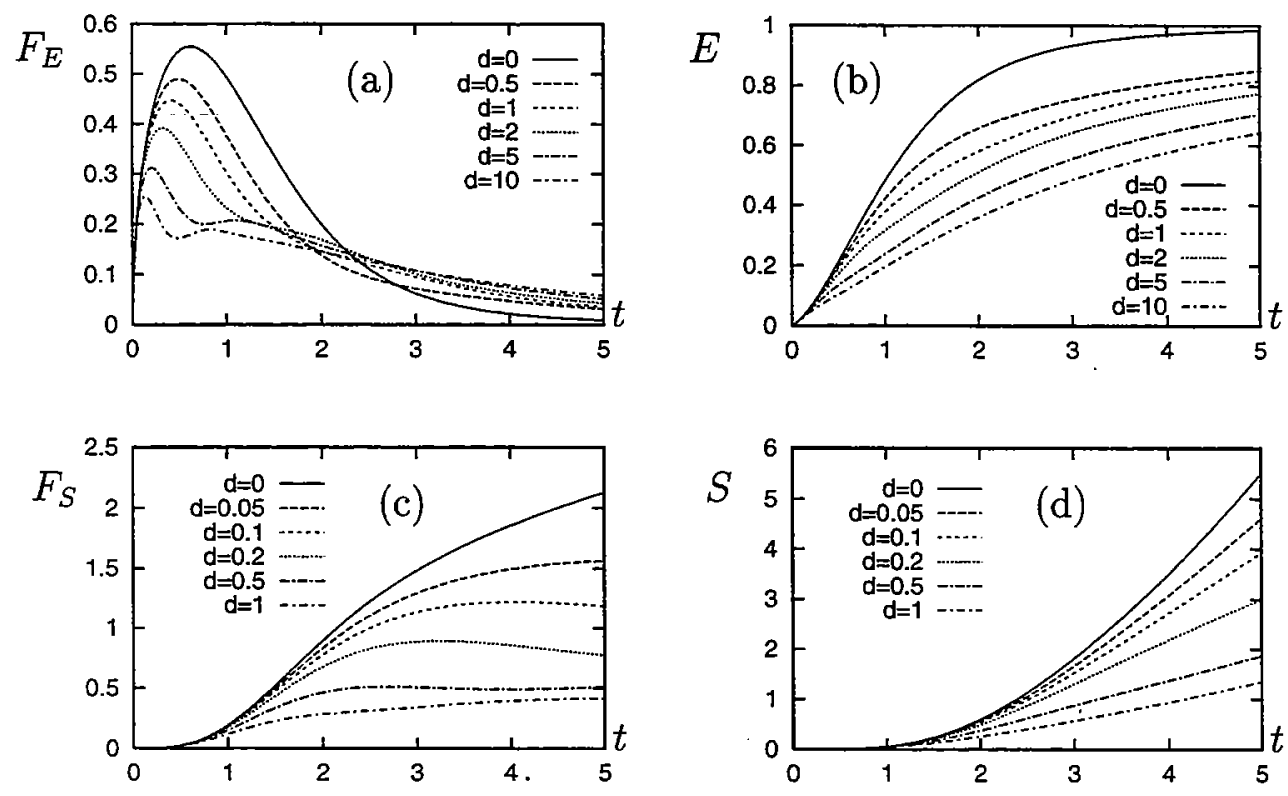

Figure 1: (a) $F_{E}(t ; d)$, (b) $E(t ; d)$, (c) $F_{S}(t ; d)$, and (d) $S(t ; d)$ for different distances $d$ below the base of the mixed layer. These show instantaneous and integrated fluxes of energy and shear; see the text for precise definitions. The solid lines (labelled $d=0$ ) give results at $z=0^{-}$.

\subsection{Analytical Solution for the Mixed Layer}

Expanding $B(z, t)=B_{0}(z, t)+\epsilon^{2} B_{2}(z, t)+\mathcal{O}\left(\epsilon^{4}\right)$ for the interval $0<z<1$,

$$
\begin{aligned}
& A_{0}(y, z, t)=e^{-i y t} B_{0}(z, t) \equiv e^{-i y t} \tilde{B}_{0}(z, T), \\
& A_{2}(y, z, t)=e^{-i y t} B_{2}(z, t) \equiv e^{-i y t} \tilde{B}_{2}(z, T),
\end{aligned}
$$


where $T=t^{3} / 3$ as before. Equation (18) then implies that

$$
\tilde{B}_{2 z z T}-\frac{i}{2} \tilde{B}_{0}=0 \quad 0<z<1 .
$$

This is Laplace transformed to give

$$
p b_{2 z z}-\tilde{B}_{2 z z}(z, 0)-\frac{i}{2} b_{0}=0
$$

where $b_{2}=\mathcal{L}\left[\tilde{B}_{2}\right]$ and $b_{0}=\mathcal{L}\left[\tilde{B}_{0}\right]$. The initial condition within the mixed layer is $A_{z z}=\epsilon^{2}$, so $A_{2 z z}=1$ at $t=0$; thus $\tilde{B}_{2 z z}(z, 0)=1$. Now, $A$ is continuous across $z=0$, and $A=A_{0}$ to leading order in $\epsilon$. This implies that $\tilde{B}_{0}\left(0^{+}, T\right)=\tilde{B}\left(0^{-}, T\right)$ to leading order in $\epsilon$. Also, $A_{0}$ and hence $\tilde{B}_{0}$ are independent of $z$. Laplace transforming, we conclude that the value of $b_{0}$ for $0<z<1$ is equal to $b\left(0^{-}, p\right)$. Using all of this and equation (31) evaluated at $z=0^{-}$in equation (41),

$$
b_{2 z z}=\frac{1}{p}-\frac{i}{2 \alpha} \frac{1}{\sqrt{p}+\alpha} \Rightarrow B_{2 z z}=e^{\alpha^{2} t^{3} / 3} \operatorname{erfc}\left(\frac{\alpha}{\sqrt{3}} t^{3 / 2}\right)
$$

Thus,

$$
A_{2 z z}(y, t)=e^{-i y t} e^{\alpha^{2} t^{3} / 3} \operatorname{erfc}\left(\frac{\alpha}{\sqrt{3}} t^{3 / 2}\right)
$$

The local HKE is, to leading order, $\left|A_{2 z z}\right|^{2}=\left|A_{z z} / \epsilon^{2}\right|^{2}$; the total HKE contained within the mixed layer is

$$
\int_{\mathrm{ML}}\left|\frac{A_{z z}}{\epsilon^{2}}\right|^{2} d V=\int_{\mathrm{ML}}\left|A_{2 z z}\right|^{2} d V .
$$

Using $\alpha^{2}=i / 2$, the amount of HKE per unit volume within the mixed layer is

$$
\epsilon_{\mathrm{ML}} \equiv\left|\operatorname{erfc}\left(\frac{\alpha}{\sqrt{3}} t^{3 / 2}\right)\right|^{2}
$$

From [1],

$$
\begin{aligned}
e^{\alpha^{2} t^{3} / 3} \operatorname{erfc}\left(\frac{\alpha}{\sqrt{3}} t^{3 / 2}\right) & \sim \sqrt{\frac{3}{\pi}} \frac{1}{\alpha t^{3 / 2}} \quad t \rightarrow \infty \\
\Rightarrow \quad \epsilon_{\mathrm{ML}} & \sim \frac{6}{\pi t^{3}} \quad t \rightarrow \infty .
\end{aligned}
$$

This asymptotic relationship is confirmed in Figure 2. Since $A_{2 z z}$ is independent of $z$, to leading order in $\epsilon$ the shear within the mixed layer is zero. 

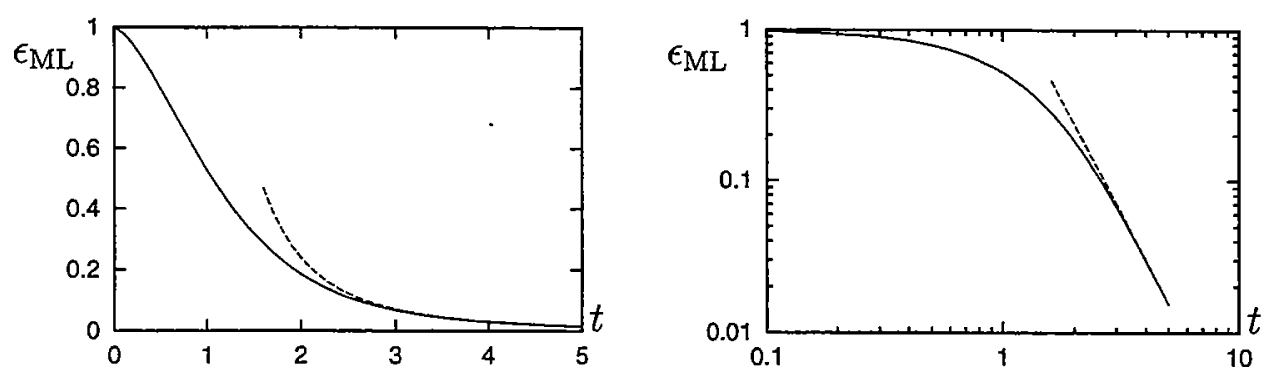

Figure 2: $\epsilon_{\mathrm{ML}}$ gives the HKE per unit volume in the mixed layer. The solid line shows the exact result and the dashed line shows the asymptotic result, shown both for linear and logarithmic axes.

\subsection{Solution for Other Depths}

A tractable analytical form for the inverse Laplace transform of equation (31) for $z \neq 0$ could not be found; however, it may be inverted numerically as described in this section. The inverse Laplace transform is given by

$$
\tilde{B}(z, T)=-\frac{1}{2 \pi \alpha i} \int_{\mathcal{B}} \frac{\exp \left(\frac{\alpha z}{\sqrt{p}}+p T\right)}{\sqrt{p}+\alpha} d p,
$$

where $\mathcal{B}$ is the Bromwich contour. Here the branch cut for the square root is taken to be along the negative real axis, and the principal branch is chosen. The integrand does not have any poles on this sheet of the Riemann surface. Such a pole would satisfy $\sqrt{p}+\alpha=0$, and would be given by $p_{p}=\alpha^{2}=e^{i \pi / 2} / 2$; however, $\sqrt{p_{p}}+\alpha=\sqrt{2} e^{i \pi / 4} \neq 0$ and thus there is no pole. It is useful to make the change of variables (valid for $z \neq 0$ and $T \neq 0$ )

$$
p=\left(-\frac{z}{T}\right)^{2 / 3} w, \quad \xi \equiv\left(z^{2} T\right)^{1 / 3}, \quad \eta \equiv\left(-\frac{z}{T}\right)^{1 / 3}
$$

Then,

$$
\begin{aligned}
\tilde{B}(z, T) & =-\frac{\eta^{2}}{2 \pi \alpha i} \int_{\mathcal{B}} \frac{\exp \left[\xi\left(-\frac{\alpha}{\sqrt{w}}+w\right)\right]}{\eta \sqrt{w}+\alpha} d w \\
& \equiv-\frac{\eta^{2}}{2 \pi \alpha i} \int_{\mathcal{B}} g(w ; \xi, \eta) d w .
\end{aligned}
$$

Defining the contour $\mathcal{C}$ as in Figure 3 and using the facts that the contributions from $\mathcal{C}_{1}$ and $\mathcal{C}_{2}$ vanish and that there are no poles,

$$
\int_{\mathcal{C}} g(w ; \xi, \eta) d w=\left\{\int_{\mathcal{B}}+\int_{\mathrm{AB}}+\int_{\mathrm{BC}}+\int_{\mathrm{CD}}\right\} g(w ; \xi, \eta) d w=0 .
$$

For the path $\mathrm{AB}, w=r e^{i \pi}$ and

$$
I_{\mathrm{AB}} \equiv \int_{\mathrm{AB}} g(w ; \xi, \eta)=\int_{1}^{\infty} \frac{e^{-r \xi} e^{i \alpha \xi / \sqrt{r}}}{\eta i \sqrt{r}+\alpha} d r
$$




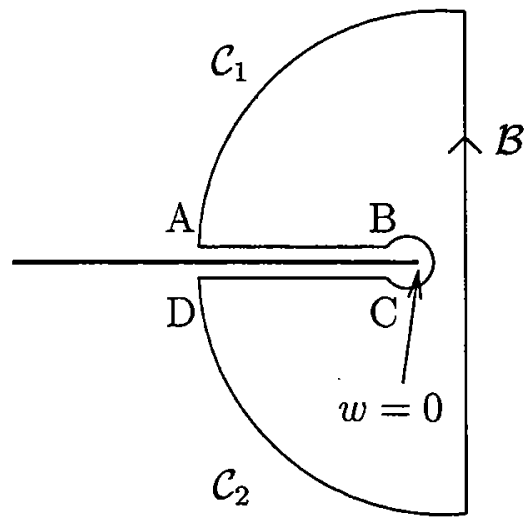

$w$

Figure 3: Contour used to determine inverse Laplace transformations. Here $A=\left(-\infty, 0^{+}\right)$, $\mathrm{B}=\left(-1,0^{+}\right), \mathrm{C}=\left(-1,0^{-}\right)$, and $\mathrm{D}=\left(-\infty, 0^{-}\right)$.

For the path $\mathrm{BC}, w=e^{i \theta}$ and

$$
I_{\mathrm{BC}} \equiv \int_{\mathrm{BC}} g(w ; \xi, \eta)=-i \int_{-\pi}^{\pi} \frac{\exp \left[\xi\left(-\alpha e^{-i \theta / 2}+e^{i \theta}\right)\right] e^{i \theta}}{\eta e^{i \theta / 2}+\alpha} d \theta
$$

Finally, for the path CD, $w=r e^{-i \pi}$ and

$$
I_{\mathrm{CD}} \equiv \int_{\mathrm{CD}} g(w ; \xi, \eta)=-\int_{1}^{\infty} \frac{e^{-r \xi} e^{-i \alpha \xi / \sqrt{r}}}{-\eta i \sqrt{r}+\alpha} d r
$$

Specifying $z$ and $T$ fixes $\xi$ and $\eta$. The integrals $I_{\mathrm{AB}}, I_{\mathrm{BC}}$, and $I_{\mathrm{CD}}$ are well-behaved and can be calculated numerically. Then

$$
\tilde{B}(z, T)=\frac{\eta^{2}}{2 \pi \alpha i}\left(I_{\mathrm{AB}}+I_{\mathrm{BC}}+I_{\mathrm{CD}}\right) .
$$

Differentiating (31) with respect to $z$,

$$
\begin{aligned}
b_{z}(z, p) & =-\frac{1}{p+\alpha \sqrt{p}} \exp \left(\frac{\alpha z}{\sqrt{p}}\right), \\
b_{z z}(z, p) & =-\frac{\alpha}{p(\sqrt{p}+\alpha)} \exp \left(\frac{\alpha z}{\sqrt{p}}\right), \\
b_{z z z}(z, p) & =-\frac{\alpha^{2}}{p(p+\alpha \sqrt{p})} \exp \left(\frac{\alpha z}{\sqrt{p}}\right) .
\end{aligned}
$$

Using the contour in Figure 3, we find

$$
\begin{aligned}
\tilde{B}_{z}(z, T) & =\frac{\eta^{2}}{2 \pi i}\left(I_{\mathrm{AB}}^{(1)}+I_{\mathrm{BC}}^{(1)}+I_{\mathrm{CD}}^{(1)}\right) \\
\tilde{B}_{z z}(z, T) & =\frac{\alpha \eta^{2}}{2 \pi i}\left(I_{\mathrm{AB}}^{(2)}+I_{\mathrm{BC}}^{(2)}+I_{\mathrm{CD}}^{(2)}\right) \\
\tilde{B}_{z z z}(z, T) & =\frac{\alpha^{2} \eta^{2}}{2 \pi i}\left(I_{\mathrm{AB}}^{(3)}+I_{\mathrm{BC}}^{(3)}+I_{\mathrm{CD}}^{(3)}\right)
\end{aligned}
$$


where

$$
\begin{gathered}
I_{\mathrm{AB}}^{(1)}=\int_{1}^{\infty} \frac{e^{-r \xi} e^{i \alpha \xi / \sqrt{r}}}{-\eta^{2} r+\eta \alpha i \sqrt{r}} d r, \quad I_{\mathrm{BC}}^{(1)}=-i \int_{-\pi}^{\pi} \frac{\exp \left[\xi\left(-\alpha e^{-i \theta / 2}+e^{i \theta}\right)\right]}{\eta^{2}+\eta \alpha e^{-i \theta / 2}} d \theta \\
I_{\mathrm{CD}}^{(1)}=\int_{1}^{\infty} \frac{e^{-r \xi} e^{-i \alpha \xi / \sqrt{r}}}{\eta^{2} r+\eta \alpha i \sqrt{r}} d r, \quad I_{\mathrm{AB}}^{(2)}=-\int_{1}^{\infty} \frac{e^{-r \xi} e^{i \alpha \xi / \sqrt{r}}}{\eta^{3} i r^{3 / 2}+\eta^{2} \alpha r} d r \\
I_{\mathrm{BC}}^{(2)}=-i \int_{-\pi}^{\pi} \frac{\exp \left[\xi\left(-\alpha e^{-i \theta / 2}+e^{i \theta}\right)\right]}{\eta^{3} e^{i \theta / 2}+\eta^{2} \alpha} d \theta, \quad I_{\mathrm{CD}}^{(2)}=\int_{1}^{\infty} \frac{e^{-r \xi} e^{-i \alpha \xi / \sqrt{r}}}{-\eta^{3} i r^{3 / 2}+\eta^{2} \alpha r} d r \\
I_{\mathrm{AB}}^{(3)}=\int_{1}^{\infty} \frac{e^{-r \xi} e^{i \alpha \xi / \sqrt{r}}}{\eta^{4} r^{2}-\eta^{3} \alpha i r^{3 / 2}} d r, \quad I_{\mathrm{BC}}^{(3)}=-i \int_{-\pi}^{\pi} \frac{\exp \left[\xi\left(-\alpha e^{-i \theta / 2}+e^{i \theta}\right)\right]}{\eta^{4} e^{i \theta}+\alpha \eta^{3} e^{i \theta / 2}} d \theta \\
I_{\mathrm{CD}}^{(3)}=-\int_{1}^{\infty} \frac{e^{-r \xi} e^{-i \alpha \xi / \sqrt{r}}}{\eta^{4} r^{2}+\alpha \eta^{3} i r^{3 / 2}} d r .
\end{gathered}
$$

All of these integrals are well-behaved and can be calculated numerically.

\subsection{Results}

\subsubsection{Fluxes and Integrated Fluxes}

The quantities $F_{E}(t ; d), F_{S}(t ; d), E(t ; d)$, and $S(t ; d)$ may now be calculated and results are shown in Figure 1. From Figure 1(b), as noted in Section 4.3, by $t=1$ nearly half of the total horizontal near inertial energy has left the mixed layer; only about $38 \%$ of the total energy has penetrated below $z=-1$. By $t=2,82 \%$ of the total energy has left the mixed layer, but only $58 \%$ of the total energy has penetrated below $z=-1$. Thus, at $t=2$ (using the typical values quoted in Section 3.1, about 23 days after the storm) nearly a quarter of the total energy is contained in the distance $H_{\text {mix }}$ immediately beneath the mixed layer. Figure 1 (d) demonstrates that the shear tends to be localized just below the base of the mixed layer. For example, by $t=5$ the integrated shear flux which has entered the mixed layer is, in nondimensional units, about 5.5. The integrated shear flux which has penetrated below $z=-0.05$ is 4.6 , and the integrated shear flux which has penetrated below $z=-1$ is 1.35 .

\subsubsection{Vertical Profiles}

Using the expressions from Section 4.5 and equation (22) it is now also possible to calculate vertical profiles of physically relevant quantities. Figure $4(\mathrm{a}, \mathrm{b})$ shows the vertical dependence of the HKE, $u^{2}+v^{2}=\left|A_{z z}\right|^{2}$, and the vertical shear, $u_{z}^{2}+v_{z}^{2}=\left|A_{z z z}\right|^{2}$, at different times for $y=0$. For both quantities, as time increases the instantaneous distribution becomes more sharply peaked near the base of the mixed layer. The maximum value of $u_{z}^{2}+v_{z}^{2}$ increases without bound as time increases, but the maximum value of $u^{2}+v^{2}$ remains bounded (asymptotically approaching unity) because of energy conservation. Figure 4(c,d) shows the vertical dependence of the fluxes $F_{E}$ and $F_{S}$. 

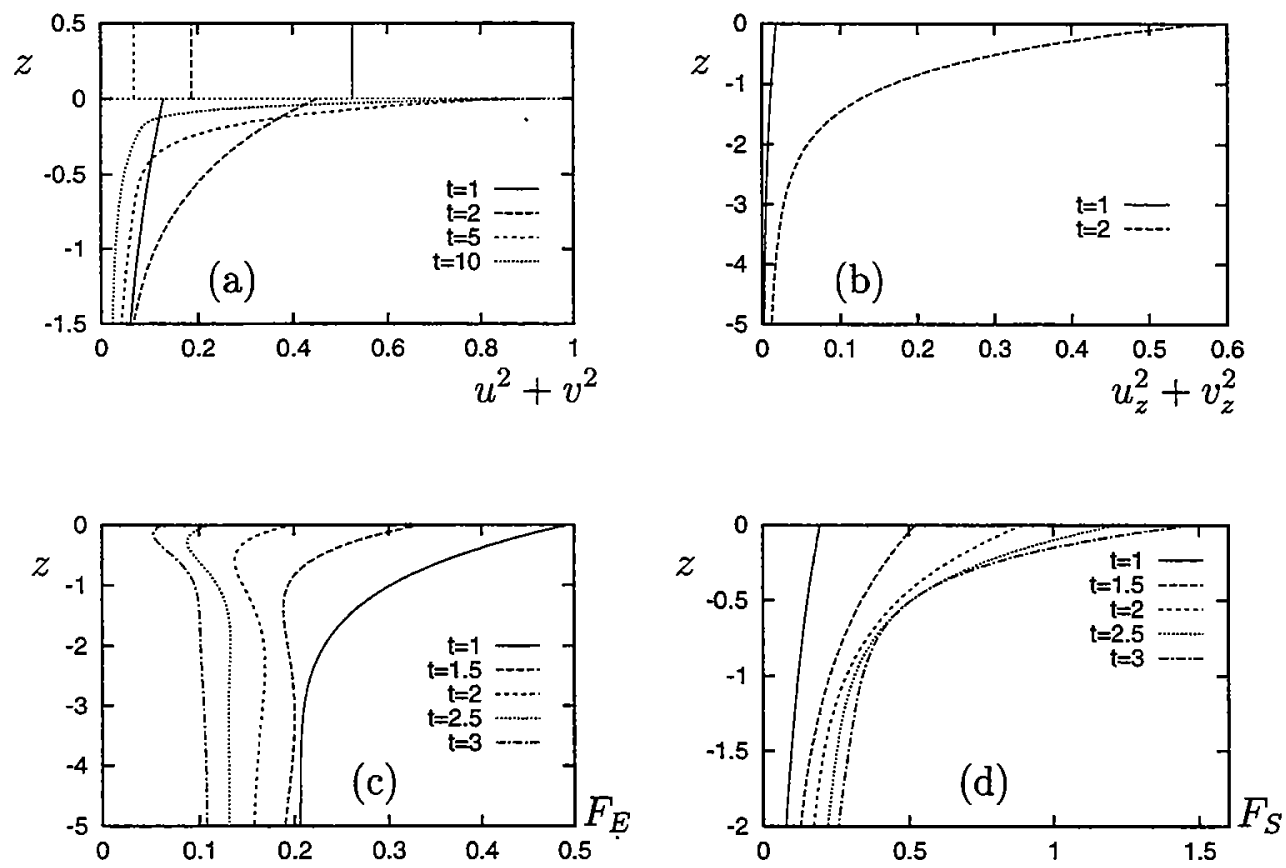

Figure 4: Vertical profiles of (a) $u^{2}+v^{2}$ and (b) $u_{z}^{2}+v_{z}^{2}$ at $y=0$ for different times showing the decay of energy from the mixed layer $(0<z<1)$ and resultant behavior in the interior $(z<0)$. Note that $u_{z}^{2}+v_{z}^{2}=0$ in the mixed layer to leading order in $\epsilon$. Also, vertical profiles of (c) $F_{E}(t,|z|)$, and (d) $F_{S}(t,|z|)$ for different times. Note the different vertical scales.

\subsubsection{Back-Rotated Velocity and Shear}

Finally, consider the back-rotated velocity $A_{z z}=e^{i f_{0} t}(u+i v)$. This filters out the purely intertial motion at frequency $f_{0}$. Similarly, the back-rotated shear is defined to be $A_{z z z}=$ $e^{i f_{0} t}\left(u_{z}+i v_{z}\right)$. The amplitudes of the back-rotated velocity and shear at different depths are shown as time series in Figure 5. At a given depth, the magnitude of the back-rotated velocity reaches a peak value shortly after the storm, then decays away, while the magnitude of the back-rotated shear increases monotonically with time. Note that Figure 4, may also be interpreted in terms of the back-rotated velocity and shear.

Back-rotated velocity and shear may be represented by hodographs which respectively show the vectors $\left(\operatorname{Re}\left(A_{z z}\right), \operatorname{Im}\left(A_{z z}\right)\right)$ and $\left(\operatorname{Re}\left(A_{z z z}\right), \operatorname{Im}\left(A_{z z z}\right)\right)$ as curves parametrized by time. For $f_{0}>0$, if these curves are traced out in a clockwise (counterclockwise) fashion, the corresponding motion has frequency larger (smaller) than $f_{0}$. Figure 6 shows the backrotated velocity and shear at $y=0, z=-1$. The hodographs for both quantities start at the origin and are traced out in a clockwise fashion. The back-rotated velocity starts out in the third quadrant, reaches a peak in magnitude in the second quadrant, then decays in magnitude spiralling back to the origin. The back-rotated shear also starts out in the third quadrant, and monotonically increases in magnitude while spiralling outward. Note that the lines labelled $z=-1$ in Figure 5 give the radii of these hodographs as functions of time. The depth dependence of the back-rotated velocity is seen by comparing Figure 6 
with Figure 7(a), where both have $y=0$ and thus the same value of the Coriolis parameter. Qualitatively the results are the same, but closer to the mixed layer the direction change of the back-rotated velocity becomes slower, meaning that the frequency is closer to $f_{0}$. An idea of the latitudinal dependence is seen by comparing Figure 6 with Figure $7(\mathrm{~b}, \mathrm{c})$. At $y=1$ the hodograph is traced out in a clockwise fashion as for $y=0$, but at $y=-2$ it is traced out in a counterclockwise fashion.
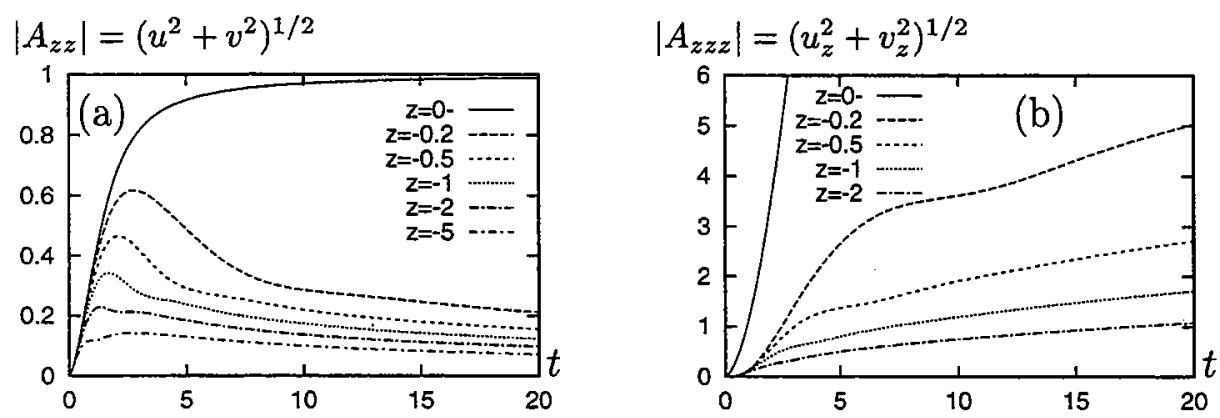

Figure 5: Time series for $\left|A_{z z}\right|$ and $\left|A_{z z z}\right|$ at different fixed $z$ values.

\subsection{Asymptotic Behavior}

Making the change of variables (44), the inverse Laplace transforms of $b, b_{z}, b_{z z}$, and $b_{z z z}$ can be written in the form

$$
\begin{aligned}
I(\xi) & =\int_{\mathcal{B}} f(w, \eta) e^{\xi h(w)} d w \\
h(w) & \equiv-\frac{\alpha}{\sqrt{w}}+w .
\end{aligned}
$$

The asymptotic behavior of this in the limit of large $\xi$ with $\eta$ fixed can be determined by the method of steepest descents. This involves determining the saddle points of $h(w)$ (i.e., points satisfying $h^{\prime}(w)=0$ ) and deforming the contour $\mathcal{B}$ so as to pass through each saddle point along a path of constant $\operatorname{Im}(h(w))$ such that $\operatorname{Re}(h(w))$ has a local maximum at each saddle point. Supposing that there is a simple saddle $\left(h^{\prime \prime}\left(w_{0}\right) \neq 0\right)$ at $w=w_{0}$,

$$
I(\xi) \sim \frac{\sqrt{2 \pi} f\left(w_{0}\right) e^{\xi h\left(w_{0}\right)} e^{i \gamma}}{\left|\xi h^{\prime \prime}\left(w_{0}\right)\right|^{1 / 2}} .
$$

Here $\gamma$ is the angle relative to the positive real axis at which the path satisfying the above conditions passes through the saddle. Letting $h^{\prime \prime}\left(w_{0}\right)=a e^{i \sigma}, \gamma=-\sigma / 2+\pi / 2$ or $\gamma=$ $-\sigma / 2+3 \pi / 2$; the appropriate choice is determined by making sure that $h(w)$ only has local maxima at the saddle points and no where else along the deformed contour.

Taking the branch cut for the square root along the negative real axis, for this problem, there are saddles at

$$
w_{1}=\frac{1}{2} e^{-i \pi / 2}, \quad w_{2}=\frac{1}{2} e^{i \pi 5 / 6}
$$



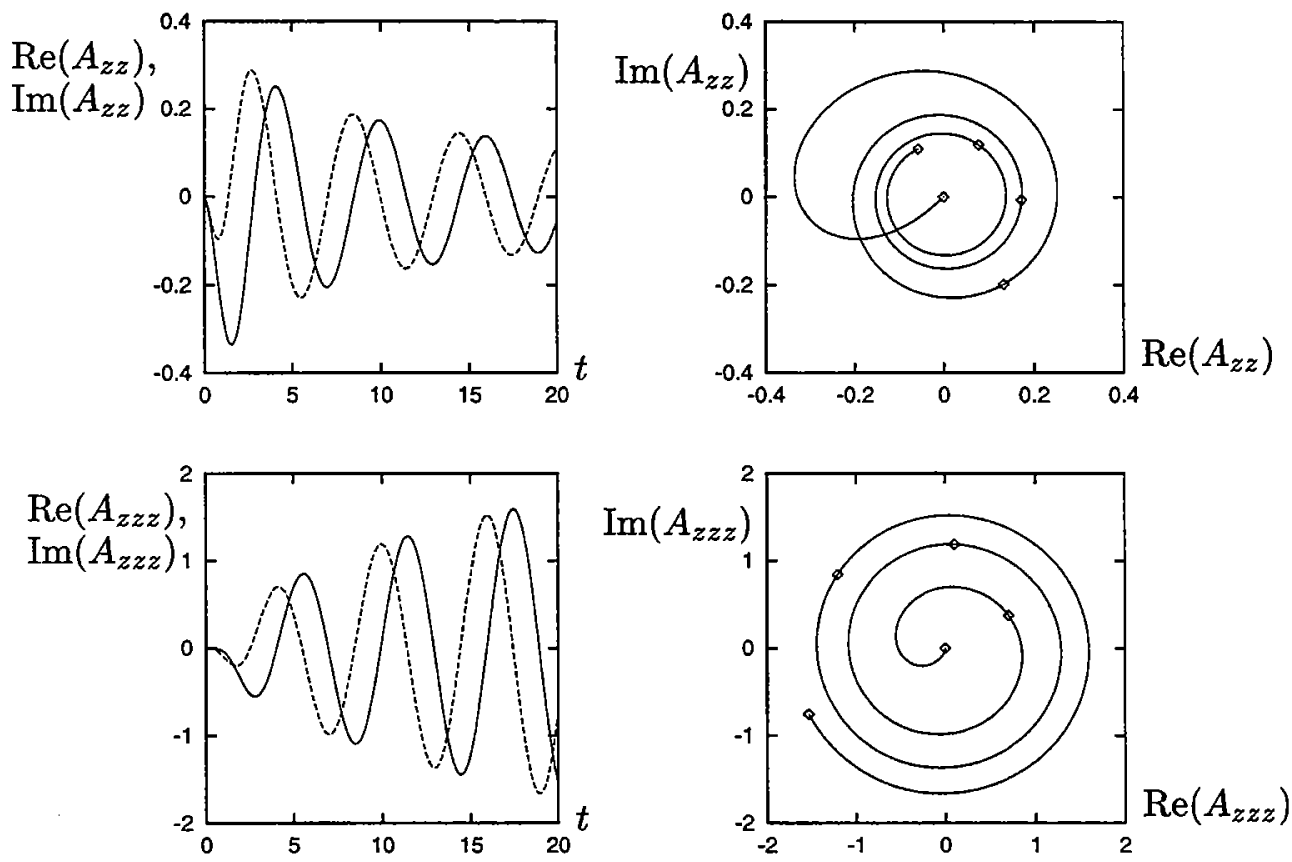

Figure 6: Back-rotated velocity and shear at $z=-1, y=0$. In these and the time series in Figure 7, the solid and dashed lines show real and imaginary parts, respectively. The diamonds are drawn at $t=0,5,10,15,20$.

with

$$
\begin{aligned}
h\left(w_{1}\right) & =-\frac{3}{2} i, & h\left(w_{2}\right)=-\frac{3 \sqrt{3}}{4}+\frac{3}{4} i \\
h^{\prime \prime}\left(w_{1}\right) & =3 i, & h^{\prime \prime}\left(w_{2}\right)=3 e^{-i \pi 5 / 6} .
\end{aligned}
$$

Figure 8 shows the contours of constant real and imaginary parts of $h(w)$. The deformed contour is taken to pass through both of these saddles, and passes to the right of the origin in order to avoid the branch cut. Since $h\left(w_{1}\right)$ is purely imaginary and $h\left(w_{2}\right)$ has negative real part, it is immediately seen that the contribution from $w_{2}$ will be exponentially small compared with the contribution from $w_{1}$ in the limit $\xi \rightarrow \infty$. Using $\gamma=\pi / 4$ for the passage through $w_{1}$, in the limit $\xi \rightarrow \infty$ with $\eta$ fixed

$$
I(\xi) \sim \sqrt{\frac{2 \pi}{3}} \xi^{-1 / 2} f\left(w_{1}\right) e^{i(-3 \xi / 2+\pi / 4)}
$$



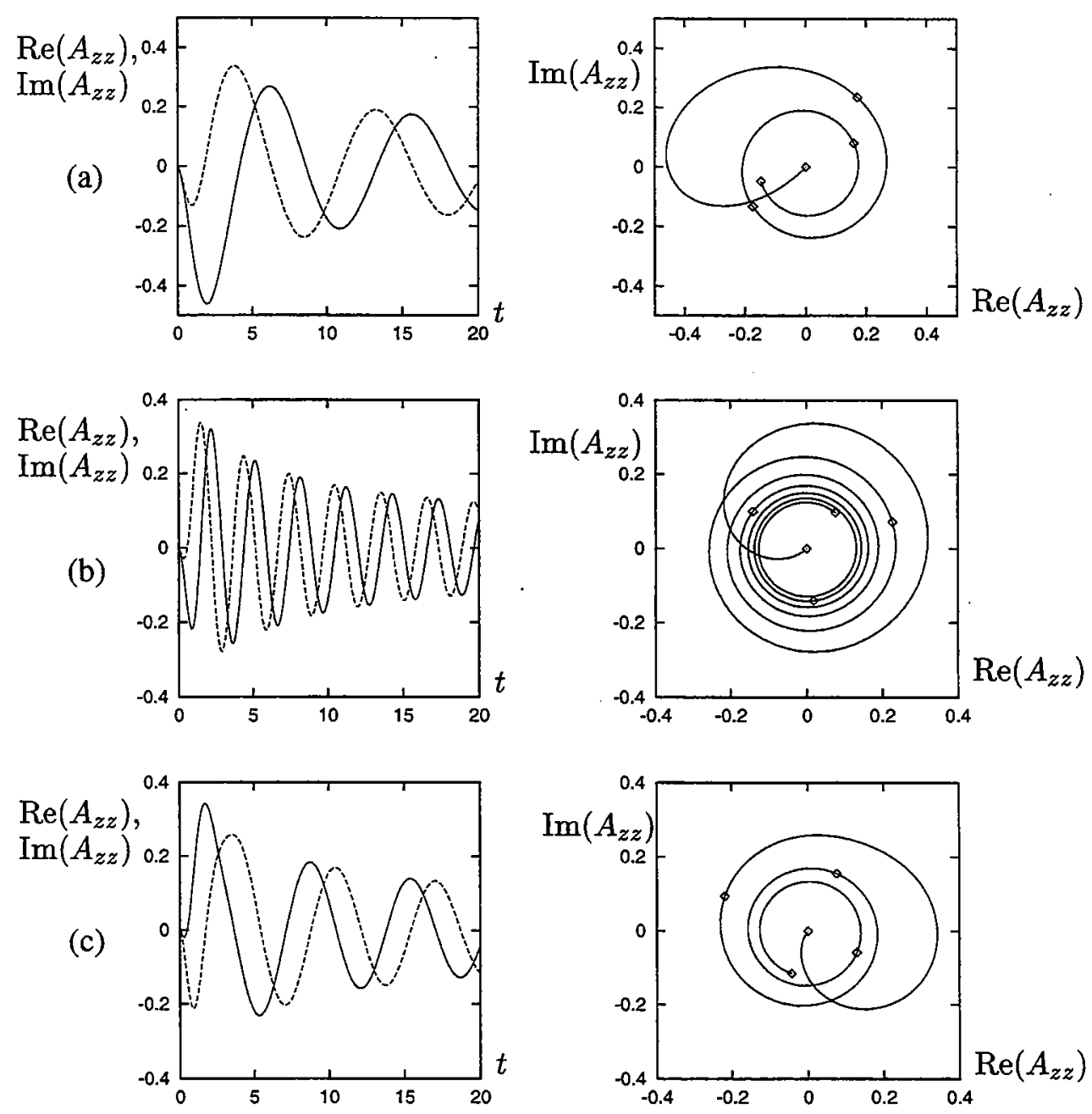

Figure 7: Back-rotated velocity for (a) $z=-0.5, y=0$, (b) $z=-1, y=1$, and (c) $z=$ $-1, y=-2$. The conventions of Figure 6 are also used for this Figure. 


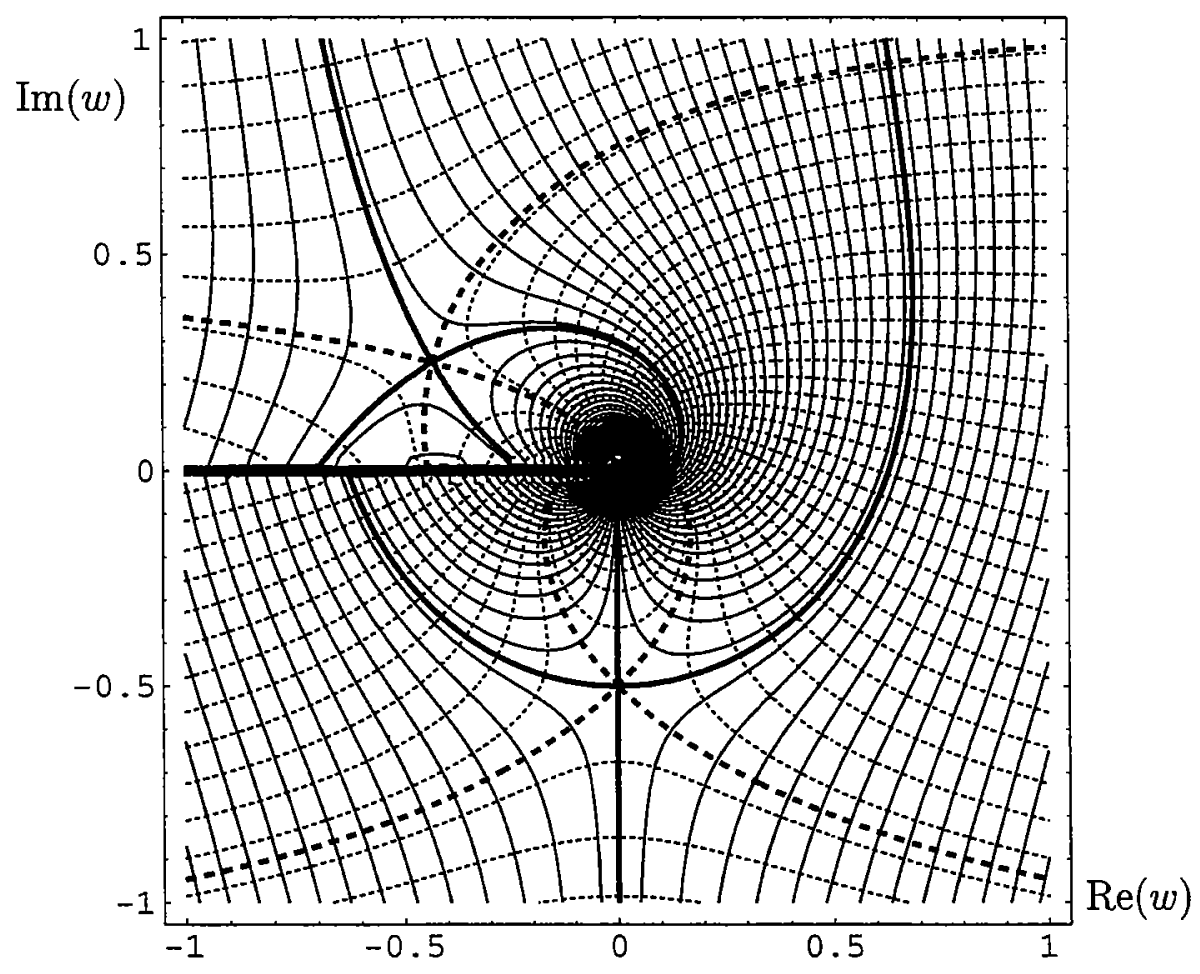

Figure 8: Contours of constant $\operatorname{Re}(h(w))$ (solid lines) and $\operatorname{Im}(h(w))$ (dashed lines) in the complex $w$ plane. The thick lines are the contours passing through the saddle points. The branch cut is clearly seen along the negative real axis. In the method of steepest descents, the Bromwich contour is deformed to pass through $w_{1}=e^{-i \pi / 2} / 2=-i / 2$ with angle $\gamma=\pi / 4$, to the right of the origin avoiding the branch cut, and through $w_{2}=e^{i \pi 5 / 6} / 2$ with angle $\gamma=11 \pi / 12$. 
This implies that

$$
\begin{aligned}
B & \sim \frac{\eta^{2}(1+i \eta)}{\pi\left(1+\eta^{2}\right)} \sqrt{\frac{2 \pi}{3}} \xi^{-1 / 2} e^{i(-3 \xi / 2+\pi / 4)} \\
B_{z} & \sim \frac{\eta(i-\eta)}{\pi\left(1+\eta^{2}\right)} \sqrt{\frac{2 \pi}{3}} \xi^{-1 / 2} e^{i(-3 \xi / 2+\pi / 4)} \\
B_{z z} & \sim-\frac{1+i \eta}{\pi\left(1+\eta^{2}\right)} \sqrt{\frac{2 \pi}{3}} \xi^{-1 / 2} e^{i(-3 \xi / 2+\pi / 4)} \\
B_{z z z} & \sim \frac{\eta-i}{\eta \pi\left(1+\eta^{2}\right)} \sqrt{\frac{2 \pi}{3}} \xi^{-1 / 2} e^{i(-3 \xi / 2+\pi / 4)}
\end{aligned}
$$

Now, using $\xi=\eta^{2} T=\eta^{2} t^{3} / 3$ and taking the constant $\eta$ to be $\eta_{0}$, along the "rays" $z=$ $-\eta_{0}^{3} t^{3} / 3$

$$
\begin{aligned}
u^{2}+v^{2} & =\left|A_{z z}\right|^{2}=\left|B_{z z}\right|^{2} \sim \frac{2}{\left(1+\eta_{0}^{2}\right) \pi \eta_{0}^{2} t^{3}} \\
u_{z}^{2}+v_{z}^{2} & =\left|A_{z z z}\right|^{2}=\left|B_{z z z}\right|^{2} \sim \frac{2}{\pi \eta_{0}^{4}\left(1+\eta_{0}^{2}\right) t^{3}}, \\
F_{E} & =\left.t^{2}\left[\operatorname{Im}\left(B_{z}\right) \operatorname{Re}(B)-\operatorname{Re}\left(B_{z}\right) \operatorname{Im}(B)\right]\right|_{z=-\eta_{0}^{3} t^{3} / 3} \sim \frac{2 \eta_{0}}{\pi\left(1+\eta_{0}^{2}\right) t} \\
F_{S} & =\left.t^{2}\left[\operatorname{Im}\left(B_{z z}\right) \operatorname{Re}\left(B_{z}\right)-\operatorname{Re}\left(B_{z z}\right) \operatorname{Im}\left(B_{z}\right)\right]\right|_{z=-\eta_{0}^{3} t^{3} / 3} \sim \frac{2}{\pi \eta_{0}\left(1+\eta_{0}^{2}\right) t} .
\end{aligned}
$$

These asymptotic relationships are confirmed in Figure 9. A more useful way to represent the asymptotic results is to write $\eta_{0}$ in terms of $z$ and $t$ and then draw contour plots of quantities of physical interest in the $(z, t)$ plane; this is shown in Figure 10. Note that $\xi$ is large for sufficiently large $z$ and/or $t$. For example, this shows that in the asymptotic limit for constant $z$, as time increases, $u^{2}+v^{2}$ and $F_{E}$ decrease, while $u_{z}^{2}+v_{z}^{2}$ and $F_{S}$ increase.

\section{Conclusion}

In this paper, a simplified model has been developed and studied for the decay of near-inertial currents excited in the mixed layer by a passing storm. This decay occurs due to the radiation of downward propagating NIOs into the interior of the ocean. The main assumptions of the model are that the background flow does not vary in the longitudinal direction and has no associated vorticity, the ocean has a simple (piecewise constant) buoyancy frequency profile, and the storm has moved very quickly over the ocean causing a horizontally uniform nearinertial current concentrated in the mixed layer. The $\beta$ effect is included in the analysis. Because the depth of the mixed layer is much smaller than the total depth of the ocean, the problem is formulated in the limit of an effectively infinitely deep ocean; the resultant initial value problem is solved by Laplace transforms. Analytical and numerical results are given for quantities of physical interest including horizontal kinetic energy, vertical shear, energy and shear flux, and back-rotated velocity and shear. Also, asymptotic behavior is determined by the method of steepest descents. 

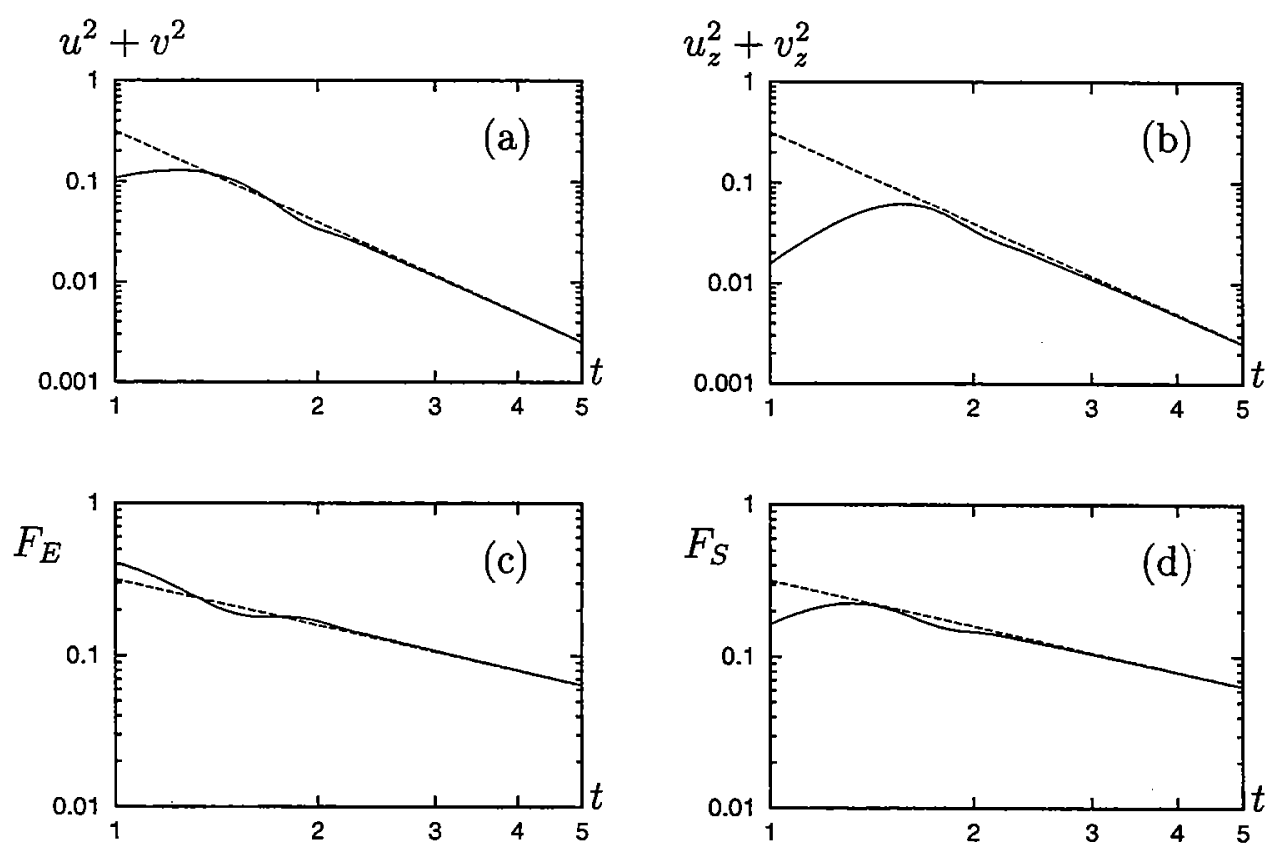

Figure 9: Comparison of numerically calculated (solid lines) and asymptotic (dashed lines) results along the ray $z=-t^{3} / 3$, i.e., $\eta_{0}=1$. (a) $u^{2}+v^{2}$, (b) $u_{z}^{2}+v_{z}^{2}$, (c) $F_{E}$, (d) $F_{S}$.

Although this simplified model cannot be expected to capture the full complexity of the aftermath of a storm passing the ocean, it does capture much of the observed behavior. Most importantly, the decay of mixed layer energy is found to occur on the appropriate timescale (approximately twenty days). It would be interesting to compare the results obtained for this simplified model with observations and numerical simulations. Also, from both a computational and a more philosophical perspective, it would be interesting to compare this method of solution with the standard approach of projecting onto normal modes (e.g., [2, 3]). In the latter, the decay must be viewed as a complicated interference between normal modes, while in the method presented in this paper it is more naturally viewed as a radiation problem. Extensions to a more realistic ocean and storm would involve including a more realistic buoyancy frequency profile (for example, the profile used by [10]), considering the effect of different initial velocities (including both horizontal and vertical structure), and considering the effect of background flow. The study of all of these could use the same formalism of [18] and an approach similar to that presented here.

\section{Acknowledgments}

I would like to thank Bill Young and Stefan Llewellyn Smith for providing so much useful guidance on this project. I enjoyed learning some oceanography, and it was nice to be reminded that linear problems can be quite interesting and rich. Thanks also to Neil Balmforth for directing a great program, and to all the other fellows and staff for an enjoyable summer. 

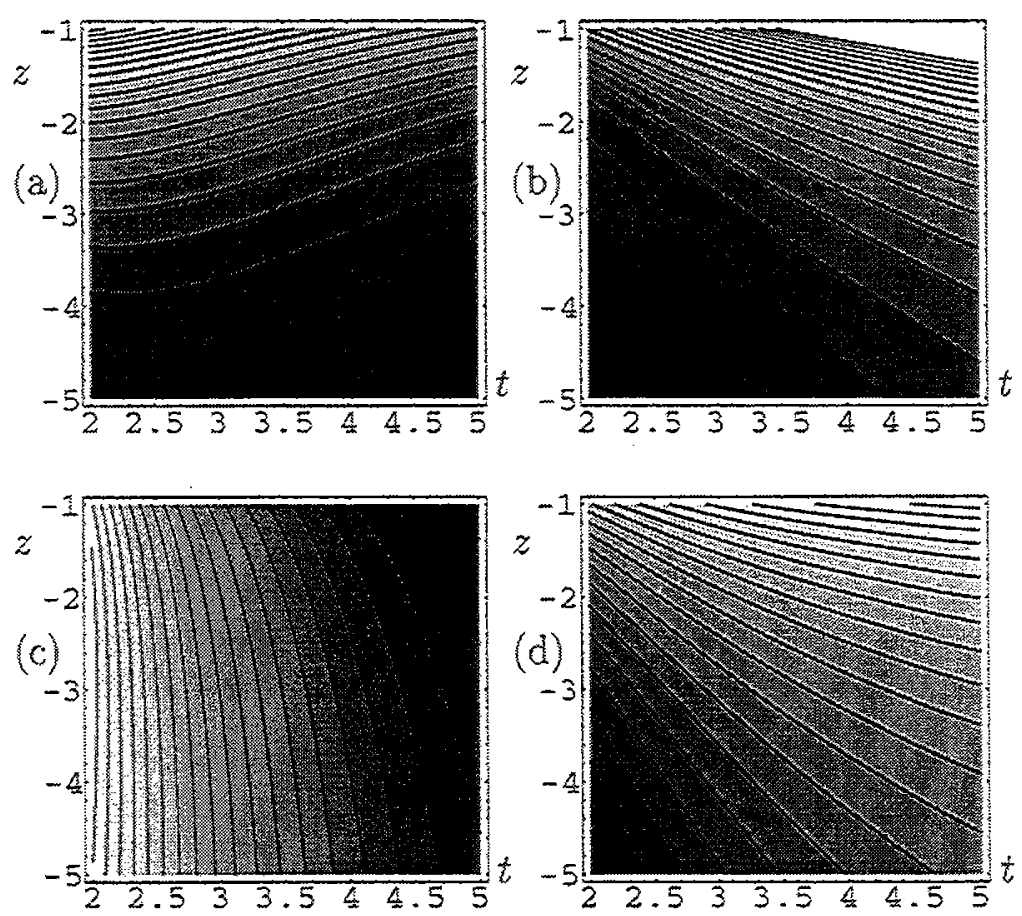

Figure 10: Contour plots of the asymptotic results for (a) $u^{2}+v^{2}$, (b) $u_{z}^{2}+v_{z}^{2}$, (c) $F_{E}$, (d) $F_{S}$. Darker shading corresponds to smaller values.

Finally, thanks to my wife Allison for making our honeymoon in Woods Hole so wonderful.

\section{References}

[1] Abramowitz, M. and Stegun, I. A. (1972) Handbook of Mathematical Functions, Wiley Interscience Publications, $1046 \mathrm{pp}$.

[2] Balmforth, N. J., Llewellyn Smith, S. G. and Young, W. R. (1998) Enhanced dispersion of near-inertial waves in an idealized geostrophic flow. J. Mar. Res., 56:1-40.

[3] Balmforth, N. J. and Young, W. R. (1999) Radiative damping of near-inertial oscillations in the mixed layer, preprint.

[4] Cushman-Roisin, B. (1994) Introduction to Geophysical Fluid Dynamics, Prentice Hall, $320 \mathrm{pp}$.

[5] D'Asaro, E. A. (1985) The energy flux from the wind to near-inertial motions in the surface mixed layer. J. Phys. Oceanogr., 15:1043-1059.

[6] D'Asaro, E. A. (1989) The decay of wind-forced mixed layer inertial oscillations due to the $\beta$ effect. J. Geophys. Res., 94:2045-2056. 
[7] D'Asaro, E. A. (1995) Upper-ocean inertial currents forced by a strong storm. Part II: Modelling. J. Phys. Oceanogr., 25:2937-2952.

[8] D'Asaro, E. A., Eriksen, C. C., Levine, M. D., Niiler, P., Paulson, C. A., and van Meurs, P. (1995) Upper-ocean inertial currents forced by a strong storm. Part I: Data and comparisons with linear theory. J. Phys. Oceanogr., 25:2909-2936.

[9] Garrett, C. (1999) What is the "near-inertial" band and why is it different?, preprint.

[10] Gill, A. E. (1984) On the behavior of internal waves in the wakes of storms. J. Phys. Oceanogr., 14:1129-1151.

[11] Hebert, D. and Moum, J. N. (1993) Decay of a near-inertial wave. J. Phys. Oceanogr., 24:2334-2351.

[12] Henyey, F. S., Wright, J. A., and Flatté, S. M. (1986) Energy and action flow through the internal wave field: an eikonal approach. J. Geophys. Res., 91(C7):8487-8495.

[13] Levine, M. D. and Zervakis, V. (1995) Near-inertial wave propagation into the pycnocline during ocean storms: observations and model comparison. J. Phys. Oceanogr., 25:28902908.

[14] Pollard, R. T. and Millard, R. C. Jr. (1970) Comparison between observed and simulated wind-generated inertial oscillations. Deep-Sea Res., 17:813-821.

[15] Qi, H., De Szoeke, R. A., Paulson, C. A., and Eriksen, C. C. (1995) The structure of near-inertial waves during ocean storms. J. Phys. Oceanogr., 25:2853-2871.

[16] van Meurs, P. (1998) Interactions between near-inertial mixed layer currents and the mesoscale: the importance of spatial variabilities in the vorticity field. J. Phys. Oceanogr., 28:1363-1388.

[17] Webster, F. (1968) Observation of inertial-period motions in the deep sea. Rev. Geophys., 6:473-490.

[18] Young, W. R. and Ben Jelloul, M. (1997) Propagation of near-inertial oscillations through a geostrophic flow. J. Mar. Res., 55:735-766.

[19] Zervakis, V. and Levine, M. D. (1995) Near-inertial energy propagation from the mixed layer: theoretical considerations. J. Phys. Oceanogr., 25:2872-2889. 


\title{
Stirring and Mixing by Vortical Modes
}

\author{
Jennifer MacKinnon
}

\begin{abstract}
Tracer release experiments in coastal and open ocean settings have revealed unexpectedly large isopycnal diffusivities. The stirring and mixing effects of small scale eddies (vortical modes) are discussed as a possible candidate for enhancing diffusivity. A simple analytical model of vortical modes is used to evaluate their mixing potential.
\end{abstract}

\section{Introduction}

Recent oceanic dye release experiments have provided a venue for evaluating existing models of passive tracer horizontal dispersion. In the Coastal Mixing and Optics (CMO) experiment and the North Atlantic Tracer Release Experiment (NATRE), anthropogenic dye was injected along constant density surfaces (isopycnals) in streaks a few $\mathrm{km}$ long. Subsequent surveys through the evolving dye patches with towed instruments were used to look at the qualitative nature of dye patch evolution and calculate quantitative measures of isopycnal diffusion $[1,2]$.

In the CMO experiment, several mid-water-column dye releases were conducted between 1995 and 1997 in $\sim 80 \mathrm{~m}$ deep water on the continental shelf south of New England. Post release surveys were conducted over the several days following each release. By assuming that dye streak evolution was governed by a balance between horizontal diffusion and strain induced stretching [3], Sundermeyer 98 calculates an observed horizontal diffusivity of .3-5 $\mathrm{m}^{2} / \mathrm{s}$ [1]. A traditional way of estimating horizontal diffusivity in the ocean is by looking at the enhanced diffusivity that comes from combining vertical diffusion with shear from the internal wave field [4]. Applying this method to the measured CMO velocities gives diffusion estimates that are a factor of 1-10 below observed values[1].

The NATRE experiment was conducted in open ocean $1200 \mathrm{~km}$ west of the Canary Islands during May 1992 [2]. The dye was sampled five times during several subsequent years. Over this extended period, the qualitative evolution of the dye agrees reasonably well with the model of horizontal diffusion presented by Garrett 83 [1]. He proposes that a dye patch will initially be stretched into long twisted streaks, the width of which is governed by a balance between an effective isopycnal diffusivity and exponential stretching. The observed dye at 6 months is indeed streaky in nature and calculations give an estimated effective diffusivity of $3 \mathrm{~m}^{2} / \mathrm{s}$. The Young, Rhines and Garrett shear dispersion model applied to this situation gives an estimate of $.08 \mathrm{~m}^{2} / \mathrm{s}$ [1]. For longer times, Garrett predicts that dye streaks will coalesce into a more homogeneous patch that grows with an effective diffusivity related to the Lagrangian velocity autocorrelation time scale. During later dye surveys (1-2 years), the tracer has expanded to encompass hundreds of kilometers and is more homogeneous. The 
observed diffusivity for this scale is $10^{3} \mathrm{~m}^{2} / \mathrm{s}$, and agrees with estimates calculated from float velocities.

In both experiments, observed diffusivities on 1-10 km scales are larger than predicted by shear dispersion alone. Both Sundermeyer and Polzin et al.99 propose that the small scale eddies known as vortical modes could be an alternate diffusion mechanism. In brief, vortical modes are thought to be generated when a vertical mixing event creates a relatively well mixed patch of water within a stratified fluid [5]. This density anomaly adjusts to pressure and Coriolis forces by spreading radially outward and beginning to rotate anticyclonically. A steady rotational state can exist until the anomaly diffuses away or some event or instability breaks it apart. Both the adjustment and equilibrium phases can act to enhance horizontal diffusivity.

Sundermeyer considers dimensional arguments and concludes that in the coastal ocean at least, horizontal spreading from vortical mode generation and adjustment may be of an appropriate magnitude to explain observed diffusivities [1]. Polzin et al. evaluate evidence of vortical modes in NATRE by looking at spectral shear and strain values that are not well explained by an internal wave field. Using inferred vortical mode spectra, they calculate estimates for shear dispersion and stirring contributions to effective diffusivity and also get results potentially large enough to explain observations[6].

To tackle the time dependent picture of diffusivity due to vortical modes, we start with a broad qualitative description of what might happen to an initially small dye patch in an ocean where small eddies randomly appear and disappear in various locations near the patch. We ignore the initial vertical mixing event that generates the vortical mode as beyond the scope of this project. Instead we assume that we start with a round (axisymmetric) density anomaly that abruptly appears near a small patch of concentrate. Over the lifetime of a single vortex, a small dye patch located nearby feels a net (center of mass) displacement both outward due to the initial adjustment and around the vortex. The patch also feels a distortion due to the stretching effects of radial shear and molecular and shear-enhanced diffusion. On a longer timescale, the patch will witness the appearance and disappearance of many vortices appearing at different positions around it. Additionally, as it grows in size it eventually becomes large enough to feel several spatially separated vortices at the same time. The larger the number of vortices felt, the more their net displacements will cancel out to produce little net patch movement. However, the net movements felt by smaller segments of the patch stretch, twist, and fold the patch; increase gradients; and enhance diffusion to cause the patch as a whole to grow in size. We seek an understanding of diffusion that will encompass both small and large time limits. For small times, we'll have a more time dependent story of patch growth. For larger times and larger spatial scales we hope that the net effects of smaller motions can be parameterized by an effective diffusivity. Our goals hence are threefold: to understand the time dependence of small time evolution, to estimate a long time eddy diffusivity, and to figure out an appropriate time scale for transition between these two regimes.

Toward these goals, we develop a a simple analytical model that we hope replicates some of the essential stirring and mixing characteristics of oceanic vortical modes. Such an analytical approximation allows us to look at the role small eddies may play in the different stages of horizontal tracer dispersion, get some bounds on the relative importance of different 
diffusive mechanisms, and explore how these results are functions of fundamental problem parameters. In section 2 we begin by looking at numerically integrated full numerical solutions for vortical mode generation. Based upon these solutions, in Section 3 we suggest an analytical approximation for a vortical mode field and extend it into a time dependent stochastic model. In section 4 we evaluate the expected effect of an idealized single vortex velocity field on the evolution of a dye patch. We expand the time scale to consider a time

dependent blinking vortex field in section 5 . Throughout, we try to integrate different ways of looking at diffusion and consider dependence on a few oceanographic parameters of interest. Finally, in section 6 we re-dimensionalize our results and make some simple comparisons to the observed ocean values.

\section{Vortical Mode Solutions}

Before we can develop an appropriate analytical model, we must first get a better feeling for physically realistic vortex velocity fields. We start with the generation of the density anomalies that become vortical modes. We then numerically solve for the equilibrium solutions that describe the velocity field after an adjustment period. Finally, we consider physical arguments for time dependence.

\subsection{Generation Possibilities}

Many observations have shown diapycnal mixing in the ocean to occur episodically [7]. Energetic events such as internal wave breaking and wave wave interaction can lead to shear instabilities and overturning. Such mixing events produce local regions of relatively unstratified water. Most observations of mixed patch height in the CMO coastal area range from 2-10 $\mathrm{m}$ [1]. In the NATRE experiment, observed vertical patch sizes were much larger, on the order of $10-50 \mathrm{~m} \mathrm{[6]}$. It is difficult to measure the horizontal extent of mixing events. A starting guess is that the mixed patches have roughly the same aspect ratio as the internal waves that generate them, $N / f$ (where $N$ is the buoyancy frequency and $f$ the local inertial frequency). Microstructure sections made during the CMO experiment show mixing patches on the order of a kilometer in horizontal extent [8].

Following Garrett and Munk, we estimate the frequency of vertical mixing events by considering net observed vertical diffusivity[1]. We assume that vertical diffusion of tracer is due to the sum of discrete identical vertical mixing events which occur with a frequency $\nu$, are characterized by height $h_{*}$, and by stratification change $\Delta N^{2}$. Using potential energy arguments we can infer the frequency of event occurrence given an observed vertical diffusivity of $K_{z}$,

$$
\nu=3 \frac{N^{2}}{\Delta N^{2}} \frac{1}{h^{2}} K_{z}
$$

\subsection{Adjustment}

A density anomaly of sufficient size in a stratified fluid will evolve according to the pressure and Coriolis forces it feels until a state of balance is achieved. In studying the equilibrium 
solution reached after adjustment of an initial density anomaly, we follow McWilliams tactic of combining the thermal wind equations with conservation of potential vorticity and mass [9]. He starts by non-dimensionalizing as follows:

$$
\begin{aligned}
q_{d} & =f N_{*}^{2} q \\
\theta_{d} & =\frac{\rho_{*} v_{*} f l_{*}}{g h_{*}} \theta \\
r_{d} & =l_{*} r \\
z_{d} & =h_{*} z \\
p_{d} & =\rho_{*} v_{*} f l_{*} \\
\rho_{d} & =\frac{\rho_{*} N_{*}^{2} h_{*}}{g} \rho \\
v_{d} & =v_{*} v,
\end{aligned}
$$

where the subscript ' $d$ ' indicates the dimensional form of a variable. Variables without this subscript (now and throughout this paper) are assumed to be dimensionless. Variable $q$ is Ertel's potential vorticity, $\theta$ is the density. anomaly (deviation from constant stratification) and the other variables have their traditional meaning (radial and vertical distance, pressure, density and velocity respectively). Important non-dimensional parameters are given by

$$
\begin{aligned}
R & \equiv \frac{4 v_{*}}{f l_{*}} \\
B & \equiv\left(\frac{N_{*} h_{*}}{f l_{*}}\right)^{2} \\
\beta & \equiv \frac{R}{4 B} .
\end{aligned}
$$

$\mathrm{R}$ and $\mathrm{B}$ are the Rossby and Burger numbers. $\beta$ can be shown to be a measure of the strength of the density anomaly, with initial density and stratification profiles given non-dimensionally by

$$
\begin{aligned}
\rho_{i} & =-z+\beta \theta \\
N_{i}^{2} & =1-\beta \frac{\delta \theta}{\delta z} .
\end{aligned}
$$

(We use $\beta$ instead of McWilliams $\gamma$ because the later is used as a stretching rate in later sections.) Following McWilliams, we assume an initial density anomaly of the form

$$
\theta=2 z e^{-\left[z^{2}+\left(r / r_{0}\right)^{2}\right]} .
$$

At this point, parameters $r_{0}$ and $\mathrm{B}$ both describe an aspect ratio of the problem. Without loss of generality, we can set $\mathrm{B}=1$ and vary $r_{0}$.

For simplicity, we adopt these non-dimensionalizations for the remainder of our paper. Physically, all horizontal distances, angular velocities and times discussed are fractions of 
the internal Rossby radius, intertial frequency and inertial period respectively. To convert any result back into dimensional coordinates, one only needs to specify $f, N$ and an anomaly height scale $h_{*}$. Dimensional quantities are then given by

$$
\begin{aligned}
r_{d} & =\frac{N}{f} h_{*} r \\
\Omega_{d} & =f \beta \Omega \\
t_{d} & =\frac{1}{f \beta} t .
\end{aligned}
$$

We'll return briefly to the world of dimensions at the end of the paper to compare our results with oceanographic data.

The actual process of geostrophic adjustment will involve internal waves which radiate energy away in a time roughly given by $1 / f[10]$. After the transients have disappeared, the system will achieve an equilibrium state where Coriolis, pressure and centrifugal forces are in balance. Equilibrium density and potential vorticity are given by

$$
\begin{aligned}
& \rho_{f}=-z-\beta \frac{\partial p}{\partial z} \\
& q_{f}=Z N_{s}^{2}-\frac{R \beta}{4 S}\left(\frac{\delta^{2} p}{\delta r \delta z}\right)^{2}
\end{aligned}
$$

with

$$
\begin{aligned}
S & \equiv \sqrt{1+\frac{R}{r} \frac{\partial p}{\partial r}} \\
v & \equiv \frac{2 r}{R}[S-1] \\
Z & \equiv 1+\frac{R}{4 r} \frac{\partial(r v)}{\partial r} .
\end{aligned}
$$

Knowledge of one variable, $p(r, z)$, is enough to specify the whole system. This final state is related to the initial state through the net displacements felt by each Lagrangian water parcel. Non-dimensional displacement variables are defined as

$$
\begin{aligned}
& \zeta\left(r_{f}, z_{f}\right) \equiv r_{f}-r_{i} \\
& \eta\left(r_{f}, z_{f}\right) \equiv z_{f}-z_{i} .
\end{aligned}
$$

These parcels conserve their density and potential vorticity values during adjustment

$$
\begin{aligned}
\rho_{f}(r, z) & =\rho_{i}(r-\zeta, z-\eta) \\
q_{f}(r, z) & =q_{i}(r-\zeta, z-\eta) .
\end{aligned}
$$



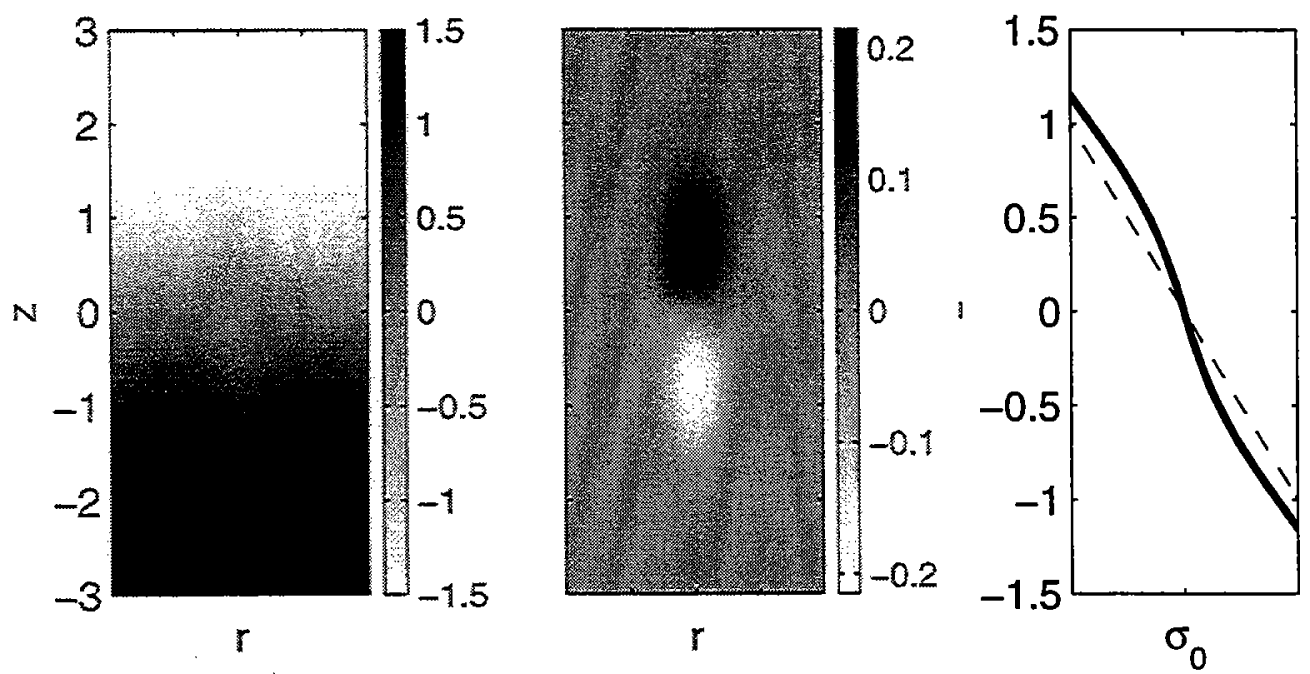

Figure 1: Pre-adjustment density field used for numerical calculations. The full density field is shown in (a), the density anomaly in (b), and a vertical profile at $r=0$ in (c). Also shown in (c) is a reference linear stratification profile. All values are non-dimensional.

Plugging forms for $\rho_{i}, \rho_{f}, q_{i}, q_{f}$ into equations (8) and (9) gives us two equations and three unknowns $(p, \zeta, \eta)$. The final equation comes from requiring that the adjustment be incompressible, or equivalently that the Jacobian of the lagrangian transformation is identically one:

$$
\frac{r_{i}}{r_{f}}\left(\frac{\delta r_{i}}{\delta r_{f}} \frac{\delta z_{i}}{\delta z_{f}}-\frac{\delta r_{i}}{\delta z_{f}} \frac{\delta z_{i}}{\delta r_{f}}\right)=1
$$

where $r_{i}, r_{f}, z_{i}, z_{f}$ can be written in terms of $\zeta$ and $\eta$ following equations 6-7. As McWilliams suggests, an iterative method is necessary to solve the non-linear set of equations $8-10$ for $\mathrm{p}, \zeta, \eta$. There are only two free parameters in this system, $r_{0}$ and $\beta$, which control the aspect ratio and strength of the initial anomaly, respectively. Physically, $r_{0}=1$ corresponds to an anomaly with aspect ratio $f / N$. Larger $r_{0}$ corresponds to a flatter anomaly and vice versa. The anomaly strength is controlled by $\beta$, which ranges from 0 (no anomaly, or background stratification) to .5 (minimum of zero stratification). Figure 1 shows an initial density field with $r_{0}=1, \beta=\frac{1}{4}$, the associated density anomaly, and a profile of density at $r=0$ (with linear stratification for comparison). These values of $r_{0}$ and $\beta$ will be used as a good first estimate.

We numerically integrated solutions to equations (8), (9), and (10) ${ }^{1}$. Boundary conditions

\footnotetext{
${ }^{1}$ Numerical integration was done using routines AVINT and HSTCYL, available from the NIST Guide to Available Math Software
} 

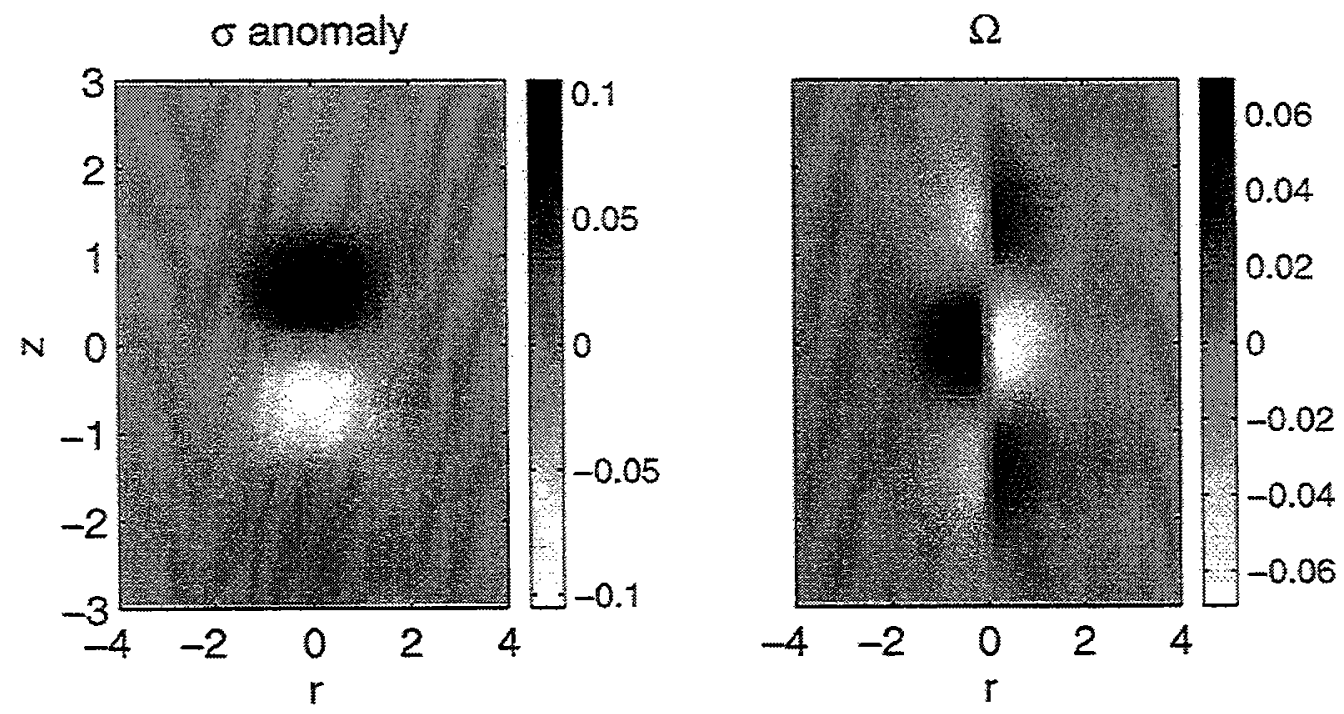

Figure 2: Equilibrium solutions for the density anomaly (a) and angular velocity (b).

are given by

$$
\begin{array}{ccc}
\eta, \zeta, p \rightarrow 0 & \text { as } & r, z \rightarrow 0 \\
\zeta=\frac{\partial p}{\partial r}=0 & \text { at } & r=0 \\
\eta=\frac{\partial p}{\partial z}=0 & \text { at } & z=0 .
\end{array}
$$

As expected, equilibrium solutions consist of a slumped density anomaly rotating anticyclonically. Figure 2 shows non-dimensional equilibrium density and angular velocity fields. Above and below the main anomaly, there are smaller, cyclonically rotating vortices that correspond to regions of enhanced stratification that border the well mixed patch. We ignore these smaller vortices for now and hope to come back to them in future work.

Figure 3 shows profiles at $z=0$ of radial adjustment displacements, $\zeta$, and equilibrium angular velocities. Adjustment displacements are largest at the edge of the initial anomaly $\left(r=r_{0}=1\right)$, and have a maximum value that approaches 1 as $r_{0}$ becomes large. Angular velocity is roughly given by solid body rotation out to $r \sim .5$, and exponentially decays for larger r. Also shown is an analytical approximation of velocity that will be used in later sections. Further dependence on parameters $r_{0}$ and $\beta$ is considered by McWilliams. The effect of varying these parameters on our diffusion calculations may be discussed in future work.

\subsection{Time dependence}

As isolated vortical mode will exist in its equilibrium state until the density anomaly diffuses away, another vertical mixing event occurs on top of it, or it succumbs to some type of 

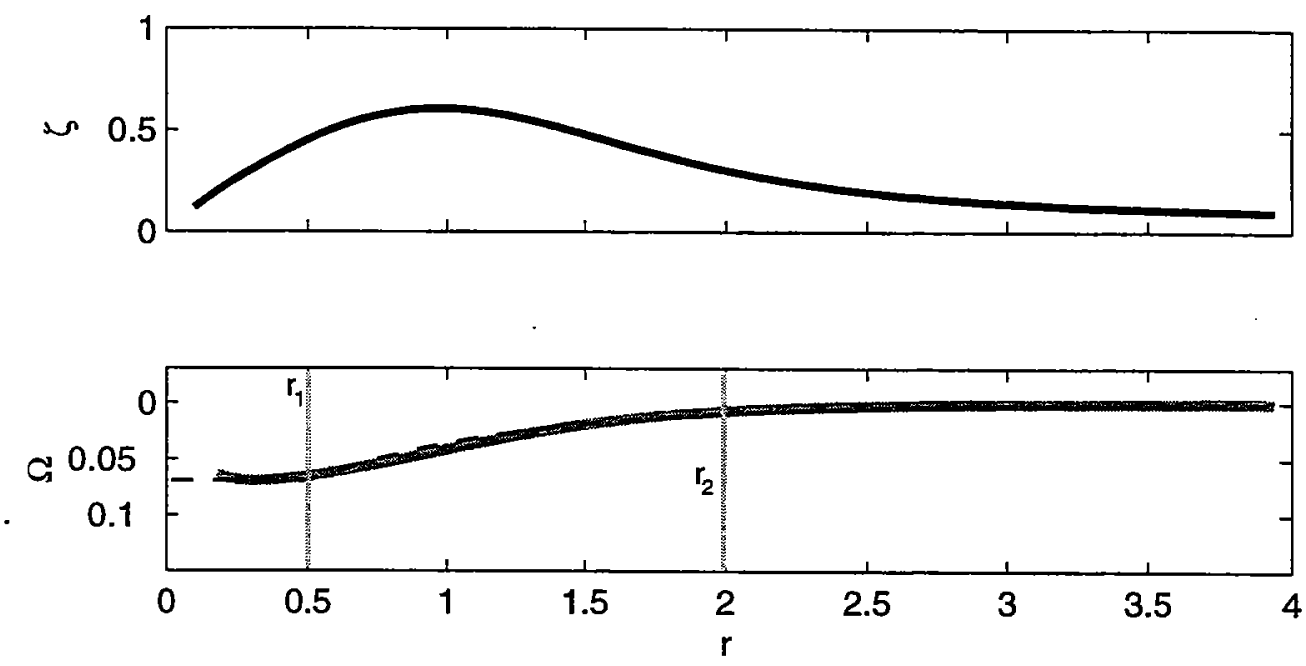

Figure 3: Outward adjustment displacements (a) and equilibrium radial velocity (b) at $\mathrm{z}=0$ for $r_{0}=1$ and $\beta=.25$. Also shown are distances $r_{1}$ and $r_{2}$ which are the edge of solid body rotation and an effective edge of vortex influence.

instability or large scale strain. The time scale for vertically mixing an anomaly of height $h$ away by a molecular vertical diffusivity $K_{z m}$ is

$$
\tau_{1}=\frac{h^{2}}{K_{z m}}
$$

A new vortex will on average appear at a particular site with a frequency given by (1). Since molecular diffusivity will always be smaller than observed diffusivity, we expect the maximum lifetime for vortices will be given by

$$
\tau \equiv 1 / \nu
$$

For the coastal ocean observed patch size of $2-10 \mathrm{~m}$ and observed vertical diffusivity of $10^{-5} \mathrm{~m}^{2} / \mathrm{s}$, the appropriate dimensional timescale is $\tau_{d}=1-20$ days . In non-dimensional units, $\Omega \tau=.01-.3$. In most cases, significantly less than one rotation is completed. In the open ocean, patch heights of $10-50 \mathrm{~m}$ and diffusivities of $.5-1 * 10^{-5} \mathrm{~m}^{2} / \mathrm{s}$ give nondimensional $\Omega \tau=.3-15$ (All calculations assume $\Omega=.07$.) In the later case vortices can exist in equilibrium form for many rotations. This difference in the time scale of vortical modes in different regions is in itself a major result. We expect that the ways in which vortical modes contribute to diffusion may be significantly different in these two situations. Hence, it will be a primary goal of subsequent calculations to consider how vortical mode enhanced diffusion both qualitatively and quantitatively depends on our choice of $\tau$.

\section{Analytical Model}

We now wish to consider the diffusive effects of a field of such vortices. To make our task tractable, we incorporate an analytical approximation to the solutions in section 2 into a 
simple Random Renovating Vortex model that allows us to stochastically approach diffusion quantities of interest. We posit a field of vortices, each of which appears in a random location, exists for a set time $\tau$, identical for all vortices, and disappears. Associated with the appearance of any vortex are instantaneous adjustment displacements $\zeta$ outward from the vortex center. During the subsequent interval $\tau$ of vortex existence, water parcels follow a steady azimuthal velocity field, $\Omega(r)$. For simplicity, we basically ignore $\mathrm{z}$ dependence from now on (except when considering vertical shear dispersion). We hope a first order picture of the dispersive abilities of vortical modes will emerge from considering the radial dependence alone. Future work may include a more baroclinic model. The numerical solutions to McWilliams equations suggest analytical forms as follows. Let the azimuthal velocity field a distance $r$ from the center of the vortex be given by

$$
\Omega(r) \equiv\left\{\begin{array}{cc}
\Omega_{0} & r<r_{1} \\
\Omega_{0} e^{-\alpha\left(r-r_{1}\right)} & r \geq r_{1}
\end{array}\right.
$$

The free parameters are $r_{1}$, the edge of the solid body rotation part of the vortex, $\Omega_{0}$, the velocity scale, and $\alpha$, the exponential decay rate for the outer vortex velocity field. Fitting this model to the numerical solution shown in Figure 3 yields $r_{1}=.5, \alpha=1.3$, and $\Omega_{0}=.07$. Also noted on the graph is a distance $r_{2}$, which we define as a length-scale of vortex influence. It should scale roughly with the exponential decay scale, $r_{2} \sim 1+1 / \alpha$. For simplicity, we set $r_{2}=2$.

Simplifying further, we assume a parcel at any given location feels only one vortex at a time and that vortices do not interact with each other. Despite these caveats, we want vortices to be evenly distributed in space, in some statistical sense. Hence, we propose that the probability of a vortex appearing within $r$ and $r+d r$ of a given parcel is given by the Holtzmark distribution,

$$
P(r) \equiv \frac{2}{r_{2}^{2}} r e^{-\left(r / r_{2}\right)^{2}} d r
$$

Intuitively, this probability is proportional to the area of the strip between $r$ and $r+d r$, multiplied by the probability that there isn't a closer (within a circle r) vortex. The probability is normalized using the vortex spatial scale, $r_{2}$. Finally, we assume that these spatially uncorrelated vortices blink in and out of existence simultaneously over units of time $\tau$. At small scales, the steady flow field of the single, closest vortex will be felt during each time interval. For objects of larger scales, several spatially separated vortices may be felt during each time step.

To account for the variability associated with different realizations of vortex placement, we ensemble average many quantities of interest. For any quantity which is a function of distance to the vortex center, $r$, we define

$$
\langle f(r)\rangle=\int_{0}^{\infty} f(r) P(r) d r
$$

Ensemble averaging can be problematic, as no particular oceanic realization will resemble the smoothness of an ensemble average. However, such averaging is necessary to make our problem tractable and we hope that the most salient features are preserved. 


\section{Short Times / One Vortex}

Using this model we can consider what happens to a small dye patch during short times, in which it feels only the effects of a single vortex. The center of mass of the patch will be displaced as a point parcel would be. This displacement is made up of two components, the quick adjustment radial displacement and the slow azimuthal displacement from the equilibrium velocity field. A patch will also be stretched by radial shear, and for long times and high shear could be wrapped up around the vortex center several times. Finally, the patch will be diffusing all this time, both from molecular diffusion and under some circumstances from an enhanced horizontal shear dispersion. Each of these items will be considered in turn below.

\subsection{Outward Displacements}

The first displacement comes during the adjustment phase and is given by $\zeta(r)$. The ensemble average adjustment displacement felt by a parcel will take into account the probability of being a given distance away from the closest vortex center and is given by

$$
\begin{aligned}
\left\langle\zeta(r)^{2}\right\rangle & =\int \zeta^{2}(r) P(r) d r \\
& \approx .01
\end{aligned}
$$

where the approximate form was obtained by integrating the numerical $\zeta(r)$ profile shown in Figure 3. As a reminder, these numbers should be scaled by $\left(h_{*} N / f\right)^{2}$ to return to dimensional units of $m^{2}$. Since we don't have a good analytical approximation for $\zeta(r)$ and since it doesn't vary with the main parameter of interest, $\tau$, we aim for just an order of magnitude estimate.

\subsection{Azimuthal Displacements}

The average azimuthal displacements felt by a parcel moving around a vortex will depend not only on the distance to the vortex center, but also on the length of time $\tau$ it has to move. For a given $\tau$ and $r$, the displacement is shown in Figure 4 and is given by

$$
\begin{aligned}
l^{2} & =2 r^{2}[1-\cos (\Omega(r) \tau)] \\
\left\langle l^{2}\right\rangle & =\int_{0}^{\infty} P(r) 2 r^{2}[1-\cos (\Omega(r) \tau)] d r
\end{aligned}
$$

With $\Omega(r)$ given by 16 , it is difficult to solve for $\left\langle l^{2}\right\rangle$ analytically. However, there are a few limits of interest that are more approachable. For small vortex lifetimes, we can Taylor expand(19) in powers of $(\Omega \tau)$.

$$
\left\langle l^{2}\right\rangle \approx \sum_{n=1}^{\infty}\left(\Omega_{0} \tau\right)^{2 n} \frac{4(-1)^{n-1}}{(2 n) !} \int_{r_{1}}^{\infty} r^{3} e^{-\left(\frac{r}{r_{2}}\right)^{2}} e^{2 n \alpha\left(r_{1}-r\right)} d r
$$




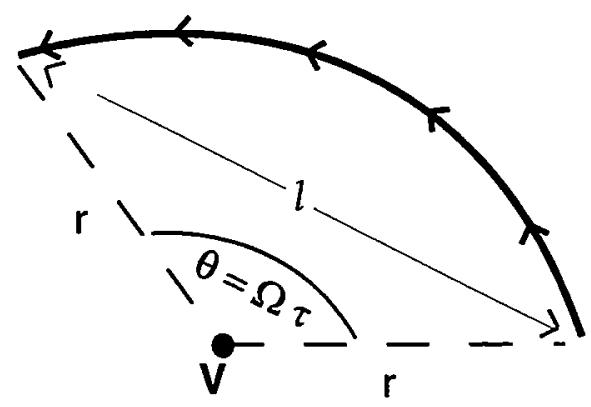

Figure 4: Azimuthal distance traveled during a time $\tau$ by a particle a distance $\mathrm{r}$ away from the center of the closest vortex (V).

Taking only the first expansion term and integrating gives

$$
\left\langle l^{2}\right\rangle \approx 2\left(\Omega_{0} \tau\right)^{2} e^{-\left(\frac{\tau_{1}}{\tau_{2}}\right)^{2}} \Gamma
$$

where we define

$$
\begin{aligned}
\Gamma \equiv & \frac{r_{2}^{2}}{4}\left[\sqrt{\pi} \alpha r_{2} e^{\alpha^{2} r_{2}^{2}+2 \alpha r_{1}+\left(r_{1} / r_{2}\right)^{2}}\left(3+2\left(\alpha r_{2}\right)^{2}\right)\left(\operatorname{erf}\left(\frac{\mathbf{r}_{1}}{\mathbf{r}_{2}}+\alpha \mathbf{r}_{2}\right)-1\right)\right. \\
& \left.+2\left(\left(\frac{r_{1}}{r_{2}}\right)^{2}-\alpha r_{1}+\left(\alpha r_{2}\right)^{2}+1\right)\right] .
\end{aligned}
$$

The upper bound on (19) is obtained by noting that

$$
1-\cos [\Omega(r) \tau] \leq 2
$$

and thus

$$
\left\langle l^{2}\right\rangle \leq \frac{r_{2}^{2}}{2}\left(r_{1}^{2}+r_{2}^{2}\right) e^{-\left(\frac{r_{1}}{r_{2}}\right)^{2}} .
$$

A numerical solution to equation (19) together with the limits given in (21) and (23) is plotted in Figure 5a. The outward displacement magnitude, $\zeta^{2}$, is also plotted for reference. Figure $5 \mathrm{~b}$ shows expected squared displacements divided by $\tau$, which is related to the diffusivity expected for a random walk process of given step length. For increasing $\tau$, average displacements approach steady values, but expected diffusivities peak and then decline.

The relative importance of azimuthal versus radial displacements depends on the magnitude of $\tau$. From Figure 5, the two effects achieve equal magnitudes for $\Omega \tau \sim .6$. In coastal areas, we expect outward displacements to be more important, and vice versa for the open ocean. More precisely, the true ensemble average displacement is $\left\langle(l+\zeta)^{2}\right\rangle$, which is even less analytically tractable. But since

$$
\left\langle l^{2}\right\rangle+\left\langle\zeta^{2}\right\rangle \leq\left\langle(l+\zeta)^{2}\right\rangle
$$

calculating them separately gives us an upper bound on average displacement magnitude. 

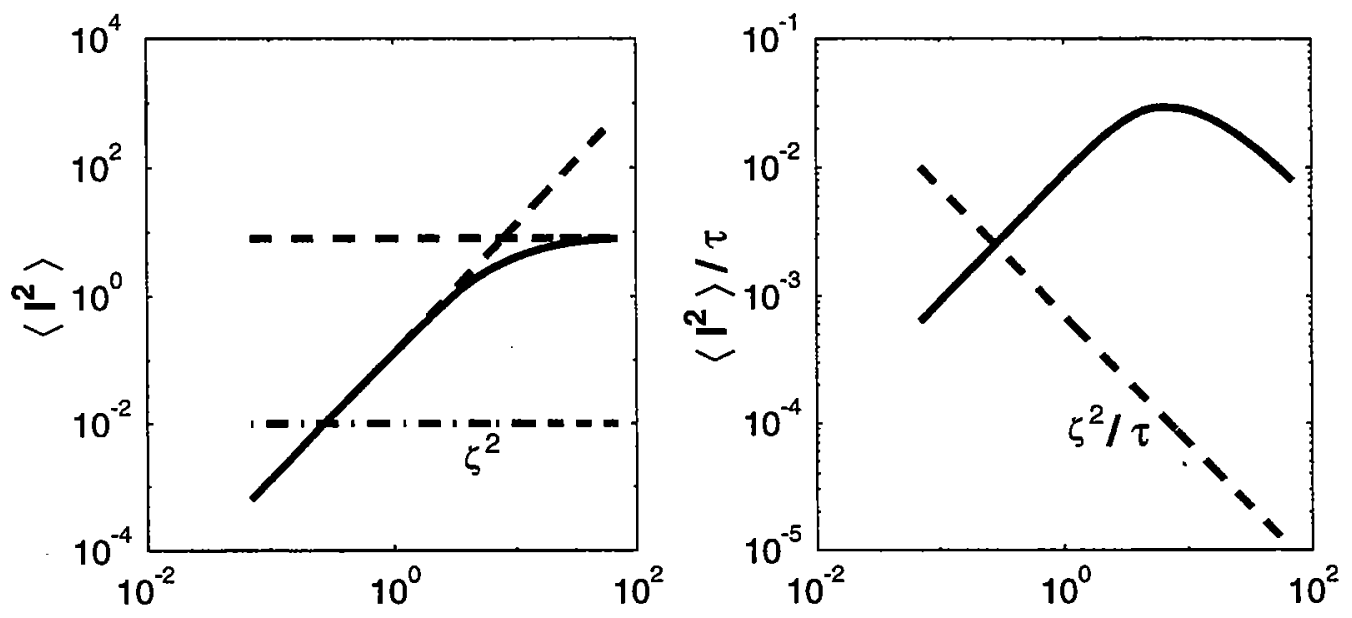

Figure 5: Numerically integrated average squared displacements along with analytically calculated limits are shown in (a). Also shown are adjustment displacements squared for comparison. Random walk diffusivities based on such displacements are shown in (b).

\subsection{Azimuthal stretching}

While a dye patch moves around a vortex center, it is sheared by radial gradients in velocity. The direction of shear is likely to be different in different periods of time $\tau$. Hence, in calculating stretching we must consider not only the distance to a vortex center but also a dye streak orientation with respect to a vortex. The increase in length of a small line element during one time interval $\tau$ is shown in Figure 6 and given by

$$
\delta s^{2} \equiv \frac{s_{1}^{2}}{s_{0}^{2}}=\left[1+\tau r \frac{d \Omega(r)}{d r} \sin (2 \phi)+\left(\tau r \frac{d \Omega(r)}{d r} \cos \phi\right)^{2}\right]
$$

Ensemble averaging must be done over $r$ and $\phi$. We assume that $\phi$ is evenly distributed between 0 and $2 \pi$. After $N$ period of length $\tau$ have passed, a streak of initial length $s_{0}$ will have grown to

$$
\begin{aligned}
& \left\langle s_{N}^{2}\right\rangle=s_{0}^{2} \quad\left[\left\langle\left\langle\delta s(r, \phi)^{2}\right\rangle_{\phi}\right\rangle_{r}\right]^{n} \\
& =s_{0}^{2}\left[\left\langle 1+\frac{\tau^{2}}{2}\left(r \frac{\delta \Omega(r)}{\delta r}\right)^{2}\right\rangle_{r}\right]^{n} \\
& =s_{0}^{2} e^{-n\left(r_{1} / r_{2}\right)^{2}}\left[1+\Gamma \alpha^{2}\left(\Omega_{0} \tau\right)^{2}\right]^{n} \text {. }
\end{aligned}
$$

Equation (24) can be written in a simple exponential form

$$
\left\langle s_{N}^{2}\right\rangle=e^{2 \gamma t},
$$




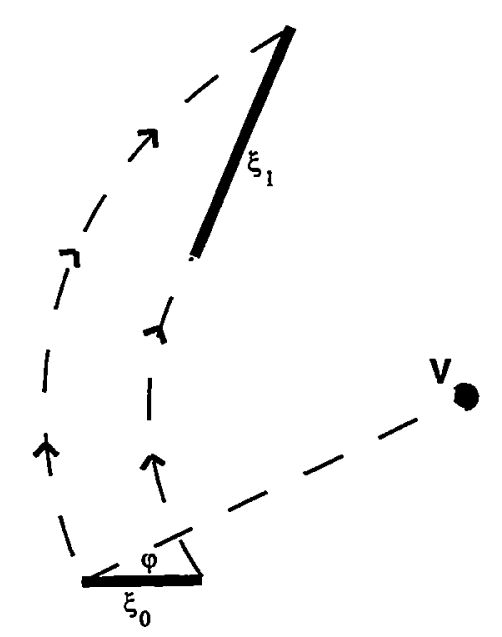

Figure 6: Stretching of a line element.

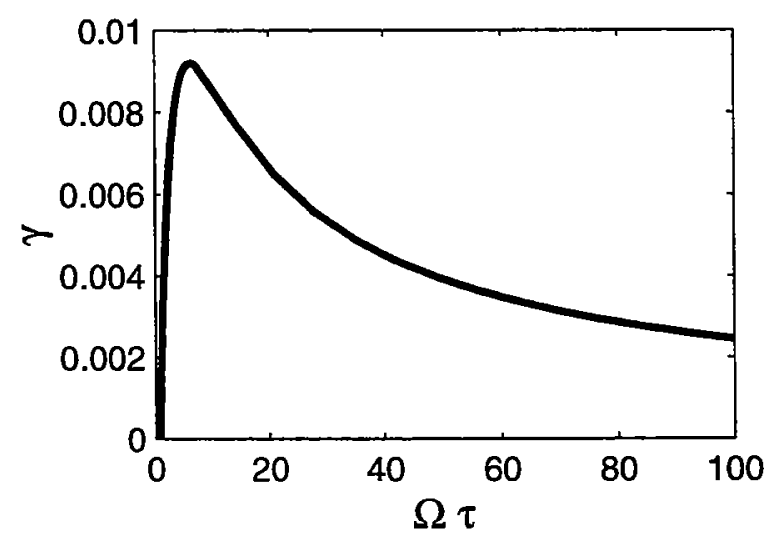

Figure 7: Exponential stretching rate felt over many vortex lifetimes.

by defining

$$
\gamma \equiv \frac{1}{2 \tau}\left[\ln \left(1+\Gamma \alpha^{2}\left(\Omega_{0} \tau\right)^{2}\right)-\left(\frac{\mathbf{r}_{1}}{\mathbf{r}_{2}}\right)^{2}\right]
$$

One might note the similarity of this to the Lyapunov exponents calculated for the Random Renovating Wave model developed in the principle lectures. The stretching rate, $\gamma$, is plotted in Figure 7 as a function of $\Omega \tau$.

\subsection{Diffusion/Shear Dispersion}

As a dye patch stretches and moves around a vortex center, its area will increase due to horizontal diffusivity. Molecular diffusion in a sheared velocity field is enhanced by the interaction between horizontal or vertical shear and a background horizontal or vertical diffusivity. In a steady shear field, this interaction leads to an anomalously fast diffusion at large times[4]. 
In an oscillatory flow field, the anomalous diffusion of steady shear is appropriate only for time-scales smaller than the oscillation time. Over several periods, diffusion regains a Fickian character, with an enhanced effective diffusivity which is averaged over the oscillation time of the anomalous diffusion. Young et al. considered the vertical shear associated with typical open ocean values of the internal wave field and found that horizontal diffusion was enhanced over it's molecular values by a factor of $N^{2} / f^{2}$. Our flow similarly contains a finite time scale. In our case, shear is steady for the vortex existence time, $\tau$, after which it may change direction and magnitude. However, unlike the Young et al. model in which the advective solution returns to it's original state at the end of each oscillation, our flow is circular within each time period. Therefore, Rhines and Young's work on two-dimensional dispersion for closed streamlines is also helpful[11]. The shear in their case is radial shear of a circular flow. To get the best shear dispersion estimate for our problem, we start with the Rhines and Young approach and add a vertical component of shear and time dependence.

Following their approach, we start with the advection diffusion equation for concentration, $\theta$ in a steady velocity field. For a circular velocity field with azimuthal velocity given by

$$
\frac{1}{r} u_{\phi}=\Omega(r, z)
$$

and distinct vertical and horizontal diffusivities, the governing equation is

$$
\theta_{t}+\Omega \theta_{\phi}=K_{H}\left[\frac{1}{r}\left(r \theta_{r}\right)_{r}+\frac{1}{r^{2}} \theta_{\phi \phi}\right]+K_{z} \theta_{z z}
$$

If we assume that there is an independent internal wave field with a faster time scale superimposed on our vortical mode field, a plausible relationship between $K_{H}$ and $K_{z}$ is given by $[4]$

$$
K_{H}=\frac{N^{2}}{f^{2}} K_{z} .
$$

We assume the solution can be written as a sum of components with different azimuthal wavenumbers, each of which is given by

$$
\begin{aligned}
\theta & =A(r, z, t) e^{i n \hat{\phi}} \\
\hat{\phi} & \equiv \phi-\Omega(r, z) t
\end{aligned}
$$

Plugging (30) into (28) and grouping terms gives an equation for the rate of concentration change in the framework of the advective solution

$$
\begin{aligned}
A_{t}= & K_{H}\left[\frac{1}{r}\left(r A_{r}\right)_{r}+\frac{n^{2}}{r^{2}} A\right]+K_{z} A_{z z} \\
& -i\left\{K_{H}\left[\frac{A}{r}\left(r \bar{\Omega}_{r}\right)_{r}+2 A_{r} \bar{\Omega}_{r}\right]+K_{z}\left[A \bar{\Omega}_{z z}+2 A_{z} \bar{\Omega}_{z}\right]\right\} \\
& \left.-\left\{K_{H} A \bar{\Omega}_{r}^{2}+K_{z} \bar{\Omega}_{z}^{2}\right)\right\}
\end{aligned}
$$




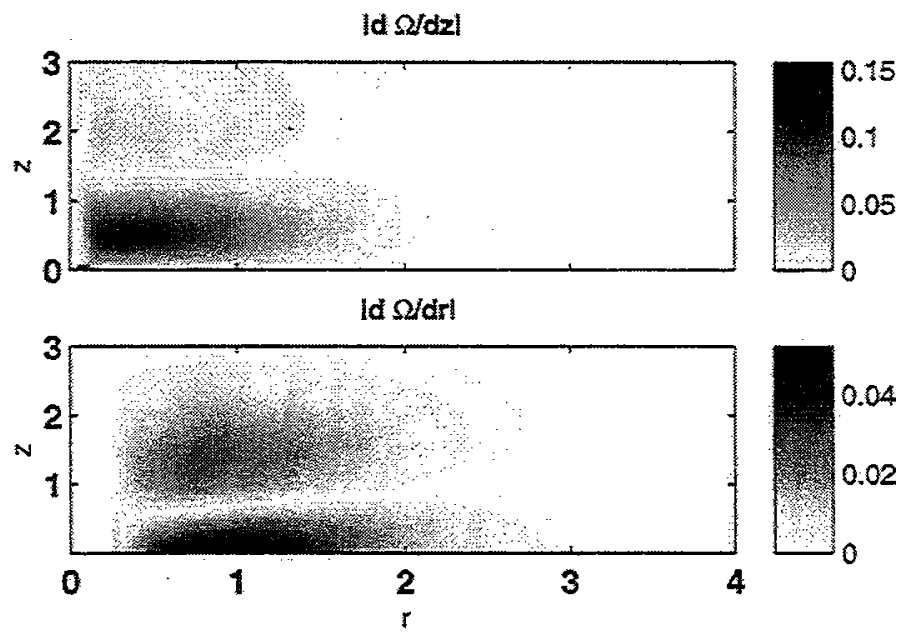

Figure 8: Non-dimensional magnitude of vertical and horizontal shear from the full numerical solutions for an equilibrium vortex velocity field.

with

$$
\bar{\Omega} \equiv n t \Omega .
$$

The rhs terms of (32) are grouped by powers of ( $n t \Omega)$, which is physically related to distance traveled around the vortex center. Analysis in section 2 indicated that oceanic vortical modes can have existence times that vary from much shorter to much longer than their eddy turnover time such that we are interested in solutions for a range of $\bar{\Omega}$ values. The first term in (32) is simply molecular diffusion, and in the limit of $\widetilde{\Omega} \ll 1$, this is the dominant term. In the opposite limit, $\bar{\Omega} \gg 1$, the third term of (32) dominates. This is the limit considered by Rhines and Young. Following their analysis, the solution to (32) for large $\bar{\Omega}$ becomes

$$
A \sim e^{-\frac{1}{3} n^{2} t^{3}\left[K_{H} \Omega_{\tau}^{2}+K_{z} \Omega_{z}^{2}\right]} .
$$

For intermediate values of $\bar{\Omega}$, we need to use the full version of (32). Such precision is beyond the scope of this paper, and unnecessary to get the sort of magnitude estimates we are interested in. The above approximations suggest that for vortices with short lifetimes (coastal regions), vortical shear dispersion is not significant, and we can define an appropriate effective horizontal diffusivity, $K_{s}$ which is in this case equal to the traditionally used internal wave enhanced value given by (29). In areas with long lived vortices (open ocean), this traditional value is likely to be further enhanced by vortical mode shear dispersion. Roughly following methods of dealing with oscillatory waves in Rhines and Young and Young et al., we pull from (33) an effective diffusivity of the form

$$
K_{s} \sim \frac{1}{6} n^{2} \tau^{2}\left\langle K_{H} \Omega_{r}^{2}+K_{z} \Omega_{z}^{2}\right\rangle
$$




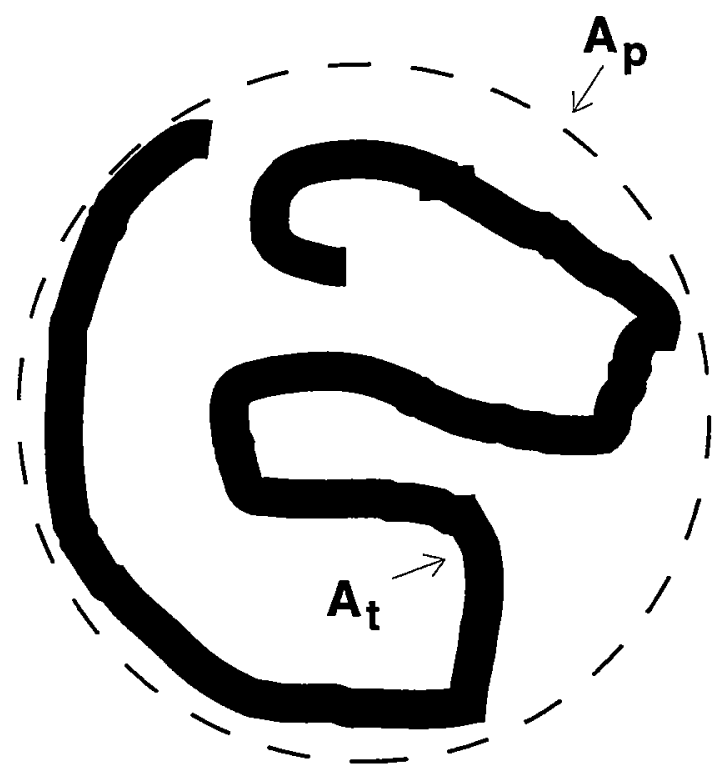

Figure 9: Schematic of different growth rates that characterize tracer distribution.

$K_{H}$ is given by (29) and $K_{z}$ is perhaps a molecular diffusivity. Radial and vertical shears from the McWilliams model are shown in Figure 8. The reader will recall that these shears are non-dimensional and dimensional forms of $\Omega_{r}^{2}$ and $\Omega_{z}^{2}$ will have a relative scaling factor of $N^{2} / f^{2}$. Hence, the roughly comparable magnitudes shown in Figure 8 indicate comparable importance of the two terms in (34).

All of the abovementioned effects (stretching, shear dispersion, net displacement) occur at the same time for a dye patch feeling a single vortex field. To gain some insight into how these various effects fit together into a more coherent picture of diffusion, we now turn to longer time scales.

\section{Longer Times}

During longer time periods, a dye patch will experience the effects of multiple vortices, both because we are considering times greater than an individual vortex lifetime and because over long times a patch grows spatially to feel many vortices. As a dye patch is stretched by several randomly oriented vortices in turn, it stretches and folds as illustrated in the cartoon in Figure 9. There are now two types of diffusion of interest. First, one might like to know the size of the bounding circle, designated as $A_{p}$ in Figure 9. This represents the area in which one has a chance of encountering concentrate. As suggested by Garrett, this area grows as the separation between discrete parcels in the flow field[3]. The second quantity of interest is the actual area occupied by dye, $A_{t}$, which will depend on the stretching rate $\gamma$ from equation (26). We now approach both these quantities more specifically for our RRV flow field. 


\subsection{Multiparticle Dispersion}

The bounding area, $A_{p}$, will roughly grow like the size of a group of discrete water parcels initially close together. Qualitatively, the dispersion rate of a collection of particles is a function of how close they are to each other. A group of closeby particles will all be feeling a similar velocity field and will move more or less together, dispersed only by the small velocity differences between their positions. As they move apart, they feel a larger velocity difference and move apart more quickly. In our case there comes a point when they are so far separated that (on average) they no longer feel the same vortex, at which point their velocities become completely uncorrelated. At this point each particle moves in its own random walk and the particle separation distance should increase as twice that of a single random walker.

Quantitatively, the area of a group of particles is described by the second moment,

$$
\sigma^{2} \equiv \frac{1}{n} \sum_{i=1}^{n}\left(x_{i}-\bar{x}\right)^{2}=\frac{1}{n} \sum_{i=1}^{n} x_{i}^{2}-\bar{x}^{2}
$$

with

$$
\bar{x} \equiv \frac{1}{n} \sum_{i=1}^{n} x_{i} .
$$

Parcel position $x_{i}$ at any time $t$ can be obtained by integrating the Lagrangian velocity field

$$
x_{i} \equiv x_{i 0}+\int_{0}^{t} u_{i}\left(t^{\prime}\right) d t^{\prime}
$$

Plugging (36) into (35) gives

$$
\begin{aligned}
\sigma^{2}= & \sigma_{0}^{2}+\frac{1}{n} \sum_{i=1}^{n}\left[\int_{0}^{t} u_{i}\left(t^{\prime}\right) d t^{\prime}\right]^{2} \\
& -\frac{1}{n^{2}} \sum_{i=1}^{n} \sum_{j=1}^{n} \int_{0}^{t} u_{i}\left(t^{\prime}\right) d t^{\prime} \int_{0}^{t} u_{j}\left(t^{\prime \prime}\right) d t^{\prime \prime} .
\end{aligned}
$$

For our model, $t=N \tau$, where $\mathrm{N}$ is the number of vortex lifetimes experienced. The net displacement, $x_{i}(t)$, is simply the sum of displacement from each successive vortex

$$
\int_{0}^{t} u_{i}\left(t^{\prime}\right) d t^{\prime}=\sum_{k=1}^{N} \int_{0}^{\tau} u_{i}^{(k)}\left(t^{\prime}\right) d t^{\prime} .
$$

Next, we simplify by ensemble averaging (37). Consider how variance increases on average during one period

$$
\begin{aligned}
\left\langle\sigma_{N}^{2}\right\rangle-\left\langle\sigma_{N-1}^{2}\right\rangle= & \left\langle\frac{1}{n} \sum_{i=1}^{n}\left[\int_{0}^{\tau} u_{i}\left(t^{\prime}\right) d t^{\prime}\right]^{2}\right\rangle \\
& -\left\langle\frac{1}{n^{2}} \sum_{i=1}^{n} \sum_{j=1}^{n} \int_{0}^{\tau} u_{i}\left(t^{\prime}\right) d t^{\prime} \int_{0}^{\tau} u_{j}\left(t^{\prime \prime}\right) d t^{\prime \prime}\right\rangle
\end{aligned}
$$


which can be re-written as

$$
\begin{aligned}
& =\frac{1}{n} \sum_{i=1}^{n}\left\langle l^{2}\right\rangle-\frac{1}{n^{2}} \sum_{i=1}^{n} \sum_{j=1}^{n}\left\langle l_{i} l_{j}\right\rangle \\
& =\frac{1}{n} \sum_{i=1}^{n}\left\langle l^{2}\right\rangle\left[1-\frac{1}{n} \sum_{j=1}^{n} \frac{\left\langle l_{i} l_{j}\right\rangle}{\left\langle l^{2}\right\rangle}\right] .
\end{aligned}
$$

Here $\left\langle l^{2}\right\rangle$, from (19) is the same for each parcel and $\left\langle l_{i} l_{j}\right\rangle$ is a measure of correlation between a pair of parcels and must be summed over all pair combinations. Physically, (38) states that the increase in particle area goes like the single particle diffusivity with a correction term that accounts for correlation between particles. If all particles are moving with the same, perfectly correlated velocity, $\left\langle l_{i} l_{j}\right\rangle=\left\langle l^{2}\right\rangle$, then no relative dispersion occurs. In our flow, the degree of correlation is a function of the particle separation, $\left\langle l_{i} l_{j}\right\rangle=\mathcal{F}\left[\left\langle\sigma_{N-1}^{2}\right\rangle\right]$.

To make this calculation more tractable, consider the evolution of distance between only two particles. Let $\left\langle\xi^{2}\right\rangle=\mathbf{x}_{2}-\mathbf{x}_{1}$ be the distance between two parcels. The ensemble averaged increase in $\left\langle\xi^{2}\right\rangle$ during any single time period is given by

$$
\left\langle\xi_{N}^{2}\right\rangle-\left\langle\xi_{N-1}^{2}\right\rangle=2\left\langle l^{2}\right\rangle\left[1-\frac{\left\langle l_{i j}^{2}\left(\xi_{N-1}\right)\right\rangle}{\left\langle l^{2}\right\rangle}\right]
$$

For two parcels a distance $\xi$ apart, $\left\langle l_{i} l_{j}\right\rangle$ is given by

$$
\left\langle l_{i j}^{2}\right\rangle=\int_{0}^{\infty} \int_{0}^{2 \pi} P(r) 2 r(r+\delta r) \sqrt{[1-\cos (\Omega(r) \tau)][1-\cos (\Omega(r+\delta r) \tau)]} d \phi d r,
$$

with

$$
\delta r \equiv \sqrt{\frac{\xi^{2}}{4}+r^{2}}-\sqrt{\frac{\xi^{2}}{4}-\xi r \cos (\phi)+r^{2}} .
$$

We define a spatial correlation function

$$
\mathcal{R}_{i j} \equiv \frac{\left\langle l_{i} l_{j}\right\rangle}{\left\langle l^{2}\right\rangle}
$$

As is, this representation assumes that as particles grow further apart their velocity becomes less correlated but they still feel different parts of the same vortex field. It is more realistic to say that significantly separated particles will feel the effects of uncorrelated vortices. From Section 2, the lengthscale of vortex influence is $r_{2}$. We manually impose this constraint by defining a new correlation function in which particles greater than $r_{2}$ away feel different vortices

$$
\mathcal{R}^{\prime}(\xi) \equiv(\mathcal{R})(\xi) f(\xi)
$$

where $f(\xi)$ is some function that goes from 1 at $r=0$ to 0 for large $r$. There are several forms $f(\xi)$ can take. We could impose that correlation abruptly goes to 0 when the distance exceeds $r_{2}$, so $f(\xi)$ takes the form of a Heavyside step function 


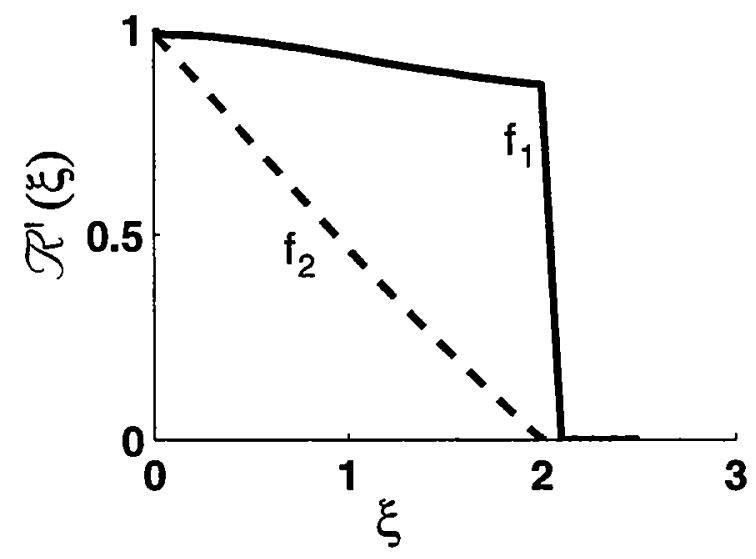

Figure 10: Two different possibilities for multiparticle spatial correlation.

$$
f_{1}(\xi)= \begin{cases}1 & r<r_{2} \\ 0 & r \geq r_{2}\end{cases}
$$

This is straightforward but a little abrupt. Another possibility is to make the transition very smooth by assuming that the probability of nearby particles being in different vortices goes from 0 to 1 linearly:

$$
f_{2}(\xi)=1-\frac{\xi}{R_{0}}
$$

Both possibilities for $\mathcal{R}^{\prime}$ are plotted in Figure 10, where $R_{i j}$ has been numerically calculated from (19) and (40) for different values of $\xi$. Reality is likely to be somewhere in between. The second choice has the advantage of a continuous derivative. It also provides an approximate lower bound for parcel spatial correlation and hence an upper bound for diffusion rate. We will use the form in (44) from now on.

The real quantity of interest is the time evolution of $\xi$ for particles initially separated by a distance $\xi_{0}$. We numerically integrate (39) for different values of $\xi_{0}$ and $\tau$ and plot the results in Figure 11. For reference, we also plot the separation growth expected for uncorrelated particles based on (19) alone, assuming $\left\langle l_{i} l_{j}\right\rangle=0$. At large times, particle separation goes as twice the single particle dispersion rate, $\left\langle l^{2}\right\rangle$, as expected. For a given value of $\tau$, the initial particle separation, $\xi_{0}$, controls the time it takes to settle into linear growth, but not the qualitative nature of the transition or any aspect of the large time solution. For different values of $\tau$, several things change. First, the final diffusivity (the intercept of our log-log plot) is different, as expected from the relationship in Figure 5. Second, the transition to linear growth has a different character. For $\tau=1,10$, the initial growth in $\left\langle\xi^{2}\right\rangle$ is slower than linear, while for $\tau=100,1000$ it grows with a faster than linear growth rate. 

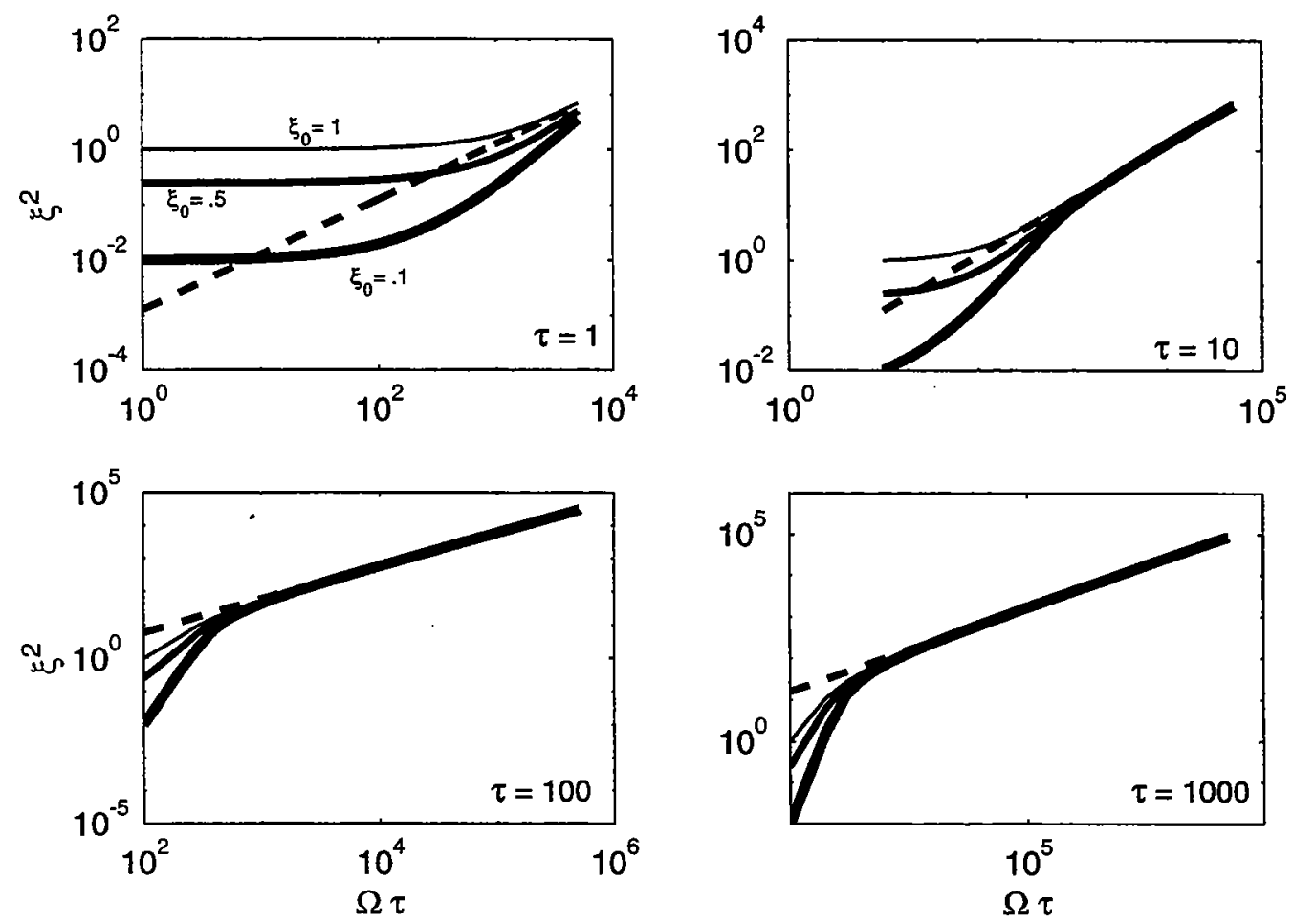

Figure 11: Squared pair separation distance obtained by iterative integration. $\tau$ ranges from 1 to 1000 . In all subplots the solid lines represent $\xi_{0}$ values of $.1, .5$ and 1 . The dotted line is the growth rate expected for uncorrelated randomly walking particles. 


\subsection{Streak Area}

The other way to characterize the growth of a dye streak is by the actual area of the tracer. As described by Ledwell et al. and Sundermeyer, the streak will go through an initial period of stretching during which it gets thinner, cross-streak gradients get larger and cross-streak diffusive fluxes grow. Eventually a balance between stretching and diffusion is reached, at which point the streak continues to grow larger, but its width remains the same. This equilibrium width is related to the stretching rate $\gamma(26)$, by

$$
\Delta l=\sqrt{\frac{K_{s}}{\gamma}}
$$

where $K_{s}$ is an appropriate horizontal diffusivity (section 4.4).

After a width is established, the streak area grows with its increasing length

$$
\begin{aligned}
A_{t} & =(\Delta l)(l) \\
& \sim l_{0} \sqrt{\frac{K_{s}}{\gamma}} e^{\gamma t}
\end{aligned}
$$

for some initial length $l_{0}$.

\subsection{The Big Picture}

During the initial period of stretching and folding of a dye patch, the containing area $A_{p}$ will be significantly larger than the actual dye area $A_{t}$. However, tracer area grows exponentially, (46), and will catch up. Physically, as the tracer area depicted in Figure 9 grows, different parts of the tracer streak get close enough together that they start to merge and the tracer fills in the area bounded by $A_{p}$. From then on, the dye patch is fairly homogeneous, and continues to grow in a Fickian manner with $A_{p}$. In Figure 12, we plot $A_{p}(t)$ and $A_{t}(t)$ together for $\gamma=.008, \tau=100, \xi_{0}=.1$. We have assumed that both quantities start with the same initial area,

$$
A_{0} \equiv l_{0} \sqrt{\frac{K_{s}}{\gamma}}=\xi_{0}^{2} .
$$

In this case, tracer area is much smaller than the bounding area up until a time $\Omega \tau_{0} \sim 70$, after which tracer area grows linearly as $A_{p}$.

\section{Ocean comparisons and Conclusions}

Our goal in this work has been to evaluate whether sub-mesoscale eddies could make a significant contribution to horizontal diffusion in the coastal or open ocean. Because it was not immediately apparent which of the potential stirring and mixing abilities of vortical modes would be important, we undertook a step by step look at the time dependent diffusion of an initially small patch of passive tracer. To get a realistic form for a single vortical mode, we 


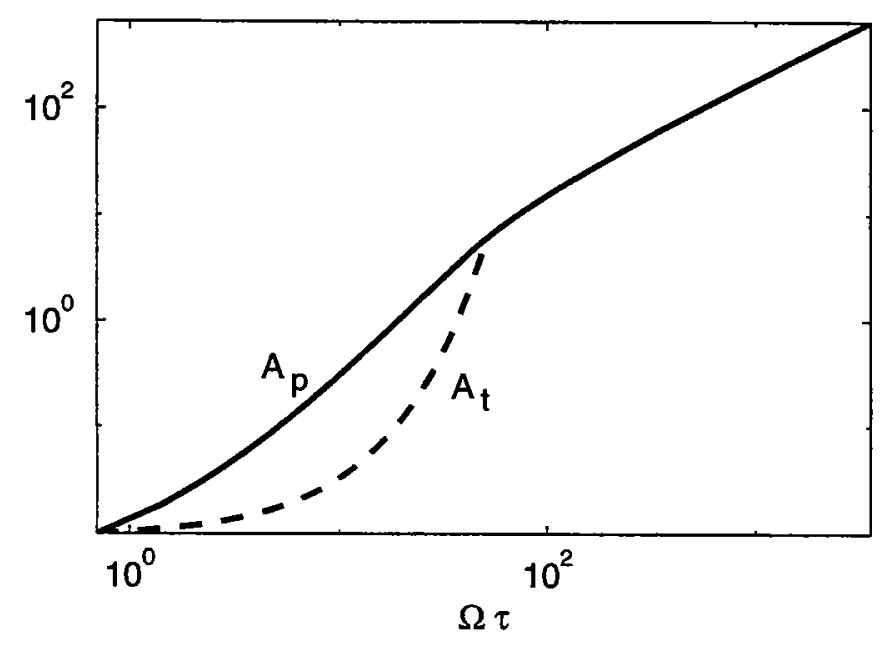

Figure 12: Growth rates for multiparticle dispersion and tracer area. They merge

looked at numerical solutions to the non-linear adjustment of an isolated density anomaly, and incorporated an idealized version of these results into a simple stochastic renovating vortex model. Most of the qualitative features of qualitative tracer evolution we discuss are the same as those described by Garrett for a two-dimensional turbulent field. Using our analytical model, we're able to calculate exact values for some of the stages he talks about more qualitatively. The picture that emerges is that at space and time scales of the same order of magnitude of vortex scales and lifetimes, a patch of dye twists and folds with exponentially increasing area, filling a larger bounding area governed by the multiparticle dispersion rate. At larger space and time scales, the tracer area grows in a fickian manner with the effect of vortical modes incorporated into an effective diffusivity.

In a realistic ocean, the added complexities of stirring processes operating on a variety of time and space scales will prohibit comparison of the distinct stages of diffusion we've discussed. The most practical results to emerge from our work are estimates of an effective (Fickian) diffusvity, and bounds on when such a diffusivity is appropriate. In the coastal ocean, vortex lifetimes are likely to be shorter than their turnover timescale. In this limit, the displacements, stretching distortion and shear dispersion associated with azimuthal motion may not be as important as displacements from the initial vortex adjustment. We calculate a non-dimensional effective diffusivity for this random-walk like motion of .01 (Figure 5.) To redimensionalize, we multiply by $\left(N^{2} h_{*}^{2} f \beta\right) / f^{2}$. Using $N=10 \mathrm{cph}, f=1 \mathrm{day}^{-1}, \beta=.25$, and $h_{*}=3-10 \mathrm{~m}$, we get dimensional diffusivities of up to $.1 \mathrm{~m}^{2} / \mathrm{s}$. While this is an enhancement over the estimates from shear dispersion alone, it is still an order of magnitude smaller than observations. In the open ocean, vortices may exist for many rotations, in which case the mechanisms of Section 5 are more appropriate. Using $h_{*}$ up to $30 \mathrm{~m}$, we calculate dimensional diffusivities of order $1 \mathrm{~m}^{2} / \mathrm{s}$, the same order as observations. This value will be appropriate only after a dye patch has grown to scales larger than the vortex scale (approximately an internal Rossby radius). Our analysis suggests the time necessary to get there is highly 
dependent on the initial dye patch size and the vortex lifetime (Figure 11).

There are numerous additional complexities that could be included in future work. First, given the importance of the vortex lifetime, $\tau$ in our results, we should try to get better estimates of realistic ocean values. We should consider the potential effect of instabilities as a limit on expected lifetime. Second, we could consider a model with a continuous distribution of vortex lifetimes. Third, we could use a the full baroclinic vortex form suggested by the numerical adjustment results. Such an approach would include the effects of cyclonically rotating vortices, which could significantly enhance stirring motions. Finally, we could compare our analytical results with full numerical simulations, such as those being performed by $\mathrm{P}$. Lelong [12].

\section{$7 \quad$ Acknowledgements}

I'd like to thank Jim Ledwell and Miles Sundermeyer for suggesting this project and being patient and helpful throughout. I also thank Bill Young, Glenn Flierl, Antonello Provenzale, Claudia Pasquero, Jean-Luc Thiffeault, and Stefan Llewellyn Smith for numerous helpful discussions. I'm indebted to Neil Balmforth, Bill Young and the entire GFD staff for giving me the opportunity to participate in the summer program and for all the wisdom they generously share. The summer was infinitely enhanced by the company of the other fellows for lively (and only occasionally squalid) dinners, triathalons, beach trips, and softball games. Thanks especially to Nifer, Claudia and J-Luc for their friendship and support.

\section{References}

[1] M. A. Sundermeyer, Ph.D. thesis, WHOI, 1998.

[2] J. Ledwell, A.J.Watson, and C. Law, "Evidence for slow mixing across the pycnocline from an open-ocean tracer-release experiment," Nature 364, 701 (1993).

[3] C. Garrett, "On the initial streakiness of a dispersing tracer in two- and three-simensional turbulence," Dyn. Atmos. Oceans 265 (7).

[4] W. Young, P. Rhines, and C. Garrett, "Shear-flow dispersion, internal waves and horizontal mixing in the ocean," J. Phys. Ocean. 12, 515 (1982).

[5] J. McWilliams, "Submesoscale, coherent vortices in the ocean," Rev. Geophys. 23, 165 (1985).

[6] K. Polzin, E. Kunze, J. Toole, and R. Schmitt, in progress (unpublished).

[7] M.C.Gregg, E. D'Asaro, T. Shay, and N. Larson, "Observations of persistent mixing and near-inertial internal waves," J. Phys. Ocean. 16, 856 (1986).

[8] N. Oakey, personal communication.

[9] J. McWilliams, "Vortex generation through balanced adjustment," J. Phys. Ocean. 18, 1178 (1988). 
[10] W. Blumen, "Geostrophic adjustment," Rev. Geophys. and Space Phys. 10, 485 (1972).

[11] P. Rhines and W. Young, "How rapidly is a passive scalar mixed within closed streamlines?," J. Fluid Mech. 133, 133 (1983).

[12] P. Lelong, personal communication. 


\title{
Stationary vortices in a Keplerian shear flow
}

\author{
P. Garaud
}

\section{Introduction}

\subsection{Planetary formation}

Science in it's most general definition began as a quest to answer the fundamental questions on the origin of humanity and it's relation to the surrounding Universe. One of the keys to understanding the origin of Life is the mechanism of formation of our own Solar system, and especially the formation of planets. This subject has gained a new interest in the past few years with the discovery of giant planets orbiting some of the nearest neighbouring stars. The generally accepted theory of planet formation consists in the following steps:

- Due to the onset of a large scale gravitational instability, the core of a dense molecular cloud collapses into a protostar; the conservation of its initial angular momentum results in the gradual flattening of the collapsing gas into an accretion disk around the protostar.

- The gas in the accretion disk has two components: a molecular gas, composed mainly of $\mathrm{H}_{2}$ and other small molecules, and a dust gas, composed of particles of sizes ranging from a few microns to a few centimeters. The interaction between these components takes place mainly via Stokes drag. The vertical stratification in the accretion disk relies on the balance between pressure and the vertical component of the gravitational force. As a result, since the thermal pressure of the dust gas is much smaller than that of the molecular gas, the dust settles into a very thin disk within the accretion disk.

- The dust particles then coalesce into larger and larger grains, up to sizes of a few kilometers; as they grow in mass, the dynamics of these "planetesimals" gradually decouple from that of the molecular gas.

- The planetesimals continue aggregating into planets. Giant planets may accrete some of the molecular gas still left in the accretion disk.

However, although the dust aggregation into larger grains is known to take place, the exact mechanism is poorly understood. The time-scale for this aggregation process has an upper limit of a few Myr $\left(10^{6} \mathrm{yr}\right)$ set by the evolution of the protostar into a T-Tauri star. Indeed, T-Tauri stars are observed to have intense magnetic activity and strong stellar winds which scatter all non-gravitationally bound dust and gas into the interstellar space. Random encounters of the dust particles due to thermal agitation is not sufficient to account for the growth of the dust grains into planetesimals within the T-Tauri evolution time-scale. 
In an attempt to remedy this problem, it has been shown in the case of two-dimensional barotropic turbulence in a rotating fluid that dust particles may migrate to the center of anticyclonic vortices [1]. They concentrate there for the lifetime of the vortex. As a result, provided the vortices are long lived, it is possible to greatly increase the aggregation rate, and reach the required sizes of dust grains before the T-Tauri phase. However, it is not yet clear whether a Keplerian flow can undergo self-sustained turbulence. Indeed, from the Rayleigh inflexion theorem, we see that the accretion flow is stable to linear shear instability, and the latest numerical simulations seem to indicate that the primordial solar nebula may be stable to nonlinear hydrodynamic instabilities too [2]. It has been shown that even a very small magnetic field may trigger some linear instability [3], but in this case it is not clear how the magnetic forces would influence the existence or stability of the vortices.

Although the problem of hydrodynamical stability of the accretion flows is not yet fully understood, there has been evidence in two-dimensional decaying turbulence for the spontaneous apparition and the persistence of vortices on time-scales much larger than the turnover time-scale. There is therefore hope in the Keplerian case that even if the turbulence is not self-sustained, the initial anisotropies in the flow are large enough to create these long-lived vortex structures. The work presented in this report is an attempt at finding steady state solutions for vortices in Keplerian accretion flows. If these solutions exist and are found to be stable, they would explain the persistence of the vortices, and therefore solve the remaining dust aggregation problem.

\subsection{Mathematical setup}

We will always take $\mathbf{u}$ to be the velocity field, $\psi$ the corresponding stream function and $\omega$ the potential vorticity. In the work presented here, we have chosen to simplify the problem greatly by considering only 2-dimensional, incompressible fluid motion. As a result of this approximation, we can now write

$$
\mathbf{u}=-\nabla \times\left(\psi \hat{\mathbf{e}}_{\mathbf{z}}\right)=\hat{\mathbf{e}}_{\mathbf{z}} \times \nabla \psi \text { and } \boldsymbol{\omega}=\omega \hat{\mathbf{e}}_{\mathbf{z}}=\nabla^{2} \psi \hat{\mathbf{e}}_{\mathbf{z}} .
$$

We will consider the vortex to be a perturbation on the main Keplerian accretion flow. The unperturbed shear flow $\mathbf{u}_{\mathrm{K}}$ is given by the Keplerian rotation law, which describes the equilibrium between the centrifugal and gravitational forces:

$$
\mathbf{u}_{\mathrm{K}}=v_{\mathrm{K}} \hat{\mathbf{e}}_{\theta}=\sqrt{\frac{G M}{R}} \hat{\mathbf{e}}_{\theta}
$$

where $R$ is the distance from the central accreting object of mass $M$. The corresponding vorticity is $\omega_{\mathrm{K}}=\frac{1}{2} \sqrt{\frac{G M}{R^{3}}}$. The vortex studied will be placed at a distance $R_{0}$ from the center, at $\theta=0$. In the following work, we will often have to change from the polar coordinate system around the central mass, $(R, \theta)$ to that around the vortex, $(r, \varphi)$. We chose to take $\varphi=0$ where $\theta=0$; this change of coordinate is represented in Fig.1.

The perturbed vorticity and flow are represented by dashed quantities. The equation for the evolution of the vorticity perturbation is

$$
\frac{\partial \omega}{\partial t}+\mathbf{u} \cdot \nabla \omega=\frac{\partial \omega^{\prime}}{\partial t}+\mathbf{u}_{\mathrm{K}} \cdot \nabla \omega^{\prime}+\mathbf{u}^{\prime} \cdot \nabla \omega_{\mathrm{K}}+\mathbf{u}^{\prime} \cdot \nabla \omega^{\prime}=0
$$




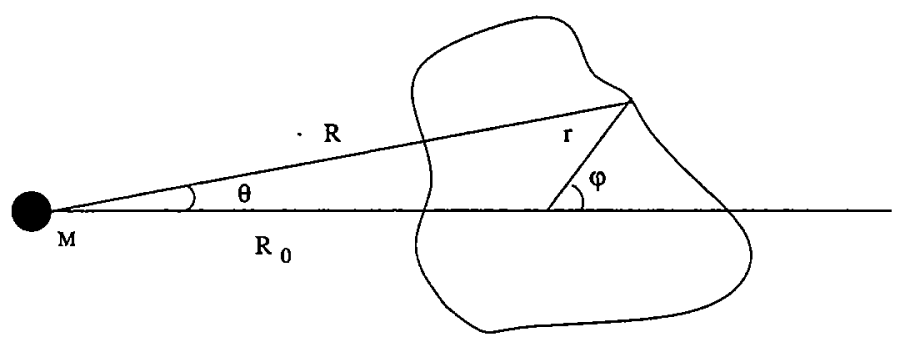

Figure 1: Change of coordinate

This can be rewritten in cylindrical coordinates around the vortex patch as

$$
\frac{\partial \omega^{\prime}}{\partial t}+v_{\mathrm{K}} \frac{1}{r} \frac{\partial \omega^{\prime}}{\partial \varphi}-\frac{1}{r} \frac{\partial \omega_{\mathrm{K}}}{\partial r} \frac{\partial \psi^{\prime}}{\partial \varphi}+\frac{1}{r} \frac{\partial \psi^{\prime}}{\partial r} \frac{\partial \omega^{\prime}}{\partial \varphi}-\frac{1}{r} \frac{\partial \omega^{\prime}}{\partial r} \frac{\partial \psi^{\prime}}{\partial \varphi}=0
$$

The vorticity perturbation and the perturbed stream function are related by

$$
\omega^{\prime}=\nabla^{2} \psi^{\prime}
$$

\subsection{Dimensionless quantities}

In order to simplify the expressions, we will now introduce the following new units system:

$$
M=1, R_{0}=1, \text { and } T_{0}=1
$$

where $T_{0}$ is the revolution time around the central object at radius $R_{0}$, namely $T_{0}=2 \pi \sqrt{\frac{R_{0}^{3}}{G M}}$. As a result, the Keplerian velocity becomes $v_{\mathrm{K}}=2 \pi R^{-1 / 2}$.

\subsection{Change of reference frame}

We will be looking for vortex solutions where the vortex is rotating around the central star with a Keplerian velocity. Steady state solutions then only have a meaning when taken in the rotating coordinate frame. We use a frame of reference rotating with velocity which is that of the center of the vortex patch. The relative shear around this point is given by

$$
v_{\mathrm{K}}(R)=2 \pi\left(R^{-1 / 2}-R\right)
$$

The corresponding stream function is

$$
\psi_{\mathrm{K}}(R)=2 \pi\left(2\left(R^{1 / 2}-1\right)-\frac{1}{2}\left(R^{2}-1\right)\right)
$$

Without loss of generality, we have chosen $\psi_{\mathrm{K}}(1)=0$. The Keplerian vorticity and its gradient are given by

$$
\omega_{\mathrm{K}}=\pi R^{-3 / 2}-4 \pi, \text { and } \frac{\partial \omega_{\mathrm{K}}}{\partial R}=-\frac{3}{2} \pi R^{-5 / 2}
$$




\section{Top hat vortices in a Keplerian shear}

In this section we will use an approximation which consists in neglecting the background Keplerian vorticity gradient in the vorticity equation. This approximation is valid primarily for small vortices. In this case, there exists a solution of the steady state problem with $\omega^{\prime}$ piecewise constant. We will therefore try to find solutions of the type

$$
\begin{aligned}
\omega^{\prime}=\nabla^{2} \psi^{\prime} & =q \text { inside the vortex patch } \\
& =0 \text { outside the vortex patch }
\end{aligned}
$$

Equation (10) can now be rewritten as

$$
\nabla^{2} \psi^{\prime}=q \mathcal{H}(a+\eta(\varphi)-r)
$$

where $a$ is the average radius of the patch, and $\eta$ is the departure from that average. We will linearize this equation by considering $\eta \ll a$, so that

$$
\nabla^{2} \psi^{\prime}=q \mathcal{H}(a-r)+q \eta(\varphi) \delta(a-r)+O\left(\eta^{2}\right)
$$

Replacing $\omega^{\prime}$ in equation (4) by this ansatz, we get the contour dynamics equation (provided we neglect the term involving the vorticity gradient)

$$
\frac{\partial \eta}{\partial t} \delta+\frac{v_{\mathrm{K}}(r)}{r} \frac{\partial \eta}{\partial \varphi} \delta+\frac{1}{r} \frac{\partial \psi^{\prime}}{\partial r} \frac{\partial \eta}{\partial \varphi} \delta+\frac{1}{r} \frac{\partial \psi^{\prime}}{\partial \varphi}\left(\delta+\eta \delta^{\prime}\right)+O\left(\eta^{2}\right)=0
$$

where $\delta \equiv \delta(a-r)$. Taking the steady state part of this equation, we integrate it once across the boundary $r=a$ to get

$$
\left(\frac{\partial \psi_{\mathrm{K}}}{\partial r}+\frac{\partial \psi^{\prime}}{\partial r}\right)_{r=a} \frac{\partial \eta}{\partial \varphi}+\frac{\partial \psi^{\prime}}{\partial \varphi}+O\left(\eta^{2}\right)=0
$$

The condition for no fluid to enter or leave the vortex (which defines it as a localized vortex patch) is that the total stream function should be constant along the boundary

$$
\left.\frac{\partial}{\partial \varphi}\left(\psi_{\mathrm{K}}+\psi^{\prime}\right)\right|_{r=a}+O\left(\eta^{2}\right)=0
$$

so that if we integrate equation (14) along the boundary, we get

$$
\psi_{\mathrm{K}}(a)+\psi^{\prime}(a)+\left(\frac{\partial \psi_{\mathrm{K}}}{\partial r}+\frac{\partial \psi^{\prime}}{\partial r}\right)_{r=a} \eta=\psi_{\eta}
$$

where $\psi_{\eta}$ is a constant. Since this implies that the velocity field is everywhere parallel to the boundary, there is no net force exerted on the vortex.. 


\subsection{Solution to zeroth order}

Let $\psi_{\mathrm{c}}$ be a solution of the zeroth order in $\eta$ of equation (12):

$$
\nabla^{2} \psi_{\mathrm{c}}=q \mathcal{H}(a-r) \text {. }
$$

We can integrate this on either sides of $r=a$, which yields the solution

$$
\begin{aligned}
\psi_{\mathrm{c}}(r) & =\frac{q r^{2}}{4}+c_{1} \ln r+c_{2} \text { for } r<a \\
& =c_{3} \ln r+c_{4} \text { for } r>a
\end{aligned}
$$

Regularity at the origin requires that $c_{1}=0$, and we can choose $c_{4}=0$. Note that the stream function diverges at infinity, but the velocity field is well behaved. Matching the function and its derivative at $r=a$ yields

$$
\begin{aligned}
\frac{q a^{2}}{4}+c_{2} & =c_{3} \ln a \\
\frac{q a}{2} & =\frac{c_{3}}{a}
\end{aligned}
$$

so that finally, we have

$$
\psi_{\mathrm{c}}(r)=\left[\frac{q r^{2}}{4}+\frac{q a^{2}}{2}\left(\ln a-\frac{1}{2}\right)\right] \mathcal{H}(a-r)+\frac{q a^{2}}{2} \mathcal{H}(r-a) \ln r .
$$

\subsection{Solution to first order}

If we define $\psi^{\prime}=\psi_{c}+\tilde{\psi}$, subtracting equation (17) from equation (12) yields

$$
\nabla^{2} \tilde{\psi}=q \eta(\varphi) \delta(a-r) .
$$

Write that $\tilde{\psi}=\sum_{n} \psi_{n} e^{i n \varphi}$, and $\eta=\sum_{n} \eta_{n} e^{i n \varphi}$, then equation (21) becomes

$$
\frac{1}{r} \frac{\partial}{\partial r}\left(r \frac{\partial \psi_{n}}{\partial r}\right)-\frac{n^{2}}{r^{2}} \psi_{n}=q \eta_{n} \delta(a-r)
$$

so the solution will be of the kind

$$
\begin{aligned}
& \psi_{n}(r<a)=a_{1}(r / a)^{|n|} \\
& \psi_{n}(r>a)=a_{2}(r / a)^{-|n|}
\end{aligned}
$$

provided $n \neq 0$; we have implicitly imposed regularity of the solutions at the origin and at infinity. Matching the solution at $r=a$ requires that $a_{1}=a_{2}$. Finally, integrating equation (21) across the discontinuity, we get

$$
\left.\frac{\partial \psi_{n}}{\partial r}\right|_{a-} ^{a+}=q \eta_{n}
$$

For $n \neq 0$, this condition yields $a_{1}=a_{2}=-\frac{q \eta_{n} a}{2|n|}$. We therefore get

$$
\tilde{\psi}=-\sum_{n \neq 0} \frac{q \eta_{n} a}{2|n|}\left((r / a)^{|n|} \mathcal{H}(a-r)+(r / a)^{-|n|} \mathcal{H}(r-a)\right) e^{i n \varphi}
$$

The case of $n=0$ is discussed in the next session. 


\subsection{Matching the vortex patch to the Keplerian flow.}

The function $\eta$ is given by equation (16), which corresponds to the requirement that the shape of the vortex patch remains steady. We rewrite it here

$$
\psi_{\mathrm{K}}(a)+\eta \frac{\partial \psi_{\mathrm{K}}}{\partial r}(a)+\psi_{\mathrm{c}}(a)+\eta \frac{\partial \psi_{\mathrm{c}}}{\partial r}(a)+\tilde{\psi}(a)=\psi_{\eta}+O\left(\eta^{2}\right)
$$

The $\tilde{\psi}$ term, as we saw, is of first order in $\eta$. This equation provides all the $\eta_{n}$ 's but $\eta_{0}$. A last condition arises from the normalization of the total vorticity of the patch, which is equivalent to fixing the area of the patch. If we require that the area be $A=\pi a^{2}$ (i.e. the area of a corresponding circular patch), then we have

$$
\begin{aligned}
A & =\int_{0}^{2 \pi} \int_{0}^{a+\eta} r \mathrm{~d} r \mathrm{~d} \varphi=\int_{0}^{2 \pi} \frac{(a+\eta)^{2}}{2} \mathrm{~d} \varphi=\pi\left(a+\eta_{0}\right)^{2}+\pi \sum_{n>0} \eta_{n}^{2} \\
& =\pi\left(a+\eta_{0}\right)^{2}+O\left(\eta^{2}\right)
\end{aligned}
$$

The normalization condition on $A$ is therefore $\eta_{0}=0$, and is valid to first order in $\eta$. The only terms left to evaluate are $\psi_{\mathrm{K}}(a)$ and $\eta \frac{\partial \psi_{\mathrm{K}}}{\partial \tau}(a)$.

\subsubsection{The linear shear case}

Before starting on the Keplerian shear flow case, let's treat the simple linear shear case; in any case, one would expect that the results of the linear shear case are recovered in the limit where the size of the vortex patch $a$ is much smaller than the distance of the patch to the center of the Keplerian shear flow $R$.

A linear shear is given by $u_{\mathrm{L}}(R)=s(R-1$ ), (taking the velocity to be 0 at the position of the center of the vortex patch) so that the corresponding stream function is $\psi_{\mathrm{L}}(R)=$ $\frac{s}{2}(R-1)^{2}$. Since, to a first approximation $R=1+r \cos \varphi+O\left(r^{2}\right)$, we have $\psi_{\mathrm{L}}(r)=$ $\frac{s}{2} r^{2} \cos ^{2} \varphi=\frac{s r^{2}}{8}\left(e^{2 i \varphi}-2+e^{-2 i \varphi}\right)$. The matching condition then yields

$$
\begin{aligned}
\frac{s a^{2}}{8}\left(e^{2 i \varphi}-2+e^{-2 i \varphi}\right) & +\left(\sum_{n \neq 0} \eta_{n} e^{i n \varphi}\right) \frac{s a}{4}\left(e^{2 i \varphi}-2+e^{-2 i \varphi}\right) \\
& +\frac{q a^{2}}{2} \ln a+\left(\sum_{n \neq 0} \eta_{n} e^{i n \varphi}\right) \frac{q a}{2}-\sum_{n \neq 0} \frac{q \eta_{n} a}{2|n|} e^{i n \varphi}=\psi_{\eta}
\end{aligned}
$$

An important point is that the first term in that expression is potentially much larger than the other ones. In order for this term to be balanced, one requires that $\eta q \approx s a$. Since $\eta \ll a$, this condition is equivalent to $q \gg s$. Hence this work is only valid for vortex patches with vorticity much larger than the local Keplerian vorticity. We also see that the second term in that expression is of order of $\eta / a$ compared to the other ones, and will be neglected in the coming analysis. As a result, if we take $\int_{0}^{2 \pi}(28) e^{-i m \varphi} \mathrm{d} \varphi / 2 \pi$, we get

$$
\psi_{\eta}=\frac{q a^{2}}{2} \ln a-\frac{s a^{2}}{4} \text { and } \eta_{ \pm 2}=-\frac{s a}{2 q}
$$


and all other $\eta_{n}$ are zero. We see again that the condition $\eta \ll a$ is equivalent to the condition $s \ll q$. We therefore have

$$
\eta(\varphi)=-\frac{s a}{q} \cos (2 \varphi)
$$

To first order in $\eta$, this corresponds to an elliptical shape ${ }^{1}$.

\subsubsection{The Keplerian shear case}

As a first approximation, let's take $R=1+r \cos \varphi$. This approximation will be discussed later. In this case, if we define

$$
\psi_{\mathrm{K}}(a) \equiv \psi_{\mathrm{K}}(1+a \cos \varphi) \equiv \sum_{n} I_{n}^{\mathrm{K}} e^{i n \varphi}
$$

we have, from the matching condition given by equation (26)

$$
\begin{aligned}
& \psi_{\eta}=I_{0}^{\mathrm{K}}+\frac{q a^{2}}{2} \ln a \\
& \eta_{n}=\frac{2 I_{n}^{\mathrm{K}}|n|}{q a(1-|n|)} \text { for }|n|>1
\end{aligned}
$$

Because of the symmetry in $\varphi \rightarrow-\varphi$ of the Keplerian shear flow, we know that $I_{n}^{\mathrm{K}}=I_{-n}^{\mathrm{K}}$, which is confirmed by expression (33). The case $n=1$ corresponds to a translation of the vortex along the $\theta$-direction (azimuthally around the central mass), so that $\eta_{1}$ can always be taken to be 0 by an appropriate change of referential ${ }^{2}$. The $I_{n}^{\mathrm{K}}$ are given by

$$
\begin{aligned}
I_{n}^{\mathrm{K}} & =\int_{0}^{2 \pi} \psi_{K}(\sqrt{1+a \cos \varphi}) e^{-i n \varphi} \frac{\mathrm{d} \varphi}{2 \pi} \\
& =2 \pi \int_{0}^{2 \pi}\left[2(\sqrt{1+a \cos \varphi}-1)-\frac{1}{2}\left(a^{2} \cos ^{2} \varphi+2 a \cos \varphi\right)\right] e^{-i n \varphi} \frac{\mathrm{d} \varphi}{2 \pi}
\end{aligned}
$$

In order to solve this integral, we need to expand it as a Taylor series (which is necessarily convergent since we have $a<1$ ). So

$$
I_{n}^{\mathrm{K}}=-\pi a^{2} \int_{0}^{2 \pi} \cos ^{2} \theta e^{-i n \varphi} \frac{\mathrm{d} \varphi}{2 \pi}+4 \pi \int_{0}^{2 \pi} \sum_{k>1} \frac{(1 / 2)_{k}}{k !} a^{k} \cos ^{k} \varphi e^{-i n \varphi} \frac{\mathrm{d} \varphi}{2 \pi}
$$

where we define $(\nu)_{k}=\nu(\nu-1)(\ldots)(\nu-k+1)$, and $(\nu)_{0}=1$. Then

$$
I_{n}^{\mathrm{K}}=-\pi a^{2} J_{2, n}+4 \pi \sum_{k=|n|}^{\infty} \frac{(1 / 2)_{k}}{k !} a^{k} \frac{1}{2^{k}} C_{\frac{k-n}{2}}^{k}
$$

\footnotetext{
${ }^{1}$ Indeed, the equation for an ellipse being $r=b{\sqrt{1-a \cos ^{2} \varphi / e}}^{-1}$, if the eccentricity $e$ is very small, then $r \approx b\left(1+e \cos ^{2} \theta / 2 a\right)=r_{0}+\frac{b e}{2 a} \cos (2 \varphi)$.

${ }^{2}$ Indeed, let's take the example of the displacement of a circular patch: the equation for a circle centered on $x=\eta$ (instead of $x=0$ ) is $(x-\eta)^{2}+y^{2}=a^{2}$. Expanding this to first order in $\eta$ and changing coordinates from $(x, y)$ to $(r, \varphi)$, we get $r=a+\eta \cos \varphi$, which corresponds to an $n=1$ deformation mode.
} 

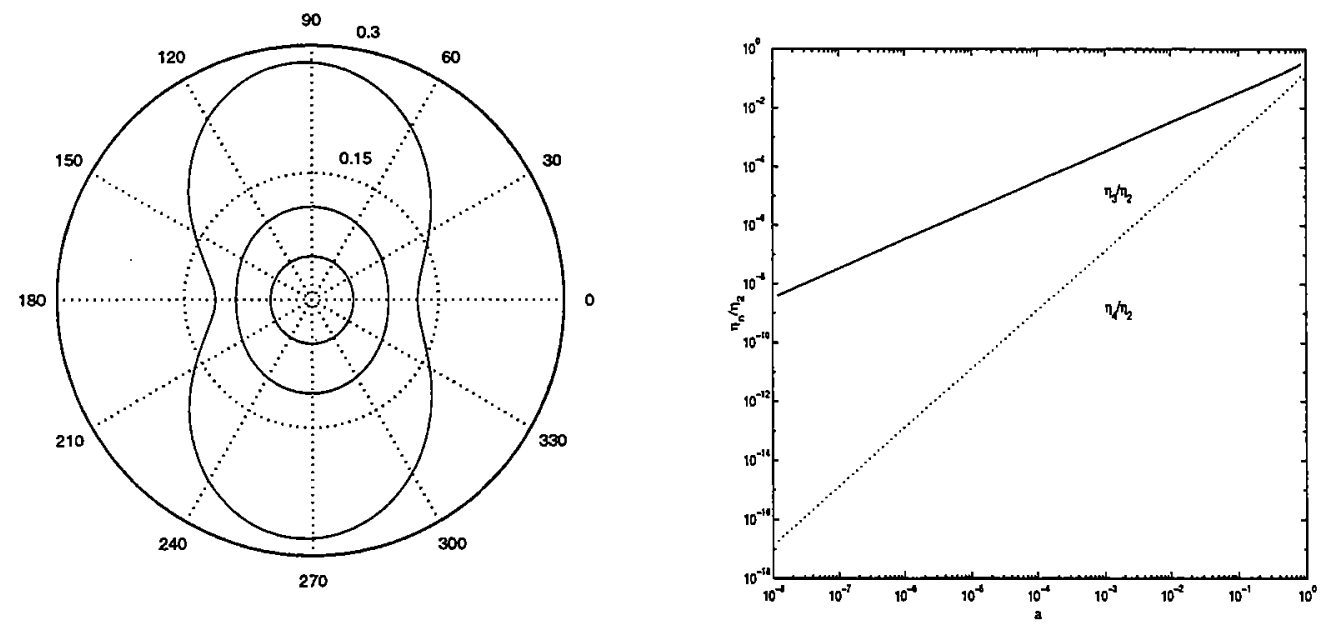

Figure 2: On the left: steady-state shape of the vortex patch, for $a=0.05,0.1$ and 0.2 in the Keplerian shear case, with the approximation $R=1+r \cos \varphi$. In all 3 plots $q=1$. On the right: Ratio of the $3 \mathrm{rd}$ and 4 th order of deformation to the $2 \mathrm{nd}$ as a function of $a$.

where the $C_{n}^{k}$ are the binomial coefficients. Finally, we compute the deformation by adding the Fourier coefficients as

$$
\eta(\varphi)=\sum_{n>0} 2 \eta_{n} \cos (n \varphi)
$$

Fig. 2 present the results for some values of $a$ and $q$. One can however guess (and check) that:

- The larger $q$, the smaller the deformation from a circular patch. Since $q$ only appears in the "normalization" of $\eta$ rather than in the relative amplitude of the modes $\eta_{n}$ of deformation, changing $q$ only amounts to changing the total amplitude of the deformation. In the following plots, a small value of $q$ was chosen on purpose to let the vortex deformation be more easily identifiable. In reality, we should take $q \gg 1$ to have the required $\eta \ll a$.

- On the other hand, the value of $a$ will influence the relative importance of the $\eta_{n}$, and will dictate the shape of the vortex. The larger $a$, the larger the higher order modes of deformation, and the more difficult the convergence.

We can see in Fig. 2 that for $a$ ( $a / R_{0}$ in real units) small, the dominant term in the deformation $\eta$ is $\eta_{2}$, the ellipsoidal term. The ratio of the 3rd and 4rd order deformation modes to the second is also shown. As we can see from this plot, for $a / R_{0}<0.1$, the shape of the vortex patch is very well approximated by an ellipse. 


\subsubsection{Validity of the approximation $R=1+r \cos \varphi$.}

The full expression for $R$ is

$$
R=\sqrt{1+r^{2}+2 r \cos \varphi} \approx 1+r \cos \varphi+O\left(r^{2}\right)
$$

therefore the approximation is only valid for $r \ll 1$. Let's study again the example of the linear shear case: this time, we have

$$
\psi_{\mathrm{L}}(R)=\frac{s}{2}(R-1)^{2}=\frac{s}{2}\left(2+r^{2}+2 r \cos \varphi-2 \sqrt{1+r^{2}+2 r \cos \varphi}\right)
$$

Following the same procedure as before (Taylor expansion + Fourier decomposition) we can obtain the Fourier coefficients of $\psi_{\mathrm{L}}(a)$ : successively

$$
\begin{aligned}
\psi_{\mathrm{L}}(a) & =-s \sum_{k>1}\left(a^{2}+2 a \cos \varphi\right)^{k} \frac{(1 / 2)_{k}}{k !} \\
& =-s \sum_{k>1} \frac{(1 / 2)_{k}}{k !} \sum_{p=0}^{k} C_{p}^{k} a^{k+p} 2^{k-p} \cos ^{k-p} \varphi
\end{aligned}
$$

so that

$$
I_{n}^{\mathrm{L}}=-s \sum_{k>1} \frac{(1 / 2)_{k}}{k !} \sum_{p=0}^{k} 2^{k-p} C_{p}^{k} a^{k+p} \frac{1}{2^{k-p}} C_{\frac{k-p-n}{2}}^{k-p}
$$

The $\eta_{n}$ are given by equation (33). The resulting steady-state shape of the vortex patch is shown in Fig. 3 This deformation is due to the fact that the flow is not a plane parallel flow, but rather curves around the central accreting object. When the size of the patch is large, this curvature acts to deform it.

To conclude, if we interpolate the linear shear results to the Keplerian case, it is likely that the approximation will fail for $a>0.1$. The full expression for $R$ should therefore be kept. The results for the Keplerian shear, using equation (38) in expression (35), are the following:

$$
I_{n}^{\mathrm{K}}=-4 \pi \sum_{k>1} \frac{(1 / 4)_{k}}{k !} \sum_{p=0}^{k} a^{k+p} 2^{k-p} C_{p}^{k} C_{\frac{k-p-n}{2}}^{k-p}
$$

The corresponding vortex patches are presented in Fig. 4, for both a cyclonic and an anticyclonic vortex.

\subsection{Discussion.}

Assuming that the background vorticity is constant, it has been possible to calculate the steady state shape of top-hat (i.e. constant piece-wise) vortices. In a linear shear, it is a well known result that the shape of the vortices should be elliptical [4]. For very small vortices, for which the variation of the background vorticity is negligible, we could expect, and saw 

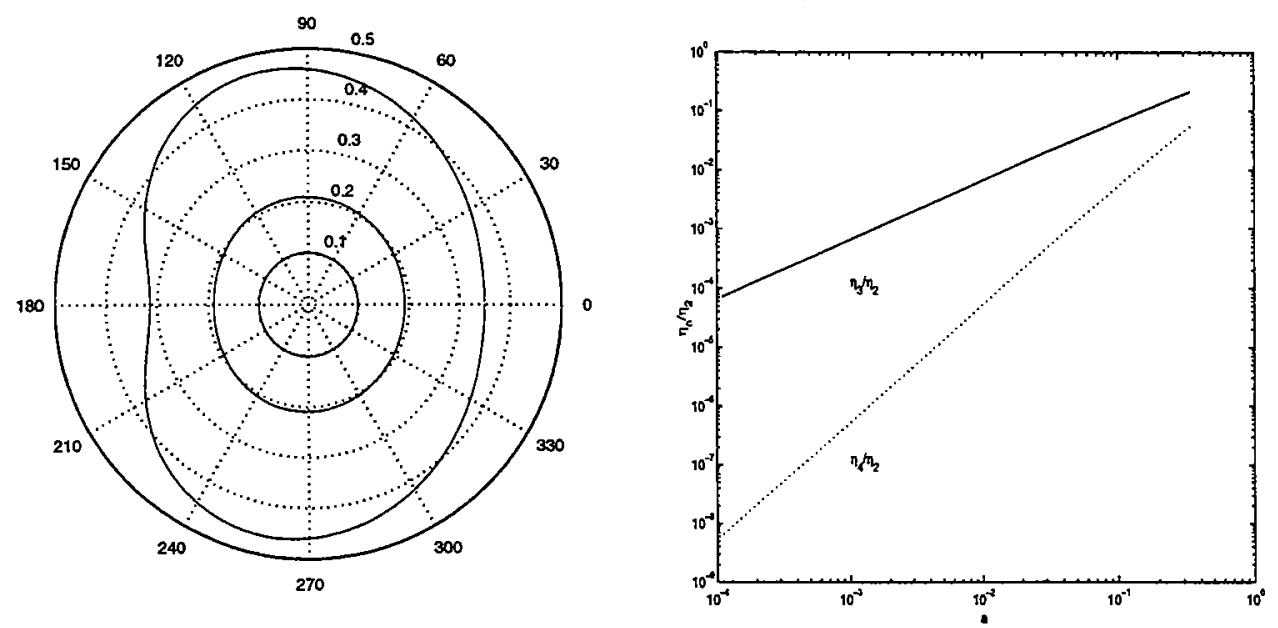

Figure 3: On the left: steady-state shape of the vortex patch, for $a=0.1,0.2$ and 0.4 in the linear shear case. In all 3 plots $q=1$. On the right: relative amplitude of the 3rd and 4th moment of deformation compared to the second, for varying values of $a$.

that the vortices were mainly elliptical in shape. However, for larger vortices ${ }^{3}$, there is a systematic variation from elliptical, and this has two main causes: firstly, the curvature of the Keplerian flow around the central star, and secondly (and this is the dominant effect), the background variation in the velocity field. The next step in this analysis would be to consider the stability of these vortices, in a similar way as has been done by Meacham et al.[5]. This is not in the scope of this project.

\section{Including the main flow vorticity gradient}

In the previous section, the vorticity gradient term in the vorticity equation (4) has been neglected in order to allow solutions with piece-wise constant vorticity. However, including the vorticity gradient term forbids this solution. In particular, when the nonlinear terms can be neglected, we will see that there exist stationary wave-like solutions: the lee waves. These have to be taken into account in their interaction with the vortex patch. In the following work, we will therefore study two main regimes:

- Far from the vortex, the perturbation induced by the vortex is small; the vorticity equation becomes a linear equation for stationary lee waves. The far field of the vortex can then be obtained by studying the lee waves which are created around a point vortex.

- Near the vortex, the perturbation is much stronger than the Keplerian flow. By rescaling the coordinate system to emphasize the region near the vortex, we will see that to a first approximation, the gradient of the vorticity can be neglected. This zeroth

\footnotetext{
${ }^{3}$ In fact the approximation of constant vorticity around the vortex is no more valid for the larger vortices anyway.
} 

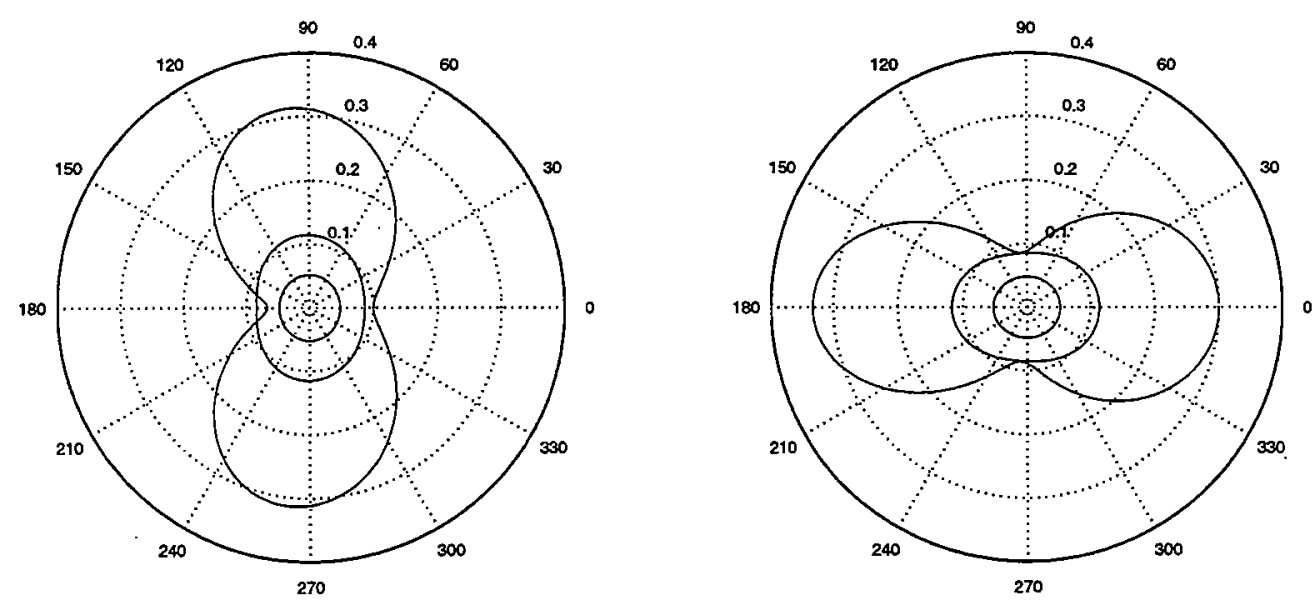

Figure 4: Deformation of a vortex patch in a Keplerian shear for $a=0.05,0.1,0.2$, and $q=1$.

order solution resembles closely that presented in Section 1 for the linear shear. The deformation of the vortex patch is then given by the next order in the approximation.

In the steady state, equation (4) becomes

$$
J\left(\psi_{\mathrm{K}}+\psi^{\prime}, \omega_{\mathrm{K}}+\omega^{\prime}\right)=0
$$

where the Jacobian $J$ is, in the cylindrical coordinate system

$$
J(A, B)=\frac{1}{R} \frac{\partial A}{\partial R} \frac{\partial B}{\partial \theta}-\frac{1}{R} \frac{\partial A}{\partial \theta} \frac{\partial B}{\partial R}
$$

Let's now use the new coordinate system

$$
\begin{aligned}
\theta & =-X \\
R & =\exp (Y)
\end{aligned}
$$

The Keplerian velocity and stream function in this new ordinate system is then (using equations (7), (8), (9) and $\Gamma=3 \pi$ )

$$
\begin{aligned}
v_{\mathrm{K}} & =\frac{2 \Gamma}{3}\left(e^{-\frac{1}{2} Y}-e^{Y}\right) \\
\psi_{\mathrm{K}} & =\frac{2 \Gamma}{3}\left(2 e^{\frac{1}{2} Y}-\frac{1}{2} e^{2 Y}-\frac{3}{2}\right) \\
\omega_{\mathrm{K}} & =\frac{\Gamma}{3} e^{-\frac{3}{2} Y}-\frac{4 \Gamma}{3}
\end{aligned}
$$

The Jacobian equation then becomes (dropping the primes on the perturbed quantities)

$$
J\left(\frac{2 \Gamma}{3}\left(2 e^{\frac{1}{2} Y}-\frac{1}{2} e^{2 Y}-\frac{3}{2}\right)+\psi, \frac{\Gamma}{3} e^{-\frac{3}{2} Y}-\frac{4 \Gamma}{3}+e^{-2 Y} \nabla^{2} \psi\right)=0
$$

where we now have $J(A, B)=\partial_{X} A \partial_{Y} B-\partial_{Y} A \partial_{X} B$, and the Laplacian operator is $\nabla^{2}=$ $\partial_{X X}+\partial_{Y Y}$. 


\subsection{The far field: a linear approach}

\subsubsection{Assumptions and equations}

We will assume that the perturbation is much smaller than the Keplerian stream function. This should be valid everywhere but around $Y=0$, where $\psi_{\mathrm{K}}$ vanishes. Equation (49) implies that

$$
\frac{\Gamma}{3} e^{-\frac{3}{2} Y}-\frac{4 \Gamma}{3}+e^{-2 Y} \nabla^{2} \psi=F\left[\frac{2 \Gamma}{3}\left(2 e^{\frac{1}{2} Y}-\frac{1}{2} e^{2 Y}-\frac{3}{2}\right)+\psi\right]
$$

The function $F$ is not unique. This means that there are many steady state solutions to the problem we are considering, each of them depending on the type of forcing, the symmetries required, the behaviour far from the vortex. We must choose the function $F$ carefully to represent the physics of the system considered. We want to represent the presence of a small vortex patch, and its influence on the Keplerian accretion flow. Far from the vortex (the region considered here), we hope that there exist solutions in which the disturbance caused by the vortex is very small, so that the Keplerian stream lines are merely displaced by a small amount. Taking these two ideas in consideration, we see that a possible prescription for $F$ is

$$
F\left(\psi_{\mathrm{K}}+\psi\right)=F_{\mathrm{K}}\left(\psi_{\mathrm{K}}+\psi\right)+Q \delta(X) \delta(Y)
$$

where $Q$ is the total vorticity of the patch, and the function $F_{\mathrm{K}}$ is defined as

$$
\omega_{\mathrm{K}}=F_{\mathrm{K}}\left(\psi_{\mathrm{K}}\right), \Leftrightarrow \frac{\Gamma}{3} e^{-\frac{3}{2} Y}-\frac{4 \Gamma}{3}=F_{\mathrm{K}}\left[\frac{2 \Gamma}{3}\left(2 e^{\frac{1}{2} Y}-\frac{1}{2} e^{2 Y}-\frac{3}{2}\right)\right]
$$

Putting this ansatz back into equation (50) we get

$$
\begin{aligned}
e^{-2 Y} \nabla^{2} \psi & =F_{\mathrm{K}}\left(\psi_{\mathrm{K}}+\psi\right)-F_{\mathrm{K}}\left(\psi_{\mathrm{K}}\right)+Q \delta(X) \delta(Y) \\
& \approx \psi F_{\mathrm{K}}^{\prime}\left(\psi_{\mathrm{K}}\right)+Q \delta(X) \delta(Y)
\end{aligned}
$$

since we assumed that $\psi \ll \psi_{\mathrm{K}}$. The function $F_{\mathrm{K}}^{\prime}$ can easily be obtained by taking the $Y$-derivative of equation (52), and is

$$
F_{\mathrm{K}}^{\prime}=\frac{\frac{\partial \omega_{\mathrm{K}}}{\partial Y}}{\frac{\partial \psi_{\mathrm{K}}}{\partial Y}}=-\frac{3}{4} e^{-\frac{3}{2} Y}\left(e^{\frac{1}{2} Y}-e^{2 Y}\right)^{-1}
$$

We finally get

$$
\left(e^{\frac{3}{2} Y}-1\right) \nabla^{2} \psi=\frac{3}{4} \psi+Q \delta(X) \delta(Y)\left(e^{\frac{7}{2} Y}-e^{2 Y}\right)
$$

Note that equation (53) is an equation for stationary waves, the lee waves. 


\subsubsection{Localized solutions to the point vortex problem}

The periodicity in $X$ suggests the expansion $\psi=\sum_{m} \psi_{m} e^{i m X}$. The symmetry of the system as $X \rightarrow-X$ limits the sum to $m \geq 0$. In this case, we will have to solve

$$
\left(e^{\frac{3}{2} Y}-1\right)\left(\frac{\partial^{2} \psi_{m}}{\partial Y^{2}}-m^{2} \psi_{m}\right)=\frac{3}{4} \psi_{m}+\frac{Q}{2 \pi} \delta(Y)\left(e^{\frac{7}{2} Y}-e^{2 Y}\right)
$$

Asymptotically, we see that there exists solutions to this equation which are localized in the radial direction:

- for $Y \gg 1$, we get

$$
e^{\frac{3}{2} Y}\left(\frac{\partial^{2} \psi_{m}}{\partial Y^{2}}-m^{2} \psi_{m}\right) \approx \frac{3}{4} \psi_{m}
$$

This equation can be solved exactly by using the change of variable $t=e^{-\frac{3}{4} Y}$, which leads to the solutions

$$
\psi_{m}=I_{ \pm \frac{4}{3} m}\left(\sqrt{\frac{4}{3}} t\right)
$$

For $Y \gg 1, t \rightarrow 0$ so we must keep the $I_{+\frac{4}{3} m}$ solution to ensure the decay of the solutions. Note that when $m=0$, there is no decaying solution. This will be discussed later.

- for $Y \ll-1$, which corresponds to the center of the accretion disk, the equation becomes

$$
\left(\frac{\partial^{2} \psi_{m}}{\partial Y^{2}}-m^{2} \psi_{m}\right) \approx-\frac{3}{4} \psi_{m}
$$

which has the decaying solutions

$$
\dot{\psi}_{m} \propto \exp \left(\sqrt{m^{2}-\frac{3 \Gamma}{4}} Y\right)
$$

when $m \neq 0$. When $m=0$, we get oscillatory solutions.

Near the point vortex $(X, Y$ small) the equation becomes

$$
Y\left(\frac{\partial^{2} \psi_{m}}{\partial Y^{2}}-m^{2} \psi_{m}\right) \approx \frac{1}{2} \psi_{m}+Y \frac{Q}{2 \pi} \delta(Y)
$$

By changing the variable to $t=\alpha Y$, we get, for the homogeneous part

$$
\frac{\partial^{2} \psi_{m}}{\partial t^{2}}-\frac{m^{2}}{\alpha^{2}} \psi_{m}-\frac{1}{2 \alpha t} \psi_{m}=0
$$


which corresponds to a Whitaker equation with coefficients $\kappa=-1 / 2 \alpha$, and $\mu^{2}=1 / 4$ (cf. Abramovitz \& Stegun [6]) provided $\frac{m^{2}}{\alpha^{2}}=1 / 4$. The solutions are the Whitaker functions $M_{\kappa, \mu}$ and $W_{\kappa, \mu}$ such that

$$
\begin{aligned}
& M_{\kappa, \mu}=e^{-\frac{1}{2} t} t^{\frac{1}{2}+\mu} M\left(\frac{1}{2}+\mu-\kappa, 1+2 \mu, t\right) \\
& W_{\kappa, \mu}=e^{-\frac{1}{2} t} t^{\frac{1}{2}+\mu} U\left(\frac{1}{2}+\mu-\kappa, 1+2 \mu, t\right)
\end{aligned}
$$

where $M$ and $U$ are the regular and singular confluent hypergeometric functions. Since we are actually solving the problem of stationary waves in a shear flow, we know that the point at which the velocity vanishes is a critical layer for the waves. The singularity of the equations near $Y=0$ reflects the presence of this critical layer. As a result we expect solutions of the kind $[7]$

$$
\begin{aligned}
& \psi_{r} \propto t P_{1}(t) \\
& \psi_{s} \propto t \ln |t| P_{1}(t)+P_{2}(t)
\end{aligned}
$$

with $P_{1}(t)=a_{0}+a_{1} t+\cdots$ and $P_{2}(t)=b_{0}+b_{1} t+b_{2} t^{2}+\cdots$ near the origin, we know that we should take $\mu=1 / 2[6]$. The expansion of the functions near the origin is then

$$
\begin{aligned}
M_{\kappa, 1 / 2} & =e^{-\frac{1}{2} t} t M(1-\kappa, 2, t)=e^{-\frac{1}{2} t} t \sum_{n=0}^{\infty} \frac{(1-\kappa)_{n} t^{n}}{(n ! 2)_{n}} \\
W_{\kappa, 1 / 2} & =e^{-\frac{1}{2} t} t U(1-\kappa, 2, t) \\
& =\frac{e^{-\frac{1}{2} t} t}{\Gamma(-\kappa)}\left[M(1-\kappa, 2, t) \ln t+\sum_{n=0}^{\infty} \frac{(1-\kappa)_{n} t^{n}}{(2)_{n} n !}\left(\psi_{\Gamma}(1-\kappa+n)\right.\right. \\
& \left.\left.-\psi_{\Gamma}(1+n)-\psi_{\Gamma}(2+n)\right)+\frac{\Gamma(-\kappa)}{\Gamma(1-\kappa)} \frac{1}{t}\right]
\end{aligned}
$$

where $\psi_{\Gamma}(a)=\Gamma^{\prime}(a) / \Gamma(a)$. We see that in order for $W_{\kappa, \mu}$ to be well defined, we need to choose $t$ positive everywhere, which means taking $\alpha_{+}=2 m$ for $Y>0$ and $\alpha_{-}=-2 m$ for $Y<0$. This also means that the $Y>0$ and $Y<0$ branches will have different values of $\kappa$ : $\kappa_{+}=-1 / 4 m$ and $\kappa_{-}=1 / 4 m$.

Let's now write the full solutions:

$$
\begin{aligned}
& \psi_{m}(Y>0)=A M_{\kappa_{+}, \frac{1}{2}}(2 m Y)+B W_{\kappa_{+}, \frac{1}{2}}(2 m Y) \\
& \psi_{m}(Y<0)=C M_{\kappa_{-}, \frac{1}{2}}(-2 m Y)+D W_{\kappa_{-}, \frac{1}{2}}(-2 m Y)
\end{aligned}
$$

Since $M_{\kappa, \frac{1}{2}}(0)=0$, and $W_{\kappa, \frac{1}{2}}(0)=\frac{1}{\Gamma(1-\kappa)}$, the continuity of $\psi_{m}$ across the origin implies

$$
\frac{B}{\Gamma(1+1 / 4 m)}=\frac{D}{\Gamma(1-1 / 4 m)}
$$

Integrating equation (56) across $Y=0$, we get the jump condition

$$
\left.\frac{\partial \psi_{m}}{\partial Y}\right|_{0-} ^{0+}=\int_{0-}^{0+} \frac{\psi_{m}}{2 Y} \mathrm{~d} Y+\frac{Q}{2 \pi}
$$


The integral term on the RHS is mathematically ill-defined. However, if we assume that it should really be the principal value of this integral, then we can show that this term is 0 using the expansion in $Y$ of $\psi$ near $Y=0$, and we are left with the simple jump condition

$$
\left.\frac{\partial \psi_{m}}{\partial Y}\right|_{0-} ^{0+}=\frac{Q}{2 \pi}
$$

Since we have $M_{\kappa, \frac{1}{2}}^{\prime}(0)=1$ and $W_{\kappa, \frac{1}{2}}^{\prime}(0)=\frac{1}{\Gamma(-\kappa)} \ln t+c(\kappa)$ where $c(\kappa)$ is a constant term, we see that the singular part of the derivative is continuous across the origin when the function $\psi_{m}$ itself is: indeed, the derivative is

$$
\begin{aligned}
& \psi_{m}^{\prime}\left(0_{+}\right)=2 m\left(A+B \frac{\ln (2 m Y)}{\Gamma\left(-\kappa_{+}\right)}+B c\left(\kappa_{+}\right)\right) \\
& \psi_{m}^{\prime}\left(0_{-}\right)=-2 m\left(C+D \frac{\ln (-2 m Y)}{\Gamma\left(-\kappa_{-}\right)}+D c\left(\kappa_{-}\right)\right)
\end{aligned}
$$

so that the continuity of the singular part of the derivative implies

$$
\frac{B}{\Gamma(1 / 4 m)}=-\frac{D}{\Gamma(-1 / 4 m)} \Leftrightarrow \frac{B}{\Gamma(1+1 / 4 m)}=\frac{D}{\Gamma(1-1 / 4 m)}
$$

using the property $\Gamma(1+x)=x \Gamma(x)$.

This comment implies that although the derivative of the function $\psi_{m}$ becomes singular near the origin, it is still possible to have a finite jump of the derivatives across the origin. The asymptotic behaviour and the jump condition define uniquely the four coefficients $A, B$, $C$ and $D$ for each value of $m$ but 0 , to yield a unique solution for the far field depending only on the vortex strength $Q$.

\subsubsection{The axisymmetric $(m=0)$ case}

In this case, the solutions do not decay at infinity. In fact, we see that for $Y \gg 1$, the equation becomes $\psi_{0}^{\prime \prime}=0$, which has the general solutions $\psi_{0}=a Y+b$, and for $Y \ll-1$ there is an oscillatory solution $\psi=c \cos \left(\sqrt{\frac{3}{4} Y}\right)+d\left(\sin \left(\sqrt{\frac{3}{4} Y}\right)\right.$. There is here an arbitrariness in the choice of the boundary conditions, which is solved by the matching with the inner solution. For the purpose of plotting the results only, we chose to take the following boundary conditions $\psi_{0}\left(Y_{\mathrm{c}}\right)=\psi_{0}\left(-Y_{\mathrm{c}}\right)=0$. Again, there exists a unique solution fulfilling these 2 boundary conditions and the jump condition at the origin.

\subsubsection{Numerical procedure and results}

Having established that there exists a unique solution to the problem, it is now easy to find it numerically. We start by integrating equation (56) from $+\infty$ and $-\infty$ towards the origin using the asymptotic behaviour as a first boundary condition. We define a free parameter $h$ as $\psi_{m}(0)=h$, and use this as a second boundary condition for both branches of the solution. We then calibrate this parameter $h$ so that the jump across the origin is indeed $Q / 2 \pi$. Since 
both parts of the solution are linear, increasing $h$ by a factor of 2 amounts to increasing $Q$ by a factor of 2: there is a linear relation between $h$ and $Q$, namely

$$
\frac{Q}{2 \pi}=s(m) h
$$

The coefficients $s(m)$ can be found numerically by fixing $h=1$. The result is shown in Fig.5, on the left. On the right, the resulting solutions for $Q=-50$ are shown. Note the slow convergence of the modes for large $m$. This is due to the fact that the point vortex is a logarithmic singularity, and the amplitude of the modes vary as $1 / \mathrm{m}$.
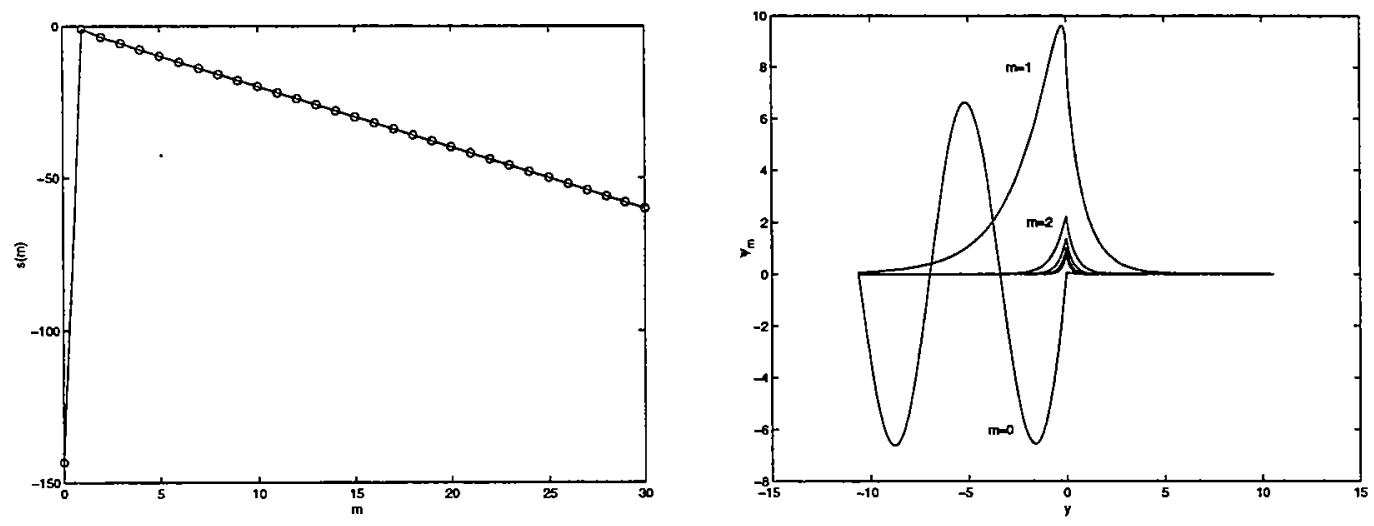

Figure 5: On the left, the coefficients $s(m)$ have been calculated and are represented as a function of $m$. On the right, this calibration has been used to calculate the functions $\psi_{m}$ for $m=0,1,2,3,4,5$ for $Q=-50$. Only the first few have been labelled. The cutoff for $\psi_{0}$ has been chosen at $Y_{\mathrm{c}}=10$.

Finally, we are left to sum the Fourier coefficients to reconstruct the function: we have

$$
\psi=\psi_{0}+2 \sum_{m=1}^{\infty} \psi_{m}(Y) \cos (m X)
$$

The contour lines of the total stream function (the perturbation and the Keplerian shear) have been plotted for 4 values of $Q$, and are represented in Fig.6. In all cases, the summation over $m$ has been truncated at $m=20$.

\subsubsection{Discussion}

The solutions obtained correspond well to what might have been expected. There are here two main features to the result. Firstly, the presence of a point vortex in any shear flow induces the deformation of stream lines seen in Fig.7. This type of deformation is also seen in the results presented here. The second feature corresponds to the presence of the critical layer at the radius $R=1$, and is characterized by the discontinuity in the velocities at that radius. This is qualitatively similar to the case of the Cat's Eyes patterns seen in the plane parallel shear flows [7]. The linear approximation theoretically fails as $Y \rightarrow 0$, and a full 

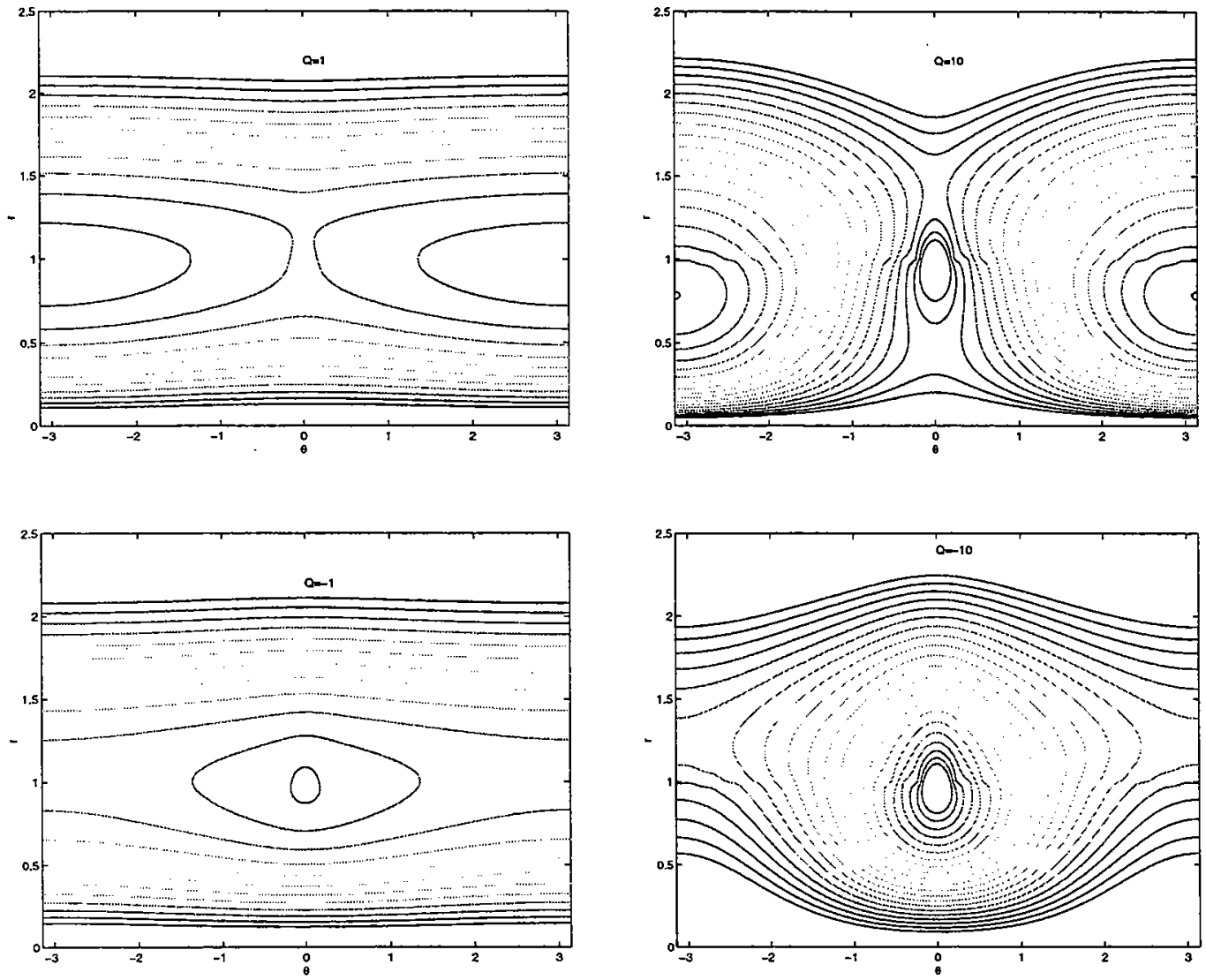

Figure 6: Stream line contours of around an anticyclonic point vortex in Keplerian shear flow, $Q=1$, (upper left), $\mathrm{Q}=10$ (upper right), $\mathrm{Q}=-1$ (lower left) and $\mathrm{Q}=-10$ (lower right)

non-linear theory would normally be necessary; however, it was shown that the nonlinear boudary layer simply connects to the linear branches of the solution far from the critical layer, without change in the phase of the logarithm, so that the solution found here is a good approximation to the nonlinear solution provided $Y \gg \epsilon$

\subsection{Close to the vortex}

In this case, we want to chose a new scaling to represent the region near the vortex. Let's chose to take $Y=\epsilon y$ and $X=\epsilon x$, and expand the equations in $\epsilon$, assuming that $\epsilon \ll 1$. We also assume the following form for the stream function $\psi$ and the vorticity:

$$
\begin{aligned}
& \psi=\epsilon^{2}\left(\psi_{0}+\epsilon \psi_{1}\right) \\
& \omega=\omega_{0}+\epsilon \omega_{1}
\end{aligned}
$$



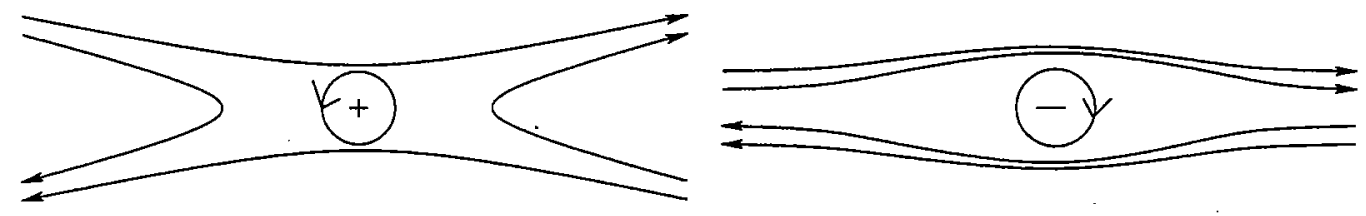

Figure 7: Stream lines around a cyclonic (left) and an anticyclonic (right) vortex positioned at a zero-velocity point

The Jacobian equation becomes

$$
\begin{array}{r}
J\left(-\frac{\Gamma}{2} y^{2}-\frac{5}{12} \Gamma \epsilon y^{3}+\psi_{0}+\epsilon \psi_{1}+O\left(\epsilon^{2}\right),\right. \\
\left.\frac{\Gamma}{3}\left(1-\frac{3}{2} \epsilon y\right)+(1-2 \epsilon y) \nabla^{2} \psi_{0}+\epsilon \nabla^{2} \psi_{1}+O\left(\epsilon^{2}\right)\right)=0
\end{array}
$$

and we also have

$$
\begin{aligned}
& \omega_{0}=\nabla^{2} \psi_{0} \\
& \omega_{1}=\nabla^{2} \psi_{1}-2 y \nabla^{2} \psi_{0}
\end{aligned}
$$

The successive orders in $\epsilon$ from the Jacobian yield

$$
\begin{aligned}
J\left(\psi_{0}-\frac{\Gamma}{2} y^{2}, \nabla^{2} \psi_{0}\right) & =0 \\
J\left(\psi_{0}-\frac{\Gamma}{2} y^{2},-\frac{\Gamma}{2} y+\omega_{1}\right)+J\left(\psi_{1}-\frac{5}{12} \Gamma y^{3}, \nabla^{2} \psi_{0}\right) & =0
\end{aligned}
$$

\subsubsection{Zeroth order solutions}

The solutions to

$$
J\left(\psi_{0}-\frac{\Gamma}{2} y^{2}, \nabla^{2} \psi_{0}\right)=0
$$

are well known, and have been calculated in the previous section: taking piece-wise constant solutions, we get an elliptical vortex patch of constant vorticity. This suggests the use of the elliptical orthogonal coordinate system $(\chi, \zeta)$, such that

$$
\begin{aligned}
& x=f \chi \zeta \\
& y=f \sqrt{\chi^{2}-1} \sqrt{1-\zeta^{2}}
\end{aligned}
$$

with $1<\chi$ and $-1<\zeta<1$. The boundary of the vortex is given by $\chi=a$ so that the solution can be written as

$$
\omega_{0}=q \mathcal{H}(\chi-a)
$$


The stream function $\psi_{0}$ is then given by equation (82). The Laplacian in the elliptical coordinate system is

$$
\begin{aligned}
\nabla^{2} \psi_{0} & =\frac{1}{f^{2}\left(\chi^{2}-\zeta^{2}\right)}\left[\sqrt{\chi^{2}-1} \frac{\partial}{\partial \chi}\left(\sqrt{\chi^{2}-1} \frac{\partial \psi_{0}}{\partial \chi}\right)\right. \\
& \left.+\sqrt{1-\zeta^{2}} \frac{\partial}{\partial \zeta}\left(\sqrt{1-\zeta^{2}} \frac{\partial \psi_{0}}{\partial \zeta}\right)\right]=q \mathcal{H}(a-\chi)
\end{aligned}
$$

Looking for separable solutions, such that $\psi_{0}(\chi, \zeta)=\sum_{n} G_{n}(\chi) H_{n}(\zeta)$, we must solve

$$
\begin{aligned}
& \sqrt{\chi^{2}-1} \frac{\partial}{\partial \chi}\left(\sqrt{\chi^{2}-1} \frac{\partial G_{n}}{\partial \chi}\right)=\lambda_{n}^{2} G_{n}+q f^{2} \chi^{2} \\
& \sqrt{1-\zeta^{2}} \frac{\partial}{\partial \zeta}\left(\sqrt{1-\zeta^{2}} \frac{\partial H_{n}}{\partial \zeta}\right)=-\lambda_{n}^{2} H_{n}-q f^{2} \zeta^{2}
\end{aligned}
$$

The homogeneous part generates the Chebyshev polynomials for both $\chi$ and $\zeta$ with $\lambda=n$. The polynomials form the basis for the regular solution. The $H_{n}$ solutions must always be regular, so that we simply have

$$
H(\zeta)=\sum_{n=0}^{\infty} b_{n} T_{n}(\zeta)
$$

where the $T_{n}$ are the Chebyshev polynomials of the first kind. However, because of the matching of $\Psi$, we also need to find the singular solution for $G$ outside the vortex. In order to do this, let's perform the change of variables $\chi=\cosh \alpha$

$$
\frac{\partial^{2} G_{n}}{\partial \alpha^{2}}=n^{2} G_{n}
$$

We have the solutions, for $n>0$

$$
\begin{aligned}
G_{n}=a_{n} e^{n \alpha}+\tilde{a_{n}} e^{-n \alpha} & =a_{n}\left(\chi+\sqrt{\chi^{2}-1}\right)^{n}+\tilde{a_{n}}\left(\chi+\sqrt{\chi^{2}-1}\right)^{-n} \\
& \equiv a_{n} R_{n}+\tilde{a}_{n} S_{n}
\end{aligned}
$$

which defines the functions $R_{n}$ and $S_{n}$, and and for $n=0$

$$
G_{0}=a_{0} \ln \left(\chi+\sqrt{\chi^{2}-1}\right)+\tilde{a_{0}}=a_{0} S_{0}+\tilde{a_{0}}
$$

which defines $S_{0}$, and $R_{0} \equiv 1$. The special solution, necessary inside the vortex, is a second order polynomial in the variables $\chi$ or $\zeta$ respectively, so that the final solution is

$$
\begin{aligned}
\psi_{0}^{\text {in }} & =\sum_{n=0}^{\infty}\left(A_{n} T_{n}(\chi)+\left(\chi^{2}-2\right) \frac{q f^{2}}{2}\right)\left(B_{n} T_{n}(\zeta)+\left(\zeta^{2}-2\right) \frac{q f^{2}}{2}\right) \\
\psi_{0}^{\text {out }} & =\sum_{n=0}^{\infty} C_{n} S_{n}(\chi) T_{n}(\zeta)
\end{aligned}
$$


where we have used the fact that the solutions must be regular inside the vortex, and that they should decay outside the vortex. Note that we can rewrite $x^{2}=\left(T_{0}(x)+T_{2}(x)\right) / 2=$ $\left(1+T_{2}(x)\right) / 2$. The matching condition at the boundary $\chi=a$ yields the following relation between the coefficients ${ }^{4}$ :

$$
\begin{aligned}
\left(A_{n} T_{n}(a)+\left(a^{2}-2\right) \frac{q f^{2}}{2}\right)\left(B_{n}+\frac{q f^{2}}{4} \delta_{n, 2}\right) & =C_{n} S_{n}(a) \text { for } n>0 \\
\left(A_{0}+\left(a^{2}-2\right) \frac{q f^{2}}{2}\right)\left(B_{0}-3 \frac{q f^{2}}{4}\right) & =C_{0} S_{0}(a) \text { for } n=0
\end{aligned}
$$

The matching of the derivatives yields a similar system,

$$
\begin{aligned}
\left(A_{n} T_{n}^{\prime}(a)+q f^{2}(a-1)\right)\left(B_{n}+\frac{q f^{2}}{4} \delta_{n, 2}\right) & =C_{n} S_{n}^{\prime}(a) \text { for } n>0 \\
q f^{2}(a-1)\left(B_{0}-3 \frac{q f^{2}}{4}\right) & =C_{0} S_{0}^{\prime}(a) \text { for } n=0
\end{aligned}
$$

Using equation (82) with the fact that $\frac{\partial \omega_{0}}{\partial \zeta}=0$ yields

$$
\left.\frac{\partial}{\partial \zeta}\left(\psi_{0}-\frac{1}{2} \Gamma y^{2}\right)\right|_{\chi=a}=0
$$

or rather,

$$
\psi_{0}-\left.\frac{1}{2} \Gamma y^{2}\right|_{\chi=a}=\psi_{0}(a, \zeta)-\frac{1}{2} \Gamma f^{2}\left(a^{2}-1\right)\left(1-\zeta^{2}\right)=c
$$

where $c$ is a constant. This implies that we must take

$$
C_{n} S_{n}(a)=\frac{\Gamma}{2} f^{2}\left(a^{2}-1\right)\left(\delta_{n, 0}-\frac{1}{2} \delta_{n, 2}\right)
$$

so we see that only 2 coefficients are non-zero, namely $C_{0}$ and $C_{2}$.

If we were to match this with the Keplerian shear flow, and ignore the far field solution, we would then obtain a unique relation between the size of the vortex $a$ and it's vorticity $q$.

\subsubsection{First order solutions}

We now have to solve equation (83). The Jacobians in this equation can directly be transformed into Jacobians for the new coordinate system: with

$$
\mathcal{J}(A, B)=\frac{\partial A}{\partial \chi} \frac{\partial B}{\partial \zeta}-\frac{\partial A}{\partial \zeta} \frac{\partial B}{\partial \chi}
$$

\footnotetext{
${ }^{4}$ In order to derive these conditions, we use the orthogonality relation between the Chebyshev polynomials

$$
\begin{aligned}
\int_{-1}^{1} T_{n}(\zeta) T_{m}(\zeta) \frac{\mathrm{d} \zeta}{\sqrt{1-\zeta^{2}}} & =\frac{\pi}{2} \delta_{m, n} \text { if } n \neq 0 \\
& =\pi \delta_{m, n} \text { if } n=0
\end{aligned}
$$


we get

$$
\mathcal{J}\left(\psi_{0}-\frac{\Gamma}{2} y^{2},-\frac{\Gamma}{2} y+\omega_{1}\right)+\mathcal{J}\left(\psi_{1}-\frac{5}{12} \Gamma y^{3}, \nabla^{2} \psi_{0}\right)=0
$$

Since $\nabla^{2} \psi_{0}=q \mathcal{H}(a-\chi)$, we see that

$$
\mathcal{J}\left(\psi_{0}-\frac{\Gamma}{2} y^{2},-\frac{\Gamma}{2} y+\omega_{1}\right)=-q \delta(a-\chi) \frac{\partial}{\partial \zeta}\left(\psi_{1}-\frac{5}{12} \Gamma y^{3}\right)_{\chi=a}
$$

This equation suggests the ansatz $\omega_{1}=q \eta(\zeta) \delta(a-\chi)+\omega_{2}$, so that we have the condition

$$
\frac{\partial \eta}{\partial \zeta} \frac{\partial}{\partial \chi}\left(\psi_{0}-\frac{\Gamma}{2} y^{2}\right)_{\chi=a}+\frac{\partial}{\partial \zeta}\left(\psi_{1}-\frac{5}{12} \Gamma y^{3}\right)_{\chi=a}=0
$$

which can be integrated along the boundary to yield

$$
\eta(\zeta) \frac{\partial}{\partial \chi}\left(\psi_{0}-\frac{\Gamma}{2} y^{2}\right)_{\chi=a}+\psi_{1}(a, \zeta)-\frac{5}{12} \Gamma f^{3}\left(a^{2}-1\right)^{3 / 2}\left(1-\zeta^{2}\right)^{3 / 2}=\psi_{\eta}
$$

The equation for $\omega_{2}$ is

$$
\mathcal{J}\left(\psi_{0}-\frac{\Gamma}{2} y^{2},-\frac{\Gamma}{2} y+\omega_{2}\right)=0
$$

which implies

$$
\omega_{2}=G\left(\psi_{0}-\frac{\Gamma}{2} y^{2}\right)+\frac{\Gamma}{2} y
$$

As in the far field solution, we must chose the function $G$ to represent the presence of a vortex. Ideally, the functions $F$ should be the linear continuation of $G$ when $\psi \ll \psi_{\mathrm{K}}$. As a first guess, we chose to take simply $G \equiv 0$, so that

$$
\omega_{2}=\frac{\Gamma}{2} y
$$

We now have to express the stream function $\psi_{1}$ as a function of $y$. This can be done by solving the equation

$$
\nabla^{2} \psi_{1}=2 y q \mathcal{H}(a-\chi)+q \eta(\zeta) \delta(a-\chi)+\frac{\Gamma}{2} y
$$

Set $\psi_{1}=\phi_{1}+\phi_{2}+\phi_{3}$, where the $\phi_{i}$ satisfy respectively

$$
\begin{aligned}
\nabla^{2} \phi_{1} & =q \eta(\zeta) \delta(a-\chi) \\
\nabla^{2} \phi_{2} & =\frac{\Gamma}{2} y \\
\nabla^{2} \phi_{3} & =2 q y \mathcal{H}(a-\chi)
\end{aligned}
$$


In all three cases we will have to solve the homogeneous equation $\nabla^{2} \phi_{h}=0$. This has already been done in the zeroth order case, and the result is

$$
\phi_{h}^{\text {out }}=\sum_{n=0}^{\infty}\left(a_{n}^{\text {out }} R_{n}(\chi)+b_{n}^{\text {out }} S_{n}(\chi)\right) T_{n}(\zeta)
$$

outside the vortex, where the singular solution must be kept, and

$$
\phi_{h}^{\text {in }}=\sum_{n=0}^{\infty} a_{n}^{\text {in }} T_{n}(\chi) T_{n}(\zeta)
$$

inside the vortex. For $\phi_{2}$ and $\phi_{3}$, the special solutions are easy to find and we get

$$
\begin{aligned}
& \phi_{2}=\phi_{h}+\phi_{2, s}=\phi_{h}+\frac{\Gamma}{12} y^{3}+c_{1} y+c_{0} \\
& \phi_{3}=\phi_{h}+\phi_{3, s}=\phi_{h}+\left(\frac{q}{3} y^{3}+d_{1} y+d_{0}\right) \mathcal{H}(a-\chi)
\end{aligned}
$$

Note that the solutions are divergent for large $y$, and that the true solution is obtained by matching the near-vortex solution to a far field, wave-like solution. Inside the vortex, however, the solutions must be regular.

To summarize, renormalizing the coefficients $a_{n}$ and $b_{n}$, we have

$$
\begin{aligned}
\psi_{1}^{\text {in }} & =\sum_{n=0}^{\infty} a_{n}^{\text {in }} T_{n}(\chi) T_{n}(\zeta)+\phi_{2, s}+\phi_{3, s} \\
\psi_{1}^{\text {out }} & =\sum_{n=0}^{\infty}\left(a_{n}^{\text {out }} R_{n}(\chi)+b_{n}^{\text {out }} S_{n}(\chi)\right) T_{n}(\zeta)+\phi_{2, s}
\end{aligned}
$$

where by definition, $R_{0}(\chi)=1$. The continuity of the function across the boundary of the vortex implies that

$$
\frac{\pi}{2} a_{n}^{\text {in }} T_{n}(a)+\int_{-1}^{1} \frac{\phi_{3, s}(a, \zeta)}{\sqrt{1-\zeta^{2}}} T_{n}(\zeta) \mathrm{d} \zeta=\frac{\pi}{2}\left(a_{n}^{\text {out }} R_{n}(a)+b_{n}^{\text {out }} S_{n}(a)\right)
$$

for $n>0$ and

$$
\pi a_{n}^{\text {in }}+\int_{-1}^{1} \frac{\phi_{3, s}(a, \zeta)}{\sqrt{1-\zeta^{2}}} \mathrm{~d} \zeta=\pi\left(a_{0}^{\text {out }}+b_{0}^{\text {out }} S_{0}(a)\right)
$$

for $n=0$. For $\phi_{1}$, the function must be continuous across the boundary, but the derivative has a jump given by equation (112). Integrating (112) across the boundary, we get

$$
\frac{1}{f^{2}} \frac{a^{2}-1}{a^{2}-\zeta^{2}}\left[\frac{\partial \phi_{1}}{\partial \chi}\right]_{a-}^{a+}=q \eta(\zeta)
$$

Let's write $\eta(\zeta)=\sum_{n=0}^{\infty} \eta_{n} T_{n}(\zeta)$. The matching condition of the derivatives therefore implies that, for $n>0$

$$
\left(a_{n}^{\text {out }} R_{n}^{\prime}(a)+b_{n}^{\text {out }} S_{n}^{\prime}(a)\right)-a_{n}^{\text {in }} T_{n}^{\prime}(a)=q \eta_{n}
$$


and for $n=0$,

$$
b_{0}^{\text {out }} S_{0}^{\prime}(a)=q \eta_{0}
$$

The coefficients $\eta_{n}$ can actually be determined from self-consistently using equation (105), provided we know $a_{n}$ and $b_{n}$ for all $n$. If we truncate the system at the order $N-1$, there are in total $3 N+3$ coefficients to solve for, and $2 N$ matching conditions. The remaining coefficients are given by the matching of this solution to a far field.

\subsection{Matching of the far field to the vortex solution.}

The behaviour of the far-field is mostly determined by the total vorticity $Q$ of the vortex patch (with the exception of the axisymmetric component). In order to be consistent between the far-field and the close-field, we require that $Q=\epsilon^{2} q$, since we assumed the size of the vortex patch to be of order of $\epsilon$. The aim of this section is more to assess whether such a matching is possible rather than to perform it. The actual matching, as we shall see, can only be done numerically, and will be the aim of future work.

In order to do this matching, it is necessary to study the behaviour of the inner solution for $\chi \rightarrow \infty$ and the outer solution as $\chi \rightarrow 1$. The elliptical coordinate system asymptotically tends to the polar coordinate system as $\chi \gg 1$. Indeed, we then have $r^{2}=x^{2}+y^{2} \approx f^{2} \chi^{2}$ and $\zeta \approx \cos \varphi=x / \sqrt{x^{2}+y^{2}}$. Also, we use the property that $T_{n}(\cos \varphi)=\cos (n \varphi)$, and that $\chi+\sqrt{\chi^{2}-1} \approx 2 \chi$. As a result, for $\chi \gg 1$, the inner solution tends to

$$
\begin{aligned}
\psi^{\text {inner }} & =\epsilon^{2} \sum_{n=0}^{\infty} C_{n}\left(\frac{2 r}{f}\right)^{-n} \cos (n \varphi)+\epsilon^{3}\left[a_{0}^{\text {out }}+b_{0}^{\text {out }} \ln \left(\chi+\sqrt{(} \chi^{2}-1\right)\right) \\
& \left.+\sum_{n=1}^{\infty}\left(a_{n}^{\text {out }}\left(\frac{2 r}{f}\right)^{n}+b_{n}^{\text {out }}\left(\frac{2 r}{f}\right)^{-n}\right) \cos (n \varphi)+\phi_{2, s}\right]
\end{aligned}
$$

On the other hand, as we saw, the outer solution tends to

$$
\begin{aligned}
\psi^{\text {outer }}=\alpha_{ \pm} \psi_{0}^{\text {outer }}(|Y|) & +\sum_{n=1}^{\infty}\left(A_{m, \pm} M_{ \pm 1 / 4 m, 1 / 2}(|Y|)\right. \\
& \left.+B_{m, \pm} W_{ \pm 1 / 4 m, 1 / 2}(|Y|)\right) \cos (n X)
\end{aligned}
$$

where the \pm sign refers to the difference in the $Y>0$ and $Y<0$ branches. All the coefficients $A_{m, \pm}$ and $B_{m, \pm}$ are uniquely defined, with the exception of the $m=0$ mode where we imposed some additional boundary conditions to determine them. These may be taken as free parameters if necessary to perform the matching on to the inner solution.

Let's study the various terms that appear in the inner and the outer, and that may cause problems in the matching. The most obvious term is the axisymmetric term, which has the main component as $(\Gamma / 12) \epsilon^{3} y^{3}$ in the inner, and that can be shown to behave as $c_{0}+c_{1} Y+c_{2} Y^{2}+c_{3} Y^{3}+\ldots+$ logarithmic terms in the outer. The $Y^{3}$ terms can be matched, since we can to choose the coefficients $c_{3}$ on either sides of $Y=0$ to be $\Gamma / 12$. This is possible since we had the freedom of varying the boundary conditions on the axisymmetric mode to fit 
this requirement. Next, we must fit the logarithmic terms. The main logarithmic dependence in the inner comes from the $O\left(\epsilon^{2}\right)$ term. As we take $y \rightarrow \infty$, this term can be assimilated to the contribution from a point vortex only in the outer region. We expect this term to match exactly onto the outer solution for a point vortex only, which has been studied in Section 3.2.5. Finally, it can be shown that the remaining difference between the point vortex case and the vortex+waves case is non-singular, so that this could possibly be matched onto the $O\left(\epsilon^{3}\right)$ term in the inner. This last matching would yield the coefficients $a_{n}$ and $b_{n}$, and therefore determine the shape of the boundary by determining $\eta$.

\section{Conclusion}

In an attempt to understand the dynamics of vortices in accretion flows, we have been looking for steady state solutions of such a system, since the existence of stable steady states might be reason for the observed longevity of the vortices. The first part of the project was a simple attempt at finding such solutions using the rather crude assumption of a constant vorticity field, which is only truly justified in the case small vortex patches. This assumption allowed us to consider top-hat vortex solutions, and study their steady state shapes. The second part of the project was an attempt at dropping this assumption. In that case, it has been shown that a general stationary lee wave solution must be added to the vortex solution in order to satisfy the vorticity equation. This problem can only be solved asymptotically in two limits: far from the vortex, it is possible to find linearized solutions. Closer to the vortex, an expansion in the small parameter $\epsilon$ which is really the ratio of the size of the vortex to the distance to the center of the shear flow, yields results very similar to the first section: to zeroth order, we recover the elliptical vortex solution, and to first order, the deformation of the vortex matches onto the "background flow", which consists of the Keplerian flow and the lee waves. The possibility of the matching between the two solution has been considered, and will be the purpose of future work.

\section{References}

[1] A. Provenzale, "Transport by coherent barotropic vortices," Ann. Rev. Fluid Mech. 31, 55 (1995).

[2] S. A. B. J. F. Hawley and W. F. Winters, "Local hydrodynamic stability of accretion disks," ApJ 518, 394 (1999).

[3] S. A. Balbus and J. F. Hawley, "A powerful local shear instability in weakly magnetized disks. i - linear analysis. ii - nonlinear evolution," ApJ 376, 214 (1991).

[4] S. Kida, "Motion of elliptic vortex in a uniform shear flow," Journ. Phys. Soc. Japan 50, 3517 (1981).

[5] G. R. F. S. P. Meacham and U. Send, "Vortices in shear," Dynamics of Atmospheres and Oceans 14, $333(1990)$. 
[6] M. Abramowitz and I. A. Stegun, Handbook of Mathematical Functions (Wiley Interscience Publications, New York, 1972).

[7] P. G. Drazin and W. H. Reid, Hydrodynamic stability (CUP, Cambridge, 1981). 


\title{
Some analysis of a two-dimensional double diffusion experiment
}

\author{
David Osmond
}

\section{Introduction}

Convection driven by double diffusion occurs when two properties contributing to the density of a fluid diffuse at different rates. In the ocean, the density of water is governed primarily by heat and salt, and the heat diffuses about 100 times faster than salt. It is now clear that double diffusion is an important process driving convection in the ocean, especially given its ability to move fluid particles across isopycnals.

Despite the primary mechanisms of double diffusion being formulated back in 1960 by Stern [1] as he considered the 'perpetual salt fountain' experiment conceived by his colleagues a few years earlier [2], there remains a great deal to be learnt on the subject. The two simplest examples of double diffusive convection are salt fingers and diffusive layers. Both types involve a stable stratification of one component, and an unstable stratification of the other, although the overall density field is always stable. Fingers occur when the faster diffusing component is stably stratified. If we consider the heat-salt situation present in oceans, salt fingers occur when the salt is unstably stratified, so there is hot salty water overlying cold fresh water. If a perturbation moves a fluid parcel downwards, it finds itself in cooler and fresher surroundings than was previously the case. It loses both heat and salt to the surrounding fluid; however, since temperature diffuses more rapidly, it experiences a net. increase in density and thus continues its downwards motion. The surrounding fluid gains heat from this descending finger, and it in turn becomes lighter and moves upwards. Eventually the region becomes filled with fingers moving in alternating upwards and downwards directions.

Diffusive layers result when the slower diffusing substance is stably stratified. Thus in the ocean they occur when cool fresh water over lies hot salty water. Temperature diffuses faster than salt, so the bottom layer is heating the top layer, thus driving convection in a similar manner to Rayleigh Bénard convection. The top layer also cools the bottom, which also drives convection. The convection tends to homogenise the fluid above and below the diffusive interface so that a very sharp interface results.

These two processes are primarily one-dimensional. The substances in the mean state have gradients only in the vertical direction. The situation becomes more complicated when there are gradients in both horizontal and vertical planes, and the physics of this process are less well understood.

In 1996 Turner and Veronis resumed work on an experiment Turner had been considering for a long time, and which was designed to look at one example of the two-dimensional double 


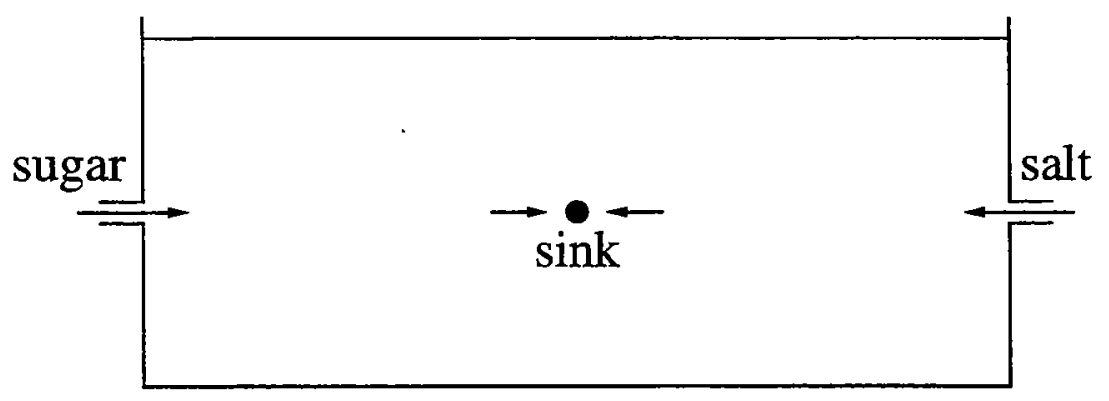

Figure 1: The experimental set-up. Salt and sugar are slowly pumped into the left and right hand side respectively of a long tank. Both source rates are $5 \mathrm{ml}$ per minute. The volume of fluid in the tank is kept constant by using a constant head overflow device in the very center of the tank.

diffusive problem. Veronis' suggestion was to consider the simplest configurations possible, and here we consider one of them.

\section{The Experiment}

A long thin tank $(1820 \times 80 \times 120 \mathrm{~mm})$ is constructed with an inlet at the center of each end, and a constant pressure head outlet at mid height in the center of the tank. It is pictured in figure 1. The tank is filled with a 50-50 mixture of salt and sugar with a density $\rho=1100 \mathrm{~kg} / \mathrm{m}^{3}$. Sugar is chosen as the second diffusing component, as it does not diffuse through the side walls of the tank as does heat. Sugar has a diffusivity about $1 / 3$ that of salt, and so in this system plays the role that salt does in the ocean, while the salt plays the part of heat in the real ocean.

The experiment commences by slowly pumping a salt solution with density $\rho=1100 \mathrm{~kg} / \mathrm{m}^{3}$ through the inlet at the left end of the tank, and a sugar solution with the same density in through the right hand inlet. Both flow rates are very close to $5 \mathrm{~cm}^{3} / \mathrm{min}$. Initially, when the salt emerges from the source at the left inlet, it is much saltier than the fluid in the tank, and thus salt diffuses out of it. Sugar also diffuses from the fluid in the tank into the salt plume, but at a slower rate. The plume gets lighter as salt diffuses from it, while a sheath of fluid surrounding the plume gets denser as the salt from the plume diffuses into it. Thus the plume separates into a light core which convects up towards the surface, and a dense sheath which sinks towards the base. The opposite process occurs at the sugar source, so that the core sinks and the sheath rises.

As time continues, the density difference between the the top and bottom of the tank increases. The rate of increase slows however, and after some time (a few days in this experiment), a steady state density field is formed. Active double diffusive convection still continues however, although it does not alter the density field significantly. To first order, the steady state convection consists of a region of fingering in the top left quadrant of the tank above the salt plume, and again in the lower right quadrant beneath the sugar source. In the top right and bottom left quadrants, diffusive layers are visible. 

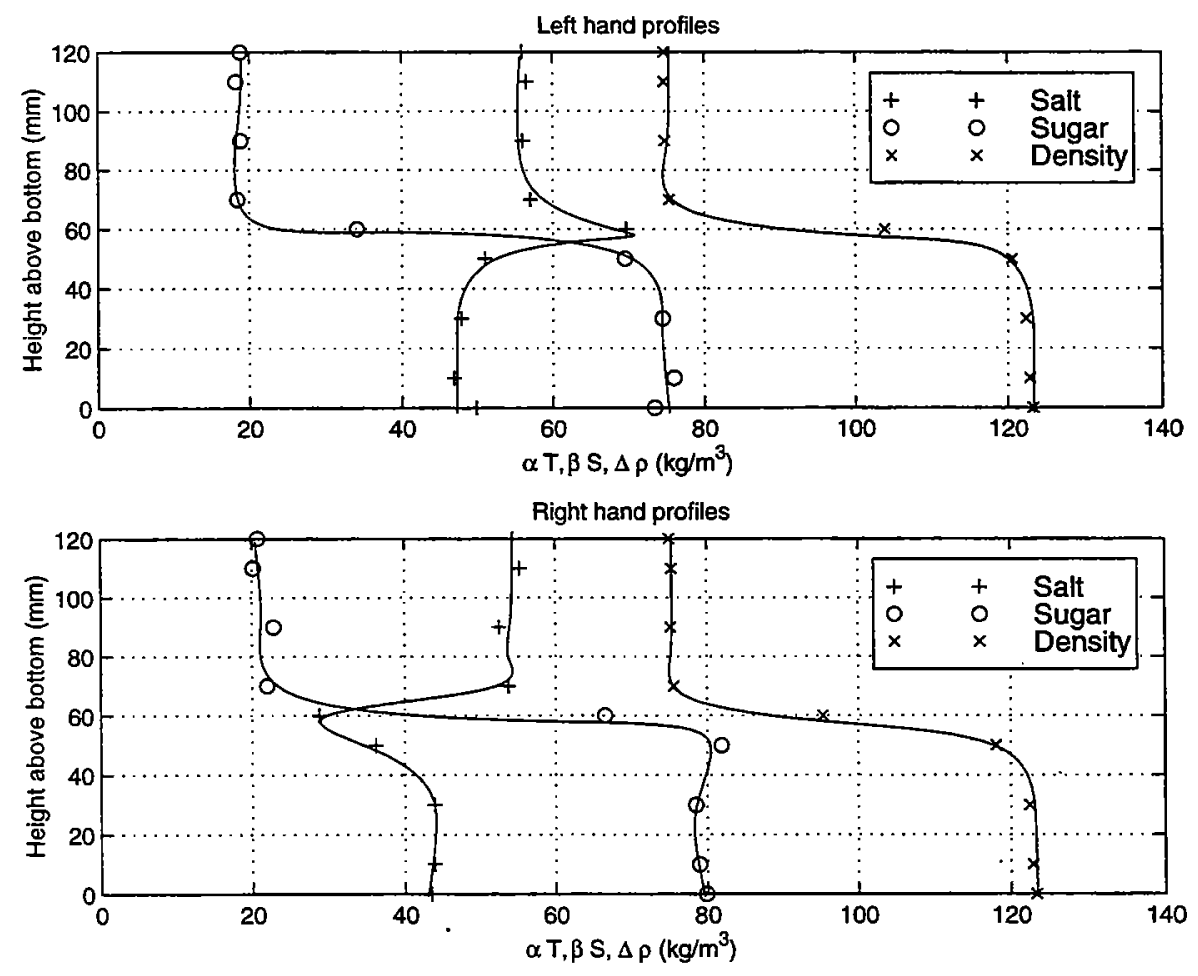

Figure 2: Sugar, salt and density profiles of the left and right hand side of the experimental tank. Salt and sugar concentrations are given in units of density 
The steady state sugar, salinity and density profiles are shown in Figure 2. There are distinct differences between the left and right hand side of the tank, as would be expected from the antisymmetric convective patterns, so they are profiled separately. The most dominant feature of the profiles is the rapid increase of density at mid height. In particular, in both the left and right hand sides of the tank most of this increase has arisen from the sugar having settled predominantly to the bottom half of the tank. In contrast, the salt is marginally unstable in both sides of the tank, having slightly higher concentrations in the top half than in the bottom, and rapidly changing extrema at mid height.

Looking more carefully at the profiles, we can broadly divide each side of the tank into 4 layers. Starting with the left hand side, on top we have fairly constant concentrations for about 50mm. As mentioned earlier, fingers were observed in this region, which in this experiment are associated with a stable salt gradient, and an unstable sugar gradient. There are some indications of these gradients, although they are clearly very small compared to most of the other features.

The next $10 \mathrm{~mm}$ contain rapid increases of both salt and sugar, thus leading to a very stable density profile. This acts as a very strong barrier to any kind of vertical convection through this layer, and indeed one would only expect pure diffusion to transfer properties from one side to the other. Measurements of the profile from Figure 2 leads us to estimates of the diffusive salt flux to be of order $0.14 \mathrm{mg} / \mathrm{s}$, while the sugar flux is about $0.06 \mathrm{mg} / \mathrm{s}$. The rate at which both salt and sugar are being pumped into the tank through the source is approximately $8.33 \mathrm{mg} / \mathrm{s}$, or about 60 and 150 times larger than the pure salt and sugar diffusive fluxes respectively.

From $50-60 \mathrm{~mm}$ above the bottom of the tank there is a very stable sugar gradient, and an unstable salt gradient. We associate this with a strong diffusive layer, and indeed that is what is seen in the experiment. In the lowest $50 \mathrm{~mm}$ the sugar concentration gently increases and the salt decreases downward as in the diffusive layer above.

The layers are reversed in the right hand side of the tank. There is a region of weak diffusing layers on top of a sharp diffusive interface, followed by a layer stable in both properties, while weak fingering occurs in the lowest layer. The level of the sources and sink corresponds very closely to the interface between the strong diffusive layer and the stable layer.

As mentioned earlier, the dominant feature of the profiles was the fact that most of the sugar was in the bottom half of the tank, while the salt is marginally unstable. This is very similar to the profile one would expect if one had run a diffusive layer experiment by placing a salt solution above a sugar one. The unstable salt stratification drives the diffusive layers until the salt is nearly evenly distributed between the upper and lower halves, in the process raising some of the sugar, but not as much as the salt that is lowered. Diffusive layers do a better job of reducing the potential energy of a fluid than do fingers, so from energy considerations it is not surprising that the density field resembles that of a diffusive layers experiment rather than a fingers experiment. But given that both diffusive layers and fingers are active in this experiment, it is not immediately clear from a dynamic viewpoint as to why the diffusive layers dominate the concentration profiles.

Given that the sugar travels upwards through the diffusive layers, and downwards through the fingers, we present Figure 3 as a simplistic picture of the salt and sugar pathways through the tank. 


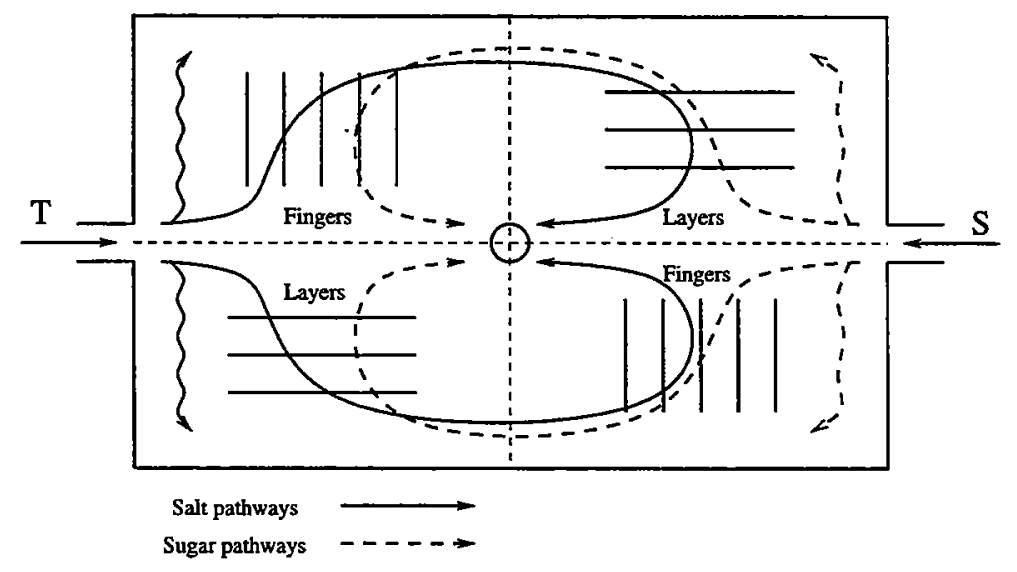

Figure 3: Simplistic picture of salt and sugar pathways through tank.

One aspect of double diffusion experiments that has been looked at quite closely is the ratio of the fluxes through either the fingers or the diffusive layers. For salt-sugar fingers the ratio of sugar to salt fluxes is around 0.9 , provided the density ratio, $R_{\rho}=\alpha \bar{T}_{z} / \beta \bar{S}_{z}$ is not too close to one. For salt-sugar diffusive layers, the ratio of salt to sugar fluxes is around 0.6 , again provided the density ratio is not too close to one. Given that we have four dominant flux pathways indicated in Figure 3, and four diffusive regions, we may construct a series of 4 equations with 4 unknowns. Alas, the 4 equations are not independent, nor are they even consistent, so there is no solution other than the trivial zero solution. We may illustrate this through an analogy with two connected water wheels. One water wheel is powered by a descending sugar solution, and it in turn raises a salt solution. There is friction in the system, so that it can only raise $9 \mathrm{~kg}$ of salt for every $10 \mathrm{~kg}$ of sugar that falls through it. We shall call this the finger wheel. The second water wheel is powered by the descending salt solution, power it uses to raise the sugar solution. This water wheel has a greater friction, so that it can only raise $6 \mathrm{~kg}$ of sugar for every $10 \mathrm{~kg}$ of salt that powers it. This wheel is called the diffusive wheel. The two wheels are connected so that the salt raised by the finger wheel powers the diffusive interface wheel, which in turn raises the sugar to drive the first wheel. As the wheels are not $100 \%$ efficient, the system slows to a halt. Clearly we need some other mechanism to get either salt or sugar or both to the top of the system to drive the interconnected components so that they run continuously.

Figure 3 hints at one possibility. In the early stages of the experiment, while the tank was still close to being homogeneous, the plumes rapidly split into a core and sheath, one part rising up to the top of the tank, and the other half descending to the bottom. These are represented by the wiggly lines in Figure 3. In the final steady state, this process is not nearly as obvious, but never-the-less there are possible signs of it still occurring. Another option is the fact that most of the diffusive layers are inclined at an angle. This can be explained through the experimental evidence that fluid is moving parallel to these interfaces. Fluid immediately underneath the diffusive layer is continually getting denser, and so as it moves laterally underneath the interface, it also tends to sink, thus causing the interface to slope downwards. The fluid above the interface is continually getting lighter, thus it 
must be moving in the opposite direction to the fluid underneath the interface so that in its direction of motion the interface is sloping upwards to accommodate its increased buoyancy. This advection can transport sugar or salt vertically (as it travels horizontally), without ever crossing the diffusive layer and thus being constrained to the flux transport ratios.

\section{Theory}

Before delving into the theory of diffusive fingers and layers, let us continue with the coupled water wheels analogy. For the wheels to turn, we require an additional driving force to overcome the friction of the system. Let us derive a formula for the ratio of the flux of the driving to the flux transport of the wheels.

Let us define $F_{T 1}$ to be the salt flux indicated in figure 3 for the path that travels from the source, up through the fingers, down through the layers, and then out through the outlet. $F_{S 1}$ is the corresponding value for the sugar flux. The two wiggly lines flowing upwards from the sources represent the driving terms $F_{T 2}$ and $F_{S 2}$. The driving salt flux $F_{T 2}$ bypasses the fingers, using a different mechanism to get to the top, but then joins up with the salt flux $F_{T 1}$ in traveling down through the layers. Similarly, the driving sugar flux $F_{S 2}$ bypasses the layers, but joins up with $F_{S 1}$ to pass through the fingers. Let $\gamma_{f} \approx 0.9$ be the ratio of the sugar to salt flux through the fingers, and $\gamma_{d} \approx 0.6$ be the ratio of the salt to sugar flux through the diffusive layers. Thus we have the following relationships:

$$
\frac{F_{T 1}}{F_{S 1}+F_{S 2}}=\gamma_{f}, \quad \frac{F_{S 1}}{F_{T 1}+F_{T 2}}=\gamma_{d}
$$

We rearrange to get

$$
F_{T 1}=\frac{\gamma_{f}\left(F_{S 2}+\gamma_{d} F_{T 2}\right)}{1-\gamma_{d} \gamma_{f}}, \quad F_{S 1}=\frac{\gamma_{d}\left(F_{T 2}+\gamma_{f} F_{S 2}\right)}{1-\gamma_{d} \gamma_{f}}
$$

We consider three situations.

- $F_{T 2}=0$ :

$$
\frac{F_{T 1}}{F_{S 2}}=\frac{\gamma_{f}}{1-\gamma_{d} \gamma_{f}} \approx 2.0, \quad \frac{F_{S 1}}{F_{S 2}}=\frac{\gamma_{f} \gamma_{d}}{1-\gamma_{d} \gamma_{f}} \approx 1.2
$$

- $F_{S 2}=0$ :

$$
\frac{F_{T 1}}{F_{T 2}}=\frac{\gamma_{f} \gamma_{d}}{1-\gamma_{d} \gamma_{f}} \approx 1.2, \quad \frac{F_{S 1}}{F_{T 2}}=\frac{\gamma_{d}}{1-\gamma_{d} \gamma_{f}} \approx 1.3
$$

- $F_{T 2}=F_{S 2}$ :

$$
\frac{F_{T 1}}{F_{T 2}+F_{S 2}}=\frac{\gamma_{f}\left(1+\gamma_{d}\right)}{2\left(1-\gamma_{d} \gamma_{f}\right)} \approx 1.6, \quad \frac{F_{S 1}}{F_{T 2}+F_{S 2}}=\frac{\gamma_{d}\left(1+\gamma_{f}\right)}{2\left(1-\gamma_{d} \gamma_{f}\right)} \approx 1.2 .
$$

It is clear that in all situations, the driving flux is able to generate more convection than itself in the fingers - diffusive layers system; however the ratio is not more than about two. 


\subsection{Salt Fingers}

The linear stability analysis of the perturbations that grow into salt fingers is a well-studied problem, and reveals the wavelength of the disturbance which grows fastest. There remains some debate as to whether in the equilibrium model the fingers remain with this width (as proposed by Schmitt [3]), or whether they obtain a different width which maximises the buoyancy flux (see Stern [4] and Howard and Veronis [5]). Most theories appear to agree that the salt flux through fingers scales as the formula

$$
\beta F_{S} \sim \frac{\kappa_{T}(\beta \Delta S)^{2}}{\alpha \bar{T}_{z} L^{2}}
$$

where $L$ is the buoyancy-layer scale defined as

$$
L=\left(\frac{4 \nu \kappa_{T}}{g \alpha \bar{T}_{z}}\right)^{1 / 4}
$$

The discrepancy between most formulas comes into the formula for $\bar{T}_{z}$. It is the unstable salt field which drives the fingers, and thus presumably controls the gradients in the finger zone, so ideally one would like to rewrite the temperature gradient $T_{z}$ that appears in (6) in terms of the salinity difference in order to determine the flux law solely as a function of $\beta \Delta S$. Many people favour a 4/3 power law (see Stern [4], section 11.4), and there is experimental evidence in support of this (Turner [6]). However, as we have profiles of both the $T$ and $S$ field, we are able to try either formula, and see if they give consistent results. The formula we shall use is that derived by Howard and Veronis [5] in the form of (6) given by

$$
\beta F_{S 1}=0.1578 \sqrt{\frac{g \kappa_{t}}{\nu}} \frac{(\beta \Delta S)^{2}}{\sqrt{\bar{T}_{z}}}
$$

together with Stern and Turner's [7] empirically fit curve to the $4 / 3$ power law

$$
\beta F_{S 2}=C(\beta \Delta S)^{4 / 3}
$$

where $C=10^{-4} \mathrm{~m} / \mathrm{s}$

In both cases we assume the heat flux $F_{T}$ is a factor of 0.91 [7] times the salt flux.

\subsection{Diffusive Layers}

Turner [8] first suggested that since convection through diffusive layers was similar to Rayleigh Bénard convection, the formula for the heat flux should scale in a similar fashion. That is, the nondimensional heat flux through diffusive layers, given by the Nusselt number, $N u_{T}=F_{T} d / \kappa_{T} \Delta T$, should be proportional to the Rayleigh number to the power of $1 / 3$. The reasoning behind this relationship is that the length scale of the convective rolls is not in general governed by the size of the tank, and it is this relationship that removes the external length scale dependence. It follows that the heat flux is given by

$$
\alpha F_{T}=C\left(R_{\rho}\right)\left(g \kappa_{T}^{2} / \nu\right)^{1 / 3}(\alpha \Delta T)^{4 / 3}
$$




\begin{tabular}{|l|l|}
\hline 1:Fingers & $5:$ W. Diffusion \\
\hline 2:Stable & $6:$ S. Diffusion \\
\hline 3:S. Diffusion & $7:$ Stable \\
\hline 4:W. Diffusion & $8:$ Fingers \\
\hline
\end{tabular}

Figure 4: The geometry of the 8 Box model

The function $\mathrm{C}$ has quite a strong dependence on $R_{\rho}$, especially for heat salt systems. Shirtcliffe [9] found his experimental results fit the formula

$$
C=2.6 R_{\rho}^{-12.6}
$$

He also provided an estimate for the flux ratio, $\beta F_{S} / \alpha F_{T}=0.60$.

\section{The Flux box model}

As mentioned previously in section 2, the experimental profiles drawn in Figure 2 suggest the tank can be divided up into eight primary regions, four on each side. We draw them in Figure 4.

Table 1 provides all the data necessary to solve the flux equations given in the preceding section. To get the total flux of either solute, we must multiply by the area of the region where the fingering or diffusive layer is present. We assume this area is constant for all six of the interfaces we consider, and non-dimensionalise it by half the actual physical area of the box $A$, writing the non-dimensional area by $A_{f}$. We then non-dimensionalise the fluxes by the input salt and sugar flux, and the answers are listed in Table 1.

One clear result is that the flux through box 1 is much smaller than any of the other fluxes. This results from the extremely small unstable sugar gradient which is driving it. There is a large amount of uncertainty in the value for $\beta \Delta S$, indeed it may be up to a factor of three bigger than the best fitting line used to generate the value listed. This would increase the values of $F_{S 1}$ and $F_{T 1}$ by a factor of 9 , but they would still remain negligible compared to the other terms.

Another clear result is that nearly all the fluxes are much larger than physically reasonable. Although we have not substituted in a value for $A_{f}$, we would expect it to be of order one, meaning that the entire left hand of the tank is fingering in the top layer, diffusing in the bottom, and vice-versa in the right hand side. The dimensionless flux values listed imply the dimensional fluxes are often over 100 times greater than the input flux. Referring to our previous water wheel analogy, a small amount of driving can generate fluxes through 


\begin{tabular}{lrrrrrrrr}
\hline Property & Box 1 & Box 2 & Box 3 & Box 4 & Box 5 & Box 6 & Box 7 & Box 8 \\
\hline height (m) & 0.05 & 0.01 & 0.01 & 0.03 & 0.05 & 0.01 & 0.01 & 0.03 \\
$\alpha \Delta T$ & 0.8 & 13.2 & -19 & -4 & -1.4 & -24.2 & 7 & 8 \\
$\beta \Delta S$ & -0.13 & 15.5 & 36 & 6 & 1.9 & 44.6 & 15 & -3.5 \\
$R_{\rho}$ & 6 & - & 1.9 & 1.5 & 1.4 & 1.8 & - & 2.3 \\
$\alpha \bar{T}_{z}\left(m^{-1}\right)$ & 16 & 1320 & -1900 & -133 & -28 & -2420 & 700 & -267 \\
\hline$\alpha F_{S 1}^{*} / A_{f}$ & 0.7 & - & - & - & - & - & - & 130 \\
$\alpha F_{T 1}^{*} / A_{f}$ & 0.6 & - & - & - & - & - & - & 110 \\
$\alpha F_{S 2}^{*} / A_{f}$ & 0.015 & - & - & - & - & - & - & 11 \\
$\alpha F_{T 2}^{*} / A_{f}$ & 0.013 & - & - & - & - & - & - & 9.7 \\
$\alpha F_{S 3}^{*} / A_{f}$ & - & - & 54 & 130 & 110 & 20 & - & - \\
$\alpha F_{T 3}^{*} / A_{f}$ & - & - & 90 & 210 & 190 & 34 & - & - \\
\hline
\end{tabular}

Table 1: Properties of the Eight Boxes. The fluxes have been normalised by the source input fluxes. $A_{f}$ is the area of the convecting region divided by the area of half the tank. The subscripts on the fluxes refer to whether they were predicted by equation (8), (9) or (10) respectively.

the coupled diffusive system greater than the driving term, but by no more than a factor of two. Clearly a factor of 100 or more is out of the question, even if it were possible for the entire input fluxes to reach the top of the tank by some unidentified mechanism to become the driving flux. Thus there must be a problem with our application of the theory. There are a few obvious suggestions. Firstly, the formula by Howard and Veronis was derived for two fluids which have vastly different diffusivities. It was designed for the heat-salt system rather than our sugar-salt system. They allowed the salt to pass through the fingers without diffusing, so one would expect their formula to predict a larger salt flux than the sugar-salt experiment produces. Secondly, our profiles of the both the sugar and salt in most cases just do not have the resolution required to gain accurate values of the salt and sugar contrasts across the interfaces. The contrasts that appear in the theories refer to the contrasts that occur across the finger or layer interfaces, while we are using instead the contrasts that occur across the whole box. In the one dimensional theories the fluid above and below the interfaces are usually well mixed, so this difference is not important. In our experiment, the regions are distinctly two dimensional. This is most obvious in the experiment through the observation that the diffusive layers are generally inclined at some angle to the horizontal. This slope is associated with advection parallel to the interface, and we seem not to have the well mixed regions above and below the interfaces that the theories assume. Thus the difference between the sugar or salt contrasts across the interfaces, and the corresponding differences across the boxes that we used to apply the theories may be significant. To conclude, we suggest that the simple theories derived from one dimensional models do not adequately describe this complicated two dimensional system. 


\section{The box model}

In the previous section, we divided the tank up into the eight well defined regions observed in Figure 2. In that model, we used the temperatures and salinities found on the boundary of the boxes to determine the fluxes within each box. Let us now construct a similar box model corresponding to those eight regions, but now assign a mean temperature and salinity to each box rather than boundary values. The idea in this model is to try to calculate the fluxes between the boxes as opposed to the fluxes within each box.

In the previous section, we found that the simple one dimensional formulas for fingering and diffusion did not adequately describe the experimental system. Let us discard these theories and return to the simple assumption that the flux between two boxes is proportional to the difference in concentration between those boxes. Writing a formula for the rate of change of salinity in box 1 , we get

$$
V_{1} \dot{T}_{1}=b_{12}\left(T_{2}-T_{1}\right)+b_{15}\left(T_{5}-T_{1}\right)+T_{i 1} C-Q_{1} T_{1} \equiv 0
$$

The final equivalence is due to the fact that we are interested in finding the steady state solution. The first two terms on the right hand side of this equation are the fluxes of sugar into box 1 from box 2 and box 5 respectively, where the $b$ terms are unknown flux transfer coefficients. The third term represents a source input term. For the moment we will assume that the salt source is able to directly inject fluid into all four boxes on the left hand side of the tank (boxes 1-4). C is the salinity of the salt source, also equal to the concentration of sugar in the sugar source, while $T_{i 1}$ is an unknown outflow coefficient. The fourth term represents the rate of outflow of salt from box 1 , where $Q_{1}$ represents the rate at which fluid is leaving box 1 , which has salinity $T_{1}$. Finally $V_{1}$ is the volume of box 1 . We choose to use units of $\mathrm{kg} / \mathrm{m}^{3}$ for both salt and sugar concentrations, so the transfer coefficients $b_{i j}$ have units of $\mathrm{m}^{3} / \mathrm{s}$. It is tempting to interpret these as the volume flux between box $\mathrm{i}$ and box $\mathrm{j}$; however this is would only be correct if the flux transports were due to advection only, which is not true. To further emphasise this, we use different transfer co-efficients for the corresponding sugar equations, $a_{i j}$.

Inherent in the above equation is the assumption that each box is homogeneous. Thus, the flux of either solute to the outflow is equal to the the volume flux of the fluid leaving that box times the concentration of that solute. In addition, we assume that fluid entering each box from the inflow has the concentration of the reservoirs, and thus the flux of either salt is proportional to the volume flux times the concentration.

There are similar equations for rate of change of salinity for the remaining 7 boxes, as well as the 8 corresponding equations for sugar, and they may be found in the appendix. In addition, there are two more conservation equations. They are simply that the sum of the individual salt sources is equal to the flux of the salt source, $C \sum_{j} T_{i j}=Q C$, as is the sum of the individual sugar sources, $C \sum_{j} S_{i j}=Q C$. There are also two similar conservation equations for the sum of the outflow terms, however they are already implicitly expressed in the previous 18 equations.

The experimental data gives us the values of the sugar and salt concentrations in the boxes. Thus the unknowns are the 10 transfer parameters for each of sugar and salt, the 8 output flux terms $Q_{j}$, and the 4 input flux terms for each of salt and sugar - a total of 36 
unknowns.

As of the moment, we have 19 equations - just over half the number of unknowns - a highly under-determined problem. We can introduce more equations, for example by relating the transfer coefficients in some way, or reducing the number of unknowns by restricting inflow and outflow from some boxes, but for the moment, let us discuss a method of finding solutions to under-determined pröblems, described by Veronis in General Ocean Circulation [10].

Our eighteen equations may be written in matrix form $\mathbf{A x}=\mathbf{b}$. Here $\mathbf{A}$ has 19 rows and 36 columns, or more generally $m \times n$, where $m$ is less than $n$. The sizes of $\mathbf{x}$ and $\mathbf{b}$ are $n \times 1$ and $m \times 1$ respectively.

The key to the method is to assume we can write $\mathbf{x}=\mathbf{A}^{\mathbf{T}} \mathbf{f}$, where $\mathbf{A}^{\mathbf{T}}$ is the transpose of $\mathbf{A}$, and $\mathbf{f}$ is a yet to be determined matrix of size $m \times 1$. $\mathbf{f}$ satisfies the following equation, $\mathbf{A} \mathbf{A}^{\mathbf{T}} \mathbf{f}=\mathbf{A} \mathbf{x}=\mathbf{b}$. Now $\mathbf{A} \mathbf{A}^{\mathbf{T}}$ is square $(m \times m)$, and if it has a non-zero determinant, we may solve for $\mathbf{f}=\left(\mathbf{A} \mathbf{A}^{\mathbf{T}}\right)^{-1} \mathbf{b}$, and thus $\mathbf{x}=\mathbf{A}^{\mathrm{T}}\left(\mathbf{A} \mathbf{A}^{\mathbf{T}}\right)^{-\mathbf{1}} \mathbf{b}$.

We have thus determined a unique solution to an undetermined problem. The apparent paradox is explained through the writing of $\mathbf{x}=\mathbf{A}^{\mathbf{T}} \mathbf{f}$. This means we are writing the solution vector $\mathbf{x}$ as a linear combination of the $\mathbf{m}$ vectors that make up the rows of $\mathbf{A}$. $\mathbf{x}$ has $\mathbf{n}$ components, and thus defines a point in an $\mathbf{n}$ dimensional space. We cannot write all the points in the $n$ dimensional space through the summation of $m$ vectors. It turns out that the solution this method returns is a projection of the (unknown) true solution in $n$ dimensional space, onto a $\mathrm{m}$ dimensional space defined by the $\mathrm{m}$ rows of $\mathbf{A}$.

Let us take the example of $x+y=10$, so $\mathbf{A}=[1,1]$. We do not know what the true solution is, other than it lies on the line $y=10-x$, but its projection onto the one dimensional vector space defined by the one row of $\mathbf{A}$ is $x=y=5$. It is clear in the formulation of the problem that we have not treated $x$ any differently to $y$, and that is reflected in the identical values returned. This is important, as if we distinguish between them somehow, then that can make a great difference. For example, let us non-dimensionalise $x$ by $L$, and $y$ by $2 L$, writing $X=x / L$ and $Y=y /(2 L)$. The original equation may now be written $X+2 Y=10 / L$, and the returned solution is $X=2 / L, Y=4 / L$, which lies on the space defined by the vector $(1,2)$, the row of $\mathbf{A}$. In terms of the original variables, the solution is $x=2, y=8$, vastly different to the previous solution $(5,5)$ even though the equation solved was identical. This has important consequences for how we scale our problem. We must choose consistent scales for all quantities, as otherwise the solution will be biased towards those quantities that were scaled by values too large. It is clear that to use this method, if there is no reason to favour any unknown value over any other, then it is important to reflect that in the formulation of the equations, so that their co-efficients are equal.

Figure 5 shows how the solutions to the box model equations do not change dramatically as we increase the number of equations until they equal the number of unknowns. Plotted are the inflow, outflow and cross box salt and sugar flux transports. The fluxes have been normalised so that the sum of each of the salt and sugar inflows is 100 , as is the sum of each of the outflows. In picture (a), only the 19 equations listed in the appendix have been used. The main point to note in this picture is that the horizontal transports are an order of magnitude bigger than the vertical transports. In addition, the inflows and outflows to the two middle layers are also an order of magnitude larger than the corresponding values for the 
Salt
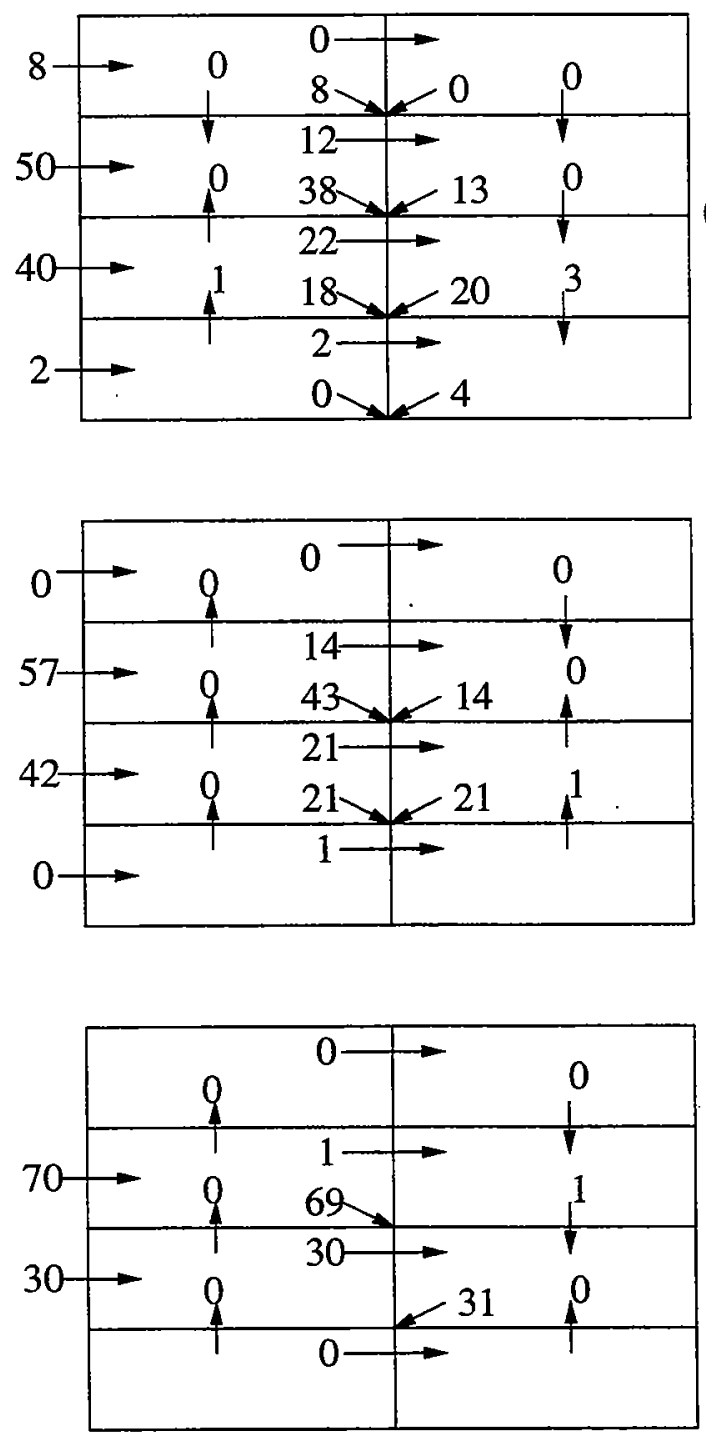

(a)

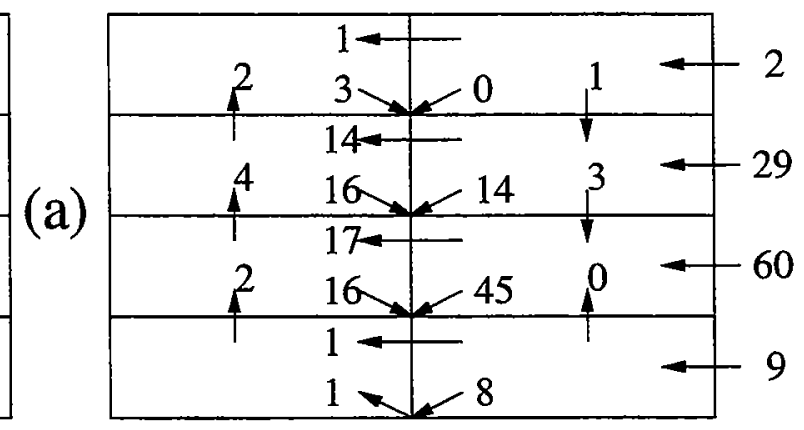

(b)

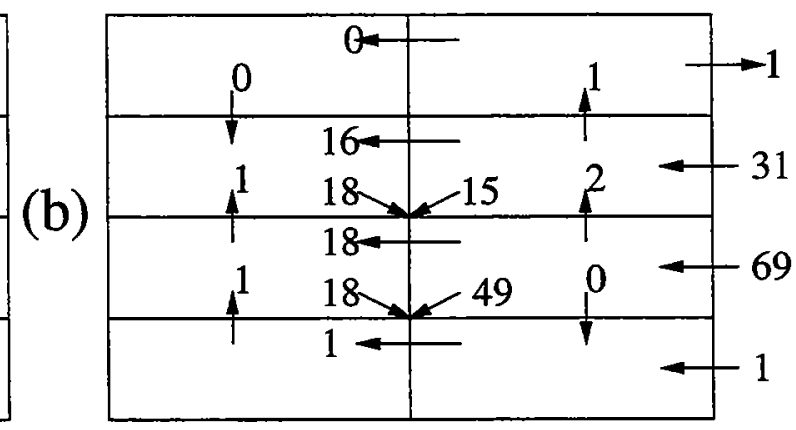

\section{Sugar}

(c)

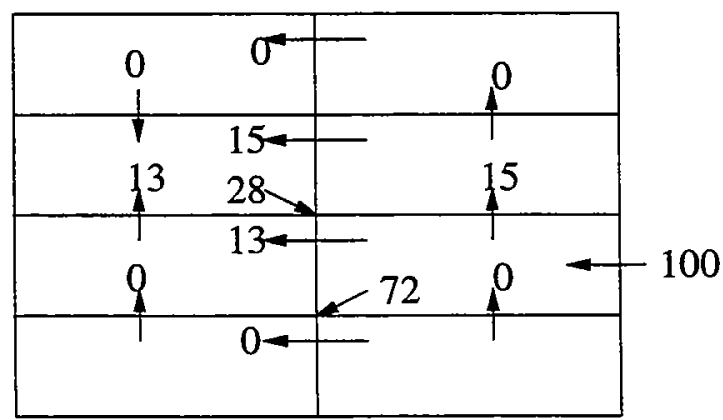

Figure 5: Salt and sugar fluxes given by the solution to the box model equations. The fluxes have been normalised so that the sum of each of the inflows is 100 , as is the sum of the outflows. In (a), only the original 19 equations are solved. In (b), there are an additional 10 equations, corresponding to limiting the outflows to the middle two layers, and applying the six vertical dynamic constraints. (c) is an exact solution, as it contains the same number of unknowns as equations. It differs from (b) through the inflows being limited to the 3 boxes indicated, while the outflows have been limited further to just the two middle layers. 
Salt
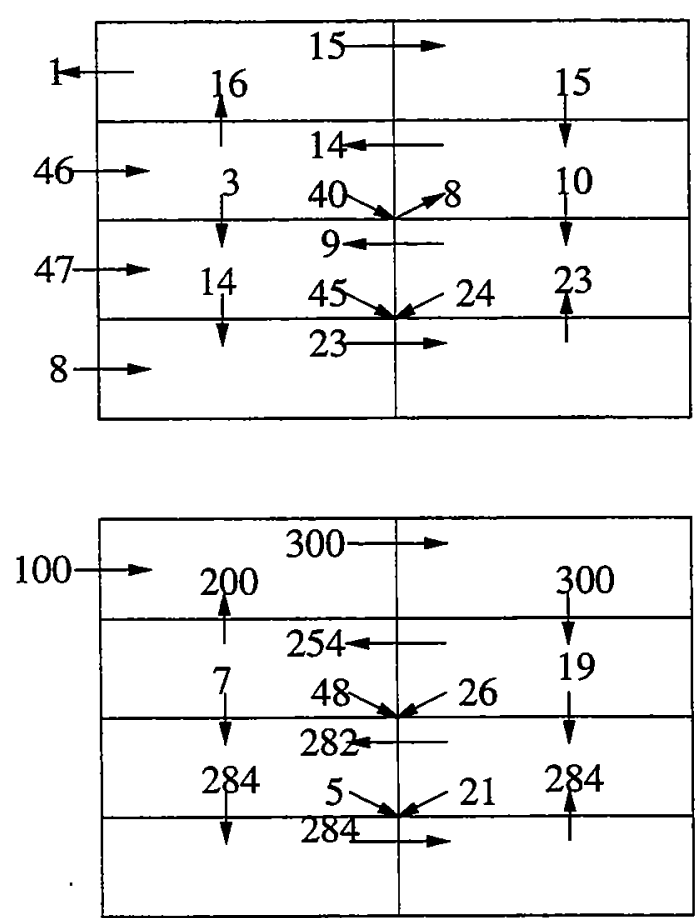

Sugar
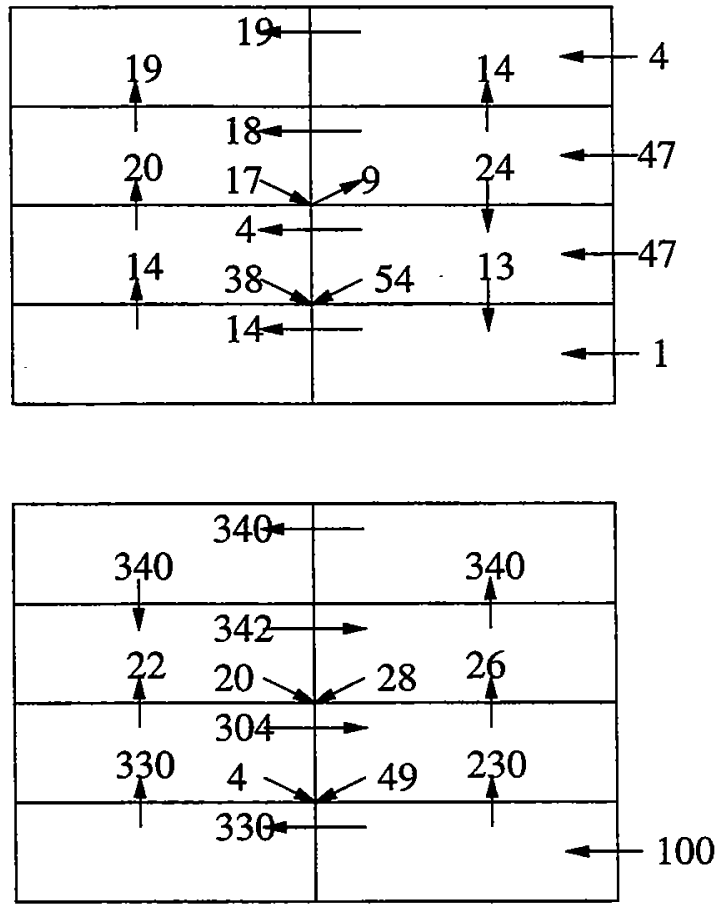

Figure 6: Fluxes of salt and sugar given by the solutions to the box model equations. Fluxes normalised as in figure 5 . In (a), the coefficients of the inflows have been reduced from $C_{0}$ to $C_{0} / 50$. In (b) the source inputs are limited to the top left and bottom right hand boxes. In both cases the outflows are limited to the middle two boxes on each side, and the six vertical dynamical constraints described in the text are applied. 
upper and lower layer. As a result, there is very little transport of sugar or salt occurring in the upper and lower layers. In (b), there are an additional 10 constraints, consisting of restricting the outflow from 8 to four layers, and adding 6 vertical dynamical constraints. These are based on symmetry arguments and are $a_{12}=a_{78}, b_{12}=b_{78}, a_{23}=a_{67}, b_{23}=b_{67}$, $a_{34}=a_{56}$ and $b_{34}=b_{56}$. Notice the fluxes are very similar to those plotted in (a). In (c), the solution is exact, as the number of unknowns is the same as the number of equations. To achieve this match, we have had to restrict the inflows to just the 3 boxes indicated, and the outflows to the two boxes indicated. We feel these constraints are too harsh, and we provide the result only to show the the exact solution is not too dissimilar to those plotted in (a) and (b).

Our reason for the unexpectedly small fluxes in the upper and lower layers, particularly in Figure 5 (a) where we have not forbidden inflow or outflow to the top and bottom layers, is that we have formulated our original 19 equations in a manner that is biased against fluxes in these regions. This is due to assuming the input source enters all boxes at the source concentration. This is a reasonable assumption for the middle two layers, but not so for the upper and lower layers. The main reason for allowing inflow and outflow from the upper and lower layers is to allow some mechanism to drive the diffusive 'water wheels'. While we have not specified what that mechanism is, although we suspect it is due to vertical transport along the sloping diffusive layers, it is highly improbable that the flux from the source to the upper and lower layers via this mechanism arrives with the concentration of the inflow. We have seen in the examples provided earlier that if we non-dimensionalise a term in the under-determined system of equations by an large quantity, then that term dominates the equation. Thus we expect the inflow terms to dominate the equations. For conservation arguments, this term must be balanced by the sum of the other terms, and the co-efficient of the outflow is the next biggest term, so it is the next most dominant term. Thus we see the cross-box transport seems to play a minor role in these conservation equations.

To see how changing the scaling for the input terms makes a difference, in Figure 6 (a) we show the solution to the system of equations where the source term is now the source concentration divided by 50 . We can see in this figure that the vertical fluxes is now a similar order of magnitude to the horizontal fluxes, and we have convection in the upper and lower layers. This solution matches nicely the schematic drawing of the fluxes drawn in Figure 3 , however it is not realistic, as we should really only rescale the input terms to the upper and lower boxes in this way. If we were to do that however, we would somehow have to redistribute the lost concentration to the two middle layers, the method by which to do so is currently not clear.

As a final test of our box model, we try restricting the source terms to solely the upper left and lower right boxes. The resulting fluxes are pictured in Figure 6 (b). Notice the very large recirculations apparent in the upper and lower halves. This bears a nice similarity to the water-wheel analogy. A driving flux can generate larger fluxes in the coupled double diffusive regions that the driving flux itself. In this case the factor is greater than the maximum factor of two predicted by the theory, but we have not specified any flux ratio parameters for the finger or diffusive regions, and thus we would not expect a match. This example also helps to explain why we see very little inflow or outflow from the upper or lower layers. It does not take much of an inflow in these layers to drive large amounts of recirculation in the middle 
layers. It seems logical that this model tends to reject these high-energy solutions when there is a much simpler solution with no flux in the upper and lower layers.

\section{Conclusion}

We have seen that a coupled system of fingers and diffusive layers cannot be sustained adjacent to each other without some other driving mechanism to transport either salt or sugar to the top of system. With driving, the resulting fluxes through the fingers and diffusive layers can be up to twice as large as the original driving term.

The one dimensional flux laws predict fluxes through the salt fingers and diffusive layers up to a few hundred times the source fluxes. These are much too large to be considered possible. While the flux laws used are not without question, it is more likely that the two dimensional problem we are analysing is too far removed from the one-dimensional theories to be of use, in addition to the experimental data being a little too sparsely separated.

Our box model only permits the driving flux to enter the top of the system at the same concentration as the source. The large co-efficient of this source term dominates the vector space of the possible solution set, and to first order it is the outflow that matches this term. The cross box transports play only a minor role in the solutions. Small amounts of source flux terms in the upper and lower layers produce large amounts of recirculation in the middle layers, which the model rejects in favour of the less energetic solutions that contain very little input terms to the upper and lower layers. To generate non-negligible amounts of convection in the upper and lower layers, we require a theory for the mechanism behind the driving term, which we may then use to scale the source terms for the upper and lower layers.

\section{Acknowledgements}

I would like to thank George Veronis greatly for his endless patience in guiding me through this project, for introducing us to the wonderful game that is social softball, and for some wonderful evenings enjoying life at Crooked Pond (thanks also Kim). Thanks also to Stuart Turner for his help during the two weeks he was here, in addition to dreaming up and carrying out the original experiment, and for providing me with the experimental results.

A very special thank you to all the other fellows who helped to make this summer so enjoyable (perhaps more than it was supposed to be): Roberto, Pascale, Jen, Nifer, Jeff, Meredith, Yuan-Nan and Raffaele. In addition, thanks to the pseudo-fellows who went above and beyond their call of duty in keeping us smiling: Claudia Pasquero, Jean-Luc Thiffeault, Allison Moehlis, Stephan Llewellyn Smith and Francesco Paparella.

Finally, thanks to Neil Balmforth for not visibly regretting the decision to invite me in the first place. 


\section{Appendix: The box model equations}

The model has nineteen basic equations, comprised of a sugar equation for each box,

$$
\begin{gathered}
\dot{S}_{1}=a_{12}\left(S_{2}-S_{1}\right)+a_{15}\left(S_{5}-S_{1}\right)+C S_{i 1}-S_{1} Q_{1} \equiv 0 \\
\dot{S}_{2}=a_{12}\left(S_{1}-S_{2}\right)+a_{23}\left(S_{3}-S_{2}\right)+a_{26}\left(S_{6}-S_{2}\right)+C S_{i 2}-S_{2} Q_{2} \equiv 0 \\
\dot{S}_{3}=a_{23}\left(S_{2}-S_{3}\right)+a_{34}\left(S_{4}-S_{3}\right)+a_{37}\left(S_{7}-S_{3}\right)+C S_{i 3}-S_{3} Q_{3} \equiv 0 \\
\dot{S}_{4}=a_{34}\left(S_{3}-S_{4}\right)+a_{48}\left(S_{8}-S_{4}\right)+C S_{i 4}-S_{4} Q_{4} \equiv 0 \\
\dot{S}_{5}=a_{15}\left(S_{1}-S_{5}\right)+a_{56}\left(S_{6}-S_{5}\right)-S_{5} Q_{5} \equiv 0 \\
\dot{S}_{6}=a_{26}\left(S_{2}-S_{6}\right)+a_{56}\left(S_{5}-S_{6}\right)+a_{67}\left(S_{7}-S_{6}\right)-S_{6} Q_{6} \equiv 0 \\
\dot{S}_{7}=a_{37}\left(S_{3}-S_{7}\right)+a_{67}\left(S_{6}-S_{7}\right)+a_{78}\left(S_{8}-S_{7}\right)-S_{7} Q_{7} \equiv 0 \\
\dot{S}_{8}=a_{48}\left(S_{4}-S_{8}\right)+a_{78}\left(S_{7}-S_{8}\right)-S_{8} Q_{8} \equiv 0
\end{gathered}
$$

a salt equation for each box,

$$
\begin{gathered}
\dot{T}_{1}=b_{12}\left(T_{2}-T_{1}\right)+b_{15}\left(T_{5}-T_{1}\right)-T_{1} Q_{1} \equiv 0 \\
\dot{T}_{2}=b_{12}\left(T_{1}-T_{2}\right)+b_{23}\left(T_{3}-T_{2}\right)+b_{26}\left(T_{6}-T_{2}\right)-T_{2} Q_{2} \equiv 0, \\
\dot{T}_{3}=b_{23}\left(T_{2}-T_{3}\right)+b_{34}\left(T_{4}-T_{3}\right)+b_{37}\left(T_{7}-T_{3}\right)-T_{3} Q_{3} \equiv 0 \\
\dot{T}_{4}=b_{34}\left(T_{3}-T_{4}\right)+b_{48}\left(T_{8}-T_{4}\right)-T_{4} Q_{4} \equiv 0 \\
\dot{T}_{5}=b_{15}\left(T_{1}-T_{5}\right)+b_{56}\left(T_{6}-T_{5}\right)+C T_{i 5}-T_{5} Q_{5} \equiv 0 \\
\dot{T}_{6}=b_{26}\left(T_{2}-T_{6}\right)+b_{56}\left(T_{5}-T_{6}\right)+b_{67}\left(T_{7}-T_{6}\right)+C T_{i 6}-T_{6} Q_{6} \equiv 0 \\
\dot{T}_{7}=b_{37}\left(T_{3}-T_{7}\right)+b_{67}\left(T_{6}-T_{7}\right)+b_{78}\left(T_{8}-T_{7}\right)+C T_{i 7}-T_{7} Q_{7} \equiv 0 \\
\dot{T}_{8}=b_{48}\left(T_{4}-T_{8}\right)+b_{78}\left(T_{7}-T_{8}\right)+C T_{i 8}-T_{8} Q_{8} \equiv 0
\end{gathered}
$$

plus three conservation equations,

$$
\begin{gathered}
Q_{1}+Q_{2}+Q_{3}+Q_{4}+Q_{5}+Q_{6}+Q_{7}+Q_{8}=2 Q \\
S_{i 1}+S_{i 2}+S_{i 3}+S_{i 4}=Q \\
T_{i 1}+T_{i 2}+T_{i 3}+T_{i 4}=Q
\end{gathered}
$$




\section{References}

[1] M. Stern, "The 'salt fountain' and thermohaline convection," Tellus 12, 172 (1960).

[2] H. Stommel, A. Arons, and D. Blanchard, "An oceanongraphical curiosity: The perpetual salt fountain," Deep-Sea Res. 3, 152 (1956).

[3] R. Schmitt, "The growth rate of super-critical salt fingers," Deep-Sea Res. 26, 23 (1979).

[4] M. Stern, Ocean Circulation Physics (Academic, London, 1975).

[5] L. Howard and G. Veronis, "The salt-finger zone," J. Mar. Res. 3, 265 (1948).

[6] J. Turner, "Salt fingers across a density interface," Deep-Sea Res. 14, 599 (1967).

[7] M. Stern and J. Turner, "Salt fingers and convecting layers," Deep-Sea Res. 16, 497 (1969).

[8] J. Turner, "The couple turbulent transports of salt and heat across a sharp density interface," Int. J. Heat Mass Tranfer 8, 759 (1965).

[9] T. Shirtcliffe, "Double diffusive convection with similar diffusivities," J. Fluid Mech. 57, 27 (1973).

[10] General Circulation of the Ocean, edited by H. Abarbanel and W. Young (SpringerVerlag, New York, 1987). 


\title{
Scalar Dispersion in a Two Dimensional Random Flow Field
}

\author{
Meredith M. Metzger \\ Department of Mechanical Engineering \\ University of Utah
}

\section{Introduction}

Experimental observations of the vertical propagation and decay of near inertial oscillations (NIO) through the oceanic mixed layer has stimulated a desire to understand the effect of NIO activity on mixing processes in the upper ocean. Ocean surface forcing due to the passage of large scale wind events or storms instigates the formation of coherent NIO structures which tend to migrate in a helical trajectory as evident from near surface buoy drifters. Observations also indicate that NIO mixed layer activity eventually decays to background levels approximately 20 days after the initial onset of the storm [1]. A major challenge to oceanographers has been to explain the primary mechanisms responsible for the observed time scales of NIO propagation and decay. Young and Ben Jelloul [2] hypothesized that advective distortion by the geostrophic eddy field decreases the NIO horizontal coherence scale. From a multiple time scale analysis, they formulate a reduced NIO equation linearized about the geostrophic flow. This analysis effectively filters out inertial oscillations allowing focus on the near inertial component of the motion. Their NIO equation combines the effects of advection by the geostrophic velocity, wave dispersion, and refraction due to the geostrophic vorticity. Subsequent work by Balmforth et al. [3] investigated results from the NIO equation for the case of a background geostrophic shear flow. The present study extends this work by considering the fundamental properties of the NIO equation for the specific case of linearized NIOs superposed on a two stage random wave model of the background geostrophic eddy field.

We begin the paper with an overview of near inertial oscillations and describe the methodology used to obtain the reduced NIO equation that provides the basis for the present study. We will see that the NIO equation is characterized by a parameter termed the dispersivity, analogous to the diffusivity associated with passive scalar diffusion processes. We then discuss the random wave model used to represent the background geostrophic flow and some fundamental properties associated with this type of model flow. We then study some limiting parameter cases of the governing equation, specifically, zero and infinite dispersivity. We present data from the numerical solution of the governing equation for a range of dispersivity values. Finally, we take a look at the decay of energy in the large scales and conclude with comments on directions for future research. 


\section{Near Inertial Oscillations (NIO)}

Inertial oscillations describe fluid motions arising from a force balance between fluid inertia and Coriolis acceleration. The reduced horizontal momentum equations, in a reference frame rotating with the earth, are

$$
u_{t}-f_{o} v=0 \quad \text { and } \quad v_{t}+f_{o} u=0 .
$$

where the subscript $t$ represents partial differentiation with respect to time and $f_{o}=2 \Omega \sin \theta$ is the inertial frequency with $\theta$ and $\Omega$ denoting the latitude and the earth's rotation rate, respectively. Throughout the paper, we follow the convention that $(u, v)$ describe the horizontal velocity components in the easterly $(x)$ and northerly $(y)$ directions, respectively, and $z$ refers to the vertical direction. The solution to (1), assuming constant $f$, is simply $\mathcal{U}=\tilde{\mathcal{U}} \mathrm{e}^{-i f_{o} t}$ where $\mathcal{U}=u+i v$ and $\tilde{\mathcal{U}}$ is the initial velocity. The corresponding particle trajectories, $x+i y=\frac{i \overline{\mathcal{U}}}{f_{o}} \mathrm{e}^{-i f_{o} t}$, form closed loops. As a simple model of the passage of a storm front, we assume that at time $t=0$, an instantaneous, homogeneous wind event occurs thereby exciting the entire horizontal domain to move with a uniform velocity of $\tilde{\mathcal{U}}$. The ensuing motions, described by (1), are referred to as inertial oscillations. Using data from mid latitude ocean buoy drifters [1], estimates of the typical diameter of inertial oscillations is of the order of $5 \mathrm{~km}$. In reality, however, (1) only represents the leading order behavior of the flow; and therefore, ensuing motions are actually near inertial oscillations that are more accurately characterized by helical type trajectories and have a finite lifespan in the mixed layer of approximately 20 days.

Note, in the solution of (1), a constant inertial frequency $f_{o}$ was assumed. This assumption ceases to be valid if a coherent fluid motion spans a large enough horizontal extent; then the latitude difference (and, hence, the change in $f_{0}$ ) between the most northerly and southerly points of the coherent motion can no longer be neglected. For simplicity, we neglect these so-called $\beta$ effects in the remainder of the paper.

\section{Reduced Linearized NIO Equation}

The previous work of Young and Ben Jelloul [2] regarding near inertial oscillations provides the basis for the present study. We briefly summarize the relevant points of that work here. We begin by assuming hydrostatic, Boussinesq, inviscid, incompressible flow. The velocity is linearized about the background geostrophic flow which can be written compactly in terms of a streamfunction, $\Psi(x, y, z, t)=P_{g} /\left(f_{o} \rho_{o}\right)$ with $P_{g}$ denoting the geostrophic pressure field and $\rho_{o}$, the mean density. Further reduction of the linearized governing equations (not reproduced here) is achieved through a multiple time scale analysis with the requirement that internal waves be nearly inertial. The general dispersion relation for internal waves can be written as $\omega^{2}=\left(N_{o}^{2} \kappa_{h}^{2}+f_{o}^{2} \kappa_{v}^{2}\right) / \kappa^{2}$ where $\omega$ is the oscillation frequency, $\left(\kappa_{h}, \kappa_{v}\right)$ denote the horizontal and vertical wavenumbers, respectively, $\kappa^{2}=\kappa_{h}^{2}+\kappa_{v}^{2}$, and $N_{o}$ is the characteristic buoyancy frequency associated with the vertical density stratification of the fluid (for further discussion of internal waves, see Gill [4], pp. 258). We define a small parameter $\epsilon \equiv\left(N_{o} \kappa_{h}\right) /\left(f_{o} \kappa_{v}\right)$. Physical estimates of $N_{o}$ in the North Pacific and North Atlantic indicate $N_{o} / f_{o}=\mathcal{O}\left(10^{2}\right)$ 
[5]. Therefore, in order for $\epsilon \ll 1$, the characteristic vertical wavelengths of the motion must be several orders of magnitude smaller than the characteristic horizontal wavelengths, i.e. $\kappa_{v} \gg \kappa_{h}$. This is entirely consistent with our use of the hydrostatic approximation (see Gill[4],pp. 159 for a discussion on the equivalence between longwave and hydrostatic approximations). The internal wave dispersion relation then reduces to $\omega^{2} \approx f_{o}^{2}\left(1+\epsilon^{2}\right)$; whereby, inertial oscillations are recovered at leading order. Departures from perfect inertial oscillations become appreciable on the slow time scale $t_{s} \equiv \epsilon^{2} f_{o} t$.

In the multiple time scale analysis, the complex velocity $\mathcal{U}$ is expanded in powers of $\epsilon^{2}$ giving $\mathcal{U}=\mathcal{U}_{0}+\epsilon^{2} \mathcal{U}_{2}+\ldots$ where the leading order solution $\mathcal{U}_{0}$ is simply the velocity associated with the inertial oscillations governed by (1). For convenience we write the leading order solution as $\mathcal{U}_{0}=M_{z}\left(x, y, z, t_{s}\right) \mathrm{e}^{-i f_{0} t}$ which allows for trivial integration of the incompressibility condition to obtain the leading order vertical velocity explicitly. If we further define a new complex field $A$ such that $M \equiv\left(f_{o}^{2} N^{-2}\right) \mathcal{A}_{z}$, then it is also possible to explicitly calculate the leading order pressure by integrating the hydrostatic equation along with the mass conservation equation. In essence, $\mathcal{A}$ incorporates all of the relevant physical quantities of interest. Therefore, we prefer to work solely with the dependent variable $\mathcal{A}$. Substituting the definition of $\mathcal{A}$ into the leading order horizontal velocity solution yields an expression for the demodulated velocity of the NIO

$$
u+i v=\mathrm{e}^{-f_{o} t} L \mathcal{A}
$$

where

$$
L \mathcal{A} \equiv\left(f_{o}^{2} N^{-2} \mathcal{A}_{z}\right)_{z}
$$

We find that the $\mathcal{O}\left(\epsilon^{2}\right)$ equation contains resonant terms proportional to $\mathrm{e}^{-i f_{o} t}$. To prevent related secular terms from arising in the higher order correction, we require that

$$
L \mathcal{A}_{t}+J(\Psi, L \mathcal{A})+\frac{i}{2} f_{o} \nabla^{2} \mathcal{A}+\frac{i}{2} \nabla^{2} \Psi L \mathcal{A}=0
$$

where $\nabla^{2}=\partial_{x x}+\partial_{y y}$ represents the horizontal Laplacian operator and $J(\Psi, L \mathcal{A})=\Psi_{x}(L \mathcal{A})_{y}-$ $\Psi_{y}(L \mathcal{A})_{x}$ is the Jacobian. One advantage of $(4)$ is that the first term on the left hand side has the direct physical interpretation of being the time rate of change of the horizontal velocity. The vertical boundary conditions demand zero vertical velocity at the top and bottom of the ocean, translating into

$$
\mathcal{A}_{z}(x, y, 0, t)=\mathcal{A}_{z}(x, y,-H, t)=0
$$

where $H$ is the depth of the ocean. This condition follows the rigid lid approximation that assumes the typical amplitude of surface waves are negligible compared to the vertical wavelength of the propagating NIOs. Normalized horizontal boundary conditions are $2 \pi$ periodic. The initial condition depends on how one chooses to model the passage of the storm or other instigating event. We will specify this condition later. The present study will focus on investigating some of the fundamental characteristics of the NIO equation (4) in the specific context of a simple random wave model of the background turbulent geostrophic eddy field. 


\section{Vertical Normal Modes}

The top and bottom boundaries of the ocean have the effect of confining wave energy to a region of finite vertical extent. Thus, the ocean can be considered as a waveguide causing energy to propagate along the horizontal direction. With this notion, we proceed in assuming a solution to (4) in terms of a superposition of vertical normal modes

$$
\mathcal{A}=\sum_{m=1}^{\infty} A_{m}(x, y, t) P_{m}(z) \sigma_{m}
$$

where $m$ denotes the vertical wavenumber, $P_{m}(z)$ represents the eigenfunctions, and $\sigma_{m}$ represents the projection of the initial condition onto the vertical normal modes. Note, the expansion in (6) relies on the assumption that the background geostrophic flow is barotropic, i.e. $\Psi_{z}=0$. Applying the differential operator $L$ to (6) gives

$$
L \mathcal{A}=L\left(A_{m} P_{m}\right)=\left(A_{m}\right) L P_{m}
$$

Substituting (7) into (4),

$$
\frac{L P_{m}}{P_{m}}=\frac{-i\left(f_{o} / 2\right) \nabla^{2} A_{m}}{A_{m_{t}}+J\left(\Psi, A_{m}\right)+i / 2\left(\nabla^{2} \Psi\right) A_{m}}=-R_{m}^{-2}
$$

For historical reasons [6], eigenvalues are represented as $R_{m}^{-2}$, where $R_{m}$ (dimensions of length) symbolizes the Rossby deformation radius. From (8), we obtain a partial differential equation for $A_{m}$

$$
A_{m_{t}}+J\left(\Psi, A_{m}\right)+\frac{i}{2}\left(\nabla^{2} \Psi\right) A_{m}=\frac{i \hbar_{m}}{2} \nabla^{2} A_{m}
$$

where $\hbar_{m}=f_{0} R_{m}^{2}$ will be referred to as the dispersivity ${ }^{\dagger}$ associated with the $m^{\text {th }}$ vertical mode. Since the initial condition of $A$ has been projected onto vertical normal modes, the initial condition associated with each $A_{m}$ is simply $A_{m}(x, y, 0)=1$. Exact numerical values of $\hbar_{m}$ depend on the eigenvalues of (8) which, in turn, depend on the shape of the buoyancy frequency profile, $N=N(z)$. If we assume $N=$ constant, then $P_{m}(z) \propto \cos \left(N^{2}\left(f_{o} R_{m}\right)^{-2} z\right)$ and $R_{m}=N / f_{o} \sqrt{H /(m \pi)}$. A constant buoyancy frequency profile, however, is not a reasonable physical model; and therefore, we chose something slightly more realistic. In this regard, we follow the work of Gill [6]. Figure 1 shows the model $N$ profile used here. The corresponding eigenvalues are computed numerically and plotted in figure 2 using a value of $f_{o}=1 \times 10^{-4} \mathrm{~s}^{-1}$.

${ }^{\dagger}$ This is appropriate nomenclature since (9) begets a dispersion relation. Consider only the time derivative and Laplacian terms; assuming a solution of the form $e^{k x+\ell y-\omega t}$ yields the real-valued dispersion relation $\omega=\hbar\left(k^{2}+\ell^{2}\right)$. This is particularly interesting since (9) "looks like" an advection-diffusion equation but because of the $i$ multiplying the Laplacian term, a real-valued dispersion relation is obtained analogous to a wave equation. 


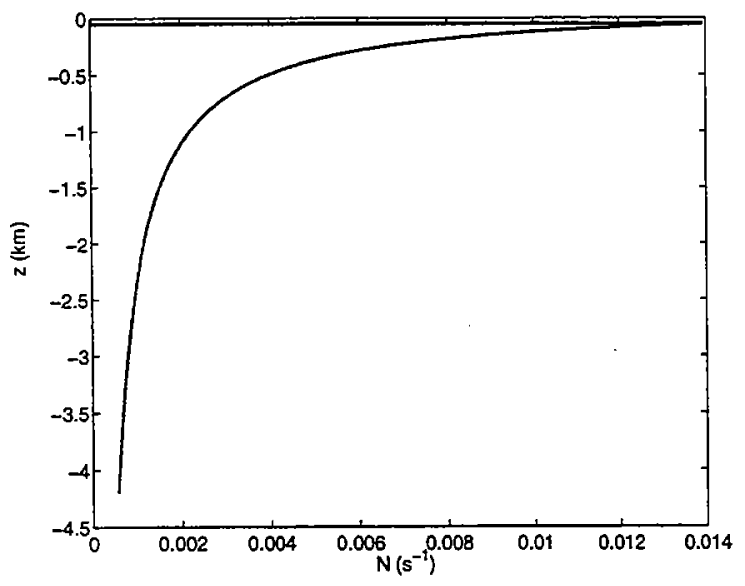

Figure 1: Buoyancy frequency profile used to calculate $h_{m}$.

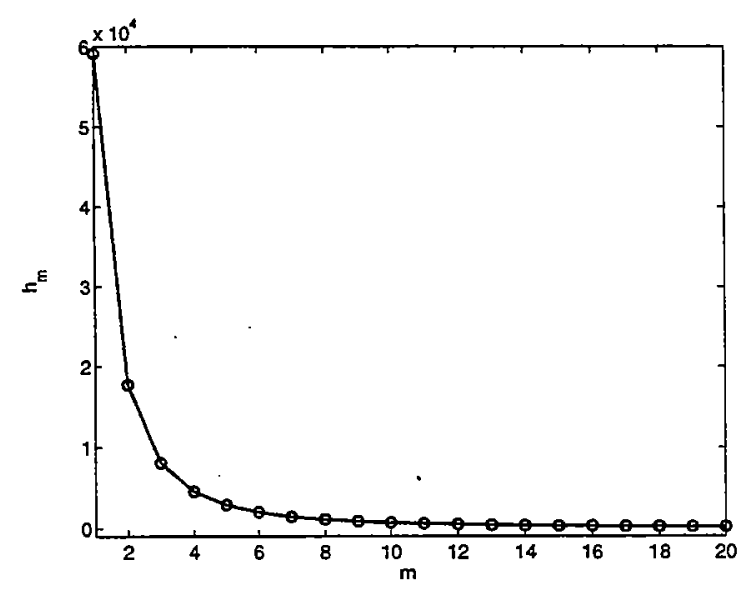

Figure 2: Dispersivity as a function of vertical wavenumber $m$.

Of interest is the relatively large range of $\hbar$ values (over four orders of magnitude) apparent just within the first 10 vertical modes. We expect this to play an important role in the developing structure of $A$. Also, note that for the $N$ profile in figure $1, \hbar \rightarrow m^{-2}$ as compared to $\hbar \rightarrow m^{-1 / 2}$ for the case of $N=$ constant.

Except for the dependence of the dispersivity $\hbar$ on the vertical wavenumber $m, A$ apparently satisfies the same initial value problem regardless of the specific vertical normal mode under consideration. Therefore, in the remainder of the paper, we drop the subscript $m$ on $A$. Focus is placed on understanding the general behavior of the initial value problem given by

$$
A_{t}+J(\Psi, A)+\frac{i}{2}\left(\nabla^{2} \Psi\right) A=\frac{i \hbar}{2} \nabla^{2} A, \quad A(x, y, 0)=1
$$

for a range of parameter values $0 \leq \hbar<\infty$ and a specific form of $\Psi$ detailed in section 6 . Equation (10) will be referred to as the passive scalar dispersion equation.

\section{Scalar Dispersion Equation}

An important aspect of $(10)$ is that the quantity $|A|^{2}$ is conserved over the spatial domain. To see this, we start by writing $A$ in terms of a magnitude $R$ and phase $\theta$

$$
A=R \mathrm{e}^{i \theta}
$$

Substituting (11) into (10), separating real and imaginary parts and dividing by $\mathrm{e}^{i \theta_{n}}$ yields evolution equations for $R$ and $\theta$, respectively, 


$$
\begin{aligned}
R_{t}+J(\Psi, R) & =\frac{-\hbar}{2}\left[2 \nabla R \nabla \theta+R \nabla^{2} \theta\right], \\
\theta_{t}+J(\Psi, \theta) & =\frac{-\zeta}{2}+\frac{\hbar}{2}\left[\frac{\nabla^{2} R}{R}-(\nabla \theta)^{2}\right],
\end{aligned}
$$

where $\zeta=\nabla^{2} \Psi$ represents the vorticity. Multiplying (12) by $R$ and integrating over the two dimensional spatial domain gives

$$
\frac{D}{D t} \int_{\mathcal{S}} \frac{R^{2}}{2} d S=-\hbar \int_{\mathcal{S}} \nabla\left(\frac{R^{2}}{2} \nabla \theta\right) d S=-\hbar \int_{\mathcal{C}} \frac{R^{2}}{2} \nabla \theta \cdot \hat{r} d C=-\hbar \int_{\mathcal{C}} \frac{R^{2}}{2} \frac{\partial \theta}{\partial r} d C
$$

where $D R / D t \equiv \partial R / \partial t+J(\Psi, R)$ and $\hat{r}$ is the outward normal unit vector at the boundary. In obtaining (14), we have used the product rule $\nabla R^{2} / 2 \nabla \theta=\nabla\left(R^{2} / 2 \nabla \theta\right)-R^{2} / 2 \nabla^{2} \theta$ and the divergence theorem. Far-field boundary conditions are utilized, which translates into $\nabla A \cdot \hat{r}=0$, on the boundary; or in polar representation, $\frac{\partial R}{\partial r}=0$ and $\frac{R^{2}}{2} \frac{\partial \theta}{\partial r}=0$, on the boundary. Note, periodic boundary conditions in a two dimensional box automatically satisfy the far-field conditions due to the fact that the gradient of the function at one end of the periodic domain is exactly equal and opposite to the gradient at the other end. Application of the far-field boundary condition to the last expression of (14) leaves

$$
\frac{D}{D t} \int_{V}|A|^{2} d V=0
$$

which proves our initial statement at the beginning of the section.

We contrast (15) with the case of passive scalar diffusion. The equation governing the evolution of a scalar concentration field $c$ in a background flow can be written as

$$
c_{t}+J(\Psi, c)=\nu \nabla^{2} c, \quad c(x, y, 0)=c_{o}
$$

where $\nu$ is the molecular diffusivity coefficient, in analogy to the dispersivity of the scalar dispersion equation (10). Following the same procedure for $A$, we find

$$
\frac{D}{D t} \int_{V} \frac{c^{2}}{2} d V=-\nu \int_{V}(\nabla c)^{2} d V
$$

We conclude that the diffusion process tends to minimize the squared scalar concentration whereas the dispersion process conserves this same quantity. What does this say about the generation of small scales or the cascade of energy from large to small scales? Does $A$ exhibit a Batchelor scale/spectrum [7]? These questions have motivated, to some extent, the work herein. At this point, we present the specific streamfunction model used for the remainder of the calculations in the paper. 


\section{Two Stage Random Wave Model}

We will investigate the behavior of $(10)$ for the specific two dimensional, random flow field described by the following streamfunction $\Psi$

$$
\Psi_{n}= \begin{cases}\sin \left(y+\phi_{n}\right), & 2 n \tau<t \leq(2 n+1) \tau \\ \cos \left(x+\chi_{n}\right), & (2 n+1) \tau<t \leq(2 n+2) \tau\end{cases}
$$

for $n=0,1, \ldots$, where $\phi, \chi$ are uniform random phases between 0 and $2 \pi$ and $\tau$ represents a characteristic decorrelation time of the turbulence. A variation of this model has been used in the past, [8]. Note, we have nondimensionalized $\Psi$ by a characteristic streamfunction $\Psi_{o}$ and the spatial coordinates by a characteristic horizontal wavenumber $\kappa_{h}$. The spatial domain is thus $2 \pi$ periodic. In order for the model to have physical relevance to the oceanic geostrophic eddy field, we have taken $\Psi_{o}=3000 \mathrm{~m}^{2} / \mathrm{s}$ and $\kappa_{h}=6 \times 10^{-5} \mathrm{~m}^{-1}$, based on the data of [1]. Together, these give a characteristic decorrelation time scale of $\tau^{*}=\mathcal{O}$ (1day). The velocity components, $\vec{u}=(u, v)$, follow from the definition of the streamfunction as $u=-\Psi_{y}$ and $v=\Psi_{x}$. The corresponding particles trajectories are

$$
\begin{aligned}
& x_{n+1}=x_{n}-\cos \left(y_{n}+\phi_{n}\right) t, \quad 2 n \tau<t \leq(2 n+1) \tau \\
& y_{n+1}=y_{n}+\cos \left(x_{n+1}+\chi_{n}\right) t \quad(2 n+1) \tau<t \leq(2 n+1) \tau .
\end{aligned}
$$

As apparent, the flow model is characterized by a two stage advection process. In the first stage, during time intervals $2 n \tau<t \leq(2 n+1) \tau$, particles are advected in the $x$ direction only for a time $\tau$; while in the second stage, during $(2 n+1) \tau<t \leq(2 n+1) \tau$, particles are advected in the $y$ direction only for a time $\tau$. The combination of these two advection stages constitutes a single step in the random wave model. The total time to complete $n$ steps is then $t=n(2 \tau)$. The main advantage of the two stage random model stems from simplifications in the subsequent mathematics as will be described later. It is worthwhile to analyze the two stage random flow field in terms of the effect on material line stretching and fluid particle diffusivity. We compare the two stage model of (18) with a one stage model such as the rennovating random wave model,

$$
\Psi_{n}=\cos \left[x \cos \phi_{n}+y \sin \phi_{n}+\chi_{n}\right], \quad(n-1) \tau<t \leq n \tau,
$$

where advection is performed in a single stage.

\subsection{Material line stretching}

We investigate material line stretching induced by (19-20) in the context of Lyapunov exponents. Lyapunov exponents are used extensively in the study of nonlinear dynamical systems as a measure of whether two initial conditions diverge exponentially in time, thus possibly leading to the onset of chaos. The same concept is often applied to neighboring fluid particles 
in the study of fluid turbulence. In this manner, the Lyapunov exponent gives some indication of the stretching of differential line elements in the flow. In the case of advection of a real-valued passive scalar, this can be directly related to the development of spatial gradients in the scalar field. We will consider later whether an analogy to the complex-valued scalar $A$ exists.

\subsubsection{Lyapunov exponent: background}

We follow the general definitions and methodology of Seydel [9] regarding the Lyapunov exponent calculations. An initially small ring of fluid particles with initial radius $\rho_{o}$ deforms into an ellipse with major axis $\mu$ due to regions of localized strain in the flow. Linearizing the flow about the origin $\rho_{o}=0$, we obtain $\vec{h}_{n}=\mathrm{J}^{n} \vec{h}_{o}$ where $\mathrm{J}$ denotes the Jacobian matrix of the random map given by (19-20), and $\vec{h}_{o}, \vec{h}_{n}$ describe the particle positions in the original (circular) and deformed (elliptic) configurations, respectively. Strictly, $\vec{h}_{n}$ and $\vec{h}_{o}$ must be differential vectors for the linearization to be valid. We look for exponential stretching of the form $\left\|\vec{h}_{n}\right\|^{2}=\left(\left\|\vec{h}_{o}\right\| \mathrm{e}^{L t}\right)^{2}$ where $L$ denotes the Lyapunov exponent. Rearranging and taking the limit as $t \rightarrow \infty$ leads to

$$
L=\lim _{n \rightarrow \infty} \frac{1}{4 n \tau} \ln \left(\frac{\| \mathbf{J}^{n} \vec{h}_{o}}{\left\|\vec{h}_{o}\right\|^{2}}\right),
$$

where we have substituted in $t=2 n \tau$ with $2 \tau$ denoting the decorrelation time associated with the two stage random wave model of (19-20). Since $\mathbf{J}$ describes a random process, we ensemble average (denoted as $\langle\cdot\rangle$ ) over the random variables $\phi_{n}$ and $\chi_{n}$ so that $L$ does not depend on a particular realization. Additionally, we use the identity $\left\|\mathbf{J}^{n} \vec{h}_{o}\right\|^{2}=\vec{h}_{o}^{\mathrm{T}}\left(\mathbf{J}^{n^{\mathrm{T}}} \mathbf{J}^{n}\right) \vec{h}_{o}$ to simplify (22). The resultant definition of the Lyapunov exponent used herein is

$$
L=\lim _{n \rightarrow \infty} \frac{1}{4 n \tau} \ln \left(\frac{\vec{h}_{o}^{\mathrm{T}}\langle\mathbf{K}\rangle \vec{h}_{o}}{\vec{h}_{o}^{\mathrm{T}} \vec{h}_{o}}\right),
$$

where $\mathbf{K}=\mathbf{J}^{n^{T}} \mathbf{J}^{n}$. Note, for convenience, we have taken $\langle\mathbf{K}\rangle$ rather than $\langle\ln \mathbf{K}\rangle$. The ramifications of this subtle difference stem from the fundamental differences between additive and multiplicative random walks as detailed by Redner [10]; but it is not a primary concern of the present study.

\subsubsection{Lyapunov exponent: two stage random wave model}

We now want to explicitly calculate the Lyapunov exponent defined in (23) for the two stage random wave model given by (19-20). During the $n^{\text {th }}$ step of the random walk, $\mathrm{J}_{n}=$ $\mathbf{J}_{\mathrm{II}}\left(\chi_{n}\right) \mathbf{J}_{\mathrm{I}}\left(\phi_{n}\right)$, where $\mathbf{J}_{\mathrm{I}}$ and $\mathbf{J}_{\mathrm{II}}$ describe advection in the $x$ direction (first stage) and $y$ direction (second stage), respectively. From (19-20),

$$
\mathbf{J}_{\mathbf{I}}\left(\phi_{n}\right)=\left[\begin{array}{cc}
1 & \sin \left(y_{n}+\phi_{n}\right) \tau \\
0 & 1
\end{array}\right], \quad \text { and } \quad \mathbf{J}_{\mathrm{II}}\left(\chi_{n}\right)=\left[\begin{array}{cc}
1 & 0 \\
-\sin \left(x_{n+1}+\chi_{n}\right) \tau & 1
\end{array}\right]
$$


The independence of each step allows the ensemble average of $\mathbf{K}$ after $n$ steps to be written as

$$
\langle\mathbf{K}\rangle=\left\langle\mathbf{J}_{\mathrm{I}}^{\mathrm{T}}\left(\phi_{1}\right)\left\langle\mathbf{J}_{\mathrm{II}}^{\mathrm{T}}\left(\chi_{\mathrm{I}}\right) \ldots\left\langle\mathbf{J}_{\mathrm{I}}^{\mathrm{T}}\left(\phi_{n}\right)\left\langle\mathbf{J}_{\mathrm{II}}^{\mathrm{T}}\left(\chi_{n}\right) \mathbf{J}_{\mathrm{II}}\left(\chi_{n}\right)\right\rangle \mathbf{J}_{\mathrm{I}}\left(\phi_{n}\right)\right\rangle \ldots \mathbf{J}_{\mathrm{II}}\left(\chi_{1}\right)\right\rangle \mathbf{J}_{\mathrm{I}}\left(\phi_{1}\right)\right\rangle
$$

Introducing the diagonal matrix $\mathbf{\Gamma}=\left[\begin{array}{ll}a & 0 \\ 0 & b\end{array}\right]$, the inner most ensemble average of $\langle\mathbf{K}\rangle$ can be written as $\left\langle\mathbf{J}_{\mathrm{II}}^{\mathrm{T}}\left(\chi_{n}\right) \Gamma \mathbf{J}_{\mathrm{II}}\left(\chi_{n}\right)\right\rangle$. Upon calculating several sequential ensemble averages, the recurrsion relation for $\vec{a}=(a, b)$ becomes apparent. After $n$ steps, we find

$$
\left[\begin{array}{l}
a_{n+1} \\
b_{n+1}
\end{array}\right]=\left[\begin{array}{cc}
1 & \frac{\tau^{2}}{2} \\
\frac{\tau^{2}}{2} & 1+\frac{\tau^{4}}{4}
\end{array}\right]\left[\begin{array}{l}
a_{n} \\
b_{n}
\end{array}\right]
$$

where $a_{0}=b_{0}=1$. With this, the general form of the Lyapunov exponent (23) reduces to

$$
L=\lim _{n \rightarrow \infty} \frac{1}{4 n \tau} \ln \left(\left[h_{1} h_{2}\right]\left[\begin{array}{cc}
a_{n} & 0 \\
0 & b_{n}
\end{array}\right]\left[\begin{array}{l}
h_{1} \\
h_{2}
\end{array}\right]\right) .
$$

In order to represent $\vec{a}_{n}$ in terms of $\vec{a}_{0}$ we need to solve the corresponding eigenproblem, $\mathbf{M} \vec{v}=\lambda \vec{v}$, with $\mathbf{M}$ as given in (26). Due to space limitations, we do not provide the details of this calculation. The main result is $\vec{a}_{n}=\mathbf{R} \mathbf{\Lambda}_{n} \mathbf{R}^{\mathrm{T}} \vec{a}_{0}$, where $\mathbf{R}$ is the rotation matrix,

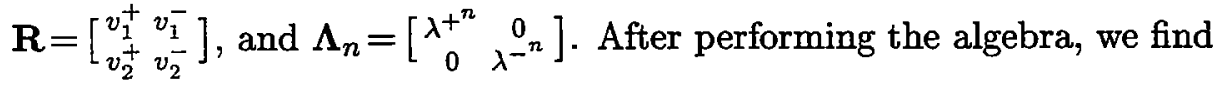

$$
\begin{gathered}
a_{n}=\frac{2^{-1-3 n}}{16+\tau^{4}}\left[\left(16+\tau^{4}+\left(\tau^{2}-4\right) \sqrt{16+\tau^{4}}\right)\left(8+\tau^{4}-\tau^{2} \sqrt{16+\tau^{4}}\right)^{n}+\right. \\
\left.\left(16+\tau^{4}-\left(\tau^{2}-4\right) \sqrt{16+\tau^{4}}\right)\left(8+\tau^{4}+\tau^{2} \sqrt{16+\tau^{4}}\right)^{n}\right] \\
b_{n}=2^{-1-3 n}\left[\frac{\left(4+\tau^{2}+\sqrt{16+\tau^{4}}\right)\left(8+\tau^{4}+\tau^{2} \sqrt{16+\tau^{4}}\right)^{n}}{\sqrt{16+\tau^{4}}}-\right. \\
\left.\frac{\left(\tau^{2}-4+\sqrt{16+\tau^{4}}\right)\left(8+\tau^{4}-\tau^{2} \sqrt{16+\tau^{4}}\right)^{n}}{16+\tau^{4}+\tau^{2} \sqrt{16+\tau^{4}}}\right] .
\end{gathered}
$$

Notice, $a_{n}$ and $b_{n}$ are not equal; therefore, stretching is anisotropic in this flow field. In fact, looking at the simple case of $n=1, a_{1}=1+\tau^{2} / 2$ and $b_{1}=1+\tau^{2} / 2+\tau^{4} / 4$; we see that more stretching occurs in the $y$ direction as compared to the $x$ direction. However, at long times, taking the limit as $n \rightarrow \infty$, we find that the stretching does become isotropic as shown in the following. We rewrite (27) as

$$
L=\lim _{n \rightarrow \infty} \frac{1}{4 n \tau} \ln \left(h_{1}^{2} \frac{a_{n}}{b_{n}}+h_{2}^{2}\right)+\lim _{n \rightarrow \infty} \frac{1}{4 n \tau} \ln \left(b_{n}\right)
$$




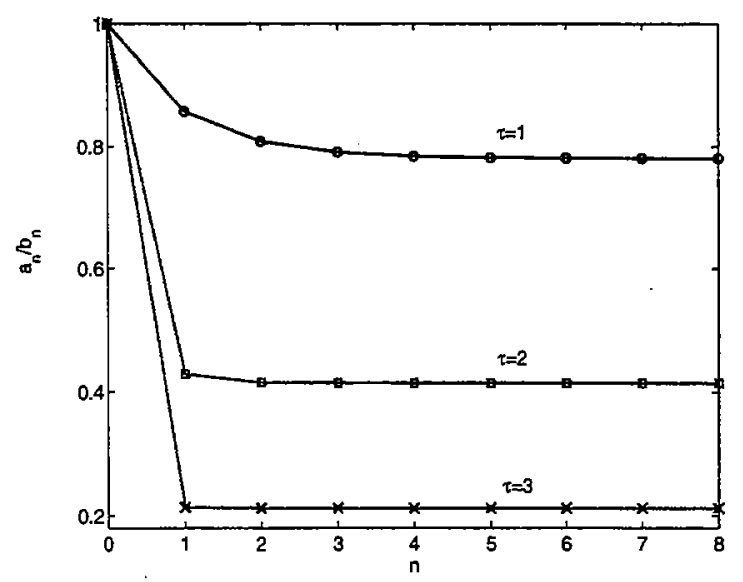

Figure 3: Ratio of the two diagonal terms in the stretching matrix as a function of steps $n$ in the random map.

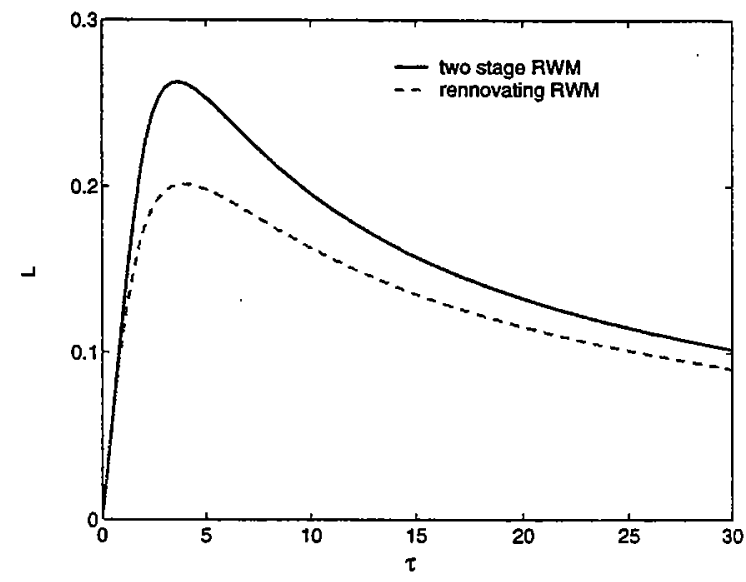

Figure 4: Lyanpunov exponent $L$ as a function of turbulent decorrelation time $\tau$.

As $n \rightarrow \infty, \frac{a_{n}}{b_{n}}=-\frac{\tau^{2}}{4}+\frac{\sqrt{16+\tau^{4}}}{4}$. Figure 3 shows $a_{n} / b_{n}$ as a function of $n$ for a family of three $\tau$ values. We see that for any given $\tau, a_{n} / b_{n}$ asymptotes to a constant value. Therefore, in the limit as $n \rightarrow \infty$, the first term in (30) goes to zero. At large $n, b_{n}$ remains as the only contribution to $L$ and thus stretching becomes isotropic. From figure 3 , one can determine how quickly the flow becomes isotropic for a given $\tau$. We see that for $\tau=3$, stretching becomes isotropic after the first step.

With further manipulation of (27) we obtain the functional relation between $L$ and $\tau$

$$
L=\frac{1}{4 \tau} \ln \left[\left(1+\tau^{4} / 8\right)+\frac{\tau^{2}}{8} \sqrt{\tau^{4}+16}\right]
$$

Figure 4 shows the graphical representation of (31) compared with that obtained for the rennovating random wave model of (21). Maximum stretching in the two stage random wave model occurs at $\tau=3.64$ in contrast to $\tau=3.94$ for the one stage random model. In general, the two stage random wave model generates more stretching of the fluid elements.

\subsection{Diffusivity of fluid particles}

We follow Einstein's theory of Brownian movement [11] to determine the diffusivity associated with the movement of fluid particles in the random wave model of (19-20). For a random walk processes in two dimensions, Einstein showed that

$$
{\overline{\Delta^{2}}}_{n}=4 D_{\text {eff }} t
$$

where $\Delta$ is the particle displacement, an overline denotes an average performed over $n$ steps in the random walk, and $D_{\text {eff }}$ is the effective diffusion coefficient that appears in the scalar 
diffusion equation. Since each of the steps in the random walk is independent, $\overline{\Delta^{2}}{ }_{n}=n \Delta^{2}=$ $\Delta^{2} t / \tau$, where $t$ is the total time and $\tau$ represents the decorrelation time of the random walk. In our particular case, particle displacements during the $n^{\text {th }}$ step can be written as $r_{n}^{2}=r_{I_{n}}^{2}+r_{I I_{n}}^{2}$, where $r_{I}, r_{I I}$ are the displacements during the $x, y$ stages of advection, respectively. Without loss of generality, we can make the coordinate translation $\left(x_{n}, y_{n}\right) \rightarrow(0,0)$ before each $n^{\text {th }}$ step. Applying this simplification to (19-20) gives

$$
r_{n}^{2}=\tau^{2} \cos ^{2}\left(\phi_{n}\right)+\tau^{2} \cos ^{2}\left(-\cos \left(\phi_{n}\right) \tau+\chi_{n}\right) .
$$

Because we want the calculation to be independent of the random phase angles associated with a particular realization, we ensemble average over both $\phi$ and $\chi$ to yield $\left\langle r^{2}\right\rangle_{\phi, \chi}=\tau^{2}$. Note, in analogy to (32), $\Delta^{2}=\left\langle r^{2}\right\rangle_{\phi, \chi}$. Taking the average of $\left\langle r^{2}\right\rangle_{\phi, \chi}$ over $n$ total steps in the random walk and using (32) gives the resultant particle displacement diffusivity of $D_{\text {eff }}=\frac{\tau}{8}$. It is interesting to note that this diffusivity is identical to that of the rennovating random wave model (21) where advection is performed in a single step. At this point, there is some confidence in our understanding of the model flow field; we, therefore, proceed to study the behavior of the scalar dispersion equation (10) under the two limiting parameter conditions of $\hbar=0$ and $\hbar \rightarrow \infty$.

\section{Zero Dispersion Limit}

In the case $\hbar=0$, the scalar dispersion equation (10) along with (18) reduces to

$$
\begin{aligned}
A_{t}-\cos (y+\phi) A_{x}-\frac{i}{2} \sin \left(y_{+} \phi\right) A & =0, \quad 2 n \tau<t \leq(2 n+1) \tau, \\
A_{t}-\cos (x+\chi) A_{y}-\frac{i}{2} \sin (x+\chi) A & =0, \quad(2 n+1) \tau<t \leq(2 n+2) \tau .
\end{aligned}
$$

We solve the above set of equations using the method of characteristics. In the first stage of the random wave model, we define new variables $\tilde{x}=x+c_{\mathrm{I}} t, \tilde{t}=t, \tilde{y}=y$; while in the second stage, we define $\tilde{y}=y+c_{\mathrm{II}} t, \tilde{t}=t, \tilde{x}=x$ where $c_{\mathrm{I}}=\cos (y+\phi)$ and $c_{\mathrm{II}}=\cos (x+\chi)$. This effectively removes the advective terms from (34) simplifying the problem to two, uncoupled, first order ordinary differential equations, one at each advection stage. The solutions valid in the first and second stages are

$$
A_{\mathrm{I}}=A_{o} \mathrm{e}^{i / 2 \sin (\tilde{y}+\phi) \tilde{t}} \text { and } A=A_{\mathrm{I}} \mathrm{e}^{i / 2 \sin (\tilde{x}+\chi) \tilde{t}},
$$

respectively, where $A_{o}$ represents the initial condition at the beginning of the $n^{\text {th }}$ step. Recall, at $n=0, A(x, y, t=0)=1$. Using the fact that $\tilde{x}$ and $\tilde{y}$ remain constant along characteristic curves, the solution can be written in terms of an iterated map

$$
\begin{aligned}
& 2 n \tau<\underline{K}(2 n+1) \tau: A_{\mathrm{I}_{n+1}}=A_{n} \mathrm{e}^{i / 2 \sin \left(y_{n}+\phi_{n}\right) \tau}, \quad x_{n+1}=x_{n}-\cos \left(y_{n}+\phi_{n}\right) \tau, \\
& (2 n+1) \tau<t \leq(2 n+1) \tau: A_{n+1}=A_{I_{n+1}} \mathrm{e}^{i / 2 \sin \left(x_{n+1}+\chi_{n}\right) \tau}, \quad y_{n+1}=y_{n}+\cos \left(x_{n+1}+\chi_{n}\right) \tau \text {. }
\end{aligned}
$$



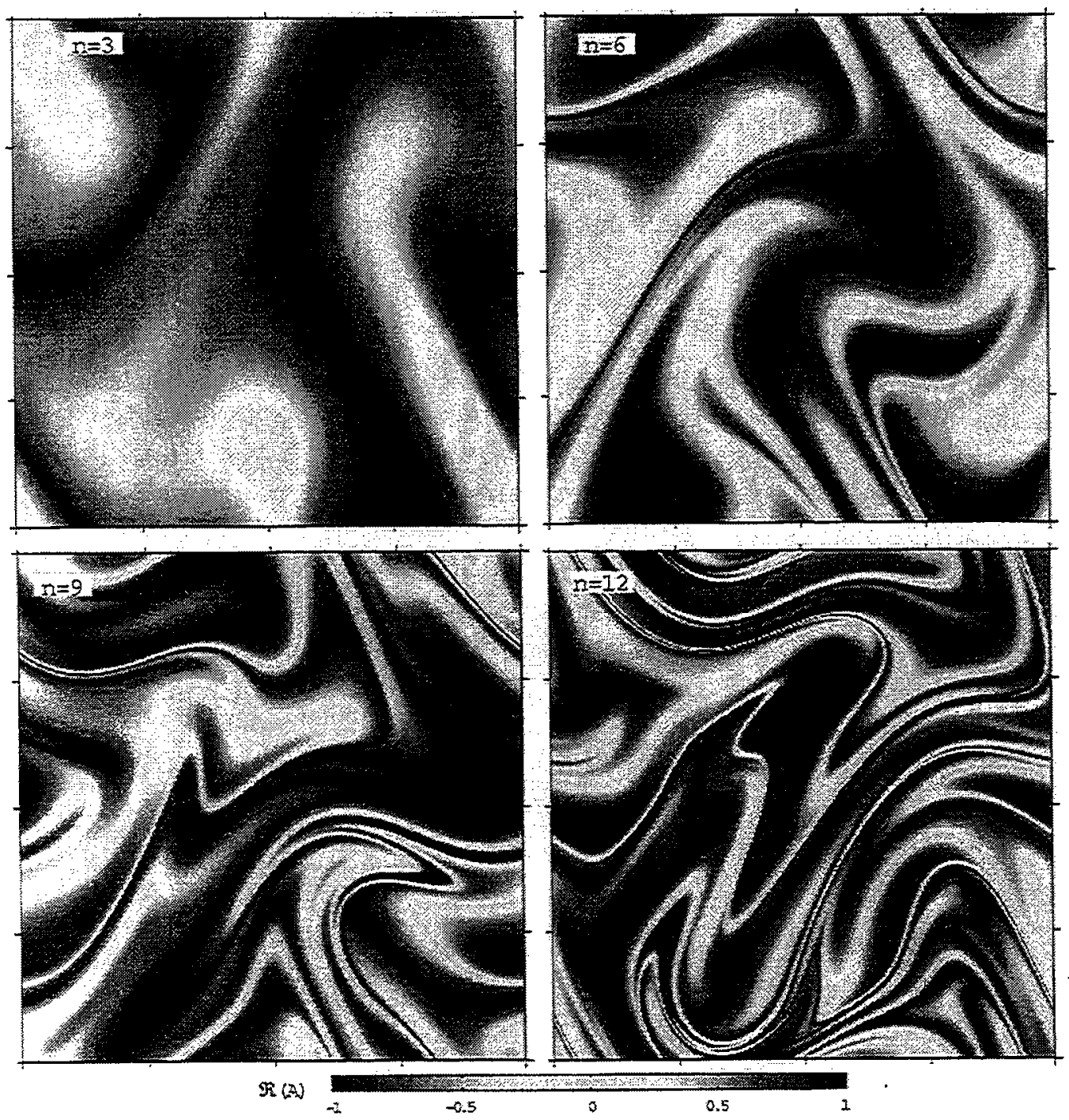

Figure 5: Snapshots of the spatial structure of $\Re(A)$ for the $\tau=1, \hbar=0$. Recall that at $n=0, \Re(A)=1$.

Figure 5 displays four snapshots of $\Re(A)$ as computed from the map above for the case of $\tau=1$. The resolution is $1028 \times 1028$. Later, we will compare these pictures to the structure of $\Re(A)$ for $\hbar \neq 0$. From (37) and (38), it is apparent that no mechanism exists to instigate changes in $|A|$; therefore, $|A|$ remains constant at its initial value of unity. This agrees with the previous results of section 5. However, the phase of $A$, denoted by $\theta$ as in (11), does exhibit interesting behavior. The iterated map for $\theta$ follows directly from (37-38) as

$$
\theta_{n+1}=\theta_{n}+\sin \left(y_{n}+\phi_{n}\right) \frac{\tau}{2}+\sin \left(x_{n+1}+\chi_{n}\right) \frac{\tau}{2},
$$

where $x_{n+1}$ and $y_{n+1}$ are given as in (37) and (38). We observe that for $\hbar=0, \theta$ undergoes 
a random walk process and therefore has an associated diffusivity $D_{\theta}$ (not to be confused with the diffusivity of particle trajectories). To calculate $D_{\theta}$, we follow the procedure in section 6.2. The ensemble averaged variance of $\theta$ is defined as $\left\langle\theta^{\prime 2}\right\rangle_{\phi, \chi}=\left\langle\left(\theta_{n+1}-\theta_{n}\right)^{2}\right\rangle$. Substituting in (39) and performing the average yields $\left\langle\theta^{2}\right\rangle=\tau^{2} / 4$. Making the analogy with Einstein's theory in (32), we have $\Delta^{2}=\left\langle\theta^{\prime 2}\right\rangle_{\phi, x}$. Taking the average of $\Delta^{2}$ over $n$ total steps in the random walk and using $(32)^{\dagger}$ gives the resultant phase diffusivity $D_{\theta}=\tau / 16$.

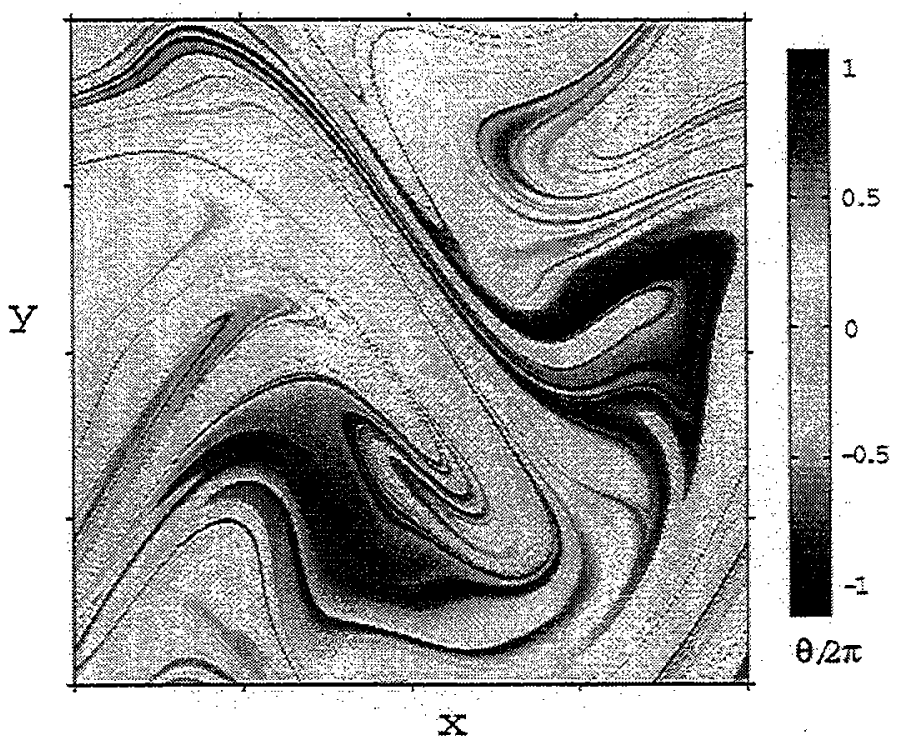

Figure 6: Phase of $A$ for the case of $\hbar=0, \tau=1$ after 25 random advection steps.

This is an interesting result in that the behavior of $\theta$ for zero dispersivity undergoes a normal diffusion process analogous to that of a real passive scalar. The structure of $\theta$ after 25 iterations of the map in (39), for $\tau=1$, is shown in figure 6 . Note, in order to obtain a continous field, results are plotted as $\theta / 2 \pi$. The initial condition at $t=0$ is $\theta=0$ and the resolution is $1028 \times 1028$. This picture looks surprisingly similiar to the stirring of a passive scalar as presented in [8] (figure 1 of that paper), for a random velocity field nearly equivalent to that of (18).

\section{Strong Dispersion Limit}

We use a simple multiscale analysis to investigate the large $\hbar$ behavior of (10) by introducing a small parameter $\epsilon$ and a slow time $t^{\prime}$ such that $\hbar=\mathcal{O}(1 / \epsilon)$ and $t^{\prime}=\epsilon t$. Applying these scalings to (10) and multiplying by $\epsilon$ gives

$$
A_{t^{\prime}}+\epsilon J(\Psi, A)+\epsilon \frac{i \zeta}{2} A=\frac{i}{2} \nabla^{2} A
$$

\footnotetext{
${ }^{\dagger}$ In the case of $\theta$, we have a one dimensional random walk given by $\overline{\Delta^{2}}{ }_{n}=2 D_{\text {eff }} t$.
} 
We assume that the solution can be written as a power series expansion in $\epsilon, A=A_{0}+$ $\epsilon A_{1}+\epsilon^{2} A_{2}+\ldots$ The $\mathcal{O}(1)$ equation is obtained by substituting this expansion into (40) and taking the limit as $\epsilon \rightarrow 0$

$$
\mathcal{O}(1): \quad A_{0_{t^{\prime}}}=\frac{i}{2} \nabla^{2} A_{0}
$$

with the initial condition $A_{0}(t=0)=1$. The solution $A_{0}=1$ trivially satisfies this initial value problem. The $\mathcal{O}(\epsilon)$ equation is obtained by substituting the $\mathcal{O}(1)$ solution into (40), dividing by $\epsilon$, and taking the limit as $\epsilon \rightarrow 0$

$$
\mathcal{O}(\epsilon): \quad A_{1_{t^{\prime}}}+\frac{i \zeta}{2}=\frac{i}{2} \nabla^{2} A_{1}
$$

with the initial condition $A_{1}(t=0)=0$. Note, $J\left(\Psi, A_{0}\right)=0$. Because of the explicit form of $\zeta$, see (18), we need to consider the two stages of the advection process separately. This complicates the problem in that the initial condition for each advection stage depends on the final state at the end of the previous advection stage. We start by considering the first time interval $0<t \leq \tau$, during which $\zeta=-\sin (y+\phi)$ and (42) reduces to

$$
A_{1_{t^{\prime}}}-\frac{i}{2} \nabla^{2} A_{1}=-\frac{i}{2} \sin (y+\phi)
$$

with $A_{1}(t=0)=0$. We require both the homogeneous and particular parts of the solution to vanish at $t=0$. Therefore, the homogenous part of the solution is simply 0 . We assume a particular solution of the form $A_{1}=\tilde{a}\left(t^{\prime}\right) \sin (y+\phi)$. Substituting this into (43), we obtain an ordinary differential equation for $\tilde{a}\left(t^{\prime}\right)$, the solution of which is $\tilde{a}\left(t^{\prime}\right)=1-\mathrm{e}^{-i t^{\prime} / 2}$. Thus, in the first time interval,

$$
A=1+\frac{1}{\hbar}\left(1-\mathrm{e}^{-\frac{i t}{2 \hbar}}\right) \sin (y+\phi)+\mathcal{O}\left(\epsilon^{2}\right), \quad 0<t \leq \tau
$$

In the subsequent time interval $\tau<t \leq 2 \tau, \zeta=-\sin (x+\chi)$ and the initial condition is $A_{1}(t=\tau)=\left(1-\mathrm{e}^{-\frac{i \tau}{2 \hbar}}\right) \sin (y+\phi)$. Following the same procedure used above, we find

$$
A=1+\frac{1}{\hbar}\left[1+\mathrm{e}^{-\frac{i}{2 \hbar}(t-\tau)}\left(\left(1-\mathrm{e}^{-\frac{i \tau}{2 \hbar}}\right) \sin (y+\phi)+1\right)\right] \sin (x+\chi)+\mathcal{O}\left(\epsilon^{2}\right), \quad \tau<t \leq 2 \tau
$$

One thing to notice in the strong dispersion limit is that spatial structure in $A$ develops very slowly, in stark contrast to the case of $\hbar=0$ where, after only 12 iterations, the structure of $A$ has become highly stretched and contorted (see figure 5). We will return to these results later in section 10 to ascertain how the energy contained in the large scales of $A$ varies as a function of $\hbar$. We now consider the regime $\hbar \neq 0$. 


\section{$9 \quad$ Numerical Solution for $\hbar \neq 0$}

Both the spatial domain and the streamfunction are $2 \pi$ periodic; therefore, we seek a general solution to (10) in the form of a Fourier series expansion

$$
A=\sum_{k, \ell=-\infty}^{\infty} a_{k, \ell}(t) \mathrm{e}^{i k x+i \ell y}, \quad a_{k, \ell}(0)= \begin{cases}1, & \text { if } k=\ell=0 \\ 0, & \text { otherwise }\end{cases}
$$

where $\vec{\kappa}=(k, \ell)$ describes the wavenumber vector and $a_{k, \ell}(t)$ are the corresponding Fourier coefficients. Substituting (46) into (10) yields

$$
\sum_{k, \ell=-\infty}^{\infty}\left[\dot{a}_{k, \ell}+i \ell \Psi_{x} a_{k, \ell}-i k \Psi_{y} a_{k, \ell}+\frac{i}{2} \nabla^{2} \Psi a_{k, \ell}\right] \mathrm{e}^{\vec{k} \cdot \vec{x}}=-\frac{i \hbar}{2} \sum_{k, \ell=-\infty}^{\infty}\left(k^{2}+\ell^{2}\right) a_{k, \ell} \mathrm{e}^{\vec{k} \cdot \vec{x}}
$$

In order to eliminate the summations, we utilize the fact that the Fourier modes are orthogonal. To exploit this, we multiply (47) by $e^{-i q y-i p x}$ and integrate over $y=0-2 \pi$ and $x=0-2 \pi$. Integrations are performed in detail in the Appendix. It is worth noting that if we did not chose a two stage random wave model for the velocity field, but rather used a single stage model such as (21), then it would not be possible to analytically integrate the terms resulting from (47). Performing the integrations yields two sets of coupled, first order ordinary differential equations for $a_{k, \ell}(t)$, each valid in one of the two stages of the advection process

$$
\begin{gathered}
\dot{a}_{k, \ell}+a_{k, \ell+1} \mathrm{e}^{-i \phi}\left(\frac{1-i 2 k}{4}\right)-a_{k, \ell-1} \mathrm{e}^{i \phi}\left(\frac{1+i 2 k}{4}\right)=\frac{-i \hbar}{2}\left(k^{2}+\ell^{2}\right) a_{k, \ell} \\
\dot{a}_{k, \ell}+a_{k+1, \ell} \mathrm{e}^{-i \chi}\left(\frac{1+i 2 \ell}{4}\right)-a_{k-1, \ell} \mathrm{e}^{i \chi}\left(\frac{1-i 2 \ell}{4}\right)=\frac{-i \hbar}{2}\left(k^{2}+\ell^{2}\right) a_{k, \ell},
\end{gathered}
$$

for $n=0,1, \ldots$ and $k, \ell=-\infty, \ldots,-1,0,1, \ldots, \infty$ where (48) and (49) are valid during the time intervals $2 n \tau<t \leq(2 n+1) \tau$ and $(2 n+1) \tau<t \leq(2 n+2) \tau$, respectively. The apparent coupling between nearest neighbors of $a$ results directly from the fact that the imposed velocity field contains only one Fourier component, the lowest nonzero wavenumber component. A convenient aspect of the two stage random wave velocity field is that, in the first stage, coupling occurs only between $\ell$ wavenumbers; while, in the second stage, coupling occurs only between $k$ wavenumbers.

We solve (48-49) numerically to obtain the time evolution of the Fourier coefficients $a_{k, \ell}$, then utilize an inverse fast Fourier transform (FFT) algorithm to perform the resummation in (46) to obtain $A$. In the numerical solution, $k, \ell$ must be truncated at the $N^{\text {th }}$ Fourier mode, i.e. the summation appearing in (46) occurs over $-N \leq k, \ell \leq N$. We chose $N$ such that the amplitudes of the corresponding Fourier modes at $|k|,|\ell| \geq N$ have decreased below a set tolerance. In practice, though, we typically over resolve the Fourier domain by a substantial amount since we favor a grid size of $2^{p} \times 2^{p}, p=0,1,2, \ldots$. 

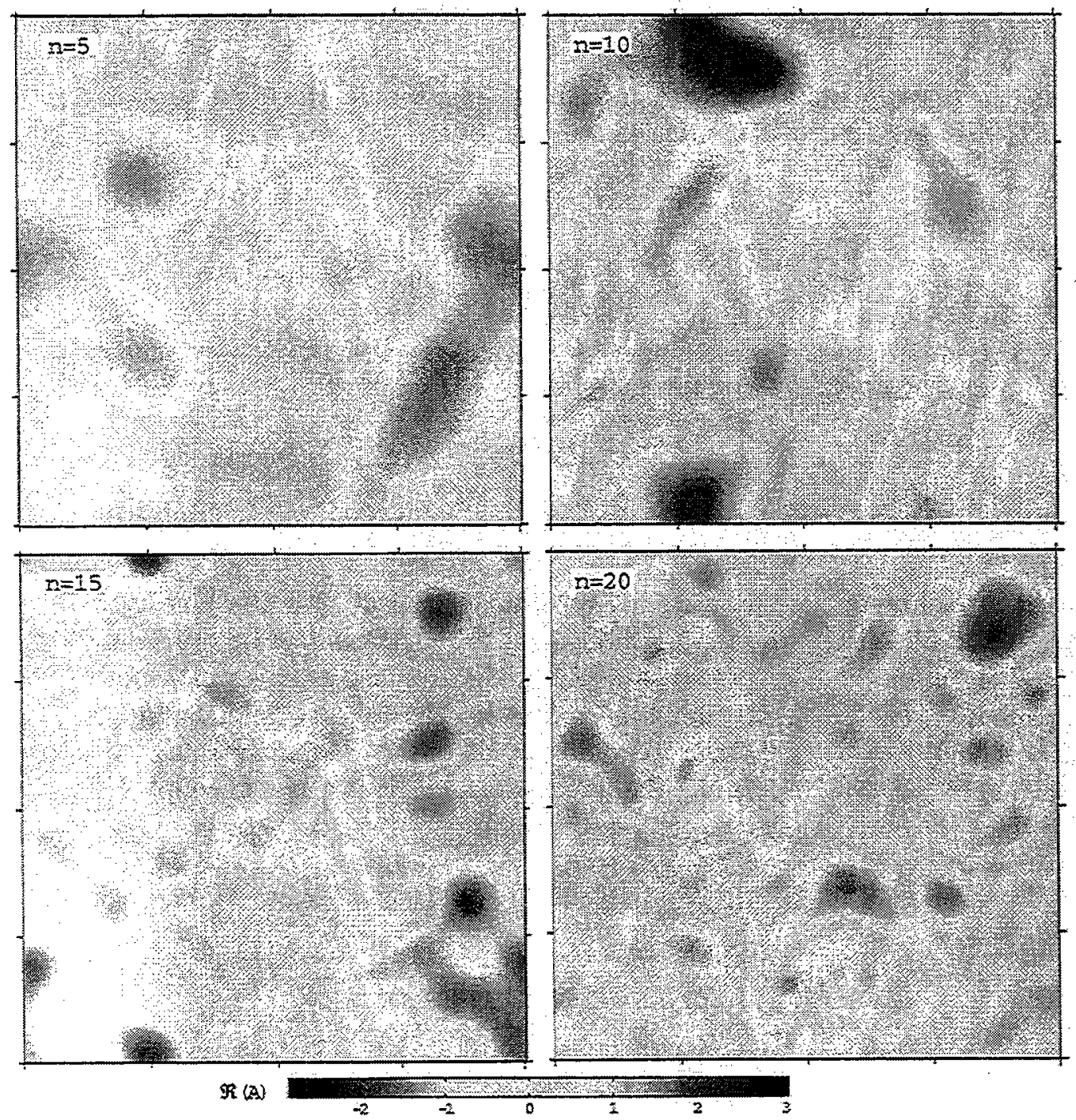

Figure 7: Snapshots of the spatial structure of $\Re(A)$ for the $\tau=1, \hbar=1$. Recall that at $n=0, \Re(A)=1$.

The two truncated systems can each be described by a matrix equation, written in the general, compact form

$$
\dot{\vec{y}}(t)=\vec{f}(t, \vec{y}(t)) \text {. }
$$

For example, considering the simple case of $N=2,(50)$ becomes 


$$
\left[\begin{array}{c}
\dot{a}_{-2} \\
\dot{a}_{-1} \\
\dot{a}_{0} \\
\dot{a}_{1} \\
\dot{a}_{2}
\end{array}\right]=\left[\begin{array}{ccccc}
c & d & 0 & 0 & 0 \\
b & c & d & 0 & 0 \\
0 & b & c & d & 0 \\
0 & 0 & b & c & d \\
0 & 0 & 0 & b & c
\end{array}\right]\left[\begin{array}{c}
a_{-2} \\
a_{-1} \\
a_{0} \\
a_{1} \\
a_{2}
\end{array}\right]
$$

where $b=(1+i 2 k) / 4 \mathrm{e}^{i \phi_{n}}, c=-i \hbar / 2\left(k^{2}+\ell^{2}\right), d=-(1-i 2 k) / 4 \mathrm{e}^{-i \phi_{n}}$, in the first stage; and $b=(1-i 2 \ell) / 4 \mathrm{e}^{i \chi_{n}}, c=-i \hbar / 2\left(k^{2}+\ell^{2}\right), d=-(1+i 2 \ell) / 4 \mathrm{e}^{-i \chi_{n}}$, in the second stage. We use the tridiagonal structure of (51) to our advantage in selecting a discretization method. The second order, fully implicit Adams-Moulton method is used in the present study. Applying this discretization method to $(50)$ gives

$$
\frac{\vec{y}_{m+1}-\vec{y}_{m}}{\delta t}=\frac{\vec{f}_{m+1}-\vec{f}_{m}}{2}
$$

The numerical method conveniently perserves the tridiagonal structure of the original truncated system. At each time step, we solve two, uncoupled linear, tridiagonal systems for $a_{k, \ell}(t)$ (one during each advection stage) with an efficient tridiagonal system solver. A numerical $\mathrm{C}$ code was written to compute the time evolution of $a_{k, \ell}$ and perform the subsequent inverse FFT to obtain the spatio-temporal structure of $A$. Since $\mathrm{C}$ does not have built-in capability for handling complex numbers, special functions were written to deal with complex number operations.

There are some additional comments worth mentioning regarding the present numerical scheme. At each time step we verify that the code conserves $|A|^{2}$ over the spatial domain (refer to section 5) by tracking $a_{0,0}$. The deviation of $a_{0,0}$ from the expected value of unity is never greater than $1 \times 10^{-10}$. Additional calculations regarding the stability and accuracy of the present numerical scheme were performed; however, due to space limitations, we do not provide those details. The analyses stem from a comparison between the solution to the discretized equation and the analytical solution obtained in section 8 . We found the numerical method to be unconditionally stable. Furthermore, we found that the minimum time step required to achieve a specified accuracy depended on $\hbar$ and the magnitude of the wavenumber $\kappa^{2}$. For example, to achieve $98 \%$ accuracy in the highest wavenumber component of the numerical solution for $\hbar=1$, a minimum time step of 0.005 is required.

Figure 7 displays four snapshots of the spatial structure of $\Re(A)$ for the case of $\hbar=1$, $\tau=1$. The resolution in each picture is $1028 \times 1028$. Figure 8 displays the numerical results from a comparison study between four different parameter values of $\hbar=0,0.1,1$, and 10 . Only the real part of $A$ is shown, although the imaginary part exhibits similar structure. The snapshots are taken at $n=10$ with $\tau=1$. All computations utilized the same random data set for $\phi$ and $\chi$.

\section{Energy in Large Scales}

We now take a look at how the energy in the large scales decays in time as a function of $\hbar$. Recall from section 5 that $\left\langle A A^{*}\right\rangle$ represents a conserved quantity where $\langle\cdot\rangle$ denotes a spatial 

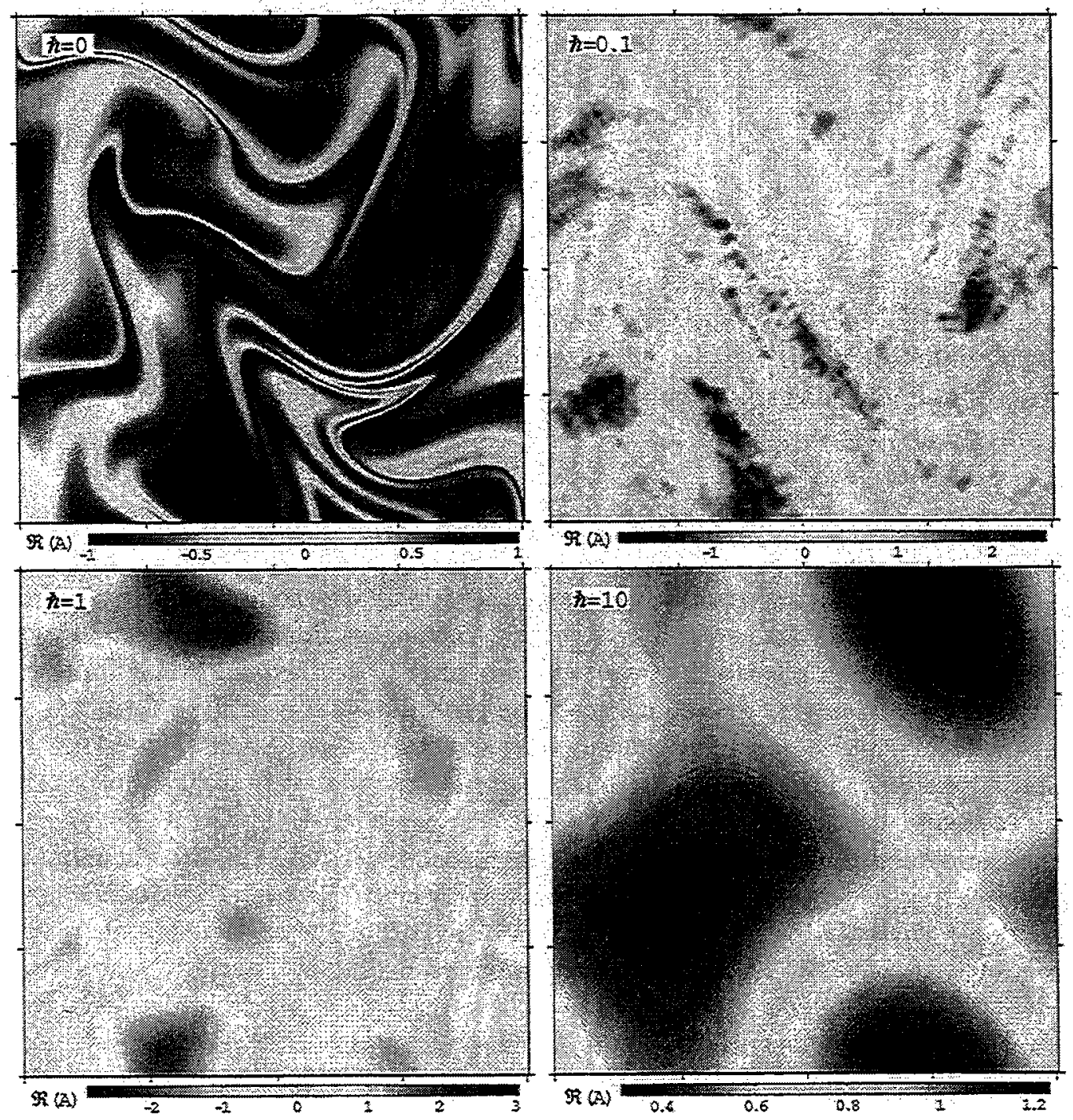

Figure 8: Comparison of the spatial structure of $\Re(A)$ for four different $\hbar$ values of $0,0.1,1$, 10. All snapshots were taken at $n=10, \tau=1$ and utilize the same random data set for $\phi, \chi$.

average and the superscript * denotes the complex conjugate. Therefore, we will define the energy associated with the complex scalar $A$ as

$$
\varepsilon=\langle A\rangle\left\langle A^{*}\right\rangle
$$

Figure 9 shows the results of $\varepsilon$ for three different cases of $\hbar=0,0.01,1$ as computed from the numerical code presented in section 9 . The results are for one particular realization only. The exponential prediction shown stems from the hypothesis that the spatial average in the definition of (53) can be replaced by an ensemble average, i.e. $\langle A\rangle=E\left[\mathrm{e}^{i \theta}\right]$, where $E[\cdot]$ denotes the expectation of the random process. For the case of $\hbar=0$, we showed in section 7 


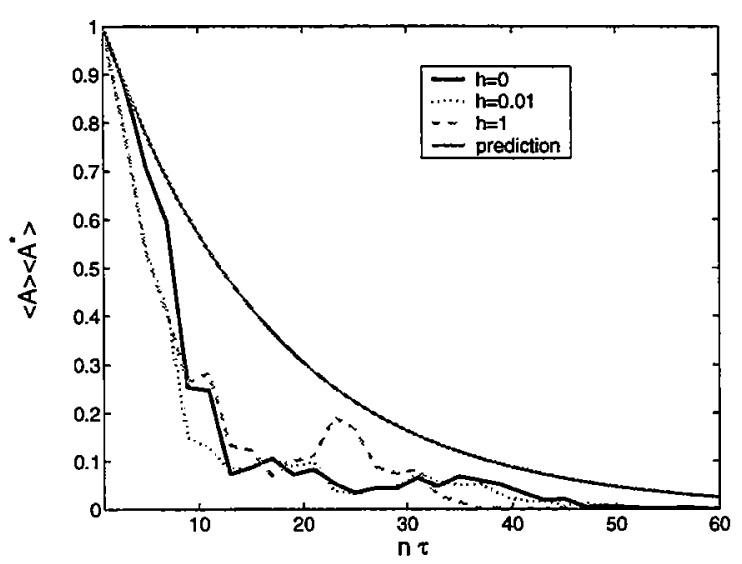

Figure 9: Decay of energy in the large scales of $A$ for $\tau=1$.

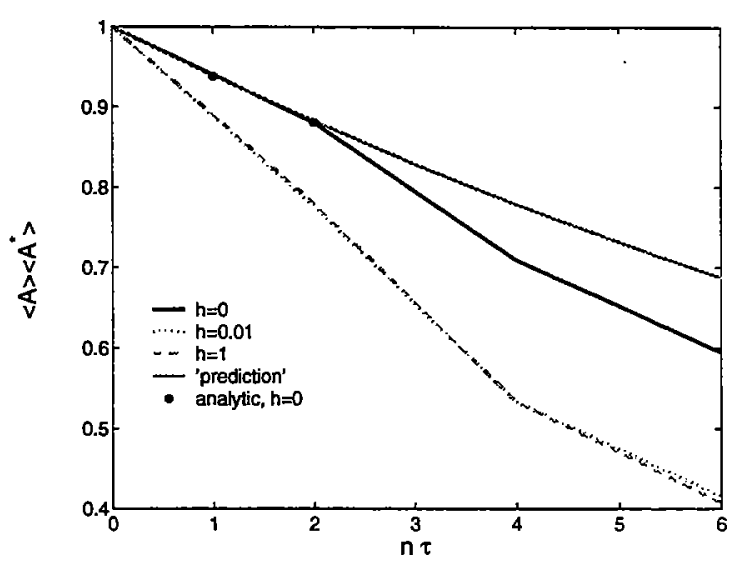

Figure 10: Expanded view of figure 9 in the region near $t=0$.

that $\theta$ obeys a random walk; therefore, the probability of $\theta$ is Gaussian. According to the prediction,

$$
\langle A\rangle=\int_{-\infty}^{\infty} \frac{\mathrm{e}^{-\theta^{2} / 2 \sigma^{2}}}{\sqrt{2 \pi \sigma^{2}}} \mathrm{e}^{i \theta} d \theta=\mathrm{e}^{-\sigma^{2} / 2}=\mathrm{e}^{-D_{\theta} t},
$$

where $D_{\theta}$ is the diffusivity of the phase as calculated in section 7 . Obviously from figure 9 , the prediction (54) fails to describe the actual behavior of $\varepsilon$.

From our analysis of the strong dispersion limit, we know that as $\hbar \rightarrow \infty, \varepsilon$ remains constant at the initial value of 1 . This yields an interesting picture of the decay of the large scale energy as a function of $\hbar$. At $\hbar=0$, the rate of decay of $\varepsilon$ is faster than exponential. As $\hbar$ increases from 0 , the rate of decay becomes faster than that for $\hbar=0$. However, at some critical value of $\hbar>1$, the rate of energy decay becomes slower than exponential and eventually becomes zero, since $\varepsilon=1$ for all time as $\hbar \rightarrow \infty$.

\section{Conclusion and Future Work}

The main focus of the present study has been the attempt to understand some of the fundamental properties of the passive scalar dispersion equation (10) in the context of a random wave model (18) for the two dimensional background turbulent velocity field. We have found that the dispersivity parameter $\hbar$ greatly affects the spatiotemporal structure of the complex scalar $A$. For $\hbar=0$, both the real and imaginary parts of $A$ become highly stretched and contorted even after only 10 iterations of the random wave field. In contrast, for the case of $\hbar=1$, the spatial structure of $A$ looks blotchy with little indication of stretching or amplification of the gradient of $A$. As $\hbar$ increases, the time evolution of the spatial structure of $A$ becomes increasingly slower. Below a critical value $\hbar_{c}$, energy in the large scales of $A$ decays faster than exponential; while for cases of $\hbar>\hbar_{c}$, the energy in the large scales decays slower than exponential. In fact, as $\hbar \rightarrow \infty$, the energy in the large scales remains constant at a 
value of unity. A useful extension of the present study would be to quantify the structure of $A$, visualized herein, using probability density functions. Another natural direction for future work would be to determine whether $A$ exhibits a Batchelor scale. In other words, is there a limit to the smallest scales of $A$ achievable in the flow? Additionally, if there is a cascade of energy from large to small scales, then what are the relevant scalings associated with the spectra and how do these compare with the case of passive scalar diffusion?

Finally with regard to near inertial oscillations, from the vertical normal mode decomposition presented in section 4 along with figure 2 , we recognize that large $\hbar$ corresponds to low wavenumber vertical motions while $\hbar \rightarrow 0$ corresponds to high wavenumber vertical motions. It remains somewhat unclear, though, how one can directly apply the present observations regarding the variation in the decay of energy of $A$ with $\hbar$ toward further understanding the behavior of the NIO velocity field in the oceanic mixed layer. Recall that in order to relate the NIO velocity field to the results of $A$ presented here, we first need to compute $\mathcal{A}$ using the superposition of vertical normal modes and then apply the operator $L$. This is left as a task for future work.

\section{Acknowledgments}

This work was performed in collaboration with W. R. Young and S. G. Llewellyn Smith. The author would like to thank J.-L. Thiffeault for many helpful discussions and E. Chassignet for providing additional computational resources. The author would also like to thank N. Balmforth, the GFD Fellowship Committee, and all of the 1999 GFD Fellows and participants for a wonderful experience at Walsh Cottage, WHOI.

\section{Appendix}

Here, we detail the integrations resulting from the Fourier series expansion of $A$ as described in section 9. The corresponding terms in (47) will be referred to sequentially as (I)-(V) starting from left to right. Each term is multiplied by $e^{-i q y-i p x}$ and integrated over $y=0-2 \pi$ and $x=0-2 \pi$. Due to orthogonality of the Fourier modes,

$$
\int_{x=0}^{2 \pi} \mathrm{e}^{i(k-p) x} d x=2 \pi \delta_{k p} \quad \text { and } \quad \int_{y=0}^{2 \pi} \mathrm{e}^{i(\ell-q) y} d y=2 \pi \delta_{\ell q}
$$

where $\delta$ is the kronecker delta. The two stages of the advection process are considered separately. We only outline integrations for the first stage; those for the second stage follow in a similar manner. The first advection stage occurs during time intervals, $2 n \tau<t \leq(2 n+1) \tau$, $n=0,1, \ldots$ with the streamfunction given by $\Psi=\sin (y+\phi)$. Terms in (47), excluding term (II) which is identically zero, are as follows:

(I) $\int_{0}^{2 \pi}\left[\int_{0}^{2 \pi} \sum_{k, \ell=-\infty}^{\infty} \dot{a}_{k, \ell}(t) \mathrm{e}^{i(k-p) x} d x\right] \mathrm{e}^{i(\ell-q) y} d y=(2 \pi)^{2} \dot{a}_{k, \ell}$ 


$$
\begin{aligned}
& \text { (III) }-\int_{0}^{2 \pi}\left[\int_{0}^{2 \pi} \sum_{k, \ell=-\infty}^{\infty} i k \cos (y+\phi) a_{k, \ell}(t) \mathrm{e}^{i(\ell-q) y} d y\right] \mathrm{e}^{i(k-p) x} d x= \\
& -\int_{0}^{2 \pi}\left[\sum_{k=-\infty}^{\infty} i 2 \pi k\left(a_{k, \ell+1}(t) \frac{\mathrm{e}^{-i \phi}}{2}+a_{k, \ell-1} \frac{\mathrm{e}^{i \phi}}{2}\right)\right] \mathrm{e}^{i(k-p) x} d x= \\
& -i k(2 \pi)^{2}\left[a_{k, \ell+1}\left(\frac{\mathrm{e}^{-i \phi}}{2}\right)+a_{k, \ell-1}\left(\frac{\mathrm{e}^{i \phi}}{2}\right)\right], \\
& \text { (IV) }-\int_{0}^{2 \pi}\left[\int_{0}^{2 \pi} \frac{-i}{2} \sin (y+\phi) \sum_{k, \ell=-\infty}^{\infty} a_{k, \ell}(t) \mathrm{e}^{i(\ell-q) y} d y\right] \mathrm{e}^{i(k-p) x} d x= \\
& -\int_{0}^{2 \pi} 2 \pi\left[\frac{\mathrm{e}^{-i \phi}}{4} \sum_{k=-\infty}^{\infty} a_{k, \ell+1}(t)-\frac{\mathrm{e}^{i \phi}}{4} \sum_{k=-\infty}^{\infty} a_{k, \ell-1}(t)\right] \mathrm{e}^{i(k-p) x} d x= \\
& (2 \pi)^{2}\left[a_{k, \ell+1}\left(\frac{\mathrm{e}^{-i \phi}}{4}\right)-a_{k, \ell-1}\left(\frac{\mathrm{e}^{i \phi}}{4}\right)\right], \\
& \text { (V) } \int_{0}^{2 \pi}\left[\int_{0}^{2 \pi} \frac{-i \hbar}{2} \sum_{k, \ell=-\infty}^{\infty}\left(k^{2}+\ell^{2}\right) a_{k, \ell}(t) \mathrm{e}^{i(\ell-q) y} d y\right] \mathrm{e}^{i(k-p) x} d x= \\
& -(2 \pi)^{2} \frac{i \hbar}{2}\left(k^{2}+\ell^{2}\right) a_{k, \ell}(t) \text {. }
\end{aligned}
$$

Note, terms similar to $\cos (y+\phi) \mathrm{e}^{i(\ell-q) y}$ can be easily integrated by rewriting the trigonometric part as an exponential, e.g. $1 / 2\left(e^{i(y+\phi)}+e^{-i(y+\phi)}\right)$.

\section{References}

[1] E. A. D'Asaro, C. C. Eriksen, M. D. Levine, P. Niiler, C. A. Paulson, and P. Van Meurs, "Upper-ocean inertial currents forced by a strong storm. Part I: Data and comparison with linear theory," J. Phys. Ocean. 25, 2909 (1995).

[2] W. R. Young and M. Ben Jelloul, "Propagation of near-inertial oscillations through geostrphic flow," J. Mar. Res. 55, 735 (1997).

[3] N. J. Balmforth, S. G. Llewellyn Smith, and W. R. Young, "Enhanced dispersion of near-inertial waves in an idealized geostrophic flow," J. Mar. Res. 56, 1 (1998).

[4] A. E. Gill, Atmospher-Ocean Dynamics (Academic Press, London, 1982).

[5] W. J. Emery, W. G. Lee, and L. Magaard, "Geographic and seasonal distributions of Brunt-Väisälä frequency and Rossby radii in the North Pacific and North Atlantic," J. Phys. Ocean. 14, 294 (1984). 
[6] A. E. Gill, "On the behavior of internal waves in the wakes of storms," J. Phys. Ocean. 14, 1129 (1984).

[7] G. K. Batchelor, "Small-scale variation of convected quantities like temperature in turbulent fluid," J. Fluid Mech. 5, 113 (1958).

[8] R. Pierrehumbert, "Tracer microstructure in the large-eddy dominated regime," Chaos, Solitons, and Fractals 4, 1091 (1994).

[9] R. Seydel, Practical bifurcation and stability analysis: from equilibrium to chaos (Springer-Verlag, New York, 1994).

[10] S. Redner, "Random multiplicative processes: An elementary tutorial," American Journal of Physics 58, 267 (1990).

[11] A. Einstein, "On the movement of small particles suspended in a stationary liquid demanded by the molecular kinetic theory of heat," Annalen der Physik 17, 549 (1905). 


\title{
The temperature-salinity relationship in the mixed layer
}

\author{
Raffaele Ferrari
}

\section{Introduction}

In the surface mixed layer (ML) of the ocean there is a remarkable correlation between the horizontal temperature and salinity gradients. The goals of this project are (1) to investigate if these correlations are the result of processes at work within the ML, (2) to develop parameterizations of these processes to be used in large-scale ocean models.

Observations show abundant examples of horizontal fronts with temperature and salinity that oppose in their joint effect on density on scales of $10 \mathrm{~m}$ to $100 \mathrm{~km}$ [1], [2], [3]. A useful measure of the degree of compensation is the density ratio $R$, defined as the ratio of the relative effect of temperature and salinity on a density front. Stommel and Chen ([4], [5]) computed density ratios from large scale meridional temperature-salinity $(T-S)$ gradients in the range of latitudes between $20^{\circ}$ and $50^{\circ}$ and concluded that $R$ has a mean close to 1.7, even though individual fronts can have a density ratio markedly different from 1.7. These results are obtained from climatological data sets and refer to density ratios on scales of thousands of kilometers averaged over a number of years. On smaller horizontal scales, between $20 \mathrm{~m}$ and $10 \mathrm{~km}$, Rudnick and Ferrari find that the ML density ratio is 1 and not 2 [3]. These observations imply that the mean density ratio on large scale is 1.7 , even though typical thermohaline gradients at small scale have an instantaneous density ratio of 1 .

One possible explanation of the correlations between thermohaline gradients in the ML is that atmospheric forcing and entrainment of thermocline waters create and juxtapose water masses with compensating $T-S$ gradients. However the ratio of heat to freshwater buoyancy fluxes is variable in large scale maps and in time series at a point and there is no evidence that these fluxes force a small scale frontal density ratio of 1 and a large annual mean density ratio of 1.7. In this project we propose an alternative interpretation that relies on regulating mechanisms in the ML to create correlations between temperature and salinity, regardless of the large scale atmospheric forcing. Furthermore we show that the processes responsible for these correlations are not properly represented in large-scale ocean models and we suggest more appropriate parameterizations.

\section{Nonlinear diffusive parameterizations of eddy transfer of heat and salt in the mixed layer}

The basic idea of this study is straightforward: temperature and salinity are dynamically active in ML because they contribute to density gradients. All processes that depend on density gradients, and not on temperature and salinity gradients separately, are potentially 
capable of creating $T-S$ relations, because they act only on $T-S$ fluctuations that reinforce in their joint effect on density. In order to test this idea, we first derive parameterizations of the $\mathrm{ML}$, which use density gradients as the driving field, and then we examine the consequences of these parameterizations on the distributions of temperature and salinity in numerical models.

Let us consider the dispersion of some tracer of concentration $\theta(x, y, z, t)$ in the ML. We model the ML as a vigorously mixed shallow layer characterized by a high aspect ratio, i.e. with a depth $H$ much less than the horizontal scale $L$. The main point here is that there are two very different time scales: a fast time scale over which the layer is mixed vertically and a slow time scale associated with horizontal transports. We argue that in order to describe the lateral dispersion of the tracer on the slow time scale, it is not necessary to resolve the details of the processes active on the fast time scale. The combined action of small scale stirring stirring and vertical mixing can be parameterized as a diffusion of the vertically averaged tracer.

A mathematical model for the horizontal transport of tracers in the ML is formulated decomposing $\theta$ in a "mean" - denoted by $\bar{\theta}$ - defined as the average over the depth $H$ of the ML and over a period long compared to the time scale of vertical mixing, and in an "eddy" denoted by $\theta^{\prime}$ - defined as the departure from that mean. The Reynold's averaged equation for a conserved tracer is

$$
\bar{\theta}_{t}+\overline{\mathbf{u}} \cdot \nabla_{H} \bar{\theta}=-\nabla \cdot \overline{\mathbf{u}^{\prime} \theta^{\prime}}+\bar{F} .
$$

Here $\mathbf{u}=(u, v)$ denotes the horizontal velocity of the incompressible Boussinseq fluid. The first term in the RHS of (1) is called the eddy flux divergence. Its net effect is to redistribute the tracer within the body of the fluid. The second term, $\bar{F}$, represents the averaged fluxes of tracer from the surface of the ocean (atmospheric forcing) and through the base of the ML (entrainment of thermocline waters). The challenge of this section is to derive a long-term, large-scale equation for the mean tracer, by expressing the eddy fluxes in (1) in terms of mean quantities.

Eddy fluxes of a conserved tracer can be parameterized with closures based on local mean gradients. The argument goes that a fluid particle carries the value of $a$ conserved, and hence transferable, tracer for some length $\mathbf{l}^{\prime}$, before it is mixed with its new surroundings. If the particle was initially typical of its surroundings then the eddy flux of tracer $\theta$ is given by

$$
\overline{\mathbf{u}^{\prime} \theta^{\prime}}=-\overline{\mathbf{u}^{\prime} \mathbf{1}^{\prime}} \cdot \nabla \bar{\theta}
$$

where it is assumed that $\nabla \bar{\theta}$ varies little over distances comparable with the mixing length $\mathbf{l}^{\prime}$. The tensor $\overline{\mathbf{u}^{\prime} \mathbf{l}^{\prime}}$ defines the eddy diffusivity. The most commonly used parameterization of horizontal eddy transports in the ML is to assume a down-gradient Fickian diffusion,

$$
\overline{\mathbf{u}^{\prime} \theta^{\prime}}=-k \nabla \bar{\theta}
$$

where $k$ is set to an a priori value constant both in space and time. However down-gradient diffusion follows from (2) only if the statistics of the eddy field are horizontally homogeneous and isotropic. In the ML lateral eddy transports on time scales longer than the vertical mixing time result from hydrodynamical instabilities that release the gravitational potential energy stored in horizontal density gradients. The dynamics is simple; lateral density gradients 
slump under the action of gravity and drive horizontal eddy fluxes. The eddy velocity $\mathbf{u}^{\prime}$ and the eddy displacement $\mathrm{l}^{\prime}$ associated with the release of potential energy must be, on average, in the direction of the density gradients and larger when the horizontal density gradients are larger. Therefore in the ML the statistics of the eddy field are not homogeneous and isotropic and a down-gradient Fickian closure is not appropriate. It seems natural to look instead for closures that include the effect of large scale density gradients. A general expression for the eddy diffusivity tensor can be written in the form,

$$
\overline{\mathbf{u}^{\prime} \mathbf{l}^{\prime}}=\gamma f(|\nabla \bar{\rho}|) \nabla \bar{\rho} \nabla \bar{\rho}
$$

where $\rho$ is the density of the fluid, $\gamma$ a constant and $f(|\nabla \rho|)$ a function whose form depends on the details of the hydrodynamic instabilities that dominate in the eddy field. The corresponding flux of tracer is,

$$
\overline{\mathbf{u}^{\prime} \theta^{\prime}}=-\gamma f(|\nabla \bar{\rho}|)(\nabla \bar{\rho} \cdot \nabla \bar{\theta}) \nabla \bar{\rho}
$$

Notice that, even though the flux is in the direction of the mean density gradient, $\overline{\mathbf{u}^{\prime} \theta^{\prime}} \cdot \nabla \bar{\theta}<0$; thus the flux of tracer tends to be down the tracer gradient. With (5) we can write down an equation that describe the dispersion of the mean tracer,

$$
\theta_{t}+\mathbf{u} \cdot \nabla \theta=\gamma \nabla \cdot[f(|\nabla \rho|)(\nabla \rho \cdot \nabla \theta) \nabla \rho]+F
$$

where we dropped the overbars. Hereinafter we assume that all variables are averaged over the depth of the ML and over times longer than the vertical mixing time.

We can now turn to the case where the tracers are temperature $T$ and salinity $S$. Let us express density as $\rho=\rho_{0}\left[1-g^{-1} B\right]$, where $B$ is buoyancy. Assuming that the equation of state is linear, and using suitable definitions, the buoyancy is

$$
B=T-S
$$

With our definitions, $S, T$ and $B$ all have the dimensions of acceleration. The nonlinear diffusion equations that describe the buoyancy driven dispersion of heat and salt in the ML follow from (6),

$$
\begin{aligned}
T_{t}+\mathbf{u} \cdot \nabla T & =\gamma \nabla \cdot[f(|\nabla B|)(\nabla B \cdot \nabla T) \nabla B]+F_{T} \\
S_{t}+\mathbf{u} \cdot \nabla S & =\gamma \nabla \cdot[f(|\nabla B|)(\nabla B \cdot \nabla S) \nabla B]+F_{S}
\end{aligned}
$$

where $F_{T}$ and $F_{S}$ represent the forcings on temperature and salinity.

Parameterizations in which the dynamics depends exclusively on horizontal density gradients are not new to the oceanographic literature. The idea descends from Stommel's two-box idealization of the thermohaline circulation [6]. Stommel posited a transport law in which the exchange of mass between boxes is proportional to the square of the density difference. Some thirty years later Stommel and Young used the same model to study the $T-S$ relation in the ML [7]. Stommel's model is essentially a two-grid points discretization of (8) and (9) with $f(|\nabla B|)=|\nabla B|^{-1}$. Other classes of nonlinear diffusion models have been developed for the full range of space and time scale that are of interest to oceanographers: from the 
planetary scale of the thermohaline circulation, through the deformation scale dynamics of baroclinic eddies, down to the ageostrophic circulations in shallow water systems. Here we limit our attention to models for the upper ocean. The redistribution of temperature and salinity in the $\mathrm{ML}$ is the result of processes that release the potential energy stored in horizontal stratification; viz. buoyancy driven shear dispersion at scales below the deformation radius and baroclinic instability at larger scales. Our goal is now to emphasize the unity of the physical ideas underlying the two processes and to show that both can be described by the equations in (8) and (9).

\subsection{Buoyancy driven shear dispersion}

W. R. Young, in the first part of this volume, discussed a class of models to parameterize the transport of heat and salt in a ML idealized as a shallow system with strong vertical mixing. He derived a set of nonlinear diffusion equations for $T$ and $S$, where the nonlinearity arises because the horizontal transport of heat and salt is by shear dispersion, and the shear flow doing the dispersing is driven by slumping horizontal buoyancy gradients. The main point of his presentation was that the lateral diffusivity in such a ML is proportional to the horizontal buoyancy gradient squared. Young's model is a particular case of (8) and (9), with $f(|\nabla B|)=1$ and no vertically averaged flow, $\mathbf{u}=(0,0)$,

$$
\begin{aligned}
& T_{t}=\gamma \nabla \cdot[(\nabla B \cdot \nabla T) \nabla B]+F_{T}, \\
& S_{t}=\gamma \nabla \cdot[(\nabla B \cdot \nabla S) \nabla B]+F_{S} .
\end{aligned}
$$

The constant $\gamma$ depends on the depth $H$ of the ML and on the details of the processes doing the vertical mixing. If mixing is parameterized as an intermittent process that homogenizes the ML at intervals of time $\tau$, one finds that $\gamma=H^{2} \tau^{3} / 96$ [8]. Different parameterizations agree on the functional dependence of $\gamma$ on the depth $H$ and the characteristic time of vertical mixing $\tau$.

It is important to discuss the range of oceanic parameters for which the nonlinear diffusion equations (10) and (11) might apply. Shear dispersion mechanisms can act only at horizontal scales larger than $H$, say $100 \mathrm{~m}$, and shorter than the deformation radius, typically $10 \mathrm{~km}$ in the ML, and at timescales longer than the vertical mixing time, say a day. Motions with smaller lengths and higher frequencies are parameterized as "vertical mixing" (e.g. Langmuir circulations and convective overturning). At larger scales the effects of rotation become important and the dynamics change substantially as shown in the next section. For $H=100$ $\mathrm{m}$ and $\tau=1$ day one obtains $\gamma=10^{17} \mathrm{~m}^{2} \mathrm{~s}^{3}$; we use this as a reference value throughout the report whenever we make quantitative statements about our results.

\subsection{Buoyancy fluxes produced by baroclinic instability: Green, Stone and Held}

At scales larger then the Rossby radius of deformation eddies produced at baroclinically unstable buoyancy gradients can dominate the transports of heat and salt in the ML. Green used the arguments reviewed at the beginning of this section to show that the transfer properties of baroclinic eddies can be parameterized in terms of their large scale structure [9]. Supposing 
that the transfer of buoyancy occurs in the growing phase of baroclinic eddies, when energy is extracted by the eddy from the baroclinic zone of the large scale flow, Green deduced the expected form of the eddy diffusivity in the limit of large Richardson number. He then used linear stability analysis of the baroclinic wave to determine the direction of the eddy fluxes. Stone derived a similar expression from linear stability analysis and extended Green's results to small Richardson numbers [10]. Green and Stone imagined that diabatic processes, like vertical mixing, restore the baroclinic zone, replenishing the supply of available potential energy until it is discharged again by baroclinic instability. This repeated conversion of energy leads to a diapycnal eddy flux. Green and Stone attempted to relate this flux to large-scale parameters in zonally averaged models of the atmosphere,

$$
\overline{v^{\prime} \theta^{\prime}}=-\frac{\alpha}{N} l^{2}\left|B_{y}\right| \theta_{y}
$$

where $v^{\prime}$ is the meridional eddy velocity, $N$ is the Brunt-Väisälä frequency, $\alpha$ a universal constant of proportionality, and $l$ a measure of the meridional eddy displacement. Green argues that this distance is set by the width of the baroclinic zone. Stone, however, suggest that the deformation radius is the appropriate length scale. The ideas of Green and Stone, however, converge on predicting that the baroclinic eddy fluxes across a buoyancy gradient are driven by the absolute value of the diapycnal buoyancy gradient. It then makes good physical sense to extend (12) to two dimensions as,

$$
\overline{\mathbf{u}^{\prime} \theta^{\prime}}=-\frac{\alpha}{N} l^{2}|\nabla B|^{-1}(\nabla \theta \cdot \nabla B) \nabla B
$$

With this parameterization of lateral eddy transports, the dispersion of temperature and salinity in the ML is once again described by nonlinear diffusion equations of the form in (8) and $(9)$; in this case $f(|\nabla B|)=|\nabla B|^{-1}$ and $\gamma=\alpha l^{2} N^{-1}$.

Pavan and Held used the results of numerical simulations to test diffusive parameterizations of the buoyancy fluxes produced by baroclinic instability [11]. They integrated a two layer model of a baroclinically unstable jet to obtain a series of statistically steady states for different jet widths and evaluate diffusive approximations of the eddy buoyancy fluxes. In their simulations the flow is forced by relaxation to an unstable prescribed buoyancy gradient. Pavan and Held found that a flux-gradient relationship, deduced by a simple fit on numerical results, predicts the magnitude and the shape of the eddy fluxes in the unstable jet flows remarkably well. The functional form of the relationship differs from that suggested by Green and Stone; for large buoyancy gradients, that is for temperature gradients larger than the climatological mean of $6^{\circ} \mathrm{C}$ over $1000 \mathrm{~km}$, the effective diffusivity is proportional to the fourth power of the buoyancy gradient. In the presence of two stratifying components, like heat and salt, one obtains a set of coupled equations for temperature and salinity of the form in (8) and (9) with $f(|\nabla B|)=|\nabla B|$.

The main conclusion of this brief review is that different diffusive parameterizations of tracer fluxes produced by baroclinic instability agree on one point: diapycnal baroclinic fluxes can be described with nonlinear diffusive closures where the diffusivity is proportional to some power of the buoyancy gradient. Differences emerge only in the power law that relates the buoyancy flux to its gradient, but all parameterizations are captured by the general form in (8) and (9). The parameterizations discussed so far describe the eddy transports across and 
not along isopycnal surfaces. A full parameterization of baroclinic instability should account for the epipycnal fluxes as well. However our goal is to explain how a density ratio of one is maintained in the ML at small scales, even though the ratio of heat and freshwater fluxes is extremely variable in the external forcing. Isopycnal eddy fluxes do not release the energy stored in horizontal buoyancy gradients and thus cannot explain compensation. Therefore we do not attempt a parameterization of epipycnal fluxes in this project, but it remains an open question to verify the role of these fluxes in creating correlations between two dimensional distributions of temperature and salinity.

\section{Temperature-salinity correlations at small scales as a result of nonlinear diffusive parameterizations}

We begin by exploring the implications of the nonlinear diffusive parameterization of the ML in an idealized setting. Suppose that temperature and salinity variations are created at some instant by atmospheric forcing or entrainment of thermocline waters and that these horizontal nonuniformities disappear as a result of nonlinear diffusion. This is a rundown problem in which there is no external forcing and the down-gradient diffusion eventually erases all the initial fluctuations in temperature and salinity. There is no mechanism to produce new randomness: all the randomness comes from the initial condition. Equations (8) and (9) reduce to

$$
T_{t}=\gamma \nabla \cdot[f(|\nabla B|)(\nabla B \cdot \nabla T) \nabla B], \quad S_{t}=\gamma \nabla \cdot[f(|\nabla B|)(\nabla B \cdot \nabla S) \nabla B] .
$$

Observe that the free decay of temperature and salinity is perfectly symmetric in this system. If the statistics of temperature and salinity are identical in the initial conditions, then no asymmetries in the thermohaline fields can develop at later times. One might then expect that this rundown problem shows only the progressive decay of the initial temperature and salinity fluctuations. However apart from the trivial decay, well before all fluctuations are erased, nonlinear diffusion creates nontrivial correlations between the thermohaline fields and between their gradients. The physical reason is that in the initial conditions there will be regions in which the temperature and salinity gradients will happen to partially compensate in their joint effect on buoyancy. In those regions the nonlinear diffusion will be small and the initially compensating gradients will persist. Likewise, the initial conditions will also contain regions in which the thermohaline gradients accidentally produce large buoyancy changes; those regions are then subject to strong diffusion and the gradients will quickly disappear. The consequence of this selective decay is that only compensated thermohaline gradients persist as the system runs down and a density ratio of 1 is established.

\subsection{One-dimensional model}

We start by considering the simplest problem in which the temperature and salinity have spatial variations in only the $y$-direction. Equations (14) then reduce to

$$
T_{t}=\gamma\left(\left|B_{y}\right|^{n} T_{y}\right)_{y}, \quad S_{t}=\gamma\left(\left|B_{y}\right|^{n} S_{y}\right)_{y}
$$


where $n$ is an integer that can take any value from 1 to 3 according to the nonlinear closure under consideration.

The one-dimensional case is sufficient to illustrate the development of thermohaline correlations from random initial conditions. Numerical simulations of the system in (15) are carried in non dimensional variables. Buoyancy, temperature and salinity are measured in terms of the initial RMS buoyancy variations $\left(\mathcal{B}_{0}\right)$, lengths in terms of the initial correlation length $\left(\ell_{0}\right)$, and time in terms of $\tau_{\gamma} \equiv \ell_{0}^{4} / \gamma \mathcal{B}_{0}^{2} \cdot \tau_{\gamma}$ is the time it takes to mix tracers over a distance $\ell_{0}$ using nonlinear diffusion driven by the initial RMS buoyancy gradients. In terms of non dimensional variables one simply sets $\gamma=1$ in (15). The initial conditions are established by selecting $T$ and $S$ uncorrelated at each grid point using a uniform probability density function with zero mean such that

$$
\left\langle B^{2}\right\rangle=1, \quad\left\langle T^{2}\right\rangle=\left\langle S^{2}\right\rangle=\frac{1}{2}
$$

where \langle\rangle is an integral over the domain

$$
\left\langle B^{2}\right\rangle \equiv \frac{1}{L} \int_{0}^{L} B^{2} \mathrm{~d} y
$$

The numerical calculations are performed by integrating the non dimensional coupled equations for temperature and salinity. Global conservation of heat and salt is satisfied by requiring that the gradients $T_{y}$ and $S_{y}$ vanish at both ends of the domain (i.e., 'no-flux' boundary conditions). We solve the system in (15) on a discrete spatial grid with an explicit Euler forward scheme in time and central differencing in space. The time step, $\Delta t$, is short enough to accurately solve the set of ordinary differential equations obtained by the spatial discretization of (15).

The creation of positive correlations between temperature and salinity and their gradients is shown in a series of $T-S$ and $T_{y}-S_{y}$ scatterplots. Figures 1,2 and 3 show the results of simulations with different nonlinear power diffusivities, respectively $n=1, n=2$ and $n=3$ in (15). In all three cases the thermohaline compensation is evident as an extension of the cloud of points along the "compensation line" $T_{y}=S_{y}$ at time $t=3$. A cloud of points collapsed along the "compensation line" is equivalent to a density ratio of 1 ; thus diffusive parameterizations with a diffusivity that depends on the horizontal buoyancy gradient correctly predict the observed density ratio of 1 at small scales. The reader interested in a more rigorous analysis of these simulations is referred to [8], where the nonlinear diffusion equation with diffusivity $n=2$ is analyzed both numerically and analytically. Here we want to stress the fact that compensation is typical of all parameterizations that have a diffusivity that grows proportionally with the horizontal gradients of buoyancy. Changing the power law in the diffusive parameterization affects the aspect ratio of the cloud of points in the scatterplots, but not the orientation of the major axis.

Finally let us discuss the characteristic time over which compensation occurs. After the passage of a hurricane, typical temperature gradients can be as large as $0.1{ }^{\circ} \mathrm{C}$ over a kilometer. The shear dispersion model for a mixed layer $100 \mathrm{~m}$ deep and a vertical mixing time of the order of one day, shows compensation over scales of a kilometer after only one hour. Choosing a vertical mixing time scale of a third of a day gives that compensation is 
created in one day. More importantly compensation on scales of $10 \mathrm{~km}$ is established in a time 100 times slower. Therefore we expect compensation to be restored after each forcing event on scales of a kilometer, while on larger scales compensation and external forcing act on comparable time scales. This explains why a density ratio of 1 is observed only up to scales of at most 10 kilometers.

\subsection{Two-dimensional model}

In the previous section we investigated nonlinear diffusive parameterizations in one dimension. We now extend our analysis to two dimensional models. A second spatial dimension adds new flavor to the problem, because the nonlinear diffusion depends not only on the magnitude but also on the relative orientation of the thermohaline gradients.

Numerical simulations of the nonlinear diffusion equation in (14) are carried in dimensionless variables as discussed in the previous section. Conservation of heat and salt is satisfied by imposing periodic boundary conditions at the edges of a square domain. The initial conditions have random and uncorrelated $T$ and $S$ profiles. The numerical integrator uses a third order Adams-Bashford scheme in time and central differences in space. The time step is chosen short enough to ensure stability and accuracy of the solutions.

The two dimensional rundown problem shows that correlations develop between the magnitudes of the thermohaline fields in much the same way described for the one dimensional case. Scatterplots of the thermohaline gradients, computed along an arbitrary path within the domain, collapse along the compensation line during the first phases of the simulation and look exactly like those in figures 1,2 and 3. The new result is that correlations develop also between the orientation of the thermohaline gradients. Figure 4 shows histograms of the angles between the temperature and salinity gradients at each grid point. The process of "thermohaline alignment" is extremely fast and by time $t=1$ (of the order of one day or less in dimensional units for typical ML gradients) $T$ and $S$ gradients are nearly everywhere parallel. Note that the number of aligned gradients increases with time, proving that nonlinear diffusion actively tilts the $T$ and $S$ isolines so as to create a uniform buoyancy field. In one dimension compensation is the result of dissipation of all buoyancy gradients. In two dimensions nonlinear diffusion can also create compensated gradients not present in the initial conditions, by selectively dissipating those components of thermohaline gradients that project on density gradients. The results shown in figure 4 are obtained for a shear dispersion closure, that is by substituting $f(|\nabla B|)=1$ in (14). Analogous results are obtained for the other forms of $f(|\nabla B|)$ introduced in section 2 .

The main result of the two dimensional simulations is that nonlinear diffusive parameterizations of the ML produce compensation between the gradients of temperature and salinity along any direction. This is consistent with the density of ratio of 1 measured by profiling the ML along one dimensional paths [3]. It remains to understand why compensation and a density ratio of 1 are observed only at small scales and not at scales of thousands of kilometers. We address this point in the next section. 

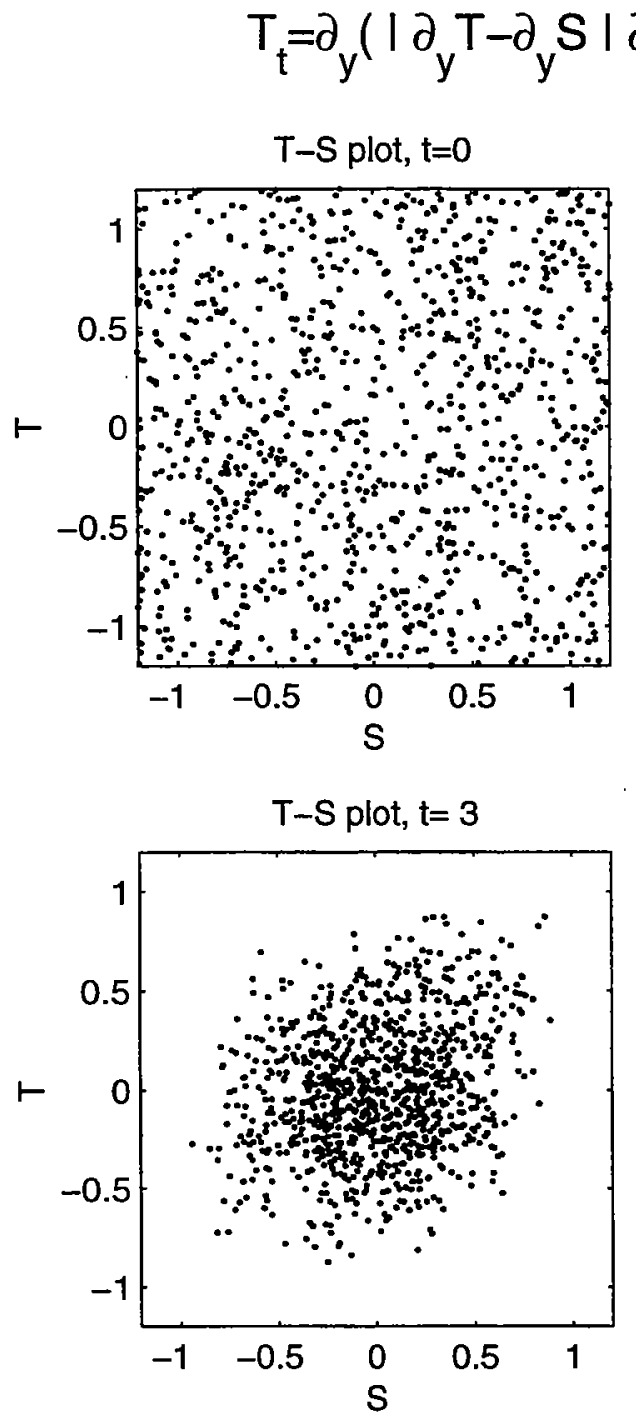
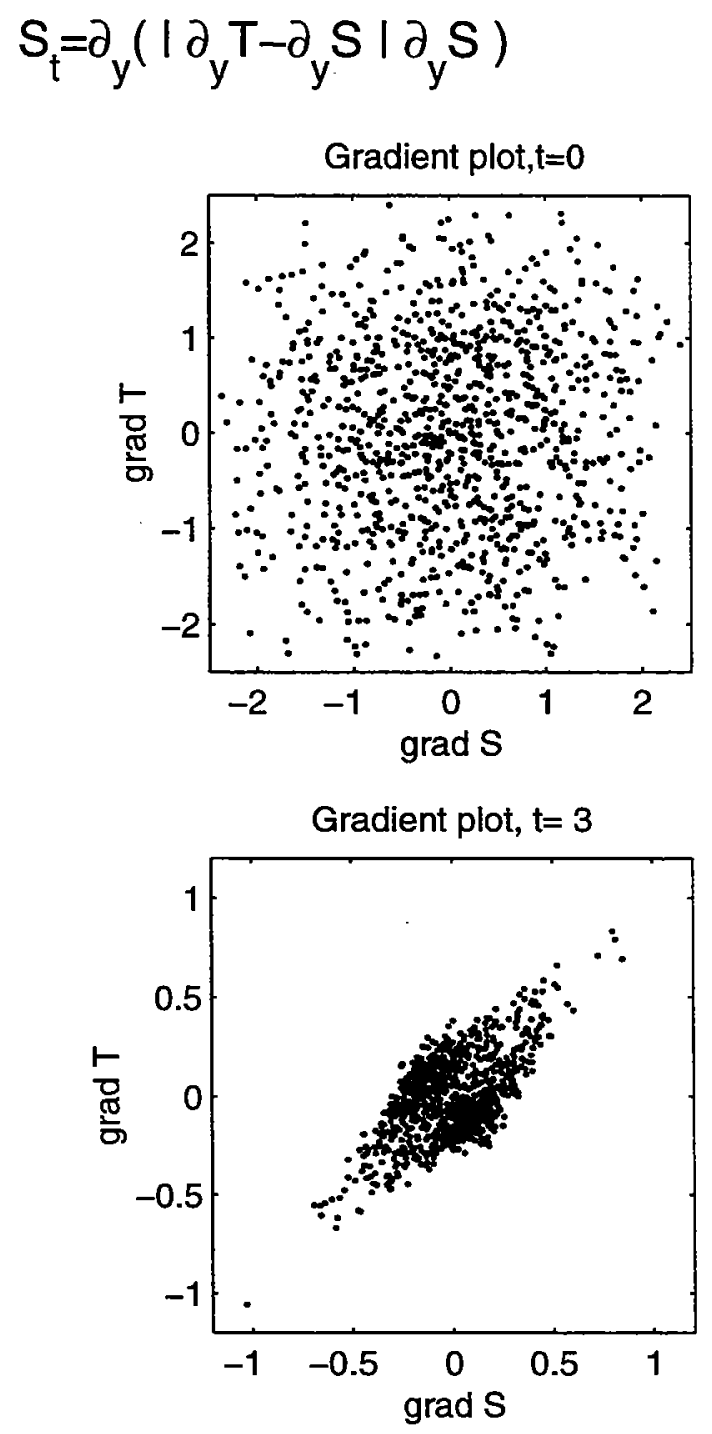

Figure 1: This figure shows the results of a simulation in which 1000 points in the $(S, T)$ and $\left(S_{y}, T_{y}\right)$ planes are created by picking uncorrelated temperature and salinity from a uniform probability density with variance $1 / 2$; thus the variance of $B=T-S$ is one. The nonlinear diffusivity is of the form $\left|B_{y}\right|=\left|T_{y}-S_{y}\right|$. The upper panels show scatterplots at time $t=0$. The lower panels scatterplots at $t=3$. 


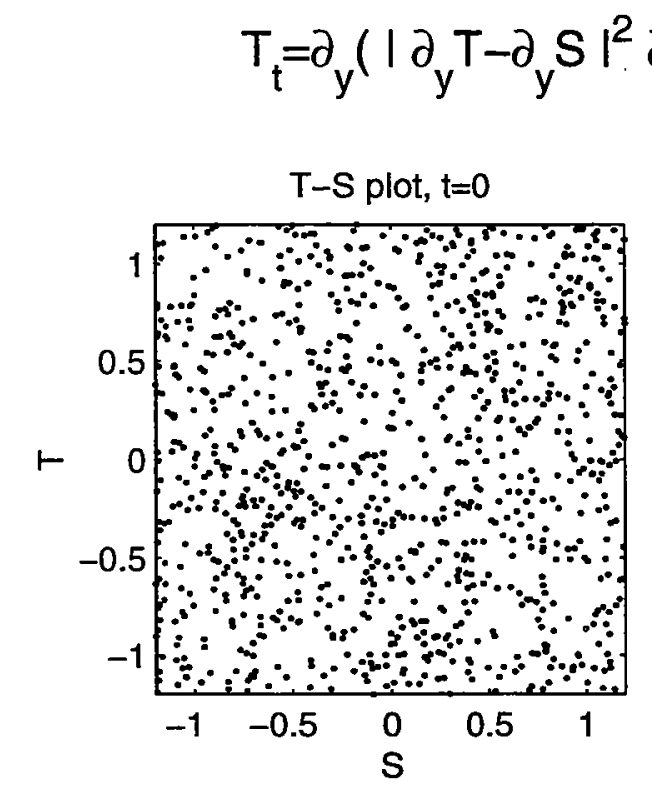

$S_{t}=\partial_{y}\left(\left|\partial_{y} T-\partial_{y} S\right|^{2} \partial_{y} S\right)$
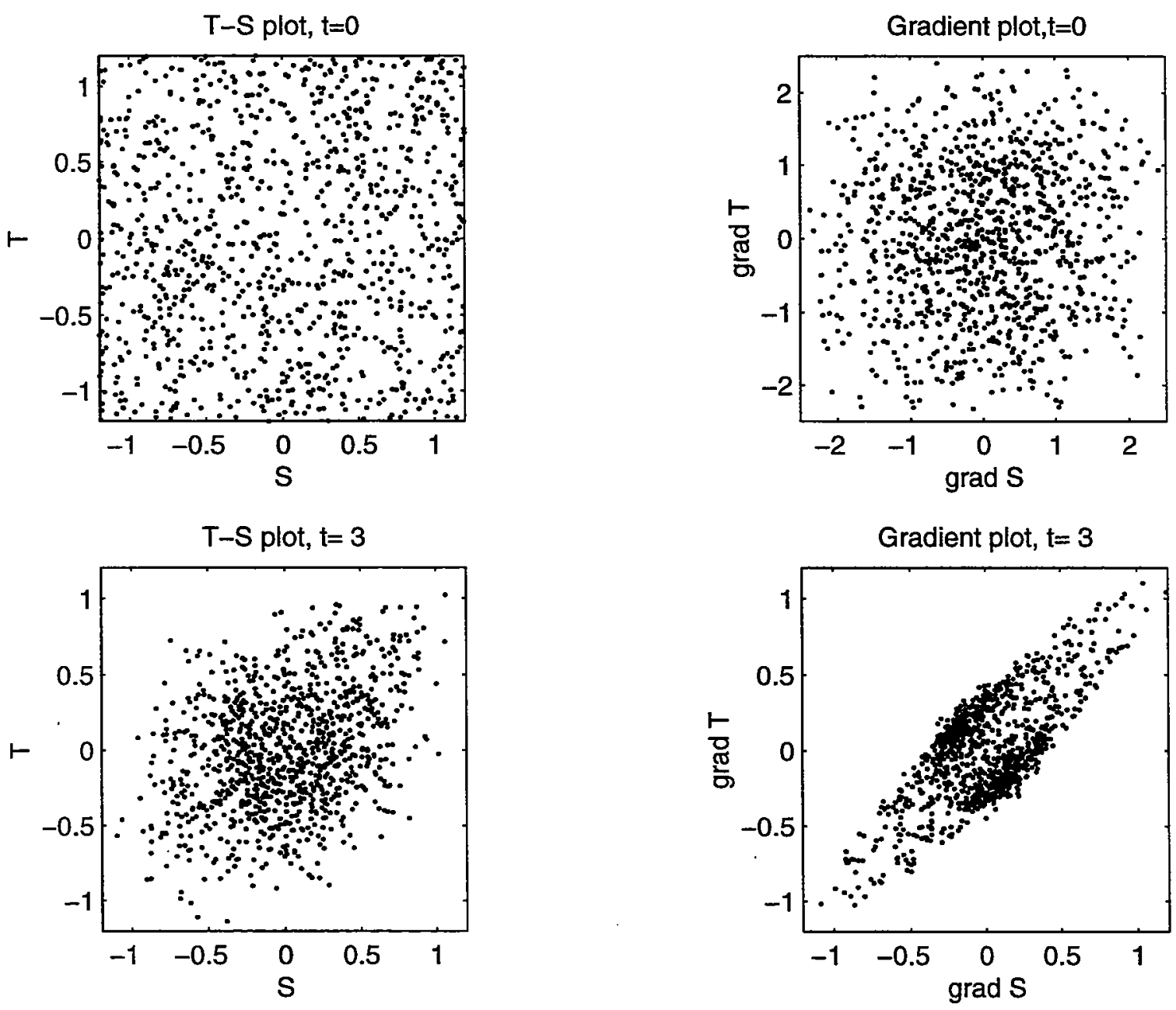

Figure 2: As in figure (1), but with a nonlinear diffusivity of the form $\left|B_{y}\right|^{2}=\left|T_{y}-S_{y}\right|^{2}$. 


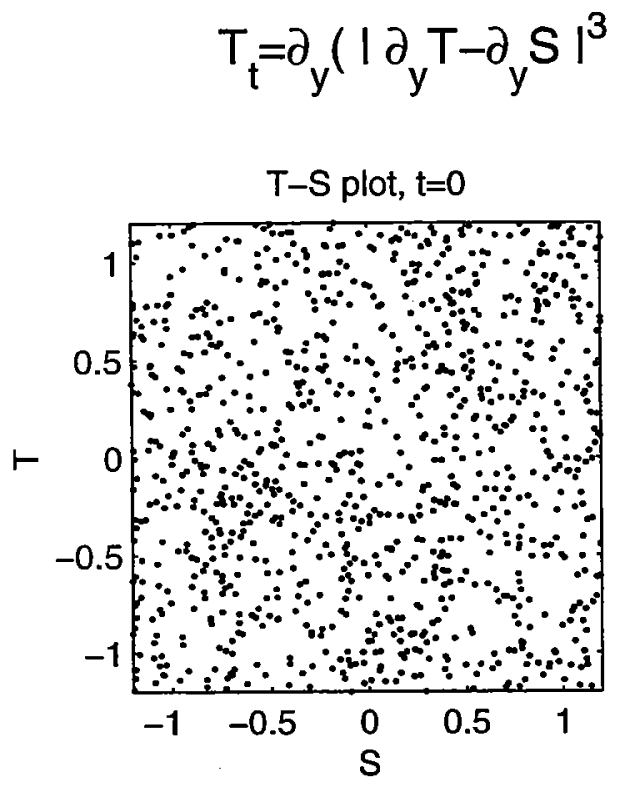

$$
\dot{S}_{t}=\partial_{y}\left(\left|\partial_{y} T-\partial_{y} S\right|^{3} \partial_{y} S\right)
$$
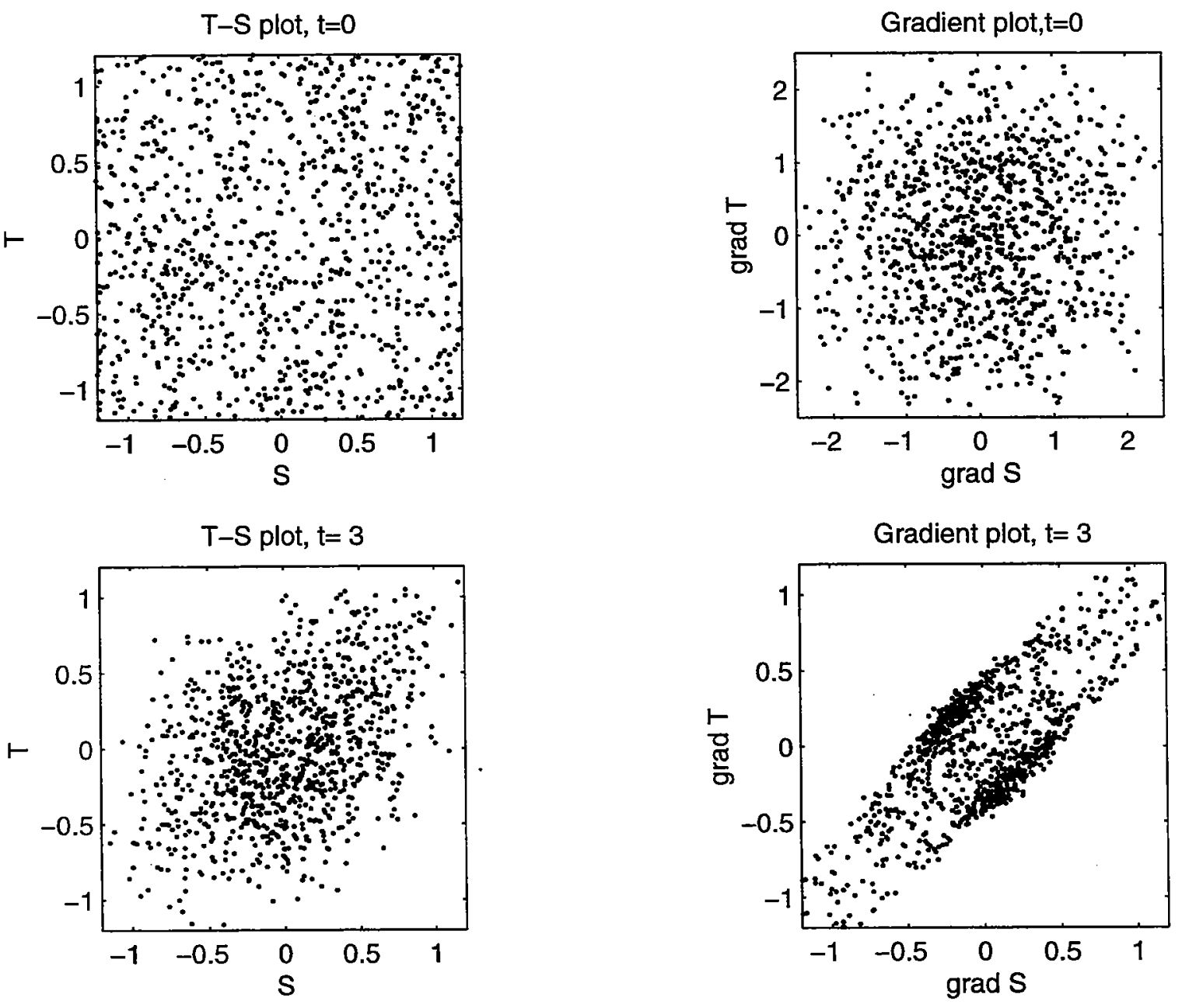

Figure 3: As in figure (3), but with a nonlinear diffusivity of the form $\left|B_{y}\right|^{3}=\left|T_{y}-S_{y}\right|^{3}$. 

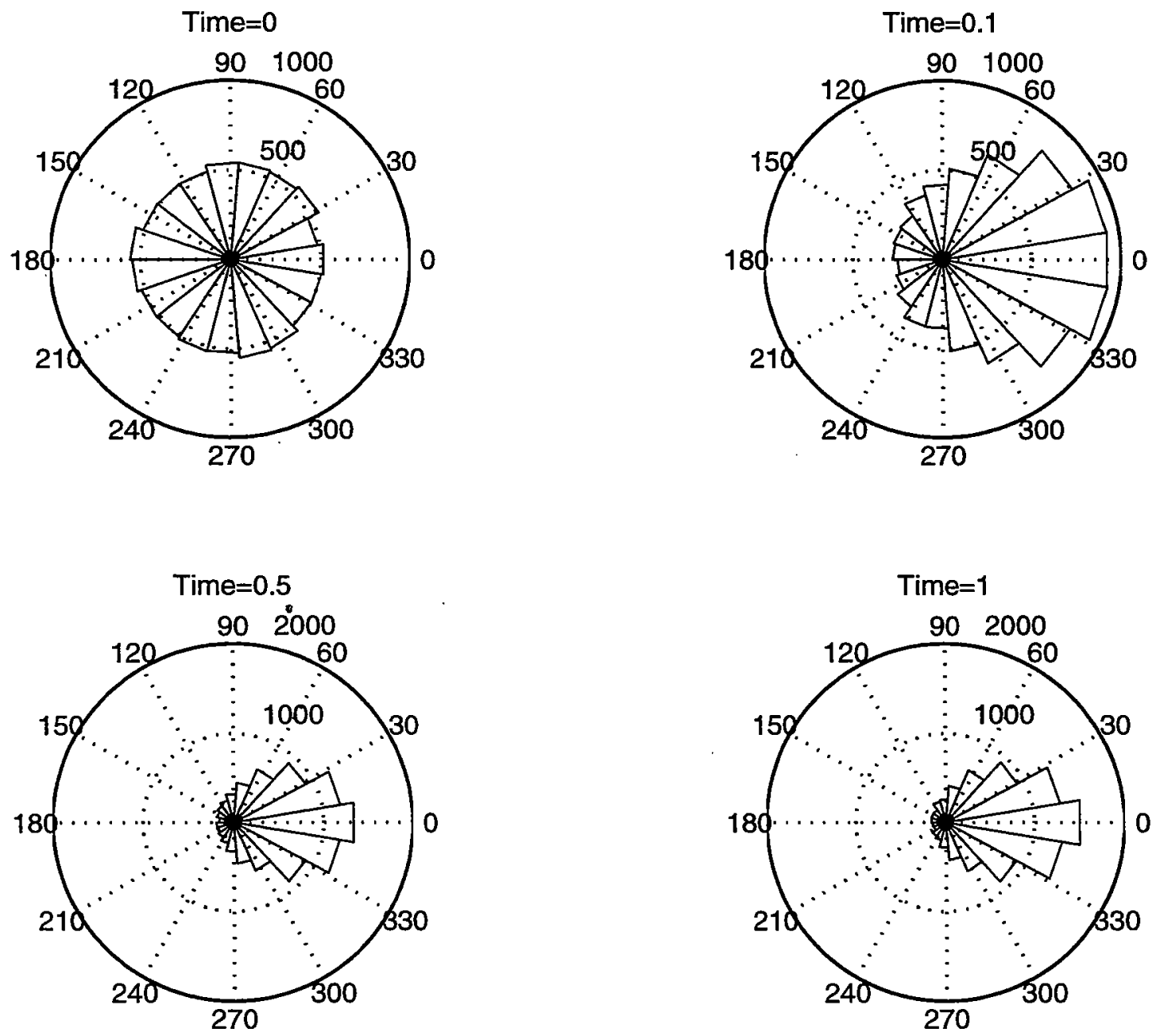

Figure 4: Histograms of the angles between the temperature and the salinity gradients at four different times in a simulations on a grid of $100 \times 100$ points. The initial conditions are created by picking uncorrelated temperature and salinity from a uniform probability density with variance $1 / 2$; thus the variance of $B=T-S$ is one. The thermohaline fields evolve according to the equations in (14) with $\gamma=1$ and $f(|\nabla B|)=1$. 


\section{Temperature-salinity relation at large scales explained with a forced nonlinear diffusion equation}

We showed how correlations develop between the thermohaline fields as a result of nonlinear diffusive parameterizations by solving initial value problems in which temperature and salinity are prepared as random and uncorrelated distributions. For typical ML thermohaline gradients these correlations are established on time scales of days for length scales of a kilometer and much slower for longer length scales. One then expects that horizontal density fluctuations on scales of a few kilometers disappear soon after they are created by forcing events like storms or wind bursts. This is consistent with the experimental evidence that typical thermohaline gradients on scales shorter than $10 \mathrm{~km}$ are compensated [3]. At scales of tens of kilometers nonlinear diffusion is not as efficient and it does not restore compensation between one forcing event and the next. The long term climatological $T-S$ relationship and the large scale density ratio of the $\mathrm{ML}$ are then the result of the competition between nonlinear diffusion, that dissipates horizontal density gradients, and thermohaline forcing, that continuously creates thermohaline anomalies.

The combined action of surface forcing and nonlinear diffusion on large scales in the ML is investigated with a model of the form given in (8) and (9). We restrict temperature and salinity to have spatial variations only in the meridional $y$-direction. A one dimensional model enables us to perform extensive Monte Carlo simulations and to obtain stable statistics of the thermohaline fields under the action of forcing. At the same time the one dimensional case capture the essential dynamics of thermohaline compensation; we verified that introducing a second dimension in simulations of the rundown problem does not change the dynamics that lead to the decay of density gradients. Only the process of thermohaline alignment cannot be captured in one dimension, but a characterization of the relative orientation of $T$ and $S$ gradients goes beyond the goal of this study. Here we limit our analysis to one dimensional statistics like the density ratio.

Numerical simulations of the nonlinear diffusion equations with forcing must be run for long times to achieve stable statistics. In order to save on CPU time, we limited our analysis of the equations in (8) and (9) to only one form of nonlinear diffusion. We chose the quadratic nonlinear diffusion that follows from a shear dispersion closure and set $f(|\nabla B|)=1$. Only a few results depend on this particular choice and we will point them out. In one dimension the equations for $T$ and $S$ reduce to

$$
\begin{aligned}
T_{t} & =\gamma\left(B_{y}^{2} T_{y}\right)_{y}-\alpha(T-\theta(y))+G(y, t) . \\
S_{t} & =\gamma\left(B_{y}^{2} S_{y}\right)_{y}+F(y, t)
\end{aligned}
$$

In the temperature equation (18), $\theta$ is the long time averaged atmospheric temperature determined by climatological data and $\alpha(T-\theta)$ represents the atmospheric feedback on the temperature of the ML: the larger $T-\theta$, the larger the heat flux from the atmospheric boundary layer into the ocean ML. $G(y, t)$ represents all other sources of heat such as daily and seasonal temperature variations. In the following numerical calculations $G(y, t)$ is set to zero under the assumption that the long term surface temperature is dominated by the climatological forcing rather than by the short term temperature fluctuations. The parameter 
$\alpha$ sets the time scale needed for the ocean ML to adjust to the atmospheric temperature $\theta$. When this time scale is small compared to the characteristic time scale of nonlinear diffusion, the equation for temperature reduces to

$$
T=\theta(y)
$$

The parameter $\alpha$ is determined by the time averaged heat flux from the atmosphere to the ocean and for a $100 \mathrm{~m}$ deep $\mathrm{ML}$ it is taken to be $10^{-7} \mathrm{~s}^{-1}$ [12]. For climatological gradients $\left(6{ }^{\circ} \mathrm{C}\right.$ over $\left.1000 \mathrm{~km}\right)$ the nonlinear diffusion term is three orders of magnitude smaller than the relaxation term and (20) is a very good approximation of the equilibrated temperature field. Numerical simulations will show that this is indeed the case.

In the salinity equation $(19), F(y, t)$ represents the combined action of salinity fluxes across the air-sea interface (evaporation and precipitation) and across the base of the ML (entrainment of thermocline waters). The forcing on salinity $F(y, t)$ is modeled in the form of localized sources and sinks randomly distributed in space and time. Each forcing event create a salinity anomaly of the order of $0.01 \mathrm{psu}$ in one day, equivalent to $3 \mathrm{~cm}$ of freshwater dumped in a $100 \mathrm{~m}$ deep ML or equivalently to an entrainment of $2 \mathrm{~m}$ of water with a salinity larger/smaller of $0.5 \mathrm{psu}$. We adjust the size and frequency of the forcing events so that we obtain the equivalent of a precipitation of $1 \mathrm{~m}$ per year, a value typical at midlatitudes in the North Pacific and in the North Atlantic [13], [14]. In order to conserve salinity the integral of the forcing on salinity must have zero average over long times,

$$
\lim _{T \rightarrow \infty} \int_{0}^{T} \mathrm{~d} t \int_{0}^{L} \mathrm{~d} y F(y, t)=0 .
$$

We impose this constraint by prescribing $F(y, t)$ as the sum of five positive and five negative Gaussians, all of the same amplitude, so that their sum is identically zero at all times. The positions of the Gaussians within the $L=1000 \mathrm{~km}$ of the domain is sampled from a uniform probability distribution and is changed every day. This ensures that the long time average forcing is zero at each point, even though several Gaussians can hit in the same place over a short period of time and produce strong local salinity and buoyancy gradients.

The key element of the problem set in (18) and (19) is that there is an asymmetry in the large scale forcing of temperature and salinity. Temperature is relaxed to some atmospheric climatological mean, while salinity is forced by random rainfall, evaporation and entrainment. The idea that asymmetries in the thermohaline forcing together with nonlinear parameterizations of eddy fluxes produce the observed large scale mean density ratio has been raised by Stommel [4]. Stommel tested this hypothesis with an idealized two-box model of the ML, but did not attempt any comparison with observations. Here we explore the process in the context of a continuous model of the $\mathrm{ML}$ and we quantify its effect for realistic oceanographic parameters.

\subsection{Numerical algorithm}

For the simulations we use dimensionless variables. Temperature and salinity gradients are measured in terms of of the climatological temperature gradient $\left(6^{\circ} \mathrm{C}\right.$ over $\left.1000 \mathrm{~km}\right)$, lengths 
in terms of the domain length $(1000 \mathrm{~km})$ and the time in terms of the time it takes to mix tracers across the domain (3000 years).

The numerical calculations are performed by integrating the non dimensional equation for the temperature and salinity gradients, obtained by differentiating (18) and (19) in $y$,

$$
\begin{aligned}
& \left(T_{y}\right)_{t}=\left(B_{y}^{2} T_{y}\right)_{y y}-\alpha\left(T_{y}-\theta_{y}\right)-\mu\left(T_{y}\right)_{y y y y} \\
& \left(S_{y}\right)_{t}=\left(B_{y}^{2} S_{y}\right)_{y y}+F_{y}-\mu\left(S_{y}\right)_{y y y y}
\end{aligned}
$$

The hyperviscosity is introduced to dissipate small scale gradients and to allow longer time stepping in the simulations. In this section we are interested in the effect of nonlinear diffusion on large scales and we do not need to resolve small scale structures. We use a grid spacing of $1 \mathrm{~km}$ and choose a value for $\mu$ that filters out all fluctuations with length scales shorter than $3 \mathrm{~km}$. Global conservation of heat and salt is satisfied by requiring that $T_{y}=T_{y y y}=0$ and $S_{y}=S_{y y y}=0$ at both ends of the domain (i.e., no-flux boundary conditions). We solve the system in (22) and (23) on a discrete spatial grid with an explicit Euler forward scheme in time and a dealiased sine spectral code in space. The time step is set by the decorrelation time of the stochastic forcing acting on salinity, chosen to be one day.

\subsection{The effect of stochastic forcing on salinity}

The vision of the large scale $T-S$ relation in the ML is that the enormously variable forcing creates salinity anomalies that are eliminated by nonlinear diffusion. The combination of strong forcing on salinity and nonlinear eddy fluxes holds the average density ratio to a constant value. It is instructive to study the decay of a localized salinity anomaly to understand how the $T-S$ relationship is established.

Let us study the evolution of $T$ and $S$ after the forcing has created a large scale salinity anomaly. We prescribe a linear climatological gradient $\left(\theta_{y}=1\right)$ and we assume that the relaxation time scale is so fast (large $\alpha$ limit) that temperature is always in equilibrium $\left(T_{y}=1\right)$. In this limit the nonlinear diffusion equation for salinity reduces to,

$$
S_{t}=\left[\left(S_{y}-1\right)^{2} S_{y}\right]_{y}-\mu\left(S_{y}\right)_{y y y y}
$$

We take the initial salinity profile to have the same width and shape of the Gaussian salinity anomalies created by $F$ in (19). Figure 5 shows the profiles of $S$ and $S_{y}$ at time zero and at some later time. Salinity is diffused faster where the initial profile has a negative slope than where it has a positive slope. This asymmetry is easily explained if we consider that for a gradient of given magnitude $\left|S_{y}\right|$ the nonlinear diffusivity $\left(S_{y}-1\right)^{2}$ is larger where $S_{y}$ is negative than when $S_{y}$ is positive. A look at the profiles of salinity gradients confirms that $S_{y}$ diffuses at a slower rate when it has a value close to 1 . As expected, in the absence of forcing, salinity decays to the only stable solution $S_{y}=0$ (the solution $S_{y}=1$ is only semistable), but regions where the salinity gradient is close to one decay slowly. We speculate that averaging the solution over many independent forcing events will reflect this asymmetry and produce a positive average salinity gradient $\left\langle S_{y}\right\rangle$; i.e., the presence of a climatological gradient $\theta_{y}=1$ breaks the symmetry in the decay of the salinity gradients and determines a finite mean density ratio $R=1 /\left\langle S_{y}\right\rangle$. 


$$
S_{t}=\partial_{y}\left(\left|\partial_{y} S-1\right|^{2} \partial_{y} S\right)
$$
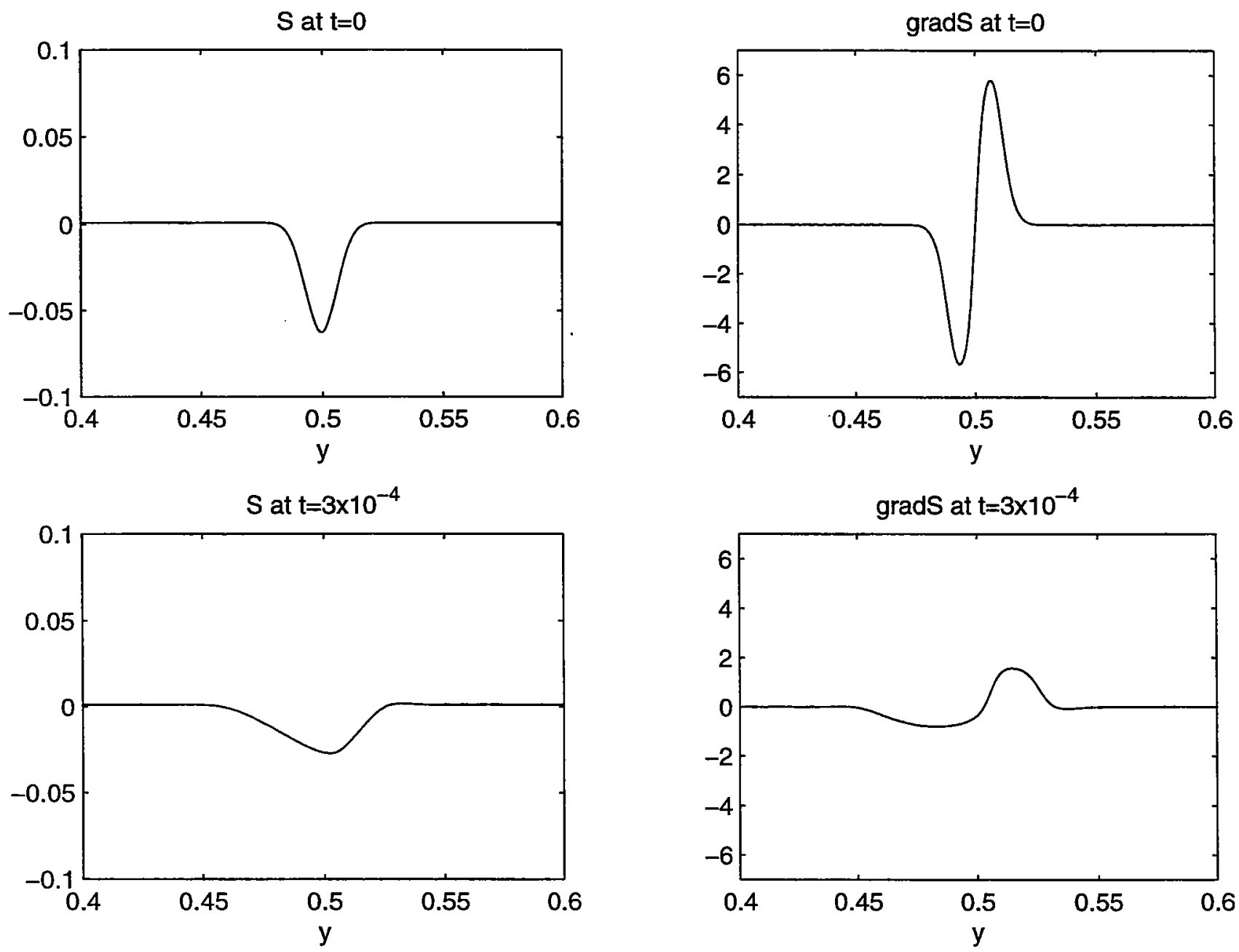

Figure 5: Evolution of a Gaussian salinity anomaly subject to the nonlinear diffusion equation in (24). The upper panels show the initial salinity and salinity gradient and the lower panels the same variables at a later time. Only the central part of the integration domain is shown. 
Some general properties of the evolution of the salinity gradient are better revealed by writing equation (23) in variational form,

$$
\left(S_{y}\right)_{t}=\partial_{y}^{2}\left[\frac{\delta \Phi}{\delta S_{y}}\right]+F_{y}
$$

where $\Phi\left(S_{y}\right)$ is the functional

$$
\Phi\left(S_{y}\right) \equiv \int_{0}^{1}\left[H\left(S_{y}, y\right)+\frac{1}{2} \mu\left(S_{y y}\right)^{2}\right] \mathrm{d} y
$$

and $H\left(S_{y}, y\right)$ is the function

$$
H\left(S_{y}, y\right)=\frac{1}{4} S_{y}^{4}-\frac{2}{3} \theta_{y} S_{y}^{3}+\frac{1}{2} \theta_{y}^{2} S_{y}^{2}
$$

In (25) we introduced the variational derivative of the functional $\Phi\left(S_{y}\right)$,

$$
\frac{\delta \Phi}{\delta S_{y}}=H_{S_{y}}-\mu S_{y y}
$$

In the absence of forcing, the form (25) can be used to show that the system has only one stable equilibrium which minimizes the functional $\Phi\left(S_{y}\right)$, i.e., $S_{y}=0$ (figure 27). Furthermore the shape of the potential is such that the decay towards equilibrium is slower around the saddle line $S_{y}=\theta_{y}$. We then expect that, even though the random forcing creates positive and negative $S_{y}$ anomalies with the same probability, salinity gradients with values close to zero and $\theta_{y}$ are more persistent and the long time averaged salinity gradient at each point settles to some value between zero and $\theta_{y}$.

\subsection{Numerical simulations}

We begin our numerical experiments of the system in (22) and (23) by relaxing temperature towards a linear climatological gradient; i.e. we set $\theta_{y}=1$ in (22). The initial temperature and salinity gradients are chosen to be zero. The model is run continuously for 3200 years.

During the first stages of the simulation the thermohaline fields evolve from the initial conditions towards a statistically steady state. Temperature relaxes to the climatological mean in a few years. After that fluctuations from the equilibrium solution $T(y, t)=\theta(y)$ are negligible. The spin up time for salinity is approximately 200 years. In the following all statistics are computed discarding this initial spin up time.

Figure 7 shows the mean thermohaline fields averaged over 3000 years. In the upper left panel, $T$ and $S$ profiles are plotted as a function of latitude. Salinity develops a large scale structure, even though the random forcing is uniformly distributed in the domain. The long time average profile of salinity is, to a good approximation, $S=(2 / 3) y$. The analog of the oceanographic mean horizontal density ratio for this model is $R=\overline{T_{y}} / \overline{S_{y}}=3 / 2$, where the overbars denote the temporal averaging. A mean density ratio of 1.5 on a scale of $1000 \mathrm{~km}$, the size of our domain, is consistent with the observational results of Stommel and Chen [4], [5]. Notice though that our averages are carried over 3000 years, while both Stommel and 


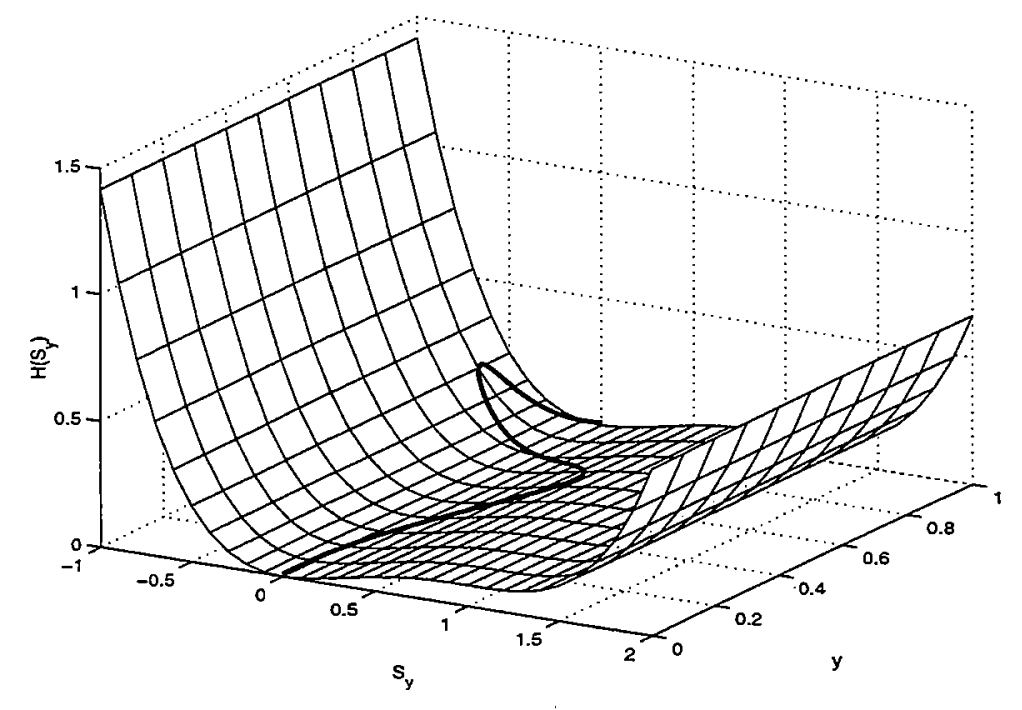

Figure 6: The function $H\left(S_{y}\right)$ in the case of a linear climatological temperature gradient, i.e., $\theta_{y}=1$. The dark line is a possible $S_{y}$ profile.

Chen used climatologies averaged over less than 20 years. We return to this point at the end of the section.

Simple arguments based on a Reynold's decomposition of the thermohaline fields are now used to interpret the density ratio of $3 / 2$ obtained in the numerical simulations. Let us decompose $S$ and $T$ as the sum of a temporal mean plus fluctuations about that mean; i.e., $S=\bar{S}+S^{\prime}$ and $T=\bar{T}+T^{\prime}$. Numerical experiments show that we can safely neglect $T^{\prime}$ in the Reynold's decomposition of $T$ and write $T=\bar{T}=\theta$. Taking the time average of the nonlinear diffusion equation for salinity then gives,

$$
\overline{S_{t}}=\left(\overline{S_{y}^{\prime 3}}+\overline{S_{y}^{\prime 2}}\left(3 \overline{S_{y}}-2 \theta_{y}\right)+\overline{S_{y}}\left(\overline{S_{y}}-\theta_{y}\right)^{2}\right)_{y}+\bar{F}
$$

Both $\bar{F}$ and $\overline{S_{t}}$ vanish if the average is carried over a long enough time. The sum of the first three terms in the RHS of (29) must then vanish as well. Notice that this sum cannot be a constant different from zero, because of the no-flux boundary conditions. Furthermore, the third term, $\overline{S_{y}}\left(\overline{S_{y}}-\theta_{y}\right)^{2}$, is likely to be smaller than the first two, because the typical amplitude of the fluctuations created by the forcing is larger than the mean gradients; numerical results show that this is indeed the case (last panel of figure 7). Therefore the leading order balance involves only the first two terms. Setting to zero $\overline{S_{y}^{\prime 3}}+\overline{S_{y}^{\prime 2}}\left(3 \overline{S_{y}}-2 \theta_{y}\right)$ gives an estimate of the mean salinity gradient,

$$
\overline{S_{y}}=\frac{2}{3}-\frac{\overline{S_{y}^{\prime 3}}}{\overline{S_{y}^{\prime 2}}} .
$$

The numerical simulations show that $\overline{S_{y}^{\prime 2}}$ settles to a constant profile different from zero, while $\overline{S_{y}^{\prime} 3}$ keeps decreasing throughout the domain. This explains why $\overline{S_{y}}$ asymptotes $2 / 3$ when 
averaged over long times. Finally we should remark that we did not include the hyperviscosity term in this analysis, because its contribution in the long term balance is maintained small by choosing an appropriate value for $\mu$.

The Reynold's decomposition analysis of the equations in (22) and (23) shows that a large scale mean salinity gradient is required if all nonlinear fluxes are to balance when averaged over long times. This balance depends on the particular form of nonlinear diffusion chosen. That is nonlinear closures different from $f(|\nabla B|)=1$ in (8) and (9) would still produce a large scale salinity gradient, but its dependence on $\theta_{y}$ would not be that given in (30). Stommel came to the same conclusion for his two box model [4]. He obtained that, for a diffusivity between the two boxes of the form $\left|B_{y}\right|^{n}$, the salinity gradient becomes linearly proportional to the climatological temperature gradient and the mean density ratio approaches $(n+1) / n$. We verified that Stommel argument applies also in the continuous limit for the diffusivity $B_{y}^{2}$.

So far we showed thermohaline profiles averaged over 3000 years. Averaging over shorter times gives results that are not in statistical equilibrium. Results averaged over two different subintervals of 30 years from the same simulation shown in figure 7 give a density ratio close to $3 / 2$ in one case (figure 8 ) and 1 in the second case (figure 9 ). The differences arise because over 30 years the leading order balance in (29) involves both $\overline{S_{t}}$ and $\bar{F}$. It is necessary to average over longer times to include a number of forcing events large enough to obtain stationary statistics. Over a 3000 year time span, each point on the grid is hit on average by $10^{6}$ forcing events, while over a 30 year time span this number is one hundred times smaller and as a consequence the average of the forcing is 10 times larger: it is the number of independent forcing events that sets the time required to have stable statistics. In this sense our results are consistent with observations, because in their analysis Stommel and Chen compute averages over a wide range of longitudes in space so that they include many realizations of forcing events per unit time.

\section{Discussion}

The main result of this project is that nonlinear diffusive parameterizations of the ML, which use buoyancy as the driving field, can explain the different values of density ratio observed in oceanographic measurements. At scales shorter than $10 \mathrm{~km}$ the nonlinear fluxes are so strong that a horizontal density ratio of 1 is restored in a few days whenever a forcing event creates an anomaly in $T$ and $S$. At larger scales the correlations between temperature and salinity emerge as a balance between nonlinear diffusion and thermohaline forcing. The large scale forcing on temperature is modeled as a relaxation of temperature towards a climatological mean profile to account for the atmospheric feedbacks on thermal anomalies. There is no reason to include an analogous term in the equation for salinity and the forcing on salinity is purely stochastic. The difference in the forcings breaks the symmetry between $T$ and $S$. The large scale temperature gradient relaxes in a few years towards the climatological mean, while salinity develops a large scale gradient that on average is proportional to that of temperature even though the forcing on salinity is uniformly distributed over the domain. The ratio of the large scale mean gradients of $T$ and $S$ is close to $3 / 2$ for the specific nonlinear diffusion parameterization used in the simulations. This value is consistent with observations. 

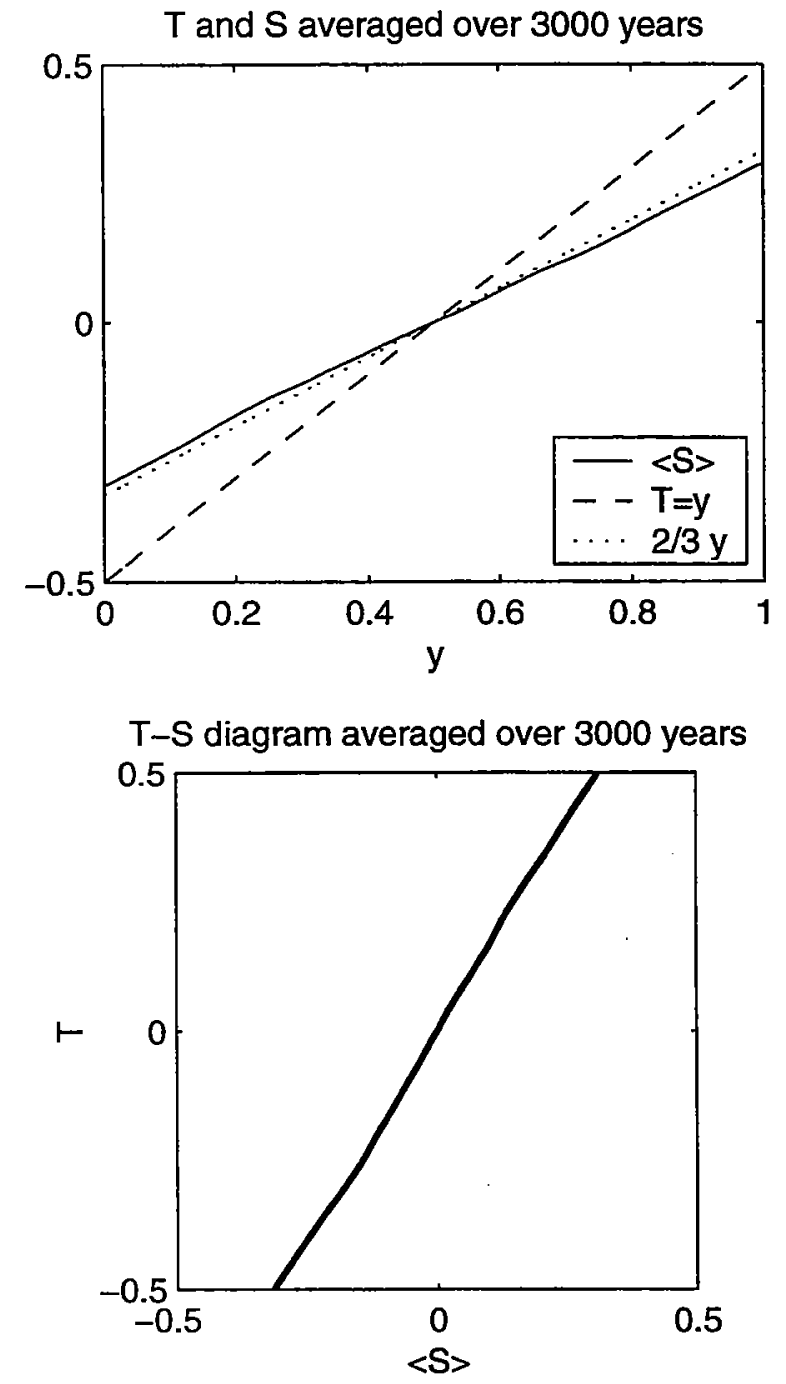

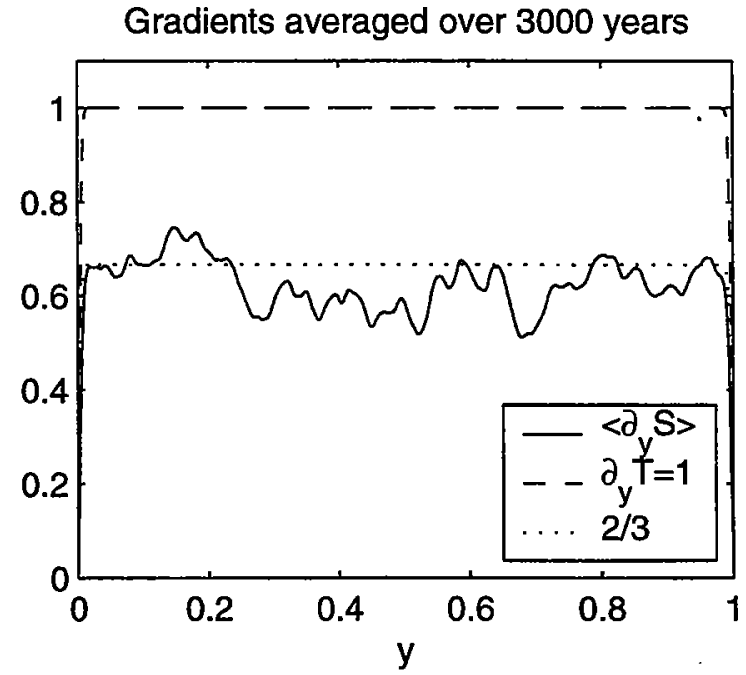

Reynolds decomposition of $\partial_{y}\left(\left|\partial_{y} S-1\right|^{2} \partial_{y} S\right)$

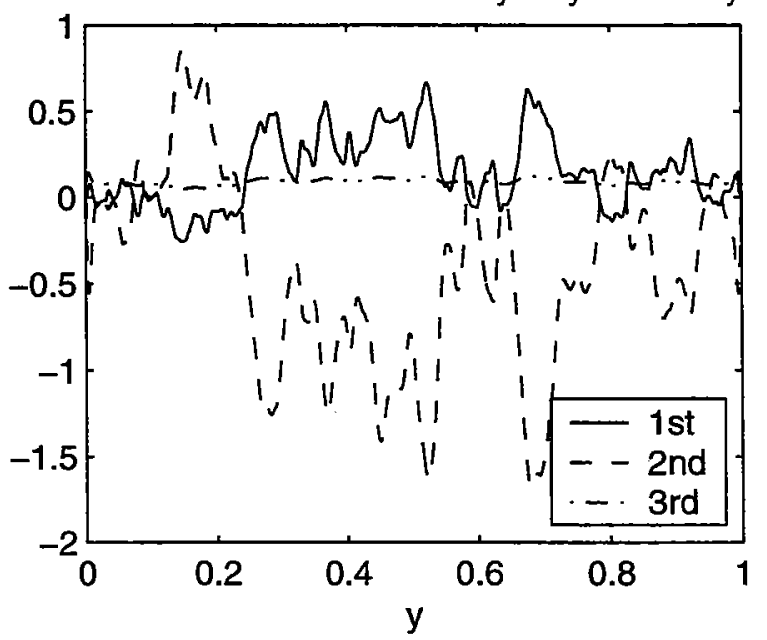

Figure 7: Results of a numerical simulation of the system in (22) and (23) with $\theta=y$ and 1000 grid points. The upper panels show the $T, S, T_{y}$ and $S_{y}$ profiles as a function of latitude $y$, averaged over 3000 years. The average salinity compensates $2 / 3$ of the climatological temperature gradient. The lower left panel is the $T-S$ diagram of the averaged $T$ and $S$ profiles. The lower left panel is a plot of the first three terms in the RHS of (30); $\overline{S_{y}^{\prime 3}}$ is the continuous line, $\overline{S_{y}^{\prime 2}}\left(3 \overline{S_{y}}-2 \theta_{y}\right)$ is the dashed line and $\overline{S_{y}}\left(\overline{S_{y}}-\theta_{y}\right)^{2}$ is the dashdotted line. 

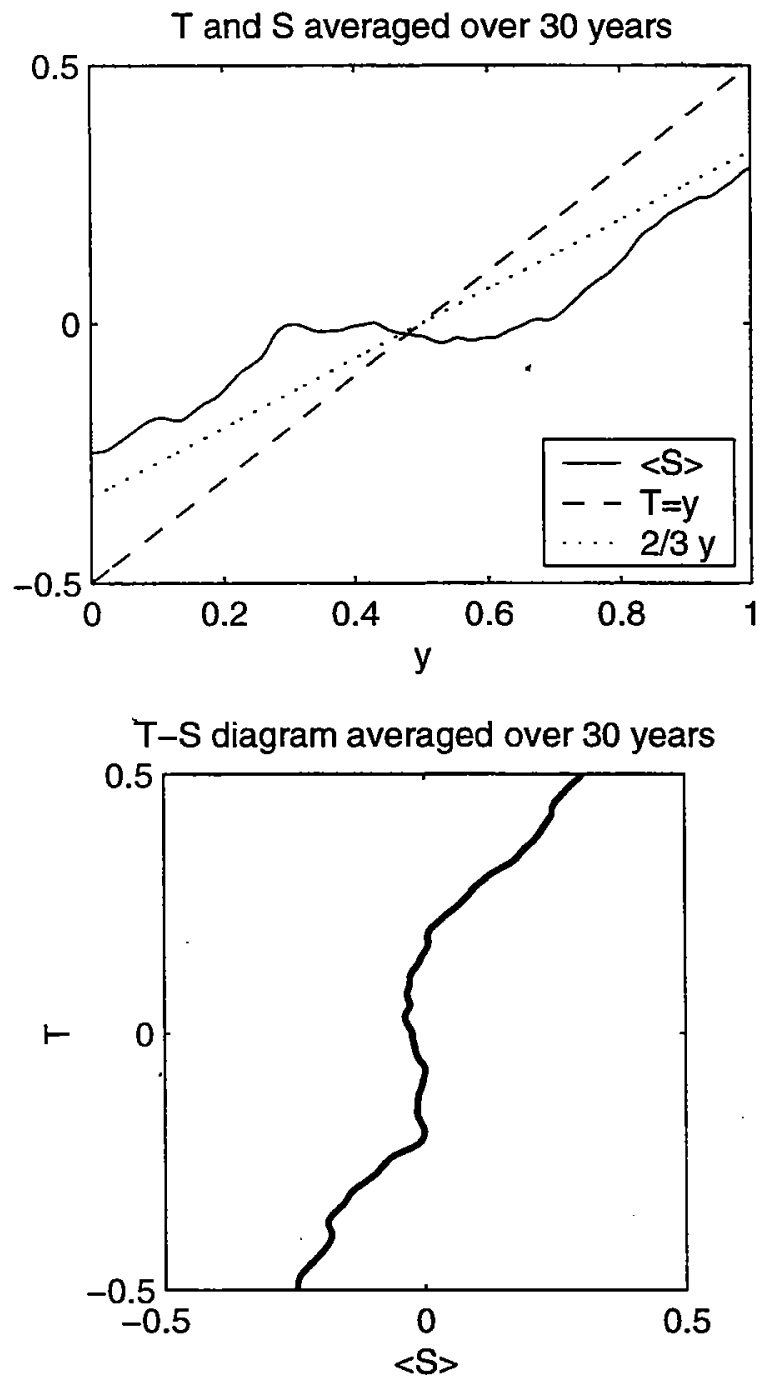

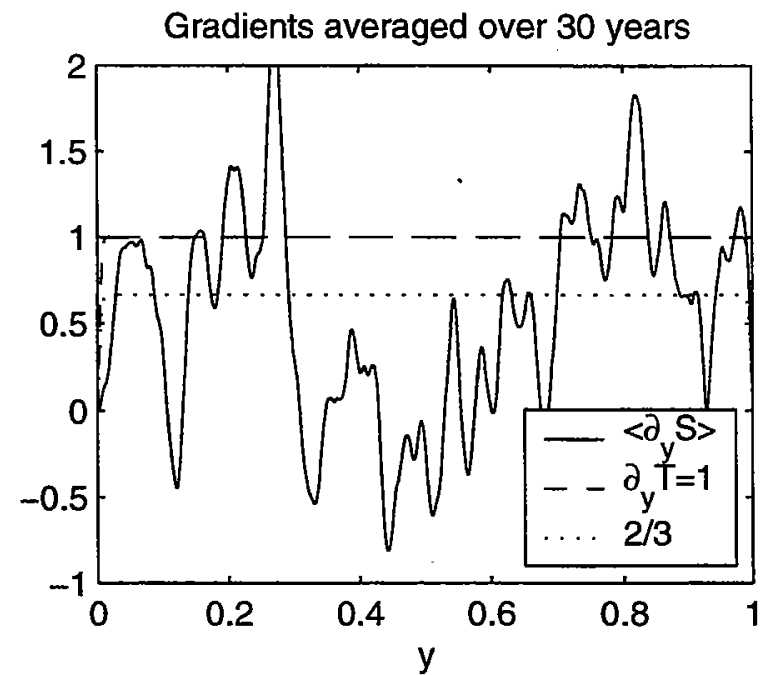

Reynolds decomposition of $\partial_{y}\left(\left|\partial_{y} S-1\right|^{2} \partial_{y} S\right)$

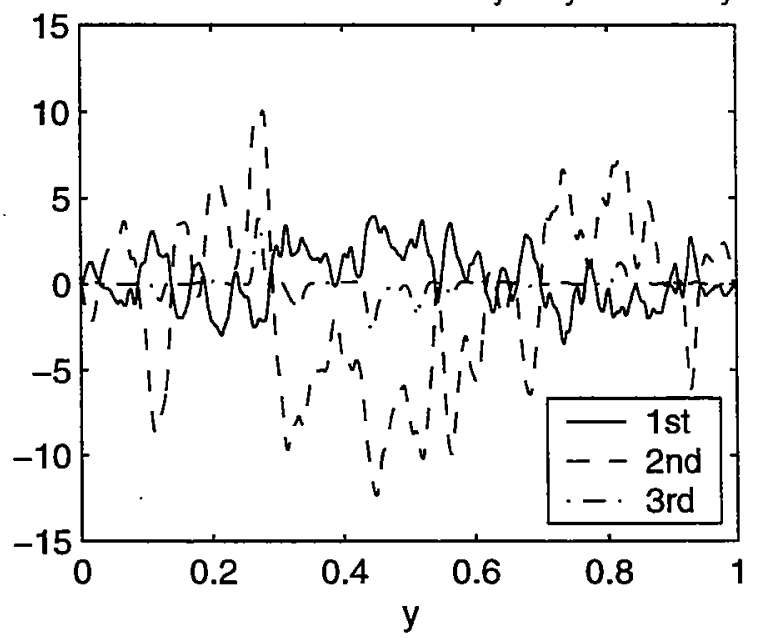

Figure 8: Results from the same simulation shown in figure 7 averaged over a subinterval of 30 years. 

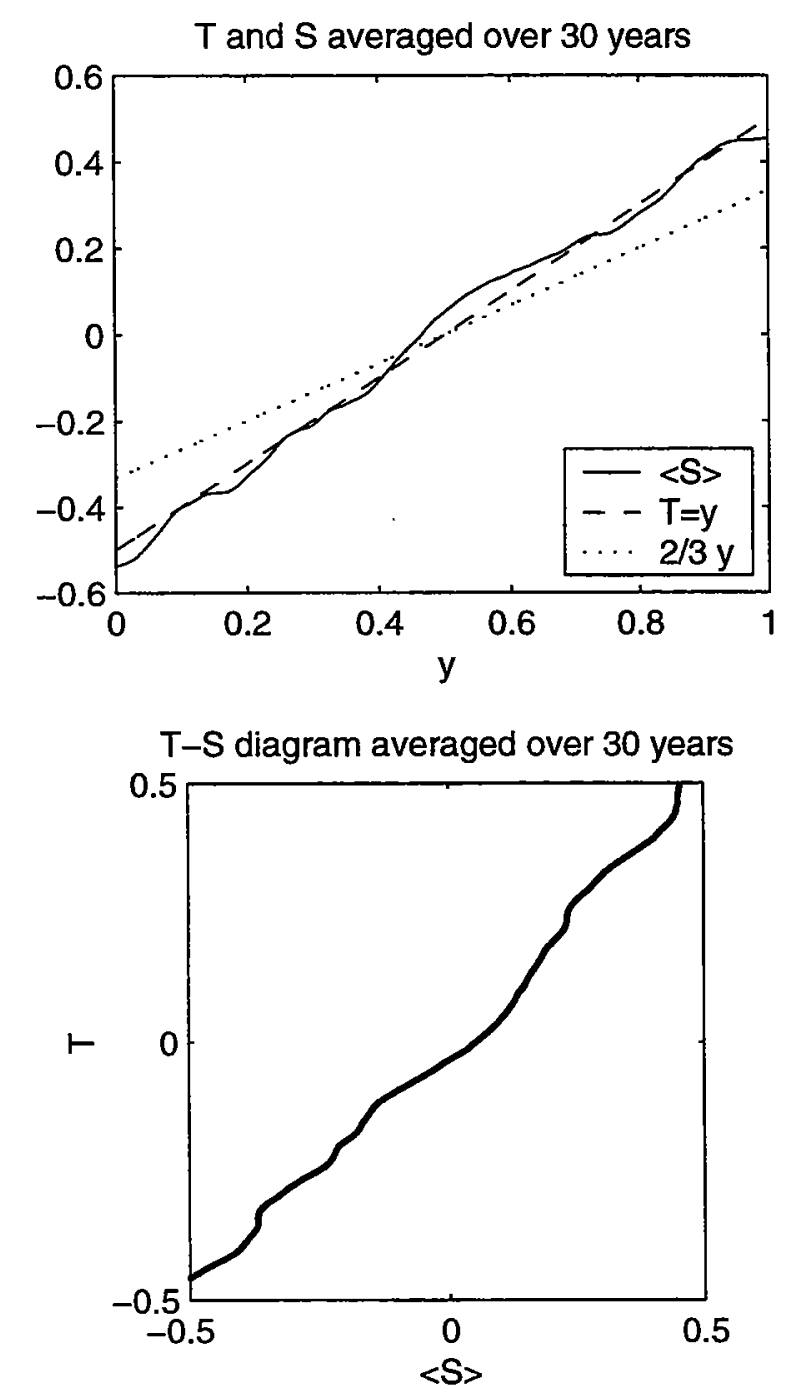

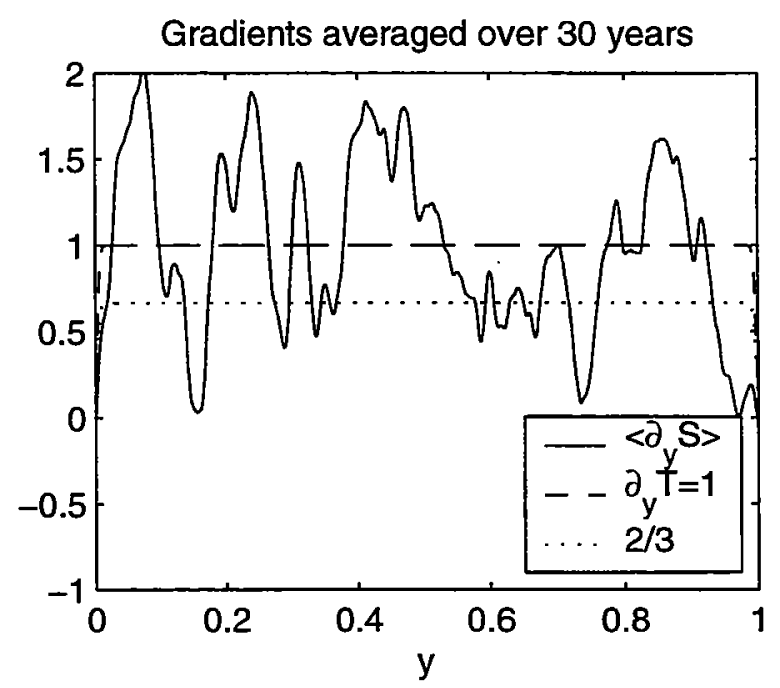

Reynolds decomposition of $\partial_{y}\left(\left|\partial_{y} S-1\right|^{2} \partial_{y} S\right)$

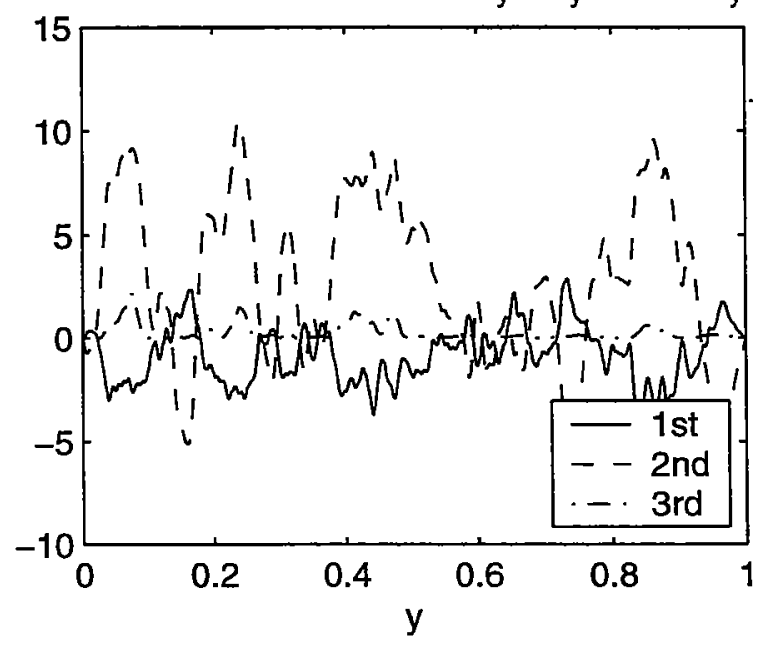

Figure 9: Results from the same simulation shown in figure 7 averaged over a subinterval of 30 years. 
In order to show both a density ratio of 1 at small scales and a density ratio of 1.5 at large scales, we are running numerical simulations of the equations in (18) and (19) with stochastic noise on temperature and salinity; that is $G(y, t)$ and $F(y, t)$ have a white spectrum in space and time. Preliminary results show that compensation develops over a few grid points and produce a small scale density ratio of 1 , while at large scales salinity compensates only part of the temperature gradient and the density ratio settles to 1.5 .

In our model the instantaneous distribution of buoyancy gradients at large scale is determined by the most recent forcing events. On the other hand observations in the ML of all oceans show that buoyancy fronts tend to concentrate in regions of Eckman convergence. The analysis of nonlinear diffusion models with a large scale advection that has regions of convergence is a direction for future research. The hypothesis is that the stochastic forcing creates buoyancy anomalies uniformly throughout the domain and the advective field collects them towards regions of convergence. The result is that the average density ratio is still $3 / 2$, but it is all due to a few localized gradients.

Finally, it would be instructive to implement nonlinear diffusive parameterizations of the thermohaline eddy fluxes in large scale ocean models. It is well known that ocean models tend to produce unrealistic distributions of salinity in the ML, because there is no feedback mechanism that maintains large scale salinity anomalies within reasonable bounds. Typically the cure is to introduce some ad hoc relaxation to observations. Nonlinear diffusive parameterizations obtain the same result by introducing an indirect feedback through the climatological temperature gradient. Furthermore the parameterizations we have discussed in this project are the results of closures based on sound physical arguments and are not dictated by numerical necessity. An obvious goal is to test the different nonlinear parameterizations against the observed density ratio to infer which are more appropriate.

\section{Acknowledgments}

I am gratefful to all the staff and fellows of the GFD Summer Program for a most enjoyable and instructive summer. I am especially thankful to Joe Keller and Eric Chassignet for many enlightening conversations, Paola Cessi for her invaluable help with the numerics and Bill Young for numerous suggestions in some critical moments. Thanks to Ed Spiegel, George Veronis, Jean-Luc Thiffeault, Francesco Paparella and our director Neil Balmforth for all their help and encouragement.

\section{References}

[1] G. I. Roden, "The vertical thermohaline structure in the argentine basin," J. Geophys. Res. 94, 877 (1989).

[2] D. L. Rudnick and J. R. Luyten, "Intensive survey of the azores front. 1. tracers and dynamics," J. Phys. Ocean. 101, 923 (1996).

[3] D. L. Rudnick and R. Ferrari, "Compensation of horizontal temperature and salinity gradients in the ocean mixed layer," Science 283, 526 (1999). 
[4] H. M. Stommel, "A conjectural mechanism for determining the thermohaline structure of the oceanic mixed layer," J. Phys. Ocean. 23, 142 (1993).

[5] L. G. Chen, "Mixed layer density ratio from the levitus data," J. Phys. Ocean. 25, 691 (1995).

[6] H. M. Stommel, "Theromhaline convection with two stable regimes of flow," Tellus 13, 224 (1961).

[7] H. M. Stommel and W. R. Young, "The average t-s relation of a stochastically forced box model," J. Phys. Ocean. 23, 151 (1993).

[8] R. Ferrari and W. R. Young, "On the development of theromhaline correlations as a result of nonlinear diffusive parameterizations," J. Mar. Res. 55, 1069 (1997).

[9] J. A. Green, "Transfer properties of the large-scale eddies and the general circulation of the atmosphere," Quart. J. Roy. Meteorol. Soc. 96, 157 (1970).

[10] P. H. Stone, "A simplified radiative-dynamical model for the static stability of rotating atmosphere," J. Atmos. Sci. 29, 405 (1972).

[11] V. Pavan and I. M. Held, "The diffusive approximation for eddy fluxes in baroclinically unstable jets," J. Atmos. Sci. 53, 1262 (1996).

[12] R. L. Haney, "Surface thermal boundary conditions for ocean circulation models," J. Phys. Ocean. 1, 241 (1971).

[13] R. K. Reed and W. P. Elliott, "New precipitation maps for the north atlantic and north pacific oceans," J. Geophys. Res. 84, 7839 (1979).

[14] P. S. B. R. W. Schmitt and C. E. Dorman, "Evaporation minus precipitation and density fluxes for the north atlantic," J. Phys. Ocean. 19, 1208 (1989). 


\section{DOCUMENT LIBRARY}

\section{Distribution List for Technical Report Exchange - July 1998}

University of California, San Diego

SIO Library 0175C

9500 Gilman Drive

La Jolla, CA 92093-0175

Hancock Library of Biology \& Oceanography

Alan Hancock Laboratory

University of Southern California

University Park

Los Angeles, CA 90089-0371

Gifts \& Exchanges

Library

Bedford Institute of Oceanography

P.O. Box 1006

Dartmouth, NS, B2Y 4A2, CANADA

NOAA/EDIS Miami Library Center

4301 Rickenbacker Causeway

Miami, FL 33149

Research Library

U.S. Army Corps of Engineers

Waterways Experiment Station

3909 Halls Ferry Road

Vicksburg, MS 39180-6199

Marine Resources Information Center

Building E38-320

MIT

Cambridge, MA 02139

Library

Lamont-Doherty Geological Observatory

Columbia University

Palisades, NY 10964

Library

Serials Department

Oregon State University

Corvallis, OR 97331

Pell Marine Science Library

University of Rhode Island

Narragansett Bay Campus

Narragansett, RI 02882

Working Collection

Texas A\&M University

Dept. of Oceanography

College Station, TX 77843
Fisheries-Oceanography Library 151 Oceanography Teaching Bldg. University of Washington

Seattle, WA 98195

Library

R.S.M.A.S.

University of Miami

4600 Rickenbacker Causeway

Miami, FL 33149

Maury Oceanographic Library

Naval Oceanographic Office

Building 1003 South

1002 Balch Blvd.

Stennis Space Center, MS, 39522-5001

Library

Institute of Ocean Sciences

P.O. Box 6000

Sidney, B.C. V8L 4B2

CANADA

National Oceanographic Library

Southampton Oceanography Centre

European Way

Southampton SO14 3ZH

UK

The Librarian

CSIRO Marine Laboratories

G.P.O. Box 1538

Hobart, Tasmania

AUSTRALIA 7001

Library

Proudman Oceanographic Laboratory

Bidston Observatory

Birkenhead

Merseyside L43 7 RA

UNITED KINGDOM

IFREMER

Centre de Brest

Service Documentation - Publications

BP 7029280 PLOUZANE

FRANCE 



\begin{tabular}{|c|c|c|}
\hline $\begin{array}{l}\text { REPORT DOCUMENTATION } \\
\text { PAGE }\end{array}$ & $\begin{array}{l}\text { 1. REPORT NO. } \\
\text { WHOI-2000-07 }\end{array}$ & 3. Recipient's Accession No. \\
\hline \multirow{2}{*}{\multicolumn{2}{|c|}{$\begin{array}{l}\text { 4. Title and Subtitle } \\
\text { Stirring and Mixing: } 1999 \text { Program of Summer Study in Geophysical Fluid } \\
\text { Dynamics }\end{array}$}} & $\begin{array}{l}\text { 5. Report Date } \\
\text { July } 2000\end{array}$ \\
\hline & & 6. \\
\hline \multicolumn{2}{|c|}{ 7. Author(s) Neil J. Balmforth, Director } & $\begin{array}{l}\text { 8. Performing Organization Rept. No. } \\
\text { WHOI-2000-07 }\end{array}$ \\
\hline \multirow{2}{*}{\multicolumn{2}{|c|}{$\begin{array}{l}\text { 9. Pertorming Organization Name and Address } \\
\text { Woods Hole Oceanographic Institution } \\
\text { Woods Hole, Massachusetts } 02543\end{array}$}} & 10. Project/Task/Work Unit No. \\
\hline & & $\begin{array}{l}\text { 11. Contract(C) or Grant(G) No. } \\
\text { (C) OCE-9810647 } \\
\text { (G) NO0014-97-1-0934 }\end{array}$ \\
\hline \multirow{2}{*}{\multicolumn{2}{|c|}{$\begin{array}{l}\text { 12. Sponsoring Organization Name and Address } \\
\text { National Science Foundation } \\
\text { Office of Naval Research }\end{array}$}} & $\begin{array}{l}\text { 13. Type of Report \& Period Covered } \\
\text { Technical Report }\end{array}$ \\
\hline & & 14. \\
\hline
\end{tabular}

15. Supplementary Notes

This report should be cited as: Woods Hole Oceanog. Inst. Tech. Rept., WHOI-2000-07.

16. Abstract (Limit: 200 words)

The central theme of the 1999 GFD Program was the stirring, transport, reaction and mixing of passive and active tracers in turbulent, stratified, rotating fluids. The problem of mixing in fluids has applications in areas ranging from oceanography to engineering and astrophysics. In geophysical settings, mixing spans and unites a broad range of scales -- from micrometers to megameters. The mixing of passive tracers is of fundamental importance in environmental and industrial problems, such as pollution, and in determining the large-scale heat and salt balance of the worlds oceans. The transport of active tracers, on the other hand, such as vorticity, plays a key role in the turbulence that occurs in most geophysical and astrophysical fluids. William $\mathrm{R}$. Young (Scripps Institution of Oceanography) gave a series of principal lectures, the notes of which as taken by the fellows, appear in this volume. Reports of the projects of the student fellows makes up the second half of this volume.

17. Document Analysis a. Descriptors

Fluids

Mixing

Transport

b. Identifiers/Open-Ended Terms

c. COSATI Field/Group

18. Availability Statement

Approved for public release; distribution unlimited.

\begin{tabular}{|c|}
\hline $\begin{array}{c}\text { 19. Security Class (This Report) } \\
\text { UNCLASSIFIED }\end{array}$ \\
\hline 20. Security Class (This Page) \\
\hline
\end{tabular}

21. No. of Pages

22. Price 
